\title{
ABSTRACTS
}

\section{Accepted for \\ POSTER PRESENTATIONS}

60th Annual Cancer Symposium

Society of Surgical Oncology

March 15-18, 2007

Washington, D.C. 


\section{P1}

Down-regulation of Sphingosine 1-Phosphate receptor-1 in intestinal tumorigenesis S. Zani, ${ }^{*}$ M. Momoi, J. Paik, T. Hla, W.A. Frederick. University of Connecticut Health Center, Farmington, CT.

Introduction: The bioactive sphingolipid, Sphingosine 1-Phosphate (S1P) is implicated in the regulation of cellular proliferation, migration, and survival via its $G$ protein-coupled receptors S1P1-5. While animal models of intestinal neoplasia have demonstrated a beneficial effect of dietary sphingolipids in chemoprevention, the role of S1P in colon cancer is still unclear. The purpose of this study was to help define the role of the S1P receptor-ligand system in colon cancer. Methods: Small intestine specimens of bigenic S1P1+/-Apcmin and S1P1+/+Apcmin mice were compared to determine effect of S1P1 heterozygosity on polyp number. Growth inhibition of RIE-1 cells was assessed using enforced expression of S1P1 receptor by adenoviral vector followed by treatment with S1P. Matched human normal and cancer colon tissue were obtained from surgical specimens. Differential expression of S1P1 between the tissues was evaluated utilizing western blot analysis and immunohistochemistry. Results: Bigenic S1P1+/-Apcmin mice revealed a $27 \%$ increase in polyp number when compared to control mice. Induced expression of S1P1 in RIE-1 cells caused growth inhibition with treatment of S1P. Western blot analysis and immunohistochemistry revealed an increased expression of S1P1 in the human normal tissue as compared with tumor tissue. Conclusions: Our results suggest that S1P1 receptor functions in the intestinal epithelium to inhibit tumorigenesis. Down-regulation of S1P1 in colorectal cancer may have functional consequences in the proliferation and or metastatic spread of cancer. Further evaluation of Sphingosine 1-phosphate receptor- 1 is necessary to determine its potential for therapeutic intervention in colon cancer.

\section{P2}

Targeting the Tumor Microenviroment to Treat Human Ocular Melanoma in Nude Mice Using the Tyrosine Kinase Inhibitors (TKIs) Sunitinib or Sorafenib alone and in Combination with Metronomic Doses of Cyclophosphamide (CTX) J.A. Blansfield, * H.R. Alexander, III, S. Morita, S.K. Libutti. Tumor Angiogenesis Section, Surgery Branch, National Cancer Institute, NIH, Bethesda, MD.

INTRO: Ocular melanoma is an aggressive form of melanoma that can metastasize widely and is refractory to many standard therapies. Targeting multiple components of the tumor microenvironment such as tumor cells, the vasculature and the immune system may be a viable strategy for treating patients with ocular melanoma. We studied the effects of TKIs alone and in combination with oral metronomic CTX on the growth and metastases of a highly aggressive xenograft model of ocular melanoma. METHODS: Nude mice were inoculated subcutaneously with a human ocular metanoma cell line and the tumors were allowed to grow to approximately $200 \mathrm{~mm} 3$. All mice were treated on IACUC approved protocols. Mice were randomly assigned to groups and treated with a daily oral gavage of sorafenib $(60 \mathrm{mg} / \mathrm{kg} /$ day $)$ alone, sunitinib $(80 \mathrm{mg} / \mathrm{kg} /$ day $)$ alone, CTX $(25 \mathrm{mg} / \mathrm{kg} /$ day $)$ alone or with sorafenib plus CTX or with sunitinib plus CTX. Control mice were treated with an oral carrier vehicle. Tumors were measured twice weekly by a blinded investigator and all animals were sacrificed on Day 15. At that time the lungs of the mice were harvested to count surface lung metastases. RESULTS: All treatment groups demonstrated a statistically significant delay in subcutaneous tumor growth when compared to control (See Figure 1). There was a significant difference between treatment groups when surface lung metastases were counted. This difference favored the groups containing sunitinib when compared to the other groups. The sunitinib groups both had a median of 0 surface lung metastases compared to 18 in the control group $(\mathrm{p}<0.001)$. CONCLUSIONS: In a highly metastatic murine model of ocular melanoma, sunitinib, sorafenib, and oral CTX alone and in combination inhibited the growth of subcutaneous tumors. Furthermore, sunitinib alone and in combination with CTX, dramatically decreased the number of metastatic pulmonary depostits. Combinations of these TKIs and low dose metronomic chemotherapy regimens may be a viable strategy for treating advanced malignancies.

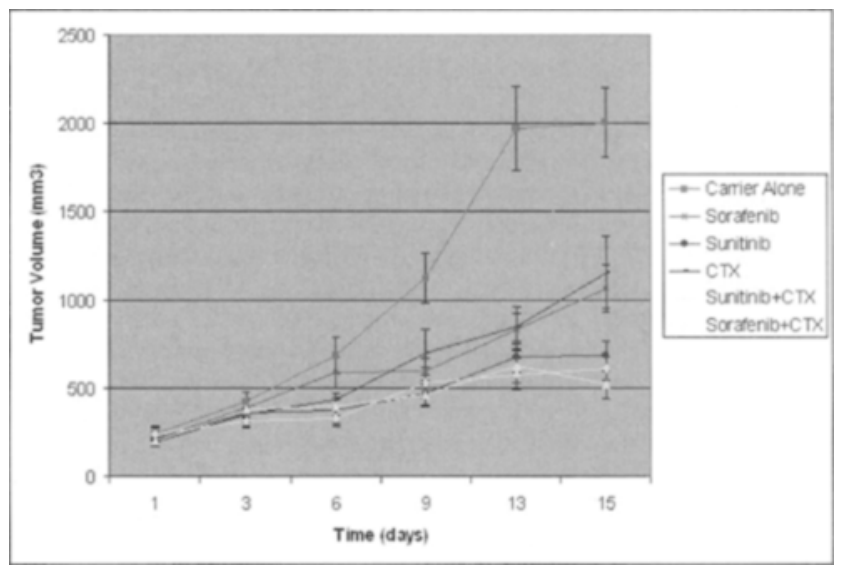

\section{P3}

Immune Response to an MVA Vaccine Expressing Human p53 G.Y. Song, D.J. Diamond, J. Ellenhorn.* City of Hope National Medical Center, Duarte, $C A$.

Vaccines targeting cellular proteins like p53 can target specific epitopes or full-length proteins. We wished to evaluate the diversity of the immune response to full-length p53 antigen as expressed by an attenuated poxvirus (MVA) vaccine (MVAp53). PBMC from cancer patients were subjected to an in vitro stimulation (IVS) with MVAp53 or control MVA infected antigen presenting cells (APC). An additional IVS was performed using an overlapping 15 mer peptide library derived from full-length $\mathrm{p} 53$. The peptide library contained 9615 mers which overlapped by 4 amino acids each. p53 specific immunity was evaluated using an intracellular cytokine (ICC) flow cytometry assay to enumerate IFN- $\gamma$ producing cells, and $\mathrm{CD} 107 \mathrm{a} / \mathrm{b}$ staining to evaluate degranulation. Neither IFN- $\gamma$ production nor CD107a/b staining was seen following IVS with a control MVA followed by IVS with the p53 peptide library. p53 specific responses were identified following stimulation of PBMC from 6 of 8 HLA A2.1+ cancer patients with MVAp 53 and the $p 53$ peptide library. We compared the percentage of IFN- $\gamma$ producing cells by ICC assay following a 16 hour stimulation with the p53 peptide library compared to the percentage of IFN- $\gamma$ producing cells by ICC assay following a 16 hour stimulation with the two known HLA A2.1 restricted epitopes of p53, 149-157 and 264-272. $1.5 \%$ of cells were positive by ICC assay using the p 53 peptide library for the assay compared to only $0.2 \%$ of cells positive for IFN- $\gamma$ production when using the two HLA-A2.1 restricted epitopes in the assay. To evaluate the response following MVAp53 stimulation, the ICC assay was repeated using 8 sequential fractionated pools of overlapping peptides, each derived from a 59 amino acid segment of the $\mathrm{p} 53$ protein. Positive responses were generated using 7 of the sub-pools. This suggests that stimulation with a full-length $\mathrm{p} 53$ antigen expressing vaccine generates an immune response far more diverse than that which might be generated following stimulation with a limited number of epitopes. Furthermore, this data suggests the importance of evaluating vaccine approaches which target whole antigens, using overlapping peptide libraries which cover the full-length antigen target.

\section{P4}

Surgery Derived Reactive Oxygen Species Produced by PMN's Promote Tumor Recurrence W. Van Grevenstein, * A.G. Aalbers, J. Jeekel, C.H. Van Eijck. Surgery, Erasmus MC, Rotterdam, Netherlands.

INTRODUCTION: Tissue injury induces the acute phase response, aimed at minimizing damage and starting the healing process. Polymorphonuclear leukocytes (PMN's) respond to the presence of specific chemoattractants and begin to appear in large numbers. The aim of this study is to investigate the influence of reactive oxygen species (ROS) produced by PMN's on the interaction between colon carcinoma cells and mesothelial cells. METHODS: An experimental human in vitro model was designed using $\mathrm{Caco} 2$ colon carcinoma cells and primary cultures of mesothelial cells. Tumor cell adhesion to a mesothelial monolayer was assessed after pre-incubation of the mesothelium 
with stimulated PMN's and unstimulated PMN's. Mesothelial cells were also incubated with Xanthine/Xanthine Oxidase $(\mathrm{X} / \mathrm{XO})$ complex producing ROS after which adhesion of Caco-2 cells was investigated and the expression of adhesion molecules (ICAM-1, VCAM-1 and CD44) by means of enzyme immunoassay (EIA). RESULTS: In the control situation the average adhesion of Caco 2 cells to the mesothelial monolayers was $23 \%$. Mesothelial monolayers incubated with unstimulated PMN's showed a $25 \%$ increase of tumor cell adhesion $(p<0.05)$. The adhesion of tumor to the monolayers incubated with the FMLP stimulated PMN's increased with $40 \%(p<0.01)$. Incubation of the mesothelium with $\mathrm{X} / \mathrm{XO}$ resulted in an enhancement of adhesion of Caco-2 cells of $70 \%$ and an upregulation of expression of ICAM-1, VCAM-1 and CD44. CONCLUSION: This study reveals an increase of tumor cell adhesion to the mesothelium induced by incubating the mesothelial monolayers with PMN's. PMN's are producing a number of products, like proteolytic enzymes, cytokines and reactive oxygen species (ROS). These factors upregulate the expression of adhesion molecules and in that way stimulate the adhesion of tumor to the mesothelium.

\section{P5}

The Effect of Extra-cellular Matrix Components on Cathepsin D Expression, Processing and Secretion D.E. Abbott, ${ }^{*} \mathrm{Z}$. Ellis, M.J. Hendrix. Childrens Memorial Research Center, Northwestern University/Feinberg School of Medicine, Chicago, IL.

Introduction Cathepsin D, a well described lysosomal hydrolase, is found to be over-produced, secreted and dysregulated in malignant mammary epithelial cells when compared to benign cells. As a potent aspartyl protease, this could have important implications in local invasion and metastases. Our objective was to investigate the effects of specific extra-cellular matrix components on cathepsin $\mathrm{D}$ intra-cellular production, processing and secretion. Methods MDA MB-231 and MCF 10a mammary epithelial cells, of malignant and benign origin respectively, were cultured on different matrices, i.e. Matrigel, type I collagen, and type I collagen supplemented individually with laminin, fibronectin, and type IV collagen. The media and whole-cell lysates from each well were subjected to Western blot analysis, and changes in gene regulation were analyzed by RT-PCR. Results Compared to controls, MDA MB231 malignant mammary epithelial cells cultured on varying extra-cellular matrices exhibited altered cathepsin $\mathrm{D}$ gene expression, lysosomal processing to an active form and secretion. When plated on specific matrices, cathepsin D mRNA was modestly decreased compared to controls (RT-PCR). Cells cultured on collagen type I and collagen type I supplemented with laminin, fibronectin and type IV collagen showed a dramatic shift towards cleavage of the pro-form of cathepsin $\mathrm{D}(52 \mathrm{kD})$ to the catalytically active isoform $(34 \mathrm{kD})$, demonstrated by Western blot analysis; see Figure 1. Additionally, cells cultured on Matrigel secreted a higher proportion of the pro-form of cathepsin D, in turn cleaved to the active isoform of cathepsin D. This is not observed in cultured media from cells grown on other matrices. Conclusions To our knowledge, this is the first demonstration of differential cathepsin D secretion and intra-cellular processing dependent upon extra-cellular matrix composition. These data and further investigation into the mechanisms of these interactions may provide targets for therapeutic agents aimed towards disrupting matrixtumor cell interaction.

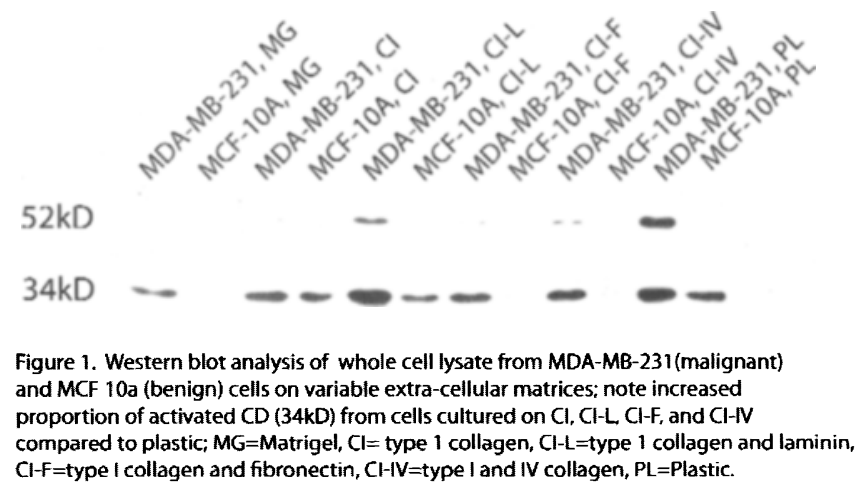

\section{P6}

The Development and Characterization of a Human Mid-gut Carcinoid Cell Line G. Van Buren, ${ }^{1 *}$ A. Rashid, ${ }^{2}$ A.D. Yang, ${ }^{1}$ E.K. Abdalla, ${ }^{1}$ M.J. Gray, ${ }^{1}$ W. Liu, ${ }^{1}$ F. Fan, ${ }^{1}$ E.R. Camp, ${ }^{1}$ J.C. Yao, ${ }^{3}$ L.M. Ellis. ${ }^{1}$ 1. Department of Surgical Oncology, MD Anderson Cancer Center, Houston, TX; 2. Department of Pathology, MD Anderson Cancer Center, Houston, TX; 3. Department of Gastrointestinal Oncology, MD Anderson Cancer Center, Houston, TX.

Introduction: Gastrointestinal neuroendocrine tumors (NETs) are a rare heterogeneous collection of tumors that hypersecrete neuropeptides leading to hormonal disability. The lack of reliable gastrointestinal NET models has limited our ability to study therapeutic regimens in vitro and in vivo. Our goal was to develop a human mid-gut carcinoid tumor cell line (CNDT2). Methods: Tumor was harvested from a resected liver specimen in a patient with a primary ileal carcinoid tumor. Following initial collagenase digestion, the CNDT2 cells were plated in vitro. After 9 weeks in culture, the cells were plated in soft agar. CNDT2 cells were injected subcutaneously into nude mice. The cell line and tumor tissue were characterized by immunohistochemistry(IHC), electron microscopy, RT-PCR, and Western blotting for NET markers and potential therapeutic targets. Results: CNDT2 cells grew in monolayer in culture, formed colonies in soft agar, and formed solid tumors in mice. Electron microscopy of the cell line revealed round, pleomorphic, and electron-dense neurosecretory granules (Figure). Tumor xenografts exhibited the characteristic appearance of NETs with small, "salt and pepper" nuclei on H\&E staining. By IHC and RT-PCR, the xenografts expressed chromogranin A, synaptophysin, neuron specific enolase, and CD56. Western blotting demonstrated that the cultured cells expressed insulin-like growth factor receptor-1, platelet derived growth factor receptor- $\beta$, vascular endothelial growth factor receptor-1, cMET, epidermal growth factor receptor, neuropilin-1, and somatostatin receptors 15. Cytogenetic analysis confirmed that the cell line is of human origin with deletions at $2 p$ and $6 \mathrm{q}$ and numerous translocations. Conclusion: We have established the second human mid-gut carcinoid tumor cell line in the world that will serve as a model system to study cell biology and new therapeutic regimens in preclinical models.
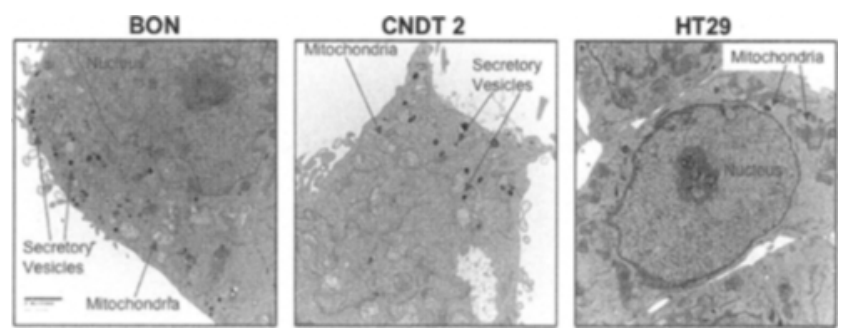

Figure: Cell lines were examined under electron microscopy at 10,000 $x$ magnification. BON pancreatic NET cells (positive control) and CNDT 2 cells exhibited electron dense neurosecretory granules. HT29 colon cancer cells (negative control) did not exhibit any electron dense granules.

\section{P7}

Fv-p53 Therapy Decreases Colorectal Liver Metastasis in a Mouse Model L.K. Fischer, ${ }^{1 *}$ R.J. Aragon, ${ }^{1}$ S.W. Baldwin, ${ }^{1}$ M.G. Minera, ${ }^{1}$ J.J. Carter, ${ }^{1}$ Y.G. Amaar, ${ }^{1}$ R.H. Weisbart, ${ }^{2}$ M.E. Reeves. ${ }^{1}$ 1. Loma Linda University and VA Medical Centers, Loma Linda, CA; 2. Sepulveda VA Medical Center, Sepulveda, $C A$.

Introduction: $75-85 \%$ of colorectal cancers have $\mathrm{p} 53$ mutations, and colorectal liver metastases have an even higher percentage of $\mathrm{p} 53$ mutations. Fvp53 is a "protein transduction" fusion protein which delivers p 53 into cells, and has been shown to kill a variety of p 53 negative cancer cells in vitro. The purpose of this study is to test the in vivo activity of Fv-p53 against colorectal liver metastases in mice. Methods: A mouse model was developed and optimized. Syngeneic colorectal cancer cells (CT26) were injected into the portal vein of Balb/c mice to produce liver metastasis. Treatment mice were injected with Fv-p53 into the spleen for delivery to the liver on days 0 and 7, while control mice were injected with an irrelevant protein. During the initial surgery the spleen was placed into a subcutaneous pocket to facilitate the second injection. Animals were euthanized on day 14 , and the liver was evaluated for 
metastasis. Results: After optimization of the mouse model, liver metastases were able to be consistently established, and the mice tolerated the procedures well. A "metastasis score" system was developed, which is based on a reproducible visual inspection of the treated liver for the number of metastases. Importantly, mice treated with Fv-p53 had a significantly lower metastasis score as compared to control $(\mathrm{P}<.004)$. Conclusion: Fv-p53 significantly decreases colorectal liver metastases in a mouse model.
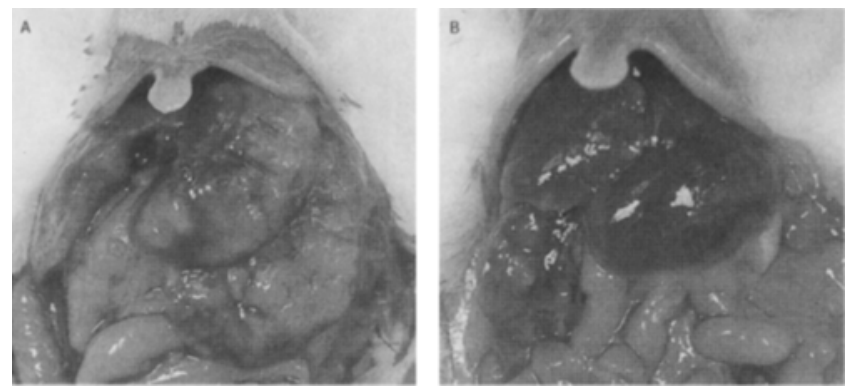

A) Control - liver infiltrated by tumor. B) Fv-p53 - minimal tumor in liver.

\section{P8}

Requirement of innate immunity in tumor-bearing mice cured by adoptive immunotherapy using tumor-draining lymph nodes J. Kim, ${ }^{*}$ H. Graor, K. Hamzeh. Division of Surgical Oncology, Case Medical Center, Cleveland, $\mathrm{OH}$.

Background: The use of culture-activated tumor-draining lymph nodes (TDLNs) for adoptive immunotherapy is an effective method of curing mice bearing a variety of solid tumors. The purpose of this study was to determine the cellular effectors of both the adoptively-transferred cells and the tumorbearing host which participate in the antitumor immune response. Methods: Tumor-draining lymph nodes harvested from mice inoculated in the mammary fat pad with 4T1 murine mammary carcinoma cells were fractionated to derive the antigen-primed L-selectin ${ }^{\text {low }}$ subpopulation, activated ex vivo with antiCD3 for 48 hours and expanded in $50 \mathrm{IU} / \mathrm{ml}$ interleukin-2 for 72 hours prior to in vitro cytokine release assays and adoptive transfer. Adoptive immunotherapy was performed into $\mathrm{BALB} / \mathrm{c}$ mice bearing 3-day established subcutaneous tumors following a 50cGy whole body irradiation conditioning regimen. SCID (lacking T, B and NK cells) and Rag2 deficient (lacking T and B cells) as well as wild-type $\mathrm{BALB} / \mathrm{c}$ mice were used as the tumor-bearing recipients. Results: L-selectin ${ }^{\text {low }} 4$ T 1 TDLN from BALB/c wild-type mice which were culture-activated in IL-2/anti-CD3 secreted significant levels of interferon-gamma in response to $4 \mathrm{~T} 1$ but not control $\mathrm{H}-2 \mathrm{k}^{\mathrm{d}}$ (Renca) tumor cells in vitro. Immunomagnetic column depletion of $\mathrm{T}$ cell subsets suggested that CD4 cells within the adoptively transfered effector cell population contributed significantly to the antitumor effect in vivo. Culture-activated L-selectin ${ }^{\text {low }}$ TDLN from BALB/c wild-type mice were able to cure Rag2 deficient but not SCID mice bearing 4T1 subcutaneous tumors, suggesting a requirement of NK cells within the innate immune system of the tumor-bearing host during the antitumor response. Conclusions: These results define the cellular effectors that participate in tumor regression following adoptive transfer and demonstrate the requirement for intact innate immmunity within the tumor-bearing host.

\section{P9}

Lymphodepletion Can Enhance the Therapeutic Efficacy of Adoptive Tumor Reactive T Cell Transfer without Promoting Proliferation or Survival of the Infused Cells S. Teitz-Tennenbaum, * Q. Li, A.E. Chang. General Surgery, University of Michigan, Ann Arbor, MI.

Introduction: We examined the proliferation and persistence of adoptively transferred effector $\mathrm{T}$ cells in tumor bearing mice utilizing a clinically relevant protocol for induction of non-myeloablative lymphodepletion. Methods: C57BL/6 mice were inoculated i.v. or s.c with D5 melanoma tumor cells on day 0. Cyclophosphamide $(1 \mathrm{mg})$ and Fludara $(2 \mathrm{mg})$ were administered i.p. on days 3 and 4. Effector T cells were derived from D5-G6 (D5 expressing GMCSF) tumor draining lymph nodes (TDLNs) induced in syngeneic mice. TDLN cells were activated ex vivo with anti-CD3 and anti-CD28 mAbs, and then expanded in IL-2 containing medium. Adoptive cell transfer was delivered i.v. on day 7 , and was followed by systemic IL-2 treatment. Anti-tumor responses were evaluated by enumerating lung metastases, measuring s.c. tumor size, and monitoring mice survival. To assess proliferation and survival of the infused cells, CFSE labeled CD45.1 effector T cells were administered to CD45.2 mice. Blood samples, spleens, and tumors were collected 1,3 , and 5 days after adoptive transfer. Tissue-derived single cell suspensions were stained using fluorochrome-conjugated $\mathrm{mAbs}$, and analyzed by flow cytometry. Results: Administration of chemotherapy mediated a profound, yet transient, reduction in total white blood cell and T cell counts in peripheral blood samples. Treatment of mice bearing 3-day established tumors with chemotherapy did not inhibit tumor growth or prolong mice survival. In both the pulmonary metastasis and s.c. tumor models, induction of lymphopenia prior to adoptive transfer significantly enhanced the therapeutic efficacy of the infused cells. Transferred cells proliferated to the same extent in lymphopenic versus control mice. Accumulation of infused CD8 + and CD4+ cells within tumors, spleens and the blood of treated mice did not differ significantly between lymphodepleted and control mice. Conclusions: Understanding the mechanisms by which a lymphopenic environment augments the anti-tumor efficacy of adoptive $T$ cell therapy might enable future design of more targeted and less toxic treatments.

\section{P10}

CD24 / FLJ13639 Expression Ratio Predicts Worse Outcome In Invasive Breast Cancer A. Meguerditchian, ${ }^{1}$ H. Huang, ${ }_{1}^{1}$ Y. Pak, ${ }^{1}$ K. Sossey-Alaoui, ${ }^{1}$ N. Watroba, ${ }^{1}$ A. Hutson, ${ }^{1}$ S. Kulkarni, ${ }^{1}$ S.B. Edge, ${ }^{1}$ J. Geradts, ${ }^{2}$ L.J. Coignet. ${ }^{1}$ 1. Roswell Park Cancer Institute, Buffalo, NY; 2. Duke University Medical Center, Durham, NC.

INTRODUCTION: Over-expressed CD24 (a ligand of P-selectin and putative actor in the metastatic cascade) is associated with aggressive cancer and worse prognosis. It correlates with decreased FLJ13639, a novel mitochondrial protein involved in cellular respiration and apoptosis that shares homologies with the family of short-chain dehydrogenase reductases. Therefore, the CD24 high / FLJ13639 low/absent expression profile may serve as a marker in assessing breast cancer prognosis. The aims of this study were: 1) determining the prevalence of CD24 and FLJ13639 abnormalities in invasive breast carcinoma samples; 2) correlating the CD24 high / FLJ13639 low/absent pathological expression profile with conventional prognostic indicators and clinical outcome in invasive breast cancer. METHODS: CD24 and FLJ13639 expression were assessed by multiplex RT-PCR in 35 human samples of invasive breast cancer. Semi-quantitative grading of expression levels of both genes as well as their ratio was then correlated with clinical data. RESULTS: CD24 had a positive correlation with tumor size $(\mathrm{p}=0.03)$, histological grade $(\mathrm{p}=0.04)$ and number of positive nodes $(\mathrm{p}<0.0001)$. FLJ13639 expression was negatively correlated with nuclear grade $(\mathrm{p}=0.009)$ and nodal positivity $(\mathrm{p}<0.0002)$. It had a statistically significant correlation with ER and PR status ( $p=0.02$ and 0.03 respectively). Forty percent of the cases showed an abnormal CD24 high / FLJ13639 low/absent expression profile (ratio $\geq 3$ ). After controlling for all other factors, this was a statistically significant predictor of decreased overall survival ( $p=0.02$ ). CONCLUSION: The CD24 high / FLJ13639 low/absent abnormal expression profile is a potential prognostic marker for breast cancer.

\section{P11}

Acidic Treatment Increases eNOS Phosphorylation and NO Production during Isolated Limb Perfusion and Activates PKA in Endothelial Cells G. Karakousis, ${ }^{1 *}$ D. Buerk, ${ }^{2}$ J. Fong, ${ }^{1}$ D. Han, ${ }^{1}$ M. Guvakova, ${ }^{1}$ D. Fraker. ${ }^{1}$ 1. Hospital of the University of Pennsylvania, School of Medicine, Department of Surgery, Philadelphia, PA; 2. University of Pennsylvania, School of Medicine, Department of Physiology, Philadelphia, PA.

Introduction: Isolated limb perfusion (ILP) with melphalan is a standard treatment for locally advanced extremity melanomas. Clinical observations and preclinical data suggest that acidifying the ILP perfusate improves response rates. Here, we use in vivo and in vitro methods to investigate the molecular basis for the effects of acidic perfusion. Methods: Nude rats with xenografted human melanoma underwent ILP with control saline (C), control+melphalan (M) or acid melphalan (AM). Nitric oxide (NO) production was measured in ILP effluents of each group $(n=4)$ using a custom-fabricated microelectrode. Immunofluorescent staining of phosphorylated endothelial NO synthase 
(pSer1177eNOS) was performed on tumor and muscle sections obtained after ILP. The effect of acidic treatment on protein kinase A (PKA) was studied by Western blotting in human microdermal endothelial cells (HMDEC) treated with acidic medium ( $\mathrm{pH} 6.5$ ) in the absence or presence of the PKA inhibitor RpcAMP. Phosphorylation at Ser 157 of vasodilator-stimulated phosphoprotein (VASP) was used as a marker for monitoring PKA activity. Results: Perfusion with AM significantly increased NO production compared to perfusion with $\mathrm{M}$ alone $(\mathrm{p}<0.012)$; this effect was observed only in tumor bearing rats. No significant difference in NO production was found between $\mathrm{M}$ and $\mathrm{C}$ groups $(\mathrm{p}=0.446)$. Mean $\mathrm{pH}$ values of $\mathrm{C}, \mathrm{M}$ and $\mathrm{AM}$ were $7.5 \pm 0.2,7.3 \pm 0.1$ and $6.5 \pm 0.2$. Immunofluorescent staining showed increased levels of pSer1177eNOS in acidic compared to non-acidic perfused tumor endothelium. There were no differences in pSer1177eNOS staining in endothelium of muscles exposed to acidic and non-acidic perfusate. Treatment of HMDEC with acidic medium caused a rapid $(<5 \mathrm{~min})$ activation of VASP at Ser 157 (5.1 fold), whereas treatment with $200 \mu \mathrm{M}$ RpcAMP inhibited this response by $50 \%$. Conclusions: ILP using acid perfusate increases pSer1177eNOS in tumor endothelium and NO production in melanoma-bearing limbs. These results may be relevant to the activation of the PKA pathway in endothelial cells and may provide a rationale for improved responses to ILP with melphalan and acid.

\section{P12}

Rational targeting of the Raf-MEK-ERK pathway in colorectal cancer using MEK inhibitor therapy J. Yeh, ${ }^{*}$ E.D. Routh, H.J. Kim, C.J. Der. Lineberger Comprehensive Cancer Center, University of North Carolina at Chapel Hill, Chapel Hill, NC.

Introduction: Ras mutations are detected in $50 \%$ of colorectal tumors. Although considerable effort has been made to target Ras for cancer treatment, anti-Ras therapies remain elusive. Treatment strategies have focused on inhibition of the activated downstream Raf-MEK-ERK mitogen-activated protein kinase cascade with inhibitors of MEK currently under clinical evaluation. It is unclear if MEK inhibitors will be effective for colorectal cancer (CRC) treatment. The aims of this study were to determine if: 1) Ras mutation status is predictive of Raf-MEK-ERK pathway activation, 2) Raf-MEK-ERK pathway activation is a predictor of response to MEK inhibitor therapy in CRC, and 3) specific gene expression changes occur in response to MEK inhibitor treatment. Methods: 10 CRC lines were analyzed for Ras and Raf mutation status. Parallel cultures were treated with inhibitor or vehicle control. Total RNA and whole cell extracts were prepared from cells at 24 hours. cDNA was hybridized to Agilent human whole genome arrays. Data was analyzed using hierarchical clustering and significance analysis of microarray (SAM). For soft agar assays, cells were suspended in soft agar and colonies were scored after 4 weeks. Results: We found that P-ERK1/2 was present at variable levels in 9/10 cell lines. There was no correlation between Ras or Raf mutation status and ERK activation. U0126 treatment significantly reduced growth in soft agar in 7 of 9 lines. However, neither mutation status nor ERK activation correlated with anchorage-independent growth inhibition by U0126. Preliminary analyses showed that hierarchical clustering was unable to differentiate CRC lines by gene mutation status or ERK activation. Using a false discovery rate of 0.05 , SAM identified 23 genes positively and 474 genes negatively regulated by U0126. Conclusions: Gene mutation status will not provide an accurate approach for patient selection for MEK inhibitor therapy. In addition, ERK activation cannot predict response to U0126 in CRC cell lines. Therefore, determining specific gene expression profiles will be useful for defining tumors that will be responsive to MEK inhibitor therapy.

\section{P13}

Permeability changes induced by IL-2 in human pulmonary microvascular endothelial cells P. Mong, D. Feng, D.W. Kim, S. KimSchulze, H.L. Kaufman, Q. Wang.* Department of Surgery, Columbia University, New York, NY.

High dose IL-2 is the most effective immunotherapy for patients with metastatic melanoma and renal cell carcinoma, and $17-20 \%$ of the patients respond to this treatment. High dose IL-2, however, induces vascular leak syndrome characterized by increased vascular permeability and, in severe cases, pulmonary and cardiovascular failure. The goal of this study was to examine the effect of IL-2 on permeability changes in primary cultures of human pulmonary microvascular endothelial cells (ECs). Permeability changes were evaluated by measuring the fluxes of FITC-labeled dextran (10kD) across a con- fluent monolayer of ECs. The effect of IL-2 at a clinically relevant dose $(10,000$ $\mathrm{IU} / \mathrm{ml})$ as well as at lower doses $(100$ and $1,000 \mathrm{IU} / \mathrm{ml})$ was examined. At 100 $\mathrm{IU} / \mathrm{ml}$, IL-2 induced a significant increase in dextran fluxes in a time-dependent manner. This effect was apparent by $4 \mathrm{hrs}$ and persisted for at least $24 \mathrm{hrs}$. At $1,000 \mathrm{IU} / \mathrm{ml}, \mathrm{IL}-2$ induced a significant increase only at $5 \mathrm{hrs}$. At 10,000 $\mathrm{IU} / \mathrm{ml}, \mathrm{IL}-2$ did not induce a significant increase at all time points (from $0.5 \mathrm{hr}$ to $24 \mathrm{hrs})$. High dose IL-2 $(6,000 \mathrm{IU} / \mathrm{ml})$ induced activation of human peripheral blood mononuclear cells (PBMC) as evaluated by CD69 expression but low dose IL-2 (100 IU $/ \mathrm{ml})$ did not. Supernatants from PBMC treated with high or low dose IL-2 did not cause a significant increase in EC permeability. These data indicate that IL-2 induces an increase in EC permeability in a dose- and time-dependent manner. These data and the clinical observations that only high dose IL-2 induces vascular leak syndrome suggest that the presence of activated leukocytes and cell-cell interaction are likely required for inducing EC injury during high dose IL-2 therapy. Understanding the mechanisms underlying EC injury and vascular leak will help identify therapeutic targets for limiting the adverse effects without compromising the therapeutic efficacy of high dose IL-2 for cancer patients.

\section{P14}

Novel multimodal therapy with TNFerade ${ }^{\mathrm{TM}}$ (Ad.Egr.TNF), radiation, and bevicizumab causes significant tumor regression in hilar cholangiocarcinoma xenografts K.A. Bickenbach, ${ }^{\mathrm{J}}$. Veerapong, M. Shao, P. Schmidt, H.J. Mauceri, M.C. Posner, R.R. Weichselbaum, K.K. Roggin. Surgery, University of Chicago, Chicago, IL.

Introduction: Cholangiocarcinoma (CC) is a lethal malignant tumor of the bile ducts. Long-term survival is dependent on complete surgical resection. Unfortunately, most $\mathrm{CC}$ are unresectable due to local extension along hepatic bile ducts. Locally-administered Ad.Egr-TNF, a replication-deficient adenoviral vector containing the Egr-1 radio-inducible promoter ligated upstream of the cDNA for human TNF- $\alpha$, may enhance locoregional treatment. Methods: $\mathrm{KMC}$ (peripheral) and $\mathrm{OZ}$ (hilar) $\mathrm{CC}$ cell lines were treated with IR, Ad.Egr-TNF, or the combination. TNF- $\alpha$ levels were measured by ELISA at $24 \mathrm{~h}, 48 \mathrm{~h}$, and $72 \mathrm{~h}$. MTT and clonogenic assays were performed with increasing doses of IR $(1,3,5,7,10 \mathrm{~Gy})$ and TNF- $\alpha$ protein $(1,5,10,20 \mathrm{ng} / \mathrm{ml})$. ELISAs were also performed to evaluate intratumoral production of VEGF Tumor regrowth studies were conducted using female nude mice bearing $\mathrm{OZ}$ hind limb xenografts treated with Ad.Egr-TNF, IR (10 Gy), and bevicizumab $\left(5 \mathrm{mcg} / \mathrm{kg}\right.$; Avastin $\left.{ }^{\mathrm{TM}}\right)$. Results: Both $\mathrm{KMC}$ and $\mathrm{OZ}$ cell lines exhibited significant TNF- $\alpha$ production following treatment with Ad.Egr-TNF \pm IR vs. controls $(p<.001)$. Both the MTT and clonogenic assays demonstrated dose-dependent cytotoxicity following TNF- $\alpha+$ IR treatment. Tumor regrowth studies in $\mathrm{OZ}$ xenografts showed that Ad.Egr-TNF + IR treatment produced increased tumor growth inhibition compared with either treatment alone. Importantly, enhanced tumor growth inhibition was achieved following triple therapy with Ad.Egr-TNF, IR, and bevicizumab up to day 45 following initiation of treatment ( $p=.0073$ vs. CTRL). Conclusions: Ad.Egr-TNF alone or in combination with IR stimulates production of cytotoxic levels of TNF- $\alpha$ protein in KMC and OZ CC cell lines. Triple treatment with Ad.Egr-TNF, IR, and bevicizumab produces enhanced tumor regression compared with all other treatment combinations. Multimodal therapy with TNFerade ${ }^{\mathrm{TM}}$, IR and Avastin ${ }^{\mathrm{TM}}$ may be exploited as a novel treatment strategy to downstage locally-advanced CC.

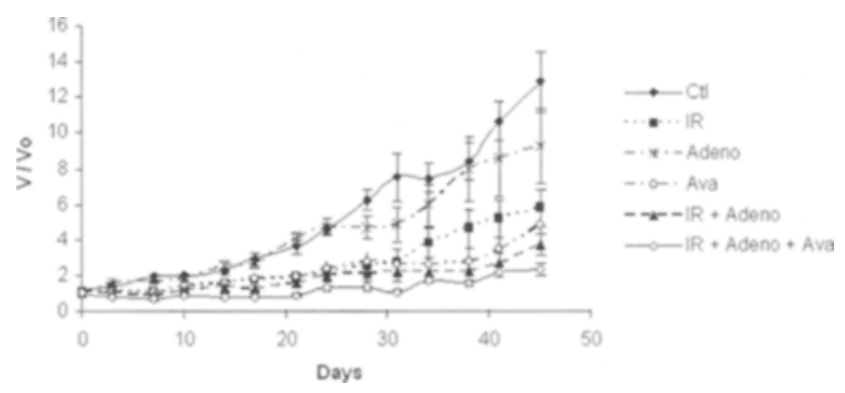

Tumor regrowth study in $\mathrm{OZ}$ cholangiocarcinoma xenografts using combination therapy with Ad.Egr-TNF, IR, \pm bevicizumab. 


\section{P15}

Rapamycin Enhances Cytotoxic Effect of Doxorubicin in Human Hepatocellular Carcinoma Cells M. Yezhelyev, ${ }^{*}$ R. Machaidze, T. Philip, V. Egnatashvili, R. O'Regan, D. Kooby. Winship Cancer Institute, Emory University, Atlanta, GA.

INTRODUCTION: Doxorubicin (dox) is the best systemic agent for treatment of advanced hepatocellular carcinoma (HCC), but provides only a 12 week survival advantage. The mammalian target of rapamycin (mTOR) pathway is central to various proliferative cellular indices and is overexpressed in HCC. Rapamycin (rapa) has anti-tumor effects in several cancers through inhibition of mTOR. We examined the combined effects of rapa and dox in human HCC cells. METHODS: Antiproliferative and cytotoxic effects of rapamycin alone and in combination with dox were performed on HepG2 cells, and measured by the Sulforhodamine B (SRB) assay. Expression of mTOR, phosphorilation of MAPK kinase, AKT, S6 kinase and p70S6 kinase (mTOR activity assay), were detected by western blotting and quantified by optical densitometry. RESULTS: Rapa had only mild antiproliferative activity compared to dox alone after 48 hours $(12.2 \%$ vs. $89.1 \%$ of proliferation inhibition). Rapa + dox inhibited proliferation completely and reduced live tumor cells by $61 \%$ $(\mathrm{p}<0.001$ versus dox alone). Monotherapy with doxorubicin resulted in phosphorilation of mTOR, S6 kinase, p70S6 in the HepG2 cells. CONCLUSIONS: Combined therapy with rapa + dox inhibits experimental HCC cell proliferation in vitro, and augments cytotoxicity of doxo alone. mTOR-S6 kinase survival pathway may play important role in the resistance of HepG2 cell towards dox. By inhibiting mTOR, rapa may interfere with dox induced activation of S6 kinase and thus enhance cytotoxic effect of dox . Anti-tumor effects of dox and rapa combination therapy against HCC should be investigated further

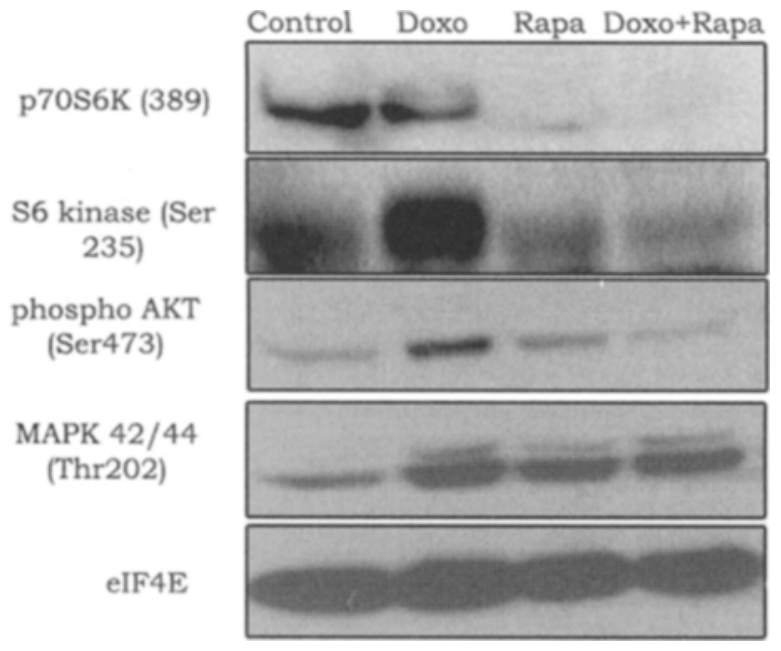

\section{P16}

Nodal metastatic breast and colon cancer cells inhibit pericyte invasion $\mathrm{H} . \mathrm{Vu},{ }^{*}$ D. Kocubinski. Surgery, VCU, Richmond, VA.

Introduction: Newly formed blood vessels in the tumor microenvironment are characterized by irregular vessel diameter, increased tortuosity and abnormal permeability. Normalization of immature vessels is believed to be modulated by pericytes. The relationship between tumors and pericytes has not been well delineated. Methods: Human tumor lines evaluated included three breast cancers, HCC1954, HCC38, HCC 1008, and three colon cancers, SW 480, SW48, and SW620. Neither HCC 1954 nor SW480 have metastasized to lymph nodes. HCC 38 and SW48 are from primary tumors with nodal metastases. HCC 1008 and SW620 are derived from nodal metastatic deposits. The tumor lines were compared against human pericyte control, HMVEC, lymphoendothelial cells and fibroblasts for modulation of in vitro pericyte proliferation, migration, and invasion. Pericyte proliferation was quantified with WST-1 reagent. Pericyte migration was assessed with scratch assay. Pericyte invasion was quantified with Boyden chambers. Statistical analysis was performed using
InStat software. Results: By 72 hours, five of the six evaluated tumor cell lines revealed statistically significant stimulation of pericyte proliferation. At 48 and 72 hours there were no observable differences among treatment groups in pericyte migration in scratch assay. Finally, at 72 hours, Boyden chamber assay demonstrated statistically significant inhibition of pericyte invasion by breast cancer cell line HCC1008 and colon cancer cell line SW620. Both lines are derived from nodal metastases. Conclusion: While evaluated human breast and colon cancer cell lines stimulated pericyte proliferation in vitro, this did not translate into an in vitro stimulation of pericyte migration on culture plate or invasion across an artificial extracellular matrix. Pericyte invasion as assayed by Boyden chamber was actually inhibited by the two tumor cell lines that were derived from nodal metastases. Further studies will be conducted to determine the molecular mechanism of observed findings. Prevention of tumordirected inhibition of pericyte recruitment may normalize tumor vasculature and may provide a novel strategy to enhance the delivery of anti-tumor agents.

\section{P17}

Characterization of fibroblasts derived from ovarian cancers in a physiologically relevant 3-dimensional matrix R.M. Quiros,*

K. Brown, E. Cukierman. Dept of Surgical Oncology, Fox Chase Cancer Center, Philadelphia, PA.

Introduction: Fibroblasts in stroma surrounding epithelial cells inhibit tumorigenesis (normal). As cancer develops, fibroblasts may promote tumor growth (primed). Fibroblasts may become irreversibly conducive to tumor growth (activated). We grew ovarian fibroblasts in a 3-dimensional (3-D) matrix mimicking the in vivo environment. We analyzed these fibroblasts and compared them to normal, primed, and activated cells to determine their role in tumor progression. Methods: Fibroblasts were harvested from fresh ovarian cancer tissue. Fibroblast lines were plated in 3 sets. Lysates were prepared from the 1st set after overnight incubation (2-D). On the remaining 2 sets, fibroblasts were grown for 8 days to allow 3-D matrix production. Cells from 1 set of plates were analyzed by Western blot for total/phosphorylated focal adhesion kinase (T-/P-FAK), smooth muscle actin (SMA), and vimentin. Cells from the 3rd set of plates were analyzed by immunofluorescence for fibroblast and matrix characteristics: cell density, matrix topography, fiber organization, and presence/orientation of SMA and fibronectin. Results: By light microscopy, fibroblasts appeared as 3 main types: "spindle" (needle-like); "intermediate" (elliptical); "spread" (broad/flat). By confocal microscopy, organization of cell nuclei, SMA, and fibronectin mirrored that of tumor-associated, primed, and normal fibroblasts. By Western blot, FAK activation levels varied in 2-D and 3-D samples and within "spindle," "intermediate," and "spread" cell types: "spindle" fibroblasts expressed higher levels of FAK and PFAK compared to normal fibroblast controls, while "spread" fibroblasts expressed lower levels of FAK and PFAK compared to normal fibroblast controls. Conclusions: We derived fibroblasts with characteristics resembling normal, primed, and activated types. Biochemical and morphologic characteristics of derived fibroblasts were evident only when cells were grown within their 3-D matrices and were reminiscent of their in vivo counterparts. This approach is useful in studying stromal development and permissiveness in tumor growth in ovarian and other epithelially-derived cancers.

\section{P18}

Analysis of False Negative Results with Frozen Section of Sentinel Lymph Node Biopsy for Breast Cancer R.M. Simmons, * D. Martins, P. Christos, E. Moseson, J. Pilavas, A. Colantino, J. Cataldi, M. Shafee. Weill Medical College of Cornell University, New York, NY.

INRODUCTION: Sentinel lymph node biopsy (SLNB) is used to detect breast cancer axillary metastases. The sentinel lymph node (SLN) is examined by serial sectioning and H\&E staining. Some surgeons send the SLN for intraoperative frozen section (FS) to minimize delayed axillary dissections. There has been concern that FS may discard nodal tissue and thus under-diagnose small metastases. This study examines whether evaluation of SLN by FS increases the false negativity rate of SLNB. METHODS: A retrospective analysis of SLNB from 805 patients seen at the Weill- Cornell Breast Service during the years 1997-2004 was conducted to determine the frequency of node positivity among SLNB subjected to both FS and permanent section versus permanent section alone. The chi-square test was used to compare the node positivity rate between these two groups. A logistic regression model was performed to estimate crude and adjusted odds ratios (OR) and $95 \%$ confidence 
intervals $(95 \% \mathrm{CI})$ for the effect of FS and permanent section versus permanent section alone on final node positivity. RESULTS: FS was performed in 373 patients and permanent section was performed in all 805 patients. Among patients undergoing both $\mathrm{FS}$ and permanent section $(\mathrm{N}=373)$, the final node positivity rate (based on FS positive or permanent section positive) was $31.4 \%$ compared to $16.4 \%$ among patients undergoing permanent section alone $(\mathrm{N}=432)(\mathrm{OR}=2.3 ; 95 \% \mathrm{CI}=1.7-3.3 ; \mathrm{p}<0.0001)$. After adjustment for age at diagnosis, pathological tumor diameter, estrogen and progesterone receptor status, tumor grade. nuclear grade, and predominant tumor histology in a multivariate logistic regression model, there remained an increased likelihood of a final node positivity for patients undergoing both procedures relative to permanent section alone (adjusted $\mathrm{OR}=2.6 ; 95 \% \mathrm{CI}=1.4-4.6 ; \mathrm{p}=0.001$ ). $\mathrm{CON}-$ CLUSIONS: There was a higher rate of SLN positivity in specimens evaluated by both FS and permanent section. Therefore, evaluating SLN by FS does not under-diagnose small metastases and thus produce a higher false negative rate. Intraoperative FS offers the advantage of less delayed axillary dissections.

\section{P19}

Comparing Resection Volume: Breast MRI Wire Localized Excisional Biopsy (MRIbx) vs. Mammogram Wire Localized Excisional Biopsy (Mammobx) S.H. Javid, ${ }^{1 *}$ J.W. Carlson, ${ }^{1}$ J.E. Garber, ${ }^{2}$ R.L. Birdwell, ${ }^{1}$ S. Lester, ${ }^{1}$ M. Golshan. ${ }^{1} 1$. Brigham and Women's Hospital, Boston, MA; 2. Dana Farber Cancer Institute, Boston, MA.

Introduction: Breast MRI has been implemented as a diagnostic tool in the early detection and management of breast cancer. Lesions identified by MRI but not amenable to conventional biopsy techniques require MRIbx. We reviewed our experience with MRIbx and its limitations. We hypothesized that more tissue would be resected with MRIbx compared to conventional Mammobx, since intraoperative radiography to confirm presence of the lesion isn't feasible. Methods: We reviewed consecutive cases of breast MRIbx from 2004 to 2006 performed by the breast service. MRI was performed in patients with either a synchronous breast cancer or significant risk factors. All patients first underwent mammogram and ultrasound. Lesions visualized solely by MRI underwent diagnostic MRIbx. The control group was comprised of consecutive cases that underwent diagnostic Mammobx during the same time period by the same surgeons. The volume of tissue resected, ratio of specimen:tumor volume (STVR), and log STVR were compared between groups. Results: Seventy-one patients, with a mean age of 48, underwent MRIbx. Eight patients $(11.2 \%)$ were BRCA 1 or 2 carriers. Ten $(14.1 \%)$ invasive breast cancers and eight cases $(11.3 \%)$ of DCIS were identified. The mean volume of tissue resected and $\log$ STVR was $42.1 \mathrm{~cm}^{3}$ and 1.63 , respectively (Table 1). Seventyone control patients, with a mean age of 55, underwent Mammobx. A density, mass or calcifications was seen in 69 patients $(97 \%)$. Twelve (16.9\%) invasive breast cancers and ten cases (14.1\%) of DCIS were identified. The mean volume of tissue resected and $\log$ STVR were $31.6 \mathrm{~cm}^{3}$ and 1.56 , respectively, which did not differ from MRIbx (Table 1). Conclusions: In patients undergoing breast MRIbx, $25 \%$ were found to have DCIS or invasive breast cancer. The volume of tissue resected, STVR, and $\log$ STVR in MRIbx did not significantly differ from Mammobx.

Table 1

\begin{tabular}{|c|c|c|c|}
\hline Measures* & MRIbx $(\mathrm{n}=71)$ & Mammobx $(\mathrm{n}=71)$ & $\mathrm{p}$ value** \\
\hline Specimen volume $\left(\mathrm{cm}^{3}\right)$ & 42.1 & 31.6 & 0.10 \\
\hline STVR & 594.8 & 375.8 & 0.59 \\
\hline Log STVR & 1.63 & 1.56 & 0.79 \\
\hline
\end{tabular}

*Measures represent mean values

${ }^{\star \star} p$ values calculated using two-tailed $T$ tests

\section{P20}

Increasing the Use of Minimally Invasive Breast Biopsy Techniques in a Community Based Teaching Hospital E. Penman, * D. DicksonWitmer, G. Pahnke, C. Whitney, D. Burbage, L. Konizer. Breast Center, Christiana Care Health System, Newark, DE.

Introduction: Minimally invasive breast biopsy techniques are being used with increasing frequency for patients with abnormalities seen on imaging or on physical exam. This review evaluated a community based teaching hospital's five year experience with performing minimally invasive breast biopsies.
This review coincides with the length of time that this community's comprehensive breast center has been open. Methods: A retrospective medical record review utilizing the surgical registry and breast biopsy registry at a community based teaching hospital was performed. Results: Records from July 1999 through June 2005 show that 23 surgeons and 3 radiologists performed breast biopsies in this community based teaching hospital. In FY 2000 , only $37 \%$ of the biopsies were performed utilizing a minimally invasive technique. In an effort to improve the care provided to patients presenting with a positive finding, the medical director and chair of the breast center leadership team embarked upon a massive campaign to increase the awareness and training of those physicians who continued to utilize an open biopsy method. Due to their efforts, in FY $2005,73 \%$ of the biopsies were performed utilizing a minimally invasive technique, an increase of slightly more than fifty percent. Conclusions: Education and training of general surgeons can increase the percentage of minimally invasive breast biopsies performed in a community based teaching hospital. Continuing education efforts will include bringing in a nationally recognized speaker at a grand rounds format, with the overall goal of increasing and maintaining the minimally invasive breast biopsy technique percentage to $90 \%$.

\section{P21}

Partial Breast Irradiation: Initial Experience with Intraoperative Radiotherapy Using the IntraBeam System in Selected Breast Cancer Patients R.L. Elliott,* M. DeLand, J.F. Head, M.C. Elliott, J. Duhon, S. Miller, A.J. Ostrowe. EEH Breast Cancer Research and Treatment Center, Baton Rouge, LA.

Introduction: Breast conserving surgery (BCS) is usually followed by total breast irradiation. However, recently there has been increased interest in partial breast irradiation (PBI). The rationale is that recurrence after BCS usually occurs at the site of the original tumor. Therefore, it is logical that if radiation is delivered to the tumor bed, better local control should be achieved. Methods: PBI should be easy to administer, convenient for the patient, free of many complications, inexpensive and practical for the community setting. Some of the techniques, such as the mammosite, require multiple visits to the radiation center and also another surgery to remove the balloon. The Veronesi's intraoperative radiotherapy (IORT) procedure is cumbersome, requires expensive special equipment, special operating rooms (OR), and it is not versatile, mobile or practical for the community setting. The IntraBeam system for IORT is versatile, easy to operate, safe, mobile and can be used in any OR without specially leaded rooms or other added expense. Results: We have performed the procedure to compliment segmental mastectomy in 33 patients. It was done in 30 patients as part of the original procedure and in 3 patients with re-exploration of the segmental mastectomy site. We have found it to be easy to use in both situations, but the techniques are slightly different. For specific reasons three patients later proceeded to total mastectomy, and 8 went on have total breast irradiation. The procedure serves as the radiation boost if total breast irradiation is indicated. Conclusions: Patient acceptance has been great and there have been no serious surgical or radiation complications. There were no wound infections, but most patients had seromas easily managed by aspiration. There have been no short-term local or systemic effects, such as depressed host immunity that is frequently seen after total breast irradiation. The follow-up is not long enough to assess the efficacy of preventing local recurrence.

\section{P22}

Patient Precautionary Behaviors and Perceptions of Lymphedema after Axillary Surgery M. Wright, * K.T. Morris, G.L. Giron, M.K. Sampson, K. Hurley, E.R. Riedel, K.J. Van Zee. Memorial SloanKettering Cancer Center, New York, NY.

Introduction: Lymphedema is the most feared complication of breast cancer surgery. With the adoption of sentinel lymph node biopsy (SLNB) to stage the axilla, it was assumed that the incidence of lymphedema would be near zero. We undertook this study to determine patients' perceptions of arm swelling after SLNB with/without completion axillary lymph node dissection (ALND) and to assess their use of precautionary measures to avoid arm swelling. Methods: We interviewed 505 patients who underwent their SLNB between 6/1/995/31/03. Time interval since SLNB was 2.7-7.1 yr (median, $4.6 \mathrm{yr}$ ). Patients were questioned regarding ipsilateral arm swelling and behaviors utilized in an attempt to avoid lymphedema. Associations between perceived arm swelling and potential factors were examined using Fisher's exact and Wilcoxon rank- 
sum tests. Results: Of the 505 patients, $316(63 \%)$ underwent SLNB alone, 189 $(37 \%)$ with ALND. Median age at operation was 55 yo for the SLNB group, 50 yo for ALND. Body-mass index was similar in the 2 groups. In the ALND group, 53/188 (28\%) reported current swelling in the ipsilateral arm compared with only $9 / 316(3 \%)$ in the SLNB group $(\mathrm{P}<0.0001)$. Factors associated with patient reported arm swelling were higher body weight $(\mathrm{P}=0.002)$, higher $\mathrm{BMI}$ $(\mathrm{P}=0.006)$, weight gain since surgery $(\mathrm{P}=0.04)$, and axillary surgery ipsilateral to the dominant arm $(\mathrm{P}=0.004)$. Regardless of extent of axillary surgery, most women utilized several precautionary behaviors (see Table). The behaviors most often avoided by both groups were intravenous catheter placement, blood pressure measurements, and blood draws in the ipsilateral arm. Conclusions: The perception of arm swelling is associated with body weight and weight gain since surgery. In addition, women with axillary surgery on their dominant side report a significantly higher incidence of arm swelling. With long-term follow-up, SLNB results in significantly lower incidence of patientperceived arm swelling than does ALND. Regardless of the low incidence of perceived arm swelling, the large majority of patients undergoing SLNB continue to dedicate significant efforts toward behaviors designed to reduce the risk of lymphedema.

Proportion of patients utilizing precautionary behaviors to avoid lymphedema

\begin{tabular}{|c|c|c|}
\hline Precautionary behaviors for ipsilateral arm & ALND & SLNB \\
\hline Avoids IVs & $187 / 189(99 \%)$ & $253 / 316(80 \%)$ \\
\hline Avoids blood pressure & $185 / 189(98 \%)$ & $185 / 189(98 \%)$ \\
\hline Avoids blood draws & $188 / 189(99 \%)$ & $259 / 312(83 \%)$ \\
\hline Avoids lifting children & $22 / 109(20 \%)$ & $36 / 209(17 \%)$ \\
\hline Avoids lifting suitcases & $70 / 180(39 \%)$ & $88 / 316(28 \%)$ \\
\hline Avoids housework & $6 / 178(3 \%)$ & $7 / 290(2 \%)$ \\
\hline Wears gloves while gardening/housework & $90 / 119(76 \%)$ & $123 / 176(70 \%)$ \\
\hline Avoids flying & $15 / 189(8 \%)$ & $13 / 315(4 \%)$ \\
\hline
\end{tabular}

\section{P23}

Atypical Ductal Hyperplasia: Improved Accuracy with the 11Gauge Vacuum-Assisted versus the 14-Gauge Core Biopsy Needle V. Sohn, ${ }^{*}$ Z. Arthurs, M. Eckert, J. Perry, J. Keylock, T. Brown. Madigan Army Medical Center, Tacoma, WA.

Intro: Percutaneous stereotactic core needle biopsy (SCNB) has become a widely used alternative to open surgical biopsy in patients with radiographically suspicious, non-palpable breast lesions. However, there still remains variable discordance of open excisional results when a diagnosis of atypical ductal hyperplasia (ADH) is rendered by SCNB. We sought to determine the benefits of upsizing biopsy needles from 14- to 11-gauge. Methods: Patients with ADH diagnosed by SCNB were included for analysis in this retrospective chart review. Patients without surgical excision were excluded. Mammographic description, number of needle passes, pathology results, and followup data were analyzed and compared to our previously published institutional results with the 14-gauge needle. Results: From June 1996 until July 2006, 4,579 SCNBs were performed at our tertiary medical facility. 78 of $88(89 \%)$ of patients diagnosed with $\mathrm{ADH}$ on SCNB with an 11-gauge vacuum-assisted needle underwent open surgical excision. Of these patients, $9(11 \%)$ were upgraded to ductal carcinoma in-situ (DCIS) while $5(6 \%)$ had infiltrating ductal carcinoma (IDC) for a total underestimation rate of $17 \%$. These results differ from our previously published series of 14-gauge SCNB which revealed an underestimation rate of $36 \%$. Mean age of our patients was 58 years, with $71(82 \%)$ undergoing percutaneous biopsy for microcalcifications discovered on routine mammography. Most common excisional diagnosis was focal ADH or atypical lobular hyperplasia (ALH) in $44(56 \%)$ patients. Mean number of passes obtained at time of biopsy, mean age of patients, and characteristic radiographic abnormality were similar for malignant and benign diagnosis. Conclusion: There is an improved accuracy and reduced sampling error with the larger, 11-gauge SCNB technique. The latest, even larger 8-gauge core needle may further improve accuracy and ultimately reduce the need for unnecessary surgical intervention. Until further elucidated, surgical excision should continue to serve as an adjunct diagnostic procedure in these patients as there still remains a risk of associated malignancy with $\mathrm{ADH}$.

\section{P24}

Desmoid Tumors (Fibromatosis) of the Breast: A 25-year Experience H.B. Neuman, ${ }^{*}$ E. Brogi, A. Ebrahim, M.F. Brennan, K.J. Van Zee. MSKCC, New York, NY.

Background:Breast desmoid tumors are rare and often clinically mistaken for carcinoma. We reviewed our institutional experience with breast desmoids Methods:A search of the pathology and sarcoma prospective databases from 1982-2006 identified 32 patients with pathologically confirmed breast desmoids. Records were retrospectively reviewed. Results:The median presentation age was 45 years (range 22-76). Two patients $(6.3 \%)$ had a history of familial adenomatous polyposis and prior desmoids, $8(25 \%)$ of breast cancer, and $15(47 \%)$ of breast surgery (augmentation[2], reduction[2], excisional biopsy[3], and mastectomy[7, 1 tissue and 3 implant reconstructions]), with tumors developing a median 24 months (range 11-179) post-operatively. All patients presented with physical findings (28-palpable mass, 2-skin retraction, 2-implant capsule contracture). Mammography visualized the mass in $6 / 16$ and ultrasound in $9 / 9$. When performed in 4 patients with a negative mammogram, MRI supported a cancer diagnosis. In 15 patients with attempted needle biopsy, fine needle aspiration was inconclusive in $9 / 9$ and core demonstrated a spindle cell lesion in 6/7. All desmoids underwent surgical excision. Median tumor size was $2.5 \mathrm{~cm}$ (range 0.3-15). Follow-up was available in 28 patients (median $28 \mathrm{mos}$ ). Eight patients recurred at a median of 15 months, with $7 / 8$ within 3 years; two had multiple recurrences. Patients who recurred were younger (median age 28 vs 46 years, $\mathrm{p}<0.05$ ). A trend toward more frequent recurrences in patients with positive margins $(\mathrm{p}=0.06)$ and larger tumors $(p=0.13)$ was observed but was not statistically significant. Recurrences were treated with surgery in $6 / 8$ patients, with 3 also receiving radiation. One patient received tamoxifen alone with complete response. Conclusion:Breast desmoid tumors often present as a palpable mass suspicious for carcinoma. Core biopsy may aid in operative planning. Surgery remains the primary therapy, with younger age and possibly positive margin status and larger size being associated with increased risk of recurrence. As not all patients with positive margins recurred, clinical judgment should be used prior to extensive and potentially deforming resections.

Recurrence After Initial Surgical Treatment, by Margin Status

\begin{tabular}{|c|c|c|c|}
\hline & Margin Positive & Margin Negative & Overall Recurrences \\
\hline Excisional Biopsy & $2 / 4(50 \%)$ & $1 / 3(33 \%)$ & $3 / 7(43 \%)$ \\
\hline Wide Local Excision & $1 / 2(50 \%)$ & $0 / 9(0 \%)$ & $1 / 11(9.1 \%)$ \\
\hline Chest Wall Resection & $2 / 3(67 \%)$ & $1 / 6(17 \%)$ & $3 / 9(67 \%)$ \\
\hline Axillary Resection & 0 & $1 / 1(100 \%)$ & $1 / 1(100 \%)$ \\
\hline Overall Recurrences & $5 / 9(56 \%)$ & $3 / 19(16 \%)$ & $8 / 28(29 \%)$ \\
\hline
\end{tabular}

\section{P25}

Body Image and Quality of Life perception in breast surgical patients H. Medina-Franco, * M.N. Garcia-Alvarez, D. Arcila, C. Trabanino. Surgery, National Institute of Medical Sciences and Nutrition, Mexico City, Mexico.

Background: Quality of Life (QoL) has become a standard measure in assessing medical and surgical interventions. The objective of this study was to compare the quality-of-life (QoL) and body image perception (BIP) differences between patients who underwent lumpectomy (L) for benign lesions or breast conservative surgery (BCS), modified radical mastectomy (MRM), and mastectomy with breast reconstruction (MRMBR) for breast cancer. Methods: All patients who underwent breast surgery from August 1st 2005 through June 30th 2006 were included. Self-administered questionnaire assessing BIP and QoL (SF-36) was assigned to all patients. Statistical analysis was performed with the aid of SPSS 12.0 statistical package. Correlation between variables was evaluated comparing means with independent-samples T-Test. Significance was considered at $\mathrm{P}<0.05$. Results: The interviewed sample comprised 202 female patients. BIP scale showed a mean score of $4.86 \pm 5.77$ for benign lesions and 8.84 \pm 7.59 for malignant lesions treated with BCS $(p=0.0001)$ evidencing favorable body image perception in the benign lesions group. For patients who underwent breast surgery for malignant lesions we found a trend to better BIP in those patients submitted to BCS $(p=0.073)$ and MRMBR $(p=0.087)$ versus those having a MRM. Regarding $Q 0 L$ there were significant differences in the following parameters: 1.Physical Function (PF) $(p=0.008)$ 
2.Physical Roll (RP) $(\mathrm{p}=0.004), 3$. Body Pain $(\mathrm{BP})(\mathrm{p}=0.002)$. 4. General Health $(\mathrm{GH})(\mathrm{p}=0.0001), 5$. Physical Scale Component $(\mathrm{PCS})(\mathrm{p}=0.0001)$ and 6 . Social Function (SF) $(\mathrm{p}=0.022)$ in favor of patients who underwent MRMBR vs. those who underwent MRM. There were no significant differences between BCS and MRM. Differences varied according to women age, educational level and marital status. Conclusions: This study supports the importance of the psychosocial impact of surgery in breast cancer patients altering BIP and QoL. Multimodal management emphasizing psychological support is imperative in breast cancer surgical patients, taking into account women age, educational level and marital status to choose the optimal surgical therapy.

\section{P26}

PEPTIDE YY (PYY) AND INOSITOL HEXAPHOSPHATE (IP6): EVALUATION OF NATURAL AND NOVEL COMPOUNDS FOR THE TREATMENT OF BREAST CANCER M.A. Robertson,

D.R. Riggs, * B.J. Jackson, C. Cunningham, D.W. McFadden. Surgery, West Virginia University, Morgantown, WV.

INTRODUCTION: PYY is a 36 amino acid peptide and a member of the peptide family of gut hormones containing pancreatic polypeptide and neuropeptide Y. Recent data shows a preponderance of PYY receptors in breast ductal cells. Our laboratory has shown that PYY can inhibit the growth of several forms of cancer in vitro. IP6 is a naturally occurring polyphosphorylated carbohydrate that is abundant in grains, cereals, nuts, and foods rich in fiber. IP6 has been shown both in vivo and in vitro to possess anti-tumor properties in breast, colon, and prostate cancers. This study examines the relationship and inhibition of breast cancer when treated in vitro with PYY and IP6. METHODS: The HTB26 breast cancer cell line was cultured using standard techniques and treated with PYY $(500 \mathrm{pmol} / \mathrm{mL})$, IP6 $(0.8 \mathrm{mM} / \mathrm{well})$, and the combination thereof. Cell viability was measured by MTT at 24,48 and 72 hours. Apoptosis was evaluated by Annexin V-FITC and results calculated using FACS analysis. VEGF production was measured in the cell supernatants by ELISA. Statistical analysis was performed by ANOVA. RESULTS: Decreased cellular proliferation was observed by the addition of PYY, IP6 and their combinations $(\mathrm{P}<0.001)$. Necrosis $(\mathrm{P}=0.008)$, early apoptosis $(\mathrm{P}=0.001)$ and late apoptosis $(\mathrm{p}=0.035)$ were all increased by the addition of combined therapy with PYY and IP6 when compared to controls. A decrease in early apoptotic activity was also observed by PYY alone ( $\mathrm{P}=0.001)$. Reductions in the VEGF concentrations were observed by PYY, IP6 and combination therapy $(\mathrm{p}<0.001)$. CONCLUSIONS: In vitro application of PYY and IP6 results in marked reductions in cellular proliferation of the breast cancer cell line HTB26. Combined therapy with PYY and IP6 exhibited modulation of both apoptotic and necrotic activity. Both PYY and IP6 significantly reduced VEGF concentrations, suggesting that in part their mechanism of action is that of anti-angiogenesis. Further studies are needed to understand completely the mechanisms by which PYY and IP6 exert their significant reductions in breast cancer growth in vitro, thereby leading to in vivo and future clinical trials.

\section{P27}

Differences in Stage IIIA Stage IIIB breast cancer in African American women M. Rizzo, ${ }^{1}$ S.G. Gabram, ${ }^{1}$ N. Hatchett, ${ }^{1}$ H.L. Bumpers, ${ }^{2}$ J. Okoli, ${ }^{2}$ O.W. Brawley, ${ }^{1}$ M.B. Lund. ${ }^{3}$ 1. Georgia Cancer Center for Excellence at Grady, Emory University, Atlanta, Georgia, United States, 30303, Atlanta, GA; 2. Georgia Cancer Center for Excellence at Grady, Morehouse School of Medicine, Atlanta, Georgia, United States, 30303, Atlanta, GA; 3. Epidemiology, Emory university, Rollins School of Public Health, Atlanta, Georgia, 30322, Atlanta, GA.

Introduction: Stage III breast carcinomas account for about $6-7 \%$ of all invasive breast cancers diagnosed annually in the US, equally distributed between IIIA (3.3\%) and IIIB (3.1\%) with inflammatory breast cancer accounting for $1-2 \%$. Locally advanced breast cancer (Stage IIIA) and inflammatory breast cancer (Stage IIIB T4d) are now recognized as two distinct clinical pathologic entities, characterized by different prognostic factors profiles and age-specific indices. The aim of this study was to compare age, axillary metastasis, nuclear grading and estrogen receptor status (ER) in women with Stage III breast cancer in an inner city Cancer Center serving a large African American population. Methods: Retrospective review was performed on all Stage III primary female breast cancers diagnosed and treated from 2000 to 2005 as recorded in our Hospital tumor registry. Results: Of the 684 primary female invasive breast cancers, 94 were Stage III $(13.7 \%)$ ages 27 to $93($ mean $=52.4)$ and $86.1 \%$ were African American. Of these 94, 43 were Stage IIIA, 33 were Stage IIIB and 14 inflammatory breast cancers. The remaining 4 cases had N3 lymph nodes metastasis (Stage IIIC). ER was unknown in 8 cases; 2 (IIIA), 5 (IIIB), and 1 (Inflammatory breast cancer). The age characteristics, ER status, nodal status and grading are summarized in Table 1. Stage IIIA and inflammatory breast cancer patients were younger than IIIB patients. ER was equally distributed among these three groups. ER negative patients were younger in Stage IIIA and Stage IIIB but not in inflammatory breast cancers. Although inflammatory breast cancer patients were overall younger and had higher nuclear grade, the incidence of axillary metastatic disease was higher in Stage IIIA patients. Conclusion: Axillary metastatic disease was more prevalent in Stage IIIA. This preliminary investigation support the finding that in African American women Stage III breast cancers are more prevalent and Stage IIIA may be worse than Stage IIIB. If confirmed, these findings would suggest Stage III breast cancers in African American women have a different clinical course.

Table 1. Age, ER status, nodal status and grading in Stage III breast cancer

\begin{tabular}{|c|c|c|c|c|c|c|}
\hline $\mathrm{N}($ Row $\%)$ & \multicolumn{2}{|c|}{ Stage IIIA $43(47.8 \%)$} & \multicolumn{2}{|c|}{ Stage IIIB $33(36.7 \%)$} & \multicolumn{2}{|c|}{$\begin{array}{c}\text { Inflammatory (T4d) } 14 \\
(15.6 \%)\end{array}$} \\
\hline \multicolumn{7}{|l|}{$\mathrm{AGE}$} \\
\hline Mean & \multicolumn{2}{|c|}{51.4} & \multicolumn{2}{|c|}{$57.3^{*}$} & \multicolumn{2}{|c|}{48.7} \\
\hline Median & \multicolumn{2}{|c|}{49.0} & \multicolumn{2}{|c|}{54.0} & \multicolumn{2}{|c|}{49.5} \\
\hline Range & \multicolumn{2}{|c|}{$27-74$} & \multicolumn{2}{|c|}{$33-85$} & \multicolumn{2}{|c|}{$33-65$} \\
\hline ER+ & \multicolumn{2}{|c|}{58.1} & \multicolumn{2}{|c|}{51.5} & \multicolumn{2}{|c|}{42.9} \\
\hline $\begin{array}{c}\text { Axillary } \\
\text { metastasis (\%) }\end{array}$ & \multicolumn{2}{|c|}{$92.7^{*}$} & \multicolumn{2}{|c|}{64.3} & \multicolumn{2}{|c|}{53.9} \\
\hline \multirow[t]{2}{*}{$\begin{array}{l}\text { High Nuclear } \\
\text { Grade }(\%)\end{array}$} & \multicolumn{2}{|c|}{51.4} & \multicolumn{2}{|c|}{57.9} & \multicolumn{2}{|c|}{$87.5^{*}$} \\
\hline & $\begin{array}{l}\mathrm{ER}+25 \\
(61 \%)\end{array}$ & $\begin{array}{l}\text { ER- } 16 \\
(39 \%)\end{array}$ & $\begin{array}{l}\mathrm{ER}+17 \\
(60.7 \%)\end{array}$ & $\begin{array}{l}\text { ER- } 11 \\
(39.2 \%)\end{array}$ & $\begin{array}{c}\mathrm{ER}+6 \\
(46.2 \%)\end{array}$ & $\begin{array}{l}\text { ER- } 7 \\
(53.9 \%)\end{array}$ \\
\hline \multicolumn{7}{|l|}{ AGE } \\
\hline Mean & 53.5 & $49.3^{*}$ & 60.2 & $55.7^{*}$ & 47.3 & 49.6 \\
\hline Median & 55.0 & 46.5 & 54.0 & 54.0 & 48.0 & 51.0 \\
\hline Range & $27-74$ & $33-63$ & $36-85$ & $33-84$ & $33-64$ & $36-65$ \\
\hline $\begin{array}{c}\text { Axillary } \\
\text { metastasis (\%) }\end{array}$ & $92.0^{*}$ & $93.8^{*}$ & 76.5 & 45.6 & 66.7 & 42.9 \\
\hline $\begin{array}{l}\text { High Nuclear } \\
\text { Grade (\%) }\end{array}$ & 40.0 & 57.1 & 60.0 & 57.1 & 80.0 & 100.0 \\
\hline
\end{tabular}

${ }^{*} p<0.05$

\section{P28}

Characteristics of BRCA1 and BRCA2 mutation carriers who chose prophylactic mastectomy J. Tchou, ${ }^{1 *}$ M. Sargen, ${ }^{1}$ S. Sonnad, ${ }^{1}$ B. Weber, ${ }^{2}$ K. Nathanson, ${ }^{3}$ S. Domchek. ${ }^{3} 1$. Surgery, University of Pennsylvania, Philadelphia, PA; 2. Glaxo Smith Kline, Upper Merion, PA; 3. Abramson Cancer Center, Philadelphia, PA.

Introduction: BRCA1 and BRCA2 mutation carriers have a $50-80 \%$ lifetime risk of breast cancer. Prophylactic mastectomy reduces this risk by $90 \%$. In the United States, more mutation carriers undergo prophylactic oophorectomy than prophylactic mastectomy to reduce their risk. However, once diagnosed with breast cancer, some will choose prophylactic mastectomy which often requires the collaborative planning between the surgical oncologist, plastic surgeon, medical and radiation oncologists. We hypothesize that early identification of this subgroup can improve coordination of their surgery and adjuvant therapy. The goal of this retrospective analysis is to identify significant clinical characteristics associated with these mutation carriers who chose prophylactic mastectomy. Methods: Chart review was performed on 141 breast cancer affected BRCA1 or BRCA2 mutation carriers who had undergone genetic testing at our breast cancer risk evaluation clinic between 1994 and 2005. Clinical characteristics were compared and analyzed between the groups who did and did not undergo prophylactic mastectomy (PM) using the chisquare or Fisher exact tests. Results: Of the 141 affected mutation carriers, 60 (43\%) underwent PM. The majority of these mutation carrier $(n=121,86 \%)$ underwent genetic testing after their breast cancer diagnosis. Of these 121 mutation carriers, 54 (45\%) underwent PM. We found that women who elected PM 
are significantly younger, have more family members affected with breast cancer, and more likely to choose mastectomy as their initial surgery treatment even though their tumors may be smaller than their counterparts (see Table). Discussion: Our results showed that disease-associated BRCA1 and BRCA2 mutation carriers who chose prophylactic mastectomy are characterized by several significant clinical characteristics. These characteristics suggest that this subset of women may be more risk averse. These women will likely benefit from early discussion of prophylactic mastectomy with emphasis on coordination of their definitive surgery, plastic surgery reconstruction options, and adjuvant therapy.

Clinical characteristics of 121 breast cancer-affected mutation carriers who had or had not undergone prophylactic mastectomy (PM)

\begin{tabular}{|c|c|c|c|c|c|}
\hline & PM & $\%$ & No PM & $\%$ & P-value \\
\hline Total & 54 & 45 & 67 & 55 & \\
\hline Median age of diagnosis & $40+1-7$ & & $44+1-10$ & & 0.01 \\
\hline \multicolumn{6}{|l|}{ Mutation Status } \\
\hline BRCA1 & 25 & 46 & 35 & 52 & 0.33 \\
\hline BRCA2 & 29 & 54 & 32 & 48 & 0.52 \\
\hline \multicolumn{6}{|c|}{ Initial Surgical treatment modality } \\
\hline BCT & 18 & 33 & 41 & 61 & 0.002 \\
\hline Mastectomy & 36 & 67 & 23 & 34 & \\
\hline \multicolumn{6}{|c|}{ No. family members affected by breast cancer } \\
\hline 0 & 4 & 7 & 19 & 28 & 0.003 \\
\hline $1-2$ & 33 & 61 & 29 & 43 & 0.051 \\
\hline 3-4 & 10 & 19 & 14 & 21 & 0.74 \\
\hline$>=5$ & 7 & 13 & 5 & 7 & 0.31 \\
\hline \multicolumn{6}{|l|}{ Tumor size } \\
\hline $\mathrm{T} 1$ & 24 & 44 & 28 & 42 & 0.77 \\
\hline T2 & 6 & 11 & 18 & 27 & 0.03 \\
\hline $\mathrm{T3}$ & 1 & 2 & 3 & 4 & 0.42 \\
\hline \multicolumn{6}{|l|}{ No. of positive Nodes } \\
\hline 0 & 26 & 48 & 31 & 46 & 0.85 \\
\hline $1-3$ & 5 & 9 & 8 & 12 & 0.65 \\
\hline$>3$ & 3 & 6 & 5 & 7 & 0.68 \\
\hline \multicolumn{6}{|c|}{ Bilateral prophylactic oophorectomy } \\
\hline yes & 32 & 59 & 39 & 58 & 0.9 \\
\hline no & 18 & 33 & 27 & 40 & \\
\hline
\end{tabular}

\section{P29}

Sentinel Lymph Node Biopsy in Breast Cancer: How Many Nodes Need to be Removed? E. Grubbs, ${ }^{1}$ K. Bennett, ${ }^{2}$ R. Westlund, ${ }^{3}$ F. Schuler, ${ }^{2}$ R. Bentley, ${ }^{2}$ L.G. Wilke, ${ }^{2}$ G. Georgaide, ${ }^{2}$ J.A. Olson, ${ }^{2}$ S.K. Pruitt. ${ }^{2}$ 1. MD Anderson Cancer Center, Houston, TX; 2. Surgery, Duke University Medical Center, Durham, NC; 3. Smith Kline Glaxo, Durham, NC.

Introduction: When performing sentinel lymph node biopsy (SLNBx) for the evaluation of breast cancer, all blue-sfained nodes as well as nodes with the highest radioactivity are routinely removed. The $10 \%$ rule is then usually employed to dictate how many additional sentinel nodes (SLNs) are removed. Collection of some of these SLNs can involve deep dissection within the axilla, risking added morbidity. The purpose of this study was to determine the need to remove multiple SLNs to accurately establish the diagnosis of metastatic breast cancer. Methods: A total of 2530 breast SLNBx procedures, using a combination of technetium-99 sulfur colloid and lymphazuran blue dye, were performed at our institution between 1/15/97 and 3/2/06. All pathology reports were retrospectively reviewed to identify patients with positive SLNs and to determine when, in the order of node removal, the first positive SLN was detected. Results: In 278 (11\%) of patients, the SLNBx procedure detected metastatic disease. The number of SLNs collected ranged from 1 to 8 . The presence of metastatic disease was established by removal of the first SLN in 79\% of patients, the second in $96 \%$, and the third in $99 \%$ (Table). Removal of more than 4 SLNs did not identify any additional patients with metastatic disease. SLN number 5 was positive in 6 patients and SLN number 6 was positive in 1 patient, but metastatic disease was detected in a higher order SLN in all 7 of these patients. Preliminary subset analysis evaluating lymphazuran blue SLN coloration, SLN radioactivity counts, and primary tumor characteristics suggests that these factors may identify specific patients in whom fewer than 4 SLNs can be removed without compromising the ability to accurately identify metastatic disease when it is present. Conclusion: When performing SLNBx for the evaluation of breast cancer patients, the collection of more than four SLNs is probably unnecessary. Limiting the number of SLNs collected may reduce morbidity associated with this procedure, without compromising accurate breast cancer staging.

Sensitivity of Removing a Given Number of Sentinel Nodes

\begin{tabular}{|c|c|}
\hline Sentinel Node Number & Fraction with Confirmation of Metastasis \\
\hline 1 & $79 \%$ \\
\hline 2 & $96 \%$ \\
\hline 3 & $99 \%$ \\
\hline 4 & $100 \%$ \\
\hline
\end{tabular}

\section{P30}

Outcome of Multiple Wire Localization for Larger Breast Cancers: When Can Mastectomy be Avoided? L.J. Kirstein, ${ }^{*}$ E. Rafferty, R. Moore, J. Rusby, M.C. Specht, K.S. Hughes, B.L. Smith. Surgical Oncology, Massachusetts General Hospital, Boston, MA.

Background: High-quality mammography may demonstrate extensive calcifications involved in a malignant process. When presented with this finding, it may be assumed that breast conservation is not feasible. In order to distinguish between patients who will have successful breast conservation and those better served by mastectomy, we examined rates of breast conservation and total number of procedures required as a function of number of localizing wires needed to delineate the lesion and size of the excised specimen. Methods: We reviewed 107 consecutive patients who underwent multiple wire localization for breast cancer and 102 controls with single wire localization. Number of localizing wires, volume and largest dimension of specimen, number of surgical procedures, and rates of breast conservation were scored. Results: $78.5 \%$ of multiple wire localizations had successful breast conservation compared to $91 \%$ of the single wire localizations. $23 / 84(27.4 \%)$ multiple wire patients required more than 1 excision to achieve clear margins (mean=2.2) with 18 requiring 2 procedures and 5 requiring 3 procedures. $31 / 93$ (33.3\%) single wire patients required 2 excisions. Of patients who went on to mastectomy, $6 / 23$ (26\%) underwent more than 1 excision before mastectomy. Of these, 5 had 2 procedures and 1 had 3 procedures prior to mastectomy. The mean volume of specimen excised in the multiple wire group was $53.7 \mathrm{cc}$ compared to $37.8 \mathrm{cc}$ for the single wire group. The greatest dimension of the specimen in the multiple wire group averaged $5.6 \mathrm{~cm}$ compared to $4.9 \mathrm{~cm}$ for the single wire group. Although specimens were larger for the multiple wire group, 91/107 (85.0\%) were removed through a single incision. Conclusion: The majority of patients whose mammographic lesions require multiple localizing wires for excision can avoid mastectomy. The extent of a mammographic lesion should not be an absolute indication for mastectomy. Although some patients will require multiple excisions to achieve breast conservation, multiple wire lumpectomy should be considered as an alternative to mastectomy.

\begin{tabular}{|c|c|c|c|c|}
\hline $\begin{array}{c}\text { Number of } \\
\text { Localizing Wires }\end{array}$ & $\begin{array}{c}\text { Total } \\
\text { Patients }\end{array}$ & $\begin{array}{c}\text { Breast Conservation } \\
\#(\%)\end{array}$ & $\begin{array}{c}\text { Median Resection } \\
\text { Volume cc }\end{array}$ & $\begin{array}{c}\text { Average Specimen } \\
\text { Length cm }\end{array}$ \\
\hline 1 & 102 & $93(91.2)$ & 29.2 & $4.9(1.6-8.5)$ \\
\hline 2 & 88 & $70(79.5)$ & 44.1 & $6.1(2.0-11.5)$ \\
\hline 3 & 17 & $12(70.5)$ & 42.2 & $6.0(1.0-10.0)$ \\
\hline 4 & 2 & $2(100)$ & 33.3 & $4.8(4.5-6.5)$ \\
\hline
\end{tabular}

\section{P31}

Patterns of Care Analysis Among Women with DCIS in North Carolina L.C. Jackson, ${ }^{*}$ F. Camacho, R.T. Anderson, J.H. Stewart. General Surgery, Wake Forest University School of Medicine, Winston-Salem, NC.

Background: Ductal carcinoma in situ (DCIS) comprises approximately $25 \%$ of breast cancer diagnoses among women in the US. Although DCIS represents a growing subset of breast cancer cases, no registry-based analysis to date has evaluated patterns of care for women with DCIS. Objectives: The aim of the study is to delineate patterns of care for women with DCIS as related 
to age, tumor characteristics, and race/ethnicity. Further study goals include the identification of predictors of breast conserving surgery (BCS), adjuvant radiation, hormonal therapy, and breast reconstruction after mastectomy. Methods: The North Carolina Cancer Registry (NCCR) was queried for primary DCIS treated in 1998 and 1999 ( $n=1905)$. Logistic regression analysis was used to define the determinants of patterns of care. Results: Thirty-five percent of the women in the study sample underwent mastectomy. Positive predictors of mastectomy included young age (age $<50$, OR 1.55, [95\% CI, 1.14-2.12]), and larger tumor size (greater than $2 \mathrm{~mm}$, OR 2.39, [95\% CI, 1.61-3.54]). Approximately $48 \%$ of women who underwent BCS received adjuvant radiation therapy. Factors associated with receiving radiation therapy following BCS include younger age (age $<50$, OR 2.11, [95\% CI 1.48-3.01]). About $18 \%$ of women who underwent $\mathrm{BCS}$ received adjuvant hormonal therapy. Positive predictors of receiving adjuvant hormonal therapy following BCS included age of 50-60 yrs (OR 2.17, [95\% CI, 1.37-3.46]) and the receipt of radiation therapy (OR 3.60, [95\% CI, 2.55-5.08]). Approximately $28 \%$ of women who underwent mastectomy received breast reconstruction surgery. Positive predictors of breast reconstruction following mastectomy include age $<50$ (OR 47.35, [95\% CI, 19.47-115.17]). Black ethnicity was negatively associated with receipt of breast reconstruction following mastectomy (OR 0.47, [95\% CI, 0.26-0.845]). Conclusions: Treatment strategies for primary surgical therapy for DCIS vary significantly by age. Inconsistencies exist within the usage of adjuvant radiation therapy following BCS in women with DCIS. Variations in approaches to reconstructive surgery following mastectomy may be related to age, ethnic/cultural norms, and/or economic constraints.

\section{P32}

Sentinel Lymphadenectomy in Breast Cancer Patients $>70$ yo M. Hulvat, ${ }^{*}$ K. Yao, P. Rajan, J. Norton, G. Aranha. Surgery, Loyola University Medical Center, Maywood, IL.

Introduction: The management of the axilla is controversial in elderly patients $>70$ yo who have small breast cancers. Sentinel lymphadenectomy in this population of breast cancer patients may have different histopathologic results than in the non-elderly population. Methods: This is a retrospective review of breast cancer patients $>70$ yo who underwent sentinel lymphadenectomy at our institution between 2000-2005. Pearson $\chi 2$ analysis and the $Z$ test of column proportions were used to compare histopathologic findings of sentinel lymphadenectomy in breast cancer patients $<70$ yo versus $>70$ yo. Results: A total of 348 patients were analyzed. Ninety-six $(26.8 \%)$ patients were $>70$ yo. Of these 96 patients, 31 (31.6\%) had a tumor positive sentinel node (SN) and $65(68.4 \%)$ were tumor negative. Extranodal extension was seen in $12(12.5 \%)$ of the patients. The number of patients with a tumor positive $\mathrm{SN}$ was greater in the patients $<70$ yo vs $>70$ yo $(42.7 \%$ vs. $31.6 \%)$ and although not significant, it trended towards significance $(\mathrm{p}=0.057)$. The number of patients with tumor positive non-SNs was significantly greater in patients $<70$ yo vs $>70$ yo $(17.4 \%$ vs. $6.4 \%, \mathrm{p}=<0.05)$. When matched for tumor size $<2 \mathrm{~cm}$, the patients $<70$ yo had a significantly higher incidence of tumor positive non-SNs than patients $>70$ yo $(11.4 \%$ vs $3.5 \%, \mathrm{p}<0.05)$ and a higher incidence of tumor positive SNs ( $34.9 \%$ vs. $24.4 \%)$ although not significant. When patients with a tumor size of $1-2 \mathrm{~cm}$ were examined, the incidence of tumor positive $\mathrm{SNs}$ and non-SNs was significantly higher in patients $<70$ yo vs $>70$ yo $(41.1 \%$ vs. $23.1 \%$, $14.9 \%$ vs. $2.6 \%$ respectively, $\mathrm{p}<0.05$ ). Conclusion: Sentinel lymphadenectomy in elderly patients with small breast cancers shows a lower rate of nodal metastases. Larger studies are needed to determine if sentinel lymphadenectomy can be omitted for breast cancer patients $>70$ yo who have small cancers.

\section{P33}

Breast Papillomas in the Era of Percutaneous Needle Biopsy V. Sohn, * Z. Arthurs, J. Keylock, J. Perry, M. Eckert, D. Fellabaum, A. Wilson, D. Smith, S. Groo, T. Brown. Madigan Army Medical Center, Tacoma, WA.

Introduction: The significance of breast papillomas detected on sterotactic core needle biopsy (SCNB) remains unclear. While those associated with malignancy or atypia are routinely excised, no clear solution exists for benign breast papillomas. We sought to determine the indication for surgical excision, incidence of malignancy, clinical significance, and natural history of breast papillomas diagnosed on SCNB. We present the largest series in literature of breast papillomas. Methods: Patients with a diagnosis of papilloma on SCNB were included for analysis in this retrospective review. Patients were divided into benign, atypical, or malignant cohorts based on the initial core biopsy results. While patients with malignant or atypical features were encouraged to undergo surgical excision, no standard recommendation was given for benign papillomas. Mammographic features, number of mammograms obtained, method of initial diagnosis, pathology results, and follow-up data were analyzed. Results: From January 1994 until December 2005, 5,257 SCNBs were performed at our tertiary level medical center. 206 patients were diagnosed with 215 breast papillomas. $172(80 \%)$ papillomas were benign, $25(12 \%)$ were associated with atypia, and $15(7 \%)$ were associated with malignancy. Three benign papillomas $(1.7 \%)$ developed into cancer (1 infiltrating ductal carcinoma, 2 ductal carcinoma in-situ) over an average of 44 months. Average follow-up of those patients not undergoing excision for benign papilloma was 41 months although we had 92 patients with greater than 2 year follow-up and 57 patients with greater than 4 year follow-up. Of patients with atypia or malignancy associated with papilloma, there was a $26 \%$ and $87 \%$ associated rate of malignancy, respectively. Conclusion: Benign breast papillomas diagnosed by SCNB have an acceptably low risk of malignancy and do not need surgical excision. However, they should be considered high risk lesions which require serial radiographic monitoring (close follow-up). Papillomas associated with atypia or malignancy should continue to be excised.

\section{P34}

Comparison of cell yield and cytology for nipple ductal lavage and random periareolar fine needle aspiration biopsies performed in the same patients $\mathrm{A} . \mathrm{Ma},{ }^{*} \mathrm{~S}$. Milchgrub, R. Ashfaq, A. Leitch, M. Bowling, R. Rao, D. Euhus. U.T. Southwestern Medical Center, Dallas, $T X$.

BACKGROUND: Cytological atypia identified in nipple aspirate fluid or random periareolar fine needle aspiration (RPFNA) samples predicts an increased risk for breast cancer. Nipple duct lavage (NDL) has been proposed as an additional approach for obtaining very cellular samples for cytological and biomarker assessment, but lavage atypia has not yet been confirmed as a marker of breast cancer risk. We compared insufficient sample rates and cytological classifications in breast epithelial cell samples obtained by both RPFNA and NDL in the same women. METHODS: NDL and RPFNA were performed in 150 women: 67 with breast cancer and 83 unaffected women. The unaffected women were equally divided between those with a 5 -year Gail risk $=>1.7 \%$ and those with a 5 -year Gail risk $<1.7 \%$ Cytology was classified as insufficient cellular material for diagnosis (ICMD), normal, hyperplasia, or atypical. Proportions were compared using $\mathrm{Chi}$-square, and general agreement between cytological classifications was measured using the kappa statistic. RESULTS Cytological assessments were available for both NDL and FNA for 279 breasts from 145 women. The insufficient sample rate was $86(31 \%)$ for NDL and 61 $(22 \%)$ for RPFNA $(\mathrm{P}=0.02)$. Cytological atypia was identified by NDL in 68 $(24 \%)$ of the breasts and by RPFNA in $18(6 \%)$ of the breasts $(\mathrm{P}<0.001)$. The kappa statistic for general agreement between cytological classifications was $\mathrm{K}=0.09,95 \% \mathrm{CI}, 0.02-0.16 ; \mathrm{P}=0.006$ suggesting a poor correlation between NDL and RP-FNA cytology. CONCLUSIONS: RPFNA obtains sufficient cells in more patients than NDL. Based on cytological classifications, it appears that RPFNA and NDL are not sampling the same population of cells.

\section{Cytology distribution of NDL and RPFNA samples}

\begin{tabular}{|c|c|c|c|c|c|c|c|}
\hline & & \multicolumn{4}{|c|}{ NDL } & & \multirow[b]{2}{*}{ Total } \\
\hline & & Normal & Hyperplasia & Atypia & ICMD & & \\
\hline & Normal & 25 & 29 & 21 & 26 & & 101 \\
\hline \multirow[t]{4}{*}{ RPFNA } & Hyperplasia & 17 & 28 & 26 & 28 & & 99 \\
\hline & Atypia & 2 & 9 & 14 & 27 & & 18 \\
\hline & ICMD & 11 & 9 & 14 & 27 & & 61 \\
\hline & Total & 55 & 70 & 68 & 86 & 279 & 279 \\
\hline
\end{tabular}

\section{P35}

The Influence of Age on the Breast Surgery Decision-Making Process R.J. Bleicher, ${ }^{1 *}$ S. Hawley, ${ }^{2}$ S.J. Katz, ${ }^{2}$ M. Morrow. ${ }^{1} 1$. Surgical Oncology, Fox Chase Cancer Center, Philadelphia, PA; 2. University of Michigan, Ann Arbor, MI.

INTRODUCTION:Variations in mastectomy rates have been assumed to be a function of physician recommendations. We have previously found that 
greater use of mastectomy correlates with greater patient involvement in decision-making(Katz,JCO 2005). The influence of age on involvement in the decision making process and impact of age on treatment choice, however, is poorly described. METHODS:All women with DCIS and a random sample of $20 \%$ of those with invasive breast cancer were identified from the Detroit \& Los Angeles Surveillance, Epidemiology \& End Results registries and surveyed a mean of 6 mo after surgical treatment(range 1-14 mo). Inclusion criteria were age $\leq 79 \mathrm{y}$, stages 0 -III, and diagnosis in 2002 . Women with contraindications to breast conserving surgery were excluded from this analysis. Women were questioned about their involvement in decision-making regarding the choice of breast conservation surgery(BCS) or mastectomy, inquiring if this was patient-based, surgeon-based, or a shared decision. RESULTS:The survey response rate was $77 \%(n=1835) .1259$ patients had in situ or localized invasive disease without contraindications to either treatment option. The median patient age was $59.4 \mathrm{y}$ (range 27-79). Patient-based decision making was reported by $54 \%$ of those aged $\leq 40,40 \%$ of those $41-50,40 \%$ of those $51-60$, $39 \%$ of those $61-70$, and $37 \%$ of those $\geq 71(p=N S)$. The mastectomy rate for the entire cohort was $20.3 \%$. In univariate analysis, women $<41$ years old were more likely to undergo mastectomy than their older counterparts $(\mathrm{p}<0.05)$. In a logistic regression model controlling for level of decision involvement, primary lesion size, race, and education, no significant effect of age on surgical treatment was observed. Lower levels of education $(p=0.006)$ and nonwhite race $(p=0.013)$ were significant predictors of mastectomy even when controlling for tumor size. CONCLUSION:Older women participate in breast cancer surgical decision making to the same degree as their younger counterparts, and are no more likely to select mastectomy as a treatment option. Our data regarding the impact of education and race on mastectomy use indicates the need for improved educational strategies targeted toward these groups.

\section{P36}

Induction of HER-2/neu Reactive CD4 T Cells Identified in Sentinel Lymph Nodes and Peripheral Blood Following Vaccination with HER-2/neu Pulsed Type I Polarized Dendritic Cells in Patients with Early Breast Cancer G.S. Son, ${ }^{*}$ U. Koldovsky, S. Xu, M. Xu, R.E. Roses, H. Nisenbaum, P. Zhang, B.J. Czerniecki. The Harrison Department of Surgery Research, University of Pennsylvania, Philadelphia, PA.

INTRODUCTION: While peripheral blood (PB) lymphocytes are generally used for assessment of $\mathrm{T}$ cell responses following vaccine therapy, rarely is there opportunity to assess these responses in tumor draining sentinel lymph nodes (SLN). We tested CD4+ T cell responses in SLN and PB from patients with ductal carcinoma in situ (DCIS) treated in a neoadjuvant setting with HER2/neu pulsed dendritic cells (DC). METHODS: An IRB approved vaccine trial was initiated for patients with HER-2/neu over-expressing DCIS using a MHC Class I and II peptide pulsed IL-12 secreting DC. Patients were administered 4 weekly vaccinations prior to surgery. This is an initial immunologic assessment of the first 13 patients treated. For 10 patients both PB and SLN T cells were available for measurement of HER- $2 /$ neu IFN- $\gamma$ specific CD4+ T cells by ELISPOT using peptide-pulsed autologous immature and mature DC. Lymph node cell composition was also assessed by three color flow cytometry. RESULTS: $11 / 12$ patients' $P B$ and 9/10 patients' SLN lymphocytes demonstrated evidence of HER-2/neu specific CD4+ T cells. Lymphocytes from PB and/or SLN in 6 patients demonstrated HER-2/neu reactivity to multiple peptides. An absolute concordance between PB and SLN was observed in only one patient. In the remaining patients there were some differences in the $\mathrm{T}$ cell reactivities to various HER-2/neu peptides. In addition, the requirement for antigen presentation between PBL and SLN was apparent. PBL demonstrated greater responses to immature DC while SLN T cells required mature DC to demonstrate reactivity to HER-2/neu peptides. The SLN contained the expected percentage of $\mathrm{T}$ and $\mathrm{B}$ cells with some donor variability. CONCLUSION: Vaccination of DCIS patients with HER-2/neu pulsed DC1 leads to the development of HER-2/neu reactive CD4+ T cells that could be detected in both PB and SLN. A challenge remains to define the physiologic state of T cells in SLN. Defining the activity of anti-tumor T cells in SLN following DC vaccination may lead to improvements in anti-cancer vaccine therapy.

\section{P37}

Microinvasive Breast Cancer: The role of sentinel node biopsy A.A. Guth, ${ }^{*}$ C. Mercado, D.F. Roses, F. Darvishian, B. Singh, J.F. Cangiarella. NYU School of Medicine, New York, NY.

INTRODUCTION: DCIS with microinvasion is an invasive breast cancer, with the ability to metastasize. The role of routine axillary lymph node dissection(ALND) in these patients is controversial, given the relatively low reported incidence of axillary metastases. Sentinel node biopsy(SNB) has virtually replaced ALND in the management of early-stage invasive breast cancer, but its role in DCIS with microinvasion is uncertain. METHODS: A retrospective analysis was made of 59 consecutive patients with DCIS with microinvasion, from among the 3601 surgical breast cancer cases treated from 1991-2005. From 1991-1997, ALND was used to stage the axilla. Since 1998, SNB has been routinely used. IRB approval was obtained. RESULTS: Results are summarized in Table 1. Forty-four patients underwent axillary staging $(24$ ALND, $20 \mathrm{SNB})$. Nodal metastases were present in 5(11.4\%). Of the two who had ALND with positive nodes, both had one positive node each. Of the three who had SNB with positive nodes; one had isolated tumor cells(itc) in one of three SN and did not undergo completion ALND, one had itc in 2 sentinel nodes and a negative axillary dissection, and one had macrometastatic disease in two sentinel nodes and micrometastatic disease in the third, with two additional positive nodes on ALND. Patients with axillary metastases tended to be younger. While there was a trend towards association with larger volumes of DCIS, this was not an absolute association; 4 of the 6 patients with DCIS measuring $>$ $100 \mathrm{~mm}$. did not manifest axillary nodal disease. DCIS presenting as a clinical mass was not associated with a higher risk of metastatic disease. CONCLUSIONS: Axillary nodal metastases in the setting of DCIS with microinvasion is not uncommon, with an incidence of $11.4 \%$ in our series, and thus axillary staging is indicated. SNB accurately detects nodal metastases, avoiding the possible morbidity of ALND.

Table 1. Patient Characteristics

\begin{tabular}{|c|c|c|}
\hline Characteristic & $\begin{array}{c}\text { \# Patients(\%) (study } \\
\text { population=59 patients) }\end{array}$ & $\begin{array}{c}\text { Patients with Axillary Node } \\
\text { Metastases(n=5) }\end{array}$ \\
\hline mean age & 57.6 years & 51.6 years \\
\hline mean size DCIS & $34.8 \mathrm{~mm}$.(range: $4-120 \mathrm{~mm}$.) & $59.8 \mathrm{~mm}$.(range: $19-120 \mathrm{~mm}$.) \\
\hline mean nuclear grade & 2.5 & 2.8 \\
\hline comedonecrosis & $42(71 \%)$ & $4(80 \%)$ \\
\hline microcalcifications & $43(73 \%)$ & $5(100 \%)$ \\
\hline palpable mass & $16(27 \%)$ & $1(20 \%)$ \\
\hline $\begin{array}{c}\text { multifocal microinvasive } \\
\text { DCIS }\end{array}$ & $25(42 \%)$ & $2(40 \%)$ \\
\hline
\end{tabular}

\section{P38}

Perioperative Antibiotic Use in MammoSite ${ }^{\mathrm{TM}}$ Breast Brachytherapy: A Comparison from a Single Center to the ASBS Registry Trial L.A. Chapleskie, ${ }^{*}$ C.D. Carruthers, T.G. Frazier. Breast Surgery, The Bryn Mawr Hospital, Bryn Mawr, PA.

INTRODUCTION: The use of the MammoSite ${ }^{\mathrm{TM}}$ brachytherapy balloon catheter for accelerated partial breast irradiation (APBI) after lumpectomy in selected patients with early stage breast cancer reduces the adjuvant treatment time from 4-6 weeks to 5 treatment days. METHODS: The American Society of Breast Surgeons (ASBS) conducted a Registry Trial(RT) of eighty seven (87) institutions comparing patient demographics, infection rates and cosmesis. The RT does not mention perioperative antibiotic use. A retrospective chart review comparing similar parameters from ninety seven (97) consecutive patients treated at our single institution was completed. Intravenous cephalosporin (or levofloxacin for penicillin-allergic patients)was administered one hour prior to open $(\mathrm{OR})$ placement with continuation of an oral equivalent for ten(10) days postoperatively. Closed(office) procedure patients received an oral cephalosporin (or levofloxacin for penicillin-allergic patients)one day prior to procedure with instructions to continue the antibiotic for seven(7)days postprocedure. RESULTS: The RT reports a patient median age of 65 ,placement ratio $44.8 \%$ open(OR):55.2\% closed(office), $85.9 \%$ excellent/good cosmesis rate and an overall infection rate of $8 \%$. Our single center results reveal patient median age of 69 , placement ratio of $75.5 \%$ open(OR): $25.5 \%$ closed(office), $88.0 \%$ excellent/good cosmesis rate and a reduced infection rate of $2.0 \%$ in the 
open(OR) group and $0.9 \%$ in the closed(office) group (overall $2.9 \%$ ). CONCLUSION: A well demonstrated predecessor to poor cosmesis is infection. The RT reported overall an infection rate of $8 \%$ with acceptable cosmesis of $85.9 \%$. The use of perioperative antibiotics at our single center produced significantly reduced rates of catheter-related infection with excellent cosmesis.

\section{P39}

Measuring Individual Surgeon Performance in Breast Cancer Surgery L.E. McCahill, * T.A. James, J. Ratliff, R. Single, H. SheeheyJones, N. Messier, M. Stanley, D. Krag, S. Harlow. Surgery, University of Vermont, Burlington, VT.

INTRODUCTION: The purpose of this study was to evaluate a data collection system capable of auditing outcomes in breast cancer surgery. Our objective was to determine institutional performance standards for breast cancer surgery, and to allow for individual surgeon comparison in order to identify areas for improvement. METHODS: Breast cancer surgeons at a single institution were assessed for 1) total mastectomy (TM) rate, 2) rate of positive and close $(<1 \mathrm{~mm})$ margins following partial mastectomy (PM) and 3) mean number of operations to obtain appropriate margins following PM. Retrospective data from the medical record was collected by a third party, and then entered into a central data processing system capable of customized queries. RESULTS: 283 initial breast cancer operations for DCIS and IDC were performed between April 2003 and March 2005, among three surgeons. Overall, the TM rate was $26.2 \%(25.8 \%$ DCIS $\{\mathrm{N}=66\}$, and $26.3 \%$ IDC $\{\mathrm{N}=217\}$. The average pathologic tumor size was $1.29 \mathrm{~cm}$ for DCIS and $1.99 \mathrm{~cm}$ for IDC. Neither tumor size nor TM rate differed significantly among individual surgeons for DCIS or IDC ( $p>0.3$ ). The overall positive margin rate with PM was $11.7 \%$, and did not differ among individual surgeons $(14.8 \%, 5.3 \%$, and $11.4 \%$ respectively, $\mathrm{p}>0.3)$. Close $(<1 \mathrm{~mm})$ margins showed a statistically non-significant trend toward being surgeon dependent $(21.6 \%, 7.9 \%$, and $26.6 \%$ respectively, $p=0.06)$. The mean number of operations to achieve negative margins following PM was 1.33 operations for DCIS, and 1.13 operations for IDC. There was no significant variation between surgeons with respect to these rates. CONCLUSIONS: Quality indicators for breast cancer surgery are identifiable and can allow for benchmarking performance among surgeons. An overall mastectomy rate of $25 \%$, positive margin rate of $11 \%$, and mean of 1.2 operations to achieve clear margins following PM, were established as our institutional benchmarks. All surgeons performed within acceptable limits of these institutional benchmarks. Variation among surgeons, although limited in this study, may offer opportunity for individualized surgeon assessment and improvement in effort to optimize patient care.

\section{P40}

Microinvasive Breast Cancer: Clinically Significant or Curiosity? D. Maibenco, ${ }^{1 *}$ G. Dombi, ${ }^{2}$ R. Severson. ${ }^{3}$ 1. Surgical Specialists of Decatur, Decatur, IL; 2. Karmanos Cancer Institute, Detroit, MI; 3. Wayne State University, Detroit, MI.

INTRODUCTION: Prior to 1997, the definition of microinvasive breast cancer was not standardized. This study was designed to determine the frequency of lymph node metastases and survival associated with the definition of microinvasive breast cancer adopted in the 1997 AJCC staging manual. METHODS: A retrospective review of breast cancer cases in the SEER database from 1997 through 2003 resulted in the identification of 1,229 cases of female microinvasive breast cancer, $=/<0.1 \mathrm{~cm}$. Subset analyses were performed based on the number of lymph nodes examined, lymph node status, tumor grade, and age. Breast cancer survival was analyzed utilizing the Kaplan-Meier survival method and Cox regression. RESULTS: Among cases with examined lymph nodes, the frequency of lymph node metastases was $10.5 \%$. The frequency of lymph node metastases varied with grade (gr.1, 6\%; gr.3, 14\%, $\mathrm{P}=.05)$, and age $(<50,15 \% ;=>>65,11 \%, \mathrm{P}=.01)$. Five-year survival was $99 \%$ among lymph node negative cases and $95 \%$ among lymph node positive cases. In univariate analysis survival varied with the lymph node status, $\mathrm{P}=.004$, but not with grade, age, or number of examined lymph nodes. CONCLUSIONS: The frequency of lymph node metastases is sufficient to warrant evaluation of the lymph nodes in cases of microinvasive breast cancer. The impact of lymph node metastases on short term survival is statistically significant, but further analysis with additional follow-up is clearly warranted.

\section{P41}

Touch Prep Analysis versus Frozen Section for Intraoperative Evaluation of Sentinel Lymph Nodes in Breast Cancer K. Vanderveen, ${ }^{1}$ * R. Ramsamooj, ${ }^{2}$ R.J. Bold. ${ }^{1}$ 1. Surgery, University of California - Davis, Sacramento, CA; 2. Department of Pathology, University of California Davis, Sacramento, CA.

BACKGROUND: Sentinel lymph node biopsy (SLNB) has largely replaced axillary dissection (ALND) for axillary staging in early breast cancer due to its decreased morbidity and enhanced pathologic examination (e.g., serial sectioning and immunohistochemistry). However, intense pathologic evaluation is not routinely available intraoperatively; therefore, patients with SLN metastasis may require a second surgery for completion ALND. We hypothesized that a single-section approach (by frozen section [FS] or touch preparation analysis [TPA]) could be accurate for intra-operative SLN evaluation. METHODS: We performed a prospective, blinded study of patients undergoing SLNB for breast cancer from September 2004 - July 2006. SLNs were bi-valved along the long axis, underwent FS and TPA of the facing halves, followed by routine sentinel node processing (serial sectioning with hematoxylin/eosin staining). A single pathologist reviewed all study slides and was blinded to the permanent section interpretation. RESULTS: We analyzed 233 nodes from 119 patients. Overall, $19 \%$ of patients $(\mathrm{N}=23$ ) had positive nodes (mean size of metastasis $=9 \mathrm{~mm})$. Single-section FS and TPA had similar sensitivities $(0.65$ and $0.63, p=N S)$ and specificities $(0.995$ and $0.995, p=N S)$ for detection of SLN metastasis, yielding equivalent accuracies (95\%). All micrometastases $(<2 \mathrm{~mm} ; \mathrm{N}=4)$ were missed by both techniques. False positives were rare only 1 in each group. CONCLUSIONS: Single-section TPA and FS have similar accuracies and can be safely employed to identify the majority of patients with SLN metastasis. Although nodal disease will be missed in $1 / 3$ of cases, this is primarily micrometastatic disease for which the role of completion ALND remains controversial.

\section{P42}

SURGERY ON THE PRIMARY TUMOR IS ASSOCIATED WITH LONGER SURVIVAL IN STAGE IV BREAST CANCER PATIENTS AFTER CONTROLLING FOR SITE OF METASTASIS R.C. Fields, ${ }^{1}{ }^{*}$ C. Arthur, ${ }^{1}$ D. Jeffe, ${ }^{2}$ K. Trinkaus, ${ }^{1}$ R. Aft, ${ }^{1}$ J. Dietz, ${ }^{1}$ T.J. Eberlein, ${ }^{1}$ W. Glanders, ${ }^{1}$ J. Margenthaler. ${ }^{1}$ 1. Department of Surgery, Washington University School of Medicine, Saint Louis, MO; 2. Department of Internal Medicine, Washington University School of Medicine, Saint Louis, MO.

INTRODUCTION: The benefits of surgery in patients presenting with primary metastatic breast cancer is not clearly established. Patients with boneonly metastases can achieve long-term survival, but without excision of the primary tumor, uncontrolled local disease could lead to higher mortality. We hypothesized that surgical excision of primary tumors in patients with stage IV breast cancer would be associated with increased survival after controlling for site(s) of metastasis and other factors associated with survival. METHODS: A retrospective chart review at our cancer center identified 406 patients with stage IV breast cancer treated from 1996-2005; 184 received surgical excision of their primary tumor and 222 did not. One hundred one patients had bone-only metastasis, 380 had metastases to other organs \pm bone, and 26 had no metastases recorded. Patient characteristics were compared between groups using the chi-squared test. Cox regression models were used to calculate adjusted hazard ratios (HR), controlling for demographic and clinical factors associated with survival in univariate tests. The log-rank test compared the differences in survival between patients who did or did not receive surgery. RESULTS: Mean age at diagnosis of all 406 patients was $57.8 \pm 15.0$ years. There were no significant differences in patient demographics or clinical variables between patients who had and did not have surgery. After controlling for age, tumor grade, tumor histology, and site of metastases, all of which were significantly associated with survival in univariate tests and the multivariable model, patients who underwent surgery had longer median survival when compared with patients who did not have surgery (Figure; 31.9 v. 15.4 months, $\mathrm{p}<0.0001$; adjusted HR 0.56 [95\% CI 0.44-0.71]). CONCLUSIONS: Surgical excision of the primary breast tumor was associated with significantly longer survival in patients with metastatic breast cancer. This association was significant even after controlling for other factors associated with survival. Randomized clinical trials are needed to validate these retrospective findings. 


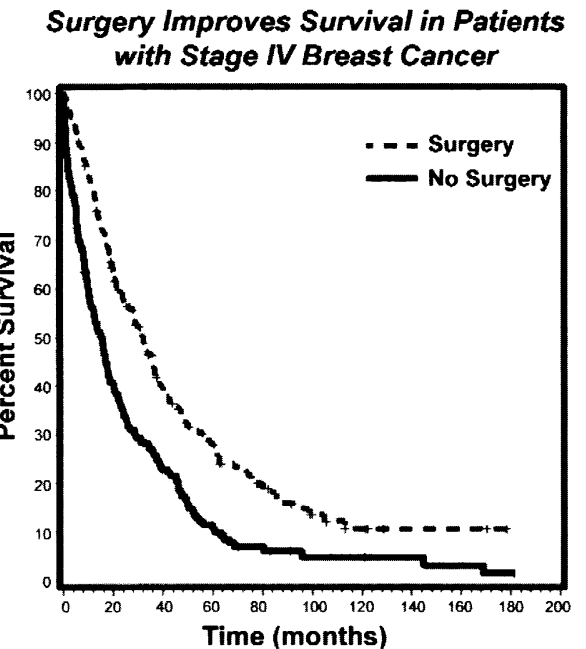

\section{P43}

Detection of enhancing lesions on contrast-enhanced MRI of the breast using real-time virtual sonography : fusion of MRI and sonography data $\mathrm{S}$. Nakano, ${ }^{1}$ M. Takasugi, ${ }^{1}$ T. Fukutomi, ${ }^{1} \mathrm{~K}$. Ohno, ${ }^{1}$ T. Mitake. ${ }^{2}$ 1. Aichi medical university, Aichi-gun, Aichi, Japan;

2. Hitachi medical corporation, Kashiwa, Chiba, Japan.

Introduction: Contrast-enhanced magnetic resonance imaging (MRI) is the most sensitive imaging method for detecting invasive breast cancer. Due to the limited specificity of MRI, we have tried to re-identify enhancing incidental lesions on sonography with the aim of assessing lesions immediately. We recently developed a real-time virtual sonography (RVS) system in cooperation with Hitachi Medical Corporation (Tokyo, Japan), enabling both breast sonography and contrast-enhanced MRI cutaway images of the same site to be displayed in real time. This system comprises a magnetic sensor fastened to a linear array probe, an image-processing workstation with display, and sonography equipment. The objective of this study was to assess the accuracy, reproducibility and clinical value of the RVS system for breast imaging. Materials and Methods: Results of sonography, contrast-enhanced MRI (1.5-T scanner, dynamic gadolinium-enhanced T1-weighted 3-dimensional flash sequence, supine position, flexible surface coil) and RVS were analyzed for 35 consecutive women with invasive breast cancer. Results were correlated with histopathological findings. Results: Analysis included 39 malignant foci in 38 cancerous breasts, of which 17 foci were palpable (median size, $14 \mathrm{~mm}$; range, $7-54 \mathrm{~mm}$ ). Sensitivity was $89 \%$ for sonography, $94 \%$ for MRI and $94 \%$ for the RVS system. Enhancing incidental lesions present in 5 cases (fibroadenoma, $\mathrm{n}=4$; small breast cancer, $\mathrm{n}=1$ ) were not seen using conventional techniques. Of these, 3 could be identified only on repeated sonography, but all 5 incidental lesions could be easily identified using the RVS system. The RVS system could project MRI imaging information onto a body surface correctly while checking sonography form image. Conclusion: The present results suggest that the RVS system offers excellent accuracy for identification of enhancing incidental lesions.

\section{P44}

Routine Operative Breast Endoscopy Reduces Local Failure in Early Stage Breast Cancer Y.C. Chang, ${ }^{2}$ J. Parker, ${ }^{1}$ W.C. Dooley. ${ }^{1 *}$ 1. Breast Institute, University of Oklahoma, Oklahoma City, OK; 2. Mackay Memorial Hospital, Taipei, Taiwan.

Introduction: The ability to endoscopically direct excision of proliferative disease in the breast at the time of therapeutic lumpectomy is now technically feasible. Clearly the endoscopic technique identifies more intra-luminal disease than other imaging techniques but the value of detecting this sub-clinical disease remains unclear. Methods: This is a retrospective review of a single surgeon's experience with endoscopically directed lumpectomy for early stage breast cancer from patients treated from 1/2000 to 2/2005. All patients were treated and followed using standard $\mathrm{NCCN}$ algorithms and data regarding local recurrence collected prospectively during follow-up care. Results: During this time period, 554 patients underwent breast conservation surgery for stage $0-2$ breast cancer with an attempt to perform endoscopy in association with therapeutic lumpectomy. Endoscopy was successful in identifying the duct connecting to the tumor in $84 \%$ (465) of cases. If endoscopy was successful, the + margin rate on the lumpectomy was $4.7 \%$. If endoscopy was not successful, the + margin rate at first lumpectomy attempt increased to $19.1 \%$. With an average follow-up of 3.7 years for the entire group, 5 of 89 patients had local failure when endoscopy was not successfully performed. Among those with successful endoscopy, 4 of 465 patients had local failure. This gives an annual hazard rate for local failure in this series for lumpectomy without endoscopic guidance of $1.3 \% / y$ r. and for lumpectomy with endoscopy of $0.2 \%$. Conclusions: Routine operative breast endoscopy leads to identification of intra-luminal proliferative disease undetected by other methods. The operative excision of this additional proliferative disease substantially reduces the early incidence of local failure in breast conservation.

\section{P45}

False negative sentinel lymph node biopsy; follow up of 391 sentinel lymph node negative breast cancer patients B.J. Wely, van, ${ }^{*}$ L.J. Strobbe, M.L. Smidt, I.M. Kievit, de. Canisius Wilhelmina Hospital, Nijmegen, Gelderland, Netherlands.

Introduction. The axillary recurrence rate following tumor-negative sentinel lymph node biopsy (SLNB) for breast cancer remains a concern. In the meta-analysis of the validation phase with completion axillary lymph node dissection (ALND), false negative rates were 5,1\%[1]. The aim of the present study was to assess the axillary recurrence rate, to identify prognostic factors and to monitor survival following recurrence. Patients and Methods. Between January 1998 and December 2004, 591 clinically node negative breast cancer patients entered a prospective database. SLNB was performed in accordance to Dutch guidelines, following a 20 patients validation phase. Sentinel Lymph Node (SLN) positive patients (including micrometastatic disease and isolated tumor cells) underwent ALND, whereas SLN negative patients underwent no further axillary surgery. Results. In 4 out of 391 SLN negative patients nonSLN's found at SLNB did show metastasis, leaving 387 patients for analysis. After a median follow up of 52 months (range 20-110) 9 patients developed an axillary recurrence. Median interval between SLNB and recurrence was 27 months (range 4-63). In one patient pathology review of the SLN was discordant, showing micrometastasis. The false negative rate in this group (definition by Estourgie et al.[2]) was $6,0 \%$. The overall recurrence rate was $2,3 \%(9 / 387)$. Patient and tumor characteristics did not differ significantly between patients with or without an axillary recurrence. Surgeon experience had no detectable influence on the recurrences found. After a median follow up of 22 months post recurrence, one patient developed systemic disease. Conclusion The incidence of axillary recurrence after SLNB increases with a longer follow-up period. The false negative rate found in this study is as high as false negative rates in validation studies on SLNB. No prognostic factors for developing axillary recurrence could be identified. Axillary recurrences will continue to occur and close monitoring of SLN negative patients is imperative. References 1. Miltenburg DM et al. J Surg Res 1999; 84:138-142. 2. Estourgie SH et al.J Surg Oncol 2003; 82:141-142.

\section{P46}

Predictors of Occult Nipple Involvement to Aid Selection of Patients for Nipple-Sparing Mastectomy J.E. Rusby, ${ }^{1 *}$ E.F. Brachtel, ${ }^{1}$ J.S. Michaelson, ${ }^{1}$ M. Othus, ${ }^{2}$ F.C. Koerner, ${ }^{1}$ B.L. Smith. ${ }^{1}$ 1. Massachusetts General Hospital, Boston, MA; 2. Harvard School of Public Health, Boston, MA.

Background: Patients requiring mastectomy could be offered nipple-sparing mastectomy to enhance cosmetic outcome. However, patients whose preoperative work-up suggests a high probability of occult involvement of the nipple by tumor should have the nipple excised. All previous studies are retrospective and too small for rigorous statistical analysis of factors that predict occult nipple involvement. This large, prospective study evaluates preoperative information and builds a predictive model to aid surgical decisionmaking. Methods: 164 consecutive mastectomy specimens without overt nipple 
involvement by breast cancer were evaluated ( 125 therapeutic and 39 prophylactic from 128 patients). Demographic, pre-operative pathology and digital imaging information was collected. At the time of mastectomy, a $2 \mathrm{~cm}^{3}$ nipple specimen was fixed and sectioned at $3 \mathrm{~mm}$ intervals. Two pathologists reviewed $\mathrm{H} \& \mathrm{E}$ sections. Logistic regression analyses were performed to identify factors associated with occult involvement and create a predictive model. Results: Nipple involvement was noted in $26 / 125(21 \%)$ of therapeutic mastectomies. 4 contained invasive ductal carcinoma, 2 invasive lobular carcinoma, 19 DCIS and 3 lymphovascular invasion. In addition, LCIS was noted in 12 nipples; these were classified as uninvolved. None of 39 prophylactic mastectomies had tumor in the nipple. In mastectomies for cancer, imaging diameter of the lesion, its distance from the nipple, and family history of breast cancer were significant on univariate analysis ( $\mathrm{p}=0.003,0.01$ and 0.03 respectively). On multivariate analysis, only lesion diameter and distance from the nipple on imaging remained as independent predictors $(\mathrm{p}=0.007$ and $\mathrm{p}=0.043)$ and were incorporated into a predictive model. The area under the ROC curve was 0.78 . Conclusions: This is the only prospective study of occult nipple involvement by breast cancer. It confirms that many patients undergoing mastectomy do not have occult nipple involvement. Furthermore, it is large enough to permit modeling by multivariate logistic regression and the development of a useful clinical tool to enhance patient selection for nipple-sparing mastectomy.

\section{P47}

Family History of Gastric Carcinoma is Prominent among Women Diagnosed with Lobular Breast Cancer B. Chikman, R. Lavy,* N. Siegelmann-Danieli, A. Halevy. Assaf Harofeh Medical center, Tel Aviv, Israel.

Background: The incidence of invasive lobular carcinoma (ILC) has been rising over the last decade. These tumors display unique biological features as compared with invasive ductal carcinoma (IDC). Sporadic reports suggest an association with a family history of gastric, prostate and rare malignancies, but clinical significance of these observations is still not obvious. In this study we assessed the family history among patients with ILC as compared with IDC. Methods: Database of all patients diagnosed with invasive breast cancer during the years 1992-2005 included 1467 patients and are the basis of this study. ILC and tumors with mixed ILC and IDC patterns were diagnosed in 173 women $(11.8 \%)$. Family history data was available in 141 patients with ILC and in randomly selected 377 patients with IDC. Statistical tests were performed against 2 -sided alternatives using SPSS computer program. Results: Of the 141 patients with ILC a family history of malignancies in first degree relatives was found in 46 pts $(32.6 \%)$ vs $62 / 377(16.4 \%)$ pts with IDC $(p<0.001)$. Distribution of the patients with family history of malignancies was as follow: breast cancer history $20 / 141(14.5 \%)$ vs $26 / 377(6.9 \%)$, $p=0.014$; ovarian cancer $0 / 141(0 \%)$ vs $6 / 377(1.6 \%), p=0.197$; gastric cancer $10 / 141(7.2 \%)$ vs $8 / 377(2.1 \%)$, $\mathrm{p}=0.012$. A family history of other malignancies among patients with ILC vs IDC was documented in 16/141 (11.3\%) and 22/377 (5.8\%), correspondently. The results were more prominent among women diagnosed with breast cancer at age $<65$ years with a family history of gastric cancer: $8.6 \%(8 / 93 \mathrm{pts})$ vs $1.9 \%(5 / 270), p=0.006$ correspondently. For comparison, breast cancer history among patients at age $<65$ was found among $13 / 93(14.0 \%)$ vs $23 / 270$ $(8.5 \%)$ pts, correspondently, $\mathrm{p}=0.158$. Conclusions: History of malignancy in a first degree family member is significantly higher among patients with ILC as compared with IDC. The family history of gastric carcinoma among women diagnosed with ILC, especially at age $<65$ years is as prominent as family history of breast cancer among those with IDC histology.

\section{P48}

Trends in Treatment and Survival of Inflammatory Breast Cancer K.E. Kilbride, ${ }^{*}$ A. James, P. Ashley, D.M. Miltenburg, M.M. Shabahang. General Surgery, Texas A\&M/ Scott \& White, Temple, TX.

Introduction: Inflammatory carcinoma accounts for $0.86 \%$ of all cases of breast cancer. Survival results are disappointing despite combined therapy. We sought to evaluate the trends in treatment and survival of this population at our institution over a 30-yr period. Methods: The institutional Tumor Registry Database was queried for patients with a diagnosis of inflammatory breast cancer from 1976-2005. Patient demographics, treatment, and recurrence/survival data were obtained. Statistical analysis was performed to determine survival data and prognostic factors. Results: 106 patients were diagnosed with inflammatory breast cancer from 1976-2005, accounting for $1.7 \%$ of all cases of breast cancer. All were female, with a white predominance. Median followup was 26 months (1-256 mo). The median age at diagnosis increased from 50 to 56 years over the 3 decades ( $24-91$ yrs). $96.3 \%$ patients were treated with chemotherapy. Modified radical mastectomy was performed in $78.5 \%$ of patients. External beam radiation was given to $71.0 \%$ of patients. In the cohort, $52 \%$ patients recurred, with mean time to recurrence 19 months. Disease free survival (DFS) at 5 years was $35 \%$, with median survival 40 months. Patients undergoing mastectomy had improved DFS when compared to those without surgery $(\mathrm{p}<0.01)$. Patients with greater than 6 lymph nodes had a significantly decreased DFS $(p=0.017)$. Hormone receptor positivity and tamoxifen also had a significant positive impact on DFS $(\mathrm{p}<0.02)$. Overall survival $(\mathrm{OS})$ at 5 years was $56 \%$, with median survival 52 months. When comparing the patients by decade, the patients in the first decade had a significantly lower 5-year OS compared to the other decades ( $28 \%$ vs $61 \%$ vs $55 \%, p=0.05$ ). Patients younger than 45 years had a significantly higher $O S(p=0.016)$. Surgical intervention, hormone receptor positivity, and tamoxifen significantly improved $O S(p<0.05)$ Grade and greater than 6 positive lymph nodes did not significantly impact OS Conclusion: Multimodality treatment including surgery and hormone receptor therapy can lead to a significantly improved overall and disease-free survival in patients with inflammatory breast cancer.

\section{P49}

A Prospective Study of Circulating Tumor Cells in Peripheral Blood and Disseminated Tumor Cells in the Bone Marrow of Patients Receiving Chemotherapy for Primary Breast Carcinoma C.D. Carruthers, ${ }^{1 *}$ L.A. Chapleskie, ${ }^{1}$ M.B. Flynn, ${ }^{1}$ M. Repollet, ${ }^{2}$ M. Hermann, ${ }^{2}$ L.W. Terstappen, ${ }^{2}$ G.V. Doyle, ${ }^{2}$ T.G. Frazier. ${ }^{1} 1$. The Comprehensive Breast Center at Bryn Mawr Hospital, Bryn Mawr, PA; 2. Immunicon Corporation, Huntingdon Valley, $P A$.

Introduction: Circulating tumor cells (CTCs) in peripheral blood (PB) and disseminated tumor cells (DTCs) in bone marrow (BM) of breast cancer patients may have prognostic significance. This was a prospective study designed to evaluate the presence and significance of CTCs and DTCs in patients treated for primary breast carcinoma with adjuvant chemotherapy. Methods: 10 consecutive pts were enrolled. BM aspirates were taken from the posterior iliac crest prior to definitive surgery, and $\mathrm{PB}$ samples were taken at 3 times during the course of therapy: prior to definitive surgery, prior to chemotherapy, and after completion of chemotherapy. These were analyzed for the presence of CTCs and DTCs using the Immunicon CellSearch ${ }^{\mathrm{TM}}$ system. Results were compared with historical prognostic indicators. Results: The median age was 49 (42-67). Tumor size ranged from $1.1 \mathrm{~cm}$ to $9 \mathrm{~cm} ; 9 \mathrm{pts}$ had IDC and 1 had ILC. 5 pts had lymph node metastases. 7 pts were ER+, 3 were PR+, and 2 were HER $2+.3$ were low NG, 5 were intermediate, and 3 were high. 1 pt had CTCs prior to surgery; this was a $2 \mathrm{~cm}$, intermediate NG, node -, ER/PR-, and HER2+ tumor. This pt also demonstrated CTCs prior to chemotherapy which were not present after chemotherapy. $1 \mathrm{pt}$ had DTCs prior to surgery; she had a $9 \mathrm{~cm}$ tumor, intermediate NG, ER+/PR-, HER2-, with massively replaced nodal metastases. This pt also demonstrated CTCs pre- and post-chemotherapy. $1 \mathrm{pt}$ had CTCs only prior to chemotherapy; this tumor was $1.7 \mathrm{~cm}$, node -, ER+/PR, and HER2-. Interestingly, $1 \mathrm{pt}$ had CTCs after chemotherapy who did not demonstrate CTCs or DTCs at any other point in therapy; the tumor was $2 \mathrm{~cm}$, node -, ER/PR+, and HER2-. Conclusions: In this small study, we demonstrate that the presence of CTCs can be followed throughout therapy for breast cancer. Further follow-up of these patients is needed to determine whether clearing of CTCs from peripheral blood following chemotherapy is a separate prognostic indicator. DTCs can also be measured in these pts, and may be a separate prognostic indicator as well.

\section{P50}

Multiple re-excisions in breast conserving surgery do not increase the risk of local recurrence M.J. O'Sullivan, ${ }^{1 *} \mathrm{G}$. Freedman, ${ }^{2} \mathrm{~T} . \mathrm{Li}^{3}{ }^{3}$ M. Morrow. '1. Surgical Oncology, Fox Chase Cancer Center, Philadelphia, PA; 2. Radiation Oncology, Fox Chase Cancer Center, Philadelphia, PA; 3. Biostatistics, Fox Chase Cancer Center, Philadelphia, PA.

Introduction Guidelines for breast conserving surgery (BCS) advise mastectomy if negative margins cannot be obtained after "reasonable" surgical attempts (Morrow M, CA Cancer J Clin, 2002). There is concern that the need for multiple re-excisions (MREs) reflects more extensive local disease that may be associated with excess local recurrence (LR). The aim of this study was to 
examine the effect of MREs on LR and to identify factors predictive of the need for MREs. Methods 2,950 patients undergoing BCS over 25 years were analyzed; 134 patients (group A) with $\geq 2$ re-excisions, 1554 patients with 1 re-excision (group B), and 1262 patients who had no re-excision (group C). An adequate margin was taken as $\geq 2 \mathrm{~mm}$. There were 386 patients with ductal carcinoma in situ (DCIS), 2142 with invasive ductal carcinoma (IDC), 243 with invasive lobular carcinoma (ILC) and 179 with other histologies. The median follow-up was 6.1 years. Chi-square or Fisher's exact tests were used for analysis. Results There were no significant differences between the three groups regarding tumor grade, site, lymphovascular invasion, receptor status, lymph node status and stage. The crude LR rate was similar in groups A, B and $\mathrm{C}$ at $5.2 \%(\mathrm{n}=7), 5.1 \%(\mathrm{n}=79)$, and $4.0 \%(\mathrm{n}=50)$ respectively $(\mathrm{p}=0.27)$. Women $>60$ years $(n=1277)$ were less likely to undergo MREs when compared to women $\leq 60$ years $(\mathrm{n}=1673 ; \mathrm{p}<0.0001)$. When compared with IDC $(\mathrm{n}=77 ; 3.6 \%)$, final histology of DCIS $(\mathrm{n}=32 ; 8.3 \% ; \mathrm{p}<0.0001)$ and ILC $(\mathrm{n}=17$; $7.0 \% ; p=0.0004)$ were more likely to result in MREs. Patients with DCIS were more likely to have MREs than those with $T 1$ or T2 tumors $(p<0.0001)$. The final margin of resection was more likely to be $>2 \mathrm{~mm}$ in patients having MREs $(\mathrm{n}=122 ; 91.0 \%)$ than in patients having a single excision $(867 ; 79.8 \% ; \mathrm{p}=0.001)$. Conclusions Multiple re-excisions in BCS do not impact on LR rates if negative margins are ultimately obtained. Conversion to mastectomy based solely on the number of excisions performed is not indicated. Subsets of patients more likely to require re-excision can be identified - these women may be candidates for a larger initial resection to avoid repeat surgery.

\section{P51}

Pre-operative axillary ultrasound can reduce the re-operation rate in node positive breast cancer M.J. O'Sullivan, ${ }^{1 *}$ P.M. Barrett, ${ }^{1}$ D. Evoy, E. McDermott, ${ }^{1}$ N. O'Higgins, ${ }^{1}$ C. Quinn, ${ }^{2}$ S. Pender, ${ }^{3}$ L. Coffey, ${ }^{3}$ A. O'Doherty, ${ }^{3}$ A.D. Hill. ${ }^{1} 1$. Department of Surgery, St Vincent's University Hospital, Dublin, Ireland; 2. Department of Pathology, St Vincent's University Hospital, Dublin, Ireland; 3. Department of Radiology, St Vincent's University Hospital, Dublin, Ireland.

Introduction Up to $30 \%$ of breast cancer patients undergoing sentinel node biopsy undergo a subsequent complete axillary dissection. Not all centers have access to intra-operative evaluation of a sentinel node biopsy. The aim of this study was to examine the role of axillary ultrasound in pre-operative axillary staging to determine if it decreased the axillary re-operation rate. Methods Over a nine month period, 232 patients with clinically node negative axillae, in whom a sentinel node biopsy was planned, had an axillary ultrasound performed by dedicated breast radiologists. Axillary core biopsy or fine needle aspirate (FNA) was performed on suspicious or indeterminate nodes on ultrasound. If either proved positive, axillary dissection was performed without preceding sentinel lymph node biopsy. Final axillary pathology was compared with initial axillary FNA or core biopsies. Results Axillary ultrasound correctly identified metastatic axillary involvement in 50\% (52/104) of patients. Of the 30 axillary ultrasound positive patients, 14 underwent FNA and $9(64 \%)$ were positive; 22 underwent axillary core biopsy and $17(77 \%)$ were positive; 6 underwent both axillary FNA and core and $5(83 \%)$ were positive. Of the 8 axillary ultrasound indeterminate patients, 6 underwent axillary FNA and none was positive; 3 underwent axillary core biopsy and $2(67 \%)$ were positive. Of the 169 negative axillary ultrasounds, $117(69 \%)$ were true negatives and $52(31 \%)$ were false negatives when compared to final histology. There was no false positive FNA or core biopsy in this study. Axillary re-operation rates were reduced by $10 \%$. Conclusions Axillary ultrasound in combination with axillary FNA or core biopsy is useful in staging the axilla and decreasing axillary re-operation rates in breast cancer.

\section{P52}

HER2 Status in Sentinel Lymph Node Negative Patients Independently Predicts Recurrence J.E. Lang, ${ }^{1 *}$ T. Jenkins, ${ }^{1}$ E.S. Hwang, L.J. Esserman, ${ }^{1}$ C.A. Ewing, ${ }^{1}$ Y. Lu, ${ }^{2}$ S.P. Leong. ${ }^{1}$ 1. UCSF Department of Surgery and Comprehensive Cancer Center, San Francisco, CA; 2. UCSF Department of Biostatistics and Comprehensive Cancer Center, San Francisco, $C A$.

Introduction: The oncogene HER2 is commonly assayed in breast cancer. Amplification of HER2 is associated with an unfavorable prognosis. We hypothesized that HER2 predicts recurrence in patients with a negative selective sentinel lymphadenectomy (SSL). We also sought to determine if HER2 status could predict a positive SSL. Methods: A retrospective review of breast cancer patients who underwent resection of the primary tumor and SSL between $12 / 97-04 / 03$ at a single institution was performed. Mean follow-up was 4.1 years. HER2 status was determined by immunohistochemistry and/or FISH. Clinical and pathologic data were analyzed by the Fisher Exact Probability Test. For multivariate analysis the Log-rank test was used. Results: Of the 438 patients, 307 had a negative SSL. Of these 307 patients, 53 were HER2 positive $(17.3 \%)$ and 198 were HER2 negative $(64.5 \%)$. HER2 results were either inconclusive or unavailable for 56 patients $(18.2 \%)$. When comparing the SSL negative/HER2 positive group to the SSL negative/HER2 negative group, HER2 positive status was associated with higher rates of loco-regional recurrence $(\mathrm{p}=0.038$, odds ratio $=5.3)$, systemic recurrence $(\mathrm{p}=0.0072$, odds ratio $=6.19)$, recurrence free survival $(\mathrm{p}=0.00008$, odds ratio $=6.95)$, and death $(\mathrm{p}=0.0072$, odds ratio $=6.19$ ). On multivariate analysis HER2 positive status was associated with worse recurrence free survival $(p=0.004$, hazard ratio 5.73), systemic recurrence $(p=0.0005$, hazard ratio $=7.13)$ and overall survival $(p=0.0034$, hazard ratio $=6.49$ ). Of the entire patient dataset, 346 were tested for HER2; there were 272 HER 2 negative patients of which 72 had a positive SSL. There were 74 HER 2 positive patients of which 21 had a positive SSL. Positive HER2 status was not associated with a higher likelihood of a sentinel node metastasis $(p=0.42)$. Conclusions: HER2 status is a significant predictor of adverse outcome as shown on both univariate and multivariate analysis, even in patients with a negative SSL. Therefore, a negative SSL should not be used as assurance of good prognosis in HER2 positive patients.

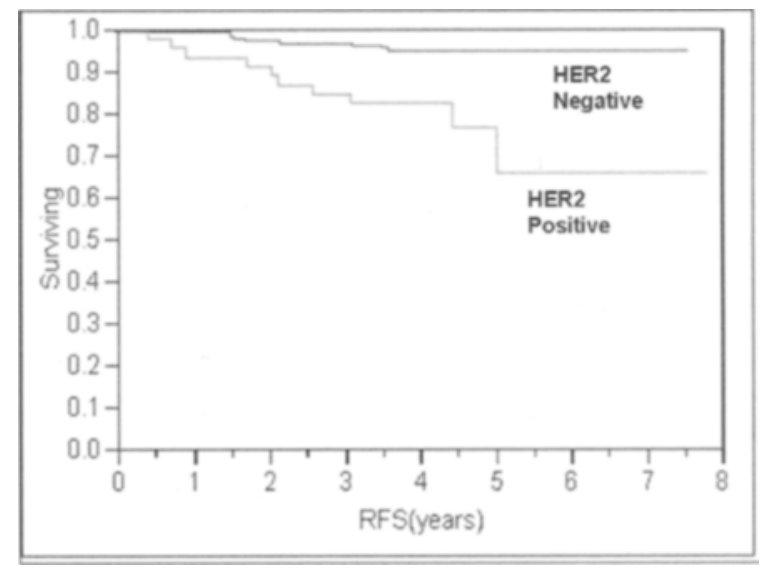

Kaplan-Meier Survival Curve for SSL negative patients stratified by HER2 status. RFS=recurrence free survival.

\section{P53}

Evaluation of CXCR4 and CCR7 expression as prognosis factors for invasive breast cancer M. Takasugi, ${ }^{1}{ }^{2}$ T. Fukutomi, ${ }^{1} \mathrm{~S}$. Nakano, ${ }^{1}$ K. Seki, ${ }^{2}$ M. Omatsu, ${ }^{3}$ T. Hasegawa. ${ }^{3}$ 1. Breast and Endocrine Surgery Division, Aichi Medical University Hospital, Aichi-Ken, Japan;

2. National Cancer Center Hospital Tokyo, Tokyo, Japan; 3. Sapporo Medical University, Hokkaido, Japan.

Background:Recently, the chemokine receptors CXCR4 and CCR7 have been reported to be potential prognosis factors for malignant tumors, particularly as markers that can predict lymph node metastases. Aithough the releavance of these markers for breast cancers has been suggested, only a few studies have been published on this matter. Subjact/Methods:Thirty-seven of 40 patients with primary invasive breast cancer who were operated on at National Cancer Center Hospital in January and February of 1997 but did not receive any preoperative treatment participated in this study. Among the 40 patients, three patients whose tissue specimens were not available for examination were excluded from the study. In order to determine the relationship between the expression of CXCR4 and CCR7 and the patients' clinicopathological factors, immunostaining was performed with 37 specimens from the 37 patients. Results:Thirty of 37 patients received modified radical mastectomies, whereas 
the rest of the patients underwent circular partial tumor resections(Bp) and radical axillary lymph node dissections. Nineteen patients were positive for axillarly lymphnode metastases, whereas 18 were negative. Among the 19 patients who were positive for axillary lymph node metastases, 12 patients were positive for CXCR4 expression and 11 patients were positive for CCR7 expression. Statistical analysis revealed a significant difference between the presence of CXCR4 expression and the presence of axillary lymph node metastases; no difference, however, was observed between the presence of CCR7 expression and the presence of the metastases. No correlation was found between the expression of CXCR4 and CCR7 and the pT stage or histological grade of the tumor. Conclusion:The results of this pilot study suggest that CXCR4 expression in breast cancer tissues is a useful marker for predicting axillary lymphnode metastases. It, however, is difficult to predict axillary lynph node metastases based on the presence of CCR7 expression alone. Furthermore, our results revealed that CXCR4 and CCR7 expression are marker that are independent of the pT stage and histological grade of the tumor.

\section{P54}

Quality Assessment in Breast Care: Three New Real-time Validation Programs for 2007 C.S. Kaufman, ${ }^{1 *}$ D.P. Winchester, ${ }^{2}$ L. Tafra, ${ }^{3}$ C.Z. Lee, ${ }^{4}$ J.K. Harness. ${ }^{5}$ 1. University of Washington, Bellingham, WA; 2. Evanston Northwestern Healthcare, Evanston, IL; 3. Anne Arundel Medical Center, Annapolis, MD; 4. C Z Lee \& Associates, Hudson, NY; 5. Comprehensive Breast Center, St. Joseph Hospital, Orange, CA.

Introduction: Since the establishment of the Commission on Cancer in 1922, there have been many attempts at setting standards and measuring breast care. Sadly, many published guidelines have languished waiting for widespread voluntary incorporation into practice. Responding to the need for ongoing breast care quality assessment, 16 major national organizations joined forces to create a new body to define standards and monitor breast cancer care both on-site and on-line. Methods: We describe the development of the National Approvals Program for Breast Care (NAPBC) that has created a trienial onsite approval program based on breast care standards. Also, two other organizations have established ongoing web-based quality assessment programs. The American Society of Breast Surgeons (ASBS) is developing a quality assessment tool for breast surgeons and the National Consortium of Breast Centers (NCBC) has developed a Quality Initiative that measures the components of care by breast centers. These three efforts complement each other. Results: The NAPBC is composed of major national organizations involved in breast care (TABLE 1). This group believes they are the best opportunity to develop uniform acceptable standards for breast care. These standards emphasize 1) maintenance of a national breast cancer database, 2) participation in research, 3) identification of a medical director and regular interdisciplinary breast conferences and 4) ongoing participation in an on-line quality assessment project with organizations such as ASBS and NCBC. These web based quality assessment tools are established to ask specific questions that reflect the quality of breast care while requiring the least amount of data mining. Data is confidential which allows private comparison with other similar centers or practitioners. Conclusions: Three new programs for 2007 will 1) create an on-site survey to measure standards of breast care (NAPBC), 2) create on-line tools to assess the quality of breast care (ASBS, NCBC), 3) expand the national breast cancer tumor registry, 4) encourage breast cancer research, 5) and may establish a mechanism by which outside organizations confirm quality breast care.

\section{Member Organizations National Approvals Program for Breast Care}

\begin{tabular}{|c|c|}
\hline American Cancer Society & $\begin{array}{c}\text { Applied Research Program, Division of Cancer Control and } \\
\text { Population Sciences, National Cancer Institute }\end{array}$ \\
\hline American College of Radiology & College of American Pathologists \\
\hline American College of Surgeons & Joint Commission on Accreditation of Healthcare Organizations \\
\hline $\begin{array}{c}\text { American Society of Breast } \\
\text { Diseases }\end{array}$ & National Consortium of Breast Centers \\
\hline $\begin{array}{c}\text { American Society of Breast } \\
\text { Surgeons }\end{array}$ & Oncology Nursing Society \\
\hline $\begin{array}{c}\text { American Society of Clinical } \\
\text { Oncology }\end{array}$ & Society of Surgical Oncology \\
\hline $\begin{array}{c}\text { American Society of Therapeutic } \\
\text { Radiation Oncology }\end{array}$ & \\
\hline
\end{tabular}

\section{P55}

The Predictive Value of Ductography and MRI in the Management of Nipple Discharge M. Morrogh, ${ }^{*}$ P.I. Borgen, T.A. King. MSKCC, New York, NY.

Although nipple discharge (ND) is associated with an underlying malignancy in up to $15 \%$ of cases, standard evaluation (PE, mammography, sonography) often fails to identify a causative lesion. The aim of this study was to evaluate clinical management patterns and determine the predictive value of ductography (DG) and MRI in this setting. Using ICD-9 codes we identified 451 patients who presented with a chief complaint of ND (1995-2005). Retrospective chart review revealed $306(68 \%)$ patients with clinically significant ND and negative standard evaluation. Of 306 patients, $187(61 \%)$ had DG and/or MRI, 35 (11\%) proceeded directly to major duct excision (MDE), and $84(28 \%)$ were followed clinically. Clinical features that predicted further evaluation (DG, MRI or MDE) included: post-menopausal status $(69 \%$ v $52 \%$, $\mathrm{p}<0.01)$, family history of breast cancer $(36 \% \vee 14 \%, \mathrm{p}<0.001)$, acute onset $(61 \%$ v $23 \%, p<0.001)$, bloody ND ( $66 \%$ v $12 \%, p<0.001)$, spontaneous ND $(85 \%$ v $20 \%, \mathrm{p}<0.001)$, single duct $\mathrm{ND}(68 \%$ v $12 \%, \mathrm{p}<0.001)$ and persistent ND $(73 \%$ v $15 \%, p<0.001)$. A total of 163 DG and 52 MRIs were obtained in 187 patients (36 had both); 140/187 (75\%) proceeded to surgery and/or biopsy, the remaining 47 were observed without event (median follow up $9.1 \mathrm{mos}$, range $0-124)$. DG was completed in $138 / 163(85 \%)$ patients and detected 12 cancers (CA) and 6 high-risk lesions (HRL) but failed to identify 4 CA and 4 HRL (PPV 18\%, NPV 47\%). MRI detected 7 CA and 3 HRL in 52 patients, but failed to identify $1 \mathrm{CA}$ and $2 \mathrm{HRL}$ (PPV 50\%, NPV 77\%). Among the 35 patients who proceeded directly to MDE, 5 CA and 3 HRL were identified. Of those that were followed clinically $1 / 84(1 \%)$ presented with an invasive cancer at $102 \mathrm{mos}$ (median follow up 6mos). Cancer was identified in 30/306 (10\%) patients presenting with clinically significant ND and negative standard evaluation. When positive, DG aids in lesion identification, however a negative DG does not reliably exclude an underlying CA or HRL. The higher predictive values of MRI in this setting may allow for improved patient selection and treatment planning however MRI should not replace MDE as the gold standard to exclude malignancy

\begin{tabular}{|c|c|c|c|c|c|c|c|}
\hline & No surgery & $\begin{array}{c}\text { Invasive } \\
\text { cancer }\end{array}$ & DCIS & HRL & $\begin{array}{c}\text { Benign } \\
\text { lesions }\end{array}$ & PPV & NPV \\
\hline $\begin{array}{c}\text { Ductogram*- Positive } \\
(\mathrm{n}=133) \text { - Negative } \\
(\mathrm{n}=25)-\text { Incomplete } \\
(\mathrm{n}=25) \S\end{array}$ & $\begin{array}{c}14(11 \%) 9 \\
(36 \%) 5 \\
(20 \%)\end{array}$ & $\begin{array}{c}6(5 \%) 2 \\
(8 \%) 0\end{array}$ & $\begin{array}{c}6(5 \%) 2 \\
(8 \%) 4 \\
(16 \%)\end{array}$ & $\begin{array}{c}6(5 \%) 4 \\
(16 \%) 2 \\
(8 \%)\end{array}$ & $\begin{array}{c}81(61 \%) 8 \\
(32 \%) 14 \\
(56 \%)\end{array}$ & $18 \%$ & $47 \%$ \\
\hline $\begin{array}{c}\text { MRI** - Birads 4/5 } \\
(\mathrm{n}=21)-\text { Birads 1/2/3 } \\
(\mathrm{n}=31)\end{array}$ & $\begin{array}{c}1(5 \%) 18 \\
(58 \%)\end{array}$ & $\begin{array}{c}3(14 \%) 1 \\
(3 \%)\end{array}$ & $\begin{array}{c}4(19 \%) \\
0\end{array}$ & $\begin{array}{c}3(14 \%) \\
2(6 \%)\end{array}$ & $\begin{array}{l}10(48 \%) \\
10(32 \%)\end{array}$ & $50 \%$ & $77 \%$ \\
\hline MDE alone (n=35) & 0 & $4(11 \%)$ & $1(3 \%)$ & $3(9 \%)$ & $27(77 \%)$ & - & - \\
\hline
\end{tabular}

$\S$ Excluded in analysis of PPV, NPV, sensitivity and specificity

* Sensitivity $69 \%$, Specificity $8 \%$.

** Sensitivity $77 \%$, Specificity $50 \%$.

$\mathrm{MDE}=$ Major duct excision. $\mathrm{HRL}=$ High risk lesion. $\mathrm{PPV}=$ positive predictive value. NPV = negative predictive value.

\section{P56}

A Specific VEGF-R Tyrosine Kinase Inhibitor Enhances the Antiproliferative Effect of Trastuzumab in ErbB-2 Overexpressing Breast Cancer Cells E. Kim, ${ }^{*}$ C.A. Lobocki, L. Dubay, V. Mittal. Providence Hospital and Medical Centers, Southfield, MI.

INTRODUCTION: Trastuzumab (Herceptin), a recombinant humanized anti-ErbB-2 monoclonal antibody, has been found to have potent antiproliferative effects in ErbB-2 overexpressing human breast tumors. Vascular endothelial growth factor receptor (VEGF-R) is overexpressed in human breast tumors and cell lines, and inhibition of VEGF-R activation has been shown to induce apoptosis in tumor cells. The purpose of this study was to evaluate the antiproliferative effect of the novel combination of trastuzumab and a specific VEGF$\mathrm{R}$ tyrosine kinase inhibitor in human breast cancer cell lines. METHODS: Increasing doses of trastuzumab $(0.125-2 \mathrm{nM})$ and a VEGF-R tyrosine kinase inhibitor $(1.25-20 \mu \mathrm{M})$ were tested alone and in combination. Three breast carcinoma cell lines were studied: two ErbB-2 overexpressing lines (BT474 and SKBR3) and one cell line that expresses low levels of the receptor (MCF7). Inhibition of growth was determined after 5 and 7 days of treatment by the 
MTT colorimetric assay, while apoptosis was assessed by quantitating Caspase- 3 levels after 48 hrs of treatment. RESULTS: A time and dose-dependent growth inhibition was demonstrated in all three cell lines tested with the VEGF-R inhibitor, while trastuzumab was only effective in the ErbB-2 positive cells. After 5 days, trastuzumab $(0.5 \mathrm{nM})$ inhibited cell growth by $35.6 \%$ and $31.9 \%$, and the VEGF-R inhibitor $(5 \mu \mathrm{M})$ by $37.4 \%$ and $54.3 \%$ in the BT474 and SKBR3 cell lines, respectively. The combination of trastuzumab and the VEGF-R inhibitor, at these doses, significantly inhibited growth in BT474 and SKBR3 cells by $60.4 \%$ and $75.8 \%$ (P $<0.001$, oneway ANOVA) compared to either agent alone. In addition, apoptosis was increased by 1.9 to 10.6-fold using the combination. CONCLUSIONS: Trastuzumab plus VEGF$\mathrm{R}$ inhibition decreased proliferation and increased apoptosis in ErbB-2 overexpressing breast cancer cell lines. Further studies in an animal model are warranted. This combination may also have important clinical therapeutic implications

\section{P57}

Intraoperative UItrasonography (US) Localization of Non Palpable Breast Cancers C. Ngo,* A.G. Pollet, S. Alran, B. Asselain, F. Thibault, R.J. Salmon. surgery, Institut Curie, Paris, France.

Introduction: Preoperative ultrasonography wire localization requires time and good coordination between departments of surgery and radiology. It is also a traumatic procedure for patients. The purpose of our study was to evaluate the accuracy of intraoperative US for localization of non palpable breast cancers by the surgeon himself. Patients and methods: 70 consecutive patients (from June 2006 to August 2006) undergoing surgical treatment of a non palpable breast cancer were prospectively evaluated. The US was performed in the operating room by the surgeon, on the patient in the operating position, just before draping, using a $12 \mathrm{MHz}$ linear probe. The localization of the lesion detected by US was marked on the skin. The findings were correlated with the preoperative US diagnostic. The margin status on the operative specimen was analyzed. When US didn't find the lesion, surgical excision was done under orthogonal localization. Results: Ultrasound correctly localized lesions at surgery in $94.3 \%$ of patients ( 66 of 70 patients). Correlation with measurements found on the preoperative ultrasound was $94.3 \%$. High BMI and large breast in cancers less than $5 \mathrm{~mm}$ diameter, usually associated, were the cause of failure of the intraoperatvive detection. Negative margins for invasive carcinoma were obtained in $98.6 \%$ of patients ( 69 of 70 ). The re-excision rate was $1.4 \%$ ( 1 of 70 patients). Conclusions: In this study, intraoperative ultrasound localization of non palpable breast cancer was always feasible. A $100 \%$ accuracy was found for cancers more than $5 \mathrm{~mm}$ diameter and good margins status was achieved. Intraoperative ultrasound localization is accurate, time efficient, improves the surgical scheduling, and saves money. Overall, it avoids a wire localization, which is painful and can lead to vasovagal episodes and/or hematoma.

\section{P58}

In Vivo Comparison of Lymphatic Drainage Patterns of Subareolar and Peritumoral Isotope Injection for Breast Cancer R.M. Fearmonti, ${ }^{1 *}$ I.W. Gayed, ${ }^{2}$ E. Kim, ${ }^{2}$ C. Warneke, ${ }^{3}$ I. Bedrosian, ${ }^{1}$ K.K. Hunt, ${ }^{1}$ E. Ghonimi, ${ }^{1}$ F. Meric-Bernstam, ${ }^{1}$ G.V. Babiera. ${ }^{1}$ 1. Department of Surgical Oncology, The University of Texas M.D. Anderson Cancer Center, Houston, TX; 2. Department of Nuclear Medicine, The University of Texas M.D. Anderson Cancer Center, Houston, TX; 3. Department of Biostatistics and Applied Mathematics, The University of Texas M.D. Anderson Cancer Center, Houston, TX.

INTRODUCTION: Subareolar injection of technetium labeled sulfur colloid to identify lymphatic drainage patterns for breast cancer poses an attractive alternative for sentinel lymph node (LN) detection. Controversy exists in the literature regarding the optimal injection site. Our study aimed to characterize the lymphatic drainage patterns within the same patient after subareo$\operatorname{lar}(\mathrm{SA})$ and peritumoral (PT) isotope injections. METHODS: In this prospective trial, 27 women with breast cancer (mean age 57 years; range 25-87 years) underwent sequential preoperative $\mathrm{SA}$ and $\mathrm{PT}$ injections of $0.5-2.7 \mathrm{mCi}$ of technetium- $-99 \mathrm{~m}$ filtered sulfur colloid $\geq 3$ days apart. Patterns of isotope uptake were reviewed independently by two radiologists, $\mathrm{A}$ and $\mathrm{B}$, each blinded to his own and the other radiologist's readings. Inter-reader agreement was assessed using the simple kappa coefficient $(\kappa)$ and injection success was assessed with the McNemar test. Reader agreement was examined in conjunction with drainage patterns to axillary and extra-axillary (supraclavicular and internal mammary) LNs. RESULTS: Almost perfect inter-reader agreement was observed on identification of axillary LN drainage by PT injection $(\kappa=.8750, \mathrm{p}=.0004)$ and substantial agreement with SA injection $(\kappa=.6167$, $\mathrm{p}=.0344$ ). SA injection appeared more likely to identify axillary LNs, while PT injection appeared more likely to identify extra-axillary LNs, though no statistically significant differences were found (Table). All patients with extraaxillary drainage on PT injection ( $\mathrm{n}=6$ patients) had only axillary drainage on SA injection. Dual drainage was observed for 6 patients with PT injection and 1 patient with SA injection. CONCLUSION: Our findings, confirmed by statistically significant inter-reader agreement, suggest that isotope injected in the SA location has a high propensity to drain to axillary LNs only. In patients for whom sentinel LN biopsy of extra-axillary sites is contemplated and/or may affect their radiation field, PT injection may be the preferable approach for lymphatic mapping.

\begin{tabular}{|c|c|c|c|c|}
\hline $\begin{array}{c}\text { Radiologist } \\
\text { Reading }\end{array}$ & $\begin{array}{c}\text { Site of Isotope } \\
\text { Injection }\end{array}$ & $\begin{array}{c}\text { Axillary Drainage } \\
\text { \#patients(\%) }\end{array}$ & $\begin{array}{c}\text { Extra-axillary Drainage } \\
\text { \#patients(\%) }\end{array}$ & $\begin{array}{c}\text { No Drainage } \\
\text { \#patients(\%) }\end{array}$ \\
\hline \multirow{2}{*}{$\begin{array}{c}\text { A (n=23 } \\
\text { patients) }\end{array}$} & SA & $20(87)$ & $1(4.3)$ & $2(8.7)$ \\
\cline { 2 - 5 } & PT & $13(56.5)$ & $5(21.7)$ & $5(21)$ \\
\hline \multirow{2}{*}{$\begin{array}{c}\text { B (n=20 } \\
\text { patients) }\end{array}$} & SA & $17(85)$ & $1(5.0)$ & $2(10)$ \\
\cline { 2 - 5 } & PT & $10(50)$ & $4(20)$ & $6(30)$ \\
\hline
\end{tabular}

\section{P59}

MammoSite ${ }^{\circledR}$ Effects on Post-Treatment Mammography J.J. Lee MD, ${ }^{*}$ L.C. Beran, MD, ${ }^{1}$ J.R. Paquelet, MD, ${ }^{2}$ W.C. Liang, DO, ${ }^{1}$ B.J. Sickle-Santanello, MD. ${ }^{1}$ 1. Departmenf of Surgery, Grant Medical Center, Columbus, OH; 2. Department of Radiology, Grant Medical Center, Columbus, $\mathrm{OH}$.

Introduction: Follow-up mammographic changes associated with whole breast irradiation are well-documented, and include scar, seroma, or hematoma. Biopsies are rarely needed to rule out occult cancer. Less well known are the mammographic changes associated with accelerated partial breast irradiation. This study was performed to evaluate imaging changes in brachytherapy with MammoSite ${ }^{\circledR}$ Radiation Therapy System. Materials and Methods: The records of 72 patients who had MammoSite ${ }^{\circledR}$ balloon catheters placed from June 2003 to March 2006 were retrospectively reviewed. 14 patients did not meet the MammoSite ${ }^{\circledR}$ criteria for brachytherapy, mostly secondary to inadequate balloon-to-skin distance. 3 patients did not have follow-up studies. Hence, mammograms of 55 patients were reviewed at $6,12,18$, and 24 months post-procedure and are the subject of this abstract. The BIRADS scores were tabulated for these follow-up intervals. Results: A total of 99 mammograms were evaluated over 3 year follow-up in 55 patients who received MammoSite ${ }^{\circledR}$ brachytherapy. 47 mammograms were evaluated at 6 month follow-up $(47 / 55,85.5 \%), 37$ mammograms at 12 months $(67.3 \%), 8$ at 18 months $(14.5 \%)$, and 6 at 24 months $(10.9 \%)$. The majority of the mammographic studies were assigned to the BIRADS category $2(51 / 99 ; 52 \%)$ and category $3(32 / 99 ; 32 \%)$, with the findings of scarring, hematoma or seroma, which were either stable or decreasing. Only 2 studies were BIRADS category 4 , and none were category 5 . The two category 4 mammograms were followed up with benign 6 month follow-up studies. 5 mammograms were assigned to the BIRADS category 0 at 6 months, for lack of prior films, collections resembling hematoma/seroma as confirmed by ultrasound, and abnormality which was observed with a benign 6 month follow-up study. There were 5 mammograms which were not assigned to the BIRADS category. No questionable mammographic features were biopsied. Conclusion: Imaging findings after breast brachytherapy appear to be similar to that of whole breast irradiation. Further study or biopsy can be avoided in most circumstances. The numbers, however, are small, and larger sample size and longer duration follow-up are needed to confirm this finding. 
Number of mammograms assigned to BIRADS category of according to follow-up months

\begin{tabular}{|c|c|c|c|c|}
\hline BIRADS Category & 6 month $\mathrm{N}=47$ & 12 month $\mathrm{N}=37$ & 18 month $\mathrm{N}=8$ & 24 month $\mathrm{N}=6$ \\
\hline 0 & 5 & 0 & 0 & 0 \\
\hline 1 & 1 & 1 & 2 & 0 \\
\hline 2 & 23 & 20 & 3 & 4 \\
\hline 3 & 15 & 12 & 3 & 2 \\
\hline 4 & 1 & 1 & 0 & 0 \\
\hline Unassigned & 2 & 3 & 0 & 0 \\
\hline
\end{tabular}

\section{P60}

Contemporary Analysis of Invasive Breast Cancer, 1987-2005 N. Coburn, ${ }^{1}$ E. Clarke-Pearson, ${ }^{2}{ }^{*}$ M. Chung, ${ }^{2}$ C. Law, ${ }^{1}$ J. Fulton, ${ }^{3}$ B. Cady. ${ }^{2}$ 1. Division of Surgical Oncology, Sunnybrook Health Sciences Centre, University of Toronto, Toronto, ON, Canada; 2. Brown University, Comprehensive Cancer Center, Rhode Island Hospital, Providence, RI; 3. Rhode Island Department of Health, Providence, RI.

Introduction: The highest rate of screening mammography is achieved in our state, according to the Behavioral Risk Factor Surveillance System, with 2004 data reflecting $86 \%$ screening in women over age fifty. Previous data from our state cancer registry demonstrated significant improvements in breast cancer presentation and mortality. We now update that data through 2005 to explore whether previous trends have stabilized or continued to improve. Methods: From 1987-2005, state cancer registry data regarding invasive breast cancer incidence, presentation, treatment, and mortality in 10,840 female residents with complete size data were analyzed for a variety of demographic and pathological factors. Data were analyzed in four time periods; 1987-1991, 1992-1995, 1996-1999, and 2000-2005. Because 1987 was not complete and 2005 data is not yet fully collected, these years were included in the nearest time period. Mortality reflects the number of cases of death due to breast cancer per 100,000 women. Student's $\mathrm{T}$ test and Chi2 tests were used for comparisons. Results: Statistically significant improvements continue to occur in age, size, pathologic grade, stage, node metastatic rate, and mortality of invasive breast cancer from 1987 to 2005 in this state (Table, $\mathrm{p}<0.05$ for all). Mortality in 1990 was $35.4 / 100,000$, which decreased to $23.9 / 100,000$ in 2003 . There has been no significant change in the incidence of invasive breast cancer over the four periods, with an overall, ageadjusted number of incident cases of 131.1/100,000 women. Conclusions: High screening rates continue to favorably impact the presentation of invasive breast cancer. From 2000-2003, there has been a remarkable 32.5\% decrease in breast cancer mortality, which should far exceed the goal of $50 \%$ reduction by 2015 which was set by the American Cancer Society.

Changes in Breast Cancer Presentation and Outcomes, 1987-2005

\begin{tabular}{|c|c|c|c|c|}
\hline & $1987-1991$ & $1992-1995$ & $1996-1999$ & $2000-2005$ \\
\hline Median Age (yrs) & 66 & 66 & 66 & 63 \\
\hline Median Size (mm) & 20 & 16 & 15 & 15 \\
\hline Node Positive & $31 \%$ & $28 \%$ & $27 \%$ & $24 \%$ \\
\hline$>3$ Nodes Positive & $15 \%$ & $13 \%$ & $11 \%$ & $9 \%$ \\
\hline Grade 1 & $12 \%$ & $13 \%$ & $16 \%$ & $19 \%$ \\
\hline Grade 3 & $58 \%$ & $47 \%$ & $39 \%$ & $35 \%$ \\
\hline Breast Conserving Therapy & $35 \%$ & $52 \%$ & $58 \%$ & $69 \%$ \\
\hline Mortality (per 100/000 women) & 37.2 & 33.6 & 29.6 & 24.6 \\
\hline
\end{tabular}

\section{P61}

Breast Carcinoma in Women 30 years and Younger Y.H. Wen, D.F. Roses, D.M. Axelrod, A.A. Guth, R.L. Shapiro, J.F. Cangiarella, N. Ziguridis, F. Darvishian, B. Singh.* New York University, New York, $N Y$.

Introduction: Breast cancer in adolescence and young adults is rare. The characteristics of breast carcinoma in women younger than 30 years are not well described. Methods: The institutional pathology database was queried for breast cancer patients younger than 30 years of age, for the years 1991-2006. Results: Seventy-five patients were identified with very early onset breast can- cer. One patient was 17 years old (1\%), 5 were between $20-24(7 \%)$, and 69 were between 25-30 years old (92\%). Data on race was available for 34 patients (65\% Caucasian, 18\% Black, 12\% Asian, 6\% Hispanic). Most (91\%) presented with a palpable mass. Family history was documented in 34 patients, and was positive in $15(48 \%)$. Three patients were pregnant at the time of diagnosis. Of the 75 cases, the diagnosis of adenocarcinoma, NOS was made by fine needle aspiration in 12 . The remaining 63 patients had histologic material for review. Fifty-five had invasive ductal carcinoma, 1 had invasive lobular carcinoma, 3 had medullary carcinoma, and 4 were DCIS. Of the 55 invasive ductal cancers, $33(60 \%)$ were poorly differentiated, $18(33 \%)$ were moderately differentiated, and $4(7 \%)$ were well differentiated. AJCC staging was assessed in 51 patients (4 Stage 0,19 Stage I, 11 Stage IIA, 6 Stage IIB, 7 Stage IIIA, and 4 Stage IIIC). Metastasis to the axillary lymph nodes was noted in 25 patients. The pathology archives showed that 6 patients recurred, 7 patients developed carcinoma in the contralateral breast, and 7 patients developed distant metastases to the liver (2), the brain (2), bones (2), and the spinal cord (1) during follow-up. Sixteen (53\%) of 30 cases with complete biomarker information, were ER negative, PR negative, HER-2 negative (triple negative), which is characteristic for basal-like carcinomas (Table 1). Conclusion: Breast carcinoma in women younger than 30 years usually presents as a mass and radiologic diagnosis is uncommon. Given the racial distribution of our surgical population there was an apparently high incidence of breast cancer in black patients. In this cohort of very young patients, the cancers were often poorly differentiated with a high incidence of triple negative tumors and poor prognosis.

\section{Biomarker Status in 30 Patients}

\begin{tabular}{|c|c|c|}
\hline Biomarker status & No. of cases & $\%$ \\
\hline ER+/PR+/Her2- & 10 & 33 \\
\hline ER+/PR+/Her2+ & 2 & 7 \\
\hline ER-/PR-/Her2+ & 2 & 7 \\
\hline ER-/PR-/ Her2- & 16 & 53 \\
\hline
\end{tabular}

\section{P62}

The utility of MIBG scintigraphy in patients with pheochromocytoma D. Y. Greenblatt, ${ }^{1}$ * S. Sehgal, ${ }^{2}$ J. Bianco, ${ }^{2}$ Y. Shenker, ${ }^{2}$ H. Chen. ${ }^{1}$ 1. Surgery, University of Wisconsin, Madison, WI; 2. University of Wisconsin, Madison, $W I$.

Introduction: 131-I-metaiodobenzylguanidine scintigraphy (MIBG) can be used to image neuroendocrine tumors including pheochromocytoma (PHEO). However, for patients with PHEO, cross-sectional imaging techniques such as computed tomography (CT) or magnetic resonance imaging (MRI) usually localize the tumor. MIBG is often obtained to rule out metastatic disease. We questioned the utility of MIBG in the diagnosis and management of PHEO. Methods: All patients who received MIBG at one academic center from 1999 to 2004 were identified. For the subset of patients who received MIBG in the work-up of possible PHEO, the following data were reviewed: age, sex, history, symptoms, results of biochemical tests, imaging studies, and other diagnostic studies, final diagnosis, and management. Results: A total of 59 patients received MIBG, including 26 patients for the evaluation of a possible PHEO. Biochemical testing was performed prior to MIBG in all 26 patients with possible PHEO. Of the 13 patients who had a negative biochemical test, 12 also had a negative MIBG. The one patient with a positive MIBG and negative biochemistry had a history of a prior paraganglionoma which was resected, and no new operation was performed on the basis of the positive MIBG. Of the 13 patients with positive biochemistry, 4 patients did not receive a final diagnosis of PHEO after complete evaluation. MIBG was negative in these 4 patients. The remaining 9 patients with positive biochemistry had their tumors imaged by CT or MRI and were found to have PHEO on pathology after surgical resection. For these 9 patients with a final pathologic diagnosis of PHEO, MIBG was positive in 7 , yielding a sensitivity of $78 \%$. MIBG did not identify any additional focus of disease in the 7 patients with positive MIBG and positive $\mathrm{CT} / \mathrm{MRI}$. Importantly, MIBG did not alter the surgical management of any patient in this series. Conclusions: MIBG does not alter the surgical management of patients with PHEO. Biochemical testing establishes the diagnosis, and CT/MRI is sufficient for tumor localization. Therefore, MIBG should be used selectively, such as for the rare patient with positive biochemistry and no visible tumor on cross-sectional imaging. 


\section{P63}

Fine-needle aspiration biopsy in pediatric endocrine tumor diagnosis: Are we afraid of needles? J. Sandoval, ${ }^{*}$ S.M. Inchauste, J. Miskulin, A.P. Ladd, F.J. Rescorla. Surgery, Indiana University School of Medicine, Indianapolis, IN.

Introduction: Endocrine malignancies are infrequent in children. We investigated the accuracy of fine-needle aspiration biopsy (FNAB) and its potential for improvement in the treatment of childhood endocrine cancers. Methods: Children with thyroid, parathyroid, pancreatic, and adrenal (excluding neuroblastoma) neoplasms were retrospectively evaluated over a 15 -year period at a single tertiary, pediatric hospital. Medical record data was reviewed for clinical presentation, workup, surgical intervention, and outcome. Results: Seventy-nine children underwent surgery for endocrine tumors. Pathology was consistent with 64 thyroid [benign (26), malignant (27), and MEN syndrome (11)], 8 adrenal [non-neuroblastoma malignant (5) and benign (3)], 4 pancreas, and 3 parathyroid (benign) neoplasms. Preoperative FNAB was utilized predominately for thyroid lesions $(\mathrm{n}=29,36.7 \%)$. One pancreatic mass was sampled by this technique (1.2\%). Aspiration for parathyroid and adrenal neoplasms was not performed. Complete concordance between cytology and histology existed in $70 \%$ of cases. Sensitivity and specificity of FNAB detecting malignancy was $80 \%$ and $83 \%$ with associated positive and negative predictive values of $85.7 \%$ and $76.9 \%$, respectively. Conclusions: We show the utility of FNAB for pediatric endocrine tumors of the thyroid gland. Despite the potential benefit of preventing unnecessary surgery in the management of endocrine neoplasms, these findings suggest this diagnostic modality is relatively underused in assessing childhood endocrine pathology.

\section{P64}

The Influence of Postoperative Morbidity on Long-term Survival Following Resection for Pancreatic Cancer V.L. Tsikitis, ${ }^{1}$ * T.J. Miner, ${ }^{1}$ S. Dessie, ${ }^{1}$ S.F. Khuri, ${ }^{2}$ W.G. Henderson. ${ }^{2}$ 1. Surgery, Brown medical School, Providence, RI; 2. Veterans Hospital, Brocton MA, MA.

IINTRODUCTION Despite advances in surgery, postoperative morbidity following pancreatic resection is still common. Although preoperative risk factors and low institutional volume have been reported to adversely affect 30 day perioperative outcomes, the impact of complications on long-term survival in patients with pancreatic cancer is incompletely described. METHODS National Surgical Quality Improvement Program (NSQIP) data from 1991-2002 was used to evaluate 1334 patients who underwent pancreatic resection for malignancy. Overall survival (OS) from the time of operation was examined using log rank and multivariate regression analysis. RESULTS The 30-day mortality and morbidity rate was $7.2 \%$ and $40 \%$ respectively. OS was not associated with previously reported preoperative risk factors (albumin $<2.5(\mathrm{p}=0.821)$, total bilirubin $>20(\mathrm{p}=0.944)$, operative time $(\mathrm{p}=0.998)$, and ASA classification $(1-3)(\mathrm{p}=0.976)$. Low volume institutions $(<20$ cases/year $)$ had higher $30 \mathrm{~d}$ mortality $(8 \%$ vs. $4.6 \%, p=0.01)$ and morbidity $(14 \%$ vs. $9.6 \% \mathrm{p}=0.01)$ compared to high volume centers. Complications within $30 \mathrm{~d}$ of operation reduced OS by $69 \%$ (median $432 \mathrm{~d}$ vs. $135 \mathrm{~d}, \mathrm{p}=0.001$ ) and diminished survival even in patients surviving complications at $30 \mathrm{~d}$ by $49 \%$ (median 440 vs. $225 \mathrm{~d}, \mathrm{p}=0.001$ ) and at $90 \mathrm{~d}$ by $32 \%$ (median $474 \mathrm{~d}$ vs. $322 \mathrm{~d}, \mathrm{p}=0.001$ ) or leaving the hospital alive by $33 \%$, (median $474 \mathrm{~d}$ vs. $320 \mathrm{~d}, \mathrm{p}=0.001$ ). OS was not associated with low versus high volume institutions (median $359 \mathrm{~d}$ vs. $361 \mathrm{~d}, \mathrm{p}=0.92$ ). $O S$ was independently associated with unplanned return to $\mathrm{OR}(\mathrm{p}=0.001)$, renal failure $(\mathrm{p}=0.001)$, cardiac arrest $(\mathrm{p}=0.001)$, systemic sepsis $(\mathrm{p}=0.001)$, reintubation $(p=0.001)$, and pulmonary embolus $(p=0.001)$. As the number of these complications accumulated, the chance of leaving to hospital alive diminished $(0$ complication $(91 \%), 1(76 \%), 2(49 \%), 3(22 \%), 4$ or more $(0 \%)$. CONCLUSIONS Independent of preoperative risk factors, postoperative complications following pancreatic resection adversely affects OS of patients that initially survive the complication. Although hospital volume played a role in short-term morbidity and mortality, it was not associated with longer-term overall survival.

\section{P65}

Pancreatic Cystic Neoplasms: The Role of Endoscopic UltrasoundGuided Fine Needle Aspiration in Surgical Decision Making

A.V. Maker,* L.S. Lee, C. Raut, T. Clancy, S. Russell, R. Swanson.

Brigham and Women's Hospital, Harvard Medical School, Boston, MA.

Introduction: Preoperative diagnosis of pancreatic cystic neoplasms is problematic. We evaluated our institutional experience with endoscopic ultrasound
(EUS) to determine the utility of fine-needle aspiration cytology (FNAC) in surgical decision making. Methods: All patients evaluated for pancreatic cysts with EUS FNA from 3/1996 - 10/2003 were included. Patients undergoing both preoperative EUS FNA and pancreatic resection were identified. FNAC read as malignant, suspicious for neoplasia, or atypical mucinous cells was classified as positive. Cytology with no malignant cells was deemed negative. FNAC read as indeterminate, atypical cells of undetermined significance, or possible contamination was classified as nondiagnostic. Results: Of 98 patients evaluated with EUS FNA, 29 underwent resection (see table). On final surgical pathology, $7 / 29$ lesions ( $24 \%$ ) were malignant ( 2 neuroendocrine tumors, 3 adenocarcinomas, 1 invasive intraductal papillary mucinous neoplasm (IPMN), and 1 metastatic uterine tumor), 4/29 (14\%) were benign ( 3 serous cystadenomas and 1 chronic pancreatitis), and 18/29 (62\%) were pre-malignant (10 mucinous cystic neoplasms and 8 IPMNs). Eight patients had positive FNAC. All 8 harbored malignant or premalignant lesions. Nine patients had negative FNAC, including $3(33 \%)$ with benign lesions and $6(67 \%)$ with premalignan lesions. Twelve of the 29 patients $(41 \%)$ had nondiagnostic FNAC with 11/12 (92\%) harboring a malignant or premalignant lesion. Sensitivity, specificity, positive predictive value, and negative predictive value were $32 \%, 100 \%, 100 \%$, and $19 \%$, respectively. FNA CEA levels were available for 12 patients. Six of 8 patients with mucinous tumors had CEA $>192 \mathrm{ng} / \mathrm{mL}$. All 8 FNAC were negative or nondiagnostic. Conclusions: In this series, a positive EUS FNAC of pancreatic cysts always indicated malignant or premalignant pathology, supporting the decision for resection. Further, the decision to proceed with nonoperative management should not be based on a negative or nondiagnostic FNAC alone, as $67 \%$ of negative and $92 \%$ of nondiagnostic specimens were associated with malignant or premalignant pathology.

Pre-operative EUS FNA Cytology of Pancreatic Cysts Compared to Final Surgical Pathology

\begin{tabular}{|c|c|c|c|c|}
\hline Surgical Pathology & \multicolumn{4}{|c|}{ EUS FNA Cytology (\# patients) } \\
\hline & Positive & Negative & Nondiagnostic & Total \\
\hline Malignant & 5 & 0 & 2 & 7 \\
\hline Benign & 0 & 3 & 1 & 4 \\
\hline MCN/IPMN & 3 & 6 & 9 & 18 \\
\hline Total & 8 & 9 & 12 & 29 \\
\hline
\end{tabular}

MCN- Mucinous cystic neoplasm, IPMN - Intraductal papillary mucinous neoplasm

\section{P66}

Cancer of the gastroesophageal junction, importance of surgical resection J.D. Amortegui,* J.L. Bell, T.E. Gaines. University of Tennessee - Knoxville, Knoxville, TN.

Introduction: The term carcinoma of the gastroesophageal junction (GEJ) has been applied to any lesion arising anatomically between the distal esophagus and the proximal stomach. Some authors have suggested these tumors should not be considered as one entity but may represent two or three types with different characteristics. The objectives of this study are to identify prognostic factors associated with survival and to determine if there are differences in the epidemiologic and clinical behavior among tumors arising in the distal esophagus, immediately at the GEJ and those in the gastric cardia. Methods: 10-year retrospective review of all carcinomas of the distal esophagus, GEJ and gastric cardia in our institution. Epidemiological and clinical variables were compared (age, gender, use of tobacco, use of alcohol, histological type, stage, therapeutic interventions, survival, and recurrence). Factors associated with survival were determined using a Cox regression analysis and the Kaplan-Meier method. Results: There were a total of 111 patients classified as follows: Distal esophagus $n=67$, GEJ $n=27$ and cardia $n=17$. Tumors of the distal esophagus had a higher incidence of squamous cell carcinoma compare to the other groups $(\mathrm{p}=0.004)$. The recurrence rate in the tumors of the cardia was higher $(\mathrm{p}=0.008)$. Resection of the tumor and node status were the only factors associated with survival ( $<<0.0001$ and $p=0.01$ respectively). In the group that had resection, age and node status were associated with survival $(p=0.04$ and $p=0.01$ respectively). Conclusion: Resection of the tumor and node status are the only factors associated with survival in this population. Although tumors of the distal esophagus, GEJ and cardia have similar epidemiological and clinical behaviors, those in the cardia have a higher recurrence rate. 


\section{P67}

Surgical resection of intrahepatic cholangiocarcinoma - a population based study J.C. Tan, ${ }^{1 *}$ N.G. Coburn, ${ }^{2}$ A. Kiss, ${ }^{3}$ N.N. Baxter, ${ }^{4}$ C.H. Law. ${ }^{2}$ 1. Division of General Surgery, University of Toronto, Toronto, ON, Canada; 2. Division of Surgical Oncology, Sunnybrook Health Sciences Center, University of Toronto, Toronto, ON, Canada; 3. Department of Research Design and Biostatistics, University of Toronto, Toronto, ON, Canada; 4. Division of General Surgery, St. Michael's Hospital, University of Toronto, Toronto, ON, Canada.

Introduction: Cholangiocarcinoma (CCA) is associated with poor survival rates and therapeutic nihilism. To date, there has not been an examination of the surgical management of CCA on a population level. Methods: Using the Surveillance, Epidemiology and End Results (SEER) database, we identified all patients with intrahepatic CCA diagnosed from 1988 through 2003. Tumors were defined as resectable if they were categorized as a single, unilobar lesion with no evidence of vascular invasion according to SEER. It was then determined whether patients received cancer directed surgery (CDS). Multivariable logistic regression was used to evaluate factors associated with CDS in patients with non-metastatic disease. The influence of CDS on overall survival (OS) was evaluated in resectable patients using Kaplan-Meier curves and Cox proportional hazards modeling. Results: Only 456 (11\%) of the 4,255 patients with intrahepatic CCA underwent CDS. On multivariable analysis, age $<70$ years $(\mathrm{p}<0.001)$ and non-Klatskin tumor $(\mathrm{p}=0.007)$ were associated with performance of CDS. There was also significant variation based on geographic location $(\mathrm{p}<0.001)$ and black race $(\mathrm{p}=0.034)$. Resectability was strongly associated with CDS $(p<0.001)$; however only $94(35 \%)$ of 266 patients deemed resectable underwent CDS. Of patients with resectable tumors, those who had CDS had significantly better survival than those who did not $(\mathrm{p}<0.0001)$, with median OS of 59 vs 9 months, and 5 year OS of $46 \%$ vs $2 \%$, respectively (figure 1). Conclusions: CDS is strongly associated with improved survival among patients with resectable CCA, with rates approaching that found in single institution studies. However, many apparently resectable patients are not offered CDS. Given the regional and racial variations in performing CDS, efforts must be made to address modifiable differences in practice patterns and patient access to specialized care.

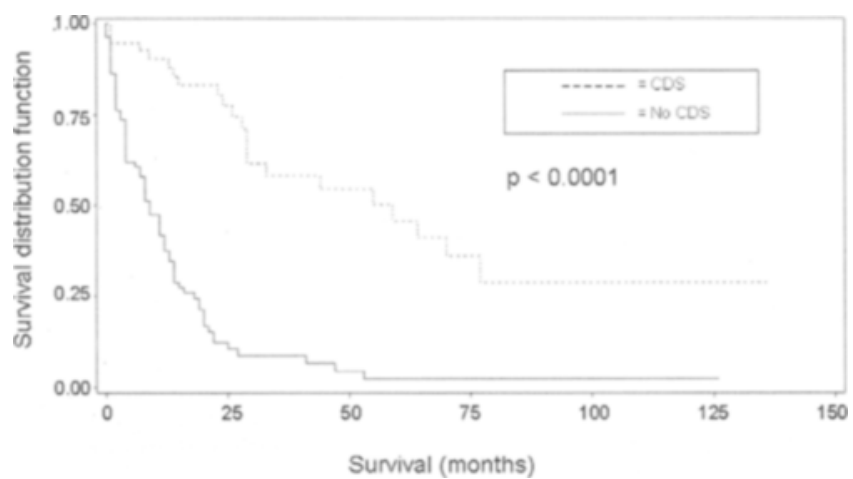

Figure 1. Influence of cancer directed surgery (CDS) on survival for patients under 70 years old with resectable intrahepatic cholangiocarcinoma

\section{P68}

Surgical issues after preoperative chemoradiation in locally advanced rectal cancer P. Delrio, ${ }^{*}$ A. Avallone, C. Guida, M. Campanile, R. Palaia, A. Petrillo, F. Tatangelo, G.L. Cascini, P. Marone, V. Parisi. INT Napoli - Colorectal Cancer Cooperative Group, Napoli, Italy.

INTRODUCTION Multidisciplinary neoadjuvant approach to locally advanced rectal cancer (LARC) is widely adopted. Many issues are raised in terms of postoperative complications and quality of life after surgery in these patients. We here present the surgical results after an highly effective regimen of neoadjuvant chemoradiation in LARC. METHODS Sixty four consecutive patients with histologically proven primary adenocarcinoma of the low rectum with an high risk of recurrence (T3N0, T4 or $\mathrm{N}+$ and/or
CRM $<5 \mathrm{~mm}$ evaluated by MRI) were included in the study. Preoperative chemotherapy (CHT) consisted of 3 biweekly cycles delivered immediately before radiotherapy on days 1 and 2 of the first, third and fifth weeks of RT. Drugs used were: Oxaliplatin, Raltitrexed, 5-fluouracil and levo-folinic acid. Conformal RT was delivered by a 3-field box technique, by a linear accelerator, to a total dose of 45 Gy (1.8 Gy x 5 fractions/week) Restaging and operations were performed at eight week after the end of preoperative treatment An anterior resection or an abdomino-perineal resection was performed on the basis of results of restaging. In cases of conservative surgery a loop ileostomy was used to divert faecal stream. RESULTS The response rate in this series was very high (almost $70 \%$ of complete response) with low morbidity and a high compliance for RT $(98 \%)$ and CHT $(93 \%)$. There were no post-operative deaths. No complications were recorded in 50 patients $(78,1 \%)$ and $14(21,9 \%)$ experienced early postoperative complications (among them 1 anastomotic dehiscence and 6 minor fistulas). No patient required an additional operation. The complication rate was higher in patients with a low response rate. Late complications included anastomotic stenosis and recto-vaginal fistulas. Continence score was poor in the first year of follow-up in $30 \%$ of patients but had a significative improvement after 2 years. CONCLUSIONS Preoperative chemoradiation in LARC can achieve significative results in terms of local response and leads to a better prognosis. Surgical approach has to be meticolous since the complication rate in pre-treated patients is high and functional results might be very unsatisfying.

\section{P69}

Short and Long Term Outcomes Following Salvage Surgery for Squamous Cell Carcinoma of the Anal Canal D.E. Schiller, ${ }^{*}$ B.J. Cummings, ${ }^{2}$ L.W. Le, ${ }^{4}$ S. Rai, ${ }^{3}$ L. Last, ${ }^{1}$ C.J. Swallow, ${ }^{1}$ A.J. Smith. ${ }^{1}$ 1. Division of Surgical Oncology, Department of Surgery, University of Toronto, Toronto, ON, Canada; 2. Department of Radiation Oncology, Princess Margaret Hospital, Toronto, ON, Canada; 3. Department of Surgery, University of Toronto, Toronto, ON, Canada; 4. Department of Clinical Study Coordination \& Biostatistics, Princess Maragaret Hospital, Toronto, ON, Canada.

Introduction: In patients with cancer of the anal canal who fail combined modality treatment (CMT), salvage surgery (SS) offers the potential for disease control and cure but patient selection can be difficult. Most of the SS literature is limited by small patient numbers and/or heterogeneous treatment protocols. We report a large series of patients treated with a standardized protocol at a university medical center. Methods: 60 patients with persistent or recurrent cancer of the anal canal following CMT who underwent SS between 1987 and 2006 were identified from institutional registries. Charts were reviewed and 19 patients were excluded from this analysis. Overall and disease-free survival (OS,DFS) curves were constructed by the Kaplan Meier method. Univariate analysis (UVA) of OS was done using the Log-Rank test, multivariate analysis (MVA) using Cox proportional hazards. Results: The study group $(\mathrm{N}=41)$ included 11 men and 30 women with a median age of 56 . The initial salvage procedure was APR $(n=39)$ or local excision $(n=2)$. Post-op mortality was 5\%. Post-op complications were seen in $68 \%$ (11 major, 27 minor); $48 \%$ had perineal wound problems. Median follow-up time was 18 mos overall and 36 mos in survivors. Median overall survival was 41 mos; OS at 3 and 5 yrs was $53 \%$ and $39 \%$ respectively. DFS at 3 and 5 yrs was $35 \%$ and $30 \%$, respectively. Recurrence was present in 22 patients at time of analysis. Failure was locoregional in $86 \%(19 / 22)$ and distant in $50 \%$ $(11 / 22)$. All patients with $\mathrm{R} 1$ resections $(\mathrm{N}=6)$ had locoregional failure. On UVA, Charlson co-morbidity score (CCS), male gender, lymphovascular invasion (LVI) and high grade histology were significant predictors of poor OS. Persistent vs. recurrent disease after CMT was not significant. On MVA, CCS $(\mathrm{HR}=2.0$ per unit increase, $\mathrm{p}=0.001)$, and LVI $(\mathrm{HR}=2.9, \mathrm{p}=0.02)$ remained significant predictors of poor OS. Conclusion: SS for anal cancer after failure of CMT is associated with acceptable post-op mortality, but significant morbidity. Long term survival was achieved in over one third of patients. Comorbidity score should guide patient selection and R0 resection should be the goal of SS. 


\section{P70}

The Pylorus: Take It or Leave It? A Systematic Review and MetaAnalysis of Pylorus-Preserving versus Standard Whipple Pancreaticoduodenectomy for Pancreatic Cancer P.J. Karanicolas, ${ }^{1 *}$ E. Davies,${ }^{2}$ R. Kunz, ${ }^{3}$ M. Briel, ${ }^{3}$ H.P. Koka, ${ }^{1}$ D.M. Payne, ${ }^{2}$ S.E. Smith, ${ }^{2}$ H.P. Hsu, ${ }^{4}$ P.W. Lin, ${ }^{4}$ G.H. Guyatt. ${ }^{1}$ 1. McMaster University, Hamilton, ON, Canada; 2. Surgery, The University of Western Ontario, London, ON, Canada; 3. Basel Institute for Clinical Epidemiology, Basel, Switzerland; 4. National Cheng Kung University Hospital, Tainan, Taiwan.

Introduction: We performed a systematic review and meta-analysis to determine the relative effects of pylorus-preserving pancreaticoduodenectomy (PPPD) and standard Whipple pancreaticoduodenectomy (SWPD) in patients with pancreatic or periampullary cancer. Methods: We searched seven bibliographic databases, conference proceedings, reference lists of articles and textbooks, and contacted experts in the field of hepatobiliary surgery. We included published and unpublished randomized controlled trials. We evaluated the methodological quality of trials and, in duplicate, extracted data regarding operative, perioperative, and long-term outcomes. We contacted all authors and asked them to provide additional information regarding the trials. We pooled results from the studies using a random-effects model, evaluated the degree of heterogeneity, and explored potential explanations for heterogeneity. Results: Six trials that included a total of 574 patients met eligibility criteria. In the pooled analysis, PPPD was 72 minutes faster ( $<<.001,95 \%$ CI 53 to $92 \mathrm{~min}$.), with $284 \mathrm{~mL}$ less blood loss ( $\mathrm{p}<.001,95 \%$ CI 176 to $391 \mathrm{~mL}$ ) and 0.66 fewer units of blood transfused ( $\mathrm{p}=.002,95 \% \mathrm{CI} 0.25$ to 1.16 ). There was a trend towards decreased perioperative mortality with PPPD (RR $0.40, p=0.09,95 \%$ CI 0.14 to 1.13). Other perioperative and long-term outcomes did not differ, although the confidence intervals include important differences. Conclusion: Moderate quality evidence suggests PPPD is a faster procedure with less blood loss and a trend toward lower mortality compared with SWPD. Large absolute differences in other key outcomes are unlikely; excluding relatively small differences will, however, require larger, methodologically stronger trials.

\section{P71}

Neoadjuvant chemoradiation impairs lymph node assessment and staging in rectal cancer A. Govindarajan, ${ }^{1}$ * A. Kiss, ${ }^{2}$ A.J. Smith, C.H. Law. ${ }^{3}$ 1. Division of General Surgery, University of Toronto, Toronto, ON, Canada; 2. Department of Research Design and Biostatistics, Institute for Clinical Evaluative Sciences, Toronto, ON, Canada; 3. Department of Surgical Oncology, Sunnybrook Health Sciences Centre, University of Toronto, Toronto, ON, Canada.

Neoadjuvant chemoradiation is increasingly used in the treatment of rectal cancer, but may affect lymph node (LN) assessment and compromise the adequacy of nodal staging. Understanding this interaction is crucial to improving the management of patients with rectal cancer. All patients $\geq 18$ years old treated curatively for primary rectal or rectosigmoid cancer at the study institution from 2000-2004 were identified. Patients were excluded if they underwent pelvic exenteration or received a non-standard course of radiation. All surgical specimens were examined for $\mathrm{LN}$ in a standardized manner. Patients were stratified into those that received neoadjuvant therapy followed by surgery(NEO) and those that had surgery as their first treatment(SURG). Multivariable analysis was used to determine the independent effect of neoadjuvant therapy on the number of $\mathrm{LN}$ assessed and the likelihood of diagnosing nodepositive disease. A total of 128 patients were identified (NEO: 49 patients; SURG: 79 patients). The mean number of $\mathrm{LN}$ assessed was 16.0 (NEO: 14.5; SURG: $17.0 ; p=0.07)$. In multivariable analysis, the NEO group had $19 \%$ fewer $L N$ assessed ( 3.2 nodes) than the SURG group $(p=0.015)$ and were 2.4 times more likely to have less than $12 \mathrm{LN}$ assessed $(\mathrm{p}=0.02)$. The degree of tumor downstaging was associated with a proportionate reduction in $\mathrm{LN}$ assessment. Within the NEO group, significantly fewer $\mathrm{LN}$ were assessed in patients classified as node-negative than in patients classified as node-positive (13.0 vs. $17.6, \mathrm{p}=0.04$ ). Importantly, the likelihood of a node-positive diagnosis in this group was dependent on the number of $\mathrm{LN}$ assessed, with a significant increase in node-positive diagnoses as more $\mathrm{LN}$ were retrieved and assessed $(p=0.03)$. Neoadjuvant chemoradiation significantly decreases the number of $\mathrm{LN}$ retrieved and assessed in patients with rectal cancer. This finding is clinically relevant as a decrease in the number of $\mathrm{LN}$ assessed may significantly impair the ability to detect patients with node-positive disease, resulting in potential understaging of $\mathrm{LN}$ status. Novel methods to improve $\mathrm{LN}$ assessment in the setting of neoadjuvant chemoradiation will need to be studied.

\section{P72}

Surveillance after initial rectal cancer treatment: minimal variation due to TNM stage K. Ode, ${ }^{3}$ K.S. Virgo, ${ }^{1}$ R.A. Audisio, ${ }^{3}$ W.E. Longo, ${ }^{2}$ F.E. Johnson. ${ }^{1 *}$ 1. Saint Louis University, St. Louis, MO; 2. Yale University, New Haven, CT; 3. University of Liverpool, Liverpool, United Kingdom.

Introduction: Many believe that follow-up testing for rectal carcinoma patients after primary curative-intent therapy should be rather intensive for high-stage lesions and less intensive for low-stage lesions. We recently carried out a survey of the members of the American Society of Colon and Rectal Surgeons (ASCRS) to quantify the strategies they use after primary treatment for their own patients. Considerable variability in surveillance exists. Here we report how initial TNM stage affects follow-up intensity. Methods: We devised vignettes succinctly describing healthy patients with rectal carcinoma (stage I treated with local excision, stage I treated with radical surgery, stage II treated with radical surgery, and stage III treated with radical surgery \pm adjuvant therapy). We mailed a questionnaire based on the vignettes to the 1,795 ASCRS members. Responses deemed evaluable were entered into a computer database. The effect of TNM stage on follow-up intensity was assessed by repeated measures ANOVA. Results: There were 566 responses (32\% response rate), of which $347(61 \%)$ were evaluable. The surveillance modality most frequently utilized was office visit. In year 1 following surgery for patients with stage I lesions treated with local resection, $3.8 \pm 1.4$ office visits (mean \pm SD) were recommended, decreasing to $1.5 \pm 0.8$ in year 5 . For patients with stage III lesions treated with radical surgery \pm adjuvant therapy, $4.0 \pm 2.8$ office visits were recommended in year 1 , decreasing to $1.7 \pm 1.2$ in year 5 . Similar results were generated for all commonly used surveillance modalities presented on the questionnaire ( 3 blood tests, 2 endoscopic procedures, 8 imaging studies). Conclusions: The intensity of follow-up after curative-intent treatment for rectal carcinoma varies minimally across TNM stages. This suggests that a controlled trial comparing high-intensity versus low-intensity follow-up testing could be carried out without stratification by TNM stage. We will outline such a trial at the meeting.

\section{P73}

Neoadjuvant and Adjuvant Chemotherapy Without Radiation for Esophageal Cancer C.P. Bowen-Wells, ${ }^{*}$ B. Ardalan, S.A. Spector, A.S. Livingstone, D. Franceschi, M. Lima, L. Sparling, E. Avisar, G. Walker, P. Ganjei-Azar. Surgical Oncology, University of Miami, Miami, FL.

Background: A Phase II trial to evaluate neoadjuvant (NAD) and adjuvant (AD) combination chemotherapy (CT) without radiation therapy (RT), for Stage III esophageal adenocarcinoma. Methods: Stage III disease by CAT scan or EUS and ECOG performance status $0-1$. The CT cycles included Cisplatin, Taxol, FUDR and Leucovorin. The chemotherapy was given for 16 weeks prior to surgery, followed by adjuvant chemotherapy for patients whose pathology at the time of surgery demonstrated at least microscopic disease. Survival was estimated via Kaplan Meier. Results: 33 patients were enrolled and initiated NAD, 15 received AD. During NAD, 14 had grade III/IV and 3 AD patients, had grade III/IV. Thirty-two (32) patients went to surgery, 30 patients were resected: 24 transhiatial and 6 transthoracic. Nineteen $(59 \%)$ demonstrated improvement in dysphagia; 11 (33\%) gained weight, and $7(21 \%)$ had no weight change. Forty-five $(45 \%)$ pathologic down-staging. Kaplan Meier estimates of overall survival at 1 and 5 years were $73 \%$ (95\% CI: 58 to $88 \%$ ) and $29 \%$ (95\% CI: 13 to $45 \%$ ). Median survival was 42 months $(95 \%$ CI: 14 to 52 months). Ten (10) patients are alive after a median follow up of 73 months (range 45 to 89 ). Comparison of the patients eligible for adjuvant therapy showed a survival benefit when at least 1 cycle of adjuvant therapy 
was given $(\mathrm{p}=0.040)$. Survival was 54 months compared with 23 months for those who received 1 cycle CT. Conclusion: This regimen of combination chemotherapy for locally advanced esophageal adenocarcinoma is safe and comparable with those regimens that contain radiation. Local recurrence was only seen in one patient in this cohort. Omission of radiation may allow for a more aggressive chemotherapy and reduction in the local complications post-surgery

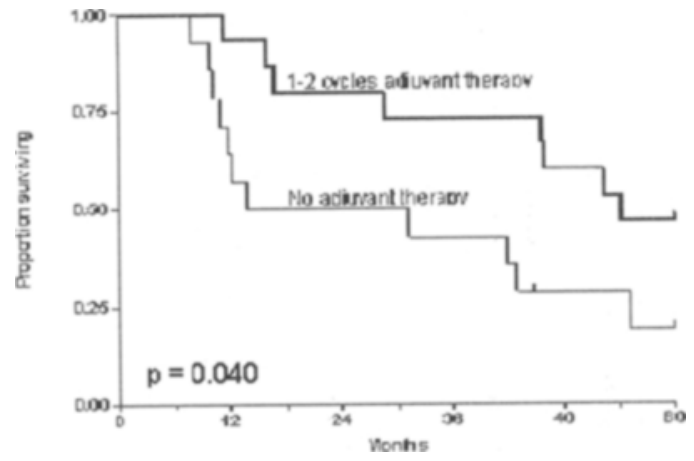

\section{P74}

A Twenty-year Experience with Adenocarcinoma of the Anal Canal R.J. Gonzalez, * J.M. Skibber, ${ }^{1}$ M.A. Rodriguez-Bigas, ${ }^{1}$ B.W. Feig, ${ }^{1}$ C. Eng, ${ }^{2}$ S. Krishnan, ${ }^{3}$ P. Das, ${ }^{3}$ C. Crane,${ }^{3}$ N. Janjan, ${ }^{3}$ G.J. Chang. ${ }^{1}$

1. University of Texas MD Anderson Cancer Center Department of Surgical Oncology, Houston, TX; 2. University of Texas MD Anderson Cancer Center Department of Radiation Oncology, Houston, TX; 3. University of Texas MD Anderson Cancer Center Department of GI Medical Oncology, Houston, TX.

Introduction: Adenocarcinoma of the anal canal is a rare malignancy with limited data regarding treatment and outcomes. The purpose of this study is to evaluate disease control and survival outcomes in patients with adenocarcinoma of the anal canal. Methods: A retrospective review of the records of all patients diagnosed with adenocarcinoma of the anal between 1983 and 2006 at a tertiary cancer center was performed. Tumor and patient characteristics and type of treatment rendered (chemoradiation (CXRT), local excision (LE) or radical surgery (RS)) were categorized. Overall survival and recurrence outcomes were evaluated using the Kaplan Meier method and the log rank test for comparisons. Results: Thirty-four patients were identified. Six patients underwent palliative treatment (stage IV, $n=4$; poor performance, $n=2$ ). Of the remaining 28 patients median follow-up was 29 months (interquartile range 16-52 months). Patient and tumor characteristics are shown in table. Twenty-five of the 28 patients received chemoradiation and 3 received local surgery plus XRT alone. The median XRT dose was 50 Gy. Procedures included RS $(n=15)$ and LE $(n=13)$. 5-FU based adjuvant chemotherapy was given for only $6 / 15(40 \%)$ RS patients and one LE patient after salvage APR for persistent disease. Median overall survival was 51 months. Actuarial 5-year survival was $42 \%$. Local failures occurred in 2 patients after RS (13\%) but only in conjunction with distant failure. Local failures occurred in 3 patients after LE $(23 \%)$ and were treated with salvage APR. The overall distant failure rate was $50 \%$ (47\% after RS, $54 \%$ after LE). Median recurrence-free survival was 45 months after RS and 12.5 months after LE $(p=0.03)$. Conclusion: Adenocarcinoma of the anal canal requires aggressive combined modality treatment but is associated with a high risk for failure. Radical surgery results in improved recurrence free survival, but improved adjuvant therapy regimens are needed to improve overall survival.
Patient Demographics and Stage at Presentation

\begin{tabular}{|c|c|c|}
\hline VARIABLE & RS & LE \\
\hline Age (median, range) & $65 \mathrm{yrs}(45-79)$ & $59 \mathrm{yrs}(32-83)$ \\
\hline Gender (M/F) & $9 \mathrm{M} / 6 \mathrm{~F}$ & $11 \mathrm{M} / 2 \mathrm{~F}$ \\
\hline T STAGE & & \\
\hline $\mathrm{T} 1$ & 0 & 3 \\
\hline $\mathrm{T} 2$ & 5 & 5 \\
\hline $\mathrm{T} 3$ & 8 & 5 \\
\hline $\mathrm{T} 4$ & 2 & 0 \\
\hline N STAGE (Incl. clinical) & & 0 \\
\hline $\mathrm{N} 1$ & 3 & 1 \\
\hline $\mathrm{N} 2$ & 2 & 1 \\
\hline $\mathrm{N} 3$ & 0 & 1 \\
\hline $\mathrm{Nx}$ & 0 & \\
\hline
\end{tabular}

\section{P75}

Prospective Comparison of The Peritoneal Cancer Index by CT scan and the Peritoneal Cancer Index at the time of Surgery in 28 patients with Peritoneal Surface Malignancies M. Gann,* A. Pitroff, J. Esquivel. St Agnes Hospital, Batimore, MD.

Introduction: The Peritoneal Cancer Index (PCI) determined at the time of surgical exploration has been shown to be an important prognostic indicator in peritoneal mesothelioma and peritoneal carcinomatosis from gastrointestinal origin. The PCI has been used to assist on patient selection for cytoreductive surgery and hyperthermic intraperitoneal chemotherapy. Evaluating the possibility of measuring this important parameter on CT scan prior to surgery constitutes the basis of this study. Materials and Methods: A prospective determination of the PCI by CT scan was recorded prior to surgery in all patients with peritoneal surface malignancies treated between November 2005 and July 2006 The PCI was determined by dividing the abdomen and pelvis into 13 regions $(0-12)$ and assigning a lesion size score to each region. The lesion size score varies from 0 if no tumor is present, to 3 , if tumor greater than $5 \mathrm{~cm}$ is present. The PCI, the summation of all lesion size scores, can vary from 0 to 39 (see diagram). At the time of the surgery, an intraoperative PCI was determined. Using a student's t-test, a comparison between the PCI by CT scan and the PCI at surgery as well as the lesion size score for each of the 13 regions was performed. Results: There were 28 patients. The mean Peritoneal Cancer Index by CT scan was 20.39 (SD 13.4) and the mean PCI at the time of surgery was 23.93 (SD 11.7). The means were not statistically significant with a p-value of 0.29 ( $95 \%$ CI: -10.3 to 3.2 ). Upon evaluating each region independently, only the central region (abdomino-pelvic region 0 ) was shown to be statistically significant with a mean lesion size score of 2.07 by $\mathrm{CT}$ scan versus 2.64 at exploration, $\mathrm{p}=0.04$ (95\% CI: -1.12 to- -0.02$)$. Conclusion: These data suggests that the reading of $\mathrm{CT}$ scans on patients with peritoneal surface malignancies should include a PCI determination. In addition, it helps to validate the PCI by CT scan as a surrogate of the intraoperative $\mathrm{PCI}$, therefore assisting in proper patient selection for cytoreductive surgery and intraperitoneal chemotherapy.

\section{Peritoneal Cancer Index}
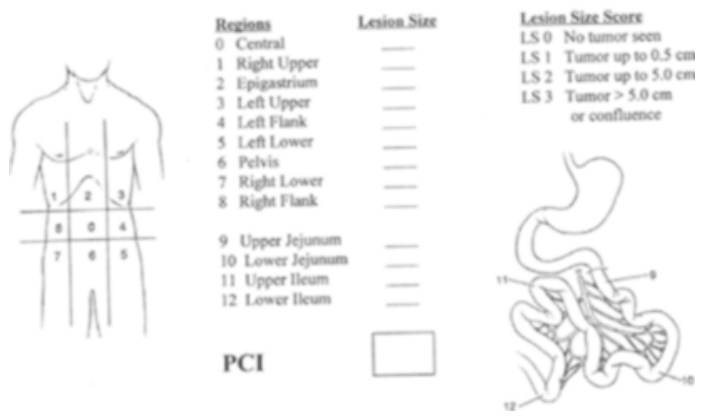


\section{P76}

In Vivo Imaging of Human Pancreatic Cancer Using a Fluorescent Antibody to CA 19-9 for Intraoperative Visualization of Tumors M. Bouvet, ${ }^{1 *}$ G.A. Luiken, ${ }^{2}$ S. Kaushal, ${ }^{1}$ M.M. Quigley, ${ }^{3}$

M.A. Talamini, ${ }^{1}$ A.R. Moossa, ${ }^{1}$ R.M. Hoffman. ${ }^{4}$ 1. University of California San Diego, San Diego, CA; 2. Fluoro-Probe, Inc., Coronado, CA; 3. Balboa Naval Hospital, San Diego, CA; 4. AntiCancer, Inc., San Diego, $C A$.

Introduction: For patients with pancreatic cancer, the initial surgical resection of all gross disease is at times incomplete. Making the malignant tissue fluorescent and therefore more easily visualized could improve the surgeon's ability to provide a complete resection. We have addressed this problem by tagging tumors in vivo with fluorophore-conjugated monoclonal antibodies. Methods: A monoclonal antibody to CA19-9 was coupled with an Oregon Green fluorophore (Molecular Probes, Inc.). The human pancreatic cancer cell lines CFPAC, BxPC-3, Panc-1, AsPC-1, Colo 357, SG, XPA-1, XPA-3, and XPA-4 and the human pancreatic ductal epithelial cell line HPDE were incubated in vitro with the antibody for 4 hours and then imaged with an inverted microscope with a GFP filter. CFPAC and BxPC-3 human pancreatic cancer cells were injected subcutaneously in nude mice to establish xenograft tumors. Mice were treated with a tail vein injection of the fluorescent antibody ( 100 micrograms in 100 microliters of PBS) and then imaged 24 hours later on the Olympus OV 100 whole mouse imaging system as well as with a simple pocket-sized $470 \mathrm{~nm}$ light-emitting diode (LED) flashlight. Results: In vitro, bright fluorescence was seen in 3 out of 9 of the pancreatic cancer cell lines but not in the negative control human pancreatic ductal epithelial cell line. In the in vivo studies, human pancreatic cancer CFPAC and BxPC-3 subcutaneous tumors were imaged through the skin of the nude mice. The brightly fluorescent tumors could easily be distinguished from the host tissue in the living mouse using the Olympus whole mouse imaging system and upon illumination with the hand-held LED flashlight. Conclusions: The CA 19-9 antibody tagged with a fluorescent probe allowed tumor detection in the living mouse. The further development of such fluorescent probes has the potential to be useful for highresolution intraoperative tumor visualization.
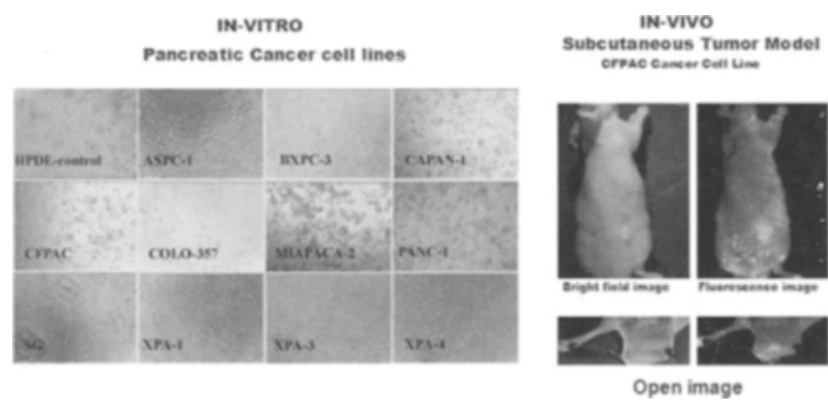

Human pancreatic cancer cells were imaged in vitro (left) or in vivo (right) after treatment with a fluorescent antibody to CA 19-9.

\section{P77}

Surveillance for Multicentric Colorectal Cancers and Other Primary Cancers in Patients with Curative Surgery for Colorectal Cancer in Japan M. Ishiguro, ${ }^{1 *}$ H. Mochizuki, ${ }^{2} \mathrm{~K}$. Kotake, ${ }^{1} \mathrm{~K}$. Sugihara. ${ }^{1}$ 1. Study Group of Surveillance after Colorectal Cancer Surgery, Tokyo, Japan; 2. National Defense Medical College, Tokorozawa, Saitama, Japan.

INTRODUCTION: Colorectal cancer patients may be at high risk to develop multicentric colorectal cancers and primary cancers of other organs because of their good prognosis. In order to consider the appropriate surveillance after colorectal cancer surgery, we conducted the multicenter retrospective study to clarify the incidences of metachronous cancers after colorectal cancer surgery. METHODS: A total of 5358 patients with colorectal cancers who underwent curative resection from January 1991 to December 1996 were studied for the incidences of multicentric colorectal cancers and multiple primary cancers of other organs. RESULTS: The median follow-up time was 7.3 years. The incidence of synchronous multiple primary cancers was $3.5 \%$ (188 cases), and that of synchronous gastric cancer was $1.6 \%$ ( 87 cases), which was higher than prevalence of gastric cancer found in mass screening in Japan $(0.2 \%)$. The incidence of synchronous gastric cancer was significantly higher in male. There were 211 cases $(3.9 \%)$ of metachronous multiple primary cancers and gastric cancer was the most frequent ( 51 cases, $0.95 \%)$. There was no difference between the incidences in our cases $(609$ and 151 per 100000 people, respectively) and the reported incidences in Japan (781 and 217 per 100000 people). The five year overall survival rate of patients with multiple primary cancers was significantly worse than that of those without multiple primary cancers, while there was no difference in colorectal cancer specific survival rate between them. The incidence of metachronous colorectal cancers after colorectal surgery (238 per 100000 people) was higher than the reported incidences (166 per 100000 people). CONCLUSIONS: Screening for gastric cancer before colorectal cancer surgery can be considered. Surveillance for metachronous multiple primary cancers may not be necessary to be included in the follow-up program after colorectal cancer surgery. The periodic follow-up for metachronous multicentric colorectal cancers should be recommended.

\section{P78}

Messenger RNA expression of vascular endothelial growth factor and its receptors in primary colorectal cancer and corresponding liver metastasis H. Kobayashi, ${ }^{1 *} \mathrm{~K}$. Sugihara, ${ }^{1}$ H. Uetake, ${ }^{1}$ T. Higuchi, ${ }^{1}$ M. Yasuno, ${ }^{1}$ M. Enomoto, ${ }^{1}$ H. Kuramochi, ${ }^{2}$ H.J. Lenz, ${ }^{3}$

K.D. Danenberg, ${ }^{4}$ P.V. Danenberg. ${ }^{2}$ 1. Department of Surgical Oncology Tokyo Medical and Dental University, Tokyo, Japan; 2. Department of Biochemistry and Molecular Biology, University of Southern California, Los Angeles, CA; 3. Department of Medical Oncology, University of Southern California, Los Angeles, CA; 4. Response Genetics Inc., Los Angeles, $C A$.

INTRODUCTION: Anti-angiogenic therapies have been recently performed in combination with traditional chemotherapy for patients with metastatic colorectal cancer. However, the correlation between the messenger RNA (mRNA) levels of angiogenesis-related genes in primary colorectal cancer and those in metastatic site was still unclear. The aim of this study was to clarify the association between the mRNA levels of vascular endothelial growth factor (VEGF) and its receptors (VEGFR) in primary colorectal cancer and those in corresponding liver metastasis. We also evaluated the correlation between mRNA levels of VEGF and those of VEGFRs. METHODS: Formalin-fixed paraffin-embedded 31 paired tumor tissues of colorectal cancer and liver metastasis were dissected by laser capture microdissection technique. After the isolation of RNA, a quantitative fluorescent dye real-time reverse transcriptionpolymerase chain reaction was used for gene expression measurement. Spearman's rank correlation and Wilcoxon signed-rank test were used for statistical analyses. RESULTS: There was a significantly positive correlation between VEGF mRNA levels in primary colorectal cancer and those in matched liver metastasis $(p=0.0083, r s=0.48)$. However, the mRNA levels of VEGFRs in primary tumor were not associated with those in liver metastasis (VEGFR-1, $\mathrm{p}=0.18, \mathrm{rs}=0.24$; VEGFR-2, $\mathrm{p}=0.086, \mathrm{rs}=0.31$ ). The mRNA levels of VEGF were associated with those of VEGFR-1 $(p=0.0026, \mathrm{rs}=0.39)$, but not with those of VEGFR-2 $(p=0.17, r s=0.18)$. The mRNA levels of VEGF were significantly higher than those of VEGFR-1 $(p<0.0001)$ or VEGFR$2(p<0.0001)$. CONCLUSIONS: We can predict the VEGF mRNA levels in liver metastasis by measuring those in primary colorectal cancer, but not VEGFRs'. The mRNA expression of VEGFRs may be more dependent on the surrounding environment than that of VEGF. Our result may also indicate that VEGF has a stronger effect on VEGFR-1 than other ligands. It would be useful to take into account these results in the management of anti-angiogenic therapies. Further studies will be necessary to validate these preliminary data. 


\section{P79}

Identification of patients who most benefit from hepatic arterial infusion combined or not with systemic chemotherapy for the treatment of unresectable colorectal liver metastases P. Pilati,* S. Mocellin, M. Forlin, S. Corazzina, M. Lise, D. Nitti. Clinica Chirurgica II, Department of Oncological and Surgical Science, University of Padova, Padova, Italy.

Background Although locoregional treatments such as hepatic arterial infusion (HAI) claim the advantage of delivering higher doses of anticancer agents directly into the affected organ, there is substantial lack of evidence for benefit in terms of overall survival (OS). Aims To test the hypothesis that systemic chemotherapy improves disease-specific OS of patients with unresectable colorectal liver metastases treated with HAI. Furthermore, we studied patient- and tumor-related predictive factors that might identify patients who most benefit from HAI regimen. Patients and methods In this retrospective study, 152 consecutive patients were considered. In group- $\mathrm{A}(\mathrm{n}=65)$, patients were treated with HAI alone (floxuridine (FUDR) $0.2 \mathrm{mg} / \mathrm{Kg}$ + leucovorin (LV) $4 \mathrm{mg} / \mathrm{m} 2$ + desamethasone $20 \mathrm{mg}$ 14 days/month) between 1996 and 1999. In group- $\mathrm{B}(\mathrm{n}=87)$, patients were treated with the same HAI regimen combined with systemic chemotherapy (5-fluorouracil (5FU) $450 \mathrm{mg} / \mathrm{m} 2+\mathrm{LV} 20 \mathrm{mg} / \mathrm{m} 2$ ) between 1999 and 2003. Results The median follow-up was 15.8 months (range: $8.5-101.7$ ). No difference in OS was observed between group-A and group-B (median OS: 18.2 and 19.1 months, respectively). None of the considered predictive factors (including p 53 expression) correlated with clinical outcome. Considering all patients (group A + group B), median OS was better in patients with less than $50 \%$ of liver parenchyma involvement $(24.3$ vs 12.4 months; $\mathrm{P}=0.0009$ ) as well as in responders (complete or partial response) vs non-responders ( 25.4 vs 12.9 morths; $\mathrm{P}<0.0001)$. The combination of low tumor load with good tumor response to HAI was the only variable retained at multivariate analysis (hazard ratio: $15.8, \mathrm{P}=0.017$ ) and was associated with a very favorable median survival (29.1 months). Conclusions Combination with 5FU+LV systemic chemotherapy did not lead to an OS benefit over FUDR-based $\mathrm{HAI}$ alone. The identification of biological markers of tumor response could select a subgroup of patients with low load but unresectable metastatic disease who most benefit from HAI therapy

\section{P80}

Comparison of operative $30 \& 90$ day mortality between General Surgeons and Cardiothoracic Surgeons in England Y. Leigh, * P. McCulloch, ${ }^{1}$ M. Goldacre, ${ }^{2}$ D. Altman. ${ }^{3}$ 1. Nuffield Department of Surgery, University of Oxford, John Radcliffe Hospital, Oxford, United Kingdom; 2. Unit of Health Care Epidemiology, Department of Public Health, University of Oxford. Old Road Campus., Oxford, United Kingdom; 3. Centre for Statistics in Medicine, Oxford, United Kingdom.

Introduction: Historically, Cardiothoracic and General Surgeons have used different approaches to perform Oesophagectomies for Cancer. The range of reported mortality results within 30 or 90 days for the 2 specialities has also differed. We hypothesised that Surgical speciality and Unit operative volume may influence post operative $30(\mathrm{~d} 30) \& 90(\mathrm{~d} 90)$ day mortality after Oesophagectomy. Methods: Hospital episode statistics (hes) case records $(n=8972)$ of Oesophagectomies for Cancer in England from 1998 until march 2003 were analysed. Research ethics approval was granted. Type of surgeon, Unit operative volume, operation type, patient socioeconomic deprivation score and post operative mortality at $\mathrm{d} 30 \& \mathrm{~d} 90$ were recorded. Cardiothoracic and General surgical mortality results were compared, using $\chi 2$ and multivariable logistic regression analysis. Results: Operative mortality for Cardiothoracic Surgeons was $33 \%$ lower and $21.6 \%$ lower at 30 and 90 days compared to General surgeons $\left(\chi_{2}\right.$ $=20.44 \mathrm{df}=1 \mathrm{p}<0.001$ and $\chi 2=12.95 \mathrm{df}=1 \mathrm{p}<0.001$ respectively). General Surgery d 30 9.06\%(95\% CI 8.37-9.76\%). Cardiothoracic Surgery d30 6.09\%(95\% CI $5.15-7.04 \%$ ). D30 difference $=2.97 \%$. Standard error of the difference (SED) between the two specialities and $95 \%$ CI $1.80 \%-4.14 \%$. General Surgery d90 $13.10 \%(95 \%$ CI $12.28-13.92 \%)$. Cardiothoracic Surgery d $9010.28 \%(95 \%$ CI $9.08-11.48 \%$ ). D90 difference $=2.83 \%$, SED between the two specialities and $95 \%$ CI $1.37 \%-4.28 \%$. Multivariable logistic regression showed that age, socioeconomic deprivation score, and Surgical speciality (Odds Ratios of death of $1.54 \& 1.32$ for General vs Cardiothoracic at $\mathrm{d} 30$ \& $\mathrm{d} 90$ respectively) were independent predictors of mortality risk. Conclusions: Mortality after oesophagectomy for cancer is independently influenced by the Surgical speciality of the operator in England. The reasons for the differences noted may be related to patient selection, surgical strategy or overall pre and post-operative patient care protocols. Further studies are required to elucidate the roles of these factors.
P81

Surgical strategy for early gastric cancer from the perspective of lymph node micrometastasis $\mathrm{H}$. Sonoda, ${ }^{*} \mathrm{H}$. Yamamoto, H. Naitoh, T. Tani. Surgery, Shiga university of medical science, Otsu, Japan.

Introduction: Patients with early gastric cancer (EGC) generally have excellent prognoses. Regional lymph node metastasis is the most important prognostic factor for patients with EGC. The recently developed endoscopic mucosal resection (EMR) procedure, endoscopic submucosal dissection (ESD), allows en bloc resection of large lesions. Selection of those patients without lymph node metastatic disease is thus important. We attempted to determine a surgical strategy for early gastric cancer from the perspective of lymph node micrometastasis (LMM). Materials and methods: We selected 33 histologically node-negative (pN0) EGC patients who underwent curative surgery in our surgery department between July 2002 and June 2004. A total of 310 lymph nodes were obtained from 33 patients. For each node, 1 piece was formalin fixed and paraffin embedded for histological examination, while another was used for RNA extraction. We used the duplex reverse transcriptase-polymerase chain reaction (RT-PCR) method with MUC2 and TFF1 genes to detect LMM in pN0 EGC. Results: Positive results were obtained for 21 of 310 lymph nodes (6.8\%) and 11 of 33 patients (33.3\%) using duplex RT-PCR. In the case of mucosal cancer, LMMs were detected in 7 of 22 patients (31.8\%) and 9 of 208 lymph nodes (4.3\%) using duplex RT-PCR. The 7 LMM-positive cases comprised 6 depressed-type carcinomas and 1 elevatedtype carcinoma. This last case had previously undergone EMR. All primary cancer cases were depressed-type carcinoma. In the depressed-type mucosal cancer, frequency of LMMs was significantly higher in patients with large tumors $(>25 \mathrm{~mm})$ than in patients with small tumors $(<25 \mathrm{~mm} ; \mathrm{P}=0.028)$. In the case of submucosal cancer, we detected micrometastasis in 4 of 11 patients $(36.4 \%)$ and 13 of 102 lymph nodes (12.7\%) using duplex RT-PCR. However, no clinicopathological factors were associated with the incidence of LMM. Conclusions: According to our study, elevated-type and small $(<25 \mathrm{~mm})$ depressed-type mucosal cancer offer good indications for EMR including ESD. However, gastrectomy with regional lymph node dissection is necessary for large $(>25 \mathrm{~mm})$ depressed-type mucosal cancer and submucosal cancer.

\section{P82}

Risk factors for complication rate after resection for carcinoma of the distal oesophagus and gastric cardia $\mathrm{E}$. Bastiaannet, ${ }^{1}$ F. Kloppenberg, ${ }^{2}$ M. Schaapveld, ${ }^{1}$ R. Otter, ${ }^{1}$ J. Plukker. ${ }^{3}$ 1. Comprehensive Cancer Centre North-Netherlands, Groningen, Netherlands; 2. Delfzicht Hospital, Delfzijl, Netherlands; 3. Surgical Oncology, University Medical Center Groningen, Groningen, Netherlands.

Objective. The only curative option for patients with carcinoma of the distal oesophagus and gastric cardia is a complex, high-risk surgical procedure. Aim of this study was to assess the complication rate and risk factors after surgery for cancer of the distal oesophagus and cardia. Method. We reviewed the medical records of 365 patients operated in the Northern Netherlands for cancer of the distal oesophagus or gastric cardia between 1993 and 2002. Data were collected on surgical procedures, complications and outcome. Major comorbidity was defined as cardiovascular, pulmonary or previous thoracotomy. Complications were divided in none, cardiac/pulmonary, infections, leakage/sepsis, bleeding and other. Univariate and multivariate logistic regression analysis were performed to determine risk factors for complications $(0-1$ versus $\geq 2)$. Results. Less than half the patients $(42.2 \%)$ had no complications after surgery; $39.7 \%$ cardiac/ pulmonary complications, $21.9 \%$ leakage/sepsis, $7.4 \%$ postoperative infections and $4.7 \%$ postoperative bleedings. Two or more complications were registered in $31 \%$ of the patients. Female patients $(\mathrm{p}=0.007$; $\mathrm{OR}=0.33$ ), patients with minor comorbidity $(\mathrm{p}=0.013 ; \mathrm{OR}=0.54)$ and patients operated by a high volume surgeon $(\mathrm{p}=0.037 ; \mathrm{OR}=0.57)$ had a decreased risk for $\geq 2$ complications. In the multivariate logistic regression of complications separately, major comorbidity was a risk factor for cardiac/pulmonary complications $(\mathrm{p}=0.008 ; \mathrm{OR}=1.92)$ and infections $(\mathrm{p}=0.04 ; \mathrm{OR}=2.52)$. The 30 day mortality was $9.3 \%$ and dependant on major comorbidity $(p=0.04$; $\mathrm{OR}=2.78)$, leakage/sepsis $(\mathrm{p}=0.001 ; \mathrm{OR}=4.47)$ and postoperative bleeding $(p=0.008 ; O R=7.09)$ in multivariate analysis. Conclusion. A majority of patients with carcinoma of the distal oesophagus and cardia experienced serious complications after surgical resection. Females, patients with minor comorbidity and patients operated by a high volume surgeon have a decreased risk of two or more complications. Major comorbidity is a risk factor for cardiac or pulmonary complications and postoperative infections. These results showed a necessity of vigilant postoperative control by a multidisciplinary team. 
P83

Can Operative Time Serve as a Quality Measure in Surgical Oncology? A.C. Freel, ${ }^{1}{ }^{*}$ K.Y. Bilimoria, ${ }^{2}$ R.S. Jones, ${ }^{3}$ C.Y. Ko. ${ }^{4}$ 1. Louisiana State University Health Sciences Center, New Orleans, LA; 2. Northwestern University, Chicago, IL; 3. University of Virginia, Charlottesville, VA; 4. University of California Los Angeles, Los Angeles, CA.

Introduction: Prolonged operative time in conventional surgery increases patient morbidity; however, longer operative time may benefit a cancer patient as a result of a more complete resection. This study investigated the effect of prolonged operative times on outcomes after abdominal cancer surgery. Methods: We searched Pubmed and Ovid using operative time and abdominal neoplasms as the search terms. Articles that included univariate and/or multivariate analysis on operative time and neoplasms were selected for analysis. Results: Twenty eight studies met inclusion criteria. Prolonged operative time independently increased thirty day mortality in two out of three studies. Seven studies addressed the effect of operative time on survival. None showed operative time as a significant independent predictor of survival. Regarding morbidity related to operative time, eight studies addressed overall postoperative morbidity. Five showed a significantly increased risk of complications when operative time was prolonged, and two suggested that longer operative times resulted in a higher complication rate. Ten other studies addressed specific complications including anastomotic leaks, intra-abdominal abscesses, infections, sepsis, and recurrence. Prolonged operative time negatively affected the majority of these results. Conclusions: Although operative time is not used as a measure of quality in surgical oncology, our research showed that prolonged operative time increased both morbidity and mortality. Operative time potentially could be utilized as a quality indicator in surgical oncology.

Multivariate Analysis of Operative Time on Mortality, Survival, and Morbidity

\begin{tabular}{|c|c|c|c|c|c|}
\hline Author & Year & $\begin{array}{l}\text { Type of } \\
\text { Cancer }\end{array}$ & Outcome & Statistical Method & P Value \\
\hline $\begin{array}{c}\text { Billingsley et } \\
\text { al }\end{array}$ & 2003 & Pancreas & Mortality & $\begin{array}{c}\text { Multivariate logistic regression } \\
\text { analysis }\end{array}$ & 0.003 \\
\hline Bufalari et al & 2006 & Colorectal & Mortality & $\begin{array}{c}\text { Multivariate logistic regression } \\
\text { analysis }\end{array}$ & 0.04 \\
\hline Alves et al & 2005 & Colorectal & Mortality & $\begin{array}{c}\text { Multivariate logistic regression } \\
\text { analysis }\end{array}$ & NS \\
\hline Schmidt et al & 2004 & Pancreas & Survival & Cox proportional hazards model & NS \\
\hline Takai et al & 2003 & Pancreas & Survival & Cox proportional hazards model & NS \\
\hline $\begin{array}{l}\text { Magistrelli et } \\
\text { al }\end{array}$ & 2000 & Pancreas & Survival & Cox proportional hazards model & NS \\
\hline $\begin{array}{l}\text { Yamaguchi et } \\
\text { al }\end{array}$ & 1999 & Pancreas & Survival & Cox proportional hazards model & NS \\
\hline Geer et al & 1993 & Pancreas & Survival & Cox proportional hazards model & NS \\
\hline Bufalari et al & 2006 & Colorectal & Survival & $\begin{array}{c}\text { Multivariate logistic regression } \\
\text { analysis }\end{array}$ & NS \\
\hline Monreal et al & 1998 & Colorectal & Survival & Cox proportional hazards model & NS \\
\hline Alves et al & 2005 & Colorectal & Morbidity & $\begin{array}{c}\text { Multivariate logistic regression } \\
\text { analysis }\end{array}$ & 0.016 \\
\hline Alves et al & 2005 & Colorectal & Morbidity & $\begin{array}{c}\text { Multivariate logistic regression } \\
\text { analysis }\end{array}$ & 0.04 \\
\hline Vignali et al & 2004 & Colorectal & Morbidity & $\begin{array}{c}\text { Multivariate logistic regression } \\
\text { analysis }\end{array}$ & 0.011 \\
\hline Kodera et al & 2005 & Gastric & Morbidity & $\begin{array}{c}\text { Multivariate logistic regression } \\
\text { analysis }\end{array}$ & 0.005 \\
\hline Shimada et al & 1998 & Liver & Morbidity & $\begin{array}{c}\text { Multivariate logistic regression } \\
\text { analysis }\end{array}$ & Significant \\
\hline Bufalari et al & 2006 & Colorectal & Morbidity & $\begin{array}{c}\text { Multivariate logistic regression } \\
\text { analysis }\end{array}$ & NS \\
\hline Chiappa et al & 2001 & Colorectal & Morbidity & $\begin{array}{l}\text { Multivariate logistic regression } \\
\text { analysis }\end{array}$ & NS \\
\hline Shimada et al & 1994 & Liver & Morbidity & $\begin{array}{c}\text { Multivariate logistic regression } \\
\text { analysis }\end{array}$ & NS \\
\hline
\end{tabular}

NS $=$ Not Significant; Significant $=$ Significant value, but $P$ value not stated in article.

\section{P84}

CD24 Expression is an Independent Prognostic Marker in Cholangiocarcinoma S. Agrawal, * B.W. Kuvshinoff, T. Khoury, J. Yu, M.M. Javle, C. LeVea, J. Groth, L.J. Coignet, J.F. Gibbs. Roswell Park Cancer Institute, Buffalo, NY.

Introduction: $\mathrm{CD} 24$ has been described as an adverse prognostic marker in several malignancies. This study evaluates CD24 expression in cholangiocarcinoma and correlates the findings with clinico-pathologic data and patient survival. Methods: Between 1996 and 2002, 22 consecutive patients with cholangiocarcinoma were treated at our institution. Demographic data, SEER stage, pathologic data, treatment, expression of CD24, MAPK, phosphorylated-MAPK and survival were analyzed. Results: The majority of the tumors demonstrated CD24 (81.8\%) and p-MAPK (87\%) expression. A negative association was noted between the expression of CD24 and p-MAPK. Median survival for patients with low expression of CD24 was 36 months and high expression 8 months (see figure). Median survival for patients who received chemotherapy with low CD24 expression was 163 months and for 7 patients with high CD24 expression 17 months $(p=0.04)$. With the addition of radiation therapy, median survival for patients with low expression of CD24 was 52 months and high expression 17 months $(p=0.08)$. On multivariate analysis, use of chemotherapy $(p=0.0014$, Hazard Ratio 0.069) and CD24 over-expression ( $p=0.02$, Hazard Ratio 7.528) were predictive of survival. Conclusion: CD24 is commonly expressed in cholangiocarcinoma and over-expression is predictive of poor survival and lack of response to chemotherapy and possibly radiation therapy. These findings may improve selection of patients for the appropriate conventional treatment modality and the development of CD24-targeted therapy.

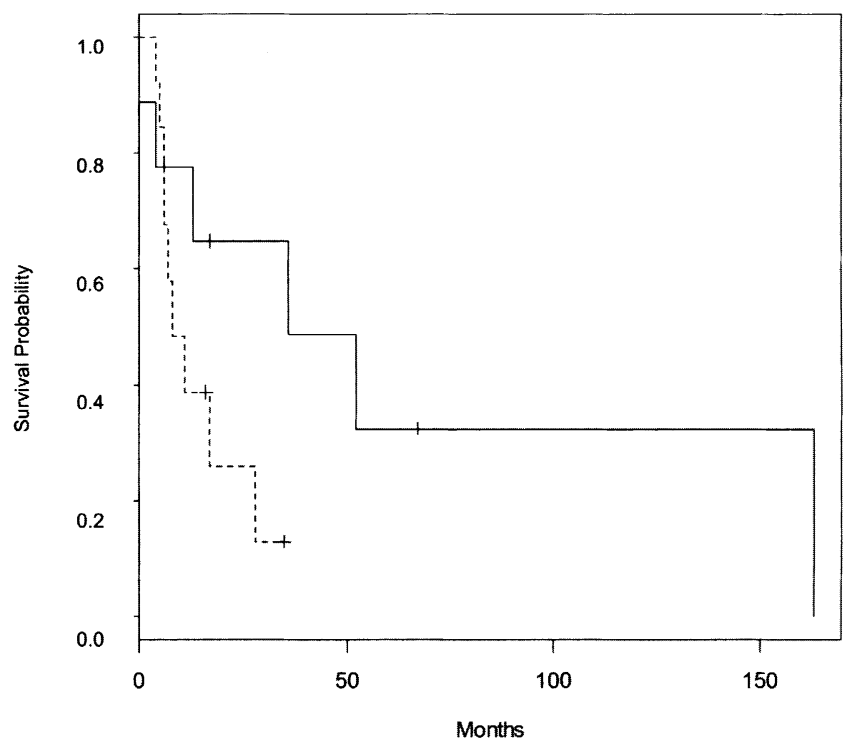

Kaplan-Meier survival curve for patients of cholangiocarcinoma with low and high levels of CD24 expression $(n=22) . P=0.02$. __ Low CD24 — High CD24

\section{P85}

Peri-hepatic cytoreduction in patients with pseudomyxoma peritonei: technical aspects and liver related trauma M. Deraco, ${ }^{*}$

D. Baratti, P. Costanzo, M. Favaro, C. Gavazzi, B. Laterza,

S. Kusamura. Surgery, National Cancer Institute, MILAN, MILAN, Italy.

INTRODUCTION: The peri-hepatic cytoreduction (PHCR) is the most complex and time consuming phase of the peritonectomy. The magnitude of liver trauma inflicted by surgical manipulation, heat and/or cytotoxic effect of chemotherapies has not been investigated so far. We aimed to evaluate in patients with pseudomyxoma peritonei (PMP) submitted to cytoreductive surgery (CRS) and hyperthermic intraperitoneal chemotherapy (HIPEC): 1) if PHCR could be a limitation for complete cytoreduction; 2) the extent of hepatocellular damage and the liver/biliary tree morbidity. METHODS: Seventy five patients (63 DPAM / 13 PMCA) submitted to 76 consecutive procedures were included in the study. Previous surgical score was $>1$ in $39 \%$ of the cases. 
The mean PCI was 20 (range: 3-39). HIPEC was performed with closed abdomen technique with Cisplatin+Mitomycin-C at $42.5^{\circ} \mathrm{C}$. The morbidity and laboratory parameters alterations were graded according to NCI CTCAE v3 criteria. RESULTS: PHCR required 70 subdiaphragmatic peritonectomy, 57 Glisson capsule resections, 51 cholecystectomies, 50 hepato-duodenal ligament dissection and 27 dissection of the retrohepatic intercavo-aortic space. The mean duration of entire procedure in the whole series was 708 minutes (range: 300 - 1320). G3-4 ALT/AST alterations were reported in 33cases mostly in the 1st postop. G3-4 alterations of bilirubin, alkaline phosphatase and GGT were observed in 3, 0 and 5 cases, respectively. Incomplete cytoreduction was observed in 4 cases (5\%), 1 of which due to the suboptimal PHCR (residual disease $>2.5 \mathrm{~mm}$ in the subdiaphragmatic and intercavo-aortic retrohepatic spaces due to previous surgical scar). G3-5 morbidity, G3-5 toxicity and mortality rates were $27 \%, 9 \%$ and $1 \%$, respectively. Two cases presented complications attributable to PHCR (biliar fistulas $1 \mathrm{G} 2$ and $1 \mathrm{G} 3$ ). CONCLUSIONS: Even with a high skilled surgical team, the completeness of cytoreduction could be potentially hampered by he PHCR phase of the peritonectomy especially in the presence of previous surgical scar. The PHCR exerted a significant hepatocellular damage that did not impacted on the emergence of G3-5 liver complications.

\section{P86}

Impact of cytoreductive surgery and hyperthermic intraperitoneal chemotherapy on systemic toxicity and liver/pancreatic morbidity S. Kusamura, * D. Baratti, P. Costanzo, M. Favaro, B. Laterza, C. Gavazzi, M. Deraco. Surgery, National Cancer Institute, MILAN, MILAN, Italy.

Introduction: The purpose of this study was to analyze the postoperative systemic toxicity, liver/pancreatic morbidity and mortality of cytoreductive surgery (CRS) and hyperthermic intraperitoneal chemotherapy (HIPEC) in the treatment of peritoneal surface malignancies (PSM). Patients and methods: Two hundred and forty two $(84 \mathrm{M} / 158 \mathrm{~F})$ patients with PSM underwent 247 consecutive procedures. The mean age was 52 years (range: 22-79). CRS was performed using peritonectomy procedures. HIPEC through the closed abdomen technique was conducted with cisplatin (CDDP $25 \mathrm{mg} / \mathrm{m} 2 / \mathrm{L}$ of perfusate) + mitomycin C (MMC $3.3 \mathrm{mg} / \mathrm{m} 2 / \mathrm{L}$ of perfusate) or CDDP $(43 \mathrm{mg} / \mathrm{L}$ of perfusate) + doxorubicin (Dx $15.25 \mathrm{mg} / \mathrm{L}$ of perfusate), at $42.5^{\circ} \mathrm{C}$. The dose of the drugs was reduced in $30 \%$ in case the patient had received systemic chemotherapy (sCT) before the CRS+HIPEC. Systemic toxicities and liver/pancreatic laboratory (ALT, AST, bilirrubin, amylase) alterations were graded according to NCI CTCAE v3 criteria. Results: G3-5 systemic toxicity rate was $11.7 \%$ and the adverse events (AEs) were 13 bone marrow suppression, 14 nephrotoxicity, 2 neutropenic infection, and 1 pulmonary toxicity. Independent risk factors for G3-5 systemic toxicity after multivariate analysis were CDDP dose for HIPEC $>240 \mathrm{mg}$ (OR: 2.78, CI95\%: $1.20-6.45$ ) and CDDP+DX schedule for HIPEC (OR: 2.36, CI95\%: $1.02-5.45$ ). G3-5 alteration of liver/pancreatic laboratory parameters were observed in $25.1 \%$ of the cases. Independent risk factors for these AEs were No-sCT before the CRS+HIPEC (OR: 2.53, CI95\%: 1.30 - 4.94), CDDP+MMC schedule for HIPEC (OR: $2.26, \mathrm{CI} 95 \%: 1.09-4.68$ ) and CRS duration $>540$ minutes (OR: 4.30 , CI95\%:2.23- 8.29). Liver/pancreatic G3 morbidities were 3 fistulas ( 2 pancreatic and 1 from the biliary tree). Operative mortality was $1.2 \%$. Conclusions: CRS+HIPEC presented acceptable systemic toxicity and mortality rates. The CDDP+DX schedule was more related with systemic toxicity than CDDP+MMC. The surgical trauma (reflected by CRS duration) contributed significantly more than the cytotoxic effect of the CDDP+MMC schedule to the emergence clinical and laboratorial AEs of the liver/pancreas.

\section{P87}

Bone marrow micrometastases and lymph node metastases are independent indicators for colon cancer cell dissemination C.T. Viehl, ${ }^{1 *}$ G. Sauter, ${ }^{2}$ U. Guller, ${ }^{1}$ I. Langer, ${ }^{1}$ A. Ochsner, ${ }^{4}$ R. Cecini, ${ }^{3}$ W.R. Marti, ${ }^{1}$ U. Laffer, ${ }^{3}$ D. Oertli, ${ }^{1}$ M. Zuber. ${ }^{4} 1$. Department of Surgery, University of Basel, Basel, Switzerland; 2. Institute of Pathology, University of Basel, Basel, Switzerland; 3. Department of Surgery, Spitalzentrum Biel, Biel, Switzerland; 4. Department of Surgery, Kantonsspital Olten, Olten, Switzerland.

Introduction: Bone marrow (BM) micrometastases can be found in $20-30 \%$ of colon cancer patients. However, little data exist on the clinical relevance of this finding. It remains to be elucidated if the presence of BM micrometastases is associated with other tumor related characteristics, particularly with lymph node metastases. Therefore, the objective of the current study was to evaluate whether BM micrometastases and lymph node metastases are independent indicators for colon cancer cell dissemination. Methods: One hundred twenty-eight patients underwent BM aspiration followed by open colon resection according to a standardized multicenter protocol. The mononuclear cell fraction of $\mathrm{BM}$ aspirates was stained with the pancytokeratin antibody A45-B/B3, and analyzed for the presence of epithelial tumor cells by an automated cellular imaging system and by visual verification by a pathologist. The Fisher's exact test was used for statistical comparison of frequencies. Results: BM analysis was positive in $43(34 \%)$ and negative in 85 patients $(66 \%)$. Fifty-four patients $(42 \%)$ had lymph node metastases. The median number of analyzed lymph nodes was 21.5 (range 8-54). Twelve primary tumors were diagnosed T1 $(9 \%)$, $17 \mathrm{~T} 2(13 \%), 81 \mathrm{~T} 3(64 \%)$, and $18 \mathrm{~T} 4(14 \%)$. One primary tumor was grade 1 $(1 \%), 96$ grade $2(75 \%)$, and 31 grade $3(24 \%)$. Twenty-three patients were stage I (18\%), 48 stage II (38\%), 44 stage III (34\%), and 13 stage IV (10\%). There was no association between the presence of nodal metastases and BM micrometastases $(\mathrm{p}=0.85$; table). Furthermore, the presence of BM micrometastases was neither associated with the T-stage $(p=1.0)$, nor with grading of the primary tumor $(\mathrm{p}=0.30)$, nor with the tumor stage according to $\operatorname{AJCC}(\mathrm{p}=0.80)$. Conclusions: Bone marrow micrometastases are not associated with the presence of lymph node metastases in colon cancer patients. Therefore, both appear to be independent indicators for colon cancer cell dissemination.

\begin{tabular}{|c|c|c|}
\hline & BM negative & BM positive \\
\hline $\mathrm{pN} 0$ & 50 & 24 \\
\hline $\mathrm{pN} 1 / \mathrm{pN} 2$ & 35 & 19 \\
\hline
\end{tabular}

\section{P88}

Impact of Pre-operative PET Scans on survival after liver resection for metastatic colorectal cancer F. Chu, ${ }^{*}$ F. Riffat, D.L. Morris. Department of Surgery, St George Hospital, Kogarah, QLD, Australia.

Positron Emission Tomography has been used to detect extrahepatic disease in patients in order to determine their suitability for liver resection from colorectal metastases. We reviewed our prospective data for patients undergoing liver resection with and without preoperative PET scanning with the hypothesis that it would result in better 5 year survival after liver resection due to better patient selection by downstaging disease. Patients with extrahepatic disease could be treated by ablative methods and neoadjuvant chemotherapy prior to undergoing liver resection. Method: Between 1990 and 2005 there were 467 patients undergoing potentially curative liver resections. PET Scanning was available to our patients without restrictions from 2002. We divided our patients into 3 cohorts. There were 215 patients in the pre-PET era (Group 1), 188 in the PET era where a PET scan was not performed (Group 2) and 64 patients with preoperative PET scanning(Group 3). Our 3 cohorts were followed up at $1,3,6$ and 12 months post-operatively with a median follow up of 24,21 and 20 months respectively. Results: The five year survival rate for patients with preoperative PET scanning was $68 \%$. The five year survival rate for patients without preoperative PET scanning was $40 \%$ in the PET era and $30 \%$ in the pre-PET era $(p$ value $=0.0113)$. The disease free interval was $35 \%$ in the PET group and $20 \%$ without PET scans at 3 years $(p<0.05)$. There were no other statistically significant differences between the cohorts on 8 other prognostic variables. Conclusion: The usage of preoperative PET scanning has led to an improved overall survival because of better patient selection by detecting occult extrahepatic disease missed with traditional staging techniques. It has reduced the number of futile laparotomies and plan better treatment strategies for patients with extrahepatic disease.

\section{P89}

Intratumoral Natural Killer Cell and Lymphocyte Infiltration: Impact and Outcomes for Pancreatic Adenocarcinoma S.M. Wilhelm, ${ }^{1}$ * X. Xiu, ${ }^{2}$ P. Gattuso, ${ }^{2}$ R.M. Quiros, ${ }^{2}$ J.A. Kim, ${ }^{1}$ E.L. Chen, ${ }^{2}$ R.A. Prinz. ${ }^{2}$ 1. Surgery, University Hospitals of Cleveland, Cleveland, OH; 2. Rush Presbyterian-St. Luke's Medical Center, Chicago, IL.

Introduction: Natural Killer (NK) cells and lymphocytes are involved in cancer immunosurveillance. The extent of intratumoral NK and lymphocyte 
infiltration has been shown to affect prognosis for gastric, colorectal, and ovarian cancer. We sought to determine if intratumoral NK or lymphocyte infiltration have prognostic significance for pancreatic adenocarcinoma. Methods: Tumors from 45 patients who underwent surgical resection for pancreatic cancer were analyzed. Immunohistochemistry was performed with CD-57, a monoclonal antibody, to assess NK infiltration. Slides were reviewed and classified as "high" NK infiltration $\geq 25$ NK/HPF or "low" $<25$ NK/HPF. Specimens were also reviewed for lymphocyte infiltration. "High" infiltration was classified as $\geq 150$ lymphocytes/HPF and "low" as $<150$ lymphocytes/HPF. Patient demographics, tumor characteristics, disease staging, and patient length of survival (LOS) were determined. Statistical analysis was performed using Student's T-test for comparison of means and regression analysis for correlations with significance of $p<0.05$. Results: Of our 45 patients, 21 were female and 24 male, ages (40-81). By AJCC staging, Stage I $(n=7)$, Stage II $(n=8)$, Stage III $(n=23)$, and Stage IV $(n=7)$. LOS for all stages ranged 1-49 months. LOS (months \pm SEM) by stage was: Stage I- $20.5 \pm 7.5$, II- $20.6 \pm 12.1$, III$12.2 \pm 2.1, \mathrm{IV}-8 \pm 3.7$. LOS correlated well with tumor stage $(\mathrm{p}=0.04)$, and tumor size $(p=0.03)$. As seen in Table 1- high levels of NK cell or lymphocyte infiltration did not statistically affect patient survival, or reduce tumor size. There was also no improvement in tumor stage or nodal metastases based on NK cell/lymphocyte infiltration. Conclusions: There is evidence that intratumoral NK cell and lymphocyte infiltration can improve patient outcomes for some cancers. Based on our study, these cells do not improve any prognostic indicators we studied for pancreatic adenocarcinoma. The lack of impact of the innate and cellular immune systems on pancreatic cancer may highlight another reason why this tumor is so uniformly lethal and why recent IL-2 trials in pancreatic cancer yield limited benefit.

\section{Table 1}

\begin{tabular}{|c|c|c|c|c|}
\hline & $\mathrm{NK} \geq 25 / \mathrm{HPF}$ & $\mathrm{NK}<25$ & Lymph $\geq 150$ & Lymph $<150$ \\
\hline LOS(months) & $15.9 \pm 2.1 \dagger$ & $13.5 \pm 3.0 \dagger$ & $14.1 \pm 2.8 \ddagger$ & $14.4 \pm 1.8 \ddagger$ \\
\hline Tumor size $(\mathrm{cm})$ & $4.1 \pm 0.4$ & $3.3 \pm 0.3$ & $4.0 \pm 0.5$ & $3.6 \pm 0.3$ \\
\hline P value & $\dagger=0.55$ & & $\ddagger=0.54$ & \\
\hline
\end{tabular}

\section{P90}

Predicting Outcomes after Surgical Therapy for Locally Recurrent Rectal Cancer E. Choi, ${ }^{*}$ J.M. Skibber, M.A. Rodriguez-Bigas, B.W. Feig, G.J. Chang. Department of Surgical Oncology, University of Texas MD Anderson Cancer Center, Houston, TX.

Background: Locally recurrent rectal cancer (LRRC) is an important problem that may be related to factors at the time of primary tumor surgery. Surgical salvage is the only potentially curative treatment. We evaluated our 17year experience with LRRC to determine factors that predict outcome. Methods: A retrospective review of patients from our institutional tumor registry who underwent surgical salvage for LRRC from 1988 to 2005 was performed. Patient and tumor characteristics and survival and recurrence outcomes were evaluated. A protocol to score all available operative reports at primary resection was developed based on NIH consensus guidelines $(n=45)$. Operative reporting elements included: findings on exploration, extent of bowel resection, level of vascular ligation, and extent of mesorectal dissection. A protocol for scoring pathology reports was based on the College of American Pathologists guidelines $(\mathrm{n}=54)$. Analysis was performed using the Kaplan-Meier method and the $\log$ rank test for categorical predictors. Results: There were 143 patients ( 81 male, 62 female), median age 57 (range 24-83). Surgery for LRRC was performed at median 30 months after the primary operation. Salvage surgery included LAR (28), APR (38), pelvic exenteration (50), pelvic mass resection (27) and en bloc bone resection in 40 patients. The 5-year overall (OS) and recurrence-free survival (RFS) for the entire cohort were $44 \%$ and $30 \%$, respectively. After a median follow-up of 38 months, failures were local in $38(27 \%)$, distant in $27(19 \%)$, and both in $3(2 \%)$. The quality of pathologic reporting did not predict outcomes. The failure to indicate an adequate mesorectal dissection indicated a trend towards shorter time to local failure (median $18 \mathrm{vs.}$ 26 mos., $\mathrm{p}=0.06$ ). Moreover, secondary recovery of any residual lymph nodes at the time of resection for recurrence predicted improved survival $(\mathrm{p}=0.006)$. Conclusions: Our results demonstrate the significance of adequate operative reporting and complete lymph node recovery at the time of primary rectal cancer resection as potential predictors of recurrence risk, and criteria for aggressive salvage surgery in patients with LRRC.

\section{P91}

Omega-3 Fatty Acids Inhibit Cellular Proliferation in Pancreatic Cancer Cells S. Garrean, ${ }^{*}$ J. Hering, T. Razzak, T. Babcock, N. Espat. Surgery, Univ. of Illinois at Chicago, Chicago, IL.

Supplementation of cancer therapies with omega-3 fatty acids (n-3 FA) has been a focus of recent investigations. Eicosapentaenoic acid (EPA), the primary biologically active component of fish oil is one such supplement that has been demonstrated to modulate intracellular events, including: the production of prostaglandins by arachidonic acid replacement, inhibition of NFKB with decreased inflammatory cytokines and induction of apoptotic factors and cell cycle mediators. In this study, the effects of n-3 FA on proliferation in 4 distinct in-vitro models were examined. Methods: MIA PaCa-2, BXPC-3, Panc1 and L3.6pl pancreatic cancer cell lines were treated with 25, 50,100 and $150 \mu \mathrm{M}$ EPA at 24 and 48 hours; (n-6 FA were used as a lipid treatment controls). Non-lipid control used media alone, for a "\% of control" calculation to be performed. Proliferation was measured by the WST-1 assay. MIA-Paca2 cell apoptosis was assessed by PARP cleavage and annexin V. Experiments were run in triplicate. Statistics by ANOVA, significance at $p \leq 0.05$. Results: Time and dose dependent decreased cellular proliferation was observed across all 4 cell lines treated with $n-3 F$. The effects were significant at 24 hours(not shown) but optimized at 48 hours (fig. 1) with $100 \mu \mathrm{M}$ as the most effective concentration. At $24 \mathrm{hrs}, 100 \mu \mathrm{M}$ EPA inhibited proliferation in MIA PaCa- 2 cells by $52 \%$, BXPC-3 53\%, Panc-1 38\% and L3.6pl 73\%; at $48 \mathrm{hrs,} \mathrm{MIA} \mathrm{PaCa-2}$ cells by $79 \%$, BXPC-3 $63 \%$, Panc- $155 \%$ and L 3.6 pl $70 \%$. MIA PaCa- 2 cells demonstrated increased PARP cleavage product and increased annexin $V$ protein (not shown). Conclusion: $4 / 4$ distinct cell lines demonstrated a time and dose dependent inhibition of proliferation with n-3FA treatment. MIA PaCa2 demonstrated mitochondrial mediated cell death and not toxicity, confirmed by PARP cleavage and annexin V. Pending experiments will address apoptosis versus toxicity for the remaining cell lines, cell cycle regulation by n-3 FA and possible chemosensitization with gemcitabine. Figure legend: pancreatic cancer cells at 48 hrs: control =media-alone treatment, significance $p \leq 0.05$ is indicated on the graph for each concentration.
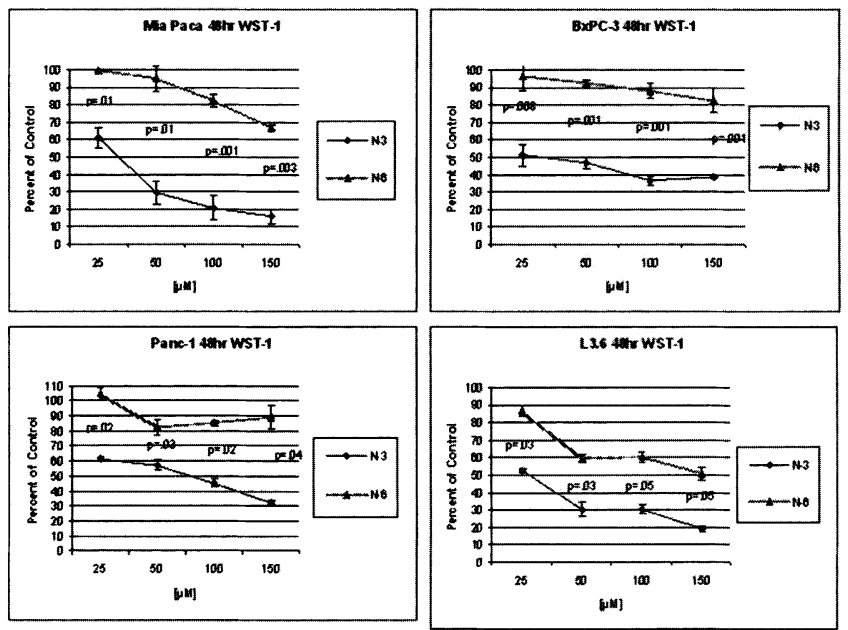

\section{P92}

Surgical Management and Outcome in Primary Adenocarcinoma of the Small Bowel S. Agrawal, * E.C. McCarron, G.E. Wilding,

J.F. Gibbs, A. Rajput. Roswell Park Cancer Institute, Buffalo, NY.

Introduction: Primary adenocarcinoma of the small bowel is a rare malignancy with poor survival outcome. Patient, tumor and treatment-related factors were analyzed for their association with recurrence and survival. Methods: Between 1971 and 2005, 64 patients with primary adenocarcinoma of the small bowel were treated at our institution. Characteristics of the primary tumor, regional lymph nodes and metastatic pattern were reviewed. Clinical presentation, operative findings, extent of surgical resection, pathologic data, additional treatment, recurrence pattern and survival were analyzed. Results: The commonest presenting clinical features included abdominal pain $(n=33 ; 51.6 \%)$ and/or bowel obstruction $(\mathrm{n}=20 ; 31.3 \%)$. The most frequently involved portion 
of the small bowel was the duodenum $(n=41 ; 64 \%)$. A segmental bowel resection was performed in 30 patients and pancreaticoduodenectomy in 14 patients. Of the 55 patients who underwent operative intervention a curative resection was performed in 30 patients $(54.5 \%)$. Postoperative mortality and morbidity were $3.6 \%(\mathrm{n}=2)$ and $14.5 \%(\mathrm{n}=8)$ respectively. The commonest sites of recurrence following a curative resection were liver and lung. Median survival for patients with adenocarcinoma of the small bowel was 18 months with a 5 -year survival of $21.1 \%$. On multivariate analysis, absence of distant metastatic disease at presentation $(n=45$, median survival 24 months, 5-year survival $30.4 \%)$, curative resection ( $\mathrm{n}=30$, median survival 56.4 months, 5 -year survival $44.8 \%$ ) and pathologic T stage 1-3 $(\mathrm{n}=22$, median survival 40.8 months, 5 -year survival 39.2\%) were independent predictors of survival (Figure 1). Conclusions: A curative resection in the absence of both distant metastatic disease and pathologic T4 provides the best survival outcome. Recurrence at distant sites is the predominant pattern of failure following a curative resection suggesting a role for improved adjuvant therapy.

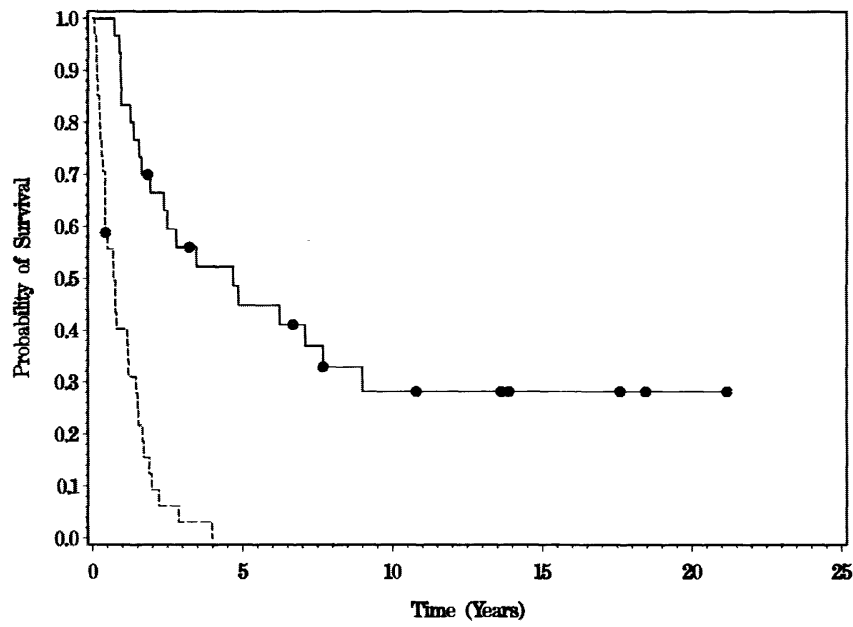

Kaplan-Meier survival curve for 30 patients who underwent a curative resection and 34 patients with residual disease. $P<0.0153$. Residual disease absent - Residual disease present

\section{P93}

Conversion of Laparoscopic Colon Resection Does Not Affect Survival in Colon Cancer J. Franko, ${ }^{2 *}$ M. Rezvani, ${ }^{1}$ B.G. O'Connell, ${ }^{1}$ S.G. Harper, ${ }^{1}$ J.H. Nejman, ${ }^{1}$ D.M. Zebley, ${ }^{1}$ S.A. Fassler. ${ }^{1}$ 1. Abington Memorial Hospital, Abington, PA; 2. Department of Surgical Oncology, University of Pittsburgh, Pittsburgh, PA.

INTRODUCTION: Laparoscopic and open resections of colon cancer are considered oncologically equivalent treatment methods based on noninferiority COST trial. Conversion of laparoscopic procedure, however, may be associated with decreased survival in colon cancer patients. We conducted this study to evaluate effect of conversion on survival. METHODS: A series of consecutive patients treated with laparoscopic resection of colorectal cancer $(n=123)$ in 1997-2002 was evaluated retrospectively for three-year survival. RESULTS: There was no statistically significant difference in all-cause mortality between laparoscopically completed and converted groups $(17 / 99,17.2 \%$ versus $5 / 24,20.8 \%$; $p=0.767)$. KaplanMeier survival analysis did not show any survival difference between both groups (Breslow, $\mathrm{p}=0.644$ ). CONCLUSIONS: Our data do not support prior evidence suggesting decreased survival in patients requiring conversion of laparoscopic colorectal resection for cancer. Laparoscopic resection of colorectal cancer can be safely offered to all patients, including those in high risk for conversion.

\section{P94}

Hilar Cholangiocarcinoma: Outcome Over 21 Years at a Tertiary Referral Center F. Ito, ${ }^{*}$ R. Rettammel, M. Been, D.M. Mahvi, L.F. Rikkers, S.M. Weber. General Surgery, University of Wisconsin, Madison, WI

Background: Hilar cholangiocarcinoma is an uncommon tumor with a poor prognosis. We sought to analyze predictors of outcome in a single institution over the last 21 years. Methods: From 1985 to 2006, patients with hilar cholangiocarcinoma referred to a tertiary surgical clinic were evaluated. Demographic data, tumor characteristics, and outcome were analyzed retrospectively. Results: A total of 91 patients with hilar cholangiocarcinoma were evaluated. At initial presentation, 22 patients $(24 \%)$ had unresectable disease. Of the 69 patients submitted to laparotomy, $31(45 \%)$ patients were unresectable due to metastases disease $(n=16)$, locally advanced disease $(n=4)$, or N2 nodal disease $(\mathrm{n}=11)$. Thirty-eight patients underwent resection, which included excision of the extrahepatic biliary system with porta-hepatis lymphadenectomy, with $(n=20)$ or without $(n=18)$ hepatic lobectomy. The operative (30 day) morbidity and mortality was $32 \%$ and $0 \%$. Of the 38 patients who were resected, 25 $(66 \%)$ had negative margins. For all patients submitted to exploration, median survival was 35 months for resected patients vs. 5 months for those with unresectable disease ( $p<0.0001$, median $\mathrm{FU}$ for surviving patients, 27 months). In resected patients, median survival for patients with negative margins was 55 months vs. 20 months for patients with positive margins $(p=0.02$ ). Factors predictive of improved disease specific survival included negative histological margins, concomitant hepatic lobectomy, lack of nodal disease, well-differentiated histology, and an earlier tumor stage $(\mathrm{p}<0.05)$. Over the years of the study, resectability increased from $25 \%(1985-1991)$ to $38.1 \%$ (1992-1998) and $46.5 \%(1999-2006, p=0.08)$. Conclusions: Hilar cholangiocarcinoma is an aggressive tumor, demonstrated by the finding that nearly half of the patients were found to have occult unresectable disease at the time of surgery. However, in patients treated with curative resection, the median survival was prolonged, indicating that aggressive operative strategy is safe and associated with improved prognosis. Further development of novel diagnostic strategies to improve early detection and to assess resectability of this disease is needed.

\section{P95}

An Epidemiologic Study of N-stage After Preoperative Radiotherapy for Rectal Cancer G.J. Chang, * M.A. Rodriguez-Bigas, J.M. Skibber. University of Texas, M.D. Anderson Cancer Center, Houston, TX.

BACKGROUND: Treatment of locally advanced rectal cancer includes preoperative chemoradiation, total mesorectal excision and systemic chemotherapy. The status of the regional lymph nodes $(\mathrm{LN})$ is the primary determinant of outcome after resection but may be affected by preoperative radiotherapy. We performed an epidemiologic evaluation of the impact of pathologic LN status after preoperative radiation for rectal cancer. METHODS: Patients with localized rectal adenocarcinoma were identified from the Surveillance Epidemiology and End Results registry from 1991 to 2002 . Overall (OS) and cancer-specific survival (CSS) by $\mathrm{pN}$ stage after radical surgery was compared between patients undergoing preoperative (PRE) or postoperative (POST) radiotherapy (XRT). Patient/tumor characteristics and number/status of $\mathrm{LN}$ were evaluated. Univariate comparisons were performed using Wilcoxon rank-sum for medians, chi-square analysis for proportions, and the log rank test for survival. Cox multiple regression was used to evaluate survival outcomes adjusted for confounders. RESULTS: Of 21,390 patients with localized rectal cancer, 7,067 $(33 \%)$ received preoperative $(n=2449$ PRE) or postoperative $(n=4618$ POST) $\mathrm{XRT}$ and radical resection. Patient/tumor characteristics are shown in the table. Among pN0 patients, 5-year OS and CSS were similar (PRE vs. POST, 73.9\% vs. $71.3 \%$, $\mathrm{p}=0.38 \mathrm{OS}, 80.5 \%$ vs. $78.8 \% \mathrm{CSS}, \mathrm{p}=0.44$ ). However, $\mathrm{pN} 1$ patients in the PRE group had worse survival $(58.4 \%$ vs. $62.4 \%, p=0.006$ OS, $63.1 \%$ vs $68.4 \% \mathrm{CSS}, \mathrm{p}=0.003)$ as did $\mathrm{pN} 2$ patients $(30.6 \%$ vs $41.7 \%$, $\mathrm{p}=0.016 \mathrm{OS}, 37 \%$ vs $45.9 \%, \mathrm{p}=0.049 \mathrm{CSS}$ ). Preoperative radiotherapy was also an important multivariate predictor of reduced OS (RR 1.43,95\% CI 1.20-1.72, $\mathrm{p}=0.0001)$ and CSS (RR 1.46, 95\% CI 1.21-1.78, $\mathrm{p}=0.0001$ ) among $\mathrm{pN1}-2$ patients. CONCLUSIONS: This population-based study identifies a subgroup of patients with rectal cancer who have an increased risk for failure after preoperative radiotherapy. These findings suggest $\mathrm{pN}+$ patients may benefit from more aggressive adjuvant chemotherapy regimens using contemporary combination agents.

\begin{tabular}{|c|c|c|c|}
\hline Variable & PRE & POST & $\mathrm{p}$ \\
\hline Age* $^{*}$ & 61 & 63 & 0.0001 \\
\hline pN0 vs pN+ & $55.8 \%$ & $38.1 \%$ & $<0.0001$ \\
\hline pN1 vs pN2 & $69.6 \%$ & $60.1 \%$ & 0.0001 \\
\hline Total \# LN* & 7 & 10 & $<0.000001$ \\
\hline \# pos LN* & 2 & 3 & $<0.000001$ \\
\hline
\end{tabular}

* median 


\section{P96}

Laparoscopic and Robotic-assisted Total Mesorectal Excision for Rectal Cancer: A 2-year experience with 38 consecutive cases M. Hellan, ${ }^{*}$ C. Anderson, A. Pigazzi. City of Hope, Duarte, CA.

Introduction: Laparoscopic total mesorectal excision (TME) is a difficult procedure and the role of minimally invasive surgery (MIS) for the treatment of rectal cancer is still undefined. Here we report our comprehensive experience with laparascopic and robotic-assisted rectal cancer surgery in a US cancer center. Methods: Clinicopathologic data from a prospectively maintained database of all rectal cases performed between November 2004 and August 2006 was reviewed. A total of 38 consecutive rectal procedures including low anterior, coloanal, and abdominoperineal resections with TME were performed. $66 \%$ of patients received neoadjuvant chemoradiation. Results: There were 37 patients with primary and one with recurrent rectal cancer. 11 cases were carried out laparoscopically (LAP), and 28 were performed with robotic-assistance (ROB). There was no mortality. There was one conversion to open surgery in each group (LAP $9.0 \%$ vs. ROB $3.7 \%$; $=$ NS). Operative time, blood loss, and length of stay were similar between the LAP and ROB groups (Table). In addition, there were no significant differences in pathologic outcomes, such as number of lymph nodes retrieved and margins. Distal and circumferential margins were negative in all cases. A TME with autonomic nerve preservation was accomplished in all patients. Major complications were similar in both groups (LAP $27 \%$ vs. ROB $22 \%$; $P=N S$ ). Overall, 4 patients had anastomotic leaks and one a pelvis abscess. Four patients required re-operation, and three drainage. One patient developed a late fistula 3 months postoperative. The use of the robot did not increase the operative time. Cases completed robotically were deemed to be technically easier by the operating surgeon. Conclusion: Both laparoscopic and roboticassisted surgery for rectal cancer can be carried out safely and according to established oncologic principles. The subjective operative experience for the surgeon appears to be superior with robotic operations. Future studies are needed to determine if robotic surgery can widen the applications of MIS in rectal cancer treatment.

\section{Operative Data}

\begin{tabular}{|l|c|c|c|c|c|}
\hline & OR time $(\mathrm{min})$ & EBL $(\mathrm{ml})$ & \#removed nodes & Distal margin $(\mathrm{cm})$ & LOS (days) \\
\hline LAP $(\mathrm{n}=11)$ & $260(168-540)$ & $120(50-1000)$ & $18(9-39)$ & $3.0(2.0-2.7)$ & $4(3-12)$ \\
\hline ROB $(\mathrm{n}=27)$ & $285(180-540)$ & $150(50-1000)$ & $14(6-28)$ & $3.4(0.3-6.4)$ & $4(3-23)$ \\
\hline
\end{tabular}

median values

\section{P97}

National Survey of Neuroendocrine Tumors of the Pancreas J.S. Hill,* J.T. McPhee, G.F. Whalen, A.C. Larkin, M.E. Sullivan, F.A. Anderson, J.F. Tseng. University of Massachusetts Medical School, Worcester, $M A$

Background: Pancreatic endocrine tumors often present as locally advanced or metastatic lesions due to their indolent nature. Surgical resection remains the only potentially curative treatment modality with superior median survival when compared to non-surgical treatments. The purpose of this study was to evaluate pancreatic endocrine tumors at the national level with regard to patient demographics, operative approach and in-hospital mortality. Methods: A retrospective survey from the Nationwide Inpatient Sample, the largest representative national database (1998-2003). Patient-discharges were identified by ICD-9 codes for a principal diagnosis of islet cell neoplasms of the pancreas. The initial cohort was characterized as operative vs non-operative. Evaluated procedure types included: pancreatectomy, hepatic resection, and bowel resection. Results: 4094 representative patients were identified with the primary diagnosis of islet cell neoplasm. $68 \%$ did not undergo operation, $27 \%$ underwent pancreatectomy, $0.7 \%$ hepatectomy, $0 \%$ had bowel-only procedures and $3.1 \%$ underwent a combination of the three. Of those who had pancreatic surgery, $54.5 \%$ underwent distal pancreatectomy, $24.8 \%$ underwent pancreaticoduodenectomy, $12.3 \%$ had nondefined pancreatic surgery and $<5 \%$ of patients underwent one of the following: proximal pancreatectomy, sub-total pancreatectomy or total pancreatectomy. $55 \%$ of all patients were female and $80 \%$ of those with race/ethnicity data were white. The majority of admissions $(63 \%)$ were seen at teaching hospitals; $77 \%$ of operations were performed at teaching hospitals. Total in-hospital mortality for all patients was $4.8 \%$. Mortality for dis- tal pancreatectomy was less than $1 \%$ and was $4.7 \%$ for pancreaticoduodenectomy. Conclusions: The majority of patients treated at U.S. hospitals for the diagnosis of pancreatic islet cell tumors will not undergo resection, presumably due to advanced disease. Prior studies have shown a survival advantage in patients undergoing primary tumor resection compared to patients that did not. Pancreatic resections for neuroendocrine tumors are associated with acceptable mortality rates and should be considered for appropriate candidates with these tumors.

\section{P98}

Real Time Intraoperative Ultrasound Guided Dissection of Superior Mesenteric and Portal Vein Improves Operative Time and Blood Loss During the Whipple Procedure R.M. Zaidi, ${ }^{2 *}$ S.C. Stain, ${ }^{1}$ A. Nigam. ${ }^{1}$ 1. Albany Medical Center, Albany, NY; 2. North Shore LIJ Hospital Systems, New Hyde Park, NY.

Introduction: Intraoperative ultrasound for directly guiding the dissecting instruments - particularly during portal vein (PV) and superior mesenteric vein (SMV) dissection - has not yet been described. This study compares the series of pancreaticoduodenectomies at a tertiary care teaching hospital prior to and following the introduction of intraoperative ultrasound guidance (IOUG) for PV-SMV vein dissection. Methods: A retrospective review was conducted of all pancreaticoduodenectomies $(n=27)$ performed from January 2005 to May 2006 by a single experienced pancreatic surgeon. Operative factors including estimated blood loss (EBL), length of operative procedure, patient outcomes, demographics, TNM staging, operative assessment of PV-SMV tumor involvement, and need for PVSMV resection were evaluated before and after initiating the use of IOUG on January 31, 2006. Results: Of a total of 27 pancreaticoduodenectomies, 5 patients were excluded for associated procedures or previous gastric surgeries. The remaining 22 patients were divided into two groups: patients with IOUG $(\mathrm{N}=11)$ and patients without IOUG $(\mathrm{N}=11)$. Patient characteristics, tumor staging, and indications for surgery did not differ between the groups. Mean operative time was significantly lower in the IOUG group (6 hours 23 minutes) vs. non-IOUG group ( 8 hours 13 minutes) with $\mathrm{p}<0.005$. EBL was also less for the IOUG group, but did not attain statistical significance $(p=0.17)$. However, the range for EBL was $100-600 \mathrm{~mL}$ in the IOUG group vs. $200-1100 \mathrm{~mL}$ in the non-IOUG group. There was no difference between the groups in outcomes or complications. Conclusions: IOUG during the PV-SMV dissection is very helpful during pancreaticoduodenectomy and significantly reduces operative time. Procedures with IOUG occur with less blood loss, but this study's sample size likely prohibits this measure from attaining statistical significance. Nevertheless, based on our experience, we have altered our practice and now use IOUG during all our pancreaticoduodenectomies.

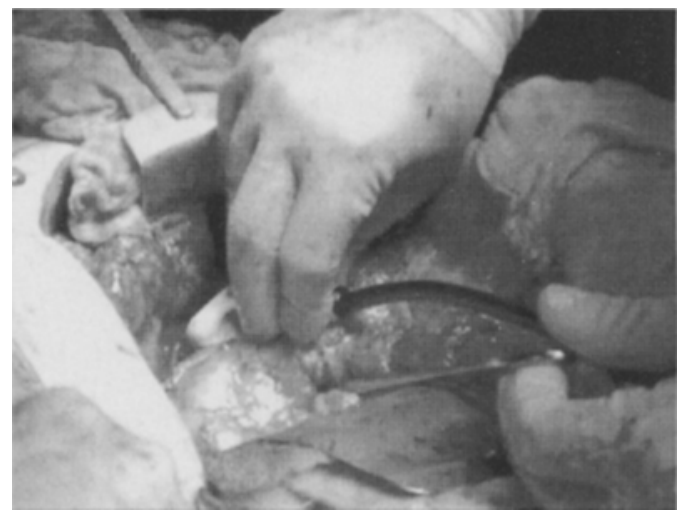

Intraoperative ultrasound guides scissors along the SMV, allowing safe directed dissection. 


\section{P99}

A Clinicogenomic Analysis of Right and Left-Sided Colon Cancers J.M. McLoughlin,* E.H. Jensen, M. Alvarado, E. Siegel, Q. Ly, J.M. Lewis, R.M. Nair, S. Eschrich, T. Yeatman, D. Shibata. Surgical Oncology, H. Lee Moffitt Cancer Center \& Research Institute, Tampa, FL.

Introduction: It is postulated that right-sided $(\mathrm{RC})$ and left-sided colon cancers (LC) may represent genetically distinct entities due to differing embryologic origins and fecal exposures. Differences in presentation, response to therapy, prognosis and epidemiology have been noted. Using a comprehensive clinicogenomic database, we have compared clinical variables and microarray gene expression (GE) profiles between RC and LC. Methods: From 1993 to 2003, colon cancer specimens were collected from patients consenting to our tissue bank protocol. Surgical specimens were flash frozen and underwent RNA extraction. We identified a total of 163 analyzable primary cancers (71 $\mathrm{RC}$ and $92 \mathrm{LC}$ ). All specimens underwent GE profiling using the Affymetrix 133 Plus GeneChip. Clinical comparisons were performed using the Fisher Exact Test. GE data were normalized and then compared using the Mann-Whitney test. A Qvalue analysis was used to estimate the false discovery rate. Using MetaCore software, potential pathways and interactions between differentially expressed genes were identified. Results: The study population consisted of 87 males and 76 females. There were no significant differences between LC and $R C$ with regard to TNM stage or histologic features. Patients with $L C$ were more likely to present with hematochezia $(24 \mathrm{vs.} 63 ; \mathrm{p}<.001)$. LC were more likely to recur locally ( 12 vs. $1 ; p=0.007)$ and in the liver (9 vs. $1 ; p=0.03)$. RC were more likely to present with metastases to the lung ( $5 \mathrm{vs.} 0 ; \mathrm{p}=0.01)$. RC displayed a 207 downregulated and 68 upregulated genes as compared to LC. Upregulated biological pathways in LC included cell adhesion ( $p=0.0003$; e.g.TIMP $3+1.52$ fold-change, Osteonectin +1.53 ) and extracellular signaling $(p=0.0004$; e.g.- PTHrp +1.26 , Collagen $1 \mathrm{~A} 1+1.39$, Collagen $1 \mathrm{~A} 2+1.43)$ Conclusion: LC and RC demonstrate clinical differences such as symptom presentation and metastatic profiles. We have demonstrated that a number of genetic differences exist between cancers from these locations and may account for some of the observed clinicopathologic differences. In this expanding era of targeted therapies, the genetic differences that exist between LC and RC may be further exploited.

\section{P100}

Laparotomy for acute complications of gastrointestinal metastases from lung cancer: Is it worthwhile or is the effort futile? B.K. Goh, ${ }^{1 *}$ A.W. Yeo, ${ }^{1}$ H.N. Koong, ${ }^{2}$ L.L. Ooi, ${ }^{2}$ W.K. Wong. ${ }^{1}$ 1. Surgery, Singapore General Hospital, Singapore, Singapore; 2. National Cancer Centre, Singapore, Singapore.

Introduction: Complications of gastrointestinal tract (GIT) metastases from lung cancer are rare and the management remains controversial. Whereas some authors advocate a non-operative policy due to the poor prognosis, others recommend aggressive surgery as it offers effective palliation. The aim of this study is to present our experience with 9 patients who underwent laparotomy for complications of GIT metastases and to determine their outcome. Methods: Between 1995 to 2005, 9 patients who underwent laparotomy for complications of pathologically proven GIT metastases secondary to lung cancer were retrospectively reviewed. Results: All patients were male with a median age of 63 years (range, $40-70$ years) at initial presentation. The sites of symptomatic GIT metastases include the ileum $(n=3)$, jejunum $(n=3)$, cecum $(n=1)$, duodenum $(n=2)$ and stomach $(n=2)$ and the patients presented with obstruction $(n=2)$, hemorrhage $(n=3)$, intussusception $(n=3)$ and perforation $(n=1)$. All the patients underwent exploratory laparotomy and the definitive surgical procedure was dependant on the site and extend of disease. These include small bowel resection with primary anastomosis $(n=5)$, subtotal gastrectomy with extended right hemicolectomy $(n=1)$, gastrojejunostomy $(n=1)$, right hemicolectomy $(\mathrm{n}=1)$ and ulcerectomy with under-running of ulcers. Eight of the 9 patients recovered from surgery and were discharged from hospital at a median time of 9.5 days. All 8 patients survived for more than 30 days and the median survival was 6 months (range, 2 to 13 months). Four of 8 patients lived for more than 6 months and all 8 patients died of advanced metastatic lung cancer with multiple sites of metastases at the time of death. Conclusion: GIT metastases should always be considered in lung cancer patients presenting with an acute abdomen. Aggressive surgical treatment is worthwhile in a selected group of patients as it provides effective palliation.

\section{P101}

Solid pseudopapillary neoplasms of the pancreas: an updated experience B.K. Goh, ${ }^{1}$ * Y.M. Tan, ${ }^{2}$ P.C. Cheow, ${ }^{1}$ Y.F. Chung, ${ }^{1}$ P.K. Chow, ${ }^{1}$ W.K. Wong, ${ }^{1}$ L.L. Ooi. ${ }^{2}$ 1. Surgery, Singapore General Hospital, Singapore, Singapore; 2. National Cancer Centre, Singapore, Singapore.

Introduction: Solid pseudopapillary neoplasms (SPPNs) of the pancreas are rare neoplasms of low malignant potential which typically occur in young females. The aim of this study is to report an update of the surgical experience at a single institution with these unusual tumors. Methods: The records of 16 consecutive patients who underwent surgery for a pathologically confirmed SPPN were retrospectively reviewed. Results: Fifteen of the patients were female and the median age at diagnosis was 30 years (range, 14-53 years). Abominal and back pain were the most common presenting symptoms. The tumors appeared on cross-sectional imaging as solid and cystic $(\mathrm{n}=14)$ or cystic $(\mathrm{n}=2)$ masses. The median tumor size was $9.5 \mathrm{~cm}$ (range, $5.0-24.0 \mathrm{~cm}$ ). All 16 patients had curative resections including 3 pancreaticoduodenectomies and 13 distal pancreatectomies. Three patients required extended resections including pancreaticoduodenectomy with portal vein resection, distal pancreatectomy with tranverse colectomy and distal pancreactomy with omentectomy. Two of the resections were R1 resections whereas 14 were R0 resections. All 16 patients were alive and disease-free at a median follow-up of 43 months (range, 3 to 186 months). Conclusion: SPPNs are rare neoplasms of the pancreas. This diagnosis should always be considered in young women presenting with a large heterogenous solid-cystic pancreatic mass. Aggressive en bloc resection should always be attempted including resection of concomitant metastases as patients demonstrate excellent long-term survival even in the presence of distant spread.

\section{P102}

Current relevance of ultrasonography of the neck in the preoperative staging of cancer of the esophagus and gastroesophageal junction L.M. Schreurs, ${ }^{1}{ }^{*}$ C.C. Verhoef, ${ }^{1}$ E. Vd Jagt, ${ }^{2}$ G. Van Dam, ${ }^{1}$ H. Groen, ${ }^{3}$ J.T. Plukker. ${ }^{1}$ 1. Dept of Surg. Oncology, University Medical Center Groningen, University of Groningen, Groningen, Groningen, Netherlands; 2. Dept of Radiology, University Medical Center, Groningen, Netherlands; 3. Dept of Epidemiology, University Medical Center, Groningen, Netherlands.

Introduction: In general, the staging algorithm for malignant tumors has been changed by current dedicated diagnostic tools. We determined the impact of ultrasonography (US) of the neck on current preoperative staging of patients with cancer of the esophagus and gastro-esophageal junction (GEJ). Methods: We analyzed the staging value of external US in 180 consecutive esophageal cancer patients without cervical lymphadenopathy on physical examination, who were treated during the period January 2001 and March 2006. Round cervical lymph nodes with clearly defined boundaries, larger than $5 \mathrm{~mm}$ and scattered internal echo pattern were suspected for malignancy and confirmed cytologically by ultrasound-guided FNA. Group A, consisted of 125 patients, who were staged according to a previous standard protocol, including endoscopic ultrasonography (EUS), multidetector computed tomography (md-CT), positron emission tomography with 18-fluorodeoxyglucose (FDG-PET) and ultrasonography of the neck. Based on the results from group A, a prospective group of 55 consecutive patients (group B) was staged following a current standardized protocol with EUS and mdCT, and an additional FDG-PET only in patients with stage III and IV disease. External US of the neck in group B was solely performed on indication. Results: In $12(7 \%)$ out of the 180 patients cervical node metastases were found (group A: 7 pts; group B: 5 pts). All nodal metastases were detected by the combination of FDG-PET and md-CT. The application of the diagnostic algorithm used in group B, showed external US to be unnecessary as no cervical metastases were missed. Conclusion: With current state of the art staging procedures, routine external US of the neck is not useful in detecting cervical lymph node metastases in patients with cancer of the esophagus and GEJ. External US is only indicated to obtain cytological proof of suspected cervical metastases. 


\section{P103}

Trends in Adjuvant Treatment of Gastric Cancer: An Analysis of 9,528 Patients U.-. Guller, ${ }^{1 *}$ N.N. Baxter, ${ }^{2}$ C.H. Law, ${ }^{3}$ C.J. Swallow, ${ }^{4}$ N.G. Coburn. ${ }^{3}$ 1. Division of Surgical Oncology, University of Toronto, Toronto, ON, Canada; 2. Division of General Surgery, St. Michael's Hospital, University of Toronto, Toronto, ON, Canada; 3. Division of Surgical Oncology, Sunnybrook Health Sciences Centre, University of Toronto, Toronto, ON, Canada; 4. Division of Surgical Oncology, Princess Margaret Hospital, University of Toronto, Toronto, ON, Canada.

Background: The Intergroup 0116 trial $(0116)$ showed that adjuvant chemoradiotherapy (ADJTx) improves survival in patients with gastric adenocarcinoma. The objective of this study is to evaluate the impact of 0116 on the use of ADJTx and the variables that influence this decision. Methods: From 1996-2003, patients 18-85 yrs who underwent curative resection of gastric adenocarcinoma were identified in the Surveillance Epidemiology and End Results (SEER) database. Patients were classified into two subsets: those resected prior to presentation of the 0116 results at ASCO (Jan 1996-April $2000=$ PRE) vs after (May 2000-Dec 2003 = POST). Univariate and multivariable models were used to determine factors associated with use of ADJTx in patients diagnosed from May 2000 - December 2003. Results: Overall, 9,528 patients were included, with a median age of $70 \mathrm{yrs}$. The percentage of patients given ADJTx in the PRE group was $15 \%$, rising to $24 \%$ POST ( $<<0.001$ ), with the most significant increase in the months surrounding the ASCO meeting. Significant increases in ADJTx usage from PRE to POST were seen in all demographic categories studied. In the POST group, patients aged 18-59 yrs were significantly more likely to receive ADJTx (46\%) than those 60-74 yrs (32\%) and 75 yrs and older $(13 \%, \mathrm{p}<0.0001)$. Higher tumor stage was associated with greater use of ADJTx $(p<0.0001)$. Patients having total gastrectomy were more likely to receive ADJTx (32.5\%) than those having proximal $(27 \%)$ or distal gastrectomy $(29 \%, \mathrm{p}=0.048)$. Married patients were significantly more likely to receive ADJTx (31.8\%) than unmarried patients $(24.9 \%, \mathrm{p}<0.001)$. The rate of ADJTx ranged from $24 \%$ to $45 \%(p<0.001)$ in the SEER regions. In multivariable analysis, age, SEER region, type of surgery, marital status, and tumor stage were all found to be significant predictors of receiving ADJTx. Conclusions: Use of ADJTx for gastric cancer significantly increased following 0116. However, the fraction of patients receiving ADJTx is still low, with significant variability according to patient demographics, resection type, and SEER region. The causes of this variability must be explored and continued educational efforts are required.

\section{P104}

Abdominosacral resection - the new face of a "historical" approach to low rectal cancer M. Bebenek, ${ }^{*}$ M. Pudelko, K. Cisarz,

W. Tupikowski, A. Balcerzak. Surgical Oncology, Lower Silesian Center of Oncology, Wroclaw, Poland.

Introduction In spite of the implementation of total mesorectal excision (TME), the prognosis in low-rectal cancer patients is still unfavorable. Consequently, there is a necessity for an alternative technique for the abdominoperineal (APR) resection presently performed in low-rectal cancer patients. The purpose of the present study is to present our experiences with abdominosacral resection (ASR) - a potential alternative for APR. Methods Low-rectal cancer ( $\leq 5 \mathrm{~cm}$ from the anal verge) patients $(\mathrm{n}=126)$ were operated on by means of ASR between May 5th, 1998 and February 23rd, 2005. In our retrospective study the percentages of five-year overall (observed and relative) survivals as well as the frequencies of local recurrence were determined for all the patients with complete follow-up documentation. Results Five-year follow-up documentation was available for all 45 patients operated on in 1998. Twenty-three of them had survived five years after surgery, which corresponded to $51.1 \%$ and $63.7 \%$ of observed and relative survivals, respectively. Overall postoperative morbidity (most commonly wound infections) amounted to $14 \%$, whereas the cumulative five-year local recurrence rate was $4.7 \%$. Conclusion The combined use of the modern TME technique and the "historical" abdominosacral excision of the rectum seems to give new, potentially useful perspectives for successful surgical treatment of low-rectal cancers.

\section{P105}

Long-term oncologic outcome following pelvic exenteration for locally advanced rectal adenocarcinoma $\mathrm{S}$. Ishiguro, ${ }^{*} \mathrm{Y}$. Moriya, T. Akasu, S. Fujita, S. Yamamoto. National Cancer Center Hospital, Tokyo, Japan.

Objective: Our aims were to (1) determine the long-term oncologic outcome for patients with rectal cancer treated with pelvic exenteration (PE), (2) identify factors predictive of oncologic outcome. Methods: One hundred seven consecutive patients with locally advanced rectal adenocarcinoma suspicious of extension to adjacent organs were treated with PE from 1976 to 2005. A collected database was retrospectively queried for long-term oncologic outcome and predictive clinicopathological factors. Results: For 99 patients, PE was carried out with curative intent, for the remaining 8 with palliative intent. Radiation therapy (preoperative, intraoperative, or postoperative) was performed for 23 patients. Total PE (including combined resection of 6 distal sacrectomies and 4 hepatectomies) was performed for 88 patients, anterior PE for 19, and posterior PE for one. With a median follow-up of 34 months, the estimated 5year overall survival (OS) was $47 \%$ and 5 year recurrence-free survival (RFS) was $42 \%$. On multivariate analysis, lateral lymph node metastasis (hazard ratio; $1.96, \mathrm{p}=0.05)$, lymphovascular-perineural invasion $(1.97, \mathrm{p}=0.04)$, and carcinoembryonic antigen level $10 \mathrm{mg} / \mathrm{dl}$ and more $(1.95, \mathrm{P}=0.02)$ were significantly associated with OS. Only lateral lymph node metastasis $(2.74, \mathrm{p}=0.002)$ was significantly associated with RFS. Conclusions: Treatment of locally advanced rectal cancer with pelvic exenteration can provide for a 5-year OS of $47 \%$ and RFS of $42 \%$. Patients with rectal cancer accompanied by no lateral lymph node metastasis were likely to be the most favorable candidates for this operation.

\section{P106}

Intra-arterial hepatic radiotherapy with 90 Yttrium Sir-Spheres in unresectable,colorectal liver metastases refractory to $i . v$. chemotherapy M. Cosimelli, ${ }^{1}$ L. Carpanese,${ }^{1}$ R. Sciuto, ${ }^{1}$ R. Golfieri, ${ }^{2}$ P. Cagol, ${ }^{3}$ F. Fiore, ${ }^{4}$ M. Cantore. ${ }^{5}$ 1. Digestive Surgery, Regina Elena Cancer Institute, Rome, Italy; 2. University of Bologna, Bologna, Italy; 3. University of Udine, Udine, Italy; 4. G.Pascale Cancer Institute of Naples, Napoli, Italy; 5. MassaCarrara Hospital, MassaCarrara, Italy.

INTRODUCTION A prospective clinical trial was aimed to evaluate role of SIRT on clinical response, time to liver progression, toxicity, median survival in patients with colorectal liver metastases progressing as only site after FOLFOX and FOLFIRI. METHODS From June 2005,35 pts. were enrolled,pretreatment evaluation included a CT scan,blood tests, a PET scan and arteriography of celiac trunk, hepatic and superior mesenteric artery;extrahepatic uptakes and pulmonary shunts more than $10 \%$ were excluded by a Scintiscan.The gastroduodenal artery was embolized before the SIR-spheres injection.The median dose administered was $1.7 \mathrm{GBq}$ (range, 0.9-2.2).The RECIST criteria for evaluating response were utilized.The median number of metastases was 4 (range, 1-15).Median diameter of lesions was $47 \mathrm{~mm}$ (range, 2097). RESULTS A median pulmonary shunt was $6 \%$. There was no mortality; $20.6 \%$ and $24.1 \%$ of pts. showed G1-2 toxic events within or after 48 hours respectively. Fever, acute pain and leucocytosis were the most observed within 48 hours while chronic pain,jaundice,nausea, the tardive ones. The partial responses were $12.5 \%$, the stable diseases $75 \%$ and progressions $12.5 \%$ after 2 months.Progression of disease was extrahepatic in $48.1 \%$ of pts. and cancerrelated deaths were $11.1 \%$. We analyzed 24 both metastatic and healthy tissue liver biopsies, before and after treatment.Morphological evaluation showed intratumoral necrosis and intensive desmoplastic reaction with lymphomonocitary flogosis in all the lesions after microspheres administration. No relevant macroscopical changes in normal liver parenchyma was found after SIRT. Immunohistochemical analysis showed an high index of proliferation (ki-67 > 40\%) in all the lesions while the Bcl-2 expression was always $<2 \%$.Decreased quantitative and qualitative phenotypical expression of p53 was observed in patients responding to treatment. CONCLUSIONS The SIRT is a safe treatment. Acute and late toxicity is acceptable.SIRT represents a good third therapeutic option when liver is the only site of disease.Longer followup time will clarify duration time of both response and stable disease. 


\section{P107}

Colorectal Tumors show increased amphiregulin expression

A.S. Caudle, * L.S. Caskey, H.S. Earp, B.F. Calvo. Surgery, University of North Carolina, Chapel Hill, NC.

Cetuximab, an antibody inhibiting EGFR ligand binding, has a $20 \%$ response rate in colorectal (CR) cancer though EGFR is not overexpressed or mutated. We have shown that EGFR is not increased using qPCR to measure EGFR mRNA levels in CR tumors and patient-matched normal colon mucosa (NCM). Extending this to the ligand family reveals increases in amphiregulin (AR) in tumors. Our goal is to confirm this in paraffin (PF) samples and study the impact of AR signaling in CR cell lines. Methods: We used quantitative PCR assays for Her1-4 and ligands (AR, TGF- $\alpha$, HB-EGF, EGF and HRG) using fluorescent probes. Full-length RNAs were transcribed and used to construct standard curves permitting absolute mRNA quantitation of RNA extracted from 9 colon cell lines and 31 paraffin-embedded colon carcinomas with patientmatched NCM. Proliferative effects of AR on cells were assayed using the colorimetric MTS assay (Promega). Time-course protein lysates were immunoprecipitated, and back blotted with anti phospho-tyrosine antibody. Results: Colorectal tumors express mean AR mRNA copy number $2.8 \mathrm{x}$ greater than matched NCM (4800 vs. 1688). While absolute mRNA copy number is lower in PF samples than in frozen samples, the mean increase over NCM is comparable (2.8 vs. 2.7$) .77 \%$ of tumors have higher AR than matched NCM and $29 \%$ have $>3 x$ increase. DLD-1 cells express high EGFR (47,727 copies) and $\mathrm{AR}(14,571$ copies) and show a dose-dependant proliferation increase with AR as well as strong EGFR and Her-2 phosphorylation likely secondary to dimer formation. AR treatment in SW480 cells, expressing average EGFR $(19,662$ copies) and low AR (272 copies), does not increase proliferation or phosphorylation of EGFR or Her-2. Conclusions: AR expression is higher in CR tumors in both frozen and PF samples. As EGFR is not overexpressed or mutated, the clinical activity of cetuximab may be explained by the ligand mileau. Cell lines' differential response to AR suggests that $\mathrm{CR}$ tumors expressing high $\mathrm{AR}$ levels may be more sensitive to EGFR blockade by agents such as cetuximab. The ability to quantify AR in paraffin samples allows us to probe archived specimens of patients treated with cetuximab to assess AR's use as a biomarker.

\section{P108}

A large-scale study of $M T 1 M M P$ as a marker for isolated tumor cells in peripheral blood and bone marrow in gastric cancer cases K. Mimori, ${ }^{1}$ T. Fukagawa, ${ }^{2}$ Y. Kosaka, ${ }^{1}$ K. Ishikawa, ${ }^{2}$ T. Etoh, ${ }^{2}$ M. Sasako, ${ }^{2}$ M. Mori. ${ }^{1}$ 1. Department of Surgical Oncology, Medical Institute of Bioregulation, Kyushu University, Beppu, Oita, Japan; 2. National Cancer Center Hospital, Chuoku, Tokyo, Japan.

Introduction: This report examines the clinical outcomes of cancer patients after 12 post-operative years to determine the prognostic value of $C E A \mathrm{mRNA}$ in peripheral blood (PB) (Mori M., JCO 1997). In addition, a novel ITC marker, identified by microarray analysis, was validated with 959 cases of gastric cancer (GC) by Taqman RT-PCR. Patients and methods: 1) The clinical outcomes of 102 cases were reinvestigated and compared to evaluate $C E A$ expression in PB. 2) Metastatic-related genes present in bone marrow (BM) were identified by cDNA microarray analysis of total RNA from the whole blood of four metastatic and four non-metastatic cases. Among the up-regulated genes in the metastatic cases, MTIMMP was chosen for further analysis. 3) $M T 1 M M P$ expression was validated by Taqman RT-PCR using the PB and BM from 959 cases of GC. Results: 1) Among 102 cases, 12 that were $C E A(-)$ showed novel distant metastasis; however, the incidence of $C E A$ in $P B$ was not associated with OS and DFS. 2) Expression levels of MTIMMP were up-regulated in metastases. MT1MMP localizes to the cell surface to regulate tumor invasion, and is expressed from cancer cells and/or interstitial cells via cell-cell interactions. 3) The positive rate of GC cases with MT1MMP expression in PB was larger in $13(72 \%)$ cases with distant metastasis than in $296(31 \%)$ cases without distant metastasis $(\mathrm{p}=0.0002)$. Five $(27.8 \%)$ cases with distant metastasis showed a higher incidence of $M T I M M P(+)$ in BM compared with $71(7.3 \%)$ cases without distant metastasis. Furthermore, $3(25 \%)$ cases with recurrence were $M T 1 M M P(+)$ in $\mathrm{BM}$, while $73(7.5 \%)$ cases without recurrence were MTIMMP (-) in BM, a significant association $(\mathrm{p}=0.020)$. Conclusion: Based on the long follow-up period, we conclude that presence of the epithelial marker $C E A$ in PB cannot be used as a prognostic or recurrent marker for GC. This large-scale study revealed that $M T 1 M M P$ expression could be an effective predictor of clinical outcome in GC cases. In GC, metastasis from primary can- cer tissues may require cancer cells with high metastatic potential and/or circumstances, such as MTIMMP expression in $\mathrm{PB}$ or $\mathrm{BM}$.

\section{P109}

Pseudomyxoma Peritonei: Clinical, Pathological and Biological Prognostic Factors in Patients Treated with Cytoreductive Surgery and Hyperthermic Intra-Peritoneal Chemotherapy D. Baratti,* D. Nonaka, S. Kusamura, B. Laterza, M. Deraco. Istituto Nazionale per la Cura e lo Studio dei Tumori, Milano, Italy.

BACKGROUND The combination of cytoreductive surgery (CRS) and hyperthermic intra-peritoneal chemotherapy (HIPEC) has been recently advocated as the standard of care for pseudomyxoma peritonei (PMP). We reviewed our ten year mono-institutional experience with PMP in an effort to identify significant prognostic factors after CRS and HIPEC. METHODS Seventy-five patients with PMP underwent CRS and closed abdomen HIPEC with mytomicin-C and cis-platinum; 20 underwent systemic chemotherapy (CT) before admission. PMP was classified into disseminated peritoneal adenomucinosis (DPAM), peritoneal mucinous carcinomatosis (PMCA) and PMCA with intermediate/discordant features (PMCA-ID). The prognostic significance of the following variables was assessed by multivariate analysis: age, sex, performance status, prior surgical score, histological subtype, prior systemic CT, disease extent, completeness of cytoreduction. Immunohistochemical stains were performed for cytokeratin (CK) 7, CK20, CDX-2, MUC-2, MUC-5AC and CD-44s in 42 patients. RESULTS Optimal CRS (residual tumor nodules $\leq 2.5 \mathrm{~mm}$ ) was performed in 72 patients $(96 \%)$. Operative mortality was $1 \%$. Sixty-two patients were diagnosed with DPAM, 13 with PMCA, none with PMCA-ID. Median follow-up was 37 months (range 1-120). Five-year overall (OS) and progression-free (PFS) survival were $78.3 \%$ and $31.1 \%$ in the overall series. Optimal CRS, no previous systemic CT and DPAM were independent predictors of better OS and PFS at multivariate analysis. CK20, CDX-2, MUC-2 were diffusely positive in all cases; CK7, MUC-5AC, CD44s were variably expressed in most cases (see table). No marker correlated to prognosis. CONCLUSIONS Favourable outcome after CRS and HIPEC can be expected in patients affected by the DPAM variant of PMP undergoing optimal surgical cytoreduction and not treated with preoperative systemic CT. Expression of MUC-2, MUC-5AC and CD44s may be related to the unique disease features of PMP

Immunohistochemical results

\begin{tabular}{|c|c|c|c|c|c|c|}
\hline \multicolumn{7}{|c|}{ N. of patients } \\
\hline Score & CK7 & CK20 & CDX2 & MUC-2 & MUC5AC & CD44s \\
\hline Negative & 4 & 0 & 0 & 0 & 0 & 2 \\
\hline$\leq 25 \%$ & 1 & 0 & 0 & 0 & 1 & 6 \\
\hline$>25 \leq 50 \%$ & 4 & 0 & 2 & 0 & 4 & 13 \\
\hline$>50 \leq 75 \%$ & 14 & 3 & 1 & 3 & 17 & 17 \\
\hline$>75 \%$ & 19 & 39 & 39 & 39 & 20 & 4 \\
\hline
\end{tabular}

\section{P110}

Smoking increases complications in patients with locally advanced or recurrent rectal cancer treated with surgery and chemoradiation J. Kasemmongkol, ${ }^{1}$ A.K. Seltman, ${ }^{1 *}$ J. Thacker, ${ }^{1}$ F.B. Enders, ${ }^{2}$ N.N. Diehl, ${ }^{2}$ K. Lopiano, ${ }^{2}$ M.G. Haddock, ${ }^{3}$ H. Nelson. ${ }^{1}$ 1. Division of Colon and Rectal Surgery, Mayo Clinic, Rochester, MN; 2. Division of Biostatistics, Mayo Clinic, Rochester, MN; 3. Department of Radiation Oncology, Mayo Clinic, Rochester, MN.

Background: In previous studies, smoking has been associated with increased surgical complications and diminished response to chemoradiation. We were concerned that patients with locally advanced or recurrent rectal cancer requiring surgical resection, chemoradiation, and intraoperative radiotherapy (IORT) (multimodality therapy) might have compromised outcomes secondary to smoking exposure. We tested the hypothesis that smoking negatively impacts surgical and oncologic outcomes in rectal cancer patients treated with multimodality therapy. Methods: 387 patients undergoing multimodality therapy for advanced or recurrent rectal cancer from 1981-2000 were identified within a prospective IORT database. Data on demographics, smoking, treatment, short and long term complications and oncologic outcomes were 
gathered. Patients were divided into nonsmoker $(\mathrm{n}=159)$, former smoker $(\mathrm{n}=172)$ and current smoker $(\mathrm{n}=56)$ groups. Research complied with HIPAA. Results: Median follow-up was 33 months (1-199) for all patients and 64 months $(1-198)$ in survivors. Smoking was more prevalent among males $(p=<0.01)$. Current smokers were younger than non- and former smokers (median age 58 vs $62,64, p=0.03$ ). Distribution of tumor, pathology, fixation, residual tumor and use of flaps were similar between groups. Primary tumors were more prevalent in current smokers $(39 \%$ vs $20 \%, 19 \%$ for nonsmoker and former smokers, $\mathrm{p}=0.01$ ). Comparable chemoradiotherapy regimens were used in all groups. Current and former smokers were significantly more likely than nonsmokers to experience one or more treatment-related complications $(86 \%, 89 \%$ and $52 \%$ respectively, $\mathrm{p}<0.01$ ). Wound, pulmonary, urinary tract, thrombotic and neurologic complication rates were higher in both former and current smokers (see table). There was no difference in incidence of bowel obstruction, abscess/leak, or flap complications. Number of recurrences, time to recurrence, and survival were equivalent between groups. Conclusions: Smoking increases short and long term treatment-related complications following multimodality therapy for rectal cancer but does not impact oncologic efficacy.

\begin{tabular}{|c|c|c|c|c|}
\hline Complications & Non-smoker n=159 & Former smoker n=172 & Current smoker n=56 & $\boldsymbol{p}$ value* \\
\hline Wound & $10(6 \%)$ & $26(15 \%)$ & $5(9 \%)$ & 0.03 \\
\hline Pulmonary & $7(4 \%)$ & $30(17 \%)$ & $10(18 \%)$ & $<0.01$ \\
\hline Urinary tract & $33(21 \%)$ & $80(47 \%)$ & $29(52 \%)$ & $<0.01$ \\
\hline DVT/PE & $5(3 \%)$ & $31(18 \%)$ & $7(13 \%)$ & $<0.01$ \\
\hline Neurologic & $16(10 \%)$ & $32(19 \%)$ & $12(21 \%)$ & 0.04 \\
\hline
\end{tabular}

${ }^{\star}$ Fisher's Exact Test

\section{P111}

Recurrence patterns in sentinel lymph node positive patients with primary cutaneous melanoma of the head and neck $\mathrm{J}$. Yeh, ${ }^{1}$ * E.D. Routh, ${ }^{1}$ J.T. Woosley, ${ }^{2}$ J.S. Frank, ${ }^{1}$ M.O. Meyers, ${ }^{1}$ D.W. Ollila. ${ }^{1}$ 1. Division of Surgical Oncology, Lineberger Comprehensive Cancer Center, University of North Carolina at Chapel Hill, Chapel Hill, NC; 2. Department of Pathology, Lineberger Comprehensive Cancer Center, University of North Carolina at Chapel Hill, Chapel Hill, NC.

Introduction: Sentinel lymph node (SLN) status is the most important prognostic factor for recurrence and survival in primary cutaneous melanoma of the trunk and extremities. Recent studies have shown that SLN biopsy (SLNB) may be performed accurately and with low morbidity in patients with cutaneous melanoma of the head and neck (CMHN) despite anatomical challenges and complex lymphatic drainage. Whether SLN status influences outcome in this patient population remains unclear. The aims of this study were to: 1) examine the frequency of SLN metastases; 2) determine the patterns of recurrence; and 3) determine if SLN metastases is associated with differences in outcome in patients with CMHN. Methods: Retrospective review of a prospective database was performed of all patients with CMHN that underwent a SLNB from 1998 to 2006. Results: 124 patients underwent SLNB for CMHN. Median age was 66 years, median thickness $1.5 \mathrm{~mm}$, and $77 \%$ of patients were male. A SLN was successfully identified in all patients. In 15/124 (12\%) patients, the SLN was positive for metastatic disease. All SLN-positive patients underwent completion lymph node dissection (CLND). Median number of lymph nodes obtained at CLND was 24 . Only $1 / 15$ (7\%) SLN-positive patients demonstrated additional nodal metastases at the time of CLND. With a median follow up of 14 months, 8/15 (53\%) SLN-positive compared to $10 / 109(9 \%)$ SLN-negative patients recurred. The pattern of recurrence was significantly different between SLN-positive and SLN-negative patients $(p=0.024)$. In SLN-positive patients, 5 patients recurred in the same nodal basin after CLND (1 patient had a nodal recurrence in an adjacent basin not evaluated at CLND). No SLN-negative patient recurred in the initial nodal basin. Median recurrence-free survival was not reached for SLN-negative patients compared to 7 months for SLN-positive patients $(p<0.001)$. Conclusion: SLNB in patients with CMHN accurately stages the nodal basins at risk for metastases. SLN-positive patients remain at high risk for recurrence in the same nodal basin despite CLND. SLN status is the most important predictor of disease recurrence in this population.
Patterns of recurrence in patients with primary cutaneous melanoma of the head and neck.

\begin{tabular}{|c|c|c|c|c|}
\hline Patients with recurrence & \multicolumn{4}{|c|}{ First site of recurrence } \\
\hline & Local & In-transit & Nodal & Distant \\
\hline SLN-negative $(\mathrm{n}=10)$ & $1(10 \%)$ & $3(30 \%)$ & $0(0 \%)$ & $6(60 \%)$ \\
\hline SLN-positive $(\mathrm{n}=8)$ & $1(13 \%)$ & $1(13 \%)$ & $5(61 \%)$ & $1(13 \%)$ \\
\hline
\end{tabular}

$\mathrm{p}=0.024$, Pearson Chi-Square test

\section{P112}

Clinical Behavior of Cutaneous Amelanotic Melanoma of the Head and Neck J.M. Bocker, ${ }^{*}$ C.N. Cordeiro, M.C. Coleman, J.O. Boyle, D.H. Kraus, A.R. Shaha, J.P. Shah, K.J. Busam, R. Delgado, S.G. Patel. Head and Neck Oncology, Memorial Sloan-Kettering Cancer Center, Manhattan, NY.

Introduction: Amelanotic melanomas have been reported to occur at a higher rate in the head and neck as compared to other regions of the body. Their clinical behavior is not clearly characterized in the literature, although these tumors have been reported to be associated with higher local recurrence. The aim of this study was to review the clinical behavior of cutaneous amelanotic melanoma of the head and neck (AMHN) treated at a single cancer care center. Methods: A retrospective chart review of 29 patients treated for AMHN between 1985 and 2004 was performed. Standard statistical methods were utilized. Results: Patients ranged in age from 27 to 91 years (median 72 years). $76 \%$ were male and $24 \%$ female. The location of the primary tumor was scalp in $18 \%$, face in $62 \%$, ear in $10 \%$, and neck in $10 \%$. The Breslow depth ranged from $0.5 \mathrm{~mm}$ to $1.4 \mathrm{~cm}$ (median $3.8 \mathrm{~mm}$ ) and Clark's level were $10 \%$ III, $45 \%$ IV, and $38 \%$ $\mathrm{V}$ (not indicated in 7\%). The surgical margins were indicated as close in $41 \%$ and negative in $38 \%$ (not indicated in $21 \%$ ). With a median follow-up interval of 43 months (1-114 months), the 3-year recurrence free survival (RFS), disease specific survival (DSS) and overall survival (OS) rates calculated by the Kaplan-Meier method were $40 \%, 80 \%$, and $68 \%$ respectively. The 3 -year local (LRR), neck and distant recurrence rates were $47 \%, 17 \%$ and $14 \%$. In univariate analysis the only variable that was significantly predictive for a higher LRR was age of the patient $(86 \%$ for $\leq 70$ years versus $23 \%$ for $>70$ years, $\mathrm{p}=0.004$ ). Gender, primary location, Breslow depth, Clark's level, and margin status did not significantly alter the 3-year LRR. On contingency analysis there was no difference between the age groups in gender, primary location, Breslow depth, Clark's location, and margin status. Conclusion: In this descriptive report we show high LRR in patients with AMHN, especially in those 70 years and younger. Local recurrence is amenable to salvage with acceptable DSS. These patients therefore need careful post-treatment surveillance to ensure early detection and intervention for local recurrences.

\section{P113}

Prognostic Implications of Regression in Thin Malignant Melanoma, and Outcome of Sentinel Lymph Node Biopsy S.E. McClain, ${ }^{1 *}$ M.E. Smolkin, ${ }^{3}$ J.W. Patterson, ${ }^{2}$ C. Slingluff. ${ }^{1}$ 1. Department of Surgery, University of Virginia, Charlottesville, VA; 2. University of Virginia - Departments of Pathology and Dermatology, Charlottesville, VA; 3. University of Virginia - Department of Public Health Sciences, Charlottesville, VA.

INTRODUCTION: Thin melanomas with histologic evidence of partial or complete regression may have induced an immune response but may have been deeper prior to biopsy. Their management remains controversial. To test an existing hypothesis that these patients have increased risk of regional metastasis, we have characterized their management and clinical outcomes. METHODS: A clinical melanoma database of 1505 patients was reviewed, along with individual chart reviews. Survival data were supplemented from the Social Security Death Index and referring physicians. RESULTS: There were 94 patients with regression in thin melanomas, within which three important subsets were identified. Group A: Thirty-five patients underwent sentinel lymph node biopsy because of regression alone, all of which were negative $(0 \%$ positive; $95 \%$ upper bound, $8.2 \%$; 5 -year survival $100 \%$; median follow up time 26 months). Group B: Of 31 patients with regression alone and without sentinel lymph node biopsies, two developed regional nodal metastatic disease $(6.5 \%), 10$ and 20 years after initial diagnosis, and both patients were disease free at last follow-up after surgical management (12 and 24 years). Five year 
survival was $92.3 \%$ (95\% CI 72.5-98.0\%)with a median follow up time of 72 months. Group C: Nine patients presented with metastatic disease clinically, with a regressed thin melanoma identified concurrently. These patients had 2year survival estimates of $50.8 \%(95 \% \mathrm{CI} 15-78 \%$; median follow up time 14.8 months). CONCLUSIONS: The poor prognosis of simultaneous metastatic disease and a regressed primary melanoma may indicate the existence of immune escape phenotypes that support melanoma progression. However, for patients with Tla melanomas and regression, we recommend that regression not be used as an indication for sentinel node biopsy but that such patients be managed with wide local excision and clinical observation for at least 20 years to evaluate for the development of metastatic disease that may be amenable to surgical resection.

\section{P114}

Single melanoma cell detection using laser induced acoustic waves J.A. Viator, ${ }^{1}{ }^{*}$ P.S. Dale, ${ }^{1}$ R.M. Weight, ${ }^{2}$ G. Gutierrez-Juarez, ${ }^{3}$ M.L. Miller, ${ }^{1}$ A.E. Lisle, ${ }^{1}$ C.W. Caldwell. ${ }^{1}$ 1. University of Missouri, Columbia, MO;

2. Kansas City University of Medicine and Biosciences, Kansas City, MO; 3. University of Guanajuato, Leon, Guanajuato, Mexico.

Detection of circulating tumor cells (CTC's) in human blood and lymph systems has the potential to aid clinical decision making in the treatment of cancer. The presence of CTC's may signify the extent of disease or presence of metastasis, indicate relapse of treated disease, or may be used to monitor response to treatment. We used photoacoustics, or laser induced acoustic waves, for detection of melanoma cells as a model for detecting circulating melanoma cells (CMC's). Melanin, a broadband optical absorber within melanoma, absorbed laser pulses at $532 \mathrm{~nm}$. The apparatus consisted of a pump feeding a customized flow chamber containing an acoustic sensor. The laser irradiated the chamber generating acoustic waves in melanoma. The acoustic sensor sent a signal to an oscilloscope indicating the presence of CMC's. The relative strength of the acoustic signal correlated to the concentration of melanoma. We tested the detection threshold by adding $10^{\wedge} 2-10^{\wedge} 6$ cultured melanoma cells per $20 \mathrm{ml}$ sample of normal saline. The acoustic signal was measured for this range of concentrations and showed a signal to noise ratio (SNR) in the range of 3 to 85. Taking an SNR of 2 as a limit, we postulate a clinical threshold of 1-5 CMC's. Additionally, we tested the photoacoustic response of 4-8 nm diameter gold nanoparticles with the goal of attaching them to cancer cells, such as the bombesin receptor for breast cancer. We found the photoacoustic peak to be at the $520 \mathrm{~nm}$ laser wavelength. The response to our apparatus for 0.018 to $0.14 \mathrm{~g} / 1$ nanoparticle suspensions was 8 to $100 \mathrm{SNR}$, indicating that attachment of gold nanoparticles can enhance the photoacoustic response of amelanotic melanomas or non-melanoma CTC's. This photoacoustic system has the requisite sensitivity to perform clinical detection of CMC's in human blood samples. In the future, we will test our hypothesis that using 532 and $633 \mathrm{~nm}$ laser wavelengths will provide the level of specificity to distinguish melanoma from any other chromophore in human blood. This specificity will enable the photoacoustic procedure to be tested on Stage I-IV melanoma patients to determine the system's clinical feasibility.
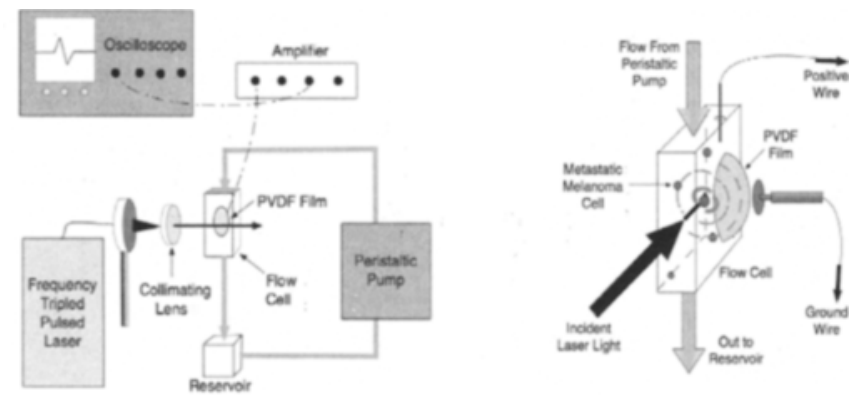

(L) The apparatus. (R) Flow chamber

\section{P115}

Cutaneous Melanoma in Asian Americans S.H. Shin, ${ }^{1 *}$ B.E. Palis, ${ }^{2}$ R.R. Perry. ${ }^{1}$ 1. Surgery, Eastern Virginia Medical School, Norfolk, VA; 2. National Cancer Database, Chicago, IL.

Background: Cutaneous melanoma is common and well described in non Hispanic white (NHW) patients. There is little information available on melanoma in non-white populations. Our objective was to characterize melanoma in Asian Americans and to compare demographics and outcomes to the NHW population. Methods: 393,413 cutaneous melanoma patients diagnosed between 1985 and 2003 were identified using the National Cancer Data Base; 983 were Asian American, and 329,822 were NHW. The impact of gender, primary site (including acral lentiginous), tumor thickness, and pathologic stage, on survival was assessed. Margin status could not be considered due to insufficient data. Significance was assessed with the chi-square test and z-test of proportions. Five-year observed survival was available on more than $60 \%$ of patients and was evaluated using the life table approach. Risk of mortality was estimated with Cox proportional hazards regression. Results: Overall survival was similar between Asians and NHW. Cox proportional hazard regression revealed pathologic stage as a significant factor affecting survival in Asians $(\mathrm{p}=0.000$, reference Stage III, hazard ratio $=0.347$, Stage II; hazard ratio $=$ 0.121 , Stage I). Gender and primary site were not significant mortality-related factors. Conclusions: This is the largest study to date on melanoma in Asian Americans. Compared to NHW, Asians are more likely to have an acral lentinginous primary site, thick tumors, and less likely to present with stage III melanoma. Despite this, their overall survival was similar to the NHW population.

\begin{tabular}{|c|c|c|c|}
\hline & Asian $(\mathrm{n}=983)$ & Non-Hispanic White $(\mathrm{n}=329,822)$ & P value \\
\hline Female & $52.2 \%$ & $43.3 \%$ & 0.000 \\
\hline Acral Lentiginous & $7.2 \%$ & $0.9 \%$ & 0.000 \\
\hline T4 & $16.6 \%$ & $8.4 \%$ & 0.000 \\
\hline Pathologic Stage I & $54.8 \%$ & $38.4 \%$ & 0.000 \\
\hline Pathologic Stage II & $28.2 \%$ & $28.0 \%$ & NS \\
\hline Pathologic Stage III & $13.3 \%$ & $25.1 \%$ & 0.000 \\
\hline 5 year Overall Survival & $69.6 \%$ & $73.7 \%$ & 0.105 \\
\hline
\end{tabular}

NS=Non-significant

\section{P116}

Burden of diagnostic tests by stage III melanoma patients E. Bastiaannet, ${ }^{1} *$ J. Hoekstra-Weebers, ${ }^{2}$ P. Jager, ${ }^{3}$ E. Van der Jagt, ${ }^{4}$

H. Hoekstra. ${ }^{1}$ 1. Surgical Oncology, University Medical Center Groningen, Groningen, Netherlands; 2. Health Psychology, University Medical Center Groningen, Groningen, Netherlands; 3. Nuclear Medicine \& PET Center, University Medical Center Groningen, Groningen, Netherlands; 4. Radiology, University Medical Center Groningen, Groningen, Netherlands.

Introduction. Patients who experience burden during diagnostic tests may disrupt these tests. The aim of this study was to describe the perception of the diagnostic tests by melanoma patients with lymph node metastases. Methods. Patients were requested to complete a self-administrated questionnaire within 1-6 weeks after the chest X-ray, CT scan and PET (Positron Emission Tomography) scan. Experienced levels of embarrassment, discomfort and anxiety were calculated, as well as (total) scores for each burden. The non-parametric Friedman test for related samples and Spearman's rank correlation were used to assess differences and correlations. Results. The questionnaire was completed by 49 stage III melanoma patients; the response rate was $86 \%$. Overall satisfaction concerning the course of the diagnostic tests was high $(8.1$ on a scale from 1 to 10 ). In total $28 \%$ of the patients experienced mild to extreme embarrassment, $36 \%$ discomfort and $30 \%$ anxiety during the diagnostic tests. Overall, $31 \%$ felt some kind of burden. There was no difference in anxiety between the three tests $(p=0.258)$. However, patients experienced more embarrassment and discomfort during the PET scan than during the other tests $(p=0.002$ and $p=<0.0001$ ). Besides, a significant correlation was found between quality of life and decision-maker: patients who indicated that the decisionmaker was predominantly the doctor reported a higher quality of life in the present study $(\mathrm{p}=0.005)$. No significant correlation was found in this study between total burden and quality of life, age or sex. Conclusion. Overall levels of burden were low. However, patients experienced more embarrassment and discomfort during the PET scan, possibly as a result of lying immobile for a long time; future PET-CT scanners could correct this discomfort as they can scan a patient in less than 30 minutes. The accuracy, costs and patients upstaged will probably be the most important to determine the additional value of FDG- 
PET and CT, but it is reassuring to know that only few patients experience severe or extreme burden.

\section{P117}

FDG uptake and markers in clinically stage III melanoma patients E. Bastiaannet, ${ }^{1}$ * P. Jager, ${ }^{2}$ A. Suurmeijer, ${ }^{3}$ I. Kema,${ }^{4}$ R. Van Ginkel, ${ }^{1}$ H. Hoekstra. ${ }^{1}$ 1. Surgical Oncology, University Medical Center Groningen, Groningen, Netherlands; 2. Nuclear Medicine, University Medical Center Groningen, Groningen, Netherlands; 3. Pathology, University Medical Center Groningen, Groningen, Netherlands; 4. Central Laboratory, University Medical Center Groningen, Groningen, Netherlands.

Introduction. Three pre-operative markers in clinically stage III melanoma patients are currently available. The potential role of the markers S100B and LDH and the standardized uptake value (SUV) of FDG-PET were evaluated in patients with locoregional disease who underwent a therapeutic lymph node dissection. Methods. Patients with clinically stage III melanoma (neck $15.2 \%$, axilla $34.8 \%$, groin $50.0 \%$ ), who were referred for a FDG-PET scan and underwent a therapeutic lymph node dissection, were prospectively studied ( 24 male, 22 female; median age 60 (range 29-93) years). Differences in patients with low SUVmean and high SUVmean (cut-off 5.97) as well as low and high preoperative LDH (cut-off 235) and low and high pre-operative S100 (cut-off 0.15) were assessed by univariate and multivariate logistic regression analysis. Results. The number of removed nodes in the neck was 22 (median, range 14-48), in the axilla 18 (11-31) and inguinal 13 (7-26). Median number of positive nodes was $2(1-7)$ in the neck, and 3 both in the axilla (1-19) and inguinal (1-12). The tumor size $(\mathrm{cm})$ in the axilla $(4.24 \pm 1.79)$, inguinal $(3.01 \pm 1.47)$ and neck region $(1.92 \pm 0.88)$ was significantly different $(\mathrm{p}=0.02)$. In $46 \%$ of the patients the LDH was elevated, in 52\% the S100 was elevated without significant correlation between these two markers. In univariate and multivariate analysis high SUVmean was associated with tumor size $(p=0.023)$. High pre-operative LDH was only associated with older age $(p=0.013)$ in multivariate analysis. Elevated $\mathrm{S} 100$ was associated with larger tumor size $(\mathrm{p}=0.007)$ in univariate analysis. However, in multivariate analysis, size was not significantly associated with high S100. Conclusion. Largest tumor size was found in the axilla, SUVmean and S100 were associated with tumor size; however, S100 only in univariate analysis. The impact of elevated SUVmean and pre-operative LDH and S100 should be further explored with respect to disease free and overall survival in clinically stage III melanoma patients who underwent a therapeutic lymph node dissection to gain more insight in the surgical treatment and biological tumor aggressiveness.

\section{P118}

Role of Radiologic Imaging at Time of Initial Diagnosis of Stage T1b-T3b Melanoma M. Yancovitz, ${ }^{1}{ }^{*}$ N. Finelt, ${ }^{1}$ M. Warycha, ${ }^{1}$ P. Christos, ${ }^{2}$ M. Mazumdar, ${ }^{2}$ R. Shapiro, ${ }^{1}$ A. Pavlick, ${ }^{1}$ I. Osman, ${ }^{1}$ D. Polsky, ${ }^{1}$ R.S. Berman. ${ }^{1}$ 1. New York University School of Medicine, New York, NY; 2. Weill Medical College of Cornell University, New York, NY.

INTRODUCTION: In patients with T1b-T3b melanoma, the utility of radiologic imaging at the time of diagnosis is unclear. This study aims to determine whether initial imaging led to a change in stage or treatment plan. METHODS: We searched our prospective melanoma database (2002-2005) for patients with $\mathrm{Tl}$ b-T3b primary lesions who were clinically N0 and asymptomatic for metastatic disease. 158 patients were identified (76 Stage I, 59 Stage II, 23 Stage III after sentinel lymph node biopsy). All radiologic studies conducted at the time of initial diagnosis (time between biopsy and surgical excision) as well as all imaging, biopsies and further interventions prompted by these initial studies were analyzed. Outcome measures included upstaging of patients, any change in initial surgical management, and true positive and false positive rates of each imaging modality. RESULTS: Of the 343 preoperative imaging studies identified (130 CXR, 57 chest CT, 57 abd/pelvis CT, 57 head CT, 42 $\mathrm{PET} / \mathrm{CT}$ ) there were 49 findings suspicious for melanoma and 134 findings suggestive of non-melanoma pathology. Only $1 / 343(0.3 \%)$ studies resulted in confirmed metastatic melanoma. Of the 49 findings suspicious for melanoma, the true positive rates (number of proven metastases out of the number of suspicious studies) were CXR 0/7 (0\%), chest CT 0/24 (0\%), abd/pelvic CT 0/11 $(0 \%)$, head CT $0 / 2(0 \%)$, PET/CT $1 / 5(20 \%)$. The false positive rates (no evidence of metastatic disease on radiologic or clinical follow-up) were CXR 5/7 (71\%), chest CT 20/24 (83\%), abd/pelvic CT 10/11 (91\%), head CT $2 / 2(100 \%)$, PET/CT 3/5 (60\%). Median follow-up was 20.4 months (range $0.72-43.2$ ). No patient was upstaged or had a change in initial surgical management based on preoperative imaging. The cost of all initial and follow-up imaging was estimated to be $\$ 357,869$ for the 158 patients studied. CONCLUSIONS: In our study cohort, imaging at the time of initial diagnosis of T1b-T3b, clinical N0, M0 melanoma is of low yield with a high false positive rate, and does not lead to upstaging or a change in initial surgical management. The findings suggest that imaging of asymptomatic patients at the time of diagnosis may not be warranted.

\section{P119}

A comparison of Melanoma and Merkel cell carcinoma: results from the California Cancer Registry J.E. Grabowski, ${ }^{1}{ }^{*}$ S.L. Blair, ${ }^{1}$ S. Saltzstein, ${ }^{3}$ W. Read, ${ }^{2}$ G. Sadler. ${ }^{1} 1$. Department of Surgery UCSD, San Diego, CA; 2. Moores' UCSD Cancer Center, San Diego, CA; 3. Department of Preventative Health UCSD, San Diego, CA

Introduction: Melanoma and Merkel cell carcinoma (MCC) are both aggressive skin malignancies associated with immunosuppression and possible UV exposure. Both tumors get similar surgical treatment; however, MCC is a relatively rare tumor for which less is known about prognosis and clinical behavior. We undertook this study to examine the behavior of these tumors to better delineate their differences and in order to better counsel patients about the diseases. Methods: The California Cancer Registry (CCR), a populationbased registry, was reviewed from the years 1988-2003. We compared Merkel cell carcinoma and melanoma with relation to gender, age at presentation, site of initial lesion, and overall survival. Results: A total of 113,187 cases of melanoma and 1,779 cases of MCC were identified in the CCR database. Though both cancers are more common in men than in women, MCC had a significantly higher incidence in men than did melanoma ( $63 \%$ vs $57 \% \mathrm{p}<.005)$. MCC tends to occur in the more elderly patient, with $73.6 \%$ of reported cases occurring in people 70 years and older. In contrast, $69 \%$ of melanoma cases occurred in people younger than 70 years. This age discrepancy is statistically significant $(p<.005)$. An additional difference is seen in site of the initial lesion. Both MCC and melanoma had a similar frequency on the extremities $(34.7 \%$ vs. $39.7 \%$, respectively). MCC, however, shows a predilection for the head and neck ( $47 \%$ of cases) while only $25.8 \%$ of melanoma presents in these areas Additionally, melanoma occurs more frequently on the trunk ( $30.2 \%$ of cases) than does MCC ( $8.7 \%$ of cases). Most importantly, the overall survival is lower for MCC than for melanoma ( $37 \%$ vs. $76 \%, p<.005$ ). Conclusion: Both tumors get similar surgical treatment and many clinicians have assumed that they had similar behaviors. However, MCC and melanoma have key differences. MCC occurs in an older population, more frequently on the head and neck, in a significantly higher percentage of men, and has a worse prognosis. Clinicians should keep these factors in mind when counseling patients about prevention, early detection, and overall outcomes.

\section{P120}

Targeted therapy for in-transit melanoma: utility of gene expression signatures to define optimal strategy C. Augustine, ${ }^{1 *}$ A.N. Potti, ${ }^{2}$ S.H. Jung, ${ }^{4}$ J. Nevins, ${ }^{3}$ D.S. Tyler. ${ }^{1} 1$. Duke Univ. Med. Center Dept. Surgery, Durham, NC; 2. Duke Univ. Med. Center Dept. Medicine, Durham, NC; 3. Duke Univ. Med. Center Dept. Molecular Genetics and Micorbiology, Durham, NC; 4. Duke Univ. Med. Center Dept. Biostatistic and Bioinformatics, Durham, NC.

Targeted therapy in combination with chemotherapy for the treatment of melanoma is a novel way to improve tumor responses and overcome resistance to chemotherapy. The success of this strategy depends on the ability to identify resistance mechanisms and deregulated signaling pathways from a single biopsy. In-transit melanoma, which usually presents with multiple nodules throughout an extremity, is an ideal setting for studying the genetic and molecular profile of melanoma. It is critical to verify that any tumor nodule is genetically representative of a patient's melanoma. The objective of this study was to evaluate the gene expression across a panel of melanoma lesions and define the degree of correlation within patients particularly with respect to patterns of deregulated intracellular signaling. Seventeen biopsies were obtained from 6 patients before isolated limb infusion treatment. RNA was isolated from each biopsy and hybridized to Affymetrix HU133plus2 chip to obtain a gene expression profile. Gene expression profiles across these lesions were strikingly similar within a patient. An unsupervised hierarchical clustering analysis of gene expression revealed six clusters, one for each patient. This is also shown by 
the principal component analysis in the figure. Correlation coefficients comparing lesions within a patient ranged from 0.95 to 0.99 demonstrating a significant degree of correlation. We defined probabilities of signaling pathway deregulation (src, Ras, $\beta$-catenin) for each lesion based on its correlation with signatures derived from cell lines. Within each patient the pattern of signaling was significantly correlated across lesions ( $p<0.01$ for lesions within a patient). These results demonstrate the marked similarity of in-transit extremity melanoma tumor nodules within a patient. Of clinical significance is the fact that these lesions share not only similar drug resistance patterns in a given patient but also similar degrees of deregulated cell signaling. Pre and post treatment tumor biopsies can help dictate the optimal treatment strategies as well as facilitate the testing of how targeted therapies contribute to improved tumor responses.

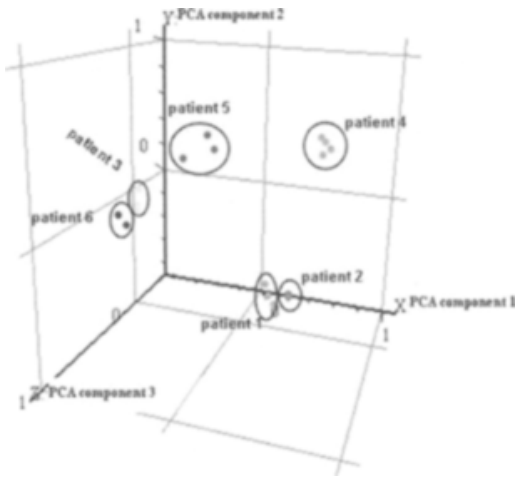

P121

The pattern of the first recurrences in melanoma patients after positive sentinel lymph node (SLN) biopsy with completion lymph node dissection (CLND) in relation to the presence of metastases in lymph nodes other than SLNs Z.I. Nowecki, P. Rutkowski, ${ }^{*}$ W. Ruka. Soft Tissue/Bone Sarcoma and Melanoma, Cancer Center Warsaw, Warsaw, Poland.

Introduction: We analyzed the pattern of the first recurrences after positive (SLN) biopsy on a large series of melanoma patients with clinically negative regional lymph nodes in relation to the presence of metastases in lymph nodes other than SLN (non-SLNs) after CLND. Material and Method: Between 05/1995 and 12/2004 we performed CLND due to positive SLNs in $262(18.8 \%)$ of 1387 consecutive patients with primary cutaneous melanoma undergoing SLN biopsy. In 81 patients $(30.9 \%)$ we identified metastases to non-SLNs after CLND (CLND/+/ group; median Breslow thickness - $4.1 \mathrm{~mm}$ ) and in 262 $(69.9 \%)$ we did not find additional nodal metastases after CLND (CLND/-/ group; median Breslow thickness $-3.1 \mathrm{~mm}$ ). Median follow-up time was 48 months. Results: The presence of metastases to non-SLNs in patients with positive SLNs correlated with higher Breslow thickness of primary tumor $(p<0.0001)$, Clark level of invasion $(p=0.003)$, presence of ulceration $(p=0.05)$ and trunk localization of primary melanoma $(\mathrm{p}=0.02)$. Disease recurrences were observed less frequently in CLND/-/ group [83/181 (45.9\%)] as compared with $\mathrm{CLND} /+$ / group [55/81 $(67.9 \%) ; \mathrm{p}=0.001]$, but the patterns of the first recurrences in CLND/// and CLND/+/ groups were similar, respectively: in-transit/local recurrences only (26/83 - 31.3\% vs $17 / 55-30.9 \%)$, lymph nodes $(6 / 83-7.2 \%$ vs $5 / 55-9.1 \%)$ and distant metastases $(51 / 83-61.5 \%$ vs $33 / 55$ $-60 \%)$. The median time to recurrences was shorter in CLND/+/group $(16.2$ months) in comparison to CLND/-/ group ( 45.6 months) [ $<<0.0001]$. The outcomes of CLND/// patients were better than CLND/+/ and 5-year disease-free survival (calculated from the date of SLN biopsy) was, respectively: $42 \%$ and
$26 \%(\mathrm{p}<0.0001)$. Conclusions: The prognosis of patients with metastases limited to SLNs is better than patients with positive non-SLNs after CLND and this group of patients is characterized by lower rate of disease recurrences, although the pattern of recurrences is similar in both groups of patients.

\section{P122}

The location of second- and higher-echelon nodes in melanoma patients with a sentinel node in the groin M.C. Van Rijk, ${ }^{*}$ O.E. Nieweg, R.A. Valdés Olmos, C.A. Hoefnagel, B.B. Kroon. The Netherlands Cancer Institute, Amsterdam, Netherlands.

Purpose: In search of ways to reduce the extent of groin dissections in melanoma patients with an involved sentinel node, a study was undertaken to determine the location of second- and higher-echelon (secondary) nodes. Patients and methods: A retrospective study was performed in 199 melanoma patients with a sentinel node in the groin. Lymphoscintigraphy for sentinel node biopsy had been routinely performed with small-sized colloid particles. Transmission scanning with a 57Co flood source was used to outline the body contour. A lead band was placed in the inguinal fold and 57Co point sources were placed over the anterior superior iliac spine and on the pubic tubercle for orientation. The images of were evaluated with regard to the location of visible lymph nodes with the aid of the anatomical information obtained by the landmarks and body contour. A sentinel node was defined as a lymph node on a direct drainage pathway from the melanoma. The groin was divided in four regions: subcutaneous and superior from the sapheno-femoral junction, inferior from the sapheno-femoral junction, the ilio-obturator basin and a central region connecting the first two regions through the femoral canal to the ilioobturator region (figure). Results: Secondary nodes were visualized in 195 of the 199 patients $(98 \%)$. These secondary nodes were either located in the same region as the sentinel node or more towards or in the ilio-obturator basin. None of the 130 patients with a sentinel node outside the superior region had secondary nodes in this particular region. None of the 42 patients with a sentinel node outside the inferior region had drainage to secondary nodes in this region. Conclusions: Secondary lymph nodes are not situated in the subcutaneous tissue superior from the sapheno-femoral junction or in the tissue inferior from this junction if there is no sentinel node in these respective regions. Knowledge of these drainage patterns may allow the surgeon to limit the extent of therapeutic lymph node dissection in case of an involved sentinel lymph node.

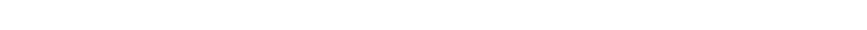




\section{P123}

Melanoma Adrenal Metastasis: Natural History and Surgical Management E.A. Mittendorf,* S.J. Lim, C.W. Schacherer, C. Ng, A. Lucci, J.N. Cormier, P.F. Mansfield, J.E. Gershenwald, M.I. Ross, J.E. Lee. Surgical Oncology, UT MD Anderson Cancer Center, Houston, TX.

Introduction: The adrenal gland is an uncommon site of melanoma metastasis. Since few data exist regarding appropriate management, we reviewed our experience with this condition to determine the natural history of disease and appropriate role for surgical intervention. Patients and Methods: Retrospective review of our institutional database of 3454 melanoma patients with a median follow-up of 2.6 years identified 787 patients with distant metastatic disease; 96 had adrenal metastasis (12\%). We reviewed the clinical presentation and outcome of these patients, including sites of extra-adrenal metastatic disease, surgical treatment and survival. Results: At the time of presentation with adrenal metastasis, 16 of 96 patients $(17 \%)$ had disease limited to the adrenal gland, while $80(83 \%)$ had synchronous distant metastases to other anatomic sites (lung 30, liver 27, other intra-abdominal 30, brain 16, subcutaneous 11). At a median follow-up of 2.6 years, 27 patients $(28 \%)$ were alive and $69(72 \%)$ had died. Median overall survival (OS) was 7 months. 18 patients underwent surgical resection of their adrenal metastasis (open 10, laparoscopic 8). Patients who underwent resection did not exhibit an improved OS compared to patients who did not undergo resection ( 7.4 mo vs $6.9 \mathrm{mo}, \mathrm{P}=.16$ ); only 2 patients who underwent surgical resection are alive without recurrence 10 and 13 months postoperatively. However, among all patients with adrenal metastasis, those with synchronous or prior metachronous metastatic disease limited to regional nodal, distant subcutaneous, and/or lung sites had an improved OS compared to patients with synchronous or prior liver, other intra-abdominal or brain metastases ( 12.2 mo vs $5.5 \mathrm{mo}, \mathrm{P}=.01$ ). Conclusions: Most patients with metastatic melanoma to the adrenal have a short survival duration regardless of presentation or therapy. Surgical treatment for melanoma adrenal metastasis should only be considered in highly selected patients, for example those with a history of limited extra-adrenal metastatic disease who are candidates for protocol-based adjuvant systemic therapy trials. To minimize morbidity, laparoscopic adrenalectomy is favored for those selected for surgical resection.

\section{P124}

Dermal Melanoma: Primary Melanoma without an Epidermal Component or Metastatic Disease? N.B. Ravipati,* B.A. Pockaj, R.J. Gray, W.J. Casey, III. Surgery, Mayo clinic Arizona, Scottsdale, AZ.

Background: Primary dermal melanoma has been described as melanoma confined to the dermis without evidence of metastatic disease elsewhere. Optimal work-up and treatment of this clinical entity has yet to be determined. Methods: A retrospective review of a prospective sentinel lymph node (SLN) database (412 patients) was performed to identify melanoma patients presenting with solitary dermal lesions without an epidermal component between 2/976/06. Results: Eighteen (4.3\%) patients were identified. Mean age was 68 years. 4 patients had a previous melanoma diagnosed at a remote site $($ a $6 \mathrm{~mm}$ melanoma 15 months prior, a $2.28 \mathrm{~mm}$ melanoma 22 months prior, a $0.2 \mathrm{~mm}$ melanoma 6 years prior, and a $0.93 \mathrm{~mm}$ melanoma 10 years prior). One patient was diagnosed with a concurrent $0.3 \mathrm{~mm}$ melanoma. Mean Breslow thickness was $3.09 \mathrm{~mm}$ (range $0.67-7.0 \mathrm{~mm}$ ). Trauma to the dermal melanoma site was documented in 6 patients (35\%). All patients had a negative basic metastatic work-up (chest x-ray, liver function tests, and LDH). Additional imaging workup was performed in 14/18 patients (77\%) and included brain MRI in $12(63$ $\%$ ), chest CT in $11(58 \%)$, abdominal CT in $10(56 \%)$, and PET scan in 12 $(63 \%)$. This additional imaging work-up was negative for metastatic disease. SLN metastasis was present in 1 patient $(5 \%)$. This patient had a prior history of melanoma and another dermal melanoma was found at the time of surgery. He was ultimately diagnosed with metastatic disease soon after surgery. Distant metastases were diagnosed in another patient who did not have a history of prior melanoma. Both were diagnosed within 3 months of their original diagnosis. Neither of these patients had undergone more than a basic metastastic work-up pre-operatively. Median follow-up of the remaining 16 patients is 37 months (range 1-75 months). There has been no evidence of metastatic disease to date. Conclusion: Dermal melanomas that present without evidence of metastatic disease behave as primary melanomas. Extensive radiographic metastatic work-up should be performed before a diagnosis of primary dermal melanoma can be made, especially in patients with a history of previous melanoma.

\section{P125}

Robotic-assisted minimally invasive surgery for oncologic procedures: a 2 yr experience at a single institution $\mathrm{C}$. Anderson, ${ }^{*} \mathrm{M}$. Hellan, K. Kernstine, J. Ellenhorn, V. Trisal, A. Pigazzi. City of Hope, Duarte, $C A$.

Introduction: Robotic surgery has advantages over conventional laparoscopy, and offers an attractive alternative modality to perform minimally invasive oncologic procedures. However, there is limited literature reporting the use of robotic surgery for non-urologic malignant disease. Two years ago a multidisciplinary robotic program was initiated at our institution. This report describes our experience with thoracic and gastrointestinal procedures. Patients/Methods: Clinicopathological data of all patients undergoing robotic procedures for cancer in our institution between November 2004 and August 2006 were retrospectively analyzed. A total of 80 procedures were identified: 12 esophageal, 18 gastric, 17 lung, and 27 rectal resections. There were 6 miscellaneous procedures including 3 mediastinal, 2 pancreatic and one pelvic tumor resections. $74 \%$ of patients with rectal cancer and $50 \%$ with esophagogastric cancers received neo-adjuvant chemoradiation therapy. Results: There was no postoperative mortality. The operative data is shown in the table below. There was one intraoperative complication requiring colonic resection. Of three conversions, one was due to obesity, another due to technical problems with the anastomosis and the third to adhesions. Major postoperative morbidity was $0 \%$ for lung, $11 \%$ for gastric, $50 \%$ for esophageal, $22 \%$ for rectal, and $33 \%$ for miscellaneous procedures. Median length of stay was 4, 9, 16 and 4 days for lung, gastric, esophageal and rectal resections respectively. At a median follow-up of 6.1 months (0-20), one patient developed a port site recurrence. Conclusion: Our comprehensive early experience suggests that robotic surgery is safe and feasible for a variety of radical oncologic surgical procedures. However, future randomized trials are needed to demonstrate the potential advantages of robotic surgery over conventional techniques.

\section{Operative Data}

\begin{tabular}{|c|c|c|c|c|}
\hline & OR time (min)* & EBL ml $)^{*}$ & Conversions & $\begin{array}{c}\text { \# removed } \\
\text { nodes* }\end{array}$ \\
\hline Lung $(\mathrm{n}=17)$ & $226(136-387)$ & $200(20-54)$ & 0 & $10(2-54)$ \\
\hline Gastric $(\mathrm{n}=18)$ & $439(160-646)$ & $300(30-1200$ & 1 & $24(6-42)$ \\
\hline Esophageal( $\mathrm{n}=12)$ & $466(387-597)$ & $400(100-1600)$ & 1 & $21(14-39)$ \\
\hline Rectal $(\mathrm{n}=27)$ & $285(180-540)$ & $150(20-1000)$ & 1 & $14(6-28)$ \\
\hline $\begin{array}{c}\text { Miscellaneous } \\
\text { Abdiastinal( } \mathrm{n}=3)\end{array}$ & $\begin{array}{c}145(74-168) \\
493(281-705)\end{array}$ & $\begin{array}{c}30(20-30) \\
350(300-400)\end{array}$ & 02 & $\mathrm{n} / \mathrm{a} 15(0-15)$ \\
\hline
\end{tabular}

*median values

\section{P126}

Peritoneal Mesothelioma: Incidence and Risk Factors for Survival C.M. Mery, ${ }^{*}$ C.P. Raut. Brigham and Women's Hospital, Boston, MA.

Introduction Peritoneal mesothelioma (PM) is a rare malignancy that is not well characterized. The objective of this retrospective study was to determine the incidence and risk factors for survival based on the Surveillance, Epidemiology, and End Results (SEER) database. Methods All cases of PM reported to SEER 1973-2003 were included. Incidence was calculated and age-adjusted, based on the 2000 standard US population. Variables were analyzed with chi-square, Kaplan-Meier, and Cox proportional hazards. Results 786 patients (age $62 \pm 14$ yrs, $57 \%$ males) presented with localized (7\%), regional (17\%), or distant (75\%) PM. Histology was available for 195 patients ( $8 \%$ fibrous, $78 \%$ epithelial, $13 \%$ mixed) and grade for $124(1: 39 \%, 2: 15 \%, 3: 36 \%, 4: 11 \%)$. Curative surgery (excision or debulking) was performed on $32 \%(\mathrm{n}=154)$. The cohort was divided into 4 age groups: $<50 \mathrm{yrs}(\mathrm{I}, \mathrm{n}=140,18 \%), 50-64$ yrs (II, $\mathrm{n}=265,34 \%$ ), 65-74 yrs (III, $n=224,28 \%$ ), $\geq 75$ yrs (IV, $n=157,21 \%$ ). The use of curative surgery decreased with age from $53 \%$ for I to $19 \%$ for IV $(p<0.0001)$. Females were more likely to receive curative surgery than males $(50 \%$ vs $20 \%, \mathrm{p}<0.0001)$. Overall median survival for the cohort was 7 mos $(1-\mathrm{yr}$ survival $38 \%, 5$-yr $14 \%$ ). Risk factors associated with worse survival were male gender $(p<0.0001)$, increasing age $(p<0.0001)$, lack of curative surgery $(\mathrm{p}<0.0001)$, distant disease $(\mathrm{p}=0.0003)$, fibrous histology $(\mathrm{p}<0.0001)$, and advanced grade $(\mathrm{p}<0.0001)$. Age was a risk factor for patients with curative surgery $(p<0.0001)$ and those without $(p<0.0001)$. Table shows 
hazard ratios for overall survival from a model with age, gender, and curative surgery. When analyzing PM-related survival, age and gender remained statistically significant but curative surgery did not. Age-adjusted PM incidence remained stable over the 30 -yr period $(0.06-0.15$ per $100,000)$. Conclusions PM is a rare disease with low survival rates. Several important risk factors for mortality (age, gender, surgery, histology, grade) were identified and will help in counseling patients with PM. The absence of information in SEER regarding details of surgery and use of chemotherapy, either intraperitoneal or systemic, may limit the conclusions of this study.

\begin{tabular}{|c|c|c|c|}
\hline Variables & Hazard ratio (HR) & $95 \%$ confidence interval & $\mathrm{p}$-value \\
\hline Age (ref: $<50$ yrs) & & & \\
\hline$-50-64$ yrs & 1.59 & $1.13-2.24$ & $0.0074^{*}$ \\
\hline$-65-74$ yrs & 2.17 & $1.55-3.05$ & $<0.0001^{*}$ \\
\hline$-75+$ yrs & 3.14 & $2.18-4.52$ & $<0.0001^{*}$ \\
\hline Male gender & 1.59 & $1.27-2.01$ & $<0.0001^{*}$ \\
\hline No curative surgery & 1.41 & $1.10-1.80$ & $0.0059^{*}$ \\
\hline
\end{tabular}

* statistically significant

\section{P127}

Intra-Lesional Injection of Granulocyte/macrophage-colony stimulating factor (GM-CSF) into Tumors pre-Treated with Radiofrequency Ablation (RFA) L.B. Riley, ${ }^{1}$ M. El Chaar, ${ }^{1}$ D.C. Desai, ${ }^{1}$ M. Habibi. ${ }^{2}$ 1. St. Luke's Hospital and Health Network, Bethlehem, PA; 2. Virginia Commonwealth University, Richmond, VA.

Radiofrequency ablated tumors induce immune responses in animals and humans. However, as an isolated treatment, RFA does not induce a sufficient immune response to diminish the rate of local or distant recurrences. Animal studies with RFA in combination with either local or systemic immunotherapy have shown synergistic effects. Previously we showed in animals and humans that the intralesional injection of small molecules into RFA tissue provides sustained, high, regional concentrations of the injected compound. The current study addresses the safety of this paracrine-based ablative vaccine (PAVAC) therapy with GM-CSF in two IRB approved clinical trials. METHODS: From 2003 to 2006, 19 patients were enrolled in one of two prospective, clinical trials using PAVAC and GM-CSF. The first trial was a dose escalation study combining RFA and intralesional GM-CSF in patients with primary or secondary hepatic tumors. The second trial used intra-lesional GM-CSF@500 mcg/patient in conjunction with systemic high-dose interleukin-2 for patients with metastatic melanoma. Postoperative side effects were monitored and reported in accordance with the NCI/CTEP guidelines. Follow-up examinations, CT scans and complete blood counts were monitored for toxicity. RESULTS: The mean follow-up for all patients was 12 months: 9 months for patients with melanoma and 15 months for the hepatic group. No patient developed any signs or symptoms of autoimmunity or known GMCSF toxicity. Follow-up CT scans showed no splenomegaly or lymphadenopathy in any of the patients. Complete blood counts done within one week of treatment revealed no leukocytosis (WBC=9.1 \pm 3.0 ). CONCLUSION: Intra-lesional injection of GM-CSF into tumors following treatment with RFA was well tolerated in all patients. The observation that $78 \%$ of the liver group is free of regional or distant recurrence within the 15 month follow-up is encouraging. Serologic and cellular studies to evaluate immunologic endpoints are currently underway. Further studies are needed to clarify the benefit of GM-CSF or other cytokines or combination of cytokines in PAVAC.

\section{Patient Follow-up}

\begin{tabular}{|c|c|c|}
\hline Status & Melanoma & Liver/Mets \\
\hline NED & $1(10 \%)$ & $7(78 \%)$ \\
\hline AWD & $4(40 \%)$ & $2(22 \%)$ \\
\hline DOD & $5(50 \%)$ & $0(0 \%)$ \\
\hline
\end{tabular}

\section{P128}

The Decision to Pursue a Surgical Oncology Fellowship: What Influences a General Surgery Resident? J.H. McCord, * R. McDonald, D.M. Mahvi, H. Chen, S.M. Weber. University of Wisconsin Hospital Department of Surgery, Madison, WI.

Background: Few studies have examined the factors influencing surgical residents to pursue subspecialty training. We sought to evaluate factors influencing residents to pursue surgical oncology training. Methods: A web survey was sent to 99 recent graduates of a university general surgery program. Respondents choosing surgical oncology fellowships (SURG ONC) were compared to those entering other fellowships (OTHER). Results were analyzed using Fisher's exact test with significance determined at $p \leq 0.05$. Results: There were a total of 78 respondents. Of these, $47(60 \%)$ completed a fellowship, including $9(19 \%)$ who chose surgical oncology. There was no difference between the SURG ONC and OTHER groups in the proportion who are currently in academic practice $(p=0.1)$. A significantly higher percentage of the SURG ONC group completed full-time research $(89 \%$ vs. $48 \%, p=0.03)$ and clinical research $(100 \%$ vs. $61 \%, \mathrm{p}=0.04)$ during residency compared to the OTHER group. Both groups were equally likely to respond that the following factors were important in choosing their specialty: interest in and intellectual appeal of that field, ability to teach, and basic science opportunities. However, the SURG ONC group was more likely to note the importance of clinical research opportunities as a factor in choosing their future career $(p=0.01)$ Although both groups were equally likely to note the importance of having an influential mentor, respondents who identified a surgical oncologist as their mentor were more likely to choose surgical oncology fellowships $(p<0.0001)$. Conclusion: Our findings show that among residents choosing to pursue specialty training, those who chose surgical oncology fellowships were more likely to participate in basic science and clinical research during residency. These residents were also more likely to be influenced by a mentor in the field of surgical oncology. Our findings suggest that providing general surgery residents with the opportunity to do basic science and clinical research and to be mentored by a surgical oncologist is important for recruiting future academic surgeons in this field.

\section{P129}

How Good Are Public Report Cards for Surgical Oncology? M.J. Leonardi, ${ }^{*}$ M. McGory, J. Tomlinson, C.Y. Ko. Surgery, UCLA, Los Angeles, $C A$.

Introduction: With the demand for increased healthcare quality by patients and payers, quality issues are being compared on publicly available websites. This study performs an objective website rating based on type of data, source, statistics/methodology of analyses, and whether data are appropriate to cancer patients undergoing surgery. Methods: A comprehensive, systematic internet search was performed on Google using "hospital quality comparison tool" in August 2006. Publicly available websites were rated on a 15-point scale for accessibility, data transparency, and appropriateness (i.e. inclusion of cancerspecific information for common cancers, appropriate amount of data, and appropriate cancer-specific outcomes [e.g. survival]). Results: 5 major quality report card websites were identified: Hospital Compare (CMS), Quality Check (JCAHO), Healthcare Advisor (Subimo), HealthGrades (HG), and Hospitalwise Professional (Medscape). Scores ranged from 6-12 (CMS 9, JCAHO 9, Subimo 6, Medscape 12, and HG 6). While all sites had some surgery-specific criteria for ranking hospitals, the criteria were not specific to cancer. Appropriate use of antibiotics, in-hospital mortality, and length of stay were included. None of the sites had cancer-specific outcomes. Only 2 sites (HG, Medscape) allowed hospital comparison based on data for a specific type of cancer surgery. All sites, except Subimo, were transparent with methodology. $\mathrm{CMS}$ and JCAHO gave unadjusted rates for quality measure compliance. Only 1 site (Medscape) provided risk-adjusted quartile rankings, while 2 sites (Subimo and HG) used risk-adjustment, but compared a hospital's actual to predicted performance. Subimo and HG required a fee for use, but did not provide any additional cancer-related information. Conclusion: Cancer patients should be able to compare hospitals on the quality of cancer surgery using the internet. However, a review of presently available comparison sites show that "surgical cancer quality" is determined by measures that are not truly cancerspecific. Cancer providers should be involved with such sites, as sufficient data are currently unavailable on public websites to appropriately inform patients on cancer surgery quality. 


\section{P130}

Targeting Bacteriolytic Therapy of Solid Tumors using Salmonella typhimurium S. Ganai, ${ }^{1}$ R.B. Arenas, ${ }^{1}$ B. Bentley, ${ }^{2}$ N.S. Forbes. ${ }^{3}$ 1. Baystate Medical Center, Springfield, MA; 2. Pioneer Valley Life Sciences Institute, Springfield, MA; 3. University of Massachusetts Amherst, Amherst, MA.

Introduction: Attenuated Salmonella typhimurium, a motile, nonpathogenic facultative anaerobe, has been demonstrated experimentally as a novel anticancer agent because of its preferential growth within the hypoxic and nutrient-rich environments of tumors. However, Phase I clinical trials demonstrated limited accumulation, raising uncertainty of its clinical utility. In order to optimize use in the clinical spectrum, the spatiotemporal dynamics of bacterial accumulation were examined. We hypothesized that bacterial chemotaxis and accumulation is determined by both nutrient and oxygen gradients, and is thus a function of distance from intact vasculature and necrosis. Methods: In a syngeneic subcutaneous murine cancer model, 4T1 mammary tumors were created in female $\mathrm{Balb} / \mathrm{C}$ mice. Mice were systemically injected via tail vein with $100,000 \mathrm{cfu} / \mathrm{g}$ VNP20009 (Vion Pharmaceuticals, New Haven, CT) or saline. Results: Bacterial accumulation was 10,000-fold greater in tumors than liver $(p<0.05)$, with an exponential relationship of accumulation within tumors with time. Bacteria exhibited a doubling time of 7.6 hours $(95 \%$ confidence interval, $6.7-8.9)$ in tumors and 11.2 hours $(95 \%$ confidence interval, 8.4 $16.6)$ in liver. Bacterial stratification occurred in regions bordering necrosis corresponding to regions with apoptosis (cleaved caspase-3 IHC). With later time points, many tumors developed evidence of intratumoral abscesses suggesting neutrophil infiltration. Regional spatial heterogeneity of bacterial accumulation within tumors in relation to regions near vasculature, necrosis, and apoptosis was observed. Conclusions: Modeling in vivo bacterial accumulation in the immune-competent host is complex due to the interplay of the bacteria against the tumor, the host against the tumor, and the host against the bacteria. VNP20009 favor dense colonies in regions bordering necrosis, with the possibility of an induction of apoptosis. Regions of accumulation suggest localization within regions of quiescence, in a margin between necrosis and viable tumor. Capitalization of this favored microenvironment will enhance its therapeutic use as a tumor-specific vector.

\section{P131}

Bevacizumab and Rapamycin for treatment of peritoneal metastases from implanted peritoneal model of ovarian cancer: An ideal combination? M. Teo, ${ }^{*}$ K.C. Soo, H.T. Hung. National Cancer Centre, Singapore, Singapore, Singapore.

Introduction: Ovarian cancer is the leading cause of death from gynaecological cancer. Often, the disease has spread to involve the peritoneal cavity. The mainstay of treatment is debulking surgery and chemotherapy with a platinum-based analogue and a taxane. However, disease recurrence in the peritoneal cavity is common. Other modalities of treatment need to be explored. In this study, we investigated the potential benefits of targeted therapy and examined the combined effects of intra-peritoneal Bevacixumab and rapamycin on peritoneal metastases from ovarian cancer. Methods: Peritoneal metastases from ovarian cancer in female SCID mice was established by peritoneal implantation via direct injection of $5 \times 106$ cells from the ovarian cell lines OV-90 and SKOV-3. Adminstration of intra-peritoneal Bevacixumab and rapamycin alone and in combination into 3 groups of 14 mice each upon development of peritoneal disease was performed, with group 1 forming the control group, receiving only intra-peritoneal injection of a similar quantity of normal saline solution. In anther set of experiments, the mice with obvious massive ascites from peritoneal metastases had removal of ascites, followed by treatment with combined Bevacizumab and rapamycin in half of these mice. Results: Combined treatment with Bevacizumab and rapamycin produced remarkable reduction in formation of ascites and peritoneal metastases when compared to the control group and the mono-therapy group. In mice with advanced disease, this combined treatment is capable of preventing re-formation of ascites and significantly reducing peritoneal tumour burden. There appeared to be no significant toxic effects from intra-peritoneal targeted therapy in the SCID mice. Conclusions: The results seen in this study, with the combined targeted therapy with Bevacizumab and rapamycin, are encouraging in the management of peritoneal disease from ovarian cancer and can prevent ascites formation and inhibit peritoneal tumour development. The above animal study paves the way for further pre- clinical studies to investigate this novel approach.

\section{P132}

Management of Hepatocellular Carcinoma with Ultrasound Guided Laparoscopic Radio Frequency Ablation A. Legaspi,* I. Sucandy. Surgical Oncology, 21st Century Oncology, Miami Beach, FL.

Resection of Hepatocellular Carcinoma (HCC) in cirrhotic patients is associated with a high morbidity and mortality. Data are presented on the effect of laparoscopic ultrasound guided radiofrequency ablation (LRFA) in this group. Methods: Retrospective review in 104 consecutive cirrhotic patients with $\mathrm{HCC}$ treated with LRFA. Complications, serum Alfa Feto Protein (AFP), and survival rates were recorded. Restaging included liver CT/MRI, and blood work 5 weeks after LRFA, and every 4 months thereafter. Patients were grouped according to the initial response to LRFA: 1) Decreased AFP and complete ablation by CT/MRI: 2) Unchanged/increased AFP and incomplete ablation by CT/MRI: 3) Recurrent AFP elevation and recurrent HCC by CT/MRI. Results:A total of $155 \mathrm{LRFAs}$ were performed in 104 patients. AFP was elevated in $87.5 \%$ $(\mathrm{N}=91)$ of 104 patients before treatment was initiated, and $12.5 \%(\mathrm{~N}=13)$ had normal AFP. Tumor size:Mean $3.5 \mathrm{~cm}$., Range 1.1 to $7 \mathrm{~cm}$. Multicentricity:A single lesion was ablated in $80.8 \%$ of the patients $(\mathrm{N}=84)$, and multiple lesions were ablated in $19.2 \%(\mathrm{~N}=20)$. Procedures: $77 \%(\mathrm{~N}=79)$ required only a single LRFA. In patients with persistent or recurrent $\mathrm{HCC}$ that were eligible for repeat LRFA, 24\% $(\mathrm{N}=25)$ underwent more than one procedure after the initial ablation because of either tumor recurrence or a second primary in a different site. Group 1, Decreased AFP: $84.6 \%(\mathrm{~N}=79)$ had a complete ablation. Group 2, Unchanged or increased AFP: In $15.4 \%(\mathrm{~N}=14)$, persistent disease as a result of an inadequate ablation was the cause of unchanged/increased AFP after LRFA Group 3, Recurrent AFP elevation ( $>9$ weeks after initial LRFA): Six patients had recurrent disease in the original site of ablation, and 19 patients had a new primary tumor in a different site. 30 -day mortality: $2.5 \%(\mathrm{~N}=4$ of 155 ablations). Complications: $6.5 \%$ had complications, one was life-threatening (Bleeding). Survival: Six years follow-up, 8 patients died from end-stage liver disease, 7 died from progressive $\mathrm{HHC}$, and 7 underwent liver transplant. Conclusions: LRFA is effective treatment for HCC in patients with underlying cirrhosis, and is associated with minimal mortality and morbidity.

\section{Alfa feto protein after laparoscopic RFA}

\begin{tabular}{|c|c|c|}
\hline stage & decrease AFP & increase AFP \\
\hline I & $5(100 \%)$ & 0 \\
\hline II & $55(84.6 \%)$ & $10(15.3 \%)$ \\
\hline III & $16(89 \%)$ & $2(11.1 \%)$ \\
\hline IV & $1(33.3 \%)$ & $2(66.7 \%)$ \\
\hline TOTAL & $77(84.6 \%)$ & $14(15 \%)$ \\
\hline
\end{tabular}

\section{P133}

Centralized implementation of accurate and efficient preoperative staging in esophageal cancer patients C.C. Verhoef, ${ }^{1 *} \mathrm{H}$. Groen, ${ }^{5}$ H.M. Van Dullemen, ${ }^{2}$ P.L. Jager, ${ }^{4}$ E.J. Van der Jagt, ${ }^{3}$ J.T. Plukker. ${ }^{1}$ 1. Dept Surgical Oncology, University Medical Center Groningen, Groningen, Groningen, Netherlands; 2. Dept of Gastroenterology. University Medical Center Groningen, University of Groningen, Groningen, Netherlands; 3. Dept of Radiology. University Medical Center Groningen, University of Groningen, Groningen, Netherlands; 4. Dept of Nucl. Med. University Medical Center Groningen, University of Groningen, Groningen, Netherlands; 5. Med.Techn.Assessment. University Medical Center Groningen, University of Groningen, Groningen, Netherlands.

Introduction: Staging esophageal cancer is notoriously difficult and often suboptimal. Endoscopic ultrasonography with fine needle aspiration (EUSFNA) and positron emission tomography (FDG-PET), which adds important information in a subset of patients, require a long learning curve. This is only attainable with high patient volume, while adequate equipment and expertise is not always available. Centralization of the staging process seems desirable. Methods: May 2005 we started an implementation study to determine the willingness of specialists in the 17 hospitals in North Netherlands to participate in a centralized diagnostic work-up for esophageal cancer using multidetector CT, EUS-FNA and FDG-PET or PET/CT, preferably within a one week 
target. They were invited to participate and to give their views on centralization of the diagnostic work-up through expanded questionnaires and dedicated hospital visits. A symposium was organised to review, discuss and summarize these views. In a pilot study of 59 patients we analyzed the feasibility of delivering an optimal diagnostic work-up within a one-week target. Results: $69.8 \%$ of specialists agreed that staging needed to improve, with $81.4 \%$ agreeing that centralization was the best step forward. Almost $90 \%$ were prepared to actively participate, though 3 large regional centers with appropriate equipment and expertise, were designated to perform work-ups. During the pilot study we noted a marked improvement in work-up time after implementation of a clear protocol and detailed agreements between the departments concerned. Completed diagnostic work-up within 5 days improved from $20 \%$ to $35 \%$ and completion within 10 days from $52 \%$ to $83 \%$. Overall staging time was reduced by 3.3 workdays, with an average of 7.0 days. Conclusion: Specialists in North Netherlands involved in the diagnosis and treatment of esophageal cancer showed broad support for centralization of staging. This can be implemented through clear diagnostic protocols and designating hospital responsibilities. Diagnostic work-ups can be accelerated to completion within 10 days, provided that protocols and interdepartmental agreements are clearly defined.

\section{P134}

A Neoadjuvant Protocol for Successful Local Control of Desmoid Tumors D.B. Zippel, * L. DiFrancesco, E. Kurien, L. Mack, N. Shachar, W. Temple. Sarcoma Group, Tom Baker Cancer Centre, Calgary, AB, Canada.

Objective: Local control of desmoids remains a significant problem. A prospective patient cohort with desmoid tumors was treated with a neoadjuvant chemoradiation protocol prior to wide surgical excision. Methods: From 1990 to 2005,25 patients with desmoids tumors were treated by a preoperative neoadjuvant regimen, using IV adriamycin $30 \mathrm{mg} \times 3$ days continuous infusion followed by $300 \mathrm{cGy} \times 10$ over 10 days, and then wide surgical excision in 4-8 weeks. Until 2000 , all patients with desmoids were included and since 2000 only those that progressed on medical treatment or recurrences were included. Tumor sites included 8 abdominal wall, 8 limb, 4 limb girdle, 4 upper trunk or breast, and 1 neck. The mean size of the lesions was $7.1 \mathrm{~cm}$. There were 21 females and 4 males and the mean age was 40 years. 9 patients had previous recurrent disease and 3 had progressed under medical therapy. Results: For the 25 patients treated, local control was $84 \%$. All recurrences $(4)$ occurred within 18 months. The remaining patients remain free of disease with a median follow up of 60 months. Of the 4 recurrences, 1 was re-excised and remains free of disease. 2 were re-radiated and stabilized. 1 was treated with systemic chemotherapy but still continues to progress. Microscopic margins were positive in 3 of 4 patients who recurred and 1 of 21 who remain free of disease. 2 patients had wound complications requiring operative revision. No amputations were performed. Conclusion: Our neoadjuvant chemoradiation protocol demonstrates the best local control to date, with low morbidity, and is the only prospective cohort study for the disease. The efficacy of this protocol is seen even in the face of the majority having a recognized high risk for recurrence, i.e. previous recurrence, failed medical Rx or positive margins.

\section{P135}

Isolated Limb Perfusion and External Beam Radiotherapy for Soft Tissue Sarcomas: Long-term Effects on Normal Tissue According to the LENT-SOMA Scoring System M.L. Hoven- Gondrie, ${ }^{*}$ K.M. Thijssens, J.H. Geertzen, R.J. Van Ginkel, H.J. Hoekstra. University Medical Center Groningen, Groningen, Netherlands.

Introduction With the combined treatment procedure of isolated limb perfusion (ILP), delayed surgical resection and external beam radiotherapy (EBRT) for locally advanced soft tissue sarcomas (STS) of the extremities, limb salvage rates of more than $80 \%$ are achieved. Long-term damage to the healthy surrounding tissue can't however be prevented. We evaluated the late effects on the normal tissue using the LENT-SOMA scoring system. Patients and Methods 24 patients (pts) with a median age of 44 (range 14-71) years were treated for a locally advanced STS with ILP, surgical resection and adjuvant 60-70 Gy EBRT. After a median follow up of 80 (range 17-152) months the pts were scored with the LENT SOMA scales for the following late tissue damage: muscle/ soft tissue, peripheral nerves, skin/ subcutaneous tissue and vessels. Not all the pts could be scored on all four items. Results For muscle/ soft tissue the mean calculated LENT score (LS) was $0.86 \pm 0.45$. A LS $<0.50$ represents no or minimal toxicities, a LS of 0.50-1.49 minor symptoms with no treatment required and a LS of 1.50-2.49 reflects moderate symptoms with only a conservative treatment required. No LENT scores higher than 2.50 were found. For peripheral nerves the mean LS was $0.62 \pm 0.58$, for skin/ subcutaneous tissue $0.68 \pm 0.32$ and for vessels the mean LS was $0.35 \pm 0.55$. The classification in subdivisions of all individual LENT scores is found in Table 1. Reviewing the individual SOM parameters of the calculated LS, $63 \%$ of pts scores grade 3 toxicity on one or more separate item, reflecting severe symptoms with a negative impact on daily activities and requiring more aggressive treatment. Moreover $13 \%$ scores grade 4 toxicity on any of the parameters, representing irreversible functional damage necessitating major therapeutic intervention. Conclusions According to the LENT- score, no severe late normal tissue damage was documented after the combined treatment procedure for STS. Evaluating the individual components of this score, however, the majority of pts has serious late toxic effects concerning any of the surrounding normal tissue.

Table 1

\begin{tabular}{|c|c|c|c|c|c|c|c|c|c|c|c|}
\hline & \multirow{2}{*}{} & $\mathrm{N}$ & \multirow{2}{*}{ Mean (range) } & \multicolumn{2}{|c|}{ LENT-score } & $0.50-1.49$ & $1.50-2.49$ & $\& \# 8807 ; 2.50$ \\
\cline { 3 - 12 } & & & $\mathrm{N}$ & $\%$ & $\mathrm{~N}$ & $\%$ & $\mathrm{~N}$ & $\%$ & $\mathrm{~N}$ & $\%$ \\
\hline muscle/ soft tissue & 23 & $0.86 \pm 0.45(0.17-1.83)$ & 5 & $22 \%$ & 15 & $65 \%$ & 3 & $13 \%$ & 0 & $0 \%$ \\
\hline peripheral nerves & 21 & $0.62 \pm 0.58(0.00-1.91)$ & 10 & $48 \%$ & 9 & $43 \%$ & 2 & $10 \%$ & 0 & $0 \%$ \\
\hline skin/ soft tissue & 20 & $0.68 \pm 0.32(0.36-1.50)$ & 5 & $25 \%$ & 14 & $70 \%$ & 1 & $5 \%$ & 0 & $0 \%$ \\
\hline vessels & 24 & $0.35 \pm 0.51(0.00-1.67)$ & 18 & $75 \%$ & 4 & $17 \%$ & 2 & $8 \%$ & 0 & $0 \%$ \\
\hline
\end{tabular}

\section{P136}

SINGLE AGENT EXPERIENCE OF HYPERTHERMIC ISOLATED LIMB PERFUSION FOR EXTREMITY SARCOMAS

M.G. Moller, * J.M. Lewis, C. Kim, M. Melis, K. Meredith, A. Sarnaik, D. Reintger, J. Zager, G.D. Letson. Surgical Oncology, H. Lee Moffitt Cancer and Resarch Institute, Tampa, FL.

Objectives: The aim of the study was to evaluate the efficacy of Melphalan, for hyperthermic isolated limb perfusion (HILP) therapy as an alternative treatment for limb salvage in patients with unresectable or recurrent soft tissue sarcomas, who otherwise will be treated with amputation or limb-sparing surgery with radiation. Methods: We report the outcome of 15 patients who underwent HILP for unresectable primary or recurrent extremity sarcomas from 1994 to 2006 at our institution. Data in regards to tumor characteristics, clinical response to treatment, recurrence time and location, disease free survival, and treatment complications were collected. Results: The overall response rate to Melphalan alone was $93.4 \%$, with $87 \%$ complete response rate. The local recurrance rate was $33 \%$ and $40 \%$ systemic recurrance rate respectively. The overall limb salvage rate was $86.6 \%$. All the patients diagnosed with advanced Angiosarcomas had a complete response with $100 \%$ limb salvage rate. We report a 45 month Medial Disease free survival. Conclusions: HILP with Melphalan is a safe and effective treatment option for selected patients with locally advanced and unresectable extremity sarcomas. The response rates are high, and comparable to those of combination with Interferon alpha, with limb salvage occurring in most patients, avoiding amputations or disfiguring surgical resections. These preliminary results are promising and additional studies of larger patient populations are warranted.

\section{P137}

Experience with the Superficial Femoral Vein as a Conduit Following Major Oncologic Resection A. McKay, ${ }^{*}$ M. Motamedi, W. Temple, L. Mack, R. Moore. Surgical Oncology, University of Calgary, Calgary, AB, Canada.

Introduction: Involvement of critical vascular structures has often been considered a relative contraindication to radical oncologic resection due to concerns of haematogenous metastasis and concerns of contamination of available conduits during contiguous gastrointestinal or genitourinary tract resections. The objective of this study is to describe the outcomes following radical oncologic resection with concomitant resection of critical vascular structures and reconstruction with the Superficial Femoral Vein (SFV). Methods: A retrospective review of all patients undergoing radical resection for malignancies 
requiring resection of major vascular structures and concomitant reconstruction using the SFV as conduit. The primary outcome measures were surgical morbidity and mortality, and the secondary measures included long-term patency of the vascular repairs and oncologic outcomes. Results: Seven patients met the inclusion criteria. Pathology included three retroperitoneal sarcomas, two groin sarcomas, and two metastatic squamous cell carcinomas to groin lymph nodes (Table 1). Minor morbidity, as defined by published criteria, was observed in the majority of patients ( 5 of 7). No major morbidity was observed. One vein graft in a patient with pre-existing chronic DVT occluded in the immediate postoperative period. No subsequent long-term venous or arterial graft occlusions have been observed after a median follow-up of 20.2 months (range 9.0 to 49.7 ). There was no peri-operative mortality. Two patients have died of tumor recurrence at 9.0 and 9.5 months after surgery. Both of theses patients had high-grade lesions; one was a radiation-induced sarcoma of the groin, and one was a retroperitoneal sarcoma. The remaining 5 patients are alive without clinical or radiological evidence of disease after a median of 37.1 months (range 9.7 to 49.7) (see Table 1). Conclusions: Involvement of critical vascular structures should no longer be a contraindication to tumor resection. The SFV conduit is a versatile option for major vascular reconstruction, as it provides good long-term patency rates with acceptable morbidity and mortality.

Table 1. Type and location of tumors, involved vascular structures, and recurrence and survival following radical resection.

\begin{tabular}{|c|c|c|c|c|c|c|c|c|}
\hline $\begin{array}{c}\text { Primary } \\
\text { Tumor }\end{array}$ & $\begin{array}{c}\text { Neoadjuvant } \\
\text { Treatment }\end{array}$ & Location & $\begin{array}{c}\text { Resected } \\
\text { Artery }\end{array}$ & $\begin{array}{c}\text { Arterial } \\
\text { Conduit }\end{array}$ & $\begin{array}{c}\text { Resected } \\
\text { Vein }\end{array}$ & $\begin{array}{c}\text { Venous } \\
\text { Conduit }\end{array}$ & $\begin{array}{c}\text { Disease } \\
\text { Status }\end{array}$ & $\begin{array}{c}\text { Survival } \\
\text { (months) }\end{array}$ \\
\hline Sarcoma & -- & Right Groin & $\begin{array}{c}\text { R Ext } \\
\text { lliac/Femoral }\end{array}$ & SFV & $\begin{array}{c}\text { R Ext } \\
\text { Iliac }\end{array}$ & $\begin{array}{c}\text { Vein } \\
\text { Ligated }\end{array}$ & $\begin{array}{c}\text { Died of } \\
\text { Recurrence }\end{array}$ & 9.0 \\
\hline $\begin{array}{c}\text { Squamous } \\
\text { Cell } \\
\text { Carcinoma }\end{array}$ & Chemo/Radiation & Left Groin & L Femoral & SFV & $\begin{array}{c}\text { L } \\
\text { Femoral }\end{array}$ & SFV & Disease-Free & 9.9 \\
\hline $\begin{array}{c}\text { Squamous } \\
\text { Cell } \\
\text { Carcinoma }\end{array}$ & -- & Right Groin & R Femoral & SFV & $\begin{array}{c}\text { R } \\
\text { Femoral }\end{array}$ & SFV & Disease-Free & 37.1 \\
\hline Sarcoma & Radiation & Retroperitoneum & -- & -- & IVC & SFV & Disease-Free & 20.2 \\
\hline Sarcoma & Radiation & Right Groin & $\begin{array}{c}\text { R Ext } \\
\text { lliac/Femoral }\end{array}$ & SFV & $\begin{array}{c}\text { R Ext } \\
\text { Iliac }\end{array}$ & SFV & Disease-Free & 38.8 \\
\hline Sarcoma & Radiation & Retroperitoneum & Aorta & Prosthesis & IVC & SFV & Disease-Free & 49.7 \\
\hline Sarcoma & Radiation & Retroperitoneum & Aorta & Prosthesis & IVC & SFV & $\begin{array}{c}\text { Died of } \\
\text { Recurrence }\end{array}$ & 9.5 \\
\hline
\end{tabular}

\section{P138}

ARTFICIAL INTELLIGENCE, SYSTEM ANALYSIS AND SIMULATION MODELING IN PREDICTION OF 5-YEAR SURVIVAL OF CARDIOESOPHAGEAL CANCER PATIENTS AFTER COMPLETE LEFT THORACOABDOMINAL ESOPHAGOGASTRECTOMIES O. Kshivets.* Surgery, Siauliai Public Hospital \& Cancer Center, Siauliai, Lithuania.

OBJECTIVE: We examined factors associated with the low- and high-risk of generalization of cardioesophageal cancer (CEC) (T1-4N0-3M0) after complete esophagogastrectomies (EG) through left thoracoabdominal incision. METHODS: We analyzed data of 150 consecutive CEC patients (CECP) $($ age $=54.9 \pm 0.7$ years; tumor size $=6.9 \pm 0.2 \mathrm{~cm}$ ) radically operated and monitored in 1975-2006 $(\mathrm{m}=116, \mathrm{f}=34$; combined $\mathrm{EG}$ with resection of pancreas, liver, diaphragm, colon transversum, splenectomies $=49$; lymphadenectomy $\mathrm{D} 2=59, \mathrm{D} 3=91 ; \mathrm{ad}=125, \mathrm{sq}=19, \mathrm{mix}=6 ; \mathrm{T} 1=16, \mathrm{~T} 2=32, \mathrm{~T} 3=58, \mathrm{~T} 4=44 ; \mathrm{N} 0=59$, $\mathrm{N} 1=18, \mathrm{~N} 2=71 ; \mathrm{N} 3=2 ; \mathrm{Gl}=42, \mathrm{G} 2=30, \mathrm{G} 3=78$ ). Variables selected for 5 -year survival (5YS) study were input levels of 45 blood parameters, sex, age, TNMPG, cell type, tumor size. Cox modeling, clustering, discriminant analysis, SEPATH, Monte Carlo, bootstrap simulation and neural networks computing were used to determine any significant dependence. RESULTS: 44 CECP (life span: $\mathrm{LS}=3545.8 \pm 238.7$ days) lived more than 5 years without CEC progressing ( $5 \mathrm{YS}=29.3 \%)$. $106 \mathrm{CECP}$ died because of generalization during the first 5 years after radical procedures $(\mathrm{LS}=593.5 \pm 32.6$ days). Cox modeling displayed that 5 YS of CECP $(n=150)$ after complete EG significantly depended on: T1-4, combined procedures, histology, G1-3, blood lymphocytes, monocytes, neutrophils, lymphoid infiltration of CEC, age $(\mathrm{P}=0.000-0.038)$. Neural networks computing, genetic algorithm selection and bootstrap simulation revealed relationships between 5 YS of CECP and combined procedures, N0-3, histology, gender, CEC growth, type of operations, P1-4, T1-4, adjuvant chemoimmunotherapy, G1-3, blood coagulation time, blood lymphocytes, thrombocytes, blood rest nitrogen, hemorrhage time, ESS, age, weight, blood chlorides, tumor size. CONCLUSIONS: Correct prediction of CECP survival after radical procedures was $90.7 \%$ by logistic regression (odds ratio $=86.7$ ), $96 \%$ by discriminant analysis and $100 \%$ by neural networks computing (area under ROC curve $=1.0$; error $=0.0012$ )

\section{P139}

A specific Guanosine-rich oligonucleotide, capable of forming stable quadraplex structures selectively blocks proliferation and induces apoptosis in esophageal tumor cells T.R. Schwartz, ${ }^{2 *}$ T.L. Bauer, E.B. Kmiec. ${ }^{2}$ 1. Surgery, Helen F Graham Cancer Center-Christiana Care, Newark, DE; 2. University of Delaware, Newark, DE.

Introduction: Single stranded DNA oligonucleotides (ODNs) can induce apoptosis and necrosis in mammalian cells with limited selectivity and specificity. Here, we report for the first time that a specific type of ODN can exhibit such selectivity and specificity when introduced into esophageal tumor cells. Methods: Various ODNs (analyzed by circular dichroism (CD) for secondary structure) were electroporated into OE19 (human malignant esophageal adenocarcinoma) and HET 1A (human non-malignant esophageal mucosal) cell lines and analyzed by FACS for cell cycle data. Caspase-3,7 activity was measured by Caspase-Glo luminescent assay. Results: At 96 hours following exposure to G20 (monomeric guanosine sequence 20 bases in length), cell cycle analysis revealed that greater than $25 \%$ of total OE 19 cells were in the sub G1 cell cycle population, demonstrating degraded chromosomal DNA consistent with apoptosis. This effect is dose- and time-dependent, requiring a treatment of $\geq 10 \mu \mathrm{M}$ G20 ODN for $>72$ hours. Other monomeric ODNs, such as C20, A20 or T20, respectively, exhibit minimal effect on cell cycle progression. Caspase-3,7 activity is $75 \%$ greater in G20 treated OE19 cells at 96 hrs than in mock treatments, also consistent with the induction of apoptosis. Conversely, the sub G1 population was less than $10 \%$ at 96 hours for the HET $1 \mathrm{~A}$ cells exposed to G20, demonstrating specificity of the ODN to tumor cells. GROs that had a thymidine nucleoside inserted every 4 or 6 base pairs failed to demonstrate a similar induction of apoptosis in the OE19 cells. CD data for these ODNs demonstrated that their secondary structure was far less stable than the G20 ODN. Conclusion: Synthetic G20 ODN exhibits anti-tumor effects with a significant degree of selectivity and specificity. G20 adopts a very stable quadruplex conformation that can modify the metabolic activity of tumor cells for sustained periods, and may be a useful anti-tumor agent for certain types of esophageal cancer.

\section{P140}

Differential Gene Expression Associated with Early Tumor Recurrrence and Long Disease Free Survival in Early Stage Non-Small Cell Lung cancer T.A. D'Amato, ${ }^{*}$ H.C. Fernando, ${ }^{2}$ B.Y. Chan, ${ }^{3}$ W.A. Ricketts, ${ }^{4}$ J.D. Luketich, ${ }^{5}$ R.J. Landreneau. ${ }^{5} 1$. Department of Surgery, Jefferson Medical College, Philadelphia, PA; 2. Boston Medical Center, Boston, MA; 3. UC Irvine, Irivine, CA; 4. Oncotech, Inc., Tustin, CA; 5. University of Pittsburgh Medical Center, Pittsburgh, PA.

Background: One-third of resected patients with Stage I non-small cell lung cancer (NSCLC) recur or develop metastases. Estimating prognosis by traditional anatomic staging and tumor histology may be inaccurate compared to a genomic profile based on gene arrays. Utilizing a cancer-focused array, tumor gene expression profiles from stage 1 NSCLC patients were determined. Methods: RNA was extracted from paraffin embedded tissue in 7 stage IA NSCLC patients who recurred within 12 months and 13 patients with a disease free survival (DFS) greater than 36 months. Amplified RNA was detected by biotinstrepavidin quantitative fluorometry after hybridization to the OHS-82 Cancer Array (SuperArray Bioscience Corp.). This array includes a set of genes controlling apoptosis, cell cycle, growth and differentiation, cell motility and signal transduction. Background corrected fluorescence was normalized against housekeeping gene signals between array data sets. Un-censored analysis of the two distributions was performed with a two sided t-test to compare gene expression levels in patients with early recurrence (R) and no recurrence (NR). Results: Of the 482 genes represented, seven genes represented in the OHS82 Cancer Array were associated with early recurrence and 9 with long DFS, 
[see Table]. Conclusions: Applying focused RNA gene arrays to paraffin embedded tumor specimens is feasible and may be a useful prognostic tool. Overexpression of genes associated with cell motility, malignant infiltration, and signal transduction likely occur in patients with early tumor recurrence. Patients with long DFS may possess tumors with increased expression of regulatory and repair genes. Future gene array studies on archived tissues from patients enrolled in clinical trials and prospective studies evaluating differential gene expression and response to therapy are warranted.

Gene Over-Expression in Resected Stage IA NSCLC

\begin{tabular}{|c|c|c|c|c|c|}
\hline - & - & & $\ldots a$ & $\begin{array}{c}\text { Mean Inensity } \\
(\mathrm{NR}, \mathrm{b}\end{array}$ & p-valuc $c$ \\
\hline HYALI & Hyaluronoglucosamminidase 1 & Cell Motiliry - Promotes Metastasis & 330 & 187 & 0.00029 \\
\hline РіКзСв & $\begin{array}{l}\text { Phosphoinositide - } 3 \text { kinasc. catalytic beta } \\
\text { polycepide }\end{array}$ & Cell Cycle Regulatory & 387 & 331 & 0.00750 \\
\hline PRKARIA & Protecin kinase, camp-dependemt. regulatory, type & Sienal Transtuction & 87 & 55 & 0.00795 \\
\hline MME & $\begin{array}{l}\text { Membrane metallo-endopoppidase (CALLA. } \\
\text { CDI0) }\end{array}$ & Cell Motility - Malignant Inffltration & 380 & 246 & 0.01151 \\
\hline WNT3 & $\begin{array}{l}\text { Wingless-ype MMTV integration site family. } \\
\text { member } 3 \text {. }\end{array}$ & Signal Transduction & 34 & 216 & 0.03775 \\
\hline SNAI2 & Snail homolog 2 (Drosopphila) & Cell Motility - Malignant Infiltration & 390 & 283 & 0.04271 \\
\hline USP7 & $\begin{array}{l}\text { Ubiquitin speceific peppidase } 7 \text { therpes virus } \\
\text { associaredil }\end{array}$ & DNA Repair. Binds p53 - Regulatory & 332 & 241 & 0.04625 \\
\hline \multicolumn{6}{|c|}{ Over expressed in Long DFS } \\
\hline CEBPG & CCAAT/Enhancer binding protein & Transcription Regulation - DNA Repair & 289 & 396 & 0.00771 \\
\hline$\overline{\text { AKRIC2 }}$ & Aldo-keto reductase family 1 & Cell Cycle Regulatory & 183 & 418 & 0.01699 \\
\hline PGAMI & Phosphoglycerate mulase I (brain) & Gilycolytic - Regulatory & 87 & 139 & 0.01899 \\
\hline NQO1 & NADAPH dehydrogenase & Ant-oxidar- - Repair / Regulation & 159 & 301 & 0.02391 \\
\hline GAPDH & Glyceraldehyde-3-Phospho dehygrogenase & Glyocolytic & 147 & 263 & 0.02557 \\
\hline TCFI & Transcription factor I. hepatic & Muational-Regularory & 236 & 334 & 0.03517 \\
\hline CANX & Calnexin & Ant-appoposis & 114 & 198 & \begin{tabular}{|l|l|l|}
0.03517 \\
\end{tabular} \\
\hline TIEI & $\begin{array}{l}\text { Tyrosine kinase with immunoglobin-like and } \\
\text { egf-like domains }\end{array}$ & Signaling - angiogenesis / remodeling & 170 & 307 & 0.04939 \\
\hline STAT3 & Signal transducer and activator of transcription 3 & $\begin{array}{l}\text { Cell Migration and Mooility - Negative } \\
\text { Regulatory }\end{array}$ & 280 & 354 & 0.04795 \\
\hline
\end{tabular}

\section{P141}

Radiation Exposure to Treatment Team from Intraoperative 125I Brachytherapy Implants as Adjuvant Therapy to Sublobar Resection in Stage I Lung Cancer M.J. Schuchert, ${ }^{1 *}$ R.P. Smith, ${ }^{2}$ K. Komanduri, ${ }^{2}$ S. Burton, ${ }^{2}$ D.E. Heron, ${ }^{2}$ H. Chen, ${ }^{2}$ Z. Zhuo, ${ }^{2}$ F. Li, ${ }^{2}$ J.D. Luketich, ${ }^{1}$ R.J. Landreneau. ${ }^{1} 1$. Heart, Lung and Esophageal Surgery Institute, University of Pittsburgh Medical Center, Pittsburgh, PA; 2. University of Pittsburgh Medical Center - Department of Radiation Oncology, Pittsburgh, PA.

125I brachytherapy mesh implantation is currently being investigated as an adjuvant modality for improved local control in the sêtting of sublobar resection for early stage lung cancer (ACOSOG Z0432). Concern has been raised regarding the potential risks of radiation exposure to operative and nursing personnel. Given the absence of published radiation exposure data in this setting, this prospective study was designed to quantitate actual radiation exposures to health care professionals during brachytherapy mesh implantation. Materials and Methods Vicryl mesh implants contained 40-60 125I seeds spaced in a grid at $1 \mathrm{~cm}$ intervals. The average radiation exposure was measured for each case using thermoluminescent dosimeters (Landauer, Inc.) during 22 cases of sublobar resection with $125 \mathrm{I}$ brachytherapy implants. At surgery, diodes were placed on the back of each hand of the radiation oncologist and surgeon. To simulate ICU staff exposure, diodes were placed on the contralateral shoulder of the patient and retrieved at 24 hours. Results The recommended yearly radiation exposure to healthcare personnel is $5 \mathrm{rems}$ (cGy). Median activity per seed was $0.511 \mathrm{mCi}$ with a median total activity implanted of $23.0 \mathrm{mCi}$. Median radiation exposure was $1 \mathrm{mrem}$ to the radiation oncologist and $2 \mathrm{mrem}$ to the surgeon (only 1-2/5000 of maximum yearly exposure). Median dose to the patient's shoulder diode simulating continuous close ICU exposure was 5.5 millirem/hour, or up to 44 millirems during continuous contact with the patient during an eight hour shift of patient care. Based on the inverse square dose relationship, actual exposures should decrease significantly at even small distances from the patient. Conclusions There is very little radiation exposure to physicians and staff during the management of patients with 125I brachytherapy mesh implants. No specific patient isolation precautions appear to be necessary following implantation, however we continue to recommend limited exposure to pregnant care givers. This modality should thus be considered a safe radiation adjunct in the setting of sublobar resection.

\section{P142}

Identification of genuine differences between experienced surgeons and cancer recurrence rates after radical prostatectomy F.J. Bianco, ${ }^{1 *}$ A.J. Vickers, ${ }^{1}$ A. Serio, ${ }^{1}$ J.A. Eastham, ${ }^{1}$ E.A. Klein, ${ }^{2}$ M.W. Kattan, ${ }^{2}$ J.E. Pontes, ${ }^{3}$ P.T. Scardino. ${ }^{1}$ 1. Surgery, Urology, Memorial Sloan Kettering Cancer Center, New York, NY; 2. Cleveland Clinic Foundation, Cleveland, OH; 3. Wayne State University, Detroit, MI.

Background: There is significant variability between surgeons on qualitative outcomes measures (perioperative, late urinary complications and long term incontinence) after radical prostatectomy (RP). These variations are attributed to differences in surgical technique and have not escaped high volume providers. In this study we hypothesized that there is marginal genuine heterogeneity in the objective outcome of cancer recurrence after RP performed by experienced surgeons. Methods: 7,850 treatment naive patients with clinically localized prostate cancer underwent open RP between 1987 and 2003. We constructed a multivariable, parametric regression survival-time model adjusting for the surgeon, its experience and casemix (preoperative characteristics, tumor stage and histology) against cancer recurrence (BCR) after radical prostatectomy. We used the I2 statistic to quantify the degree of variability between experienced surgeons, those who had conducted more than 40 post-training lifetime cases. Results: A total of 7,499 $(96 \%)$ of the operations were performed by 36 experienced surgeons. The cancer characteristics of the patients tended to be more favorable with greater surgeon experience. During the median follow up of 4 years, there were 1,167 patients with $\mathrm{BCR}$ and the 5-year freedom from BCR was $79 \%$. We encountered a significant heterogeneity in the BCR rates between the experienced surgeons $(p<0.0005)$ despite adjustment for case-mix. The I2 statistic was $55 \%$, these representing genuine differences in surgical technique rather than chance. Sensitivity analysis on surgeons whom experience exceeded 100 cases revealed a even higher genuine differences ( $(2=0.73$ ) Conclusions: There is significant variability among experienced surgeons affecting cancer control after radical prostatectomy. Moreover, the grand extent of this variation represented genuine differences, likely derived from individual surgical techniques. These results further suggest that outcomes of this operation are sensitive to small differences in performance.

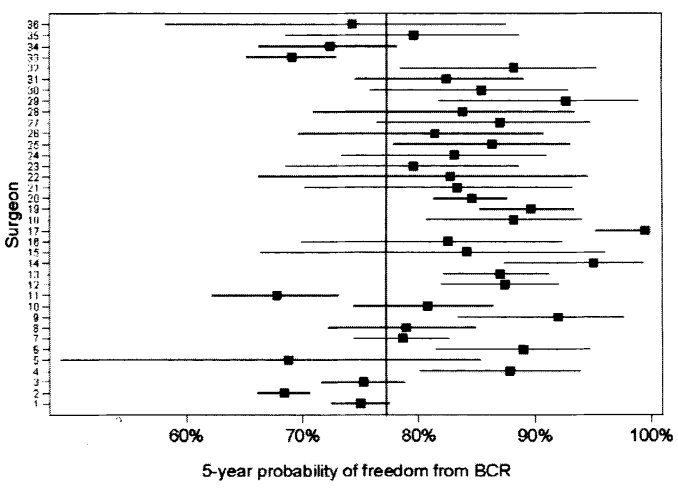

Forest plot of 5-year predicted probability of freedom from recurrence by surgeon 


\section{P143}

Iodine Impacts Pathways Governing Prostate Cancer Biology K. Shah, ${ }^{1}$ * R. Stoddard, ${ }^{1}$ B. Eskin, ${ }^{2}$ G. Johannes, ${ }^{3}$ A.D. Brooks. ${ }^{1}$ 1. Surgery, Drexel University College of Medicine, Philadelphia, PA; 2. Drexel University College of Medicine - Dept of Obstetrics and Gynecology, Philadelphia, PA; 3. Drexel University College of Medicine - Dept of Molecular Pathology, Philadelphia, PA.

Background: Although both epidemiologic and animal studies have demonstrated an association between iodine deficiency and breast cancer for over 40 years, little is known about the effects of iodine on human tumor biology at the molecular level. Our initial molecular studies in breast cancer indicated an interaction between Iodine and the estrogen pathway. In order to understand the role of Iodine in other hormone responsive tumors, we used microarray analysis to determine iodine' effects in both hormone sensitive and resistant prostate cancer cell lines. Methods: To quantify the maximum tolerable dose of iodine and its relative apoptotic effects on androgen sensitive (LNCaP) and androgen insensitive (PC3) prostate cancer cell lines, Lugols iodine solution was titrated to varying concentrations of I2 and a TUNEL apoptosis assay was performed at a number of time points. Using this data, PC3 and LNCaP cells were incubated at the maximum sub-lethal dose for up to 96 hours and microarray analysis was performed on the RNA harvested from these cells. Quantitative RT-PCR was subsequently used to verify the results of the microarrays. All arrays and RT-PCR were performed in triplicate. Results: The sub-lethal dose determined by TUNEL assay for PC3 was $1.0 \mathrm{mM} \mathrm{I2}$ and for LNCaP it was $0.25 \mathrm{mM}$ I2. Genes that saw their transcription changed by a factor greater than 1.75 fold are active in pathways including: 1 ) hormone response pathways and sex steroid catabolism (AKR1C1, CYP1B1); 2) the polyamine catabolism pathway (spermidine/spermine N1-acetyltransferase 1);3) the cell proliferative WNT pathway (FZD receptor, DKK1); 4) cell cycle and proliferation pathways (SDC1, IGFBP-3, BAG3). Conclusions: These changes in transcription indicate that iodine alters metabolic and biochemical pathways known to be involved in cancer progression in prostate cancer. Furthermore, the heightened sensitivity of $\mathrm{LNCaP}$ cells to iodine points to a potential role for iodine in hormone sensitive tumors. Elucidation of iodine's involvement in these pathways should aid in development of a new targeted pharmaceutical strategy for hormone responsive tumors.

\section{P144}

Enhancement of Active Specific Immunotherapy With Lymphodepleting Chemotherapy E.C. Hsueh,* S.M. Knebel, I.A. Collier, G.S. Smith. Surgery, Saint Louis University, St. Louis, MO.

Presence of regulatory $\mathrm{T}$ cell has been associated with non-responsiveness to immunotherapy. Pre-treatment depletion of lymphoid cells may increase the efficacy of adoptive transfer therapy for cancer. We hypothesized that prevaccination lymphodepletion can also enhance the efficacy of active specific immunotherapy. $\mathrm{C} 57 / \mathrm{BL}$ mice $(\mathrm{n}=88)$ were randomized to eight groups. B16 melanoma cells $(1 \times 105)$ were injected on Day 0 . Mice were randomized to receive saline, fludarabine $(\mathrm{F})$, cyclophosphamide $(\mathrm{C})$, or $\mathrm{F}$ plus $\mathrm{C}(\mathrm{FC})$ i.p. on Days 8 and 9 . Mice were further randomized to receive either s.q. irradiated B16 cells $(1 \times 106)$ plus IL2 $(20,000$ IU s.q. $x 4$ days) or culture media only on Day 12. Serial tumor measurement was taken. Blood was taken prior to vaccination. $\mathrm{CD} 4+\mathrm{CD} 25+\mathrm{T}$ cells were injected via tail vein into subgroups of mice prior to vaccination. Blood and spleen were harvested for cytokine array analysis and splenocyte proliferation assay, respectively. Statistical analysis was performed using Chi square test and Student's t-test. Lymphodepletion was highest in the FC group: $0.5 \pm 0.6 \mathrm{k} / \mathrm{ul}$ compared with $2.1 \pm 1.8 \mathrm{k} / \mathrm{ul}(\mathrm{C})$, $6.4 \pm 3.6 \mathrm{k} / \mathrm{ul}(\mathrm{F}), 9.7 \pm 2.2 \mathrm{k} / \mathrm{ul}$ (control), $(\mathrm{p}=0.0001)$. The combination of FC plus irradiated B16 and IL2 (V/IL2)had significantly stronger anti-tumor effect than V/IL2 alone $(p=0.02)$, or FC alone $(p=0.008)$. There was significant increase in splenocyte proliferation following irradiated $\mathrm{B} 16$ co-culture in the $\mathrm{FC} / \mathrm{V} / \mathrm{IL} 2$ group $(41 \%)$ compared with V/IL2 group (11\%) and FC group ( $8 \%$ ), $(p=0.013,0.028$, respectively). Reconstitution of CD4+CD25+ T cell did not completely abrogate the effect of lymphodepletion on immunotherapy. Serum cytokine array revealed a significant variation in the cytokine profile between groups. Notably, ICAM-1, osteopontin, and IGF-1 were up-regulated and VCAM-1, MIP-2, and CXCL16 were down-regulated in the FC/V/IL2 group compared with V/IL2 only group. These data suggest that pre-vaccination lymphodepleting chemotherapy can enhance the efficacy of active specific immunotherapy. The mechanism of action may be the depletion of regulatory $\mathrm{T}$ cell as well as a shift to favorable cytokine milieu prior to vaccination.

\section{P145}

Mutant PI3Kinase Bearing Colon Cancer Cells are More Metastatic Than Wild Type Bearing Cells in an Orthotopic Model of Colon Cancer A. Rajput, ${ }^{1}$ X. Guo, ${ }^{1}$ R. Rose, ${ }^{2}$ J. Hauser, ${ }^{1}$ A. Beko, ${ }^{1}$

K. Kuropatwinski, ${ }^{1}$ M.G. Brattain, ${ }^{1}$ J. Wang. ${ }^{1}$ 1. Surgical Oncology, Roswell Park Cancer Institute, Buffalo, NY; 2. University at BuffaloSUNY, Buffalo, NY.

INTRODUCTION: Metastases from colorectal cancer remain as a significant burden to society. PI3Kinase (PI3K), a lipid kinase, has been shown to have a gain of function mutation in $1 / 3$ of colon cancers. The purpose of this study was to determine the imapact of the wild type and mutant alleles of PI3K on metastases in an orthotopic model of colon cancer. METHODS: HCT116 human colon cancer cells which are heterozygous for the PI3K mutation were asymmetrically manipulated to knock out either the wild type (wt) or the mutan (mut) allele; thus generating either HCT116wt or HCT116mut for PI3K. The cells were labelled with green fluorescence protein (GFP) for in vivo serial imaging. $5 \times 10^{6}$ cells of each line were subcutaneously injected into male $\mathrm{BALB} / \mathrm{c}$ mice. Once xenografts were generated, they were explanted and minced into $1 \mathrm{~mm}^{3}$ pieces. Using microsurgical techniques, two pieces of the xenograft were subserosally implanted into 28 (HCT116wt) \& 29 (HCT116mut) male $\mathrm{BALB} / \mathrm{c}$ nude mice. Weekly fluorescence imaging was performed to follow tumor growth and progression. Animals were sacrificed 5 weeks post implantation and tissues were processed for histologic analysis and RNA was also extracted for PCR analysis. RESULTS: All animals in both groups showed primary tumor growth and bowel invasion. 26/29 (90\%) HCT116mut animals demonstrated distant metastases on GFP imaging and histology to either the liver and/or lungs. 20/28 (71\%) animals with HCT116wt demonstrated such metastases. HCT116mut bearing animals also showed an increased burden of metastases as compared to HCT116wt bearing animals on histology and GFP imaging as shown in the explanted lungs in the figure below. RT-PCR analysis of human specific GAPDH as a reflection of metastases also showed an increased metastatic burden in the mutant PI3K bearing cohort (Figure). CONCLUSIONS:This orthotopic colon cancer model mimics the metastatic pattern in humans. Mutant PI3K bearing HCT116 cells demostrate an enhanced metastatic potential compared to wild type PI3K bearing cells. Thus, PI3K may be a novel target for effective colorectal cancer therapies.

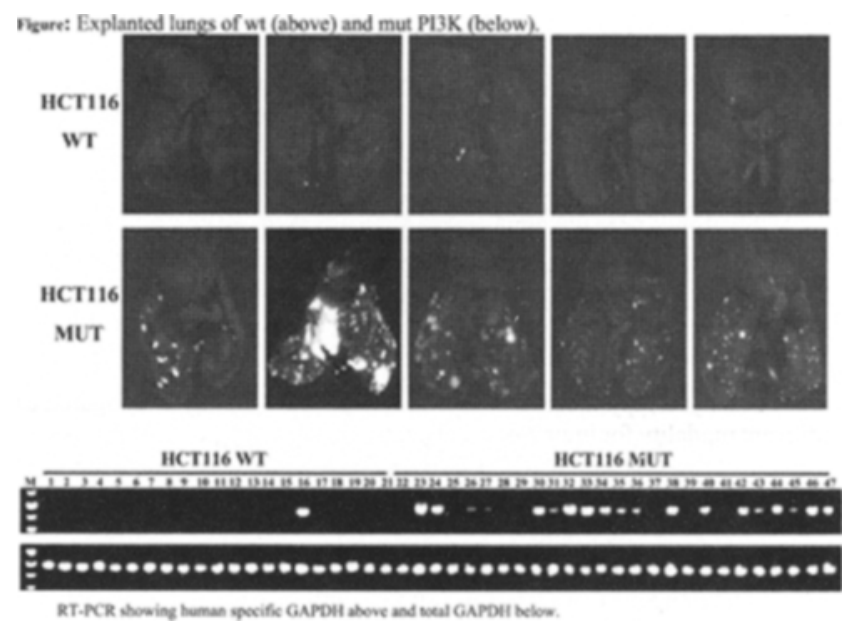

\section{P146}

Lipid Nanoemulsions for the Delivery of Combination Chemotherapy in Pancreatic Cancer F.G. Rocha, ${ }^{1}$ M.A. Abramson, ${ }^{1 *}$ S. Tiwari, ${ }^{2}$ H. Devallapaly, ${ }^{2}$ M. Amiji, ${ }^{2}$ E.E. Whang. ${ }^{1}$ 1. Surgery, Brigham and Women's Hospital, Boston, MA; 2. Northeastern University, Boston, $M A$.

Introduction: Current treatment options for pancreatic cancer are limited by a lack of efficacy and significant morbidity. Ceramide is a bioactive lipid produced by cancer cells in response to radiation and chemotherapy and serves to activate apoptosis. Paclitaxel is a drug widely used in the treatment of solid tumors that blocks tubulin polymerization to induce cell death. Because both 
agents are toxic and poorly soluble, we have developed novel oil-in-water nanoemulsions, with oils rich in omega- 3 polyunsaturated fatty acids containing these cytotoxic agents in order to enhance their delivery to pancreatic tumor cells. Methods: Pine nut oil-containing nanoemulsions were formulated with paclitaxel and C6-ceramide and the oil droplet size was reduced with ultrasound to less than $200 \mathrm{~nm}$. Pancreatic cell lines Panc-1, Capan-2, and BxPC3 were treated with ceramide $(10 \mu \mathrm{M})$ or paclitaxel $(100 \mathrm{nM})$ alone or in combination either in aqueous solution or nanoemulsions. Intracellular delivery of nanoemulsions containing cytotoxic agents was examined by using rhodaminelabeled paclitaxel and NBD-labeled ceramide in nanoemulsions. Cellular viability was measured spectrophotometrically using MTS assay at $490 \mathrm{~nm}$ and normalized to controls. Statistical analysis was conducted using ANOVA and Tukey's HSD test. Results: Nanoemulsion internalization by pancreatic cancer cell lines was confirmed by fluorescence microscopy. MTS assay results are summarized on Table 1. Panc-1, Capan-2, and BxPC-3 cells did not respond to aqueous or encapsulated ceramide. Paclitaxel in aqueous solution had a dramatic effect on the viability of BxPC-3 cells $(p<0.05)$ but was only effective on Panc-1 or Capan-2 cells when encapsulated in nanoemulsions. No synergy was seen with ceramide in aqueous solution. However, nanoemulsions containing both ceramide and paclitaxel exhibited significant cytotoxicity with synergy in Panc-1 cells $(\mathrm{p}<0.05)$ and to a lesser degree in Capan-2 cells. Conclusion: Oil-in-water nanoemulsions may be a promising strategy for treatment of chemoresistant pancreatic cancer by delivering potent cytotoxic drugs in higher doses directly to cancer cells while minimizing systemic exposure.

Table 1: Survival (\% of Control)

\begin{tabular}{|c|c|c|c|c|c|c|c|}
\hline $\begin{array}{c}\text { Cell } \\
\text { Type }\end{array}$ & Ceramide & Paclitaxel & Cer+Pax & NE-Empty & NE-Cer & NE-Pax & NE-Cer+Pax \\
\hline Panc-1 & $113.3 \pm 35.1 \%$ & $88.9 \pm 27.7 \%$ & $79.6 \pm 21.2 \%$ & $91.5 \pm 20.4 \%$ & $91.1 \pm 18.7 \%$ & $75.7 \pm 17.3 \% *$ & $21.0 \pm 24.8 \% * \#$ \\
\hline Capan-2 & $99.5 \pm 29.8 \%$ & $92.3 \pm 21.3 \%$ & $81.1 \pm 26.5 \%$ & $96.7 \pm 19.5 \%$ & $91.9 \pm 24.2 \%$ & $78.7 \pm 18.1 \% *$ & $72.7 \pm 23.7 \% *$ \\
\hline BxPC-3 & $101.2 \pm 27.6 \%$ & $27.6 \pm 13.9 \% * \#$ & $27.6 \pm 16.6 \% * \#$ & $97.4 \pm 11.2 \%$ & $95.1 \pm 14.1 \%$ & $52.6 \pm 13.5 \% *$ & $63.2 \pm 17.0 \% *$ \\
\hline
\end{tabular}

\section{${ }^{*} \mathrm{p}<0.05$ compared to Control}

$\# p<0.05$ compared to NE-Pax

\section{P147}

Delineating the Role of the Inhibitory $\mathrm{xB}$ Kinases in the Chemoresistance of Sarcomas B.K. Bednarski, * A.S. Baldwin, Jr, X. Ding, H.J. Kim. University of North Carolina Lineberger Cancer Center, Chapel Hill, NC.

Introduction: The transcription factor NF- $\mathrm{KB}$ is involved in the chemoresistance of solid tumors, including fibrosarcomas. NF- $\kappa B$ activation is mediated by phosphorylation of I $\kappa \mathrm{B} \alpha$ via inhibitory $\kappa \mathrm{B}$ kinases (IKK) that leads to its degradation by the $26 \mathrm{~S}$ proteasome. Proteasome inhibitors are clinically in use to block NF- $\mathrm{KB}$ but have other downstream effects. The role of IKKs in NF-KB-mediated chemoresistance is unclear; some studies suggest either an IKK-independent mechanism, while others argue an IKK $\beta$-dominance in response to TNF $\alpha$. Our purpose is to use a clinically relevant in vitro sarcoma model treated with doxorubicin to delineate the role of IKKs in NF- $\kappa B$-mediated chemoresistance. Methods: HT1080 fibrosarcoma cells were treated with doxorubicin $(1.5 \mu \mathrm{g} / \mathrm{ml})$. DNA binding was measured by electrophoretic mobility shift assay (EMSA) and I $\mathrm{KB} \alpha$ degradation by Western blot. With the lack of clinically useful inhibitors for IKK $\alpha, \operatorname{IKK} \alpha$ was blocked using small interference RNA (siRNA) and confirmed by Western blot; apoptotic response was measured by TUNEL. IKK $\beta$ was blocked using a novel inhibitor, DDN 3563-4-3BAY607497 (DDN; collaboration with TheraLogics, Raleigh, NC). The effects of specific IKK inhibition were evaluated using EMSA and western blots. Results: Activation of NF- $\mathrm{KB}$ in fibrosarcoma cells treated with doxorubicin was confirmed with an increase in DNA binding and I $\kappa \mathrm{B} \alpha$ degradation compared to untreated controls. IKK $\alpha$ can be effectively inhibited with siRNA strategies resulting in decreased NF$\kappa \mathrm{B}$ DNA binding; $\mathrm{I} \kappa \mathrm{B} \alpha$ was degraded but apoptotic responses were enhanced. The treatment of HT1080 cells with DDN prior to doxorubicin demonstrated decreased DNA binding and decreased degradation of I $\mathrm{KB} \alpha$ compared to doxorubicin alone (Figure). Conclusion: The results suggest that both IKK $\alpha$ and IKK $\beta$ are involved in the activation of NF- KB in a human sarcoma model in response to doxorubicin. Whether inhibition of a specific, dominant IKK or combinational approaches result in enhanced cytotoxicity remains to be established. Further studies are needed to compare these IKKinhibition strategies with the NF-KB inhibition strategies currently in clinical use.

\section{HT1080 Cells}

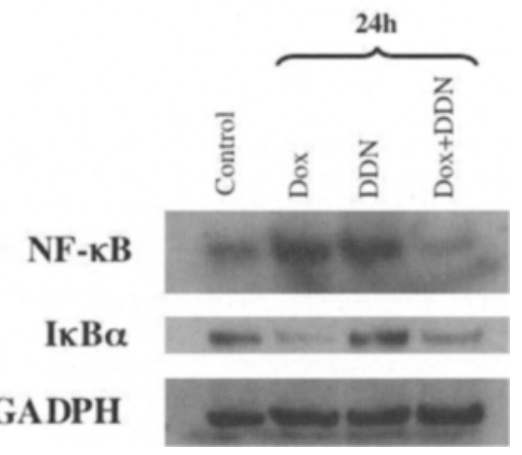

Figure: HT1080 cells treated with DDN $(1 \mu \mathrm{M})$ and Doxorubicin $(1.5 \mu \mathrm{g} / \mathrm{ml})$ for $24 \mathrm{~h}$. NF- $\kappa$ B activat ion evaluated by EMSA for DNA binding and Western blot for $\mathrm{I} \kappa \mathrm{B} \alpha$ degradation.

\section{P148}

The effect of three-dimensional growth on epithelial-to-mesenchymal transition in human breast cancer cells S. Patel, ${ }^{1 *}$ S.C. Wishnia, C. Noack, ${ }^{2}$ N. Bhogal, ${ }^{1}$ C. Klapperich, ${ }^{2}$ J.E. Rosen. ${ }^{1}$ 1. Section of Surgical Oncology, Department of Surgery, Boston University School of Medicine, Boston, MA; 2. Department of Biomedical Engineering Boston University, Boston, MA.

Introduction: The breast cancer microenvironment poses a formidable barrier to effective therapy. The pathophysiologic mechanisms underlying treatment resistance in breast cancer are not known; in part this is due to the lack of a suitable, well-characterized model. Our purpose in this study was to develop a three-dimensional (3D) breast cancer culture system capable of reproducing the tumor microenvironment. Methods: We cultured MCF7, a human breast cancer cell line, under three conditions: on polystyrene, on a two-dimensional collagen type I gel, and on a 3D collagen type I gel. The extent of phenotypic abnormality was assessed by changes in morphology on phase-contrast images and alterations in gene expression by qRTPCR. G3PDH was used as an endogenous control, and relative quantification was performed using MCF-7 cells grown on polystyrene alone as the calibrator or reference sample. Genes tested included E-cadherin, c-kit, TGFbeta, MMP-2, MMP-12 and MMP-19, chosen for their importance in cellmatrix signaling. Results: Expression of E-cadherin, c-kit, MMP-2, MMP12 and MMP-19 in MCF-7 grown on polystyrene was significantly higher than their expression in MCF-7 grown in the 3D collagen type I gel. TGFbeta expression was significantly higher in MCF-7 cells grown in the 3D collagen type I gel compared to polystyrene. Gene expression in the twodimensional gels trended towards that seen in the 3D gels but was not statistically significant. MCF-7 cells grown in 3D formed small, loose aggregates in comparison to the stellate appearance of cells grown on polystyrene. Conclusions: We can non-destructively grow and image live breast cancer cells using optical methods. We were able to build an improved, precisely defined synthetic scaffold that better replicates the gene expression changes seen in human breast cancer, demonstrate that $3 \mathrm{D}$ growth affects the expression of genes important in cell-cell adhesion and cell invasion, and nondestructively image these cells. We hope that the gene expression changes seen in this $3 \mathrm{D}$ model will provide a platform for the better understanding of treatment resistant breast cancer. 
P149

A novel phenylbutyrate-derived histone deacetylase inhibitor, $S-$ HDACi-42, mediates acid-associated gene expression changes in Barrett's esophageal adenocarcinoma cells K.A. Varker, ${ }^{1}{ }^{*}$ M.E. Baird, ${ }^{1}$ S.K. Kulp, ${ }^{1}$ H. Auer, ${ }^{1}$ K. Kornacker, ${ }^{1}$ D.G. Beer, ${ }^{2}$ C.S. Chen, ${ }^{1}$ L.A. Kresty. ${ }^{1}$ 1. Comprehensive Cancer Center, The Ohio State University, Columbus, $\mathrm{OH}$; 2. The University of Michigan, Ann Arbor, MI.

Introduction: Histone deacetylases (HDACs) modulate nucleosomal packaging of DNA, influencing multiple processes associated with carcinogenesis. We previously reported that the novel HDAC inhibitor HDACi-42 inhibited cell viability and proliferation, induced cell cycle arrest at the $\mathrm{G} 2 / \mathrm{M}$ checkpoint, and increased the percentage of SEG-1 and BIC-1 Barrett's esophageal adenocarcinoma cells undergoing apoptosis. Methods: The present study evaluated a newly developed histone deacetylase inhibitor, S-HDACi42 (the $S$-enantiomer of HDACi-42), in SEG-1 cells. HDAC activity was assessed by fluorimetric assay. Cell viability and proliferation were quantitated using the WST-1 and BrdU colorimetric ELISA kits, respectively. SEG-1 EAC cells were pre-treated with vehicle or S-HDACi-42 $(1.6 \mu \mathrm{M})$, pulsed with vehicle or acidified media ( $\mathrm{pH} 3.5$ ) for 20 minutes, replenished, and harvested 6 hours post acid exposure. RNA was isolated, reverse transcribed, and labeled cRNA was hybridized to the U133 2.0 Plus gene chip (Affymetrix). Genomewide expression was assessed by comparing relative fluorescent intensity values. Using WEDGE analysis, extreme outliers were eliminated. Results: HDAC activity was significantly greater for S-HDACi-42 than for phenylbutyrate, SAHA, or HDACi-42. S-HDACi-42 treatment inhibited SEG-1 viability and proliferation in a dose- and time-dependent manner. Treatment of acid-pulsed SEG-1 cells with S-HDACi-42 was associated with upregulation of multiple genes involved in apoptosis and MAP kinase signaling, such as BIRC3; caspase 10; cyclin A1; cyclin D2; and EGFR. Downregulation of a large number of genes involved in cell cycle progression, including BAX; BCL2; cyclins B1, $\mathrm{B} 2$, and F; cyclin-dependent kinases; and checkpoint genes was observed. Conclusion: S-HDACi-42 is a promising histone deacetylase inhibitor exhibiting increased potency as compared to HDACi-42, and may play a unique role in modulating acid effect on SEG-1 cells. Further research is warranted to assess potential therapeutic or chemopreventive applications.

\section{P150}

Improved Cytotoxicity of Vaccinia Virus in Colon Cancer Cells by Expression of Murine TRAIL and Combination with Oxaliplatin M. Ziauddin,* Z. Guo, P.J. Popovic, M.E. O’Malley, M. Kavanagh, Y. Lee, D.L. Bartlett. Surgery/Surgical Oncology, University of Pittsburgh, Pittsburgh, PA.

Introduction: We have constructed a recombinant vaccinia virus with deletion of the thymidine kinase (TK) gene and insertion of a gene for murine tumor necrosis factor-related apoptosis-inducing ligand (mTRAIL). In this study, we compare the oncolytic activity of a TK-deleted vaccinia virus (VJS6) with that of the vaccinia virus expressing mTRAIL (vvmTRAIL) in colon cancer cell lines. Given that several studies have shown synergy between chemotherapeutic agents and TRAIL, we evaluated the effects of concurrent treatment with oxaliplatin and each of the two viral constructs. Methods: Cultured colon cancer cells DLD1, HCT116, and MC38 were treated with VJS6 and vvmTRAIL, either alone or in combination with oxaliplatin. mTRAIL expression was evaluated by flow cytometry. Viral titers were determined by plaque assay. Cell viability and apoptosis were evaluated with MTS assay and Annexin V conjugated with phycoerythrin, respectively. Results: mTRAIL expression was noted as early as 6 hours after infection with vvmTRAIL at an MOI of 0.25 . The expression of mTRAIL and/or treatment with oxaliplatin reduced the viral titer by 5 8 fold at 36 hours after infection with an MOI of 0.25 . Despite the decrease in viral replication, the expression of mTRAIL increased the killing efficiency of the vaccinia virus. 96 hours after infection of DLD1 cells at an MOI of 0.5 , VJS6 caused less than $10 \%$ decrease in cell viability, whereas vvmTRAIL caused greater than $40 \%$ decrease in cell viability. The effect of the vvmTRAIL was enhanced by oxaliplatin, evidenced by a dose-dependent $46-73 \%$ reduction of the IC50 value of vvmTRAIL. A similar but less dramatic effect was seen in the other cell lines, which were relatively more sensitive to VJS6. Compared to treatment with VJS6, a 1.5- to 3-fold increase in the fraction of cells undergoing apoptosis was seen after a 48 hour treatment with vvmTRAIL. Oxaliplatin potentiated the induction of apoptosis. Conclusions: The cytotoxic effect of vaccinia virus in cultured colon cancer cells can be enhanced by the expression of TRAIL. This tumoricidal effect can be further potentiated by oxaliplatin.

\section{P151}

The Intestine-Specific Receptor Guanylyl Cyclase C (GCC) Inhibits Peritoneal Carcinomatosis A. Berger, ${ }^{1}{ }^{*}$ D. Zuzga, ${ }^{2}$ S.A. Waldman, ${ }^{2}$ G. Pitari. ${ }^{2}$ 1. Surgery, Thomas Jefferson University, Philadelphia, PA; 2. Department of Experimental Therapeutics, Thomas Jefferson University, Philadelphia, $P A$.

Introduction: GCC, the intestine-specific receptor for diarrheagenic bacterial heat-stable enterotoxins (STs), is over-expressed in colorectal cancer. We have previously demonstrated that GCC signaling effectively inhibits cell cycle progression in intestinal epithelial cells and induces colon cancer cytostasis. To investigate the utility of targeting GCC to treat patients with metastatic colon cancer, we examined a murine model of colon cancer peritoneal carcinomatosis. Methods: Human T84 colon carcinoma cells were treated in vitro in serumfree medium for $18 \mathrm{~h}$ with ST $(1 \mu \mathrm{M})$ or vehicle (control). The number of viable cells was quantified by trypan blue exclusion, and $1 \times 107$ viable cells $/ 100 \mu \mathrm{L}$ were injected intraperitoneally (IP) in nude mice to establish carcinomatosis. Human GCC expression was quantified (log GCC copy number) using realtime RT-PCR. Peritoneal carcinomatosis was quantified using the peritoneal carcinomatosis index (PCI). Results: Two weeks after IP injections, right and left mouse hemi-diaphragms were harvested and T84 colonies were quantified by GCC RT-PCR. In vitro ST treatment significantly reduced the ability of T84 cells to establish peritoneal colonies (mean GCC copy number: 0.802 for PBS and 0.211 for ST, $p=0.04$ ). Moreover, at 8 weeks, mice injected with T84 cells treated in vitro with ST exhibited a lower PCI (13.4) compared to those treated with PBS (16.6, $\mathrm{p}=0.06)$. Additionally, $25 \%$ of animals in the ST group had a low PCI (0-10) while only $8 \%$ of those in the control group did. Conclusion: Activation of GCC by ST in vitro inhibits the ability of human colon cancer cells to form peritoneal colonies in vivo, associated with a reduction in peritoneal carcinomatosis in mice. Thus, GCC is a potential target for the treatment of patients with colon cancer metastasis.

\section{P152}

Modeling malignant transformation of cancer cells: the importance of cadherins in a 3D cell culture model of the thyroid tumor microenvironment S.C. Wishnia, ${ }^{*}$ S. Patel, ${ }^{1}$ C. Noack, ${ }^{2}$ N. Bhogal, ${ }^{1}$ C. Klapperich, ${ }^{2}$ J.E. Rosen. ${ }^{1}$ 1. Section of Surgical Oncology, Department of Surgery, Boston University School of Medicine, Boston, MA; 2. Department of Biomedical Engineering, Boston University, Boston, $M A$.

Human tumors depend on their surrounding microenvironment to grow and develop; one of the earliest manifestations of malignant progression is loss of these coordinated cues. The pathophysiologic mechanisms underlying loss of structural organization and dysregulated growth behavior in thyroid cancers are not known; this is partially due to the lack of a suitable, well-characterized in vitro model. The purpose of this study was to construct a three-dimensional (3D) cell culture system to mimic gene expression changes seen in the thyroid tumor microenvironment and characterize the expression of E-cadherin in 3D thyroid cancer cell growth. Four human thyroid neoplasm cell lines (WRO, KAT5, KAK1, TPC) were cultured in three different conditions: on polystyrene (PS) alone, on a 2D collagen type I gel and in a 3D collagen type I gel for 48 hours, during which they were sequentially imaged to access changes in morphology. Trizol extraction of total RNA was performed followed by conversion to cDNA. qRT-PCR for E-cadherin (ECDH), c-kit, AP2-alpha, MMP2, MMP12, and thyroglobulin (TG) was performed using G3PDH as the endogenous control. Normal human thyroid RNA was used as the calibrator sample for relative quantification; gene expression was calculated as fold difference compared to the expression in normal thyroid. In all four cell lines, the expression of TG, MMP2, and MMP12 was significantly decreased by at least $70 \%, 60 \%$, and $90 \%$, respectively, as compared to normal thyroid. Expression of ECDH, ckit, and AP2-alpha was significantly lower in all cells studied when grown in 3D compared to PS. Cells grown in 3D also formed smaller, less cohesive aggregates than those grown on PS. In this study, we non-destructively modeled human thyroid cancer cells grown in 3D and imaged them using optical methods. We demonstrated that 3D growth alters ECDH gene expression as seen in thyroid cancer. This model is important as $3 \mathrm{D}$ growth may influence 
gene expression patterns, alter cadherin-mediated cell-cell adhesion and therefore affect other signaling pathways important in epithelial-to-mesenchymal transition.

\section{P153}

NBL1 AND COLON CANCER: CLINICAL AND GENETIC ASSOCIATIONS Q.P. Ly, ${ }^{1 *}$ E. Siegel, ${ }^{2}$ J. McLoughlin, ${ }^{2}$ J. Lewis, ${ }^{2}$ R. Nair, ${ }^{2}$ E. Jensen, ${ }^{2}$ M. Alvarado, ${ }^{2}$ S. Eschrich, ${ }^{2}$ T. Yeatman, ${ }^{2}$ D. Shibata. $^{2}$ 1. Surgery, University of Nebraska Medical Center, Omaha, NE; 2. H. Lee Moffitt Cancer Center and Research Institute, Tampa, FL.

Introduction: NBL1 is a recently discovered gene that is downregulated in colon cancers (CRC). NBL1 enhances the stimulation of neurite growth in neuroblastoma cells and contributes to cellular differentiation. NBL1's relationship to clinicopathologic (CP) parameters is unknown and knowledge of its associated signaling pathways is limited. We have used a clinicogenomic CRC database to characterize the CP and genetic features associated with NBL1 status. Methods: Between 1993-2003, over 360 patients with various stages of colorectal cancer were consented as part of our tissue banking protocol. Tumors were processed for RNA extraction and prospectively underwent gene profiling using the Affymetrix 133 Plus 2.0 GeneChip. A total of 174 primary colon cancers were identified for this current study. We have previously validated that microarray expression data for NBL1 correlates accurately with RT-PCR expression. NBL1 expression was determined for our population with comparison to a panel of normal mucosae. CP variables were compared between high and low NBL1 expressers using the Fisher Exact Test. Using linear correlation analysis at a threshold of 0.5 , we determined which genes were most frequently co-expressed with NBL1. Results: Our population consisted of 90 men and 84 women with a median age of 68 years. NBL1 expression was determined using 2 probesets and was categorized as high in 45 and low in 129 tumors. Mean NBL1 expression was reduced two-fold in malignant as compared to normal tissue. Between the two probesets, 22 genes were significantly co-expressed with NBL1, of which 6 (GSN, AHNAK, MICAL2, WASF2, CAPZB, FBLIM1) were associated with the control of cytoskeletal components. We were unable to define any differences in $\mathrm{CP}$ variables. Conclusions: We conclude that NBL1 is frequently underexpressed in colon cancer and that many of its co-expressed genes are linked to regulation of the cytoskeleton. These findings add evidence to support NBL1's role in neuronal and cellular differentiation, processes that are associated with alterations in actin and microtubular elements. Loss of NBL1 may contribute to dedifferentiation and tumorigenesis by a mechanism associated with cytoskeletal dysregulation.

\section{P154}

Tumor Lysate Pulsed Dendritic Cells Provide Protective Immunity in a Murine Pancreatic Cancer Model N. Vohra, ${ }^{1}$ S. Pilon-Thomas, ${ }^{2}$ D. Osborne, ${ }^{1}$ A. Rosemurgy, ${ }^{1}$ J. Mulé, ${ }^{2}$ E. Zervos. ${ }^{1}$ 1. University of South Florida, Tampa, FL; 2. H. Lee Moffitt Cancer Center and Research Institute, Tampa, FL.

Background: Dendritic cell (DC) based immunotherapy has shown promise in the treatment of solid tumors by inducing anti-tumor immunity. PANC02 is a murine pancreatic adenocarcinoma (PCA) that is resistant to traditional forms of treatment. The purpose of this study was to establish a model through which immune-based therapies could be evaluated in PCA. Methods: MHC class I molecule $\mathrm{K}^{\mathrm{b}}$ was measured by flow cytometry in PANC02, cultured in vitro \pm IFN- $\gamma$. Growth pattern of PANC02 in C57BL/6 mice was established by s.c. injecting $1-5 \times 10^{5}$ PANC02 cells. Tumors were measured every two days and harvested at $100 \mathrm{~mm} 3$ for flow cytometric analysis of tumor infiltrating lymphocytes (TIL). DC immunotherapy was tested by vaccinating mice three times at seven-day intervals with PANC02 tumor lysate pulsed DC. One week after the final DC vaccine, splenocytes were collected, restimulated in vitro for five days, and used as effector cells in a standard chromium release assay. Alternatively, 8 vaccinated mice were challenged with PANC02 tumor cells. Tumor size was recorded and survival calculated. Results: PANC02 cells demonstrated significant $\mathrm{K}^{\mathrm{b}}$ expression that was upregulated with IFN- $\gamma$. An optimal dose of $3 \times 10^{5}$ cells was determined for growth of PANC02 in C57BL/6 mice. High percentages of CD4+ and CD8+ T cells were measured in the TIL of PANC02 tumors. Potential immune suppressors including $\mathrm{CD} 4+\mathrm{CD} 25+\mathrm{T}$ cells and $\mathrm{CD} 1 \mathrm{lb}+\mathrm{Gr} 1+$ immature myeloid cells were also detected in TIL. Splenocytes isolated from mice vaccinated three times with PANC02 lysate-pulsed DC demonstrated spe- cific PANC02 killing in a cytotoxic assay. Negative control cells, GL26 and Yac-1, were not lysed. Finally, tumor suppression and survival were significantly enhanced in immunized mice as compared to non-immunized controls. Conclusions: Because PANC02 demonstrated significant $\mathrm{K}^{\mathrm{b}}$ expression and had significant infiltration of lymphocytes in vivo it is a suitable model to study immune-based therapies. In addition, suppression of tumor growth in vaccinated mice indicates a tumor specific immune response that could be further exploited to improve current immune-based pancreatic cancer therapy.

\section{P155}

Pro-Inflammatory and Angiogenic Serum and Tumor Profiles in Patients with Malignant Peritoneal Mesothelioma (MPM) K. Gallagher, ${ }^{1}{ }^{*}$ S. Varghese, ${ }^{1}$ J.F. Pingpank, ${ }^{2}$ S.K. Libutti, ${ }^{2}$ H.R. Alexander. ${ }^{1}$ 1. Surgery, University of Maryland, Baltimore, MD; 2. National Cancer Institute, Bethesda, MD.

Introduction: MPM is a rare primary cancer of the serosal membranes for which the prognosis is dismal. Novel approaches for both diagnosis and therapeutic intervention are needed. We analyzed pre-treatment serum and tumor tissue in a cohort of patients with MPM to identify potential angiogenic and inflammatory targets for diagnosis or intervention. Methods: Eleven patients (5 male: 6 female, median age: 46 years) with histologically confirmed MPM were treated with tumor resection and regional hyperthermic intra-operative chemotherapeutic perfusion in accordance with IRB approved protocols. Pretreatment serum was collected and stored at $-80^{\circ} \mathrm{C} ; 6$ of the patients had tumors harvested and snap frozen in liquid nitrogen. Patient serum was analyzed for levels of inflammatory and angiogenic cytokines by multiplex immunoassay and compared to 6 healthy controls. Additionally, mRNA was isolated from tumor specimens, converted to cDNA, hybridized onto $22 \mathrm{~K}$ oligonucleotide microarray chips against human universal RNA, and compared to 4 normal peritoneum samples. Average linkage agglomerative hierarchical clustering within BRB ArrayTools was performed. T-test was used for statistical analyses of cytokine levels. Results: Pre-treatment serum levels of IL-1, IL-1 receptor antagonist (IL-1Ra), IL-6, IL-8, and VEGF were significantly higher in MPM patients compared to normal controls $(\mathrm{p}<0.05)$ (Figure 1). Microarray and unsupervised hierarchical clustering of 2-fold up-or-downregulated genes differentially segregated MPM versus normal peritoneum. IL-1, IL-6, and IL8 were upregulated in most of the peritoneal tumor samples; other IL-1 pathway genes such as IL-1 receptor associated kinase- 1 and the IL-1 homologue, IL-1F7, were also upregulated. Conclusions: Patients with MPM have elevated levels of inflammatory or angiogenic cytokines that may be direct tumor products. These findings indicate that serum cytokine analysis may provide important prognostic biomarker information and that antagonists to these proteins may be useful targets for future therapeutic intervention.

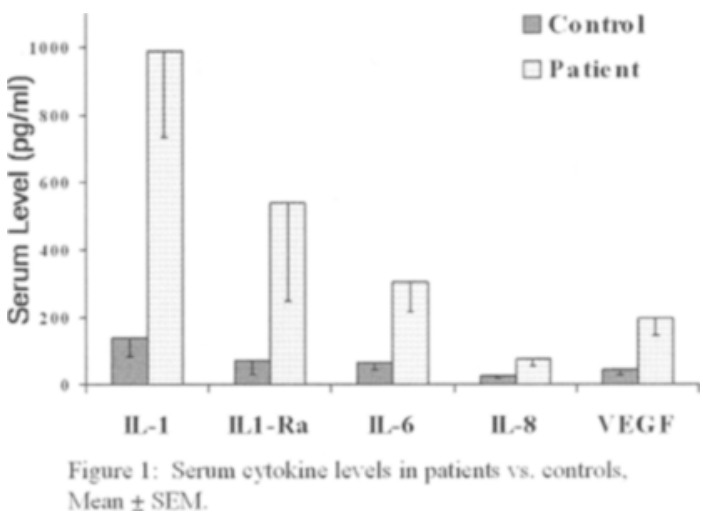




\section{P156}

Insulin-like Growth Factor 2 Expression in Colorectal Cancer K. Idrees, ${ }^{1 *}$ S.H. Park, ${ }^{1}$ J. Shia, ${ }^{1}$ Y.W. Cheng, ${ }^{2}$ R. Shattock, ${ }^{2}$ S. Khan, ${ }^{1}$ Z. Zeng, ${ }^{1}$ D. Notterman, ${ }^{3}$ F. Barany, ${ }^{2}$ P.B. Paty. ${ }^{1}$ 1. Memorial Sloan-Kettering Cancer Center, New York, NY; 2. Weill Medical College of Cornell University, New York, NY; 3. University of Medicine and Dentistry of New Jersey, New Brunswick, NJ.

Introduction Insulin-like growth factor 2 (IGF2) is a fetal growth factor that is expressed in a variety of childhood and adult cancers. Through autocrine activation of its principal receptor (IGF1R), IGF2 plays a vital role in cancer development by suppressing apoptosis and enhancing cancer cell survival. This study evaluates IGF2 expression and signaling in human colorectal cancer (CRC) \& matched normal mucosa. Methods Immunostaining of IGF2 and IGF1R (total \& phosphorylated) was performed using validated assays. The tissue microarrays contained 148 primary colorectal cancers (PCC) with paired normal mucosa, 32 adenomas, 23 liver metastases \& 22 lung metastases. Results IGF2 was overexpressed in $7 \%$ of normal mucosa, $15 \%$ of adenomas, $22 \%$ of PCC, $15 \%$ of liver metastases and $46 \%$ of lung metastases $(p=0.01)$. There were no differences in most clinicopathological features (age, tumor grade, stage \& overall survival) between IGF2(+) and IGF2(-) tumors. However, whereas $22 \%$ of the 115 IGF2(-) PCC showed microsatellite instability (MSI), none of the 33 IGF2+ PCC showed MSI $(p<.01)$. Among 33 PCC found to express IGF2, 9 did have and 24 did not have IGF2 expression in adjacent normal mucosa. When expressed in normal mucosa, IGF2 was seen in all colonocytes and at all levels of the colonic crypts. When expressed in both cancer and normal mucosa, nearly all $(88 \%)$ of the cancers were right sided tumors (proximal to splenic flexure). Conversely, when IGF2 was present in cancer but absent in normal mucosa, nearly all the cancers $(83 \%)$ were left sided $(\mathrm{p}=0.015)$. Furthermore, both IGF1R and phosphorylated IGF1R staining were detected at high intensity in IGF2+ cancers. Conclusions IGF2 is an important oncogene in $20-25 \%$ of colorectal cancers. Induction of IGF2 expression in normal mucosa appears to be a major risk factor for carcinogenesis in the right colon that is independent of the MSI pathway. On the other hand, IGF2 overexpression is acquired in a subset of left sided cancers that lack IGF2 expression in normal mucosa. These findings suggest that IGF2 plays an important role in both cancer susceptibility and cancer progression. Agents targeted to IGF2 hold great promise for the prevention and treatment of CRC.

\section{P157}

\section{Identifying peptide targets and $\mathrm{T}$ cells for CEA-directed} immunotherapy using a transgenic murine model M.E. Brown, H. Miao, J.A. Guevara-Patino, M.D. McKee.* The University of Chicago, Chicago, IL.

Introduction: Carcinoembryonic antigen (CEA) is a shared, non-mutated $\mathrm{T}$ cell target for immunotherapy of many common human adenocarcinomas. High avidity $T$ cells recognizing known peptide epitopes expressed on HLA$\mathrm{A} 2+, \mathrm{CEA}+$ tumors are rare. Altered antigenic peptides have been used clinically and in vitro to induce $T$ cell cross-reactivity against native antigen, thereby circumventing $\mathrm{T}$ cell tolerance. The purpose of this study was to identify novel HLA-A2 restricted CEA peptide epitopes and CEA-specific T cells using a transgenic murine model expressing HLA-A2 and/or CEA. Methods: Using an algorithm based on the HLA-A2 peptide-binding motif, sixteen CEA epitopes including the previously described CAP1 and CEA:691 epitopes were identified at which single amino acid changes resulted in altered peptides predicted to have moderate to high HLA-A2 binding. These peptide / altered peptide pairs were synthesized and used to stimulate T cells from HLA-A2 transgenic mice in vitro. Cells were tested for CEA peptide and tumor recognition by cytokine secretion in co-culture assays. Results: In vitro stimulations were used to identify T cells in the repertoire of HLA-A2 transgenic mice capable of recognizing the CAP1 peptide, CEA:691 peptide, and 5 additional HLA-A2 binding peptides from CEA. Altered CAP1, altered CEA:691, and 4 additional altered peptides successfully stimulated crossreactive $T$ cells that could recognize native peptides from CEA. Recognition of one novel peptide / altered peptide pair was vigorous and consistent over many independent experiments, allowing a $\mathrm{T}$ cell line to be established for further detailed study. Conclusions: The T cell repertoire of HLA-A2 transgenic mice includes cells capable of recognizing peptides expressed by HLAA2 positive human tumors. These T cell populations are suitable for studying the inter-relationships between $\mathrm{T}$ cell tolerance, self-antigen expression, and tumor growth in an HLA-A2, CEA transgenic murine model. Novel CEA peptides that stimulate tumor-reactive $\mathrm{T}$ cell populations or altered peptides capable of circumventing tolerance in CEA+ subjects may be identified using this strategy.

\section{P158}

Monocyte Derived IL-10 Expression Predicts Prognosis of Stage IV Melanoma Patients Receiving Active Specific Immunotherapy R. Essner,* H.T. Itakura, R. Scheri, Y. Huynh, D.L. Morton. Molecular Therapeutics, John Wayne Cancer Institute, Santa Monica, CA.

Introduction: There is no standard method to predict response to immunotherapy for stage IV melanoma. Cell or soluble protein based assays have not been shown to completely correlate with patient outcome. Our previous assessment of peripheral blood mononuclear cells (PBMC) from patients demonstrated that intracellular IL-10 expression might be associated with prognosis. PBMC are a mixture of $\mathrm{CD} 4+, \mathrm{CD} 8+$ cells and monocytes. This study identified the subset of PBMC responsible for IL-10 expression and evaluated the prognostic utility of IL-10 expression in stage IV patients. Methods: PBMC from 87 randomly selected patients with AJCC stage IV melanoma who had received active specific immunotherapy with a whole cell vaccine after complete resection of all clinical metastases were obtained from our database. Samples had been collected serially at 4 week intervals after the first immunization (time 0 ) administration and cryopreserved. Intracellular IL-10 expression was assessed by double staining FACS with antibody against CD 14+ monocytes. Statistical analysis was performed using student's t-test and receiveroperator characteristics (ROC) methods. Results: Predominant IL-10 expression cells in PBMC are from CD14+ monocytes, and 16 weeks after treatment (blood draw 5), IL-10 response was significantly $(\mathrm{p}=0.02)$ higher in poor survival patients than those with good outcome. Earlier time points were not significant. Patients were separated into two groups based upon the CD14+ monocyte IL-10 response: either increasing or decreasing IL-10 expression from first to week 16 blood draws. Patients with increasing IL-10 levels had significantly shorter survival $(28.1 \pm 3.9$ months $)$ than those whom IL-10 level decreased $(62.2 \pm 4.0$ months $)$ at week $16(\mathrm{p}<0.0001)$. Multivariate analysis demonstrated that changes in IL-10 levels significantly correlated with survival $(p<0.0001)$. Age, gender, M-stage, disease free interval (Stage I/II to IV) were not predictive. Conclusion: We conclude that $\mathrm{CD} 14+$ monocytes are the dominant cellular source of IL-10 among PBMC and that changes in IL-10 expression may serve as an immunologic surrogate for outcome.

\section{P159}

Axillary Lymph Node Dissection Is Not Required for Regional Control in Patients With Breast Cancer Who Have Micrometastasis in a Sentinel Node A. Kaminski, ${ }^{*}$ J.M. Guenther, L.A. DiFronzo. Surgery, Kaiser Permanente Los Angeles Medical Center, Los Angeles, CA.

Introduction: Axillary lymph node dissection (ALND) for patients with breast cancer who have a positive sentinel lymph node (SLN) provides useful prognostic information and remains the standard of care. However, the utility of a full ALND for regional control in patients with a positive SLN is unclear. Methods: A retrospective study was performed on breast cancer patients who underwent breast conserving surgery with SLN biopsy and opted to forgo ALND after metastasis in the SLN was identified. Data from 1997-2005 were reviewed. Results: Fifty patients with a positive SLN who underwent axillary observation were identified. Three patients were lost to follow-up and excluded from analysis. The mean patient age was 57 years (range $29-83$ years). The mean tumor size was $1.9 \mathrm{~cm}$ (range $0.4-5 \mathrm{~cm}$ ). The majority of patients had a grade II carcinoma; $91 \%$ of patients had tumors which were estrogen receptor positive, The mean number of sentinel nodes removed was 3 (median 2, range $1-9$ ). The mean number of positive nodes was 1.3 (median 1 , range $1-2$ ). $72 \%$ of patients had a single positive SLN. 14 patients $(30 \%)$ had macrometastatic disease $(>2 \mathrm{~mm})$, and 33 patients $(70 \%)$ had micrometastases. 17 of the patients with micrometastases had cellular metastasis only detected by immunohistological stains. All patients underwent whole breast irradiation, and 43 patients $(92 \%)$ received systemic therapy. The mean duration of follow-up was 54 
months (median 50, range 6-113). One patient (2.1\%) developed an axillary recurrence. This patient had a $4 \mathrm{~mm}$ metastasis in the SLN. ALND was performed 28 months after the initial operation, and 13 of 21 nodes were involved by tumor. No other patients had evidence of axillary recurrence or distant metastasis at time of last follow-up. Conclusions: The overall axillary recurrence rate is low after SLN biopsy demonstrates metastasis in a sentinel node and the axilla is observed. Patients with macrometastasis in a sentinel node should have a completion ALND. However ALND is not necessary for regional control in patients with micrometastatic disease.

\section{P160}

Primary Tumor Characteristics and Lymph Node Status Fail to Predict Presence of Circulating Tumor Cells in Patients with Operable Breast Cancer J.E. Lang, ${ }^{1 *}$ K. Mosalpuria, ${ }^{1}$ M. Cristofanilli, ${ }^{2}$ S. Krishnamurthy, ${ }^{3}$ J. Rubin, ${ }^{3}$ B. Singh, ${ }^{1}$ I. Bedrosian, ${ }^{1}$ F. Meric-Bernstam, ${ }^{1}$ A. Lucci. ${ }^{1}$ 1. MD Anderson Cancer Center Division of Surgical Oncology, Houston, TX; 2. MD Anderson Cancer Center Division of Medical Oncology, Houston, TX; 3. MD Anderson Cancer Center Division of Pathology, Houston, TX.

Introduction: Circulating tumor cells (CTCs) detected with the CellSearch system correlate with worse prognosis in patients with metastatic breast cancer. We hypothesized that primary tumor characteristics would predict the likelihood of identifying CTCs in patients with operable breast cancer. Methods: Clinical and pathological data from 62 patients with operable breast cancer were collected. The CellSearch system (Veridex Corporation, Warren NJ) was used to detect CTCs in whole peripheral blood. CTCs were defined as nucleated cells lacking CD45 but expressing cytokeratins 8,18 , or 19 . The presence of 1 or more epithelial cells per $10 \mathrm{~mL}$ of blood was considered a positive result. Primary tumor characteristics included: tumor size, ER, PR, HER2, tumor grade, histologic type, lymphovascular invasion (LVI) and Ki-67. Presence of lymph node metastases was also compared to presence of CTCs. Univariate analyses using the Chi-squared test of association or the Fisher's exact probability test were performed. Results: Thirty-four percent of patients $(21 / 62)$ had evidence of CTCs. Tumor and nodal staging for the 21 patients with CTCs is shown in the table. Median number of CTCs present was 1.17 CTCs per patient (range $0-18)$. Overall, 14/62 (22.6\%) patients received neoadjuvant chemotherapy. Of the patients with CTCs, $6 / 21(28.6 \%)$ had neoadjuvant chemotherapy. No primary tumor characteristic reliably predicted the presence of CTCs. No significant association was found between presence of CTCs and primary tumor size $(\mathrm{p}=0.589), \mathrm{ER}(\mathrm{p}=0.63), \mathrm{PR}(\mathrm{p}=0.53)$, HER2 $(\mathrm{p}=0.067)$, tumor grade $(p=0.30)$, histologic type $(p=0.63)$, sentinel node status $(p=0.82)$, greater than or equal to 4 positive lymph nodes $(\mathrm{p}=0.15), \mathrm{LVI}(\mathrm{p}=0.46)$ or $\mathrm{Ki}-$ $67(p=0.53)$. Conclusion: Although CTCs are found in $34 \%$ of early stage breast cancer, no single clinical or pathologic feature correlated with their presence. Long-term follow-up will be required to demonstrate the prognostic significance of CTCs in operable breast cancer.

\begin{tabular}{|cc|}
\hline Tumor Stage & $\#$ \\
T1 & 5 \\
T2 & 12 \\
T3 & 3 \\
Nodal Stage & $\#$ \\
N0 & 13 \\
N1 & 6 \\
N2 & 1 \\
N3 & 1 \\
\hline
\end{tabular}

Primary Tumor and Lymph Node Staging Distribution for 21 patients found to have CTCs in their peripheral blood by the CellSearch Assay.

\section{P161}

Body image in breast cancer patients: significantly influenced by personality and adjuvant treatment, not by surgical treatment A.F. Van der Steeg, ${ }^{1 *}$ A. Bordat, ${ }^{2}$ J.A. Roukema, ${ }^{1}$ F.W. Van der Ent ${ }^{3}$ J. De Vries. ${ }^{2}$ 1. Surgery, St Elisabeth Hospital, Tilburg, Netherlands, 2. Tilburg University, Tilburg, Netherlands; 3. Maasland Hospital, Sittard, Netherlands.

Introduction: This study was conducted to examine if satisfaction with body image changes over time and to assess which variables predict scores on body image in breast cancer patients. The effects of personality, demographic factors, and type of surgery were examined over a one-year period. Patients and methods: Women with a palpable lump in their breast or an abnormality on a screening mammogram were asked to participate prior to diagnosis was known. Seventy-three patients with early stage breast cancer were included in the study. They completed the quality of life questionnaire WHOQOL-100 prior to diagnosis and $1,3,6$, and 12 months after primary surgery. Before diagnosis the NEO-FFI personality assessment questionnaire was completed. Results: No differences in scores on body image were found between women who were treated with breast conserving therapy (BCT) or modified radical mastectomy (MRM). Scores on body image did decline significantly between measurement moment 1 (before diagnosis and treatment) and $2(1 \mathrm{~m}$ after surgery) $(\mathrm{p}<0.001$ for the MRM group and $\mathrm{p}<0.05$ for the BCT group). Body image did improve over time, but this was not significant (see figure 1). Compared with younger women, women older than 60 years of age were more satisfied with their appearance 3 months after surgery $(p<0.005)$. Single women who underwent MRM scored higher on body image compared to women with a partner 6 months after surgery $(p<0.001)$. Factors predicting scores on body image at all follow-up measurements were age $(p<0.05)$, chemotherapy $(p<0.0013 \mathrm{~m}$ after treatment and $p<0.056$ and $12 \mathrm{~m}$ after surgery), and neuroticism ( $\mathrm{p}=0.011 \mathrm{~m}$ after surgery, $p<0.05$ after $3 \mathrm{~m}$ and $p<0.0016$ and $12 \mathrm{~m}$ after surgical treatment). Conclusions: In addition to age and chemotherapy, the personality trait neuroticism plays a role in maintaining an impaired body image after breast cancer surgery. This influence is irrespective of the type of surgical treatment. During the year following surgical treatment little, though not significant, improvement was seen in body image scores for both the BCT and MRM patients.

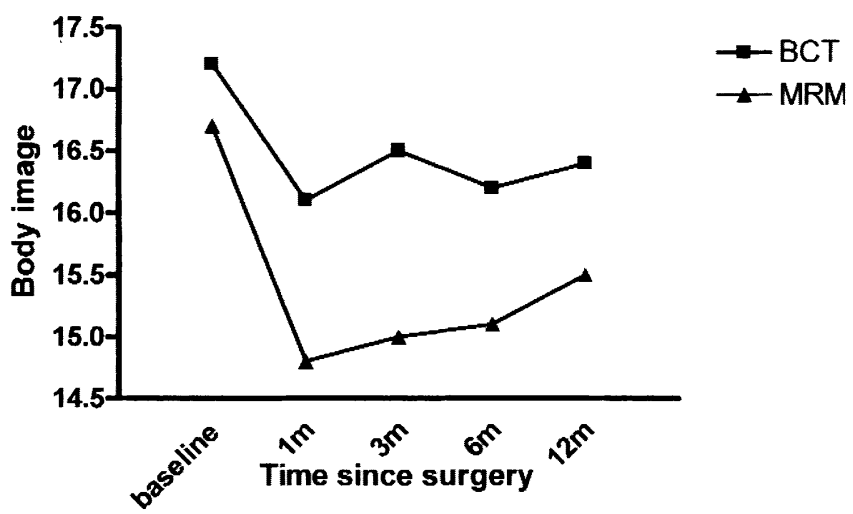

Figure 1: changes in scores on body image over time.

\section{P162}

The influence of personality on perceived social support: a prospective longitudinal study in breast cancer patients A.F. Van der Steeg, ${ }^{1}$ * K. Van der Heijden, ${ }^{2}$ J.A. Roukema, ${ }^{1}$ F.W. Van der Ent, ${ }^{3}$ J. De Vries. ${ }^{2}$ 1. Surgery, St Elisabeth Hospital, Tilburg, Netherlands; 2. Tilburg Unversity, Tilburg, Netherlands; 3. Maasland Hospital, Sittard, Netherlands.

Background: Social support is perceived as very important when coping with breast cancer and its treatment. This study was aimed at examining the relationship between personality and perceived social support in breast cancer patients, using a prospective longitudinal design. Patients and methods: Women with a palpable lump in their breast or an abnormality on a screening mammogram were included in the study and completed a set of questionnaires before diagnosis was known. Perceived social support was assessed with the World Health Organization Quality of Life Questionnaire- 
100 (WHOQOL-100). The personality characteristics extraversion, neuroticism, openness, agreeableness, and conscientiousness were assessed using the Neuroticism-Extraversion-Openness Five Factor Inventory (NEO-FFI) and trait anxiety was measured with the State-and-Trait-Anxiety Inventory (STAI). All three questionnaires were completed before diagnosis (i.e. breast cancer or benign breast disease) was known. In addition, 12 months after diagnosis and treatment the WHOQOL-100 was completed again. One-hundred and twenty-nine women were included in the study. Having children and / or a partner are (possible) confounding factors and, thus, were entered in the analyses as covariates. Multiple regression analyses were used to study the relation between personality and social support. Results: Before diagnosis, $24.1 \%$ of the variance of social support $(p<0.001)$ was explained by the personality characteristics extraversion $(\beta=-0.220)$ and agreeableness $(\beta=0.496)$, and having children $(\beta=0.219)$. One year after surgery agreeableness $(\beta=0.293)$ and trait anxiety $(\beta=-0.428)$ predicted social support $(\mathrm{p}<0.001$; total adjusted R2 $=32.7 \%)$. Conclusions: For breast cancer patients agreeableness was the most important predictor of perceived social support before treatment. A high score on agreeableness lead to more perceived social support. One year after surgery agreeableness was still important, however, trait anxiety was now the most important predictor. Patients who scored high on trait anxiety one year after diagnosis and treatment of breast cancer perceived less social support.

\section{P163}

Breast reconstruction in the setting of radiation- Determining the ideal timing and technique T. Samson, ${ }^{1 *}$ B.A. Pockaj, ${ }^{1}$ R.J. Gray, ${ }^{1}$ M.Y. Halyard, ${ }^{2}$ S.A. Vora, ${ }^{2}$ W.W. Wong, ${ }^{2}$ P.B. Garvey, ${ }^{1}$ W.J. Casey, A.M. Rebecca. ${ }^{3}$ 1. Mayo Clinic Arizona, Department of Surgery, Scottsdale, AZ; 2. Mayo Clinic Arizona, Department of Radiation Oncology, Scottsdale, AZ; 3. Mayo Clinic Arizona, Division of Plastic Surgery, Scottsdale, $A Z$

Introduction- The use of radiation therapy in the setting of breast cancer has increased. Controversy exists regarding the effects of prior or planned radiation on the reconstructed breast. We sought to identify the best type and timing of reconstruction. Methods- We performed a retrospective review of all women who underwent breast reconstruction between 1995 and 2006 at our institution. Comparisons were made between groups undergoing autologous or implant reconstruction that received radiation before (PreRT), after (PostRT), or did not receive any radiation (NoRT). Results- Four hundred eighteen patients underwent reconstruction, including 129 patients $(37 \%)$ who also received radiation therapy. In the radiation group, sixty-seven (52\%) patients underwent preoperative radiation therapy PreRT while $62(48 \%)$ patients recieved postoperative radiation therapy PostRT. Among those in the PostRT group, radiation therapy was planned at the time of reconstruction in thirty-one $(50 \%)$, with the remaining undergoing unplanned radiation therapy postoperatively. The type of reconstruction within the PostRT group was, 15 (24\%) implant-based, $22(35 \%)$ pedicled flap, and $25(40 \%)$ free-flap reconstruction. The highest rate of complications for the PostRT patients was among the implant group and the lowest rate of complications was seen in the free-flap group (Table 1). In the PreRT reconstruction patients the highest rate of complications was found in the implant reconstruction patients while the lowest rate of complications was seen in the free-flap patients. Conclusion- Autologous reconstruction, specifically free-flap reconstruction, has superior results when compared to implant reconstruction in the radiated field. Implant reconstruction in the radiated field should be avoided secondary to high rates of flap failure, capsular contracture and infection.
Breast Reconstruction Complications

\begin{tabular}{|c|c|c|c|c|c|c|}
\hline & $\begin{array}{c}\text { Flap/Implant } \\
\text { Failure (\%) }\end{array}$ & $\begin{array}{c}\text { Capsular } \\
\text { Contracture } \\
(\%)\end{array}$ & $\begin{array}{c}\text { Flap Fat } \\
\text { Necrosis } \\
(\%)\end{array}$ & $\begin{array}{c}\text { Infection } \\
(\%)\end{array}$ & $\begin{array}{c}\text { Skin } \\
\text { Dehiscence / } \\
\text { Necrosis (\%) }\end{array}$ & $\begin{array}{c}\text { Seroma / } \\
\text { Hematoma } \\
(\%)\end{array}$ \\
\hline $\begin{array}{c}\text { Implant } \\
\text { NoRT } \\
\text { (N=113) }\end{array}$ & 25 & 20 & N/A & 14 & 6 & 14 \\
\hline $\begin{array}{c}\text { Pedicled } \\
\text { Flap NoRT } \\
\text { (N=61) }\end{array}$ & 3 & N/A & 59 & 16 & 13 & 13 \\
\hline $\begin{array}{c}\text { Free-Flap } \\
\text { NoRT } \\
\text { (N=115) }\end{array}$ & 3 & N/A & 17 & 10 & 13 & 7 \\
\hline $\begin{array}{c}\text { Implant } \\
\text { PreRT } \\
\text { (N=5) }\end{array}$ & 20 & 40 & N/A & 20 & 20 & 0 \\
\hline $\begin{array}{c}\text { Pedicle Flap } \\
\text { PreRT } \\
\text { (N=18) }\end{array}$ & 28 & N/A & 56 & 17 & 5 & 11 \\
\hline $\begin{array}{c}\text { Free Flap } \\
\text { PreRT } \\
\text { (N=44) }\end{array}$ & 5 & N/A & 14 & 9 & 16 & 5 \\
\hline $\begin{array}{c}\text { Implant } \\
\text { PostRT } \\
\text { (N=15) }\end{array}$ & 7 & 40 & N/A & 27 & 20 & 20 \\
\hline $\begin{array}{c}\text { Pedicle Flap } \\
\text { PostRT } \\
\text { (N=22) }\end{array}$ & 5 & N/A & 46 & 14 & 18 & 14 \\
\hline $\begin{array}{c}\text { Free Flap } \\
\text { PostRT } \\
\text { (N=25) }\end{array}$ & 8 & N/A & 28 & 4 & 36 & 32 \\
\hline
\end{tabular}

\section{P164}

Fas- and Fas-ligand-positive breast cancers spread to the bones more frequently than negative ones do M. Bebenek, ${ }^{1}$ * D. Dus, ${ }^{2}$ J. Kozlak. ${ }^{2}$ 1. Surgical Oncology, Lower Silesian Center of Oncology, Wroclaw, Poland; 2. Institute of Immunology and Experimental Therapy, Polish Academy of Sciences, Wroclaw, Poland.

Introduction Bones belong to the most frequent localizations of breast cancer spread. Several authors have revealed that the Fas/Fas-ligand status may have a significant impact on survival in breast cancer women. The purpose of our study was to determine if these molecules might predict skeletal dissemination in radically treated breast cancer patients. Methods In this prospective study tumor samples from 147 radically-treated breast carcinoma patients were evaluated immunohistochemically for Fas/Fas-ligand expressions. The associations between the expressions of molecules studied and the skeletal spread of cancer were tested statistically. Results Both Fas $(\mathrm{p}=0.017)$ and Fas-ligand $(p=0.028)$ expressions in the primary tumor were considerably less frequent among breast cancer patients with bone metastases compared to women without skeletal spread. Moreover, either negative staining for Fas $(\mathrm{p}=0.005)$ or the lack of Fas-ligand expression $(\mathrm{p}=0.048)$ were proved to be significant predictors for bone metastases using univariate analysis. Conclusions The probability of bone metastases may be assessed on the basis of Fas/Fas-ligand expressions in primary breast cancer. Consequently, the determination of these molecules seems crucial for further prognosis and adjuvant treatment.

\section{P165}

Breast Cancer Presentation and Treatment Variation by Insurance Status N.G. Coburn, ${ }^{1}{ }^{*}$ E. Clarke-Pearson, ${ }^{2}$ J. Fulton, ${ }^{3}$ B. Cady. ${ }^{2}$ 1. Division of Surgical Oncology, Sunnybrook Health Sciences Centre, University of Toronto, Toronto, ON, Canada; 2. Rhode Island Hospital, Brown University Medical School, Providence, RI; 3. Rhode Island Department of Health, Providence, RI.

Background: Few studies have examined the relationship of insurance status to the presentation and treatment of cancer. Since 1995, there has been a state- and Centers for Disease Control-funded program to provide screening mammography and treatment to women without adequate insurance. Methods: Using a state cancer registry, the tumor presentation, treatments, and insurance status at the time of diagnosis were examined. Student's t-test and Chi2 tests were used for comparison. P-values reflect a comparison to insured patients. 
Results: From 1998 to 2004, there were 5142 cases of invasive breast cancer with either private insurance $(n=3037)$, uninsured $(n=60)$, Medicare $(n=1908)$, or Medicaid $(\mathrm{n}=137)$. The median age ( $\mathrm{yrs})$ at presentation was 55 for private insurance, 52 for uninsured, 76 for Medicare and 56 for Medicaid. The mean and median size $(\mathrm{mm})$ at presentation were 18.5 and 15 for private insurance; 30 and 18.5 for uninsured; 20.9 and 16 for Medicare; and 24.6 and 18 for Medicaid ( $\mathrm{p}<0.001$ for all). Fewer women with Medicare and Medicaid presented with node negative cancer: private insurance, $73.7 \%$; uninsured, $62.8 \%$ $(p=0.079)$; Medicare, $80 \%(p<0.001)$; and Medicaid, $57.9 \%(p=0.002)$. Significantly more women with no insurance had no surgical treatment: $15 \%$ versus $3.9 \%$ for private insurance $(\mathrm{p}<0.001)$. Of women with non-metastatic, $\mathrm{T} 1$ or T2 tumors, $72 \%$ of women with private insurance underwent breast conserving treatment $(\mathrm{BCT})$, while only $58 \%$ of uninsured $(\mathrm{p}=0.048), 66 \%$ of Medicare $(\mathrm{p}<0.001)$ and $65 \%$ of Medicaid $(\mathrm{p}=0.121)$ had BCT. Of women who had a mastectomy, the rate of reconstruction was highest in women with insurance $(16 \%)$, compared to women with no insurance $(4.5 \%, \mathrm{p}=0.145)$, Medicare $(1.6 \%, p<0.0001)$, and Medicaid $(7.7 \%, p=0.109)$. Conclusions: In women with private insurance, breast cancers are diagnosed at smaller size and earlier stage. Furthermore, there are troubling trends toward lower rates of BCT and reconstruction following mastectomy in the uninsured and Medicaid patients. Although there is a mechanism in our state to provide screening and treatment to women who are under-insured, clearly, further improvements need to be made.

\section{P166}

Black Race Is Independently Associated With Worse Outcome After Treatment For Breast Cancer S.H. Kim, ${ }^{1}$ * J.M. Ferrante, ${ }^{2}$

M. Hameed, ${ }^{2}$ B.R. Won. ${ }^{2}$ 1. Geisinger Clinic and New Jersey Medical School/UMDNJ, Wilkes-Barre, PA; 2. New Jersey Medical

School/UMDNJ, Newark, NJ.

INTRODUCTION: Recent data suggests that Black women have worse outcome after diagnosis and treatment of breast cancer. It is still unclear if this is because Black race is more often associated with known negative prognostic indicators or if it is an independent poor prognostic factor. To study this, we performed an analysis of patients from an urban university medical center where these women made up the majority of the patient population. METHODS: The results were obtained via retrospective cohort analysis of a prospectively collected database of breast cancer patients seen from October 1998 through May 2006. Time to recurrence and survival was analyzed using the Kaplan-Meier method, with statistical analysis by chi-square, log rank testing, and the Cox regression model. RESULTS: 265 female patients were diagnosed with breast cancer during the time period. We excluded patients with pure DCIS $(\mathrm{N}=50)$, leaving 215 patients with invasive disease for analysis. Of these, 124 were Black, 67 Hispanic, 21 Caucasian, 2 Arabic, and 1 AsianIndian. Age $<45$ years, insurance status, mammographic vs. clinical discovery, tumor grade, hormone receptor status, and Her2 positivity were not significantly associated with poor outcome. Univariate predictors of poor disease-free and overall survival included primary tumor size, node-positivity, and Black race (Figures 1 and 2). Multivariate analysis of these factors demonstrated that all three were independently associated with poor outcome. CONCLUSIONS: Black women appear to have worse outcome after breast cancer treatment that is independent of the usual predictors of survival. Further investigation of socio-cultural and biologic factors is necessary to identify the cause of this survival disparity.

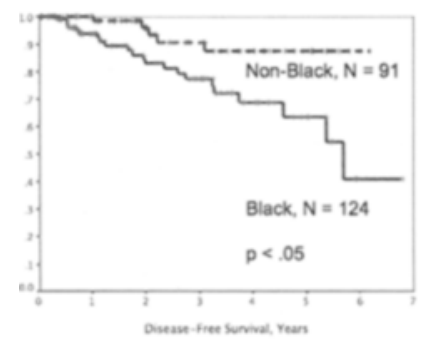

Figure 1

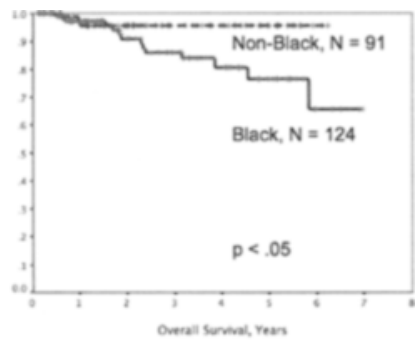

Figure 2

\section{P167}

Second Primary Male Breast Cancer - A SEER Database Review J.A. Wernberg, * S. Agrawal, A.N. Meguerditchian, J. Yap, S. Kulkarni. Roswell Park Cancer Institute, Buffalo, NY.

INTRODUCTION: Male breast cancer (MBC) is uncommon with second primary MBC being even rarer. The objective of this study is to analyze the characteristics of the MBC population diagnosed with a second breast cancer. METHODS: Using the Surveillance, Epidemiology, and End Results (SEER) database, 3077 patients diagnosed with invasive or in situ breast cancer between 1973-2002 were identified and records of patients with a second breast cancer were reviewed. Additional non-breast primary cancer diagnoses were also recorded. RESULTS: A review of 3139 records corresponding to 3077 patients revealed 2843 invasive and 296 in situ breast cancer events. Of the 3077 patients, 59 were identified with a second breast cancer. Of these 59 patients, 58 were diagnosed with a contralateral invasive or in situ breast cancer and the remaining patient with an ipsilateral in situ breast cancer. Synchronous events were noted in $27(47 \%)$ patients with the remaining $32(53 \%)$ patients being diagnosed more than six months after the initial diagnosis. The most common pathologic subtype was invasive ductal carcinoma with the majority of patients being treated with mastectomy. Of the 3139 records of patients with MBC, 835 (26.6\%) records revealed a diagnosis of an additional non-breast cancer. CONCLUSIONS: Male breast cancer is uncommon and these patients are at risk of a contralateral breast cancer and second primary non-breast cancers. This suggests that patients with MBC may benefit from continued surveillance for breast cancer and from appropriate screening for non-breast cancers.

\section{P168}

Risk for Residual Cancer After a Margin-Positive Biopsy

N.T. Holm, * K. Byrnes, J. Luke, Q.D. Chu, B.D. Li. Surgery, LSUHSCShreveport, Shreveport, LA.

Introduction: Tumor-free margin in breast cancer surgery is the standard. Yet a large percentage of specimens on re-excision fail to show residual cancer. In this study, we attempt to identify characteristics that may predict residual cancer on re-excision. Methods: 1,201 patients treated for invasive breast cancer (IDCA) were studied. Tumor-positive biopsies were divided into: 1) Focally positive IDCA, 2) DCIS at the margin, and 3) Extensive disease. Based on the histologic findings, re-excisionsubgroups were: 1) Negative for residual IDCA and/or DCIS, 2) Residual DCIS, and 3) Residual IDCA. Tumor grade, tumor size, nodal status, ER, PR, and HER2 status were collected and evaluated. Primary endpoints measured were residual disease and disease-free survival (DFS). Statistical analyses include Chi square, multivariate, Kaplan-Meier survival analysis and the log rank test. Results: In 1,201 IDCA patients, 121 $(10 \%)$ of patients had a tumor-positive margin on initial surgery. $40(33 \%)$ had focally positive IDCA margins, $28(23 \%)$ had DCIS, and $53(44 \%)$ had extensive disease at the margins. 58 patients $(48 \%)$ had no residual disease on re-excision or mastectomy. Of patients with residual cancer, $16(25 \%)$ had residual DCIS and $47(75 \%)$ had residual IDCA. Extensive IDCA at the margin and node-positive disease predicted residual cancer on re-excision $(\mathrm{p}<0.001$, $\mathrm{p}=0.02$, multivariate analysis). Tumor grade, ER, PR, and HER-2 status were not predictive for residual cancer. Patients with tumor-positive margins in the initial biopsy had a worse DFS $(\mathrm{p}=0.008, \log$-rank test) than patients with tumornegative margins. Patients with a T1 lesion $(<2 \mathrm{~cm})$ with focally positive margins did not have residual IDCA on re-excision $(\mathrm{N}=23$ patients, $\mathrm{p}<0.001$, Chisquare). Conclusion: Based on this review, there is at least a $50 \%$ risk for residual cancer on re-excision of a tumor-positive initial breast biopsy. Extensive disease at the margins and node-positive disease are associated with a higher risk for residual cancer. T1 specimens with focally positive margin may represent a low risk group. Avoiding a tumor-positive margin at initial biopsy is associated with a better DFS and should remain the standard of care.

\section{P169}

Sentinel Lymph Node Biopsy with Frozen Section Facilitates OneStage Procedure for Patients Undergoing Mastectomy with Immediate Breast Reconstruction R.O. Craft,* B.A. Pockaj, R.J. Gray, W.J. Casey, A.M. Rebecca. Mayo Clinic Scottsdale, Scottsdale, AZ, AZ.

Background: The purpose of this study was to determine the effect of concurrent sentinel lymph node (SLN) biopsy on the reconstruction outcome for patients undergoing mastectomy with immediate breast reconstruction. Methods: A retrospective review of all patients undergoing immediate reconstruc- 
tion between 1996 and 2006. Autologous tissue reconstructions performed included pedicled TRAM prior to 2001 and DIEP flaps after 2001. The microvascular reconstruction was performed to the internal mammary artery in all cases. Patients without axillary staging were excluded. Results: 266 patients underwent mastectomy with immediate reconstruction with axillary staging. 69 patients had reconstruction with an implant and 197 with autologous tissue. Mean follow-up was 26 months. One hundred fifty-six patients (59\%) underwent SLN biopsy and $110(41 \%)$ complete axillary lymph node dissection (CALND) only. Thirty-eight (24\%) patients had SLN metastases, of which 32 $(84 \%)$ were detected on intraoperative frozen section analysis. A comparison of the reconstructive outcomes among those undergoing SLN biopsy only (Group 1), SLN biopsy + immediate CALND (Group 2), SLN biopsy + delayed CALND (Group 3), and CALND only (Group 4) is presented in Table 1. Group 4 demonstrated a significant increase in fat necrosis of autologous reconstructions $(\mathrm{p}<.001)$ compared to the other groups. There were no other differences in reconstruction outcomes among the 4 groups (Pearson's Chi-Squared Test, $p>0.05$ ). Conclusions: SLN biopsy performed at the time of mastectomy with immediate reconstruction does not negatively impact the reconstruction outcome. Further, if a SLN metastasis is identified, neither immediate nor delayed CALND negatively impacts patient reconstructive outcomes.

\section{Table 1}

\begin{tabular}{|c|c|c|c|c|}
\hline & $\begin{array}{c}\text { Group 1: SLN } \\
\text { only (118 pts, } \\
122 \text { SLN } \\
\text { biopsies) }\end{array}$ & $\begin{array}{c}\text { Group 2: SLN + } \\
\text { CALND same } \\
\text { day (32 pts, 32 } \\
\text { SLN biopsies) }\end{array}$ & $\begin{array}{c}\text { Group 3: SLN + } \\
\text { delayed CALND } \\
\text { (6 patients, 6 SLN } \\
\text { biopsies) }\end{array}$ & $\begin{array}{c}\text { Group 4: } \\
\text { CALND only } \\
\text { (110 patients, } \\
110 \text { CLND) }\end{array}$ \\
\hline $\begin{array}{c}\text { Reoperation for comp } \\
<30 \text { days }\end{array}$ & $16(13.5 \%)$ & $8(25 \%)$ & $1(16.6 \%)$ & $11(10 \%)$ \\
\hline Total Days in hospital & Mean=3 & Mean=3.4 & Mean=5.2 & Mean=3.5 \\
\hline $\begin{array}{c}\text { Flap: total necrosis } \\
\text { (autologous only) }\end{array}$ & $\begin{array}{c}4(5.3 \%) \\
(\mathrm{n}=74)\end{array}$ & $2(9.5 \%)(\mathrm{n}=21)$ & $0(\mathrm{n}=2)$ & $\begin{array}{c}8(8.1 \%) \\
(\mathrm{n}=98)\end{array}$ \\
\hline $\begin{array}{c}\text { Flap: Fat necrosis } \\
\text { (autologous only) }\end{array}$ & $\begin{array}{c}13(17.8 \%) \\
(\mathrm{n}=74)\end{array}$ & $6(28.6 \%)(\mathrm{n}=21)$ & $2(100 \%)(\mathrm{n}=2)$ & $\begin{array}{c}50(51 \%) \\
(\mathrm{n}=98)\end{array}$ \\
\hline $\begin{array}{c}\text { Capsular Contracture } \\
\text { (implant only) }\end{array}$ & $\begin{array}{c}13(31 \%) \\
(\mathrm{n}=44)\end{array}$ & $4(36.4 \%)(\mathrm{n}=11)$ & 0 & 0 \\
\hline $\begin{array}{c}\text { Implant failure (implant } \\
\text { only) }\end{array}$ & $5(11.9 \%)$ & $4(36.4 \%)(\mathrm{n}=11)$ & 0 & 0 \\
\hline Flap: infection & $21(17.7 \%)$ & $7(21.8 \%)$ & $3(50 \%)$ & $14(12.7 \%)$ \\
\hline Flap: seroma/hematoma & $18(15.2 \%)$ & $9(28.1 \%)$ & $1(16.6 \%)$ & $13(11.8 \%)$ \\
\hline $\begin{array}{c}\text { Native Flap } \\
\text { Complications (skin } \\
\text { dehiscence or necrosis) }\end{array}$ & $40(33.8 \%)$ & $16(50 \%)$ & $3(50 \%)$ & $38(34.5 \%)$ \\
\hline
\end{tabular}

\section{P170}

MRI Screening in Patients with a High-Risk Family History

T.A. King, * A. Park, J. Yu, P.I. Borgen. Surgery, Memorial Sloan-Kettering Cancer Center, New York, NY.

Screening MRI is increasingly being used in high-risk patients; however, in the absence of a known BRCA mutation, guidelines for MRI screening are poorly defined. We describe our experience with MRI screening in women with a family history of breast cancer. The high-risk screening program prospective database was reviewed from $4 / 99$, when we began using screening MRI, to $7 / 06$. Patients with a high-risk family history and $\geq 1$ yr followup were identified. All patients were offered biannual clinical breast exam and annual MMG (age appropriate). Screening MRI was performed at the discretion of physician and patient. Family history profiles revealed 1019 eligible patients; stratified into 4 risk groups (table). Median follow-up (from 4/99) for all patients was 5.0 yrs (range 1-7.3yrs). Screening MRI was performed in $374 / 1019$ (37\%) patients. Patients with family history in $\geq 21$ st degree relatives were more likely to have MRI. A total of 976 MRIs were performed in 374 patients (mean 2.6 MRI, range 1-10). Among $374 \mathrm{MRI}$-screened patients, $151(40 \%)$ underwent 263 biopsies; 74/263(28\%) were based on MRI findings alone. Cancer was detected in 7/74(9\%) MRI generated biopsies and 7/976(0.7\%) MRIs performed. All 7 patients had neg MMG; median 114 days prior to MRI. One additional patient was diagnosed with Paget's, 32 mos. after neg MRI; and 1 cancer was identified on MMG, 5 mos. after neg MRI. Overall cancer was detected in 9/374(2\%) MRI-screened patients; MRI detected
7/9(78\%) cancers. Among 645 non-MRI screened patients, $188(29 \%)$ underwent 260 biopsies during the same period. Cancer was detected in 20/260(8\%) biopsies and 20/645(3\%) patients. MRI-only cancers were Stage $0-\mathrm{I}$ in 6/7(86\%) cases. Non-MRI cancers were Stage 0-I in 16/22(73\%) cases. In this series, $2 / 29(7 \%)$ cancers were in BRCA-mutation carriers. The incidence of screen-detected breast cancer in this cohort was $3 \%$. MRI detected occult cancer in $7 / 74(9 \%)$ biopsies and in $2 \%$ of all MRI screened patients. Despite a low overall positive biopsy rate, MRI facilitated earlier diagnosis and provided the most benefit to those at highest risk. More work is needed to develop guidelines for MRI screening in defined subgroups with high-risk family histories.

\begin{tabular}{|c|c|c|c|c|c|c|c|}
\hline $\begin{array}{c}\text { Family } \\
\text { Risk } \\
\text { Profiles* }\end{array}$ & $\mathrm{N}$ & $\begin{array}{c}\text { Mean } \\
\text { Age, y } \\
\text { (range) }\end{array}$ & $\begin{array}{c}\text { \# MRI } \\
\text { screened } \\
\text { patients }\end{array}$ & $\begin{array}{c}\text { \#MRI } \\
\text { generated } \\
\text { biopsies }\end{array}$ & $\begin{array}{c}\text { Positive } \\
\text { Predictive } \\
\text { Value MRI } \ddagger\end{array}$ & $\begin{array}{c}\text { \# MRI } \\
\text { detected } \\
\text { cancers }\end{array}$ & $\begin{array}{c}\text { Total \# } \\
\text { Cancers }\end{array}$ \\
\hline$\geq 2$ FDR & 192 & $\begin{array}{c}53 \\
(27-88)\end{array}$ & $110(57 \%)$ & 23 & $3 / 23(13 \%)$ & $3 / 110(3 \%) \dagger$ & $9 / 192(5 \%)$ \\
\hline $\begin{array}{c}1 \text { FDR } \\
\text { and } \\
\geq 1 \text { SDR }\end{array}$ & 421 & $\begin{array}{c}46 \\
(21-82)\end{array}$ & $152(36 \%)$ & 23 & $1 / 23(4 \%)$ & $1 / 152(0.7 \%) \dagger$ & $11 / 42(3 \%)$ \\
\hline $\begin{array}{c}1 \text { FDR } \\
\text { alone }\end{array}$ & 393 & $\begin{array}{c}50 \\
(23-85)\end{array}$ & $106(27 \%)$ & 24 & $2 / 24(8 \%)$ & $2 / 106(2 \%)$ & $8 / 393(2 \%)$ \\
\hline $\begin{array}{c}\geq 2 \text { SDR } \\
\text { only }\end{array}$ & 13 & $\begin{array}{c}41 \\
(25-59)\end{array}$ & $6(46 \%)$ & 4 & $1 / 425 \%)$ & $1 / 6(17 \%)$ & $1 / 13(8 \%)$ \\
\hline Total & 1019 & 49 & $374(37 \%)$ & 74 & $7 / 74(9 \%)$ & $7 / 347(2 \%)$ & $29 / 1019(3 \%)$ \\
\hline
\end{tabular}

* Includes family history of breast and/or ovarian cancer. FDR, first-degree relative; SDR, second-degree relative. MRI missed one cancer in each of these family risk groups. Of those with biopsy recommended by MRI.

\section{P171}

A Novel Method of Characterizing Micrometastatic Nodal Disease in Breast Cancer Using Morphometric Measurements M.M. Lomme, * A. Stuckey, D. Edmonson, C.J. Sung, D. Dizon, J. Gass. Women \& Infants Hospital of Rhode Island, Providence, RI.

Introduction: While nodal involvement is an important predictor of prognosis in breast cancer, the clinical significance of isolated tumor cells (ITCs) or micrometastatic nodal disease is not established. Conventional linear measurements (CLMs) made with a micrometer or ruler provide only a single, greatest dimension measurement and may be inaccurate or misleading. We report the use of a simple computer program to provide morphometric measurements (MMs) of tumor area as a means to better characterize minimal nodal disease. Methods: A retrospective analysis of nodal disease from patients with TxN1mic/NO(ITC) breast cancer was performed. For each positive node, the area of tumor was determined by computer-assisted morphometric analysis (Image $\mathrm{NIH} \circledast$ ). These patients will be followed to determine the significance of their nodal disease. Results: Between 1997 and 2004, 36 patients were identified. Median patient age was $51.5 \mathrm{yr}(31-83 \mathrm{yr})$. Median tumor size was $0.85 \mathrm{~cm}(0.3-11 \mathrm{~cm})$. Greater than $80 \%$ of tumors were ductal, $8.3 \%$ lobular, and $11.1 \%$ mixed ductal-lobular carcinoma. Most tumors were grade 2 and without identifed lymphatic invasion. Twelve nodes had ITCs $<0.2 \mathrm{~mm} ; 24$ had micromets $0.2-2 \mathrm{~mm}$. MMs of tumor area ranged from $0.01 \mathrm{~mm}^{2}$ to $2.7 \mathrm{~mm}^{2}$. CLMs yielded similar results for area only when tumor was present as a single uniform focus. When multiple foci or irregular nests of tumor were present, MMs proved to be much more accurate. In addition, similar greatest dimension measurements often yielded very different area measurements. For example, two separate nodes containing a $1.8 \mathrm{~mm}$ focus of tumor by CLMs had tumor areas of $2.7 \mathrm{~mm}^{2}$ and $0.9 \mathrm{~mm}^{2}$, respectively, by MMs. The overall time required to measure nodal disease with CLMs vs. computer-assisted MMs was comparable. Conclusions: Computer-assisted MMs provide a more accurate assessment of tumor present in micrometastatic nodal disease and are also useful in determining the overall area of tumor when multiple foci are present. Further follow-up is required to determine the prognostic significance of tumor area related to micrometastatic disease. Longitudinal follow-up is planned at our center. 


\section{P172}

Demographic and Pathology Trends in DCIS from 1987 to 2005 N. Coburn, ${ }^{1 *}$ M. Tente,${ }^{2}$ G. Stricsek, ${ }^{2}$ E. Clarke-Pearson, ${ }^{2}$ D. Giri, ${ }^{2}$ J. Fulton, ${ }^{3}$ M.A. Chung, ${ }^{4}$ B. Cady. ${ }^{4}$ 1. Division of Surgical Oncology, Sunnybrook Health Sciences Centre, University of Toronto, Toronto, ON, Canada; 2. Rhode Island Hospital Departments of Surgery and Pathology, Providence, RI; 3. Rhode Island Department of Public Health, Providence, RI; 4. Rhode Island Hospital Comprehensive Breast Center, Providence, RI.

Introduction: With extensive mammographic screening, ductal carcinoma in-situ (DCIS) incidence has greatly increased. However, few studies have examined changes in demographics or pathology. We hypothesized that earlier mammographic detection would result in smaller, better differentiated DCIS, and a decrease in age at presentation. Methods: Demographic data from the state cancer registry from 1987 to 2005 were analyzed. Pathology slides of all DCIS diagnosed from 1984 to 2005 at two academic hospitals ( $\mathrm{n}=566$ ) were re-examined. Results: The incidence of DCIS increased $280 \%$, from $11.6 / 100,000 / \mathrm{yr}$ in 1988 to $38.7 / 100,000 / \mathrm{yr}$ in $2004, \mathrm{p}<0.05$ ), while invasive breast cancer incidence was unchanged $(135.6 / 100,000 / \mathrm{yr}$ in 1988 and $130.9 / 100,000 / \mathrm{yr}$ in $2004, \mathrm{p}=\mathrm{ns})$. Since 2000 , over $20 \%$ of all breast cancers presented as DCIS. Both state and hospital data revealed a 4 year decline in median age ( 63 to 59 , and 61 to 57 , respectively). In the state registry, size and grade were infrequently reported. Pathology analysis revealed that median DCIS size was $7 \mathrm{~mm}$, smaller than usually cited. Neither size, histologic grade, necrosis, nor Van Nuys Prognostic Index (VNPI) scores varied over time. VNPI 4-6 occurred in 35\%, VNPI 7-9 in $62 \%$, and VNPI $10-12$ in $3 \%$ of patients. Breast conservation (BCT) rates increased in the state from $68 \%$ to $74 \%$, but did not increase further from the already high rates $(>80 \%)$ in the academic hospitals. The proportion of BCT with $1-9 \mathrm{~mm}$ surgical margins increased from $15 \%$ to over $40 \%$, while margins less than $1 \mathrm{~mm}$ decreased from over $70 \%$ to $38 \%$. Conclusions: DCIS generally presents as a very small mammographic lesion, with a markedly increased incidence over time. Age declined over time, suggesting earlier interruption in the progress of disease. Although mammography has resulted in earlier stage at presentation for invasive breast cancer, we do not find the same effect in DCIS. Pathologic features and VNPI scores did not change, suggesting a longer preclinical phase of DCIS than previously assumed. In the state population, there has been an increase in the use of BCT and an improvement in surgical margins in the tertiary care centers studied.

\section{P173}

The Significance of Lymphocytic Infiltration in Primary Breast Tumors B. Chikman, R. Lavy, ${ }^{*}$ N. Siegelmann-Danieli, A. Halevy. Assaf Harofeh Medical center, Tel Aviv, Israel.

Background: Lymphocytic infiltration (LI) of primary breast tumor is a rare pathological finding. The significance of this pathological feature is poorly studied. Methods: 1467 patients with invasive breast cancer diagnosed at our Institution during the years 1992-2005 served as the basis of this study. The data presented in this study was retrieved from a computer database created on these patients. Statistical tests were performed against 2-sided alternatives using SPSS computer program. Results: LI was found in 32/1094 pts (2.9\%) with infiltrating ductal carcinoma (IDC), in $2 / 18$ pts $(11.1 \%)$ with non-specified histological type (NST) of breast cancer. Patients with pure medullary type of breast cancer $(n=4)$ were not included in this study. Other types of invasive breast cancer $(n=351)$ were not associated with LI of primary breast tumor. Comparative analysis of clinico-pathological features was performed among 34 pts with LI and 1078 patients with IDC and NST tumors. The differences in number of patients in different subgroup of patients are due to missing data. $23 / 32(71.9 \%)$ with LI had grade 3 tumors versus $180 / 899(20.0 \%)$ in patients without LI of primary tumor $(\mathrm{p}<0.001)$. High proliferative activity of tumors $(\mathrm{Ki} 67=3-4)$ was found in $16 / 19$ pts $(84.2 \%)$ vs $94 / 475$ pts $(19.8 \%)(\mathrm{p}<0.001)$, ER-negative tumors $17 / 31(54.8 \%)$ vs $156 / 886(17.6 \%)$, PR-negative tumors $17 / 30(56.7 \%)$ vs $225 / 886(25.4 \%)$, correspondently. The unique pathological finding as squamous metaplasia was found in $2 / 34$ pts $(5.9 \%)$ with LI versus $0 / 1078(0 \%)$ of pts without LI. Although association of tumors with LI with poor prognostic factors the rate of axillary lymph node metastases was only $8 / 32(25 \%)$ in patients with LI versus $420 / 984(42.7 \%)$ in pts without LI. This difference was more prominent among patients with grade 3 tumors: 4/22 $(18.2 \%)$ vs $94 / 157(59.9 \%)$. Conclusions: LI is associated with poor prognostic factors as so high tumor grade, high proliferative activity, ER-negative and
PR-negative status but is associated with a low rate of axillary lymph node metastases. Further studies are needed for understanding this controversity.

\section{P174}

Surgical resection of the primary tumor in stage IV breast cancer and survival H.W. Hazard, * S.R. Gorla, J. Kim, D. Scholtens, W.J. Gradishar, S.A. Khan. Feinberg School of Medicine of Northwestern University, Chicago, IL.

Introduction: The concept that surgical resection of an intact primary tumor in women presenting with Stage IV breast cancer is primarily palliative was challenged based on a review of data from the National Cancer Database (Khan et. al., Surgery 2002). Two additional retrospective studies are supportive. Methods: We preformed a retrospective review of patients diagnosed with Stage IV breast cancer between January 1995 and October 2005 to examine treatment patterns and survival for this group of patients at our institution. Medical record review established the age at diagnosis, tumor size and grade, lymph node status, sites of metastatic disease at presentation, surgical intervention, systemic therapy, radiotherapy, and length of survival. Results: Ninety-nine women presented with Stage IV breast cancer between January 1995 and October 2005, and underwent systemic therapy, of whom 45 received surgical therapy (33\% wide excision and $67 \%$ mastectomy) either immediately before, or immediately following systemic therapy. The mean age was 56.3 years ( 53.3 years in the non-surgical group and 58.9 years in the surgical group, $p=0.0522$ ). Median follow-up time was 25.5 months. Women receiving surgical therapy of their primary tumor demonstrated a significant improvement in survival time, compared to women who did not receive surgical treatment $(\mathrm{p}=0.0464)$. Despite the borderline association between use of primary tumor resection and patient age, a Cox proportional hazards model demonstrated that the benefit of surgical resection was independent of age. The age-adjusted hazard of death in the surgical group was $0.613(\mathrm{p}=0.061)$. Additional Cox proportional hazards models for histology, grade, ER/PR status were not statistically significant and therefore not significant predictors of survival time. Conclusion: Surgical resection of the primary tumor in women with Stage IV breast cancer was associated with improved overall survival independent of age, histology, tumor grade and hormone receptor status. However, without a confirmatory randomized prospective trial, selection bias remains a possible explanation for these findings.

\section{P175}

Axillary Reverse Mapping (ARM) A New Concept to Enhance Lymphatic Preservation M.E. Thompson, ${ }^{*}$ K.L. Bland, K. Jackman, L. Adkins, S. Korourian, R. Henry-Tillman, K. Westbrook, V.S. Klimberg. Surgery, University of Arkansas for Medical Sciences, Little Rock, $A R$.

Introduction: The risk of developing lymphedema in patients undergoing axillary lymph node dissection (ALND) remains high. Even for those who have sentinel lymph node biopsy (SLNB) alone the risk is $2-7 \%$. We hypothesize that variations in arm lymphatic drainage put them at risk for disruption during a SLNB and/or ALND. Therefore mapping the drainage of the arm with blue dye (ARM) combined with mapping the SLNB from the breast with technetium sulfur colloid (TSC) would decrease the likelihood of inadvertent disruption of the arm lymphatics. Methods: This IRB-approved study from 05/0608/06 involved patients undergoing SLNB and/or ALND. $4 \mathrm{~mL}$ of TSC was injected in the subareolar plexus and $2-5 \mathrm{~mL}$ of lymphazurin intradermally in the ipsilateral upper extremity (ARM). The study was performed in 2 groups, Group I: positive axillas receiving SLNB and/or ALND+ARM; Group II: SLNB only+ARM. Data were collected on variations in lymphatic drainage and nonconcordance between hot breast nodes and blue arm nodes. Results: In Group I with SLNB+ALND $(n=10)$ and Group $2(n=20)$ all breast SLNs were hot but not blue. Average positive lymph nodes in Group I were 2.8 (range 0-12). In all of Group I, 8 of 14 blue lymphatics were identified in the axilla. In the first 5 cases these lymph nodes were biopsied and never positive when breast hot nodes were positive with breast cancer. In the next 3 cases the blue node was attached to a $6 \mathrm{~mm}$ blue lymphatic. In one of these cases the blue lymphatic was trapped in matted nodes and was sacrified followed by immediate postop lymphedema. In Group $2(n=20), 9(41 \%)$ blue arm lymphatics were identified including 3 with a $5 \mathrm{~mm}$ blue lymphatic that was in the bed of the hot node and protected. A total of 6 of $36(17 \%)$ cases had significant lymphatics $(\geq 5 \mathrm{~mm})$ within the surgical field. No complications of ARM were observed. Conclu- 
sions: There was non-concordance of arm and breast lymphatic drainage even in positive axillas. There were clinically significant lymphatic variations that ARM identified and enhanced preservation in all but one case. ARM added to ALND and even SLNB further delineates the axilla and may help prevent lymphedema.

\begin{tabular}{|c|c|c|c|}
\hline & Hot & Hot \& Blue & Blue Lymphatic Identified in Axilla \\
\hline Group I SLNB \&/or ALND & $10 / 10(100 \%)$ & $0 / 10(0 \%)$ & $8 / 14(57 \%)^{*}$ \\
\hline Group II SLNB Only & $22 / 22(100 \%)$ & $0 / 22(0 \%)$ & $9 / 22(41 \%)$ \\
\hline
\end{tabular}

*4 ALND without SLNB, $p<0.01$ for non-concordance of hot and blue localization.

\section{P176}

Surgical decisions in BRCA1/2 mutation carriers in a single institution D.M. Agnese, ${ }^{1 *}$ K. Sweet, ${ }^{2}$ L. Kerner. ${ }^{2}$ 1. Surgery, The Ohio State University, Columbus, $\mathrm{OH} ; 2$. Human Genetics, The Ohio State University, Columbus, $\mathrm{OH}$.

Introduction: Mutations in the BRCA1 and BRCA2 genes are associated with a $50-85 \%$ lifetime risk of breast cancer and a $10-60 \%$ lifetime risk of ovarian cancer. Our objective was to determine how BRCA mutation carriers managed this risk. Methods: Our cancer genetics database was reviewed to identify women with deleterious BRCA mutations. Data regarding cancer history/management and risk reduction strategies was recorded. Results: Between July 1997 and August 2006, 130 women with a deleterious mutation in BRCA1 (79) or BRCA2 (51) were identified. 44 (56\%) BRCA1 mutation carriers had cancer prior to testing ( 34 breast, 4 breast and ovarian, 6 ovarian). 7 had bilateral breast cancer (mean age of 1 st cancer 37) and 6 were treated with bilateral mastectomy; 31 had unilateral breast cancer (mean age 37), of whom 18 had mastectomy and 9 had contralateral prophylactic mastectomy (PM) prior to testing. 19/38 with breast cancer had prophylactic surgery after testing (1 PM,16 BSO, and 2 PM/BSO). 30 (59\%) BRCA2 mutation carriers had cancer prior to testing ( 24 breast and 6 ovarian). 5 had bilateral breast cancer (mean age of 1 st cancer 46 ), with only one choosing breast conservation for both cancers. 17/19 BRCA2 carriers (mean age 41) with unilateral breast cancer had mastectomy and 6 elected contralateral PM. 10/24 with breast cancer had prophylactic surgery after testing ( $0 \mathrm{PM}, 10 \mathrm{BSO})$. Of the 56 unaffected women with mutations, $22(39 \%)$ had prophylactic surgery after testing (mean age at results 41 ). Significantly more of the BRCA1 carriers had prophylactic surgery $(48.6 \%$; 9 PM, $13 \mathrm{BSO})$ than BRCA2 carriers $(23.8 \% ; 3$ $\mathrm{PM}, 5 \mathrm{BSO})$. The remaining 34 unaffected carriers chose increased screening (mean age at results 32 ). Three $(8.8 \%$ ) went on to develop breast cancer (mean age 42) and elected mastectomy. Conclusion: Many high risk women with breast cancer had mastectomy prior to knowledge of their mutation status. These decisions were likely based on family history and age of diagnosis. After mutation status was established, the women who chose prophylactic surgeries tended to be older. In mutation carriers who developed cancer, mastectomy was the preferred treatment strategy.

\section{P177}

Breast Tumor Detection Using Piezoelectric Fingers (PEFs) H. Yegingil, ${ }^{2 *}$ S. Jagtap, ${ }^{1}$ W.Y. Shih, ${ }^{2}$ W.H. Shih, ${ }^{2}$ J. Justin, ${ }^{1}$ A.D. Brooks. ${ }^{1}$ 1. Surgery, Drexel University College of Medicine, Philadelphia, PA; 2. Department of Materials Science and Engineering, Drexel University, Philadelphia, PA.

Introduction: For women who are below screening age, or women who don't have access to mammography, the only alternative is palpation. Self breast exam is limited because of poor compliance and user variability, and clinical breast exam has a limit of detection over $1 \mathrm{~cm}$. We developed a device that measures the 2 elements of palpation, stiffness and mobility, in order to reliably identify and characterize breast tumors. Methods: The Piezoelectric Finger (PEF) is a piezoelectric cantilever consisting of a driving and a sensing piezoelectric (lead zirconate titanate) layer bonded to a nonpiezoelectric layer (stainless steel). Using a unique dual-electrode design, a PEF can both apply a force (using the driving electrode) and detect the resultant displacement (using the sensing electrode) with one device, like a finger. We used the PEF to characterize 9 human breast tumors, immediately post-excision, with regard to tumor size, location and a prediction of malignancy based on tumor elastic modulus, E, and shear modulus, G. Results: In our first patient, a satellite tumor measuring $3 \mathrm{~mm}$ that was missed by a physician's palpation, mammography, and ultrasound imaging prior to surgery was found in the specimen by PEF prior to pathology processing. PEF successfully identified the tumor location in all 9 specimens. PEF predicted the size of each specimen to within $10 \%$ of the actual pathologic measurement. A table of the G/E ratios for the 9 specimens demonstrates that the PEF can identify a difference between our 1 benign and the remaining 8 malignant lesions with a specificity of $87.5 \%$. Conclusion: The PEF is accurate with regard to size and location of tumors down to $3 \mathrm{~mm}$ within breast specimens. The PEF may be able to differentiate benign and malignant breast lesions based on stiffness and mobility. This technology may become the platform for a low cost, user friendly adjuvant breast screening device.

\begin{tabular}{|c|c|c|c|}
\hline Type & $\mathrm{n}$ & G/E ratio & Specificity \\
\hline Benign & 1 & $0.3-0.4$ & -- \\
\hline Malignant & 8 & $0.6-0.8$ & $87.5 \%$ \\
\hline
\end{tabular}

\section{P178}

Changing Practice Patterns: Neoadjuvant Chemotherapy (NeoCTX) and Breast Conserving Surgery (BCS) in Stage II Breast Cancer J.F. Waljee, ${ }^{1 *}$ L. Patel-Parekh, ${ }^{3}$ K.I. Bland, ${ }^{2}$ L.A. Newman. ${ }^{1}$ 1. Surgical Oncology, University of Michigan, Ann Arbor, MI; 2. University of Alabama, Birmingham, AL; 3. American College of Surgeons Commission on Cancer, National Cancer Data Base, Chicago, IL.

Introduction:Prospective clinical trials reveal equivalent survival and improved BCS eligibility in Stage II pts receiving neoCTX compared to adjuvant therapy (aCTX). The extent to which neoCTX and BCS are incorporated into widespread practice is unclear, and practice patterns regarding sentinel lymph node biopsy (SLNBx) in neoCTX pts are unknown. Methods:We analyzed 104,089 Stage II breast cancer pts treated with surgery and CTX and accessioned into the National Cancer Data Base (a hospital-based registry maintained by the Commission on Cancer) 1998-2002. Of these, 8,127 (6.6\%) received neoCTX and 115,362 (93.4\%) received adjuvant CTX (aCTX). Hospital setting was defined as community facility; comprehensive cancer center; or teaching/research hospital. Geographic location was described as Northeast (NE), Southeast (SE), Midwest (MW), and West (W). Results:In 1998 most pts receiving neoCTX $(77.2 \%)$ and $\mathrm{BCS}(82.0 \%)$ were treated at comprehensive cancer centers or teaching hospitals. Rates of BCS were highest in the NE $(52.5 \%)$ and lowest in the SE (39.8\%). These trends persisted in 2002 $85.5 \%$ of neoCTX and $80.9 \%$ of BCS pts were treated in either comprehensive cancer centers or teaching hospitals. BCS increased in the SE (44.8\%), but remained lower than other regions (NE 54.7\%; MW 47.2\%; W 48.8\%). BCS frequency increased for both neoCTX and aCTX pts during the 19982002 interval (from 46.4 to $51.4 \%$ for neoCTX, and from 52.5 to $61.2 \%$ for aCTX). SLNBx also increased for both groups (from 18.5 to $39.8 \%$ neoCTX, and from 21.6 to $53.1 \%$ aCTX). Significant predictors of receiving neoCTX were tumor size and node-positivity; older age and lobular histology were associated with less frequent neoCTX use. NeoCTX pts were less likely to receive BCS $(\mathrm{OR}=0.8 ; 95 \% \mathrm{CI} 0.8-0.9)$ and SLNBx $(\mathrm{OR}=0.8 ; 95 \% \mathrm{CI} 0.7-0.8)$ compared with aCTX pts. Conclusion:Use of BCS and SLNBx has increased in Stage II breast cancer, but remains lower among women receiving neoCTX Geographic differences in utilization of BCS and neoCTX have declined, however their use is concentrated in specialized centers. Future efforts should promote these strategies across all health care settings.

\begin{tabular}{|c|c|c|c|}
\hline & & Odds Ratio & $95 \%$ Confidence Interval \\
\hline Age (yrs) & $<5151-6061-70>71$ & 1.01 .11 .00 .9 & ref. 0.95-1.03 0.82-0.91 0.82-0.91 \\
\hline Histology & Ductal Lobular & 1.00 .9 & ref. 0.78-0.93 \\
\hline Primary Tumor & T1 T2 T3 & 1.01 .711 .3 & ref. 1.58-1.85 9.96-12.88 \\
\hline Nodal Status & Negative Positive & 1.01 .3 & ref. 1.1-1.4 \\
\hline
\end{tabular}

ref.=referant value 


\section{P179}

Axillary Staging Pre- and Post Neoadjuvant Therapy for Breast Cancer is Prognostically and Therapeutically Important M.C. Lee, ${ }^{*}$ A.V. Nees, V.M. Cimmino, K.M. Diehl, M.S. Sabel, D.F. Hayes,

A. Schott, C. Kleer, A.E. Chang, L.A. Newman. University of Michigan, Ann Arbor, MI.

Background: Neoadjuvant systemic therapy (NEO) is standard for locally advanced breast cancer, and has also been advocated in earlier-stage disease, as a means of increasing lumpectomy eligibility and assessing chemosensitivity. The significance of axillary staging prior to delivering NEO is uncertain, and controversy persists regarding selection of downstaged pts that might benefit from regional and/or postmastectomy irradiation (regXRT). Methods: Medical records were reviewed for 145 pts evaluated $1999-2004$ by a multidisciplinary breast cancer tumor board and treated with NEO. Pre-NEO axillary status was determined by clinical assessment, axillary ultrasound (with imageguided needle biopsy), and sentinel node biopsy. Node-positive pts underwent complete axillary dissection after NEO. Pts were stratified into 3 groups based on pre- and post-NEO nodal status: node-negative before and after NEO (NEG); node-positive downstaged to node-negative after NEO (POS/NEG); and persistently node-positive after NEO (POS). Results: Mean age was $49 \mathrm{yrs;}$ median tumor size was $37 \mathrm{~mm}$ (range 10-120); and $97 \mathrm{pts}(67 \%)$ were node-positive at diagnosis. With a median follow-up of 38 months, 25 pts (17\%) experienced distant metastases and 16 developed locoregional recurrence $(11 \%)$. Mean time to development of metastasis was 22.3 months. Extent of axillary response was associated with outcome as shown in the Table below; distant relapse occurred in $23 \%$ of POS cases; $14 \%$ of POS/NEG cases, and only $8 \%$ of NEG cases (Spearman's rank correlation $\mathrm{p}=0.04$ ). Furthermore, a trend was noted for fewer locoregional recurrences among the POS/NEG cases if they received regXRT ( $10 \%$ vs. $5.6 \%$ ). Conclusions: Our findings indicate that comprehensive assessment of the pre- and post-neoadjuvant therapy axillary nodal status is important for both prognosis and for radiation planning. Nodepositive patients that are downstaged to node-negativity face a treatment failure risk that is intermediate between patients that present as node-negative and those that have resistant node-positive disease. Regional XRT should be considered for this subset despite their final node-negative status.

Outcome correlated with pre- and post-neoadjuvant therapy (NEO) nodal status.

\begin{tabular}{|c|c|c|c|c|}
\hline & $\begin{array}{c}\text { Node-negative pre- } \\
\text { and post NEO N=38 }\end{array}$ & $\begin{array}{c}\text { Node-positive pre-NEO, } \\
\text { downstaged to } \\
\text { Node-negative post-NEO } \\
\mathrm{N}=28\end{array}$ & $\begin{array}{c}\text { Node-positive pre- } \\
\text { and post NEO N=79 }\end{array}$ & $\begin{array}{c}\mathrm{p} \\
\text { Value }\end{array}$ \\
\hline $\begin{array}{c}\text { Distant } \\
\text { Recurrence }\end{array}$ & $7.9 \%$ & $14.3 \%$ & $22.8 \%$ & 0.04 \\
\hline $\begin{array}{c}\text { Locoregional } \\
\text { Recurrence }\end{array}$ & $7.9 \%$ & $7.1 \%$ & $13.9 \%$ & 0.26 \\
\hline Any Recurrence & $13.2 \%$ & $21.4 \%$ & $27.9 \%$ & 0.08 \\
\hline
\end{tabular}

\section{P180}

Usefulness of combined sestamibi scintimammography, axillary ultrasonography and FNA cytology in reducing the number of sentinel lymph node procedures in patients with breast cancer F. Lumachi, ${ }^{1 *}$ A. Tregnaghi, ${ }^{2}$ D. Cecchin, ${ }^{3}$ F. Bui, ${ }^{3}$ M.C. Marzola ${ }^{3}$ P. Zucchetta, ${ }^{3}$ P. Burelli, ${ }^{4}$ U. Basso. ${ }^{5}$ 1. Department of Surgical and Gastroenterological Sciences, University of Padua, 35128 Padova, Italy; 2. Radiology Section, Department of Diagnostic Medical Sciences, University of Padua, 35128 Padova, Italy; 3. Nuclear Medicine, Department of Diagnostic Medical Sciences, University of Padua, 35128 Padova, Italy; 4. U. O. di Chirurgia, Azienda Ospedaliera, 31015 Conegliano (TV), Italy; 5. Division of Medical Oncology, Istituto Oncologico Veneto, IRCCS, 35128 Padova, Italy.

BACKGROUND: The sentinel lymph node procedure (SLN) is currently performed in patients with breast cancer (BC) although the axillary nodes (AN) do not contain metastases in $60 \%$ of all patients. The aim of this study was to evaluate the usefulness of $99 \mathrm{mTc}$-sestamibi scintimammography (SSM), ultrasonography (US) and US-guided FNA cytology (FNAC) together in detecting axillary metastases, and their potential role in reducing the number of SLN. PATIENTS AND METHODS: A series of 86 consecutive women (median age 53 years, range $34-70$ ) with confirmed $\mathrm{BC}$ and clinically negative nodes (T1N0) underwent both SSM and US prior to surgery. Sonographic signs of malignancy were replacement of echogenic central sinus by hypoechoic tissue, and eccentric thickening of the cortical region of the AN. A longitudinal-to-transverse ratio higher than 2 was considered sign of benignity. US-guided FNAC was performed in patients with suspicious AN on US, or positive SSM and ultrasonographically visualized enlarged AN. A combined method using radioisotope and blue dye was used for SLN biopsy. The procedure was omitted in patients $(\mathrm{N}=14,16.3 \%)$ with FNAC suggesting AN metastases, and thus they underwent axillary dissection, as well as those ( 8 out of $72,11.1 \%$ ) with positive SLN biopsy on frozen section. RESULTS: Final pathology showed 4 pTibN0, 1 pT1bN1, 60 pT1cN0, and 21 pT1cN1 BC. AN metastases was confirmed in all patients with positive FNAC. The sensitivity, specificity, and accuracy were $59.1 \%, 93.7 \%$, and $84.9 \%$ for SSM, $63.6 \%, 90.6 \%$, and $83.7 \%$ for US, and $72.7 \%, 97.8 \%$, and $90.7 \%$ for SSM and US together. However, the results did not differ significantly ( $\mathrm{p}=\mathrm{NS}$, chi-squared test). The SLN procedure CONCLUSIONS: In patients selected by SSM or US in whom US-guided FNAC has shown the presence of AN metastases, the SLN biopsy can be avoided, and $\mathrm{AN}$ dissection should be the primary procedure. Lumachi $\mathrm{F}$ et al. Accuracy of ultrasonography and $(99 \mathrm{~m}) \mathrm{Tc}$-sestamibi scintimammography for assessing axillary lymph node status in breast cancer patients. A prospective study. Eur J Surg Oncol 2006; 32: in press

\section{P181}

Identification of Tumor Localization for Breast-Conserving Surgery after Neoadjuvant Chemotherapy using Radioactive Seed Localization A. Maaskant, * Y. Van Riet, H. Rutten, F. Janssen, C. Creemers, G. Nieuwenhuijzen. Surgery, Catharina Hospital, Eindhoven, Netherlands.

Neoadjuvant chemotherapy reduces the size of a primary tumor in the majority of cases, increasing the possibility of breast-conserving surgery. In case of a complete clinical and radiological response image-guided wire localization is no longer possible and thus it is important to mark the tumor bed before start of chemotherapy. This can be achieved by implantation of a single iodine125 labelled titanium seed into the tumor bed. After implementation and evaluation of this technique pre-clinically, we used it to mark the tumor bed in patients who were to receive neoadjuvant chemotherapy for breast carcinoma, followed by surgery. 14 patients with T2- and T3-tumors who received neoadjuvant chemotherapy after intra-lesional placement of an iodine- 125 seed were included. Radioactive seed localization was carried out by image guided techniques using a titanium seed containing $7 \mathrm{Mbq}$ of iodine-125. This is a $27 \mathrm{keV}$ source of gamma radiation with a half-life of 60 days and is therefore an excellent gamma source to use in neoadjuvant chemotherapy in combination with the technetium-99 (141 keV) used for sentinel lymph node biopsy. Radioactive seed localization was subsequently carried out using the handheld gamma probe and removal of seed and lesion were confirmed through specimen photography. Radioactive seed localization was successful in all 14 patients, position within the tumor bed was confirmed by mammography and specimen photography confirmed presence of the iodine- 125 seed within the excised lump. Overall, in $86 \%$ of the patients the tumor was no longer palpable and in $36 \%$ of the patients the original tumor could not be palpated nor visualized. In those patients the iodine- 125 seed was the only remaining evidence of the original tumor site. All 14 patients underwent breast-conserving surgery and in 12 patients the excision was pathologically radical. Conclusively, the technique of radioactive seed localization has proven useful, safe and effective in marking the tumor bed in patients who are to receive neoadjuvant chemotherapy for breast carcinoma.

\section{P182}

The Development and Microarray Analysis of a Novel Radioresistant Breast Cancer Cell Line O. Qutob, ${ }^{*}$ M.B. Watson, A.W. Beavis, M.J. Lind, P.J. Drew, L. Cawkwell. Cancer Biology Proteomics Group, Postgraduate Medical Institute of the University of Hull in association with the Hull York Medical School, Hull, United Kingdom.

Introduction: Radiotherapy resistance represents a major obstacle in the effective management of breast cancer. The aim of this study is to gain further understanding of the mechanisms underlying development of radio-resistance in an in-vitro cell culture model. Methods: We established a novel breast cancer cell line which was significantly resistant to radiotherapy by irradiating MCF-7 cells in fractionated doses of $2 \mathrm{~Gy}$ up to a total dose of 40Gy. A dose 
response curve was assessed at the end of treatment to demonstrate a statistically significant increase in radioresistance for the novel cell line when compared with parental cells. A $3 \mathrm{k}$ cancer-related oligonucleotide microarray was used to identify targets which were differentially expressed between the novel radioresistant derivative and the parental cell line. Real-time quantitative PCR was used to confirm the difference in expression of a subset of genes which demonstrated significant (at least 2-fold) differential expression. Results: Using microarray analysis, the expression of four genes was found to be significantly altered between the radiotherapy-resistant cell line and the parental cell line. The 4 genes were: GSTM3 (glutathione S transferase mu 3), LGALS3BP (galectin 3 binding protein). PSME1 (proteasome activator subunit 1) and UXT (ubiquitously expressed transcript). Real-time quantitative PCR expression analysis has so far confirmed the differential expression of GSTM3. Conclusion: The development of this novel radiotherapy-resistant breast cancer cell line and identification of candidate genes associated with radiotherapy resistance may reveal novel therapeutic targets in breast cancer. Ultimately, overcoming resistance to radiotherapy would represent a major advance in the effective management of cancer today.

\section{P183}

Stage migration in breast cancer: a trend towards better disease free survival for NO patients since the introduction of the sentinel lymph node (SLN) procedure P. Gobardhan, ${ }^{1}$ O. Bakker, ${ }^{1}{ }^{*}$ W. Fiets, ${ }^{2}$ H. Ruitenberg, ${ }^{1}$ T. Van Dalen. ${ }^{1}$ 1. Surgery, Diakonessenhuis, Utrecht, Netherlands; 2. Medical Centre, Leeuwarden, Netherlands.

Background: The extensive pathologic examination following introduction of the SLN procedure in breast cancer patients has resulted in the more frequent finding of limited lymph node involvement in breast cancer patients. We evaluated the stage migration effect on prognosis by comparing the outcome of "SLNN0"patients to N0 breast cancer patients that were treated in the "pre-SLN" era. Method: Two groups of patients were evaluated: a prospective cohort of 245 consecutive patients that were staged as N0 based on pathologic assessment (HE and IHC-staining) of the SLN, and a cohort of 182 patients treated before $2000 \mathrm{for}$ unifocal cancers and staged as N0 based on axillary lymph node dissection (ALND) specimens. Patients who had chemotherapy (in the SLN group) and patients who would nowadays have had chemotherapy (in the ALND-group) were excluded. Median follow-up was 4 years for the SLN-group and 9 years for the ALND group. Results: The SLN group and the ALND group were comparable for tumor size, the proportion of high grade cancers and the proportion that received hormonal therapy. 2- and 4-year cumulative overall survival was 95 and $91 \%$ for the ALND group and 98 and $93 \%$ for the SLN group $(P=n s) .2$ - and 4- year disease free survival was 93 and $86 \%$ for ALND-group and 97 and $91 \%$ for the SLN group $(\mathrm{P}=0.1)$ Conclusion: Stage migration appeared to have an effect on prognosis. Although not statistically significant, patients who were staged as N0 based on SLN assessment seemed to have more favorable disease free survival.

\section{P184}

Effect of tumor factors, comorbidities and treatment on survival of elderly patients $(>80)$ diagnosed with early stage breast cancer K. Avradopoulos, ${ }^{1}$ ' S.S. Sama, ${ }^{2}$ S.S. Emani. ${ }^{1} 1$. Fallon Clinic, Worcester, MA; 2. Worcester Medical Center, Worcester, MA.

Introduction: As the general population ages, and medical management improves, a greater fraction of elderly patients $(>80)$ will be diagnosed with breast cancer. The purpose of our study was to identify the impact of tumor factors, comorbidities and treatment on survival in this population. Methods: A retrospective chart analysis was done, using an electronic prospective database to identify a screened population of elderly patients $>80$, diagnosed with Stage I and II breast cancer over a 13-year period between 1985 and 1998. All patients were treated with curative intent. Patients were followed until death, or for a minimum of 6 years. Multiple variables which could influence survival, were recorded. Results: There were 91 patients included in our study with a median age of 90 and a range from 80 to 103 . The median follow-up was 11.4 years. The median survival in this patient population was 6.7 years with a range from 6 months to 16.6 years. The disease specific mortality was 12/91 (13\%). The rate of distant recurrences was 11/91 (12\%). The rate of local regional recurrences was 5/91 (5.5\%). Tumor factors associated with decreased 5-year survival by univariate analysis included T2 tumors $(\mathrm{p}<.05)$ and poorly differentiated lesions $(p<.02)$. Comorbidities associated with decreased five year survival on univariate analysis included: $\operatorname{CHF}(p<.04)$, CAD $(p<.04)$, CVA $(\mathrm{p}<.02)$, COPD $(\mathrm{p}<.05)$, and ASA score $>3(\mathrm{p}<.01)$. Axillary lymph node dissection was the only treatment factor associated with decreased 5-year survival on univariate analysis $(\mathrm{p}<.04)$. Multivariate survival analysis revealed that age was not a confounding factor. On univariate survival analysis, decreased survival was associated with poorly differentiated lesions, ASA score $>3$, and history of CVA, although these were of borderline statistical significance. A summary of breast cancer deaths is found in accompanying table. Conclusion: Poorly differentiated lesions, ASA score $>3$, and history of CVA may be associated with decreased survival in elderly patients $(>80)$ with early stage breast cancer. Further studies are warranted.

\section{Breast Cancer Deaths $\mathrm{N}=12$}

\begin{tabular}{|c|c|c|c|c|c|c|c|c|c|c|}
\hline Patient & $\begin{array}{c}\mathrm{T} \\
\text { Stage }\end{array}$ & $\begin{array}{c}\text { Grade } \\
1-3\end{array}$ & ER/PR & $\begin{array}{c}\text { Surgical } \\
\text { Procedure }\end{array}$ & \begin{tabular}{|l} 
Radiation \\
Therapy
\end{tabular} & $\begin{array}{c}\text { Hormonal } \\
\text { Therapy }\end{array}$ & $\begin{array}{c}\text { Distant } \\
\text { Recurrences }\end{array}$ & $\begin{array}{l}\text { Local/Regional } \\
\text { Recurrences }\end{array}$ & $\mid \begin{array}{c}\text { Survival } \\
\text { (Years) }\end{array}$ & \begin{tabular}{|c|} 
Co \\
morbidities \\
at \\
Diagnosis
\end{tabular} \\
\hline 1 & $\mathrm{~T} 1$ & 2 & $(+/-)$ & Lumpectomy & None & None & Lung, Bone & $\begin{array}{c}\text { Ipsilateral and } \\
\text { axilla }\end{array}$ & $\begin{array}{c}7.9 \\
\text { years }\end{array}$ & DM \\
\hline 2 & $\mathrm{Tl}$ & 3 & $(+/-)$ & Lumpectomy & None & $\begin{array}{c}\text { Tamoxifen } \\
(3 \text { years })\end{array}$ & Bone & None & $\begin{array}{c}8.5 \\
\text { years }\end{array}$ & None \\
\hline 3 & $\mathrm{~T} 2$ & 3 & $(+/-)$ & $\begin{array}{c}\text { Mastectomy } \\
\text { and axillary } \\
\text { sampling }\end{array}$ & None & Tamoxifen & Lung & None & $\begin{array}{c}1.5 \\
\text { years }\end{array}$ & $\begin{array}{l}\text { CAD, } \\
\text { COPD, } \\
\text { HTN }\end{array}$ \\
\hline 4 & $\mathrm{Tl}$ & 2 & $(+/+)$ & Lumpectomy & None & Tamoxifen & Bone & None & $\begin{array}{c}2.5 \\
\text { years }\end{array}$ & $\begin{array}{l}\text { CAD, } \\
\text { COPD, } \\
\text { HTN }\end{array}$ \\
\hline 5 & $\mathrm{~T} 2$ & 3 & $(+/+)$ & Lumpectomy & None & Tamoxifen & Bone & Ipsilateral & $\begin{array}{l}2.5 \\
\text { years }\end{array}$ & $\begin{array}{c}\text { CVA, } \\
\text { HTN, DM }\end{array}$ \\
\hline 6 & $\mathrm{~T} 1$ & 2 & $(+/+)$ & \begin{tabular}{|c|} 
Modified \\
Radical \\
Mastectomy
\end{tabular} & None & $\begin{array}{c}\text { Tamoxifen } \\
(5 \text { years })\end{array}$ & $\begin{array}{l}\text { Lung, Liver, } \\
\text { Bone }\end{array}$ & None & $\begin{array}{l}11.9 \\
\text { years }\end{array}$ & HTN \\
\hline 7 & $\mathrm{~T} 2$ & 3 & $(+/+)$ & Lumpectomy & $4680 \mathrm{cGy}$ & Tamoxifen & Liver & None & $\begin{array}{c}3.4 \\
\text { years }\end{array}$ & HTN \\
\hline 8 & $\mathrm{~T} 2$ & 3 & $(+/+)$ & Lumpectomy & 4680cGy & Tamoxifen & Bone & None & 2 years & None \\
\hline 9 & $\mathrm{Tl}$ & 2 & $(+/+)$ & Lumpectomy & $4500 \mathrm{cGy}$ & Tamoxifen & Intra-abdominal & None & $\begin{array}{c}3.1 \\
\text { years }\end{array}$ & $\mathrm{CHF}$ \\
\hline 10 & $\mathrm{~T} 2$ & 3 & $(-/-)$ & $\begin{array}{c}\text { Modified } \\
\text { Radical } \\
\text { Mastectomy }\end{array}$ & None & None & Bone & None & $\begin{array}{c}4.9 \\
\text { years }\end{array}$ & HTN \\
\hline 11 & $\mathrm{Tl}$ & 3 & $(+/+)$ & Lumpectomy & $4680 \mathrm{cGy}$ & Tamoxifen & NA & None & $\begin{array}{c}5.4 \\
\text { years }\end{array}$ & HTN \\
\hline 12 & $\mathrm{TI}$ & 3 & $(+/-)$ & Lumpectomy & None & None & Liver, Lung & Ipsilateral & $\begin{array}{c}5.6 \\
\text { years }\end{array}$ & HTN \\
\hline
\end{tabular}

$\mathrm{NA}=$ Not Available

\section{P185}

Periareolar Tracer Administration Does Not Accurately Reflect Lymph Drainage From Breast Cancer M.C. Van Rijk, ${ }^{1 *}$ P.J. Tanis, ${ }^{2}$ S.H. Estourgie, ${ }^{2}$ O.E. Nieweg, ${ }^{1}$ J.M. Klaase, ${ }^{3}$ J.W. Merkus, ${ }^{4}$ I. Dawson, ${ }^{5}$ J.L. Peterse, ${ }^{1}$ B.B. Kroon. ${ }^{1} 1$. The Netherlands Cancer Institute, Amsterdam, Netherlands; 2. Academic Medical Center, Amsterdam, Netherlands; 3. Medisch Spectrum Twente, Enschede, Netherlands; 4. Haga Hospital, The Hague, Netherlands; 5. IJsselland Hospital, Capelle aan de IJssel, Netherlands.

Background: Lymphatic drainage pathways from the breast are subject of debate. Various injection sites for tracers are used for lymphatic mapping, based on different hypotheses. The aim of this study was to investigate the hypothesis that lymph drainage from the periareolar skin to the axilla accurately reflects drainage from breast cancer. Patients and methods: The study was performed in three hospitals where sentinel node biopsy was not yet standard of care and concerned 48 patients. All had proven breast cancer, clinical stage T1-T3 N0, and all were scheduled for a modified radical mastectomy. Vital blue dye was administered intradermally around the areola at the beginning of the operation. Mastectomy was performed with in-continuity axillary node dissection. The specimen was investigated in the pathology department in a standardized way. A blue duct was identified near the injection site and then carefully dissected and followed to the lymph node into which it drained. The tumor-status of such a node was compared to the tumor-status of the other axillary lymph nodes. Multiple sections were made of larger nodes. All lymph nodes were stained with both hematoxylin-eosin and immunohistochemistry staining. Results: A blue node could not be identified in three patients. A mean number of 1.4 blue lymph nodes with lymph vessels originating in the periareolar region were identified in the other 45 patients (range 1-3). The mean number of lymph nodes identified in the remainder of dissection specimens was 13.1 (range 7-22). Twenty-five of the 45 patients were found to have a tumor-positive axilla $(56 \%)$. A blue node was involved in twenty of them (sensitivity $80 \%$ ). Five patients had tumor-positive nodes that were not stained (false-negative rate $20 \%$ ). Conclusion: This ex vivo study suggests that lymph drainage from a breast cancer 
is not accurately reflected by vital dye when administered around the areola. If this observation is substantiated by high recurrence rates in the axilla in the coming years, other injection sites must be preferred.

\section{P186}

Trends in Breast Cancer Surgery: Comparison of an Institutional Experience Between 1995 and 2005 B.A. Shanker, A.A. Guth,* D.F. Roses, D. Axelrod, B. Singh, R.L. Shapiro, T. Diflo, J.A. Cangiarella. NYU School of Medicine, New York, NY.

INTRODUCTION: Recent reviews of SEER data* have demonstrated an increase in the incidence of both non-invasive and invasive breast cancer. This has largely been attributed to improvements in breast imaging techniques. How have these changes, including the increased use of image-guided sampling techniques, influenced the clinical practice of breast surgery? We reviewed our experience over a 10 year interval, a period in which radiologic evaluation of the breast has undergone significant refinements. METHODS: The institutional pathology database was queried for all breast surgeries performed in 1995 and 2005. Cosmetic procedures were excluded. The results were tabulated on an Excel spreadsheet, and analysed utilizing the Chi-square test. IRB approval was granted. RESULTS: The results are summarized in Table 1. Indications for breast surgery changed significantly over the 10-year study period, with an increased incidence of pre-operatively diagnosed cancers undergoing definitive surgical management, and an increase in biopsies performed for abnormal or indeterminate findings on fine needle aspiration cytology. $\mathrm{ADH}$, and to a lesser extent, $\mathrm{ALH}$, became indications for surgical excision. Fewer surgical biopsies were performed for indeterminate abnormalities on breast imaging. While the rate of benign breast biopsies remained constant, there was a higher incidence of precancerous and DCIS cases in 2005. The rate of mastectomy dropped dramatically, from $36.8 \%$ in 1995 to $14.5 \%$ in 2005 . Linked to the increase in sentinel node procedures, the rate of ALND dropped significantly. Accompanying the increased recognition of early-stage cancers, the rate of positive axillary lymph node dissection also decreased significantly. CONCLUSIONS: While the rate of benign breast biopsies has remained constant over a recent 10 -year period, more preinvasive and Stage 0 cancers are undergoing surgical management. A greater percentage of patients with breast cancer or preinvasive disease have these diagnoses determined before surgery. Earlier stage invasive cancers are being detected, reflected by the lower incidence of axillary nodal metastases. * Li JAMA 2003:389; 1421

\section{Table 1}

\begin{tabular}{|c|c|c|c|}
\hline Characteristics & $1995(n=661)$ & $2005(n=1005)$ & $p$--values \\
\hline \multicolumn{4}{|l|}{$\begin{array}{c}\text { Indications for } \\
\text { surgery }\end{array}$} \\
\hline Cancer & $194(29.3 \%)$ & $394(39.2 \%)$ & $\& \# 8806 ; 0.001$ \\
\hline Abnormal $\mathrm{x}$-ray & $187(28.3 \%)$ & $169(16.8 \%)$ & $\& \# 8806 ; 0.001$ \\
\hline Palpable mass & $149(22.5 \%)$ & $262(26.0 \%)$ & NS \\
\hline Abnormal FNA & $3(0.5 \%)$ & $15(1.5 \%)$ & \&\#8806;0.05 \\
\hline ADH & $0(0 \%)$ & $31(3.1 \%)$ & \&\#8806;0.001 \\
\hline ALH & $0(0) \%$ & $8(0.8 \%)$ & \&\#8806;0.025 \\
\hline Mean Age(years) & $55+/ 214$ & $53+1-15$ & NS \\
\hline Type of surgery & $\begin{array}{l}\text { Note: } 28 \text { patients underwent } \\
\text { simultaneous multiple breast } \\
\text { procedures }\end{array}$ & $\begin{array}{c}\text { Note: incomplete } \\
\text { procedural description for } \\
20 \text { patients }\end{array}$ & \\
\hline Mastectomy & $242(36.8 \%)$ & $146(14.5 \%)$ & \&\#8806;0.001 \\
\hline Excisional biopsy & $237(36.8 \%)$ & $187(18.6 \%)$ & $\& \# 8806 ; 0.001$ \\
\hline $\begin{array}{l}\text { Needle-localization } \\
\text { biopsy }\end{array}$ & $8(1.2 \%)$ & $231(23.0 \%)$ & $\& \# 8806 ; 0.001$ \\
\hline Partial mastectomy & $201(30.4 \%)$ & $420(41.8 \%)$ & \&\#8806;0.001 \\
\hline Sentinel node & $6(0.9 \%)$ & $246(24.5 \%)$ & \&\#8806;0.001 \\
\hline Axillary dissection & $121(18.3 \%)$ & $138(13.7 \%)$ & \&\#8806;0.025 \\
\hline Core biopsy & $23(3.5 \%)$ & $3(0.3 \%)$ & \&\#8806;0.001 \\
\hline Pathology & \multicolumn{3}{|c|}{ Note: included 2 diagnoses for one specimen if significant } \\
\hline Benign & $280(42.3 \%)$ & $413(41.1 \%)$ & NS \\
\hline ADH/ALH & $94(19.2 \%)$ & $138(13.7 \%)$ & NS \\
\hline DCIS & $155(23.4 \%)$ & $322(32.0 \%)$ & \&\#8806;0.001 \\
\hline LCIS & $34(5.1 \%)$ & $75(7.4 \%)$ & NS \\
\hline IDC & $130(19.7 \%)$ & $238(23.7 \%)$ & NS \\
\hline ILC & $24(3.6 \%)$ & $43(4.3 \%)$ & NS \\
\hline IDLC & $3(0.5 \%)$ & $11(1.1 \%)$ & NS \\
\hline $\begin{array}{c}\#(+) \text { ALND/all axillary } \\
\text { staging }\end{array}$ & $54 / 127(43.3 \%)$ & $96 / 384(25.0 \%)$ & \&\#8806;0.001 \\
\hline
\end{tabular}

\section{P187}

Predictors of Residual Disease in Patients with Complete MR Response Following Neoadjuvant Chemotherapy for Invasive Breast Cancer R. Chattopadhyay, ${ }^{*}$ N. Hylton, D. Wolverton, A. Radzio, C.A. Ewing, M. Alvarado, L.J. Esserman, E.S. Hwang. Surgery, Breast Care, UCSF, San Francisco, CA.

Introduction: Breast MRI has been useful in evaluating extent of disease for invasive cancer. However, its utility in prediction of residual disease following neoadjuvant chemotherapy is uncertain. We undertook this study to define those variables associated with residual invasive breast cancer following neoadjuvant chemotherapy, despite negative presurgical MRI. Methods: A survey of a prospective neoadjuvant database (2001-2006) was undertaken. 24 consecutive unselected cases of negative MRI following neoadjuvant chemotherapy were identified. The primary outcome variable examined was residual disease at final pathology. Pathologic complete response (CR) was defined as the absence of any invasive tumor focus. Significance was determined by chi-square test. Results: Of 24 patients evaluated, 7 (29\%) had residual invasive disease. Of the 17 cases with pathologic CR, there were 3 cases with residual DCIS only and 2 cases with scattered tumor cells. The negative predictive value of MRI was $71 \%$ (17/24). Positive progesterone receptor (PR) and negative Her2-neu overexpression were highly associated with likelihood of residual invasive cancer ( $\mathrm{p}=0.005$ and 0.04 , respectively). Non-ductal histology and positive ER status approached, but did not attain statistical significance ( $\mathrm{p}=0.12$ and 0.11 , respectively). In a secondary analysis with any invasive cancer and/or DCIS as the outcome variable, both non-ductal histology and positive ER status emerged as significant predictors of residual disease $(p=0.05)$. Tumor size, grade, stage and age at presentation were not associated with the presence of residual invasive cancer and/or DCIS. A third analysis with residual nodal disease as the outcome variable revealed that lymphovascular invasion identified in tumor was predictive of residual disease in lymph nodes $(\mathrm{p}=0.015)$. Conclusion: A negative MRI is predictive for a pathologic CR; however patients with non-ductal histology, PR positive or Her2-neu negative disease have a higher likelihood of residual cancer even with a negative preoperative MRI. These factors should be considered when planning surgery following neoadjuvant chemotherapy.

\section{P188}

Retrospective Analysis of Overall Survival in Patients with Primary Carcinoma of the Breast and Concomitant Hepatic or Renal Disease F.R. Buckhold, B.P. Combs, P.M. Buchanan, P.R. Kelemen, E.C. Hsueh.* Surgery, Saint Louis University, St. Louis, MO.

Introduction: Cytotoxic chemotherapy has been shown to improve survival in patients with invasive breast cancer. However, the chemotherapeutic agents commonly employed have been associated with hepatic and nephrotoxicities. Diseases of the liver and kidney are major causes of morbidity and mortality in the U.S. We hypothesize that individuals with breast cancer and concurrent hepatic or renal disease might experience decreased survival relative to those with breast cancer alone. Methods: We studied breast cancer patients undergoing treatment from 1988-2005 at St. Louis University Hospital. Patient characteristics were obtained from chart review. Tumor information was obtained from pathology reports. Survival was defined as the interval between date of diagnosis and death or last follow-up date. Renal insufficiency was defined as $\mathrm{Cr}>1 \mathrm{x}$ normal limit or documented renal insufficiency. Hepatic disease was defined as total bilirubin $>1 \mathrm{x}$ normal limit or documented hepatic disease. The Cox proportional hazard regression model was used for regression analysis. Survival curves were calculated using the Kaplan-Meier method. Results: Of 361 breast cancer patients, $42(11.6 \%)$ had hepatic or renal disease. Median age for all patients was 61 years and median tumor size was $1.7 \mathrm{cms}$. One hundred and two patients (28.3\%) died. Univariate analysis of patients with hepatic or renal disease revealed significant decreased survival compared to those without concurrent disease for both invasive and non-invasive subtypes ( $p=0.002$ and $p=0.032$, respectively). Multivariate analysis showed that presence of renal or hepatic disease, stage, HER2 amplification, Ki-67 amplification, and age at diagnosis were significant independent prognostic indicators of survival $(\mathrm{p}<0.05)$. Conclusion: Breast cancer patients with pre-existing liver or kidney disease have decreased survival compared to patients with breast cancer alone. Given reported data on common cytotoxic chemotherapeutic agents and their association with renal and hepatic toxicity, caution is warranted in the treatment of patients with concomitant hepatic or renal disease. 


\section{P189}

Selected patients with multi-focal/multi-centric breast cancer can be safely treated by breast conservation therapy and/or SLN biopsy M.D. Fleming,* B. Pockaj, R. Gray, M. Halyard, D. Northfelt,

P. Karstaedt. Surgery, Mayo Clinic, Scottsdale, AZ.

Introduction: Controversy exists whether breast cancer patients with multifocal/multi-centric (MF/MC) disease can be treated with minimal invasive techniques such as breast conservation therapy (BCT) and/or sentinel lymph node (SLN) biopsy. Methods: Retrospective review of all patients with at least 2 separate macroscopic invasive breast cancers who underwent treatment from January 1988 - July 2006. Patients with extensive ductal carcinoma in situ were excluded. Results: 142 patients with distinct MF/MC breast cancer underwent surgery, mastectomy in $87(61 \%)$ and BCT in $55(39 \%)$. There were no differences in age, presentation, or method of diagnosis between the two groups. $68 \%$ had MF/MC disease documented pre-operatively. Mammogram demonstrated at least one tumor in $135(95 \%)$ patients but revealed MF/MC disease in only $55 \%$. Sensitivities of preoperative ultrasound and magnetic resonance imaging in detecting the MF/MC disease were $70 \%$ and $81 \%$, respectively. Infiltrating ductal carcinoma was the most common histology $(68 \%)$ and $30 \%$ of patients had more than one histologic tumor type. The cancers were located in a single breast quadrant in $91 \%$ of BCT patients. These patients were treated with a single lumpectomy. One patient underwent two separate lumpectomies. Sentinel lymph node (SLN) biopsy was performed in $101(70 \%)$ patients. A total of $31(31 \%)$ patients had a positive SLN. 27 patients underwent a complete axillary lymph node dissection with the SLN being the only lymph node(s) positive in $63 \%$. Mean follow-up is 45.8 months with $83 \%$ of patients having greater than 1 year of follow-up. Only one BCT patient $(2 \%)$ developed a local recurrence and she had refused adjuvant radiation therapy. One patient developed regional recurrence and had not undergone axillary staging at her original surgery. Seven patients $(5 \%)$ developed systemic recurrences. Conclusions: Patients with MF/MC breast cancer can be treated by BCT with a low likelihood of local recurrence. In our series, SLN biopsy was accurate with no regional recurrences to date. The indications for SLN biopsy can be expanded to include patients with $\mathrm{MF} / \mathrm{MC}$ cancer.

\section{P190}

Pre-operative assessment improves early diagnosis and treatment of lymphedema: a case-control series P.W. Soballe, ${ }^{1 *}$ N. Gergich, ${ }^{1}$ F. Washington, ${ }^{2}$ C. Pfalzer, ${ }^{3}$ C.L. McGarvey. ${ }^{4}$ 1. National Naval Medical Center, Bethesda, MD; 2. University of Maryland School of Medicine, College Park, MD; 3. University of Michigan-Flint, Flint, MI; 4. National Institutes of Health, Bethesda, MD.

INTRODUCTION. The incidence of breast-cancer related lymphedema (LE) is approximately $15 \%$ following axillary dissection and $4-8 \%$ following sentinel node biopsy. This doubles when radiation therapy is added. LE impacts quality of life, range of motion, pain, and strength. Methods used to measure LE have included limb girth using a tape measure (anthropometric), water displacement (volumetric) and infrared optoelectronic. Traditionally these are employed after the condition is clinically (visually) apparent, thus delaying treatment and requiring chronic management of irreversible LE. Complete Decongestive Therapy is the standard of care for advanced LE, but there is currently no evidence-based standard treatment for early stages. Prospective screening might therefore facilitate earlier diagnosis of LE and more effective, less costly interventions. METHODS: Twenty-five women with lymphedema, ages 51-73 (median age 60) were identified from those enrolled in a prospective breast-cancer morbidity study. Each subject's unaffected arm (UA) was used as her own control. LE was diagnosed if a $>3 \%$ limb volume change was evident in the affected arm (AA) as compared to pre-operative, measured by the optoelectronic volumeter (Perometer $\left.{ }^{\circledR}\right)$ at 1, 3, 6, 9, 12 month follow-up. Upon diagnosis of LE, intervention was instituted using an off-the-shelf compression garment of 20 to $30 \mathrm{mmHg}$, worn daily for 2 weeks. Data were analyzed using a 2-way repeated ANOVA of limb volume with arm and time as repeated factors and mean values. RESULTS. A significant $(p=0.001)$. difference in limb volume was identified between each subject's UA and AA $(p=0.001)$. Following application of a compression garment, there was a significant $(p=0.01)$ decrease in AA volume and no statistically significant difference in limb volumes between each subject's UA and AA. CONCLUSIONS. Pre-operative measurement and assessment of women with breast cancer facilitates early detection and management of LE. A cost-effective short course of compression provided excellent control of LE detected at such an early stage and may prove efficacious in prevention of the morbidity and expenses related to more advanced LE.

\section{P191}

Tumor Bed Metallic Clip Marker Utility in Patients Undergoing Neoadjuvant Therapy for Breast Cancer A. Radzio, * M. Ley, R. Chattopadyay, J. Rabban, N. Hylton, D. Wolverton, M. Alvarado, E. Hwang, C. Ewing, L. Esserman. Surgery, UCSF, San Francisco, CA

Introduction: In the setting of neoadjuvant therapy and a complete clinical response, there are different methods for approaching breast conserving surgery. We used localization of an image-directed clip placed early in the course of therapy or anatomic mapping based on the location of the tumor at the time of presentation, correlated with MRI. We hypothesized that clip placement would lead to fewer re-excisions in the setting of complete clinical response by MRI. Methods: We evaluated the charts of 157 patients who underwent neoadjuvant therapy. Patients had MRI scanning prior to treatment and at the end of treatment. 24 of these patients had a complete or near complete response by MR imaging ( $95 \%$ reduction in longest diameter). We assessed the technique used for localization and determined if there was a difference in re-excision rate. Results: Of the 24 patients with complete or near complete MR response to neoadjuvant chemotherapy, 7 patients chose mastectomy as their preferred surgical option. Of the remaining 17 patients, 7 underwent wire-localization of a previously placed clip, and 10 underwent anatomic localization based on location of tumor at presentation (size, o'clock, distance from nipple) combined with MR data. Of the 7 needle localizations, 6 patients had residual disease, 4 required re-excision. Of the 10 anatomically directed excisions, 8 had no residual disease, 2 had scattered tumor cells and none required reexcision. Conclusions: Early clip placement is emerging as a standard technique for managing neoadjuvant patients who desire breast conservation. However, clip placement by ultrasound or mammography may not provide the optimal method for resection either because of eccentric shrinkage of tumor, clip migration, or smaller volume of excision. Therefore, we are assessing all 157 patients and will present data on all, comparing clip directed exisions vs anatomic directed excisions and evaluating volume of tissue resected, re-excision rate, pathologic findings, and initial MRI characteristics (solid vs. diffuse).

\section{P192}

Pre-operative ultrasound-guided core needle biopsy of axillary sentinel nodes D.S. Nathanson, ${ }^{*}$ R. Slater, M. Burke. Surgery, Henry Ford Health System, Detroit, MI.

Hypothesis: Morphologically normal or abnormal sentinel lymph nodes (SLNs) may be identified by ultrasound (US) visualization of the largest lymph node at the level of the lowest axillary hair follicle. Core needle biopsy (CNB) of the node, with placement of a radio-opaque clip, plus $x$-ray of the node to identify the clip after SLN biopsy, would confirm the hypothesis. Materials and methods: 102 patients with biopsy-proven primary breast cancers (BRCAs) and clinically non-palpable axillary nodes were consented in this IRB-approved protocol. 81/102 had CNB and a metallic clip placed in the node. 21/102 had no biopsy because no node was seen (17) or there were anatomical difficulties (4). Operative SLN biopsy was done using standard techniques with a previously defined find rate of $>90 \%$ and a false negative rate of $<3 \%$. The excised SLN was x-rayed at the discretion of the surgeon. Results: $34 / 81(42 \%) \mathrm{CNBs}$ had metastases (mets); $4 / 34(11.8 \%$ ) had normal US morphology. $47 / 81$ (58\%) had negative cores, $11 / 47$ (23.4\%) with abnormal US morphology $(\mathrm{p}<.01)$; $41 / 47(87 \%)$ had negative, and 6/47 (13\%) had positive SLNs, 3/6 (50\%) with falsely normal US morphology. In 31/81(38.3\%) with positive CNB, a complete axillary dissection was done without a SLN biopsy and in 8 there was only one positive node corresponding to the positive CNB. In $42 / 50$ (84\%), where either the SLN was x-rayed or the core and the only node were concordant positive, the axillary node undergoing CNB was the 1 st SLN $(p=<.001)$. Sensitivity of US-guided CNB was $85 \%$, positive predictive value $=100 \%$, negative predictive value $87.2 \%$, specificity $100 \%(\mathrm{p}=<.001)$. Mets missed by CNB were $6.9+3.9 \mathrm{~mm}$ whereas those found on needle core were $19.7+15.3 \mathrm{~mm}$. $18.9 \%$ of normal-appearing nodes contained mets. $25 \%$ of abnormal appearing nodes were tumor-free. Conclusions: The correct SLN was identified preoperatively by US in $84 \%$ of BRCA cases even when the node was morphologically and/or pathologically normal. US with needle core biopsy of axillary SLNs in BRCA may allow the patient with mets to avoid an operative SLN biopsy. 


\section{P193}

Ultrasound-guided sentinel node procedure of non-palpable breast carcinoma P. Gobardhan, ${ }^{1 *}$ E. Theunissen, ${ }^{2}$ P. De Hooge, ${ }^{,}$C. Perre,${ }^{1}$ P. Davids, ${ }^{1}$ V. Bongers. ${ }^{1}$ 1. Surgery, Diakonessenhuis, Utrecht, Netherlands; 2. Mesos Hospital, Utrecht, Netherlands.

Background The sentinel node ( $\mathrm{SN}$ ) procedure has been well established for palpable breast tumours. For non-palpable tumours ultrasonography (US)can be used to guide the tracer injection. We evaluated the US guided injection technique for the SN procedure in patients with non-palpable breast tumours Method Two hundred consecutive patients with nonpalpable breast tumours had peritumoral 99Tc-nanocolloid injections using a $7.5 \mathrm{MHz}$ US probe (NPT-group). In case of ultrasonographically non-visible cancers a guide wire was placed and the tracer was given using US to aim the injection at the tip of the wire. The yield of this technique was compared to the results of the $\mathrm{SN}$-procedure in 850 patients with palpable tumours (PT group). Results SNs were identified on preoperative lymphoscintigraphy in 199 patients in the NPT group (99.5\%), and in 843 of the PT group (99.2\%). Parasternal SNs were visualized in 35 patients in the NPT group (17.5\%) and in 154 in the PT group (18.1\%). Axillary SNs were successfully retrieved in $98 \%$ of the patients in the NPT-group, and in $99 \%$ of the PT-group. Metastases were more common in the PT-group ( $35 \%$ vs. $23 \% ; \mathrm{P}<0.001)$. Conclusion Using ultrasound to guide peritumoral tracer injection for $\mathrm{SN}$ procedure in non palpable breast cancers results in lymphoscintigraphic visualization rates and surgical retrieval rates that are similar to the yield of the SN procedure in patients with palpable breast cancers.

\section{P194}

Plasminogen Activator System Localization in Ductal Carcinoma In Situ (DCIS) T.C. Hurd, ${ }^{2}$ S. Sait, ${ }^{1}$ S. Kogha,${ }^{3}$ J. Winston, ${ }^{1}$ H. Lankes, ${ }^{1}$ M. Martinick, ${ }^{1}$ R. Saxena, ${ }^{1}$ G. Markus, ${ }^{1}$ S. Harvey, ${ }^{1}$ J.F. Gibbs. ${ }^{1}{ }^{1} 1.6 I$ Surgery and Endoscopy, Roswell Park Cancer Inst., Buffalo, NY; 2. University of Texas, San Antonio, San Antonio, TX; 3. Kohga Community Hospital, Yaizu, Japan.

Background: The lack of prognostic factors in DCIS that reliably identify biologically aggressive tumors adversely effects optimal management. The uPA system comprised of urinary plasminogen activator (uPA), its receptor, uPAR and its inhibitor (PAI-1) are critical elements for tumor invasion and their expression in invasive breast cancer can predict clinical outcome. Expression of the uPA system in ductal carcinoma in situ (DCIS) may be relevant in defining histologic subsets of DCIS with invasive potential. Methods: Localization of uPA, uPAR and PAI- 1 was investigated immunohistochemically in 60 formalin fixed paraffin embedded in situ breast tumors using monoclonal antibodies and the avidin-biotin peroxidase procedure. FISH experiments were performed to determine whether uPA was present in cancer cells themselves or derived from stromal elements. Results: The median age was 55 yrs. (44-89). 22 tumors demonstrated high nuclear grade with necrosis (comedo). The remaining 38 tumors were of low or intermediate nuclear grade with or without necrosis and were classified as non-high grade (non-comedo). uPA was ubiquitously expressed in the malignant ductal epithelium of $95 \%(57 / 60)$ of DCIS tumors studied (Table 1). Morphologically normal ductal epithelium, adjacent to DCIS, stained positively for UPA and UPAR while distal normal breast epithelium did not demomonstrate any immunolocalization. uPAmRNA was detected by FISH in the malignant ductal epithelium. uPAR was expressed in $27 \%(6 / 22)$ of comedo and $24 \%(9 / 38)$ of non-comedo DCIS. In comparing co-expression, UPA and uPAR were co-expressed in only $25 \%(15 / 60)$ tumors. PAI- 1 was infrequently expressed in comedo $(3 / 22)$ and absent in non-comedo DCIS. Conclusions: This study identifies the presence of UPA and UPAR in both comedo and non-comedo DCIS. The infrequent expression of PAI-1 suggests its lack of involvement in DCIS. It may be speculated that co-expression of UPA and its receptor may identify subsets of DCIS with an increased risk for progression to invasive disease. If so, then expression of uPA system components may have prognostic and therapeutic significance in DCIS
Expression of UPA, UPAR, and PAI-1 in DCIS

\begin{tabular}{|c|c|c|c|c|}
\hline & \multicolumn{2}{|c|}{ TUMOR (\%) } & \multicolumn{2}{c|}{ Adjacent Normal Breast (\%) } \\
\hline High Grade + Necrosis (comedo; N=22)* & Present & Absent & Present & Absent \\
\hline uPA & $20(91)$ & 2 & 10 & $12^{*}$ \\
\hline uPAR & $6(27)$ & 16 & 9 & $11^{*}$ \\
\hline PAI-1 & 3 & 19 & 3 & $17^{*}$ \\
\hline uPA/uPAR Co-expression & $6(27)$ & 16 & $9(45)$ & $11^{*}$ \\
\hline Non-High Grade (non-comedo; N=38) & & & & \\
\hline uPA & $37(97)$ & 1 & 13 & 25 \\
\hline uPAR & $9(24)$ & 29 & 10 & 28 \\
\hline PAI-1 & 0 & 38 & 3 & 35 \\
\hline uPA/uPAR Co-expression & $9(24)$ & 29 & $10(26)$ & 28 \\
\hline
\end{tabular}

${ }^{\star}$ Normal breast epithelium was not present on the slide in 2 cases examined

\section{P195}

Safety of Methylene Blue Dye for Lymphatic Mapping in Breast Cancer S. Zakaria, ${ }^{*}$ T. Hoskin, A.C. Degnim. Mayo Clinic, Rochester, $M N$.

Introduction: Isosulfan blue dye is expensive and can cause anaphylaxis when used for sentinel node biopsy (SNB). The objective of this study was to determine efficacy and safety of MBD for SNB in large series. Methods: Following IRB approval, a retrospective chart review was performed on breast cancer patients undergoing SNB with MBD from 8/03-6/06. All patients received preoperative isotope injection. Data were collected for clinicopathologic factors, volume and dilution of dye, dye uptake in sentinel nodes (SNs), and complications. Mapping success and frequency of complications were determined. Results: 399 patients with breast cancer underwent 402 SNB procedures using MBD. Mean age was 61 , and mean tumor size was $1.8 \mathrm{~cm}$. The volume of dye injected was $3 \mathrm{ml}$ in $235 / 402$ cases $(58 \%), 4 \mathrm{ml}$ in $107(27 \%)$ less than $3 \mathrm{cc}$ in $42(10 \%)$, and unknown in $18(5 \%)$. Full strength dye $(10$ $\mathrm{mg} / \mathrm{ml}$ ) was injected in the first 96 cases, and the remaining 306 received increasing dilutions over the study period. Dye dilutions are known in 292 cases: $5 \mathrm{mg} / \mathrm{ml}$ in $16 \%, 3.3 \mathrm{mg} / \mathrm{ml}$ in $17 \%, 2.5 \mathrm{mg} / \mathrm{ml}$ in $31 \%$, and $1.25 \mathrm{mg} / \mathrm{ml}$ in $36 \%$. Overall technical success of SNB using both MBD and radioisotope for mapping was $99.7 \%$. Identification rate of a blue SN with MBD was $88 \%$ overall, increasing stepwise from $81 \%$ in the first year to $94 \%$ in the most recent six months. Information on complications was available in 400 cases. There were no cases of anaphylaxis or major complications. Minor complications were noted in $33 / 400$ cases $(8 \%)$. Immediate hypersensitivity reactions in the form of wheal and flare response at the site of injection occurred in two patients $(0.5 \%)$ and resolved with antihistamines. Other minor complications at the site of injection included: local inflammatory reaction (erythema or induration, $5 \%$ ), blue staining (1.5\%), and focal skin necrosis $(1.0 \%)$. Complications were more common when dye was undiluted $(13 / 95=14 \%)$ compared to cases where dye was diluted $(20 / 305=6.5 \%), p=0.02$. Conclusion: Methylene blue dye is as effective as isosulfan blue dye for sentinel lymph node mapping in breast cancer. Complications of MBD injection are not life-threatening and are less likely to occur when MBD is diluted.

\section{P196}

SKIN SPARING MASTECTOMY C.D. Robles-Vidal, ${ }^{*}$ P. VillarrealColin, M. Drucker-Zertucha. Breast Tumors, Instituto Nacional de Cancerologia de Mexico, Mexico City, Mexico city, Mexico.

BACKGROUND: Breast Cancer(BC) is one of main health problems in women in Mexico. Surgical treatment has changed, modified radical mastec- 
tomy, breast conservation and sentinel node, becoming less invasive and disfiguring. Skin-sparing mastectomy(SSM), in which the majority of a patient's breast skin and inframammary fold is preserved, greatly enhances the aesthetic results of breast reconstruction. METHODS: A retrospective review was performed in patients who underwent SSM and immediate breast reconstruction from $04 / 1997$ to $12 / 2004$. Records were analyzed for each patient, including type of tumors and treatment. RESULTS: SSM with immediate reconstruction was performed in 91 patients ( 4 patients with bilateral cancer and 10 with prophylactic mastectomy in the other breast). Average age was 40 years. $77 \%$ were pre-menopausal patients. Twenty-four $(27 \%)$ have familiar history of breast cancer. Five $(5.5 \%)$ have contralateral BC. The histological diagnoses was ductal carcinoma in 71 cases $(74.7 \%)$, lobular carcinoma in 7 cases $(7.3 \%)$, DCIS in 16 cases $(16.8 \%)$ and 1 case of tubular carcinoma (1\%). Neoadjuvant chemotherapy was performed in $32.9 \%$ (30 patients) and radiotherapy preoperative in $7.7 \%$ ( 7 patients). 58 patients underwent adjuvant chemotherapy $(63.3 \%)$ and 27 received adjuvant radiotherapy $(29.6 \%) .50$ patients had a TRAM flap reconstruction, tissue expander-implant (27 patients), implants in 23 cases and latissimus flap and implant in 5 patients. The complication rate was 36\%, 29 minor and 9 mayors. Six of 9 mayor complications associated with radiotherapy. 6 out of 7 patients with preoperative radiotherapy underwent complications ( 3 mayors) and 11 of 27 patients with adjuvant radiotherapy have any type of complication (4 mayors). The aesthetic result were: $63.3 \%$ excellent or good; $14.4 \%$ regular; $7.7 \%$ poor and $14.4 \%$ not reported. The follow-up average was 66 months (13-100 months), four recurrences were detected (4.4\%); 3 systemic and 1 local-regional and systemic. CONCLUSIONS: SSM is an oncological safe procedure in early $\mathrm{BC}$ with recurrence rates similar to non-SSM, the complication rates were comparable to conventional mastectomy, being mostly associated to radiotherapy.

\section{P197}

Immune monitoring of $\mathrm{CD}^{+} \mathrm{CD25}^{+} \mathrm{FoxP3}^{+}$regulatory $\mathrm{T}$ cells in a novel HLA Class-II HER2/neu peptide vaccine clinical trial in breast cancer patients M.T. Hueman, ${ }^{2}$ ' J.P. Holmes, ${ }^{1}$ C.E. Storrer, ${ }^{1}$ Y.H. Jama, ${ }^{1}$ A.M. Smith, ${ }^{1}$ S. Khoo, ${ }^{1}$ A. Stojadinovic, ${ }^{2}$ S. Ponniah, ${ }^{1}$ G.E. Peoples. ${ }^{3}$ 1. Department of Surgery, Building A, Cancer Vaccine Development Laboratory, Bethesda, MD; 2. Walter Reed Army Medical Center, Washington, DC; 3. Brooke Army Medical Center, Fort Sam Houston, TX.

Introduction: Regulatory $\mathrm{T}$ cells (Tregs) expressing FoxP3 which are found predominantly in the $\mathrm{CD} 4^{+} \mathrm{CD} 25^{+}$hi population $\left(\mathrm{CD}^{+} \mathrm{CD} 25^{+}\right.$hiFoxP3 $\left.{ }^{+}\right)$have been shown to be increased in cancer patients and may play a role in suppression of immune responses against cancer cells. We are currently conducting a clinical trial with a novel HLA Class-II HER2/neu peptide (AE37) in breast cancer $(\mathrm{BrCa})$ patients. To monitor the potential induction of Tregs, we have analyzed peripheral blood lymphocytes (PBL) from vaccinated patients for the presence of Tregs and correlated our findings with functional immune responses to the vaccine. Methods: $6 \mathrm{BrCa}$ patients have received 6 monthly injections of the AE37 vaccine in a dose escalation safety study. PBL obtained pre- and post-vaccination were stained with anti-CD4, CD25 and two different FoxP3 antibodies (PCH101 and 236A/E7) and analyzed by flow cytometry. Cells were also cultured ex vivo with AE37 peptide to measure proliferation (3H-thymidine-cpm) and cytokine secretion (IFN-gamma ELISPOT). Clinical DTH responses and local reactions at vaccination sites were monitored. Results: The two FoxP3 antibodies yielded very similar results which showed that Tregs were reduced in all 6 patients post-vaccination with pre- and post-vaccination levels of $1.68-2.93 \%$ (pre-) vs. $0.52-1.57 \%$ (post-) with an average significant decrease of $2.33+/-0.56 \%$ (pre-) vs. $1.04+/-0.56 \%$ (post-), $\mathrm{P}=0.02$. More importantly, the decrease in Treg levels correlated with increased AE37 vaccinespecific proliferative responses in all 6 patients, $0-6645 \mathrm{cpm}$ (pre-) vs. 1231 $19078 \mathrm{cpm}$ (post-) with an average significant increase of $3169+/-2500 \mathrm{cpm}$ (pre-) vs. $6047+/-8453 \mathrm{cpm}$ (post-), $\mathrm{P}=0.01$. Clinical DTH responses and local reactions at vaccination sites were also increased in all patients tested. Increased IFN-gamma secreting cells was seen in $4 / 6$ patient samples analyzed. Conclusion: Our results show that the AE37 vaccine does not induce Tregs. Furthermore, the reduction of Tregs in vaccinated patients appears to correlate with a robust functional immunologic response suggesting that the AE37 vaccine may be clinically useful.

\section{P198}

Risk of Breast Cancer In Women With Bloody Nipple Discharge L.J. Tesche, ${ }^{*}$ D.W. Ollila, N. Klauber-Demore, S.A. Mckenney, J. Choi, H.J. Kim, B.F. Calvo, A.A. Meyer, M.O. Meyers. Surgery, University of North Carolina, Chapel Hill, NC.

Introduction: Bloody nipple discharge has been associated with breast cancer in $20 \%$ of patients at a quaternary cancer center. We hypothesized that the risk of malignancy is lower in a comprehensive breast disease program with a broader population base. Methods: Under IRB approval, 1,891 surgical breast biopsies at our institution from July 1998 through December 2005 were reviewed. 109 were performed for bloody nipple discharge in 100 patients. Clinical, radiographic and pathologic results were collected in all cases. Data was analyzed by Fisher's exact test. Results: All patients had either bloody $(n=100)$ or guaiac positive $(n=9)$ discharge. There were 44 premenopausal and 63 postmenopausal women. 40 patients had prior breast biopsies. All had either a mammogram $(99 \%)$ or a breast ultrasound $(81 \%)$ performed. Positive findings were seen on $24 \%$ of mammograms and $46 \%$ of ultrasounds. There were 8 malignant diagnoses (7\%) of which 6 were DCIS and 2 were invasive carcinoma. Of the 54 patients with a positive radiographic finding, 7 had a breast cancer $(13 \%)$ whereas only one patient out of $56(2 \%)$ without a radiographic finding had a malignant diagnosis $(\mathrm{p}=.03)$. A positive mammogram was the only risk factor predictive of malignancy $(p=.02)$. Conclusion: This is the largest series in recent literature examining this problem. The incidence of malignancy associated with bloody nipple discharge in our series is lower than previously reported. Positive breast imaging was significantly predictive of malignancy as compared to negative findings ( $13 \%$ vs. $2 \%)$. These data are important in counseling patients with bloody nipple discharge regarding the risk of malignancy. If confirmed, it may be reasonable to observe selected patients with bloody nipple discharge who have unremarkable breast imaging.

\section{P199}

Chest Wall Resection and Reconstruction (CWRR) for Breast Cancer S. Khera, ${ }^{*}$ C.E. Cox, E.L. Dupont, J.M. Cox, T.L. Peterson, N. Allred, D.M. Hasson, M. Urish, D. Ramos, M. Meyers. Moffitt Cancer Center, Tampa, FL.

Introduction We have surgically treated a select number of patients with local chest wall involvement of breast cancer. Patients with isolated sternal, rib, and extensive deep chest wall tumors have undergone CWRR using a mesh / methylmethacrylate sandwich technique as a one-stage procedure. These selected patients may also have intractable pain or fungation as a reason for resection. This report reviews our experience and recommendations for its use. Survival and treatment outcomes are reviewed comparing patients treated with CWRR to those patients who were treated with limited soft tissue resection. Methods: We retrospectively reviewed our single-institutional experience with CWRR using the Marlex (Composix), methylmethacrylate sandwich technique. With IRB approval, all breast cancer patients who had this procedure due to extensive and/or deep chest wall involvement were entered into our retrospective chart review along with patients with superficial chest wall involvement who were treated with limited resection. All records were reviewed for age, initial staging, histology, treatment, node information, and follow-up data. The average age was 62 years for patients who had CWRR and 67 years for patients who had limited resection. Results: The results of our experience with CWRR for breast cancer are shown in Table 1. There were 44 patients who had this one-stage procedure and there were 25 patients who did not. The mean chest wall tumor size for patients who underwent CWRR was $6.05 \mathrm{~cm}$ and $2.92 \mathrm{~cm}$ for those who had limited resection. Mean survival time for patients with CWRR and for patients who had limited resection did not differ. There were $68 \%$ still alive who had CWRR and $56 \%$ still alive who had limited resection. Radiation therapy was delivered to 
$59 \%$ of patients who had CWRR and 52\% of patients who had limited resection. Conclusions: Our study establishes the survival and palliative benefit for breast cancer patients with isolated local extensive and/or deep chest wall involvement undergoing chest wall reconstruction using the Marlex methylmethacrylate sandwich technique.

\section{Chest Wall Involvement in Breast Cancer}

\begin{tabular}{|c|c|c|}
\hline & $\begin{array}{c}\text { Chest Wall Resection With No } \\
\text { Reconstruction (25 Patients) }\end{array}$ & $\begin{array}{c}\text { With Chest Wall } \\
\text { Resection/Reconstruction (44 Patients) }\end{array}$ \\
\hline Mean Age (years) & 67 & 62 \\
\hline $\begin{array}{c}\text { Mean Chest Wall } \\
\text { Tumor Size (cm) }\end{array}$ & 2.92 & 6.05 \\
\hline $\begin{array}{c}\text { Mean Total Positive } \\
\text { Nodes }\end{array}$ & 4.33 & 2.53 \\
\hline $\begin{array}{c}\text { Mean Total Nodes } \\
\text { Excised }\end{array}$ & 13.86 & 13.79 \\
\hline Initial Stage (\%): & 0.00 & 2.78 \\
\hline Stage 0 & 4.00 & 19.44 \\
\hline Stage 1 & 16.00 & 52.78 \\
\hline Stage 2 & 20.00 & 25.00 \\
\hline Stage 3 & 0.00 & 0.00 \\
\hline Stage 4 & 44 & 32 \\
\hline Dead (\%) & 56 & 68 \\
\hline Alive (\%) & 52 & 59 \\
\hline $\begin{array}{c}\text { With Radiation } \\
\text { Therapy (\%) }\end{array}$ & 48 & 41 \\
\hline $\begin{array}{c}\text { Without Radiation } \\
\text { Therapy (\%) }\end{array}$ & & \\
\hline
\end{tabular}

\section{P200}

Breast cancer subtypes; in Pre-menopausal African-American women C. Ihemelandu, * R. Dewitty, L. Leffall, H. Mezghebe, S. Siram, W. Frederick. Howard University Hospital, Washington, DC.

Introduction: - Breast cancer is currently viewed as a heterogeneous disease made up of various subtypes, with distinct differences in prognosis and response to therapy. Our goal was to study the distribution, prevalence and clinical association for the different breast cancer subtypes in pre-menopausal African-American women. Methods: - A retrospective analysis of data obtained from tumor registry, for all pre-menopausal African-American women $<50 \mathrm{yrs}$, diagnosed with breast cancer from 1998-2005 who had assessable data $(n=128)$. Results: - The luminal A subtype was the most prevalent (53.9\%), vs. basal cell-like $(20.3 \%)$, luminal B $(13.3 \%)$ and HER-2/neu $(12.5 \%)(\mathbf{P}<.001)$. However when stratified by age in each subtype, results showed the basal cell-like subtype to be more prevalent $(59.1 \%$ ), among pre-menopausal African-American women $<35 y$ rs when compared to those $>35 \mathrm{yrs}(12.3 \%)(\mathrm{P}<.001)$, and the luminal A subtype to be more prevalent among those $>35 \mathrm{yrs}(59.4 \%)$ when compared to those $<35$ yrs $(27.3 \%)(\mathrm{P}<.006)$. The luminal B and HER-2/neu subtypes showed no variation with age. P53 mutation was more prevalent in the basal like subtype compared to luminal A $(52.2 \%$ vs. $16.7 \%, \mathrm{P}<.003)$. The basal cell-like subtype showed no variation with age for P53 mutation, however in the Luminal A subtype patients $>35 \mathrm{yrs}$ were more likely not to have a P53 mutation $(85.2 \%$ vs. $14.8 \%, \mathrm{P}<.001)$. The expression of the BCL2 gene differed by subtype, with the luminal A subtype more likely to overexpress the BCL-2 gene (65.7\% luminal A vs. $14.3 \%$ basal cell-like, $12.9 \%$ luminal $\mathrm{B}$ and $7.1 \% \mathrm{Her}-2 /$ neu, $\mathrm{P}<.001)$. The expression of the BCL-2 gene also varied with age with patients $>35 y$ rs more likely to over-express the BCL2 gene. Though not statistically significant, HER-2/neu and basal like subtypes had the shortest survival time $(\mathrm{P}<.117)$. Conclusion: - Basal cell-like tumors are more prevalent in pre-menopausal women $<35 \mathrm{yrs}$, while the luminal A tumors occurred at a higher prevalence among the pre-menopausal women $>35$ yrs. An inverse relationship between the expression of BCL-2 and P53 gene mutation was noted in both groups. Further research in this area is needed.

\section{P201}

DCIS Size and Resection Volume Predict Margin Status L. Golkar, ${ }^{1 *}$ K.A. Melstrom, ${ }^{2}$ D.J. Winchester. ${ }^{3}$ 1. Department of Surgery, Northwestern University Feinberg School of Medicine, Chicago, IL; 2. Department of Surgery, Loyola University Medical Center, Maywood, IL; 3. Department of Surgery, Evanston Northwestern Healthcare, Chicago, IL.

INTRODUCTION: There is strong evidence that breast conservation therapy (BCS) with negative margins for ductal carcinoma in situ (DCIS) is associated with low rates of recurrence. In patients with positive margins, residual carcinoma can be seen in as high as $67 \%$ of re-excision specimens. In this study our goal was to identify tumor and treatment factors associated with positive margins in breast conservation therapy for DCIS. METHODS: A retrospective database review identified 741 patients diagnosed with DCIS. The current analysis included 456 of those patients treated with BCS from 2000-2005 with complete data regarding tumor and lumpectomy dimensions. Variables analyzed included tumor size $(\mathrm{cm})$, lumpectomy volume $(\mathrm{cm} 3)$, estrogen and progesterone receptor status (positive versus negative), grade, and age at diagnosis. Chi-square analysis was used to identify factors that may predict positive margins. Student's t-test was used to evaluate the relationship between tumor size, specimen volume, and surgical margin status. RESULTS: The average age of 456 patients was 60 years. $168(36.8 \%)$ had positive and $288(63.2 \%)$ had negative margins. The average size of DCIS lesions was $1.49 \mathrm{~cm}$ and was significantly larger in positive versus negative margin groups (2.02 and 1.1 $\mathrm{cm}$ respectively, $\mathrm{P}<0.001)$. The gross specimen volume was smaller in the positive versus negative margin groups $(70.8 \mathrm{~cm} 3$ and $143.4 \mathrm{~cm} 3, \mathrm{P}<0.001)$. The size to volume ratio was larger in the group with positive compared to negative margins $(0.21$ versus $0.11 ; \mathrm{P}<0.001)$. Estrogen and progesterone receptor status had no relationship to margin status $(\mathrm{P}=0.438)$. High grade lesions had a greater association to positive margin status $(\mathrm{P}=0.058)$. Age was not predictive of margin status or volume of excision ( $\mathrm{P}=0.29)$. CONCLUSIONS: Positive margins after BCS for DCIS are associated with larger lesions and a smaller volume of resection. With $36.8 \%$ of patients having positive margins, these data suggest that a more aggressive initial resection may avoid positive margins and thus lower the risk of recurrence or the need for additional surgery.

\section{P202}

The Negative Sestamibi Scan: Is a Minimally Invasive Parathyroidectomy Still Possible? A. Lal, ${ }^{*}$ H. Chen. Surgery, University of Wisconsin, Madison, WI.

Introduction: Tc-99-sestamibi scanning is utilized to determine if patients with primary hyperparathyroidism (HPT) are candidates for minimally invasive parathyroidectomy (MIP). However, if the sestamibi scan is negative, many surgeons recommend bilateral exploration because of possible multi-gland disease. The objectives of this study were to determine if patients with primary HPT and negative sestamibi scans benefit from additional imaging studies, and if they are still potential candidates for MIP. Methods: Between 3/01-4/06, 578 consecutive patients with HPT underwent parathyroidectomy by one surgeon and 458 patients had pre-operative sestamibi scans. Ninety $(20 \%)$ patients who underwent pre-operative sestamibi scans had negative results. Results: Of the 90 patients, $60(67 \%)$ had a single adenoma, $17(19 \%)$ had double adenomas and $13(14 \%)$ had hyperplasia. Seventy-four $(82 \%)$ patients underwent further localization with one or more studies including thallium subtraction scans $(n=30)$, ultrasound $(n=15)$, and intraoperative internal jugular venous sampling ( $n=49)$. Additionally the use of radioguided techniques intraperatively facilitated minimally invasive techniques. Of these 90 patients, 47 had positive localization studies, including thallium scans $(n=12)$, ultrasound $(n=5)$, radioguided detection $(n=17)$, and IJ sampling $(n=13)$. Accordingly, MIP was attempted in these patients and, $42(89 \%)$ had single adenomas and 5 were converted to bilateral exploration for double adenoma/hyperplasia. In the setting of a negative sestamibi, the sensitivity of thallium scans and ultrasound were $30 \%$ and $27 \%$ respectively. The overall cure rate in the 90 patients was $99 \%$. Conclusions: In patients with primary HPT and negative sestamibi scans, a single adenoma is the most common etiology $(67 \%)$. These patients benefit from additional localization tests as $52 \%$ will have a positive study. Therefore, even in the setting of a negative sestamibi scan, the majority of patients with primary HPT are still candidates for MIP. 


\section{P203}

Fine-needle aspiration cytology and $99 \mathrm{mTc}$-petrechnetate scintigraphy together in patients with differentiated thyroid carcinoma F. Lumachi, ${ }^{1 *}$ S. Borsato, ${ }^{2}$ A. Tregnaghi, ${ }^{3}$ L. Di Cristofaro, ${ }^{1}$ P. Zucchetta, ${ }^{4}$ M.C. Marzola, ${ }^{4}$ D. Cecchin, ${ }^{4}$ F. Bui. ${ }^{4}$ 1. Department of Surgical and Gastroenterological Sciences, University of Padua, School of Medicine, 35128 Padova, Italy; 2. Cytology Section, Department of Pathology, University of Padua, School of Medicine, 35128 Padova, Italy; 3. Radiology Section, Department of Diagnostic Medical Sciences, University of Padua, School of Medicine, 35128 Padova, Italy; 4. Nuclear Medicine, Department of Diagnostic Medical Sciences, University of Padua, School of Medicine, 35128 Padova, Italy.

BACKGROUND: Thyroid carcinoma (TC) accounts for more than $90 \%$ of all endocrine malignancies, and for less than $1 \%$ of all cancers. However, solitary thyroid nodules (TN) are common, and may be found in $5-10 \%$ of the population. The majority of the patients with TN do not have cancer, but the diagnosis of TC should be obtained prior to surgery and subsequent histological examination of the removed gland. The aim of this study was to evaluate the usefulness of fine-needle aspiration cytology (FNAC) and $99 \mathrm{mTc}$-petrechnetate scintigraphy (TS) together in patients with differentiated thyroid carcinoma. PATIENTS AND METHODS: a series of 357 consecutive patients with solitary TN of more than $10 \mathrm{~mm}$ in the greatest diameter, and no signs or symptoms of thyroid hyperfunction underwent both ultrasound-guide FNAC and TS prior to surgery. There were 284 (79.5\%) women and $73(20.5 \%)$ men, with a median age of 43 years (range 17-75 years). FNAC distinguished 3 groups of TN: benign, follicular neoplasm, thyroid cancer, while patients with "cold" TN was considered as having a thyroid tumor. RESULTS: At final pathology $61(17.1 \%)$ TC were found, of which $42(68.8 \%)$ papillary, $12(16.4 \%)$ follicular carcinomas. The sensitivity, specificity, positive predictive value, negative predictive value, and accuracy were: $95 \%, 21 \%, 22 \%, 95 \%$, and $35 \%$ for TS; $81 \%, 99 \%, 97 \%, 94 \%$, and $95 \%$ for FNAC; $98 \%, 87 \%, 92 \%, 97 \%$, and $76 \%$ for TS and FNAC together. CONCLUSIONS: Patients with "cold" TN and FNAC suggesting follicular neoplasm should be considered at risk of having TC, and thus require an adequate surgical treatment. References: Lumachi $F$ et al. Usefulness of $99 \mathrm{mTc}$-pertechenate scintigraphy and fine-needle aspiration cytology in patients with solitary thyroid nodules and thyroid cancer. Anticancer Res 2004; $24: 2531-2534$

\section{P204}

Treatment of Carcinoid Tumor Liver Metastases with Y-90 Microspheres: An Effective Cytoreduction for Disease Consolidation and Symptom Control S.A. Gulec, ${ }^{1 *}$ R. Hostetter, ${ }^{1}$ D. Schwartzentruber, K. Pennington, ${ }^{1}$ D. Bruetman, ' J. Wheeler, ${ }^{1}$ A.S. Kennedy. ${ }^{2}$ 1. Center for Cancer Care, Goshen, IN; 2. Wake Radiology Oncology, PLLC, Cary, NC.

Introduction Yttrium-90 (Y-90) microsphere therapy via hepatic arterial administration is emerging as a mainstream treatment modality in the management of patients with primary and metastatic liver cancer. The selective nature of microsphere distribution allows thè delivery of high doses of radiation to tumors while keeping the exposure of the un-involved liver at a minimum. The therapeutic ratio and outcome are more pronounced in carcinoid tumor liver metastases (CTLM)due to the hypervascular nature of these tumors as well as their protracted clinical course. Methods Treatment records and follow-up data on 20 patients (9 M, $11 \mathrm{~F}$, and ages 37 - 71) with CTLM who underwent a selective internal radiation treatment (SIRT) with Y-90 resin microspheres (SIR-SpheresTM Sirtex Medical, Lake Forest, IL) were retrospectively reviewed. Liver toxicity was assessed clinically and by liver function tests, and the response to treatment by Octreoscan, CT, and tumor markers. Results: All patients had unresectable liver disease with an average tumor volume of $484.6 \mathrm{cc}$ (Range: 30 to $1,771 \mathrm{cc}$ ). Fifteen of the twenty patients $(75 \%)$ were symptomatic despite maximal medical treatment.Seven patients received multiple treatments (6 staged lobar, 1 repeat whole liver). The average number of treatments per patient was 1.45 (range: 1-3). Median follow-up was 4 months (range: $1-20$ months). The average administered activity was $1.6 \mathrm{GBq}(0.6$ to $3.2 \mathrm{GBq})$. Liver absorbed doses ranged from $0.3 \mathrm{~Gy}$ to $99.5 \mathrm{~Gy}$ (mean: $28.9 \mathrm{~Gy}$ ). Tumor absorbed doses ranged from 19.2 Gy to 262.7 Gy (mean: 128.5 Gy). No treatment-related liver failure, clinical radiation hepatitis, or veno-occlusive disease were seen. An objective response by CT and/or Octreoscan was observed in 18/20 (90\%) and symp- tom control was achieved in 11 of $15(73 \%)$ patients. Conclusions: Yttrium90 (Y-90) microsphere therapy produced a clinically notable objective response in all patients with satisfactory symptom control and no significant liver toxicity.

\section{P205}

Surgery for pancreatic incidentilomas G.J. Lahat,* I. Shtzigovsky, I. Nachmany, N. Lubetzky, O. Zmora, M. Ben haim, R. Nakash,

J. Klausner. Sourasky medical center, Tel Aviv, Israel.

Introduction: Incidental abdominal tumors are increasingly diagnosed, mainly due to the availability of high quality imaging tools. While incidental findings in several organs such as the adrenals are well studied, pancreatic incidentilomas received little attention, therefore remained poorly characterized. Our objective was to assess the pathological characteristics of operated pancreatic incidentilomas. Methods: 415 consecutive pancreatectomies performed over the last decade were retrospectively reviewed. Asymptomatic patients with incidental findings were analyzed and compared to the rest of the cohort. Results: A total of $48(11.6 \%)$ patients presented with pancreatic incidentiloma. All patients were operated. $25(52 \%)$ located in the head and $23(48 \%)$ in the distal pancreas. 25 pancreaticoduodenectomies and 23 distal pancreatectomies were performed. Peri operative mortality rate was $2.1 \%(1 / 48)$ in the pancreatic incidentiloma group compared to $3.5 \%$ in the non incidentilomas group $(\mathrm{p}=0.03)$.Common diagnoses were mucinous cyst adenomas $(23 \%, n=11)$, IPMT $(23 \%, n=11)$ ductal carcinoma ( $23 \%, n=11)$ and neuroendocrine tumors $(18.7 \%, n=9)$. Malignancy rate for pancreatic incidentilomas was $28 \%(n=13)$ compared to $79 \%$ in the non incidentilomas group $(\mathrm{p}<0.01) .31(66 \%)$ patients had pre-malignant tumors and only $3(6 \%)$ had benign pathologies with very low malignant potential. Conclusion: Pancreatic incidentilomas are becoming increasingly common. Given its high malignant potential surgery appears to be the treatment of choice.

\section{P206}

Posterior Pelvic Resection of Recurrent Rectal Cancer Has Survival Benefit in Over 30\% of Patients, But Outcome is Governed by Strategic Tumor Biologic Factors H.J. Wanebo, ${ }^{1}$ G. Begossi, ${ }^{2}$ M. Tantorski, ${ }^{1}$ J.F. Belliveau. ${ }^{1}$ 1. Roger Williams Medical Center, Providence, RI; 2. University of Mass Medical Center, Worcester, MA.

Introduction: Locally advanced or recurrent rectal cancer represents a formidable problem for patient and surgeon. Background: Although adoption of total mesorectal excision coupled $\mathrm{w} /$ neoadjuvant/adjuvant therapy has improved outcome, local failure rates are still significant and responsible for high mortality. We have updated our series of curative abdominosacral resection (ABSR) for advanced and recurrent rectal cancer to determine prognostic factors affecting outcome. Methods: ABSR with curative intent was performed in $63 \mathrm{pts}$ w/rectal cancer ( 58 recurrence, 5 primary tumor). All pts had localized disease involving musculoskeletal pelvis. Survival was analyzed w/Kaplan-Meier method. Regression analysis compared selected prognosticators, such as preoperative CEA level, age, gender, stage, surgery of primary-tumor and adequacy of ABSR (RO-vs.-R1 resection). Results: Perioperative mortality at 60days was $6 \%(4 \mathrm{pts})$. All pts encountered various medical/surgical complications. Overall and disease-free-median survivals were 32/18 months respectively. (See table.) Conclusion: ABSR provides a curative surgical opportunity for long-term-survival. The 5-yr overall and disease-free-survival was $30 \% / 20 \%$ respectively. Prognostic factors affecting survival included type of primary surgery (APR vs. AR), CEA level ( $</>5 \mathrm{ng} / \mathrm{ml})$, performance of RO vs. R1 resection. Patient selection based on clinical and radiologic staging coupled with adjuvant therapy protocols may enhance survival in this high-risk treatment group. 
Posterior Pelvic Resection of Recurrent Rectal Cancer Has Survival Benefit in Over $30 \%$ of Patients, But Outcome is Governed by Strategic Tumor Biologic Factors

\begin{tabular}{|c|c|c|c|c|c|}
\hline & $\begin{array}{c}\text { Number } \\
\text { ofPatients }\end{array}$ & $\begin{array}{c}5 \mathrm{Y} \\
\text { KMOverall }\end{array}$ & Log-Rank/Regression & $\begin{array}{c}5 \mathrm{Y} \\
\text { KMDis-Free }\end{array}$ & Log-Rank/Regression \\
\hline Overall & 59 & $30 \%$ & & $20 \%$ & \\
\hline $\begin{array}{c}\mathrm{CEA}<5 \mathrm{nn} / \mathrm{ml} \\
\mathrm{CEA}>5 \mathrm{ng} / \mathrm{ml}\end{array}$ & 2532 & $47 \% 16 \%$ & $0.02 / 0.04$ & $28 \% 15 \%$ & $\mathrm{~N} / \mathrm{ns}$ \\
\hline $\begin{array}{c}\text { Age }<65 \mathrm{Age} \\
>65\end{array}$ & 4018 & $31 \% 29 \%$ & $0.02 / 0.04$ & $20 \% 22 \%$ & $\mathrm{Ns} / \mathrm{ns}$ \\
\hline $\begin{array}{c}\text { After APR* } \\
\text { After AR* }\end{array}$ & 2924 & $20 \% 35 \%$ & $0.02 / \mathrm{ns}$ & $13 \% 21 \%$ & $0.03 / \mathrm{ns}$ \\
\hline $\begin{array}{c}\text { Primary/Stage } \\
\text { I/II* } \\
\text { Primary/Stage } \\
\text { III* }\end{array}$ & 2724 & $34 \% 23 \%$ & $\mathrm{Ns} / \mathrm{ns}$ & $13 \% 21 \%$ & $0.03 / \mathrm{ns}$ \\
\hline $\begin{array}{c}\text { Female Male } \\
\begin{array}{c}\text { Resection RO } \\
\text { R1 }\end{array}\end{array}$ & 1939 & $35 \% 27 \%$ & $\mathrm{Ns} / \mathrm{ns}$ & $28 \% 15 \%$ & $\mathrm{Ns} / \mathrm{ns}$ \\
\hline
\end{tabular}

\section{P207}

Sphincter preserving radical proctosigmoidectomy with coloanal anastomosis for neoadjuvant treated cancers within $1 \mathrm{~cm}$ of the anorectal ring J.H. Marks, ${ }^{1 *}$ D.H. Hunt, ${ }^{1}$ M. Mohiuddin, ${ }^{2}$ L.A. Kosinski, ${ }^{1}$ G.J. Marks. ${ }^{1}$ 1. The Lankenau Hospital and Institute for Medical Research, Wynnewood, PA; 2. Geisinger Health System, Wilkes Barre, PA.

Introduction: Neoadjuvant therapy and newer surgical techniques have extended the use of sphincter preserving surgery (SPS) to include cancers in the distal one third of the rectum. Long-standing questions exist regarding the potential increased risk of local failure with SPS for the lowest lying rectal cancers, and outcome data is essential. We report the results of SPS performed following multimodal neoadjuvant therapy for patients with cancers in the distal $1 \mathrm{~cm}$ of the rectum (superior to the anorectal ring). Methods: From 1997 to 2006 as part of a prospective study, 40 consecutive patients with cancers in the terminal $1 \mathrm{~cm}$ of the true rectum underwent curative radical proctosigmoidectomy with coloanal anastomosis after neoadjuvant chemoradiation. Excluded were patients whose cancers remained fixed after therapy. External radiation (4500 to $6500 \mathrm{cGy}), 5 \mathrm{FU} \pm$ leucovorin was administered with a 6 to 10 week interval before surgery. Included were 28 men, 13 women; 38 to 83 years of age. All follow-up was conducted by the surgeons. Results: One major morbidity occured, an anastomotic leak which required maintenance of the stoma. There were no perioperative deaths. Median follow-up is 24 months. The Kaplan-Meier 5-year actuarial survival is $89.9 \%$. Local recurrence occurred in 1 patient $(2.5 \%)$ who subsequently developed metastatic disease. Three patients (7.5\%) died with disease and 3 patients $(7.5 \%)$ are alive with disease. Conclusion: For rectal cancers within $1 \mathrm{~cm}$ of the anorectal ring that are mobile after neoadjuvant therapy, SPS may be accomplished with a low rate of local recurrence and good survival.

Staging

\begin{tabular}{|c|c|c|c|c|}
\hline T - Stage & T0 & T1 & T2 & T3 \\
\hline Clinical (N) & 0 & 0 & 4 & 36 \\
\hline Post-neoadjuvant Therapy & 5 & 1 & 15 & 19 \\
\hline Pathological & 10 & 5 & 10 & 15 \\
\hline
\end{tabular}

\section{P208}

Pancreatoduodenectomy in Patients over 80 Years of Age: Do the Ends Justify the Means? B. Chung, * J.L. Peacock, L.O. Schoeniger. Surgery, University of Rochester, Rochester, NY.

It is expected that pancreatic cancer (PC) will be increasingly encountered in the very elderly patient population. A small number of studies have previously reported that pancreatoduodenectomy (PD) can be performed with adequate safety in patients over 80 years of age but an increased rate of mortality has been demonstrated. The management of this problem remains difficult and controversial. To assist in clinical decision making, we have evaluated perioperative outcomes and long-term survival after PD in a geriatric patient population. Methodology: A retrospective case review was performed using hospi- tal discharge databases and operative logs at a high-volume academic institution. Patients over the age of 80 who underwent PD between 1997 and 2006 were identified. Data collected included indications for PD, perioperative mortality and complications, type of PD, and long-term survival. Results: 19 patients over 80 years old were identified, and 2 patients were greater than 90 years of age at surgery. Chart review indicated that all patients had a preoperative diagnosis of obstructive jaundice and a mass in the head of the pancreas. The majority of patients underwent pylorus-preserving PD $(68 \%)$ versus classical PD (32\%). Major vessel resection and reconstruction was performed in $16 \%$ of cases. Final pathology revealed a malignant diagnosis in $89 \%$ with 14 patients having $\mathrm{PC}$ and 2 patients with benign diagnoses. There were no perioperative deaths and all patients were discharged from the hospital. Median length of stay was 13 days. The overall major and minor complication rate was $42 \%$, with the most common one being pancreatic fistula (26\%). 4 out 14 patients with PC received post-operative chemoradiation. Mean survival rate for allcomers was 15 months. Survival rates in all-comers at 6 months, 1 year, and 2 years were $84 \%, 53 \%$, and $26 \%$ respectively. Survival rates for patients with PC were $86 \%, 43 \%$, and $21 \%$ respectively. Conclusions: We have demonstrated that perioperative morbidity is controllable and meaningful long-term survival is possible following pancreatoduodenectomy when performed on patients 80 years of age and older at a high-volume institution.

\section{P209}

Prediction of outcome following liver resection of colo-rectal metastases: Do we need to modify our perspective? N. Lubezky, ${ }^{*} \mathrm{R}$. Geva, E. Shmueli, R. Nakckache, A. Figer, J. Klausner, M. Ben-Haim. TelAviv Sourasky Medical Center, Tel Aviv, Israel.

Introduction: Survival prediction following resection of liver colo-rectal metastases is based on the MSKCC clinical score. However, the validation was before the era of FDG PET-CT staging and before the wide use of Oxaliplatin, Irinotecan and biological agents as an adjuvant treatment. Methods: To identify risk factors for recurrence following complete R0 metastasectomy and chemotherapy, we conducted a multivariate analysis of prospectively collected data. Included are patients with colo-rectal cancer and metastases confined to the liver as defined by CT and PET-CT. factors such as synchronicity, number and size of lesions, MSKCC clinical score (0-5), treatment strategy ("neoadjuvant" vs. "adjuvant") and response to chemotherapy were examined. Chemotherapy was with Oxaliplatin (FOLFOX) or Irinotecan (FOLFIRI) together with bevacizumab (Avastin). Minimal follow-up time is of 1 year. Results: 60 patients (119 metastases) are included (2002-2005). Mean followup time was 31 months. The 1,2 and 3 years overall survival rates were $93 \%$, $91 \%$ and $76 \%$, and the disease free survival rates were $82 \%, 60 \%$ and $52 \%$, respectively. None of the traditional risk predictors including the MSKCC clinical score was found to be statistically significant in predicting outcome. The treatment strategy (sequence and type of chemotherapy and surgery) was also not an influential factor, unless complete clinical response was achieved following neoadjuvant chemotherapy $(\mathrm{n}=14)$. In this specific sub group 3 years survival and disease free survival were superior to other patients $(90 \%$ vs. $71 \%$; and $82 \%$ vs. $44 \%$, respectively). Conclusions: Current chemotherapy and staging may alter the accuracy of prognosis prediction following treatment of hepatic colo-rectal metastases. Re validation of previous factors is indicated. Response to chemotherapy should be included in the algorithm of patient selection.

\section{P210}

Impact of number of examined lymph nodes on prognosis in colon cancer: A population based study in North Netherlands W. Kelder, ${ }^{1 *}$ B. Inberg, ${ }^{2}$ M. Schaapveld, ${ }^{3}$ T. Wiggers, ${ }^{1}$ J.T. Plukker. ${ }^{1} 1$. Dept Surgical Oncology, University Medical Center Groningen, Groningen, Groningen, Netherlands; 2. Dept of Surg. Martini Hospital Groningen, Groningen, Netherlands; 3. Comprehensive Cancer Center Groningen, Groningen, Netherlands.

Introduction: Nodal metastasis is a determining factor in the adjuvant treatment and an important predictor of survival in colon cancer patients. Adequate surgical resection and punctual pathological examination of the resected specimen is desirable. We studied whether the reported number of lymph nodes at pathological examination of colon specimens has an effect on stage migration and survival in these patients. Methods: Between 1998 and 2002 a total of 2751 patients with colon cancer underwent a resection in 17 hospitals followed by examination in 7 pathological institutes in North Netherlands. The 
cancer registry of the Comprehensive Cancer Centre North Netherlands, which covers a population of 2.1 million, superintends the quality of guidelines and follow-up of cancer patients. Data of all colon cancer patients on TNM stage, tumor morphology and survival were available. The influence of possible determinants was tested in a general linear model for continuous variables and binary logistic regression analysis for nominal variables. Survival was calculated from the date of diagnosis until the date of death, the date of most recent linkage with the municipal population registries or last contact. Results: The number of harvested and examined nodes increased with a high T-stage $(\mathrm{p}<0,001)$ and mucinous morphology $(p=0,002)$, but decreased with age $(p<0,001)$. Localization was also of influence on the number of examined nodes. The proportion of node-positive patients increased with a greater number of examined nodes. T-stage and number of examined nodes had a significant influence on nodal status. Overall 5-year survival also increased with more examined nodes in both node positive $(49,6 \%)$ and node negative patients $(67,8 \%)$. Conclusions: T-stage, localization and age significantly influence the number of nodes examined by the pathologist. A greater number of examined nodes is associated with an increase in node-positivity and an improved accuracy of the pathological status. Conscientious pathological sampling with more harvested nodes improves nodal staging and may offer more patients adjuvant treatment and eventually a better survival.

\section{P211}

Positron Emission Tomography (PET) is Beneficial in the Preoperative Evaluation of Patients with Colorectal Cancer Recurrences: A Prospective Analysis G.C. Balch, ${ }^{1}$ D. D. Chessin, ${ }^{1}$ M. McCarter, ${ }^{2}$ T. Akhurst, ${ }^{1}$ N. Kemeney, ${ }^{1}$ D. Schrag, ${ }^{1}$ P.B. Paty, ${ }^{1}$ S. Larson, ${ }^{1}$ D.W. Wong, ${ }^{1}$ J.G. Guillem. ${ }^{1}$ 1. Surgery, Memorial Sloan-Kettering Cancer Center, New York, NY; 2. University of Colorado Health Science Center, Denver, $\mathrm{CO}$.

Introduction: The appropriate scenarios in which PET adds useful information following curative resection of colorectal cancer (CRC) remains controversial, largely due to insufficient prospective data. Our aims were to prospectively determine the reasons for obtaining a PET scan following CRC resection and assess the impact of PET on patient management. Methods: Over 24 months, 99 patients with a prior curative CRC resection had a PET scan ordered as part of the workup for suspected recurrent disease. The ordering physician was prospectively queried regarding the reasons for obtaining a PET scan and their management plan prior to PET. Following the PET scan, the physician was again queried regarding the post-PET management plan and whether PET had altered their plan. Patients were followed after their PET for a median of 28.2 months. Results: Overall, PET changed the management plan in 36 of 99 $(36 \%)$ patients. Twenty-eight patients had identified potentially resectable recurrence and had pre-PET plans for surgical intervention. Of these, $14(50 \%)$ had surgery plans cancelled as a result of PET findings. The most common reasons for obtaining a PET scan were abnormal CT or MRI $[n=54(55 \%)]$ or an abnormal carcinoembryonic antigen (CEA) level [ $n=35(35 \%)]$. Of the 54 patients with an abnormal CT or MRI, $22(41 \%)$ had a pre-PET plan for surgery. PET revealed occult secondary metastases in $11(50 \%)$ of these 22 . In another cohort, the pre-PET plan was either observation or treatment other than surgery, 4 patients $(7 \%)$ with abnormal anatomic imaging and 6 patients $(17 \%)$ with a rising CEA underwent operation instead as a result of PET. Overall, the prospectively recorded post-PET management plan was not carried out in 12 patients $(12 \%)$. Conclusions: In patients scheduled for surgical intervention as a treatment for CRC recurrence, PET appears to provide incremental clinical benefit. Half of these patients in our series had their operative plans cancelled as a result of the PET findings. PET should be considered as part of the preoperative evaluation prior to resection of recurrent colorectal cancer.

\section{P212}

Recurrence Patterns of Node Negative Colon Cancer: Implications for Sentinel Lymph Node Biopsy N.P. Reuter, * R.P. Sticca. Department of Surgery, University of North Dakota, Grand Forks, ND.

Introduction: Approximately one fourth to one third of stage I and II colon cancer patients develop recurrent disease. Recurrence patterns for stage I and II colon cancer are unknown and may imply hematogenous or lymphatic routes as the predominant mechanism for recurrence. If most recurrences are distant implying a hematogenous route of dissemination, sentinel lymph node biopsy (SLNB) for colon cancer may not be accurate in predicting patients who sub- sequently will recur. Methods: A multi-institutional retrospective review of all cases of stage I and II colon cancer from 1995 through 2000 were reviewed. Patients who were older than 16 , had negative margins, and were being treated for their first colon malignancy were included. A comparison of recurrence patterns of patients registered in the National Cancer Database between 1994 and 1998 was also made. SEER criteria were used for local, regional, and distant recurrence definitions. Results: 638 Patients were included from regiona databases. $40 \%$ were stage I while $60 \%$ were stage II. 59 of 638 patients $(9 \%)$ had a recurrence. $43(73 \%)$ of recurrences were distant, $7(12 \%)$ were regiona $7(12 \%)$ were local, and $2(3 \%)$ were not specified. Of 7224 recurrences in the National Cancer Database for lymph node negative patients, 5264 (73\%) were distant, $1000(14 \%)$ were regional, and $960(13 \%)$ were local. Conclusions : SLNB for colon cancer may not predict patients who will recur because greater than $70 \%$ of recurrences are distant. Based on these recurrence patterns $15 \%$ of all patients who undergo SLNB would receive additional adjuvant therapy with a survival benefit of $1-2 \%$. Because most recurrences are distant SLNB for colon cancer may lead to substantial numbers of patients receiving adjuvant therapy with minimal improvement in survival.

\section{P213}

Ultrastaging of Sentinel Lymph Nodes(SLNs) Versus Non-SLNs in Colorectal(CRca) Cancer - Do We Need Both? S. Saha, ${ }^{*}$ D. Wiese, M. Ghanem, B. Yestrepsky, S. Arora, N. Bassily, M. Patel, P. Ng, E. Quiachon, J. Badin. Mclaren Regional Medical center, Flint, MI.

Introduction: SLN mapping (M) accurately stages many solid tumors including CRca. SLNs are 3-5 times more likely to have metastases (mets) when ultrastaged by microsections and IHC as compared to non-SLNs examined by standard pathological methods. It is unknown whether ultrastaging of initially -ve non-SLNs would lead to higher incidence of +ve nodes. Hence, we retrospectively analyzed -ve non-SLNs by microsections and IHC in patients (pts) with CRca undergoing SLNM to determine its impact on nodal staging. Methods All pts underwent SLNM by circumferential subserosal injection of $1 \%$ lymphazurin. First 1-4 blue nodes were marked as SLNs, followed by standard oncologic resection and examined by 4 sections for $\mathrm{H} \& \mathrm{E}$ at 20-40 micron intervals and 1 section for cytokeratin (AE-1/AE-3). All non-SLNs were initially examined by single $\mathrm{H} \& \mathrm{E}$ section and initial staging was made as per AJCC criteria. We re-examined all initially -ve non-SLNs with 3 additional H\&E sections and one for IHC as in SLNs by a senior pathologist blinded to prior results. Results: There were 156 pts with Cca and 44 pts with Rca. SLNM was successful in $99 \%$ pts with $96 \%$ accuracy rate. A total of 2755 nodes $(13.78 / \mathrm{pt})$ were identified, of which $494(17.93 \%)$ were SLNs and $2261(82.07 \%)$ were non-SLNs. Nodal positivity was $46 \%$ and $16 \%$ for Cca and Rca pts respectively. Mets were detected in $20.9 \%$ of SLNs vs $8.6 \%$ of non-SLNs ( $p<0.0001$ ). The exclusive site of nodal mets was detected in $8.8 \%$ of SLNs, and $0.8 \%$ of non-SLNs $(\mathrm{p}<0.0001)$. Skip mets were found in $6 \%$ of pts. After ultrastaging all -ve non-SLNs, only $0.58 \%$ became +ve for mets in 12 pts. Of these, $10 \mathrm{pts}$ already had +ve SLNs, hence no change of staging occurred. Only $2 / 200$ pts with initially -ve nodes were found to have a cluster of tumor cells; one of which was +ve by IHC only. Thus, ultrastaging of 2065 initially -ve non-SLNs changed the staging from II to III only in $1 \%$ of pts. Conclusion: SLNM is highly accurate in staging CRca. The chance of finding additional mets by ultrastaging of all non-SLNs is extremely low $(<1 \%)$, hence of little benefit. Therefore, ultrastaging restricted to SLNs alone will assure accurate staging of CRca.

\begin{tabular}{|c|c|c|c|}
\hline \multirow[t]{2}{*}{200 Total Patients } & \multicolumn{2}{|c|}{ Total Lymph Nodes $=2755(13.78 / \mathrm{pt})$} & \multirow[b]{2}{*}{ p-value } \\
\hline & $\begin{array}{c}\text { SLN } \\
{[n=494(17.93 \%)]}\end{array}$ & $\begin{array}{c}\text { Non SLN's } \\
{[\mathrm{n}=2261(82.07 \%)]}\end{array}$ & \\
\hline Percent of nodes with metastases & $20.90 \%$ & $8.60 \%$ & $<0.0001$ \\
\hline $\begin{array}{c}\text { Nodes as exclusive site of } \\
\text { metastases }\end{array}$ & $8.80 \%$ & $0.80 \%$ & $<0.0001$ \\
\hline \multicolumn{4}{|c|}{ Number of non-SLNS $=2261(11.3 / \mathrm{pt})$} \\
\hline \multicolumn{4}{|c|}{ Initially negative non-SLNs $=2065$} \\
\hline \multicolumn{4}{|c|}{ Results after ultrastaging of all initially negative non-SLNs } \\
\hline \multicolumn{2}{|c|}{$12 / 2065$ became positive $(0.58 \%)$} & \multicolumn{2}{|c|}{$2053 / 2065$ remained negative $(99.4 \%)$} \\
\hline \multicolumn{2}{|c|}{$2 / 200$ pts were upstaged $(1 \%)$} & \multicolumn{2}{|c|}{$198 / 200$ pts staging unchanged ( $99 \%)$} \\
\hline
\end{tabular}


P214

Model for End-Stage Liver Disease Score Predicts Survival in Patients with Hepatocellular Carcinoma C.M. Contreras, ${ }^{*}$ J. Behrens, C.S. Cho, D.M. Mahvi, L.F. Rikkers, S.M. Weber. General Surgery, University of Wisconsin Hospital \& Clinics, Madison, WI.

Introduction: Effective treatment of hepatocellular carcinoma (HCC) remains a challenge despite multimodality therapies. We sought to evaluate predictors of outcome in patients undergoing surgical treatment of $\mathrm{HCC}$ with resection and/or ablation. Methods: Patients with HCC treated with surgical resection or ablation from 1990-2006 were identified from a prospective hepatobiliary database and hospital records. Patients undergoing liver transplantation were excluded. Demographic data, tumor characteristics, hepatitis and/or cirrhosis status, and outcome were analyzed. Results: A total of 90 patients who underwent surgical exploration with curative intent were identified, including 70 patients undergoing hepatic resection and 20 ablation. Patients serologically positive for hepatitis B, C, or both comprised $36 \%(33 / 90)$ of the group. Histological, radiological, or gross evidence of cirrhosis was identified in $54 \%(49 / 90)$ of patients at or before the time of surgery. The 30-day mortality was $6 \%(5 / 90)$. Median survival for all patients was 33 months (median follow-up for surviving patients, 20 months). Preoperative factors predictive of increased overall survival included: low Model for End-Stage Liver Disease (MELD) score $(p<0.018)$, low AFP value $(p<0.041)$, presence of viral hepatitis $(\mathrm{p}<0.037)$, and age less than 60 years at time of initial operation $(p$ $<0.049$ ). Notably, none of the following factors were found to be statistically significant predictors of survival: tumor number, AJCC stage, type of surgical treatment (resection vs. ablation), Milan criteria, and presence of cirrhosis. On multivariate analysis only a low preoperative MELD score correlated with an increased overall survival. Conclusions: Treatment of patients with hepatocellular carcinoma remains a challenging problem. More important than histological evidence of cirrhosis or serological evidence of viral hepatitis is the integrated assessment of hepatic function as reflected by the MELD score. Careful preoperative selection of patients, including the MELD score, is essential in making decisions regarding operative treatment of HCC.

\section{P215}

Is there a Difference in Survival Between Right- Versus Left-Sided Colon Cancers? R.A. Meguid, ${ }^{1 *}$ M.B. Slidell, ${ }^{2}$ D.C. Chang, ${ }^{1}$ N. Ahuja. ${ }^{1}$ 1. Surgery, Johns Hopkins University School of Medicine, Baltimore, $M D ; 2$. Georgetown University Hospital, Washington, DC.

Introduction: The incidence of right-sided colon cancers has been increasing in recent years. It is unclear if the prognosis for right-sided colon cancers is different from left-sided colon cancers. In this study, we have compared the survival of right- vs left-sided colon cancers in a longitudinal population-based database over 16 years. Methods: A retrospective survival analysis was performed using the Surveillance, Epidemiology, and End Results (SEER) prospective cohort study. Inclusion criteria were patients who underwent surgical resection between 1988 and 2003 for a primary diagnosis of pathologically-confirmed, invasive colon adenocarcinoma. Analysis was limited to patients $>=18$ years old with lymph node and staging data. Tumor location was divided into right (cecum to transverse colon, excluding appendix) vs left (splenic flexure to sigmoid, excluding rectum). Cox proportional hazards regression analysis was used to assess long-term survival outcomes. Results: 73,074 patients were identified, with a median age of 72 years, and $52.5 \%$ were female. $57.5 \%$ of these patients had right-sided cancers. Right-sided cancer patients were older ( $71.5 \mathrm{vs} 68.3$ years), more likely to be female (55.8 vs $47.9 \%)$ and unmarried $(45.3$ vs $39.3 \%)(\mathrm{p}<0.001)$. Overall median survival was 75 months (95\%CI:74-76 months). Median survival for right-sided cancers was 71 months (95\%CI:61-72 months) vs 82 months for left-sided cancers (95\%CI:80-85 months). On Cox proportional hazards regression analysis, controlling for significant confounders including age at diagnosis, race, sex, marital status, tumor stage, grade and lymph node status, right-sided colon cancers were associated with a $6 \%$ increased mortality risk compared to leftsided colon cancers (hazard ratio $=1.06$ (95\% CI:1.03-1.08)) (see Figure). Conclusions: We have shown that right-sided colon cancers have a worse prognosis than left-sided colon cancers. The reason for this difference remains unclear, and it may be due to biological and/ or environmental factors. Further research is warranted to elucidate the reasons for this difference in order to improve management and ultimately outcomes for right-sided colon cancers.

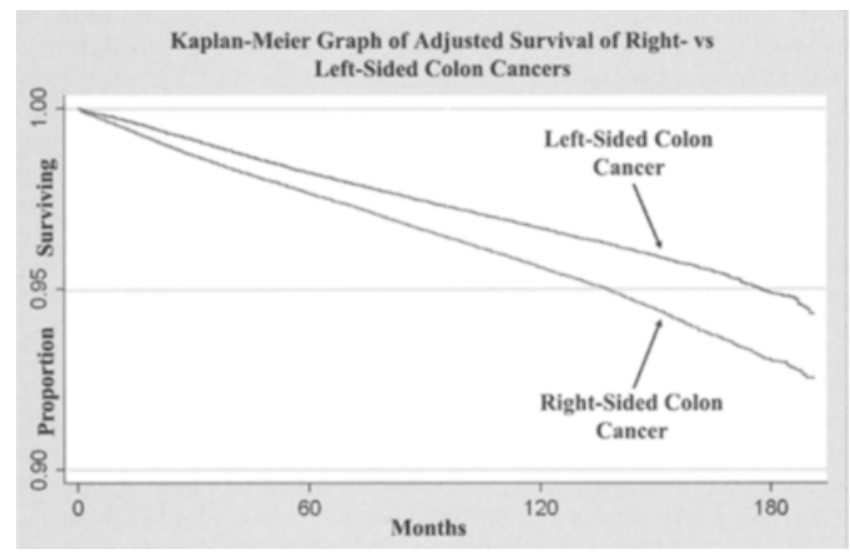

\section{P216}

Oncologic Outcome in 27 Patients Undergoing Ampullectomy for "Benign" Neoplasms S.R. Grobmyer,* C.N. Stasik, P. Draganov, A.W. Hemming, S.B. Vogel, S. Hochwald. University of Florida College of Medicine, Gainesville, FL.

Introduction: Ampullectomy may be an appropriate oncologic procedure in selected patients. Sparse data exists on procedure related complications and the relationship between histologic analysis and outcome. Methods: We retrospectively reviewed our experience with surgical ampullectomy in 27 patients with a preoperative benign histologic diagnosis over a 15 year period from 1991-2005. Presenting signs, symptoms and preoperative diagnostic studies were reviewed. Complications and follow up for recurrence were recorded. The ability of preoperative histologic biopsy, intraoperative frozen section and final histologic analysis to guide management and predict outcome were determined. Results: Median age was 63 years. Jaundice was present in 30\% of patients. Median length of stay was 9 days. $44 \%$ of patients had at least one complication and there was 1 death. Ampullary adenomatous neoplasms were present in $89 \%$ of patients. Preoperative biopsy had complete concordance with final pathology in $67 \%$ of patients. Preoperative biopsy and intraoperative frozen section correctly identified carcinoma in 0 out of 4 patients. Pancreaticoduodenectomy was performed within 7 days in the postoperative period in 3 of these patients. Following ampullectomy (median follow up $=24$ months) recurrences were identified in 2 patients $(9 \%)$ with benign tumors. No patients with high grade dysplasia $(n=3)$ have recurred. Conclusions: Preoperative biopsy and intraoperative frozen section analysis have limitations in the management of patients undergoing ampullectomy. High grade dysplasia is not an absolute contraindication to ampullectomy. Morbidity of ampullectomy is significant but long term outcome of this procedure, in those patients without invasive malignancy, is acceptable.

\section{P217}

CA 19-9 Velocity Predicts Disease-Free Survival and Overall Survival After Pancreatectomy of Curative Intent J. Hernandez,* S. Cowgill, A. Collins, S. Al-Saadi, S. Ross, J. Cooper, E. Zervos, A.S. Rosemurgy. Surgery, University of South Florida, Tampa, FL.

Introduction: Serum CA 19-9 levels are thought to predict survival after resections for pancreatic cancer, though relationships between CA 19-9 levels and survival are uncertain. This study was undertaken to correlate serum CA 19-9 levels and CA 19-9 velocity with disease-free and overall survival after resections for pancreatic adenocarcinoma. Methods: From 1997 to 2002, 102 patients underwent pancreatectomy of curative intent without adjuvant 
chemotherapy. No patients had gross residual disease. Per protocol after resection, CA 19-9 levels were drawn at: baseline, 4 weeks, and 12 week intervals thereafter. CA 19-9 velocity denotes rate of change in CA 19-9 levels (reported as $\mathrm{u} / \mathrm{ml} / 4$-weeks). CA 19-9 levels, changes in CA 19-9 levels, and CA 19-9 velocity were correlated with disease-free and overall survival utilizing regression analysis. Data are presented as median (mean $\pm \mathrm{SD}$ ). Results: AJCC tumor stage was I (32\%), II (10\%) or III (58\%). Margin status was R0 $(67 \%)$ or R1 $(33 \%)$. Disease-free survival was 7 months $(14 \pm 13.7)$ and overall survival was 12 months $(19 \pm 14.3)$ with 25 patients alive at 33 months $(33 \pm 10.4)$. Baseline CA19-9 levels predicted disease-free $(p<0.01, r=-0.35)$ and overall survival $(p<0.01, r=-0.35)$. The increase in CA 19-9 levels from baseline to disease progression did not correlate with disease-free $(p=0.4)$ or overall survival $(\mathrm{p}=0.11)$. CA 19-9 velocity predicted disease-free $(\mathrm{p}<0.01, \mathrm{r}=-0.40)$ and overall survival $(\mathrm{p}<0.01, \mathrm{r}=-0.58)$ and was a better predictor of disease-free and overall survival than baseline CA 19-9 $(\mathrm{p}<0.001)$ (Figure). CA 19-9 velocity at disease progression was $104 \mathrm{u} / \mathrm{ml} / 4$-weeks $(1663 \pm 4483)$ vs. $1.0 \mathrm{u} / \mathrm{ml} / 4$ weeks $(1.1 \pm 3.8)$ at 22 months for patients without disease progression $(\mathrm{p}=0.001$, Mann-Whitney U-test). Conclusions: While postoperative baseline CA 19-9 predicts disease-free and overall survival, CA 19-9 velocity is a better predictor of disease-free survival and overall survival. An elevated CA 199 velocity is highly predictive of imminent disease progression. CA 19-9 velocity should be utilized preferentially to CA 19-9 levels after resections for pancreatic adenocarcinoma.

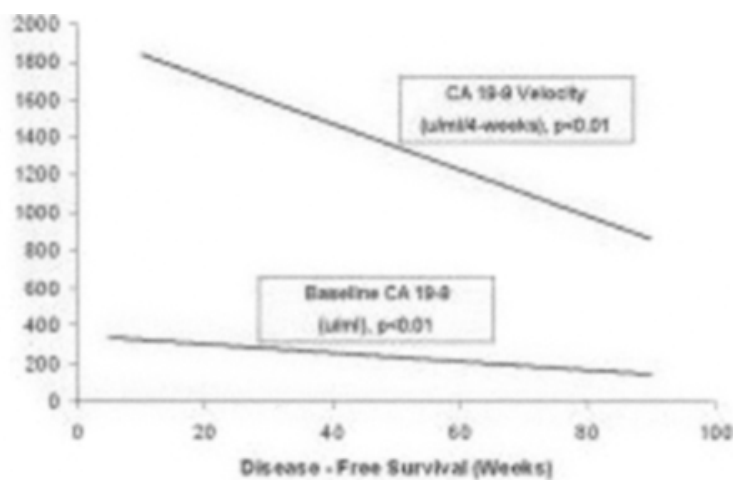

P218

Aggressive Approach to Extrahepatic Cholangiocarcinomas Warranted Because Margin Status and Stage Do Not Impact Survival After Resection S. Cowgill,* S. Al-Saadi, D. Villadolid, E. Kraemer, M. Shapiro, J. Mullinax, J. Cooper, S. Goldin, E. Zervos, A.S. Rosemurgy. Surgery, University of South Florida, Tampa, FL.

INTRODUCTION: With cholangiocarcinoma, the only hope of a cure is resection. This study was undertaken to determine the impact of margin status and tumor stage on survival after resection of extrahepatic cholangiocarcinoma. METHODS: Stage was determined using the AJCC classification. Margin status was codified as micro- / macroscopically negative, microscopically positive / macroscopically negative, or micro- / macroscopically positive. Tumor location was classified as proximal (at or above the confluence of the common hepatic duct), mid (below the confluence), or distal (below the margin of the duodenum). The impact of tumor location, concomitant hepatic resection, margin status, stage, and adjuvant therapy on survival was determined using regression analysis. Impact of margin status on survival was further evaluated by survival curve analysis. Data are presented as median, mean \pm SD. RESULTS: From 1985-2006, 91 patients, of median age 69 years, underwent resections of extrahepatic cholangiocarcinomas by pancreaticoduodenectomy $(n=36)$, extrahepatic biliary resection with concomitant hepatic resection $(n=31)$, or extrahepatic biliary resection alone $(\mathrm{n}=24)$. Overall survival after resection was 619 days, $1068 \pm 1315$. Survival was not impacted by margin status (Figure), stage, or adjuvant therapy. Survival was significantly impacted by tumor location $(\mathrm{p}=0.01)$ and the need for concomitant hepatic resection $(\mathrm{p}=0.01)$ : survival after resection of proximal tumors was 609 days, $1233 \pm 1580$, mid tumors was 1096 days, $1020 \pm 455$, and distal tumors was 575 days, $860 \pm 1034$, while survival of patients with extrahepatic biliary resection and hepatic resection was 473 days, $800 \pm 943$ vs. 1218 days, $1820 \pm 1866$ with extrahepatic biliary resection alone. CONCLUSIONS: Survival after resection of extrahepatic cholangiocarcinoma is significantly impacted by tumor location and concomitant hepatic resection; margin status, stage, and adjuvant therapy do not impact survival. Cholangiocarcinomas should be aggressively resected even if tumors appear to be of advanced stage and resection might result in positive margins.

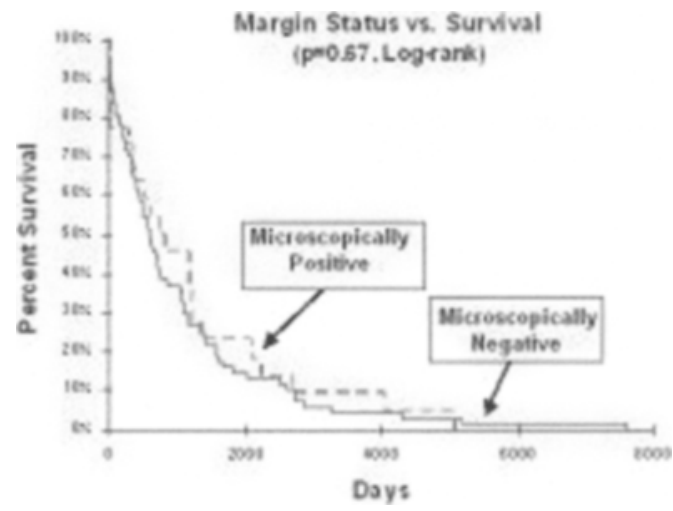

P219

Correlation of Bone Marrow Micrometastases with Nodal Status in Gastrointestinal Tumors S. Saha, ${ }^{1 *}$ S. Chivu, ${ }^{1}$ D. Wiese, ${ }^{1}$ N. Bassily, ${ }^{1}$ A. Henke, ${ }^{2}$ M. Patel, ${ }^{1}$ H. Sankaran, ${ }^{1}$ A. Misra, ${ }^{1}$ M. Arora, ${ }^{1}$ T. Singh. ${ }^{1}$ 1. Michigan State University, McLaren Regional Medical Center, Flint, MI; 2. Michigan State University, Genesys Regional Medical Center, Grand Blanc, MI.

Introduction: Presence of bone marrow micrometastases (BMM) is an important prognostic factor for patients (pts) with solid tumors. Sentinel lymph node (SLN) mapping (M) has been found to upstage patients with gastrointestinal (GI) malignancies. However, a direct correlation between the presence BMM and nodal metastases is lacking. Hence, a retrospective study was undertaken to determine the relationship between BMM and nodal status in various GI malignancies. Method: Consecutive pts with GI malignancies were analyzed. Of these $146 \mathrm{pts}, 4 \mathrm{pts}$ with non-epithelial tumors were excluded. All patients underwent bilateral posterior-superior-iliac spine bone marrow aspiration. Cytokeratin (CK) positive cells reflecting micromets were detected by CAM 5.2 monoclonal anti-cytokeratin antibody and confirmed by CK 8 and CK 18 staining. When feasible, SLNM was performed followed by standard oncologic resection $(n=94)$. The SLN's were examined by H\&E and immunohistochemical (IHC) staining. Results: A total of 142 pts were included in the study. Of these $13.4 \%(19 / 142)$ were found to have BMM (CI 8.74-19.95, $\mathrm{p}=0.05)$. When analyzed by specific sites, the incidence of BMM was $12 \%(10 / 83)$ for colon cancer, $15.4 \%(4 / 26)$ for rectal cancer, $33.3 \%(4 / 12)$ for stomach cancer and $9.1 \%(1 / 11)$ for pancreatic cancer. No BMM were detected in pts with cancer of esophagus $(n=7)$, small bowel $(\mathrm{n}=2)$ and liver $(\mathrm{n}=1)$. Interestingly, the $\mathrm{BMM}$ was positive bilaterally in $63.2 \%(12 / 19)$, and unilaterally in $36.8 \%(7 / 19)$. In pts who under- 
went SLNM ( $\mathrm{n}=94$ ), BMM were detected in $11.4 \%$ of SLN positive pts vs. $6.8 \%$ for SLN negative pts $(p=0.44)$. For a subgroup of $p$ ts with colorectal cancer who underwent SLNM ( $\mathrm{n}=86)$, BM micromets were detected in $10 \%$ of SLN positive pts vs. $5.4 \% \%$ for SLN negative pts $(\mathrm{p}=0.43)$ Conclusions: No correlation was found between BMM and nodal status for GI malignancies. This suggests that the mechanism for BMM may be different compared to that for lymphatic metastases. Detection of BMM may have a significant clinical value in SLN negative patients who may benefit from adjuvant therapy.

\begin{tabular}{|c|c|c|c|}
\hline \multicolumn{4}{|c|}{ Table 1A: Incidence of Bone Marrow Metastases in Gastrointestinal(GI) Tumors } \\
\hline GI tumor site & $\begin{array}{l}\text { Total no. of } \\
\text { Pts }\end{array}$ & $\begin{array}{l}\text { No. of pts with Bone Marrow } \\
\text { micrometastases }\end{array}$ & Percentage $(\%)$ \\
\hline Colon & 83 & 10 & $12.00 \%$ \\
\hline Rectal & 26 & 4 & $15.40 \%$ \\
\hline Stomach & 12 & 4 & $33.30 \%$ \\
\hline Pancreatic & 11 & 1 & $9.10 \%$ \\
\hline Esophageal & 7 & 0 & $0.00 \%$ \\
\hline Small Bowel & 2 & 0 & $0.00 \%$ \\
\hline Liver & 1 & 0 & $0.00 \%$ \\
\hline Total & 142 & 19 & $13.38 \%$ \\
\hline \multicolumn{4}{|c|}{ Table IB: Bone Marrow Metastases positivity versus Sentinel Lymph Node(SLN) Status } \\
\hline All GI cancers & SLN +ve & SLN-ve & $p$-value \\
\hline No. of Pts & 35 & 59 & \\
\hline $\begin{array}{l}\text { Bone Marrow } \\
\text { Micrometastases }\end{array}$ & 4 & 4 & \\
\hline Percentage & $11.40 \%$ & $6.80 \%$ & $p=0.44$ \\
\hline Colorectal Cancer & SLN +ve & SLN -ve & $p$-value \\
\hline No. of Pts & 30 & 56 & \\
\hline $\begin{array}{l}\text { Bone Marrow } \\
\text { Micrometastases }\end{array}$ & 3 & 3 & \\
\hline Percentage & $10 \%$ & $5.40 \%$ & $p=0.43$ \\
\hline
\end{tabular}

\section{P220}

Pneumoperitoneum in the Cancer Patient B. Badgwell, ${ }^{*}$ B.W. Feig, M.I. Ross, P.F. Mansfield, G.J. Chang. M.D. Anderson, Houston, TX.

Background: Pneumoperitoneum often requires emergent laparotomy. Cancer patients may be at increased risk for pneumoperitoneum due to local invasion, immunosuppression, chemotherapy, and frequent use of endoscopic procedures. The purpose of this study was to characterize the clinical presentation and management of cancer patients with pneumoperitoneum. Methods: All patients with an ICD-9 diagnosis of unspecified peritoneal disorder (568.89) or visceral perforation (569.83) treated at a tertiary cancer center between 2001 to 2006 were identified through the hospital discharge registry. A subset of patients with pneumoperitoneum on radiographic imaging were identified by chart review. Patient, tumor, treatment, and outcome variables were retrospectively recorded. Comparisons between treatment groups were performed using Chi-square and Fisher's exact test analysis. Results: The registry query yielded 647 patients of whom 91 patients had pneumoperitoneum and underwent analysis. Treatment rendered was comfort care $(n=15)$, non-operative management $(n=20)$, or surgery $(n=56)$. Cancer treatment related variables included use of chemotherapy (54\%), steroids $(31 \%)$, and abdominal radiation $(28 \%)$. All patients managed with comfort care had stage IV disease. Stage IV cancer was present in $90 \%$ managed non-operatively and $72 \%$ managed with surgery $(p=0.13)$. Factors predictive of management included asymptomatic presentation, abdominal pain and tenderness, pneumatosis, and prior abdominal radiation (table). Thirty-day mortality was $100 \%(15 / 15)$ with comfort care, $15 \%(3 / 20)$ with non-operative management, and $18 \%(10 / 56)$ with surgery. Three patients underwent laparotomy without identification of causative pathology and 30 patients $(53 \%)$ required a stoma. Conclusions: Pneumoperitoneum in the cancer patient requires a tailored approach that considers the clinical presentation and oncologic prognosis. The conventional wisdoms for surgical evaluation, such as the severity of symptoms, pain, and tenderness still apply, but in a selected subgroup of patients non-operative treatment can be successfully implemented.

\begin{tabular}{|c|c|c|c|}
\hline & Non-Operative Mgmt $(\mathrm{n}=20)$ & Operative Mgmt $(\mathrm{n}=56)$ & $\mathrm{p}$-value \\
\hline Asymptomatic Presentation & 4 & 0 & 0.003 \\
\hline Abdominal Pain & 11 & 55 & 0.00004 \\
\hline Nausea/Vomiting & 4 & 19 & 0.40 \\
\hline Fever & 5 & 7 & 0.28 \\
\hline Tenderness & 9 & 52 & 0.00005 \\
\hline Pneumatosis & 5 & 2 & 0.012 \\
\hline WBC $<1.7$ & 2 & 5 & 1.0 \\
\hline WBC $>11.0$ & 4 & 21 & 0.18 \\
\hline Chemotherapy & 11 & 29 & 0.80 \\
\hline Steroids & 8 & 18 & 0.49 \\
\hline Abdominal Radiation & 2 & 18 & 0.045 \\
\hline
\end{tabular}

\section{P221}

Ferumoxtran-10-Enhanced Magnetic Resonance Imaging for Preoperative Evaluation of Paraaortic Lymph Node Metastatasis in Patients with Pancreatic Cancer K. Takaori, ${ }^{1 *}$ N. Tanigawa, ${ }^{1}$ M. Matsuki, ${ }^{2}$ T. Agui, ${ }^{1}$ M. Iwamoto, ${ }^{1}$ Y. Miyamoto, ${ }^{1}$ I. Narabayashi. ${ }^{2}$ 1. Gastroenterological Surgery, Osaka Medical College, Osaka, Japan;

2. Osaka Medical College - Radiology, Takastuki, Japan.

BACKGROUND: Paraaortic lymph node metastasis in patient with pancreatic cancer is one of the most critical factors to determine indication for surgical resection. However, conventional imaging studies including CT often fail to discriminate metastatic lymph adenopathy from nonspecific swelling. Hence, we have introduced magnetic resonance imaging (MRI) after administration of ferumoxtran-10 (Combidex, Advanced Magnetics, Cambridge, MA), a dextran-coated ultrasmall superparamagnetic iron oxide particle that functions as a contrast agent for lymphatic tissue, and evaluated the lymph node metastasis in patients with pancreatic cancer. PATIENTS \& METHODS: Eight patients with pancreatic cancer, six invasive ductal cacrcinomas and two intraductal paillary mucinous carcinoma, enrolled in this study. The patients reeived MRI scanning 24 hours after the intravenous administration of ferumoxtran-10. The presence or absence of metastasis in lymph nodes was assessed by their enhancement patterns. The results of ferumoctran-10-enhanced MRI were compared with histologic finding and the diagnosis by CT. RESULTS: In six of the eight patients, paraaortic lymph nodes were surgically sampled. Histological finding of the paraaortic lymph nodes was negative for metastasis in five patients. Whipple resection was carried out in three and distal pancreatectomy with splenectomy in four of these patients. In contrast, intraoperative histopathological study on frozen sections of the paraaortic lymph nodes in the remaining one patient resulted in a diagnsosis of positive for metastasis and by-pass operation was performed. Sensitivity, specificity, and accuracy of ferumoxtran-10-enhanced MRI were all $100 \%$, while those of CT were $100 \%, 25 \%$, and $50 \%$, respectively, when enlarged paraaortic lymph nodes $>5 \mathrm{~mm}$ were considered positive. CONCLUSIONS: Ferumoxtran-10-enhance MRI may be of great value for preoperative evaluation of paraaortic lymph node metastasis in patients with pancreatic cancer.

\section{P222}

Combination of microsatellite instability and lymphocytic infiltration as a prognostic marker in colon cancer N.M. Johnson, ${ }^{1}$ E.Y. Chang, ${ }^{2 *}$ J.H. Frankhouse, ${ }^{1}$ P.B. Dorsey, ${ }^{1}$ W.E. Johnson, ${ }^{1}$ R.E. Lee. ${ }^{1}$ 1. Cancer Services, Legacy Good Samaritan, Portalnd, OR; 2. Oregon Health and Sciences University, porland, OR.

Introduction:Development of standard prognostic indicators for colon cancer has yet to come into common usage.Microsatellite instability (MSI) and lymphocytic infiltration of tumors have been shown to predict tumor biology.We sought to evaluate the combined prognostic significance of MSI and LI in our colon cancer population. Methods: Prospective analysis of patients undergoing definitive operation for colon cancer in community hospital system. Tumors were evaluated for MSI by assessing for instability in eleven satellite markers and classified as MSI+ if two or more satellite markers displayed instability. Tumors were classified as $\mathrm{LI}+$ if at least five lymphocytes were observed per ten high-power fields. Overall survival (OS) and disease-free survival (DFS) 
were compared according to combined MSI and LI status. Results: MSI and LI status were available for 146 patients, who were classified as follows: 89 were MSI-/LI-, 13 were MSI-/LI+, 33 were $\mathrm{MSI}+/ \mathrm{LI}-, 11$ were MSI+/LI+. Median followup was 24.8 months. Survival data are shown in the table. Conclusions: Colon cancers that were both MSI(-) and LI(-) were more aggressive with patients experiencing decreased disease free survival and overall survival. MSI and LI show promise as a combined prognostic marker for colon cancer.

\begin{tabular}{|c|c|c|c|}
\hline & MSI-/LI- & MSI+ or LI+ & $\mathrm{p}$ (log rank) \\
\hline No. of patients & 89 & 57 & \\
\hline 3-yr DFS & $57 \%$ & $73 \%$ & $0.023 *$ \\
\hline 3-yr OS & $73 \%$ & $81 \%$ & 0.198 \\
\hline 3-yr DFS (Stage III) & $59 \%$ & $100 \%$ & 0.075 \\
\hline 3-yr OS (Stage III) & $65 \%$ & $100 \%$ & 0.140 \\
\hline
\end{tabular}

\section{P223}

Intensive Follow-Up after Curative Surgery for Rectal Cancer T. Higuchi, ${ }^{*}$ M. Enomoto, K. Sugihara. Surgical Oncology, Tokyo Medical and Dental University Graduate School, Tokyo, Japan.

Introduction: The purpose of follow-up after curative surgery for rectal cancer is to improve prognosis and quality of life. A final goal is to improve survival through the earlier identification of recurrences that are still able to do curative surgery. The aim of this study is to evaluate risk factors with recurrence for rectal cancer after curative surgery and to investigate an optimal follow-up system for patients with rectal cancer after curative surgery. Patients and Methods: Consecutive 2507 patients with rectal cancer, who underwent curative resection at 16 institutions in Japan of the Study Group on Postoperative Follow-Up of Colorectal Cancer Patients from January 1991 to December 1996, were enrolled in this study. A common follow-up system consisted of carcinoembryonic antigen measurement every 3 months, ultrasonic imaging for liver every 3-6 months, chest X-ray every 6 months and colonoscopy every 1-2 years. Where recurrences were suspected, CT or/ and MRI were applied. The median follow-up period was 78 months. The 5-year overall survivals were $77.8 \%$. Recurrence time, rate, and site, treatments for relapse and outcome of re-resection were investigated. Results: The recurrences were observed in $20.6 \%$ with rectosigmoid, $21.2 \%$ with upper rectal, and $28.3 \%$ with lower rectal cancer. About recurrence site, recurrences were found $7.4 \%, 7.6 \%$, $8.9 \%$, and $1.6 \%$, in liver, lung, local, and others, respectively. Cumulative appearance rates of recurrence were $81.6 \%$ and $95.5 \%$ within 3 and 5 years after surgery, respectively. Overall 5-year survival rates of patients with surgery were better than those without surgery in each site of recurrence; $33.8 \%$ vs $2.0 \%$ in liver, $41.1 \%$ vs $2.1 \%$ in lung, $21.0 \%$ vs $9.2 \%$ in local recurrence. Multivariate analysis disclosed that lower rectum, deeper tumor depth, lymph vessel invasion, and lymph node metastasis were significantly associated with risk factors of recurrence. Conclusions: Intensive follow-up made a contribution to high rates of re-resections in patients with recurrence after curative surgery for rectal cancer. Further trials are required to evaluate the cost effectiveness of intensive follow-up and the efficacy of surveillance methods.

\section{P224}

Clinical Significance of BMP7 in Human Colorectal Cancer K. Motoyama, ${ }^{1 *}$ T. Tanaka, ${ }^{1}$ K. Mimori, ${ }^{1}$ T. Matsumoto, ${ }^{1}$ H. Inoue, ${ }^{1}$ K. Sugihara, ${ }^{2}$ M. Mori. ${ }^{1}$ 1. Surgery and Molecular Oncology, Kyushu University, Beppu, Japan; 2. Tokyo Medical and Dental University, Tokyo, Japan.

Background: Bone morphogenetic proteins (BMPs) are secreted signaling molecules belonging to the transforming growth factor (TGF)- $\beta$ superfamily, and recent studies showed that many BMPs have altered expression patterns in several tumors. The purpose of the current study was to quantify the expression of BMP7 in malignant and normal colorectal tissues, and to analyze whether BMP7 expression levels correlate with clinicopathologic variables and prognosis in patients with colorectal cancer. Methods: Paired colorectal tissue samples from cancer and corresponding non-cancer tissues were obtained from 65 patients with colorectal cancer who underwent surgical resection. The expression status of BMP7 was investigated by quantitative real-time reverse transcription-PCR (RT-PCR) and immunohistochemical studies. Results: Quantitative RT-PCR showed that BMP7 expression in cancer tissue was significantly higher than that in normal tissue $(\mathrm{P}<0.01)$. The samples were divided into high $(n=27)$ and low $(n=38)$ BMP7 expression groups. Elevated BMP7 expression was significantly correlated with liver metastasis, advanced Dukes stage and cancer-related death $(\mathrm{P}<0.01,0.04$, and 0.01 , respectively). Furthermore, the patients with high BMP7 expression showed poorer prognosis $(\mathrm{P}=0.01)$ Multivariate analysis showed that BMP7 expression status was an independent prognostic factor (relative risk, $2.28 ; 95 \%$ confidence interval, 1.07-5.31; $\mathrm{P}=0.03$ ). Immunohistochemical study revealed that $\mathrm{BMP} 7$ was predominantly expressed in cancer cells. Conclusions: BMP7 is one of the cancer-related genes, and correlates with biological aggressiveness and poor prognosis of colorectal cancer. Thus, BMP7 is a possible candidate gene for diagnosis and molecular target therapy of colorectal cancer.

\section{P225}

Effect of fixation in a lymph node revealing solution on number of lymph nodes and TNM-stage in colon cancer B. Inberg, ${ }^{1}{ }^{*} \mathrm{~W}$. Kelder, ${ }^{1}$ J. Plukker, ${ }^{2}$ H. Groen, ${ }^{2}$ P. Baas, ${ }^{1}$ A. Tiebosch. ${ }^{1}$ 1. Surgery, Martini Hospital, Groningen, Netherlands; 2. University Medical Center, Groningen, Netherlands.

Aim: To investigate the effect of modified Davidson's Fixative (mDF) on the number of lymph nodes retrieved in a specimen after colon resection and TNM-staging. Patients and methods: Specimens of 242 patients were available for analysis. Of these 242 specimens 117 were examined using gross dissection after neutral buffered formalin fixation for 24-72 hours. 125 specimens were examined using gross dissection after $\mathrm{mDF}$ fixation containing ethanol, glacial acetid acid, formaldehyde and water, for 24 -72 hours. Results: In the specimens fixed in formalin the median number of nodes retrieved was 5 (range $0-17$ ), with use of the $\mathrm{mDF}$ the median number of nodes retrieved increased to $13(0-35, p=0,000)$. Moreover the type of resection and the T-stage influenced the number of retrieved lymph nodes significantly. The percentage of node positive cases increased from $30 \%$ to $41 \%$ after the use of $\mathrm{mDF}$. $(\mathrm{p}=0,077)$ Conclusion: More lymph nodes are retrieved using $\mathrm{mDF}$ fixation in a colon resection specimen, and an increase in node positive patients $(p=0,077)$ was observed. With more patients staged as node positive, adjuvant chemotherapy can be offered to these patients which might lead to survival benefit in the future.

\section{P226}

PHOSPHOPROTEOMIC ANALYSIS OF METASTATIC COLORECTAL CANCER: INSIGHTS FOR NEW THERAPEUTIC TARGETS M. Pierobon, ${ }^{1}{ }^{*}$ V.S. Calvert, ${ }^{2}$ M. Lipsky, ${ }^{3}$ K.M. Sheehan, ${ }^{4}$ R. Speer, ${ }^{4}$ E. Mammano, ${ }^{1}$ C. Belluco, ${ }^{5}$ D. Nitti, ${ }^{1}$ L.A. Liotta, ${ }^{2}$ E.F. Petricoin. ${ }^{2}$ 1. Department of Oncological and Surgical Sciences, Padova, Italy; 2. George Mason University, Manassas, VA; 3. University of Maryland, Baltimore, MD; 4. Laboratory of Pathology, NCI, Bethesda, MD; 5. CRO Hospital, Aviano, Italy.

BACKGROUND Currently, $50 \%$ of patients with advanced colorectal cancer respond to adjuvant chemotherapies, and their median survival is about 20 months. In our study, based on the reverse phase protein microarrys technique we invented, combined with laser capture microdissection, we sought to analyze the phosphorylation state of 70 kinase substrates in colorectal cancer and its metastases in order to identify possible drug targets in the metastatic setting. PATIENTS AND METHODS We microdissected frozen tissues from 20 patients with primary colon cancer, 68 patients with matched colorectal cancer and synchronous liver metastases, 15 with lung metastases. Lysed cancer cells were subjected to reverse phase protein microarray analysis. RESULTS The comparison between the metastatic primary colon cancer (CO), its liver (LI) and lung (LU) metastases shows significant differences in the activation of pFAK Y576/577 (CO-LI t-test:0.00014; CO-LU 0.02), pcKit Y703 (CO-LI 0.001, CO-LU 0.03), Pyk2 Y402 (CO-LI 0.0004, CO-LU 0.03) between CO and the metastatic lesion; PPTEN (CO-LI 0.0001, CO-LU 5.21329E-05) and pPKCa (CO-LI 0.007, CO-LU 0.05) The comparison of non metastatic primary colon $(\mathrm{NCO})$ cancer, metastatic colon cancer $(\mathrm{CO})$ and its synchronous liver metastases (LI) highlight that there is a statistical increase of the pAKT S473 level ( CO-NCO 0,03, CO-LI 0,002) and decrease of the pPTEN level (CO-NCO 0.0001, CO-LI 0.02). pEGFR Y992 is higher in CO and in LI than in the NCO (CO-NCO 6.55683E-05, NCO-LI 4.74728E-05), while pVEGFRY951 (CO-LI 0.004), pFAK Y576/577 (CO-LI 0.0001) are significantly higher in LI than in the primary tumor. pPKCa phosphorylation increase from 
the $\mathrm{NCO}$ to $\mathrm{CO}$ and decreases in LI (CO-LI 0.007). pBAD S136 (CO-NCO 0.05 , CO-LI 0.02 ) decreases progressively from NCO to CO to the metastases. CONCLUSIONS The molecular network of colorectal cancer is significantly altered in the metastatic setting and appears to be greatly influenced by the site of metastasis. This could have profound implications for effectively treating late stage cancers.

\section{P227}

Long-term Quality of Life Following Transhiatal Esophagectomy for Esophageal Adenocarcinoma J. Sosa, ${ }^{*}$ T. Trapasso, R. Salem. Surgery, Yale University School of Medicine, New Haven, CT.

Background: Incidence of esophageal adenocarcinoma is increasing, and overall prognosis remains poor. Controversty exists about optimal managment, depending on stage of disease and general health of the patient. Our objective was to measure long-term physical, social, and emotional aspects of quality of life (QoL) among patients with esophageal adenocarcinoma following transhiatal esophagectomy (THE). Methods: Patients with esophageal adenocarcinoma referred for THE at a tertiary referral center were enrolled in this HICapproved retrospective/prospective cohort study. Patient-reported QoL was measured by validated EORTC QLQ-C30 and QLQ-OES18 questionnaires. Secondary outcomes included length of stay (LOS) and disease recurrence. Results: 31 patients with esophageal adenocarcinoma were enrolled between April 2004 and 2006. Mean age was 61 years; $87 \%$ were male, $97 \%$ white, and mean CIRS comorbidity score 20 . Half of patients had Stage II disease; $74 \%$ received neo-adjuvant and 33\% adjuvant treatment. Mean LOS was 11 days. Mean follow-up was 15 months (range, 0-60 months). 15\% had recurrent disease, with a mean time to recurrence of 15 months. CIRS score and adjuvant therapy negatively impacted post-operative global QoL (both, $\mathrm{p}=.03$ ). In particular, physical and social function significantly declined with increasing comorbidity and adjuvant treatment. Emotional function deteriorated significantly with longer LOS. Overall, global QoL after THE nadirs at 6 months, returns to pre-operative level by 12 months, and improves until 48 months, when it declines. Energy and appetite had more prolonged recovery. Age appears to have a marked effect on the durability of QoL recovery, such that patients under 70 years sustain improvement in QoL post-op, while older patients do not. Conclusions: This is a novel examination of long-term QoL following THE, as prior studies focused on the first 3 months post-op. THE has a deleterious short-term effect on QoL, but provides durable improvement by 12 months. QoL is an important outcome measure for guiding treatment selection, since patients need adequate survival to be able to recover their QoL.

\section{P228}

Evaluation with Positron Emission Tomography Before Hepatic Resection for Metastatic Colorectal Cancer Improves Survival in Patients with a High Clinical Risk Score R.C. Taylor,* T.J. Akhurst, S. Tuorto, S.M. Larson, Y. Fong. Surgery, Memorial Sloan Ketering, New York, NY.

INTRODUCTION: Positron Emission Tomography (PET) using fluoro18-deoxyglucose is used in the preoperative staging of patients with hepatic colorectal cancer metastases to improve patient selection for surgery and survival. This study was performed to determine if the impact of PET on outcome is related to clinical predictors of recurrence, as measured by the Clinical Risk Score (CRS). METHODS: From April 1999 to December 2001, 115 patients undergoing evaluation for hepatic resection of colorectal metastases following enrollment in a prospective preoperative PET protocol were compared to 134 contemporary patients not evaluated with PET. RESULTS: Of patients enrolled in the PET protocol, 45 did not undergo resection and for 11 patients $(10 \%)$ PET scan was the sole indicator of extrahepatic disease (EHD). The ability of PET to detect EHD was greatest in patients with a CRS $>2$ $(p=0.01)$. Following hepatic resection, a preoperative PET scan was associated with a significantly better overall (OS) and disease free survival (DFS) at 5 years among patients with CRS $>2$. The OS was $44 \%$ for the 70 patients evaluated with a PET scan versus $15 \%$ for the 134 patients who were not $(\mathrm{p}=0.008)$. On multivariate analysis the independent factors associated with an improved OS in patients with a CRS $>2$ were $T$ stage of the primary $(\mathrm{p}=0.06)$, CRS score $(\mathrm{p}=0.006)$ and a preoperative PET scan $(0.002)$. In patients with $\mathrm{CRS} \leq 2$ a preoperative PET scan did not improve DFS or OS $(p=0.9)$. CONCLUSIONS: The addition of PET to the preoperative evaluation of patients undergoing hepatic resection for colorectal cancer metastases is associated with a better
DFS and OS in patients with a high CRS. In this group of patients the use of PET improves patient selection for surgery and survival.

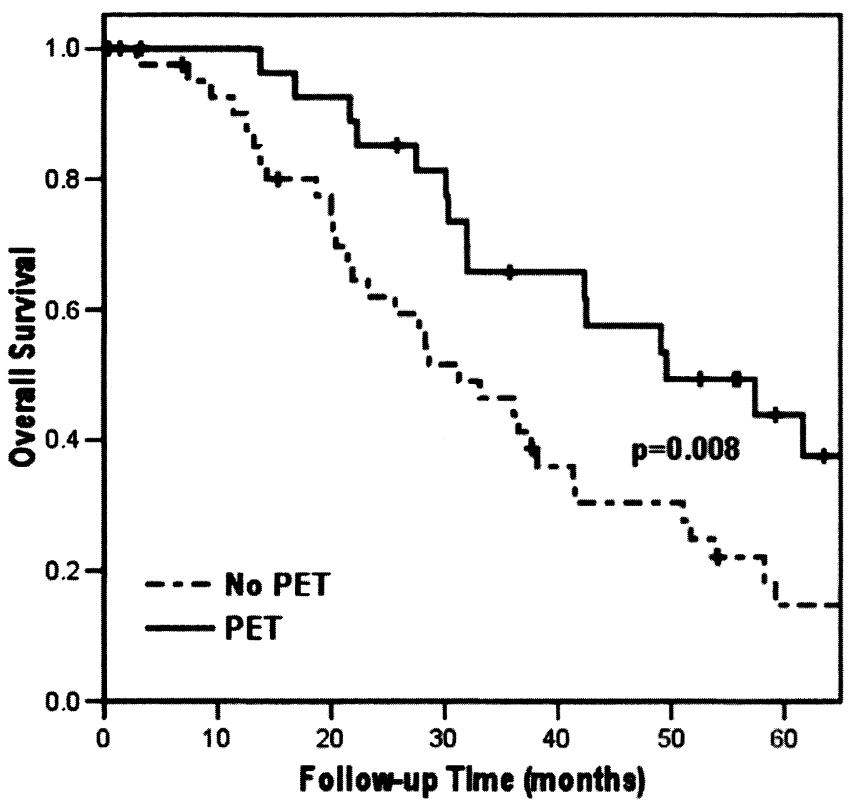

Following hepatic resection for metastatic colorectal cancer in patients with a CRS $>2$, the OS was $44 \%$ for the 70 patients evaluated with a preoperative PET scan versus $15 \%$ for the 134 patients who were not $(p=0.008)$.

\section{P229}

Serum Follistatin Levels Immediately after Hepatectomy Predict the Extent of Liver Regeneration K. Takabe, ${ }^{1 *}$ T. Tomiya, ${ }^{2}$ W. Vale. ${ }^{3}$ 1. Division of Surgical Oncology, Virginia Commonwealth University School of Medicine, Richmond, VA; 2. Department of Gastroenterology, University of Tokyo School of Medicine, Tokyo, Japan; 3. Peptide Biology Laboratory, The Salk Institute for Biological Studies, San Diego, $C A$.

[Introduction] Extensive hepatectomy is sometimes inevitable to obtain cure for a liver cancer patient, which increases the risk of liver failure. Therefore, a marker that can accurately predict the liver failure early is in need. Follistatin is a single-chain polypeptide that binds to activin and exerts its effects by neutralizing the biological activities of activin. Activin is a member of TGFbeta superfamily initially identified as a peptide that stimulate FSH secretion in the pituitary, which is now known to be one of the key factors that inhibit liver regeneration. We have previously demonstrated that the liver is maintained to its normal size by endogenous activin using adenovirus-mediated overexpression of follistatin (Endocrinology 2002;143:964, Hepatology 2003; 38, 5: 1107-1115). Based upon these results, we have hypothesized that serum follistatin levels may reflect the degree of liver regeneration after hepatectomy [Method] Fourteen patients who underwent partial hepatectomy for hepatocellular carcinoma or metastatic liver tumors were studied. Serum follistatin levels were serially measured using an enzyme-linked immunosorbent assay. The liver volume was measured by volumetric study using CT scan 28 days after surgery. [Results] The preoperative mean serum follistatin level was significantly higher than healthy adult control. Serum follistatin levels rapidly decreased after surgery in the patients who did not suffer acute liver failure, and reached a minimum in all of them within 7 days, which was below the mean level of healthy adults. The levels 1 and 3 days after hepatectomy significantly correlated inversely with the degree of liver regeneration at 28 days. Serum follistatin levels increased thereafter, and the levels between 14th and 21 st postoperative days were similar to those before surgery. In contrast, one patient who developed acute liver failure demonstrated striking elevation of the follistatin levels after surgery. [Conclusion] Serum follistatin levels decrease dramatically in the early phase of liver regeneration, which can be used to predict the degree to which liver regeneration will occur in hepatectomized patients 
P230

Postoperative morbidity after low anterior resection in patients administered preoperative chemoradiotherapy for rectal cancer: impact of colon J-pouch reconstruction S. Pucciarelli,* I. Maretto, P. Toppan, E. Urso, M. Lise, D. Nitti. Clinica Chirurgica II, University of Padova, Padova, Italy.

Purpose: to evaluate the impact of J-pouch reconstruction on post-operative morbidity after low anterior resection (LAR) in rectal cancer patients administered preoperative chemoradiotherapy (CRT). Methods: patients considered eligible for the study had, between 1996 and 2006, undergone preoperative CRT (radiotherapy: $\geq 45$ Gy and 5-FU-based chemotherapy) followed by LAR for primary rectal cancer up to $11 \mathrm{~cm}$ from the anal verge, J-pouch reconstruction being performed at the surgeon's discretion. Postoperative morbidity was evaluated by a single surgeon. Anastomotic leaks were classified as major if they: a) were life-threatening; $b$ ) required re-operation, re admission and/or a major non surgical procedure; c) involved $>50 \%$ of the anastomotic circumference. Results: of 149 patients (median age: $60 \mathrm{yrs}, \mathrm{M} / \mathrm{F}: 99 / 50$ ) who met the inclusion criteria, 31 (Group P) had J pouch and 118 (Group S) straight reconstruction. The groups were matched for sex, age, tumor location, preoperative CRT, surgeons who performed LAR and covering stoma. Postoperative morbidity was found in $14(45 \%)$ Group $\mathrm{P}$ and $58(49 \%)$ Group $\mathrm{S}$ patients. Major anastomotic leaks occurred in $3(10 \%)$ and $20(17 \%)$ patients and minor anastomotic leaks in $4(13 \%)$ and $13(11 \%)$ patients in Groups $P$ and $S$, respectively. Early re operation was required in 7 Group $S$ patients. Conclusions: the findings made suggest that J-pouch reconstruction in patients who undergo preoperative CRT followed by LAR does not reduce postoperative morbidity, but does reduce anastomotic leakage and re-operation rate.

\section{P231}

The Current Utility of Staging Laparoscopy for Pancreatic and Peri-Pancreatic Malignancy R. White, ${ }^{*}$ C.B. Winston, K.C. Conlon, M. D'Angelica, W.R. Jarnagin, Y. Fong, M.F. Brennan, P.J. Allen. Memorial Sloan-Kettering Cancer Center, New York, NY.

INTRODUCTION: The routine use of staging laparoscopy in patients with radiographically resectable pancreatic and peri-pancreatic malignancy remains controversial. METHODS: Review of a prospective database identified 1,045 patients who underwent staging laparoscopy for radiographically resectable tumors of the pancreas, distal bile duct, ampulla or duodenum between 1995 and 2005. Patients were determined to be radiographically resectable if no suspicious findings were identified within radiology reports or surgeon notes. Factors were assessed for their association with the laparoscopic identification of radiographically occult unresectable disease. RESULTS: Radiographically occult unresectable disease was identified laparoscopically in 145 patients $(14 \%)$ and included liver metastasis $(n=101)$, peritoneal metastasis $(n=34)$, locoregional disease $(n=7)$, and cirrhosis $(n=3)$. Factors associated with the laparoscopic identification of unresectable disease included the time period of the study, whether imaging had been performed at our institution, and the site of the primary tumor (Table). For the sub-group of patients with pancreatic tumors $(n=838)$, the histology of the lesion was associated with laparoscopic identification of unresectable disease. Patients with adenocarcinoma had a significantly greater risk of occult unresectable disease than patients with other histologies. Of the 900 patients in whom no unresectable disease was identified laparoscopically, only 9 patients $(1 \%)$ did not undergo resection, all due to locoregional disease. CONCLUSIONS: During the time period of this study the yield of staging laparoscopy decreased and exceeded $10 \%$ only for patients with pancreatic adenocarcinoma. When current cross-sectional imaging reveals no evidence of unresectable disease, staging laparoscopy may not be indicated for peri-pancreatic tumors other than presumed pancreatic adenocarcinoma.

\begin{tabular}{|c|c|c|}
\hline \multicolumn{2}{|c|}{ All pancreatic and peripancreatic tumors $(\mathrm{n}=1,045)$} \\
\hline Factor & & Yield of laparoscopy \\
\hline Time period & $1995-1999$ & $85 / 462(18 \%)^{*}$ \\
\hline & $2000-2005$ & $60 / 583(10 \%)$ \\
\hline Imaging & Outside & $114 / 720(16 \%)^{*}$ \\
\hline Primary site & Inside & $31 / 325(9.5 \%)$ \\
\hline & Ampulla & $3 / 125(2.4 \%)$ \\
\hline & Bile duct & $4 / 41(9.8 \%)$ \\
\hline & Duodenum & $3 / 41(7.3 \%)$ \\
\hline Factor & Pancreas & $135 / 838(16 \%)^{*}$ \\
\hline Location & Pancreatic tumors $(\mathrm{n}=838)$ & Yield of laparoscopy \\
\hline & & $93 / 631(15 \%)^{*}$ \\
\hline Histology & Head & $42 / 207(25 \%)$ \\
\hline & Body/Tail & $130 / 618(21 \%)^{*}$ \\
\hline & Adenocarcinoma & $4 / 66(7.9 \%)$ \\
\hline & Neuroendocrine & $0 / 60(0 \%)$ \\
\hline & Mucinous cystic/IPMN & $1 / 94(1.1 \%)$ \\
\hline & Other (including benign) & \\
\hline & & \\
\hline & & \\
\hline
\end{tabular}

${ }^{\star} \mathrm{P}<0.01$
Quality of life and functional outcome in patients who underwent curative surgery after preoperative chemoradiotherapy for mid-low rectal cancer S. Pucciarelli, ${ }^{*}$ P. Del Bianco, ${ }^{2}$ P. Toppan,

S. Serpentini, ${ }^{1}$ G.L. De Salvo, ${ }^{2}$ D. Nitti. ${ }^{1}$ 1. Clinica Chirurgica II, University of Padova, Padova, Italy; 2. Servizio Sperimentazioni Cliniche e Biostatistica. Istituto Oncologico Veneto, Padova, Italy.

Purpose: to evaluate Quality of Life (QoL), fecal continence and bowel function after preoperative chemoradiotherapy (pCRT) for mid-low rectal cancer. Methods: EORTC QLQ-C30, QLQ-CR38, Fecal Incontinence Score (FIS) questionnaires and a set of "ad hoc" questions related to defecatory problems were mailed to recurrence-free consecutive patients who, from 1994 to 2004 (minimum follow-up 18 months), had undergone pCRT followed by radical resection for mid low rectal cancer. Each QoL scale was separately analyzed using a regression model. Multivariate analysis was performed only for the global health status (QL) scale. A p-value $<0.01$ was considered statistically significant. Results: out of 113 eligible patients, $90(80 \%)$ returned completed questionnaires (M/F 52/38; 59 pts aged $\leq 65 \mathrm{y})$. Sphincter-saving procedures were performed in 84 patients however stoma was closed in 65 cases. Fifteen patients had early and 24 late major surgical complications. Urgency was "always" present in $42(66 \%)$ cases, incomplete evacuation in $41(64 \%)$, stool fractionation in $26(41 \%)$ and $>3$ bowel movements/day in $25(39 \%)$. Thirteen $(20 \%)$ patients were incontinent to liquids and/or solids weekly, daily or more than daily. Both at univariate and multivariate analysis, QL was impaired by stool fractionation $(p=.0003)$. Patients with urgency had poorer Role functioning than other patients $(p=.0053)$, Body image was impaired by the presence of stoma $(p=.0009)$, and sexual functioning was poorer in females than in males ( $p=.0047)$. Conclusions: defecation problems and fecal incontinence are frequent in rectal cancer patients who had undergone pCRT. Defecatory problems (not fecal incontinence) and QoL are closely associated. 
P233

Comparison of Stapled and Non-Stapled Hepatic Lobectomy

N.L. Emerson, * R. Goldstein, J.P. Lamont, J.A. Kuhn. Baylor University Medical Center, Dallas, TX.

Introduction: The transection of hepatic parenchyma has varied over the past twenty years. A novel technique of hepatic division using staplers has been suggested as an alternative. This study compares results of the stapled versus non-stapled technique in patients undergoing hepatectomy. Methods: An IRBapproved review was performed on all patients who underwent a lobectomy or partial lobectomy $(\mathrm{n}=50)$ at a single institution. Endpoints included estimated blood loss (EBL), transfusion requirements, operative time, and hospital length of stay (LOS). Results: Patients who underwent stapled right lobectomy $(S R L)(n=10)$, stapled left lobectomy $(S L L)(n=6)$,stapled left lateral lobectomy $(\mathrm{SLLL})(\mathrm{n}=4)$, or stapled partial right lobectomy (SPRL) $(\mathrm{n}=5)$, were compared to a group of patients undergoing non-stapled right lobectomy (NSRL) $(n=7)$, non-stapled left lobectomy (NSLL)( $\mathrm{n}=2)$, non-stapled left lateral lobectomy (NSLLL)( $n=11)$, or non-stapled partial right lobectomy (NSPRL) $(n=5)$. Patients who underwent a SRL had a decreased EBL compared to those who underwent a NSRL ( $373 \mathrm{cc}$ vs $764 \mathrm{cc}$ ). Similar results were noted in the left lobectomy group ( $408 \mathrm{cc}$ vs $475 \mathrm{cc}$ ), and the partial right lobectomy group (140cc vs $675 \mathrm{cc}$ ). However, the SLLL group had a higher EBL (350cc vs $211 \mathrm{cc}$ ). Fewer SRL patients required a transfusion during their first admission than NSRL patients ( $30 \%$ vs $71 \%$ ). Transfusion requirements were, on average, four units of PRBCs for NSRL patients vs two units of PRBCs for SRL patients. No patients who underwent a SLL, a SLLL, or a SPRL required transfusion during the first admission. Transfusions were required in $50 \%$ of NSLL patients, $27 \%$ of NSLLL patients, and $40 \%$ of NSPRL patients. Operative time was reduced for stapled resections of each type, with the greatest reduction being in the right lobectomy group ( $220 \mathrm{~min}$ vs $319 \mathrm{~min}$ ). Median LOS was similar between the stapled and non-stapled groups. Conclusions: Stapled hepatic transection for hepatectomy is a novel technique that allows for decreased blood loss, transfusion requirement, and operative time. This technique should be added to the armamentarium of general surgeons performing major liver resection.

\section{P234}

Positron emission tomography in patients with locally-advanced rectal cancer: Its role in detecting distant disease and defining optimal neoadjuvant therapy C.S. Nahas, ${ }^{*}$ T. Akhurst, A.J. Markowitz, B.D. Minsky, P. Paty, M. Weiser, L. Temple, W.D. Wong, S.M. Larson, J.G. Guillem. Surgery, Memorial Sloan-Kettering Cancer Center, New York, $N Y$.

Purpose: Since a number of patients with locally advanced rectal cancer present with synchronous distant metastases, the decision to pursue neoadjuvant chemoradiation versus systemic chemotherapy alone depends greatly on accurate determination of distant disease. We prospectively evaluated the ability of 18 Fluorodeoxyglucose (18F-FDG) positron emission tomography (PET) scanning for detection of distant disease in patients with locally-advanced rectal cancer otherwise eligible for combined modality therapy. Methods: 93 patients with locally advanced rectal cancer underwent a whole-body 18 F-FDG PET 2-3 weeks before combined modality therapy. Sites other than rectum, mesorectum or along the inferior mesenteric artery were considered distant and were divided into 9 groups: neck, lung, mediastinal lymph node (LN), abdomen, liver, colon, pelvis, peripheral LN, and soft tissue. Two nuclear medicine physicians blinded to clinical information used PET/CT images and a 5point scale (0-4) to determine certainty of disease. A score of $>3$ was considered malignant. Confirmation of disease was based on tissue diagnosis, surgical exploration, and subsequent imaging. Results: At a median follow-up period of 34 months, PET accuracy, sensitivity, and specificity for overall sites were $93.7 \%, 77.8 \%$, and $98.7 \%$, respectively. The best performance was obtained for liver (accuracy $=99.9 \%$, sensitivity $=100 \%$, and specificity $=98.8 \%$ ), and lung (accuracy $=99.9 \%$, sensitivity $=80 \%$, and specificity $=100 \%$ ). Of the
12 confirmed malignant sites in liver and lung, PET detected 11 of them. Conclusion: Since a baseline PET in patients with locally advanced rectal cancer can reliably detect metastatic disease in liver and lung, it should be considered in order to establish extent of distant disease and define type of neoadjuvant therapy.

\begin{tabular}{|c|c|c|c|c|c|}
\hline Sites & $\begin{array}{c}\text { Accuracy } \%(\mathrm{CI} \\
95 \%)\end{array}$ & $\begin{array}{c}\text { Sensitivity \%(CI } \\
95 \%)\end{array}$ & $\begin{array}{c}\text { Specificity } \%(\mathrm{Cl} \\
95 \%)\end{array}$ & $\begin{array}{c}\text { PPV } \%(\mathrm{CI} \\
95 \%)\end{array}$ & $\begin{array}{c}\text { NPV } \%(\mathrm{CI} \\
95 \%)\end{array}$ \\
\hline All & $93.7(86.6-100)$ & $77.8(57.5-90.0)$ & $98.6(97.5-99.3)$ & $\begin{array}{c}56.0 \\
(36.0-74.2)\end{array}$ & $\begin{array}{c}99.5 \\
(98.7-99.8)\end{array}$ \\
\hline Liver & $99.9(96.1-100)$ & $100(59.0-100)$ & $98.8(93.7-100)$ & $\begin{array}{c}87.5 \\
(47.4-99.7)\end{array}$ & $100(95.8-100)$ \\
\hline Lung & $99.9(96.1-100)$ & $80.0(28.4-99.5)$ & $100(95.9-100)$ & $\begin{array}{c}100 \\
(39.8-100)\end{array}$ & $\begin{array}{c}98.9 \\
(93.9-100)\end{array}$ \\
\hline
\end{tabular}

PPV : Positive predictive value

NPV : Negative predictive value

P235

Clinicopathologic Feature of Signet Ring Cell Carcinoma of the Colon and Rectum B. Min, ${ }^{*}$ N.K. Kim, S.H. Baek, Y.T. Ko, J.H. Cho, S.K. Sohn. Yonsei University College of Medicine Department of Surgery, Seoul, South Korea.

Introduction: Because of its rarity, there is little information about signetring cell carcinoma of the colon and rectum. The aim of the current study is to elucidate the clinicopathologic feature if signet-ring cell carcinoma arising from the colon and rectum by means of comparing with non-signet ring cell type adenocarcinoma. Methods: Retrieving a database of 4349 surgical patients with colorectal cancers operated between 1989 and 2004, twenty-seven patients with signet-ring cell carcinoma were identified. Clinicopathologic feature were compared with that of non-signet ring cell carcinoma group. Stage stratified comparison of oncologic outcome was made between different groups. Results: the mean age of signet-ring cell group was 47 years while that of non-signet ring cell carcinoma group was 59 years $(\mathrm{p}=0.000)$. Male-to-female ratio, tumor location, tumor size, rate of distant metastasis, curative resection rate, preand post-operative serum CEA levels were not significantly different between the groups. Lymph node metastasis rate was higher in signet-ring cell carcinoma group $(75.0 \%)$ than in non-signet ring cell carcinoma group $(52.9 \%)$ $(p=0.046)$. The ratio of positive lymph nodes to retrieved lymph nodes was significantly higher in signet-ring cell carcinoma group ( 48.5 vs. $22.7, \mathrm{p}=0.016)$. As to the rectal cancer, sphincter preserving procedures were less frequently used in signet-ring cell carcinoma group $(33.3 \%)$ than in the other group $(67.9$ $\%)(p=0.023)$. The frequencies of blood vessel invasion, lymphatic vessel invasion and perineural invasion were lower in signet ring cell carcinoma $(\mathrm{p}=0.000)$. Overall 5-year-survival rate of signet-ring cell carcinoma was $50.0 \%$ and $48.6 \%$ in stage II and III respectively and that of non-signet ring cell carcinoma was $81.9 \%$ and $60.8 \%$ in each. The survival curve was significantly different between the two group $(\mathrm{p}=0.019)$. Signet-ring cell carcinoma group shared similar characteristics with poorly differentiated type and showed significantly worse oncologic outcomes compared with differentiated types and mucinous type. Conclusion:Patients with signet-ring cell colorectal carcinoma have a worse prognosis. 
Perineural Invasion is a Significant Prognostic Indicator in Colorectal Cancer C. Liebig, * G. Ayala, N. Agarwal, G. Verstovsek, D.H. Berger, D. Albo. Baylor College of Medicine, Houston, TX.

Introduction: Perineural invasion (PNI) is emerging as a marker of aggressive tumor behavior. However, the clinical significance of PNI in colorectal cancer (CRC) is not well characterized. There are currently no animal models of PNI in CRC. In this study we evaluate the prognostic value of PNI in CRC and show an in vitro model of perineural colorectal tumor invasion. Methods: All cases of CRC resected at our institution during a 3 year period $(n=144)$ were reviewed. Demographic, pathology, tumor stage and outcome data (minimum 5 years) were entered into a database. All cases were re-reviewed for PNI by a pathologist. Dorsal root ganglia (DRG) from green fluorescent mice were co-cultured with KM12 (low invasive) or KM12L4 (highly metastatic) human colon cancer cells in matrigel. Neurite outgrowth was quantified using computerized image analysis. Results: Twenty-six percent (35/133) of tumors in our series were PNI positive. Only $1.5 \%(2 / 133)$ of tumors were reported as PNI positive on the initial pathology report. Five-year overall (OS) and disease-free survivals (DFS) were lower in PNI positive vs. PNI negative patients ( $17 \%$ vs. $44 \%$, p $<0.002$, and $19 \%$ vs. $50 \%$, p $<0.005$, respectively) (Fig. 1A). Median OS and DFS for PNI positive cases were also lower than PNI negative cases (55 vs. 126 months, $\mathrm{p}<0.002$ and 47 vs. 160 months, $\mathrm{p}<0.005$ ). Among patients with node-negative disease, PNI was associated with lower 5year OS and DFS $(42 \%$ vs. $75 \%, \mathrm{p}<0.05$ and $50 \%$ vs. $75 \%, \mathrm{p}<0.4)$. PNI positive cases were also three times more likely than PNI negative cases to have liver metastasis at staging $(14 \%$ vs. $43 \%, p<0.001)$. Our in vitro PNI assay showed colon tumor cell/neurite interaction as well as directional outgrowth of neurites towards cancer cells(Fig.1B). Neurite outgrowth was greater when DRG were co-cultured with KM12L 4 cells vs. less invasive KM 12 cells ( $30 \%$ vs. $12 \%, \mathrm{p}<0.05$ ). Conclusion: PNI is grossly under-reported in pathology reports. For both node-negative and positive CRC patients, PNI is a significant prognostic indicator. PNI status should be considered when determining the need for adjuvant treatment. The in vitro PNI assay could be useful in studying mediators of PNI in $\mathrm{CRC}$

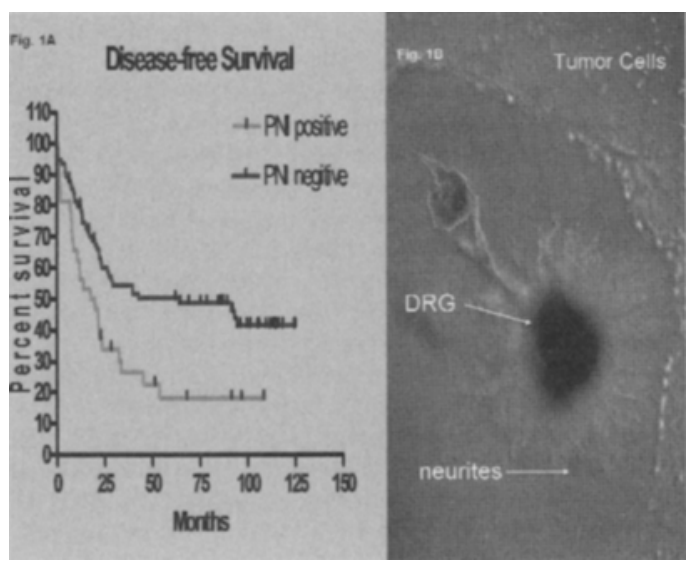

Surgery for Early Esophageal Adenocarcinoma: Analysis of Lymphatic Spread and Prognostic Factors A. Hughes, ${ }^{*}$ B.M. Smithers, J. Thomas, I. Martin, D.C. Gotley, A.P. Barbour. Surgery, Princess Alexandra Hospital, Woolloongabba, QLD, Australia.

Objective: Endoscopic treatment of esophageal cancer has been proposed as an alternative to esophagectomy. Knowledge of factors related to outcome is vital for the selection of treatment options. The aims of this study were to determine predictors and patterns of lymphatic spread and prognostic factors associated with overall survival for early (T1) esophageal and GEJ adenocarcinoma following esophagectomy. Methods: A prospective database identified 69 patients with T1 esophageal and GEJ adenocarcinoma who underwent esophagectomy without neoadjuvant therapy at a single institution between September 1987 and December 2005. Univariable and multivariable analyses were undertaken to determine factors predictive of outcome. Median follow up was 59 months. Results: Thoracoscopic 3-phase esophagectomy was performed in $61 / 68(88 \%)$ patients. There were no procedure related deaths. All patients underwent R0 resection. Of the 69 patients, 27 (39\%) had intramucosal (T1a) tumors and $42(61 \%)$ had tumors invading the submucosa (T1b) Lymph node metastases were identified in $9 / 69(13 \%)$ patients: $0 / 28(0 \%)$ with T1a tumors and $9 / 42(21 \%)$ with $\mathrm{T} 1 \mathrm{~b}$ tumors $(\mathrm{P}=0.01)$. The presence of lymph node metastases was also associated with lymphatic invasion in the primary tumor $(\mathrm{P}<0.01)$. Among the 9 patients with metastatic lymph nodes, $2(22 \%)$ had periesophageal mediastinal lymph node involvement and $7(78 \%)$ patients had positive perigastric lymph nodes. The 5 -year overall survival was $77 \%$ for the entire cohort. Univariate survival analyses identified $\mathrm{T} 1 \mathrm{~b}$ tumors $(\mathrm{P}=0.02)$ and $\mathrm{N}$ stage $(\mathrm{P}<0.01)$, but not lymphatic invasion, as prognostic factors. Multivariate analysis found that the presence of lymph node metastases was the only independent prognostic factor for overall survival ( $\mathrm{P}=0.02$, Cox method). Conclusions: The prevalence of lymph node metastases varies between Tla and $\mathrm{T} 1 \mathrm{~b}$ esophageal adenocarcinoma and may involve mediastinal or abdominal sites. For patients with tumors confined to the mucosa local resection techniques without lymphadenectomy may be an alternative to esophagectomy. Esophagectomy should remain the standard of care for patients with tumors invading the submucosa.

\section{P238}

EphB2 and EphB4 Receptor Tyrosine Kinase Expression in Primary and Metastatic Colon Cancer E. Ley, ${ }^{1}$ S.R. Kumar, ${ }^{2}$ J. Scehnet, ${ }^{2}$ A. Kaiser, ${ }^{1}$ G. Ault, ${ }^{1}$ R.W. Beart, ${ }^{1}$ P.S. Gill. ${ }^{2} 1$. Colorectal Surgery, University of Southern California, Los Angeles, CA; 2. University of Southern California, Los Angeles, CA.

Aberrant expression of certain EphB receptor tyrosine kinases (EphB2, EphB3 and EphB4) in colon cancer is increasingly acknowledged as a mechanism for transformation and progression from normal villus to adenoma to invasive cancer although the role of each of these receptors in different stages is not well characterized. We undertook a study of fresh frozen colon cancer (primary and metastatic) and matched normal adjacent tissue to address this question. EphB2 and EphB4 expression was determined by immunofluorescence in 20 human colon cancers and matched adjacent tissue, as well as 10 metastatic liver lesions with adjacent normal liver tissue. High EphB2 levels were noted in all normal colon specimens while EphB4 was detected with very low signal in nearly all the normal cases. In sharp contrast 18 of the 20 primary colon cancer specimens showed higher EphB4 expression compared to the adjacent normal tissue. EphB2 was detected in only half of the primary colon cancer tissues with signal intensity lower than adjacent normal colon. Metastatic colon cancer to the liver showed the following: EphB2 and EphB4 signals were not demonstrated in adjacent normal liver tissue, high levels of EphB4 signal was seen in all metastatic liver lesions and EphB2 was not expressed in the metastatic liver lesions. These data indicate that $1 . \mathrm{EphB} 2$ is expressed in normal colon and $50 \%$ of colon cancers, 2 . EphB4 has minimal expression in 
normal colon, but all cancers express high levels, 3. Metastatic tumors to the liver express high EphB4 levels only.

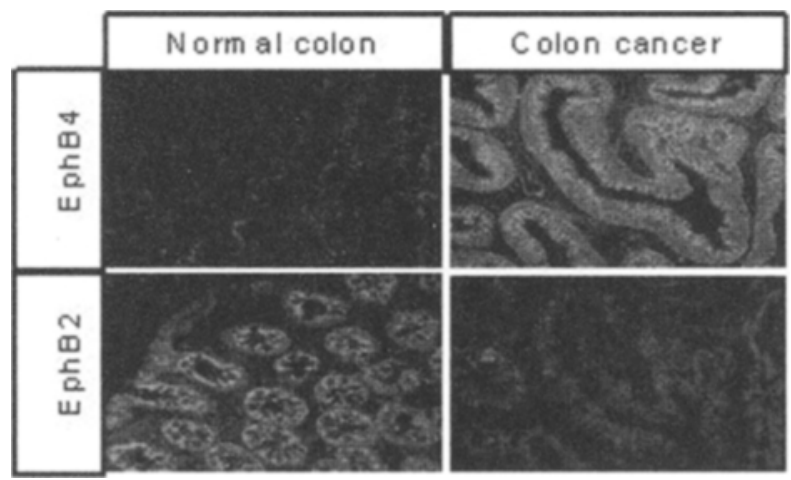

EphB4 is highly expressed in colon cancer, while minimal expression is observed in adjacent normal tissue. In contrast EphB2 is expressed in the normal colon and little appreciable expression is observed in the colon tumor tissue.

P239

Tumors of the Small Intestine: Presentation, Treatment, and Outcome S. Carpenter, * A. James, J.E. Eckford, J.P. Sullivan, J.S. Thomas, P. Ashley, M. Shabahang. Surgery, Texas A\&M University/Scott and White, Temple, TX.

Introduction: Tumors of the small intestine are uncommon and as such have not been well characterized. Methods: A prospectively collected Tumor Registry at a single institution was queried for patients with a diagnosis of small intestinal malignant tumor. The time span ranged from 1976-2005. Results: The cohort included in the study consisted of 173 patients. The median age at presentation was 54 . There was an equal distribution of males and females. Tumors included adenocarcinoma (29\%), carcinoid (38\%), GIST $(11 \%)$, lymphoma $(18 \%)$, and others $(4 \%)$. The majority of the tumors occurred in the ileum $(47 \%)$ with $24 \%$ in the duodenum and $22 \%$ in the jejunum. The predominant type of tumor in each location differed: $62 \%$ of duodenal tumors were adenocarcinoma with $21 \%$ carcinoid in contrast to $18.4 \%$ adenocarcinoma and $47 \%$ carcinoid in the jejunoileal region. Lymphoma was more prevalent in the jejunoileal region comprising $23 \%$ of the tumors vs. $7 \%$ in the duodenum. GISTs were equally distributed: $9.5 \%$ of duodenal vs. $11 \%$ of jejunoileal. The presenting symptoms in the entire cohort were abdominal pain $(37.5 \%)$, obstruction (26\%), weight loss (15\%), diarrhea (13\%), and incidental $(10 \%)$. Diagnosis was made by endoscopy in $23 \%$, CT in $9 \%$, and surgery in $61 \%$. Surgical therapy was undertaken in $83 \%$ of the patients with $88 \%$ undergoing small bowel resection and $12 \%$ having pancreaticoduodenctomy. Of the $144,48 \%$ had positive nodes. The median follow-up was 28.2 months(m). The median survival of the entire cohort was 72 months. The median survival was signifantly higher for lymphoma $(145 \mathrm{~m})$ and carcinoids $(109 \mathrm{~m}) \mathrm{vs}$. GIST $(40 \mathrm{~m})$ and adenocarcinomas $(15 \mathrm{~m}), \mathrm{p}<.001$. Location had a significant impact on survival with median survival being $103 \mathrm{~m}$ for ileal tumors compared to 76 $\mathrm{m}$ for jejunal and $19 \mathrm{~m}$ for duodenal tumors $(\mathrm{p}=.0059)$. Patients younger than 50 years had a median survival of $165 \mathrm{~m}$ compared to $59 \mathrm{~m}$ for those above 50 $(0.055)$. Nodal involvement and distant metastasis did not affect survival. Comparison to the SEER data showed similar results. Conclusions: Small bowel tumors are a variable group in which prognosis is determined mainly by type and location of tumor.
Intraoperative Ultrasound Improves Staging of Extra-Hepatic Bile Duct Malignancy J.V. Sitzmann, C.M. Schmidt.* surgery, indiana university, Indianapolis, IN.

INTRODUCTION: Aim of study is evaluation of extra-hepatic bile duct carcinoma preoperative staging tests versus intraoperative ultrasound (IOUS) staging to determine each exam diagnostic and therapeutic staging specificity, sensitivity and exam cost.Despite multiple preoperative imaging studies, surgeon operative open evaluation remains the final accurate determination of diagnosis and resectability. METHODS: From 1992 to 2004, a single surgeon performed IOUS on 130 consecutive patients with a preoperative diagnosis of hepatic biliary malignancy: cholangiocarcinoma or ampullary malignancy. For each patient, preoperative radiology tests: CT, MRI, angiography, transcutaneous and/or endoscopy ultrasound (EGDUS) were compared with IOUS. Diagnostic and therapeutic staging specificity and sensitivity and cost were assessed. RESULTS: IOUS altered staging and/or resection assessment in 90\% (115/130) patients. Most important with "cancer v. benign disease" in which the impression changed for all 60 patients. In addition, IOUS determined "resectability" $(95 \%, 125 / 130)$. IOUS significantly improved identifying previously undetected metastatic disease $(20 \%)$, determining resectability $(60 \%)$, and identifying vessel involvement $(25 \%)$. IOUS increased specificity and sensitivity determining: 1. vascular involvement than arteriography or CT; 2 . metastatic disease than CT or EGDUS. IOUS+MRI was $20 \%$ lower cost and $50 \%$ more accurate than $\mathrm{CT}+$ Arteriography+ EGDUS. CONCLUSION: IOUS has significant benefit in diagnostic and therapeutic staging of extra hepatic cholangiocarcinoma or ampullary cancers. IOUS with MRI lowers cost and increases staging accuracy compared with $\mathrm{CT}$ and invasive procedures: angiography or endoscopy. Data supports conclusion that MRI + IOUS may supplant invasive radiograph and endoscope diagnostic studies for therapeutic evaluation of extra hepatic biliary malignancy.

\section{P241}

How Does Young Age Influence the Treatment and Outcome of Colon Cancer H.M. Quah, * D. Schrag, W.D. Wong, L.K. Temple, J.G. Guillem, P.B. Paty, M.R. Weiser. Surgery, Memorial Sloan-Kettering Cancer Center, New York, NY.

Introduction: Early age at onset is often considered a poor prognostic factor for colon cancer. The aim of this study was to determine associations between age and clinicopathologic features, use of adjuvant therapy, and survival following colon cancer resection. Methods: A prospective database of 1327 surgically stage I-III colon cancer patients operated on between 1990 and 2001 was evaluated and patients grouped by age. Clinicopathologic features, use of adjuvant chemotherapy, RFS, DSS and OS were evaluated in patients less than or equal to 40 and those greater than 40 years. Results: 68 patients $(5 \%)$ were diagnosed at age 40 years or younger compared with 1259 patients diagnosed at age greater than 40 . Younger patients were more likely to have a familial colorectal cancer including HNPCC ( $7 \%$ versus $3 \%$ HNPCC by history, $\mathrm{p}=0.03$ ). Younger patients were no more likely to present with symptomatic lesions but were more apt to have left sided tumors $(66 \%$ vs. $51 \%, \mathrm{p}=0.02)$. Pathologic stage was similar in both cohorts (Table). With regard to treatment, younger patients were more likely to have extended resections as denoted by a greater number of identified lymph nodes (median 18 versus $14, \mathrm{p}=0.001$ ). Younger patients were also more likely to receive adjuvant chemotherapy and this was most pronounced in the stage II cohort: $39 \%$ vs $14 \%, p=0.003$. With a median follow-up of 55 months, 5-year DSS rate was similar in both study groups: $85 \%$ in younger patient cohort and $84 \%$ in the older patient cohort. Five-year OS was significantly higher in the young patient cohort $(84 \%$ versus $73 \%, p=0.001$ ). Age remained an independent predictor of overall survival on multivariate analysis (HR $0.5 ; 95 \%$ confidence interval $0.3-0.9 ; p=$ $0.03)$. Conclusion: Young patients undergoing complete resection of stage IIII colon cancer have similar DSS as older patients. However, young patients 
are more likely to undergo more radical resections and receive adjuvant therapy; especially for node-negative disease. These factors may have contributed to their overall favorable outcome.

Clinicopathologic features and outcome of young colon cancer patients

\begin{tabular}{|c|c|c|c|c|}
\hline & & Younger cohort ( $\leq 40$ yrs) & Older cohort ( $>40$ yrs) & p-value \\
\hline & $\mathrm{n}(\%)$ & $68(5)$ & $1259(95)$ & \\
\hline Family History & HNPCC (\%) & $5(7)$ & $35(3)$ & 0.032 \\
\hline \multirow{2}{*}{ Location } & Right & $23(34)$ & $617(49)$ & \multirow{2}{*}{0.015} \\
\hline & Left & $45(66)$ & $642(51)$ & \\
\hline \multirow{2}{*}{ Pathology } & Median \# Nodes & 18 & 14 & 0.001 \\
\hline & Poor Path Features & $25(37$ & $526(42)$ & 0.414 \\
\hline \multirow{3}{*}{ AJCC Stage } & I & $22(32)$ & $401(32)$ & \multirow{3}{*}{0.561} \\
\hline & II & $23(34)$ & $498(39)$ & \\
\hline & III & $23(34)$ & $360(29)$ & \\
\hline \multirow{2}{*}{ Chemotherapy } & Stage II & $9(39)$ & $68(14)$ & 0.003 \\
\hline & Stage III & $20(87)$ & $287(80)$ & 0.590 \\
\hline \multirow{2}{*}{ Outcome } & 5 -yr DSS $(95 \% \mathrm{CI})$ & $85 \%(75-95)$ & $84 \%(82-87)$ & 0.636 \\
\hline & $5-\mathrm{yr}$ OS $(95 \% \mathrm{Cl})$ & $84 \%(75-94)$ & $73 \%(70-76)$ & 0.001 \\
\hline
\end{tabular}

\section{P242}

Isolated Hepatic Perfusion Is Effective Therapy For Unresectable, Isolated Liver Metastases M.J. D'Alessio, ${ }^{1 *}$ R. Anunobi, ${ }^{2}$ T.C. Gamblin, ${ }^{2}$ M. Holtzman, ${ }^{2}$ S. Pappas, ${ }^{2}$ C.K. Brown, ${ }^{2}$ H.J. Zeh, ${ }^{2}$ D.L. Bartlett. ${ }^{2}$ 1. Mitchell Cancer Institute, University of South Alabama, Mobile, AL; 2. University of Pittsburgh Medical Center, Pittsburgh, PA.

INTRODUCTION: Isolated hepatic perfusion (IHP) is a novel, regional therapy for patients with unresectable, isolated liver metastases. We reviewed a single-institution experience with IHP over a 3 year period to examine the safety and efficacy of this technique. METHODS: From 4/2003 to 4/2006, 28 consecutive patients with unresctable, isolated liver metastases were treated with regional chemotherapy to the liver using IHP at a tertiary cancer center. Patients had biopsy-proven malignancy, and were deemed unresectable by experienced liver surgeons. Outcome data was obtained from the electronic medical record and radiographic studies. This study wàs conducted in concordance with IRB and HIPAA regulations. Radiographic response was judged using RECIST criteria. Survival analysis was performed using the Kaplan-Meier method. RESULTS: Patient and surgical characteristics are depicted in the Table. Pathologic types treated included colorectal adenocarcinoma, cholangiocarcinoma, and metastatic melanoma. Nineteen patients underwent IHP with melphalan; 9 received oxaliplatin. Median length of hospitalization was $10 \mathrm{~d}$ [range 5-42], with a median ICU stay of $5 \mathrm{~d}$. There were no deaths within 30 days of IHP. Two patients who received melphalan developed liver failure during the post-operative period $(10 \%)$. One patient who received oxaliplatin developed veno-occlusive disease as the dose limiting toxicity on a phase 1 trial. Of the 25 evaluable patients, $15(60 \%)$ had a radiographic response, with 3 patients $(12 \%)$ achieving a complete response. Three patients $(12 \%)$ went on to undergo complete resection of their liver tumors. At a median follow-up of $17.2 \mathrm{~m}$ [range 4.8-35.8], estimated median survival for the treated patients is $29 \mathrm{~m}$. Patients whose tumors displayed a radiographic response to IHP had significantly longer survival than nonresponders $(p=0.006)$. CONCLUSION: IHP is an effective therapy for patients with unresectable, isolated liver metastases. IHP provides excellent response and survival rates in highly pre-treated patients with significant disease burden. IHP can be delivered safely with acceptable morbidity and mortality in a tertiary care setting.
Patient and Surgical Characteristics

\begin{tabular}{|c|c|c|c|}
\hline Number of patients $(\mathrm{n})$ & & 28 & \\
\hline Age & & 55 & {$[24-77]$} \\
\hline ECOG Status & & 0 & {$[0-1]$} \\
\hline \multirow{3}{*}{ Primary Pathology } & Colorectal & $22 / 28$ & $(79 \%)$ \\
\hline & Melanoma & $4 / 28$ & $(14 \%)$ \\
\hline Prior Systemic Therapy & Cholangiocarcinoma & $2 / 28$ & $(7 \%)$ \\
\hline Number of Cycles & & $19 / 28$ & $(68 \%)$ \\
\hline \multirow{2}{*}{ Hepatic Metastases } & & 6 & {$[2-16]$} \\
\hline & \#ize of Largest Lesion & 5.5 & {$[1.4-12.1]$} \\
\hline Operative Time (hr) & $\%$ Hepatic Replacement & 20 & {$[5-60]$} \\
\hline Estimated Blood Loss $(\mathrm{mL})$ & & 5.4 & {$[3.3-9.5]$} \\
\hline Units pRBC Transfused & & 800 & {$[250-2800]$} \\
\hline Pump Time (veno-veno) $(\mathrm{min})$ & & 2.5 & {$[0-6]$} \\
\hline Flow Rate (veno-veno) $(\mathrm{mL} / \mathrm{min})$ & & 96 & {$[82-121]$} \\
\hline Hepatic Perfusion Time $(\mathrm{min})$ & & 2400 & {$[1300-4500]$} \\
\hline Hepatic Perfusion Rate $(\mathrm{mL} / \mathrm{min})$ & & 80 & {$[65-89]$} \\
\hline Max. Hepatic Temp $\left({ }^{\circ} \mathrm{C}\right)$ & & 500 & {$[280-910]$} \\
\hline
\end{tabular}

Data expressed as median [range] unless otherwise specified.
Significance of loss of interface with portal vessels in prognosis of pancreatic adenocarcinoma J.L. Wild,* J. Scruggs, R. Erickson, J. Sing, J.E. Eckford, M. Shabahang. Surgery, Texas A\&M University/Scott and White, Temple, TX.

Introduction: Loss of interface (LOI) between tumor and portal vein (PV) or superior mesenteric vein (SMV) is commonly reported by endoscopic ultrasound (EUS) and CT. The impact of this finding on survival has not been characterized. Methods: A retrospective analysis of patients who underwent successful resection of pancreatic adenocarcinoma was undertaken. Results of EUS as regards to venous involvement were compared to intraoperative findings. Results: From 1996 to 2006, 683 patients with pancreatic carcinoma were identified. Of those with pancreatic adenocarcinoma, 105 underwent successful resection. In this cohort, EUS identified LOI in 20. Of these, only 12 had venous involvement necessitating resection at time of surgery. Among the 85 patients who were not thought to have LOI on EUS, 11 did have vein involvement and resection at the time of surgery. This yielded a sensitivity of $52 \%$ and specificity of $90 \%$. Similar analysis of CT data showed a sensitivity of $19 \%$ and specificity of $97.5 \%$. Median overall survival of the entire cohort was 14 months. Patients with LOI on EUS had a median survival of 16 months compared to 13 months among those with no LOI $\left(p^{=} .633\right)$. The median survival of the patients with vein resection was 15 months compared to 12 months for those without ( $\mathrm{p}=817)$. Conclusion: In patients with pancreatic adenocarcinoma, EUS has limited success in identifying patients with portal venous 
involvement. Neither LOI on EUS or venous involvement at the time of surgery have a significant impact on survival.

\begin{tabular}{|c|c|c|}
\hline & Loss of Interface on EUS & No Loss of Interface on EUS \\
\hline $\mathrm{n}$ & 20 & 85 \\
\hline $\mathrm{M}: \mathrm{F}$ & $12: 8$ & $42: 43$ \\
\hline Median Age(years) & 70.5 & 71 \\
\hline Location (Head) & $90 \%$ & $82 \%$ \\
\hline Mean Size (cm) & 3.2 & 3.2 \\
\hline Positive Nodes & $60 \%$ & $46 \%$ \\
\hline Positive Margins & $35 \%$ & $22 \%$ \\
\hline Vein Resection & 12 & 11 \\
\hline
\end{tabular}

\section{P244}

In Vitro Drug Resistance Profiles and Biomarker Expression in 414 Freshly Resected Esophageal Cancer Specimens I. Yu, ${ }^{*}$ R. Parker, E. Mechetner. Oncotech, Inc., Tustin, CA.

INTRODUCTION: Current management of esophageal cancer includes surgery combined with chemotherapy/radiation therapies. Despite recent advances, the majority of patients develop metastatic disease with a poor prognosis. More efficient agents and patient tailored treatments are needed to improve outcomes. This study aims at identifying approaches to individualized chemotherapy through the analysis of drug response and biomarker expression in esophageal tumors. METHODS: 414 freshly resected tumors were exposed in vitro to suprapharmacologic concentrations of $5 \mathrm{FU}$, cisplatin, doxorubicin, gemcitabine, mitomycin C, and taxol in the EDR assay and analyzed by flow cytometry for S-phase/DNA ploidy or immunohistochemistry for the expression of tumor associated markers. RESULTS: Spearman rank analysis showed significant correlation of drug resistance profiles between: $5 \mathrm{FU}$ vs cisplatin (two-tailed $\mathrm{p}=0.0099)$, doxorubicin $(\mathrm{p}=0.0002)$, and mitomycin $(\mathrm{p}=0.0424)$; cisplatin vs doxorubicin $(p=0.0031)$ and mitomycin $(p=0.0023)$; gemcitabine vs doxorubicin ( $\mathrm{p}=0.0222)$ and taxol $(\mathrm{p}=0.007)$; and mitomycin vs doxorubicin $(p=0.0003)$. There was no correlation between in vitro drug response patterns for: $5 \mathrm{FU}$ vs taxol $(\mathrm{p}=0.3509)$ and gemcitabine $(\mathrm{p}=0.6622)$; cisplatin vs taxol $(\mathrm{p}=0.1647)$ and gemcitabine $(\mathrm{p}=0.102)$; doxorubicin vs taxol $(\mathrm{p}=0.3743)$; gemcitabine vs mitomycin ( $p=0.5817)$; and mitomycin vs taxol $(p=0.2648)$. No drugs showed correlation with the S-phase/aneuploidy status or with biomarker expression. Significant correlations were found for MDR 1 vs MRP $(p=0.0055)$, Ki-67 vs PCNA ( $p=0.0023)$ and S-phase $(p=0.0052)$, and between various angiogenesis markers. CONCLUSIONS: Our data suggest that traditional esophageal cancer protocols based on 5FU, cisplatin, and mitomycin can be complemented with other agents, such as taxol, gemcitabine, and doxorubicin. The taxol drug response pattern was unique compared to the other drugs, suggesting a different mechanism of growth inhibition and potential efficiency of taxol based drug combinations. New studies are warranted to prove synergy of these combinations in esophageal cancer and the role of the EDR assay in individualized therapies.

\section{P245}

\begin{abstract}
Is Pancreatic Resection Justifiable in the Presence of Gross Residual (R2 Resection) or Metastatic (M1) Disease? K.M. Brown, ${ }^{1 *}$ D.M. Heimann, ${ }^{1}$ D. Kim, ${ }^{2}$ J.C. Watson, ${ }^{1}$ J.P. Hoffman. ${ }^{1}$ 1. Surgical Oncology, Fox Chase Cancer Center, Philadelphia, PA; 2. Temple University School of Medicine, Philadelphia, PA.
\end{abstract}

Introduction: A complete resection is the goal of pancreatic surgery. Preoperative or intraoperative circumstances may however preclude this goal.
Methods: Retrospective chart review of pancreatic resections, with demographic and treatment variables abstracted from patient charts. Descriptive statistics were calculated using SPSS. Survivals were calculated using the Kaplan-Meier method. Results: Of the 300 pancreatic resections for pancreatic adenocarcinoma performed between September 1987 and April 2006, 33 (11\%) were determined to have had an R2 resection or M1 disease at the time of exploration. Median age at diagnosis was 69 years (range 45-81), and $15(45 \%)$ were male. Tumor location was in the head in 23 patients, head and body in 6 , tail in 2 , and head and tail in 2 . Two patients had a distal pancreatectomy, 28 patients had a pancreaticoduodenectomy, and 3 underwent a total pancreatectomy. In 30 patients the surgical goal was attempted cure, while 3 patients were explored for ongoing bleeding. Seven patients had previous non-resectional surgery, 2 with IORT. Eleven of the $33(33 \%)$ patients received neoadjuvant chemoradiation. Seven patients $(21 \%)$ had resections in the setting of M1 disease, 12 (36\%) had R2 resections, and $14(42 \%)$ had positive peritoneal cytology (PPC). There were 2 peri-operative deaths $(6 \%), 1$ in a patient who underwent an emergency procedure. Eleven patients had complications (33\%). Median length of stay (LOS) was 13 days (5-34). Median overall survival (OS) from diagnosis was 16 months $(2-57)$ and 10 months $(0-57)$ from resection. Five patients are still alive 6-57 months after surgery ( 2 with PPC, 3 with R2 resections); all are being treated with chemotherapy. Conclusions: We report the largest combined $\mathrm{R} 2$ and $\mathrm{M} 1$ population. These operations are unavoidable if one wishes to give patients with radiologically resectable tumors the benefit of resection. Pancreatic resections can be safely performed in this cohort with comparable complication rates and LOS. As demonstrated here, long survival is possible in this population but it is clearly dependent on successful long-term chemotherapy postoperatively.

\section{P246}

\section{Adjuvant radiation therapy improves survival for surgically} resected gastric adenocarcinoma-A population based study E.T. Castaldo, ${ }^{*}$ J.A. Greco, I.D. Feurer, C.W. Pinson, A.B. Chakravarthy, A.A. Parikh, N.B. Merchant. Vanderbilt University Medical Center, Nashville, TN.

Background: Although adjuvant chemoradiation therapy for resected gastric adenocarcinoma is extensively used in the United States, recent prospective randomized data have questioned its survival benefit. The primary aim of this study is to determine the effect of adjuvant radiation (XRT) in patients with surgically resected gastric adenocarcinoma using a population-based database. Methods: Using the Surveillance, Epidemiology, and End Results (SEER) registry, all patient records with surgically resected gastric adenocarcinoma from $1973-2003$ were queried. Patients with metastatic disease, pre operative or intra-operative radiation therapy, multiple primary malignancies or incomplete tumor grading, staging, radiation, or demographic data were excluded. A Cox regression model was tested with gender, race, tumor grade, age over 60 years, adequacy of lymph node dissection (defined as $\geq 15$ nodes resected), stage, and radiation as co-variates Results: A total of 12,345 patients were eligible for analysis (2,580 received adjuvant XRT while 9,765 did not). Mean follow-up for the entire cohort was 30 mos. When stratified by stage, adjuvant XRT resulted in an improved median survival (see table). Additionally, Cox regression revealed a significantly improved survival in patients who received adjuvant radiation therapy compared to those who did not $(\mathrm{HR}=0.72 ; 95 \% \mathrm{CI} 0.68-0.76 ; \mathrm{p}<0.01)$. Additional independent significant factors leading to improved survival included female gender $(p<0.01)$, race other than black compared to white $(p<0.01)$, and an adequate lymph node dissection $(p<0.01)$. Independent factors significantly associated with poorer survival included poor and undifferentiated histology ( $p=0.01$ and 0.03 , respectively), age $>60$ yrs. $(p<0.01)$, and increased stage $(\mathrm{p}<0.01$ for each increase in stage). Conclusion: Improved survival was demonstrated for patients receiving adjuvant radiation after resection for gastric adenocarcinoma even when adjusted for the effects of gender, race, tumor grade, age, stage, and adequacy of lymph node dissection. Additionally, adequate lymph node dissection remained an independent predictor of survival. 
Overall Survival by Stage

\begin{tabular}{|c|c|c|c|c|c|}
\hline \multirow{2}{*}{ Stage } & \multicolumn{2}{|r|}{ Resection Alone } & \multicolumn{2}{|r|}{ Adjuvant Radiation } & \multirow{2}{*}{$\mathrm{p}$ value } \\
\hline & $\mathrm{N}$ & Median Survival (months) & $\mathrm{N}$ & Median Survival (months) & \\
\hline 2 & 2,058 & 37 & 451 & 51 & $<0.01$ \\
\hline $3 \mathrm{~A}$ & 2,717 & 18 & 980 & 26 & $<0.01$ \\
\hline $3 \mathrm{~B}$ & 1,119 & 12 & 507 & 18 & $<0.01$ \\
\hline $4^{*}$ & 1,543 & 10 & 497 & 18 & $<0.01$ \\
\hline
\end{tabular}

Stages $1 \mathrm{~A}$ and $1 \mathrm{~B}$ not shown as sufficient analyses could not be performed.

*Stage 4 represents non metastatic Stage 4 disease only.

\section{P247}

The apoptosis signal ceramide (C6) significantly enhances the antitumor effects of oxaliplatin and gemacitabine on the growth of human pancreatic cancer in scid mice D. Shrayer, ${ }^{1}$ M. Resnick, ${ }^{2}$ S. Hazelwood, ${ }^{1}$ H.J. Wanebo. ${ }^{1}$ * 1 . Surgery, Roger Williams Gen Hospital, Providence, RI; 2. Rhode Island Hospital, Providence, RI.

Introduction: Ceramide gemcitabine (C6) is an analog of endogenous ceramides, a major signaling pathway for apoptosis in cells undergoing stress or exposure to chemotherapy. We have previously demonstrated synergistic anti-tumor effects of combining C 6 ceramide with Paclitaxel and Cisplatin. Background: Currently the invivo anti tumor effects of $\mathrm{C} 6$ with oxaliplatin and gemcitabine. Methods: Invivo experiments SCID/Beige/Taconic male mice inoculated S.C. with 2X106 L3.6 pancreatic cells were treated 4 days post tumor implant with thrice weekly $(3 \mathrm{x} / \mathrm{wk})$ intraperitoneal (IP) injections of paclitaxel (P) $3.0 \mathrm{~m} / \mathrm{kg}$, oxaliplatin (OX) $2.5 \mathrm{mg} / \mathrm{kg}$, cisplatin (CP) $2.5 \mathrm{mg} / \mathrm{kg}$, gemcitabine (Gem) $15 \mathrm{mg} / \mathrm{kg}$ with $/$ without ceramide $10 \mathrm{mg} / \mathrm{kg}$. Mice were observed for 6 weeks and were autopsied when near death. (All controls died by 3 rd week). Maximum tumor volume, tumor weight; body weight and survival were recorded. Preliminary mechanistic studies focused on the role of caspase activation and mitochondrial depolarization as demonstrated by Zvrad-FMK (caspase inhibitor), JC-1 fluorescence probe. Separate histopathology studies recorded short term effects on tumor necrosis, mitotic index and caspase 3 index. Results: Combination with $\mathrm{C} 6$ ceramide augmented the tumor reduction obtained by chemotherapy alone by $57 \%$ (while preserving body weight), and increased 6 week survival from $0 \%$ (Chemotherapy alone) to $60 \%$ with combined therapy. Mean survival was increased from 25 to 37 days. Although short term immunohistochemical studies suggested enhanced apoptosis and increased caspase 3 production by ceramide combinations, in vitro studies suggest that the anti-tumor effects of ceramide combinations may actually be independent of caspase activation and mitochondrial activation. Conclusion: Combination therapy with the apoptotic signal C 6 Ceramide significantly enhanced the anti tumor regression and survival induced by Oxaliplatin and gemcitabine in SCID Mice bearing L3.6 pancreatic tumor implants. The mechanism of the ceramide effect is unclear and is under continued investigation.
Effect of C6 Ceramide +/- Chemotherapy on L3-6 Growth in Scid Mice

\begin{tabular}{|c|c|c|c|c|}
\hline \multicolumn{5}{|c|}{ In Vivo Anti-tumor Response } \\
\hline Drugs & $\begin{array}{c}\text { Mean Final } \\
\text { Tumor Volume }\end{array}$ & $\begin{array}{c}\text { Mean Survival } \\
\text { Time (days) }\end{array}$ & $\begin{array}{c}\text { \% Survival @ } \\
\mathbf{3} \text { \& 6 Wks }\end{array}$ & $\begin{array}{c}\text { Mean Body Weight } \\
\text { (g)(Time of Death or } \\
\text { Sacrifice) }\end{array}$ \\
\hline Control & $1.56+/-0.2$ & $17.8+/-1 / 1$ & $0 \% / 0 \%$ & 17.8 \\
\hline Ceramide & $1.69+/-0.3$ & $20.8+/-1.1$ & $40 \% / 0 \%$ & 17.0 \\
\hline Taxol & $1.83+/-0.4$ & $23.0+/-2.4$ & $60 \% / 0 \%$ & 17.4 \\
\hline Oxaliplatin & $1.76+/-0.2$ & $27.4+/-2.2$ & $100 \% / 0 \%$ & 15.6 \\
\hline Cisplatin & $1.83+/-0.1$ & $25.6+/-3.2$ & $60 \% / 0 \%$ & 16.6 \\
\hline $\begin{array}{c}\text { Ceramide \& } \\
\text { Taxol }\end{array}$ & $1.19+/-0.1(++)$ & $35.2+/-4.0(++)$ & $\begin{array}{c}100 \% / \\
60 \%(++)\end{array}$ & $20.0(++)$ \\
\hline $\begin{array}{c}\text { Ceramide \& } \\
\text { Oxaliplatin }\end{array}$ & $0.75+/-0.01(++)$ & $35.0+/-4.4(++)$ & $\begin{array}{c}100 \% / \\
60 \%(++)\end{array}$ & $20.0(++)$ \\
\hline $\begin{array}{c}\text { Ceramide \& } \\
\text { Cisplatin }\end{array}$ & $1.16+/-0.01(++)$ & $40.6+/-1.4(++)$ & $\begin{array}{c}100 \% / 60 \% \\
(++)\end{array}$ & $20.0(++)$ \\
\hline $\begin{array}{c}\text { Ceramide } \\
\& \text { Gemcitabine }\end{array}$ & $0.693(++)$ & $38.8+/-3.2(++)$ & $\begin{array}{c}100 \% / \\
60 \%(++)\end{array}$ & $18.6(++)$ \\
\hline \multicolumn{5}{|c|}{ Significance $+\mathrm{p}<0.1,++\mathrm{p}<0.05,+++\mathrm{p}<0.01$} \\
\hline
\end{tabular}

\section{P248}

Evaluation of Multiprobe Radiofrequency and Microwave Ablation Technology W.W. Hope, ${ }^{*}$ A.E. Lincourt, W.L. Newcomb, T.M. Schmelzer, B.T. Heniford, D.A. Iannitti. Department of Gastrointestinal and Minimally Invasive Surgery, Carolinas Medical Center, Charlotte, NC.

Multiprobe Radiofrequency and Microwave ablation is a developing technology. The purpose of this study is to evaluate two new radiofrequency devices in an in-vivo animal model and the effect of clustered microwave probes in invivo perfused hepatic tumors Multiprobe ablations were performed in 12 porcine livers. Half of the ablations were performed with a Pringle maneuver. 200 watt RF generators were used. Three $3.0 \mathrm{~cm}$ active tip Cooled-Tip single probes $2.0 \mathrm{~cm}$ apart with switcher controller was compared to standard clustered Cooled-Tip probes. The liver was then removed for gross and histological assessment. Outcome measures were the ablation dimensions, time and histologic analysis. Microwave ablations to the liver ( 45 watts for 10 minutes) were performed on 11 patients in an "ablate and resect" trial from April 2003 to December 2003. Thirty-four total radiofrequency ablations were performed in the porcine livers. Diameter $(\mathrm{mm})(51.4$ vs. 40.3, p. 0001$)$, surface area $(\mathrm{cm} 2)(22.4$ vs. $16.0, \mathrm{p}, .0002)$, and volume (cc) $(66.1$ vs. $36.9, \mathrm{p}, .0001)$ were significantly increased for the combination probes with switcher controller compared to the standard clustered probes respectively. Ablation volumes for the Pringle vs. No Pringle in the combination probes were $68.0 \mathrm{cc}$ vs $64.3 \mathrm{cc}$ and for the clustered probes $40.1 \mathrm{cc}$ vs $33.7 \mathrm{cc}$ respectively. Microwave ablation average size was noted to be $5.65 \times 4.42 \times 3.67 \mathrm{~cm}$ with an average volume of $50.4 \mathrm{~cm} 3$. The use of switcher controller with combination probes consistently resulted in larger ablation diameter, surface area, and volumes when compared to a standard clustered probe. Use of the the Pringle maneuver resulted in similar sized ablations in half the time. Microwave ablation resulted in comparable overall volume to radiofrequency. Multiprobe ablation may have a role in the treatment of larger liver tumors. Further trials are needed to evaluate these emerging technologies.

\section{P249}

Treatment of Colorectal Cancer Liver Metastases with Y-90 Microspheres Selective Internal Radiation Treatment S.A. Gulec, ${ }^{1 *}$

R. Hostetter, ${ }^{1}$ D. Schwartzentruber, ${ }^{1}$ K. Pennington, ${ }^{1}$ D. Bruetman, ${ }^{1}$ J. Wheeler, ${ }^{1}$ A.S. Kennedy. ${ }^{2} 1$. Center for Cancer Care, Goshen, IN;

2. Wake Radiology Oncology, PLLC, Cary, NC.

Introduction Yttrium-90 (Y-90) microsphere therapy via hepatic arterial administration is emerging as a mainstream treatment modality in the management of patients with primary and metastatic liver cancer. The selective nature of microsphere distribution allows the delivery of high doses of radiation to tumors while keeping the exposure of the un-involved liver at a minimum. The technique has shown very promising outcomes in patients with colorectal cancer liver metastases (CRCLM) in early clinical trials. Methods 
Treatment records and follow-up data on 39 patients $(22 \mathrm{M}, 17 \mathrm{~F}$, and ages 45 - 86) with CRCLM who underwent a selective internal radiation treatment (SIRT) with Y-90 resin microspheres (SIR-SpheresTM Sirtex Medical, Lake Forest, IL) were retrospectively reviewed. Liver toxicity was assessed by liver function tests. Response to treatment was evaluated by CEA and FDG-PET and/or CT. Results Of the 39 patients, 33 were treated in a salvage setting after failing 3 lines of systemic treatment. Six patients were treated in a front-line setting in combination with a systemic chemotherapy protocol (FOLFOX$6: n=4$, FOLFIRI: $n=2)$. Average administered activity was $1.6 \mathrm{GBq}(0.4$ to 2.7 $\mathrm{GBq}$ ). Liver absorbed doses ranged from $0.4 \mathrm{~Gy}$ to $84.0 \mathrm{~Gy}$ (mean: $20.9 \mathrm{nGy}$ ). Tumor absorbed doses ranged from 43.4 Gy to $503.0 \mathrm{~Gy}$ (mean: $159.8 \mathrm{~Gy}$ ). Median follow-up was 4 months (range: 1-15 months). No treatment-related liver failure, clinical radiation hepatitis or veno-occlusive disease were seen. In the salvage group, $35 \%$ of the patients showed an objective response by CT. The metabolic response rate by FDG-PET was $91 \%$. In the front-line chemoSIRT group, all patients showed objective tumor response by imaging. Four patients had complete and 2 patients had more than $50 \%$ metabolic response by FDG-PET. Conclusions Yttrium-90 (Y-90) microsphere therapy produced a significant objective response rate in all patients with acceptable liver toxicity. The response rate and scale were markedly better in those who were treated front-line with chemo-SIRT combination. A meaningful survival data requires longer median follow-up.

\section{P250}

A Decision Analysis Model Predicts the Optimal Treatment Pathway for Colorectal Cancer Patients with Resectable Synchronous Liver Metastases T. Aloia, ${ }^{*}$ N. Barshes, N. Becker, J. Rodriguez, C. O'Mahony, J. Goss. Baylor College of Medicine, Houston, TX.

Introduction: The estimated 3,000 Americans who annually present with colorectal cancer and simultaneous resectable liver metastases encounter a wide array of surgical and medical treatment options. Due to the large number of possible treatment sequences and the absence of clinical trials comparing these various pathways, there is no consensus on the optimal therapeutic strategy. Methods: To address this issue, a decision model was developed incorporating all possible combinations of the following treatments: systemic chemotherapy, colorectal resection, hepatic resection, simultaneous colohepatic resection. Transition probabilities (morbidity, mortality, recurrence, and survivals) associated with each treatment were determined by systematic literature review. Variations in complication rates based on the extent of hepatectomy (minor: 1-2 segments vs. major: $>2$ segments) were factored into the model. Complication rates related to omission of certain treatments were also considered (e.g. risk of colonic perforation or hemorrhage in patients receiving chemotherapy prior to colectomy). Sensitivity analyses were performed to identify threshold values for study variables that altered the optimal treatment pathway. Results: Following 10,000 simulated patient trials with no bias toward any one initial treatment (i.e. current practice conditions), the global calculated 5-year survival was $29 \%$. For simulated patients with moderate hepatic tumor burden only treatment sequences that placed systemic therapy before major hepatectomy resulted in improved 5 -year survival projections ( $38 \%$ vs. $29 \%$, $p=0.001$, OR: 1.82). Initial treatment with simultaneous colohepatic resection was only favored when the operative mortality rate was adjusted to $<0.5 \%$. Conclusions: This detailed decision analysis predicts that the optimal treatment pathway for most colorectal cancer patients with simultaneous resectable liver metastases is preoperative systemic therapy followed by colohepatectomy or 2-stage resection. In the era of improved systemic therapies, major hepatic resection should be deferred until both local and systemic disease can been addressed.

Transition Probability Ranges for Major Variables

\begin{tabular}{|c|c|c|c|}
\hline Treatment & Morbidity & Mortality & Reduction in Recurrence Risk per Year \\
\hline Systemic Chemotherapy & $\mathbf{0 - 1 5 \%}$ & $\mathbf{0 - 3} \%$ & $\mathbf{0 - 2 5 \%}$ \\
\hline Colectomy & $\mathbf{0 - 1 5 \%}$ & $\mathbf{0 - 4} \%$ & $\mathbf{0 - 1 0 \%}$ \\
\hline Hepatectomy & $\mathbf{0 - 3 0 \%}$ & $\mathbf{0 - 5} \%$ & $\mathbf{0 - 1 5 \%}$ \\
\hline Colohepatectomy & $\mathbf{0 - 4 0 \%}$ & $\mathbf{0 - 8} \%$ & $\mathbf{0 - 2 5} \%$ \\
\hline
\end{tabular}

\section{P251}

Hepatic Imaging Characteristics Predict Overall Survival in Hepatocellular Cancer C.E. Woodall, ${ }^{*}$ C.R. Scoggins, J. Loehle, K.V. Ravindra, K.M. McMasters, R.C. Martin. Department of Surgery, Division of Surgical Oncology, University of Louisville, Louisville, KY.

Background: Pathologic tumor-related factors, including vascular invasion, remain the only reliable predictor of recurrence and overall survival in Hepatocellular cancer (HCC). Other pre-operative factors, such as hepatitis status, degree of liver disease (cirrhosis), number of tumors, and size of tumors have been inconsistent in predicting outcome. The aim of this study is to demonstrate that standard radiological imaging characteristics will predict overall survival in HCC. Methods: We identified 103 consecutive HCC treated in our department from $1 / 1999$ to $6 / 2005$. All images were reviewed by two blinded physicians and classified into one of three radiologic characteristics: pusher well encapsulated without parenchymal violation, invader: non encapsulated with violation of parenchyma and hanger: encapsulated with a majority of the lesion suspended from segments 2,3,4B,5, and/or 6. Results: There were 68 males and 35 females with a median age of 64 years (range 23 to 90 years). Hepatitis was seen in $38(37 \%$ ) patients (Hep B in 6 and Hep C in 31), a median AFP of 34 (range 0 to 17,000), and median MELD score of 8.9 (range 5.941.3 ). There was a median of 1 lesion (range $1-10$ ), majority had $<25 \%$ liver involvement $(50 \%), 26-50 \%(32 \%),>50 \%(13 \%)$, with a median lesion size of $6 \mathrm{~cm}$ (range 1-22cm). Surgical Therapy included hepatic resection 34(33\%), RFA $23(22 \%)$, and liver transplantation $21(20 \%)$. The distribution of radiologic characteristics at initial evaluation was $54 \%$ pushers, $41 \%$ invaders, and $4 \%$ hangers. Median survival for invaders (8.2 months) and hangers (10.0 months) were significantly less than pushers (median 29 months) $(\mathrm{p}=0.0007)$. Of the all the possible preoperative/ pre-therapy factors evaluated for recurrence and overall survival, only radiologic characteristic $(\mathrm{p}=0.006)$ and extent of tumor ( $p=0.002$ ) were significant on multivariate analysis Conclusion: Standard, reproducible radiologic characteristics are predictive of outcome in patients with HCC. Further emphases on identifying pre-operative factors remain imperative to better identify patient's biology that should undergo resection or transplantation

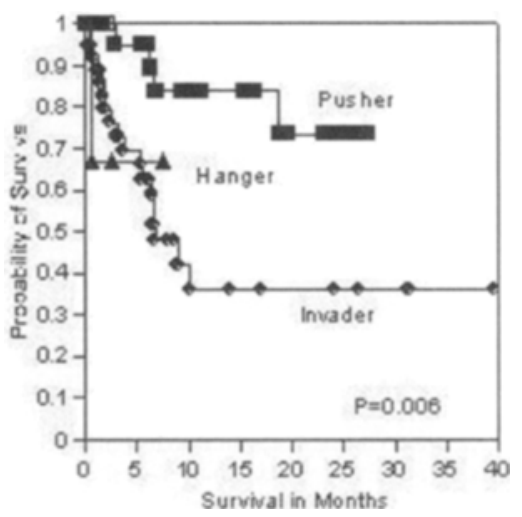

\section{P252}

Survival Benefit with Adjuvant Radiation Therapy in Surgically Resected Pancreatic Cancer J.A. Greco, ${ }^{*}$ E.T. Castaldo, I.D. Feurer, C.W. Pinson, A.B. Chakravarthy, N.B. Merchant, A.A. Parikh. Surgery, Vanderbilt University, Nashville, TN.

Introduction: Survival for patients with pancreatic cancer remains abysmal. Early studies have shown a benefit of adjuvant chemoradiation therapy. However, recent trials have shown a benefit of adjuvant chemotherapy while showing a negative effect with the addition of radiation therapy. This has led to significant controversy about the role of adjuvant radiation therapy (XRT) in these patients. The primary aim of this population-based study is to determine if adju- 
vant XRT improves overall survival in patients with resected pancreatic cancer. Methods: Using the Surveillance, Epidemiology, and End Results (SEER) registry, all patient records from 1973-2003 with surgically resected pancreatic adenocarcinoma were queried. Patients with Stage 3 or 4 disease, pre-operative or intra-operative radiation therapy, multiple primary malignancies, or incomplete tumor grading, staging, radiation, or demographic data were excluded. Kaplan-Meier methods and the log-rank test were used for survival data. A Cox regression model was tested with gender, race, tumor grade, age over 60 years, stage, and radiation as co-variates. Results: The analysis included 2,636 patients with resected pancreatic cancer $(1,123$ received adjuvant XRT and 1,513 did not). With a mean $\mathrm{f} / \mathrm{u}$ of 19 mos., median overall survival for the patients receiving XRT was 18 mos. compared to 11 mos. for the group that did not $(\mathrm{p}<0.01)$. Additionally, Cox regression demonstrated that patients who received adjuvant XRT had a statistically significant increase in overall survival when compared to patients who received no adjuvant XRT $(\mathrm{HR}=0.57$; $95 \%$ CI $0.52-0.63 ; p<0.01)$. Independent significant factors leading to decreased survival included race other than black compared to white $(p<0.01)$, moderately $(p<0.01)$ and poorly differentiated $(p<0.01)$ histology, age greater than $60 \mathrm{yrs} .(p<0.01)$ and increased stage of tumor $(p<0.01)$. Conclusion: These data suggest a survival benefit for the addition of radiation therapy following surgical resection of pancreatic adenocarcinoma. Radiation therapy was an independent predictor of survival in this model after adjusting for the effects of gender, race, tumor grade, age and stage.

\section{P253}

The Natural History of Resected Pancreatic Cancer Without Adjuvant Treatment E. Zervos, ${ }^{*}$ S. Al-Saadi, J. Hernandez, D. Villadolid, A. Collins, J. Cooper, R. Gillman, N. Vohra, A. Rosemurgy. surgery, university of south florida, tampa, FL.

Introduction: BB183, a prospective placebo controlled adjuvant trial provides a unique opportunity to study and establish the natural history of resected pancreatic cancer. Five years after completion of this trial, we report survival outcomes and patterns of recurrence in patients undergoing resection for pancreatic cancer whose adjuvant treatment was withheld until disease progression. Methods: Records of patients randomized to receive placebo in trial $\mathrm{BB} 183$ were reviewed noting tumor-related factors (AJCC stage, nodal and margin status, CA 19-9, Karnofsky Performance Score (KPS) at baseline and recurrence) and patterns of recurrence prior to initiation of salvage chemoradiotherapy. Multiple regression analysis was utilized to determine which factors impacted disease-free and overall survival. Recurrence categories were classified as: liver only, local, distant, multiple, or "clinical" (defined by absence of radiological recurrence in the presence of unexplained weight loss, intractable pain, jaundice or ascites). Follow up was $100 \%$ at 60 months. Results: 98 patients receiving placebo as adjuvant therapy until disease progression were identified. $67 \%$ of patients underwent $R 0$ resection and experienced longer $(21.2 \mathrm{mo}$ $\pm 14.41)$ overall survival than those with positive margins $(15.1 \mathrm{mo} \pm 13.67$, $\mathrm{p}=0.0173$ Log-rank test). Patients with R0 resections experienced longer disease-free survival ( $16.3 \mathrm{mo} \pm 14.36$ vs. $9.6 \mathrm{mo} \pm 11.74, \mathrm{p}=0.01)$. Multiple regression analysis documented that stage and margin status correlated with disease-free survival $(p=0.009)$ while stage, nodal status, CA 19-9 level at baseline, margin status and KPS at recurrence were significant predictors of overall survival $(p<0.05)$. Patterns of recurrence and their impact on survival are noted (Table). Conclusions: Stage and adequacy of resection remain the best predictors of outcome following resection for pancreatic adenocarcinoma. Patients dying with anatomical evidence of recurrent disease had poor survival outcomes. Patients surviving longest before succumbing to recurrent disease had no identifiable anatomic site of recurrence and died after a prolonged interval of systemic manifestations of recurrent pancreatic cancer.

\begin{tabular}{|c|c|c|c|}
\hline $\begin{array}{c}\text { Site of } \\
\text { Recurrence }\end{array}$ & $\begin{array}{c}\text { Disease Free Survival } \\
\text { (months) }\end{array}$ & $\begin{array}{c}\text { Overall Survival } \\
\text { (months) }\end{array}$ & $\begin{array}{c}\mathrm{p} \text {-Value Compared to Clinical } \\
\text { Recurrence }\end{array}$ \\
\hline Multiple $(\mathrm{n}=13)$ & $6.6 \pm 9.67$ & $7.7 \pm 10.03$ & $<0.0001$ \\
\hline Distant $(\mathrm{n}=9)$ & $7.2 \pm 5.70$ & $10.2 \pm 8.27$ & $<0.0001$ \\
\hline $\begin{array}{c}\text { Liver only } \\
(\mathrm{n}=22)\end{array}$ & $8.4 \pm 9.90$ & $12.6 \pm 11.97$ & 0.0001 \\
\hline Local $(\mathrm{n}=20)$ & $9.7 \pm 11.17$ & $12.5 \pm 10.43$ & 0.0002 \\
\hline Clinical $(\mathrm{n}=34)$ & $24.3 \pm 15.10$ & $29.2 \pm 14.80$ & N/A \\
\hline
\end{tabular}

\section{P254}

Cytoreductive surgery followed with intraperitoneal hyperthermic chemotherapy in elder patients with appendiceal carcinomatosis J. Munalula, ${ }^{*}$ C. Nieroda, P.N. Kostuik, D. Holter, B. Kalesan, V. Gushchin, A. Sardi. Institute of Cancer Care, Mercy Medical Center, Baltimore, $M D$.

Introduction: Long-term overall and disease-free survival have been reported after cytoreductive surgery and intraperitoneal hyperthermic chemotherapy (CRS/IPHC) in patients with appendiceal carcinomatosis. The aggressiveness of the procedures has historically precluded elder patients ( $\geq 65$ years) from candidacy. We review and compare our experience with CRS/IPHC in this group of patients. Methods: A retrospective review of a prospective database was performed of patients undergoing CRS/IPHC by a single surgeon between 1999 and 2005. All patients had a diagnosis of peritoneal carcinomatosis of appendiceal origin. The mean peritoneal carcinomatosis score (PCI, range 0 to 39) and disease outcomes were compared using Wilcox rank-sum and log rank tests. Results: Between 1994 and 2005, 34 patients underwent CRS and IPHC in our community practice. Of the 34 patients, 28 were under 65 (group 1 , mean 46.2) and 6 were 65 years or older (group 2, mean 70.7). The mean PCI scores were 24.3 and 29.9 , respectively $(p=.32)$. The completeness of cytoreduction index ( 0 to 3 ) was similar in both groups, 0.8 (group 1) and 1.3 (group 2), $p=.11$. Length of hospital stay was similar for both groups (mean 12.3 days, $p=.92$ ) and there was not a significant difference in the rate or severity of complications. Median survival is 38.8 months in the patients less than 65 and 18.6 months in those 65 years or older $(p=.09)$. Conclusion: Cytoreductive surgery and intraperitoneal hyperthermic chemotherapy can be performed safely and with favorable outcomes in appropriately selected patients. Advanced age should not deter the experienced surgeon from offering aggressive multi-modal therapy in the appropriate clinical setting with an expected improvement in survival as shown in our small cohort.

\section{P255}

Adequate lymph node harvest in colorectal cancer resections independent of specialty training and surgeon volume T.S. Kent, ${ }^{1 *}$ P. Sreeramoju, ${ }^{2}$ T.K. Weber. ${ }^{2} 1$. Dept of Surgery, University of Pittsburgh Medical Center, Pittsburgh, PA; 2. Albert Einstein College of Medicine, Bronx, $N Y$.

INTRODUCTION: Twelve lymph nodes are required to confirm node-negative status in colorectal cancer resections, according to the NCI. Node positivity mandates adjuvant therapy, whereas only selected high-risk patients require therapy for node-negative disease. Appropriate classification of node status is critical for directing patient care. We characterize nodal harvest in a moderate volume single institution study. METHODS: We conducted a retrospective review of a prospective database $(\mathrm{N}=152)$ of colorectal cancer specimens obtained over the most recent 18 month period for which data were available. Data were stratified by surgeon, surgeon volume, type of resection, and nodal harvest. Descriptive and comparative statistics (ANOVA and Student's t-test) were used to characterize factors influencing nodal harvest. RESULTS Specimen information was obtained from our cancer registry. Analyzable data were available for 140 specimens. $44.3 \%$ of specimens had adequate nodal harvest. Overall mean harvest was 12.4 . Mean nodal harvest was significantly greater for right colon resections than for sigmoid $(13.7$ versus $10.7, p=0.025)$ Nodal harvest was not significantly associated with either surgeon volume or fellowship training. Laparoscopic resections had the same mean lymph node harvest as open resections. CONCLUSIONS: We reviewed nodal harvest in our colorectal cancer resections, to investigate potential factors contributing to adequate harvest. Surgeons in our institution were more successful at harvesting an adequate number of lymph nodes with right-sided resections compared to sigmoid resections, consistent with the literature. Of interest, contrary to previously published literature, in our moderate-volume, university-based institution, there was no significant increase in nodal harvest with fellowship training or surgeon volume, and overall mean harvest was adequate according to NCI recommendations. We conclude factors other than fellowship training and surgeon volume may be relevant in achieving higher lymph node yield in colorectal cancer resections. These factors may differ by type of resection, and warrant further investigation. 


\section{P256}

Correlation between regulatory T cells and serum levels of IL10 and IL12 with disease outcome in HNSCC patients O. Alhamarneh,* F. Agada, L. Fletcher, D. Mistry, A. Jebreel, N.D. Stafford, J. Greenman. Department of Otolaryngology, Head and Neck Surgery, Postgraduate Medical Institute, University of Hull, Hull, East Yorkshire, United Kingdom.

Introduction: The levels of Regulatory T cells (Treg cells), IL10 and IL12 have been shown to be imbalanced in HNSCC patients when compared to normal controls. Treg cells, a suppressor cell population that dampen the immune response, and IL10, a Th2-type cytokine, are both elevated in HNSCC patients. On the other hand, the levels of IL12, a Th1-type cytokine, are reduced. Here, we investigate correlations between these immune parameters and clinical outcome. Methods: A cohort of primary, previously untreated, HNSCC patients $(n=56$; larynx 18 , oral cavity 13 , oropharynx 13 , hypopharynx 10$)$ had their serum cytokine levels (ELISA, $\mathrm{n}=56$ ) and PBMC Treg cells numbers (flow cytometry, $\mathrm{n}=46$ ) determined. Independent samples t-test, Fishers exact test and Kaplan-Meier survival curves were used to analyse associations with clinical outcome. Results: Overall tumour stage significantly correlated with death $(\mathrm{d}=15,26.7 \%, \mathrm{p}<0.001)$, but not with metastasis $(\mathrm{m}=5,8.9 \%, \mathrm{p}=0.145)$ or rate of recurrence $(\mathrm{n}=8,14.3 \%)$ when comparing patients with early (stages $1 \& 2$ ) vs. advanced (stages $3 \& 4$ ) disease. The mean follow up period was 19 months (range 8-41 months). Treg cells levels were determined as a percentage after measuring $\mathrm{CD} 4+\mathrm{CD} 25+$ high $\mathrm{T}$ cells (mean $=35 \%$ ). No significant correlation was found between the levels of Treg cells, IL 10 and IL 12 with overall tumour stage, recurrence rate, death or metastasis. However, when comparing IL10 levels in stage $1(n=13)$ with stage $4(n=29)$, significant higher levels were seen in the latter stage $(\mathrm{p}=0.016)$. Conclusion: Neither Treg cells numbers, IL 10 nor IL12 levels showed any statistical correlation with disease outcome when the whole cohort of head and neck cancer patients is considered. However, raised levels of IL10 do appear to be a potentially useful prognostic factor, in addition to the overall tumour stage in HNSCC patients.

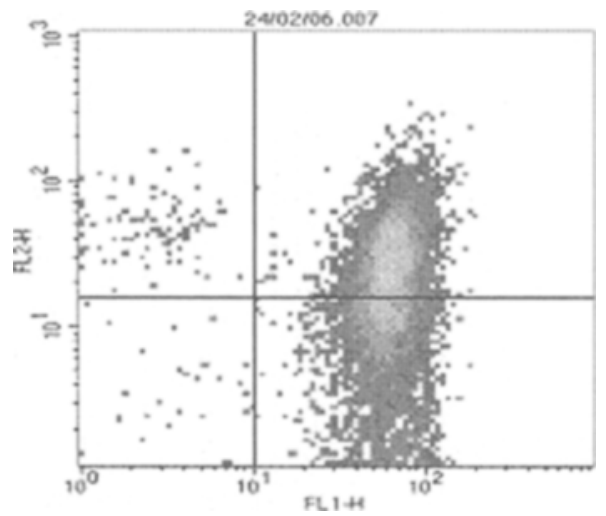

Isolated Treg cells (CD4+CD25+high) are shown in the right upper quadrant of the figure.

\section{P257}

Treatment of dysplastic oral mucosal lesions with a new cell-cycle inhibiting agent M. Pandey, ${ }^{1 *}$ R.C. Huang, ${ }^{2}$ M.K. Nair. ${ }^{3} 1$. Surgical Oncology, Institute of Medical Sciences, Varanasi, UP, India; 2. Johns Hopkins University, Baltimore, MD; 3. Regional cancer Centre, Trivandrum, Kerala, India.

Introduction Oral cancer is the sixth most common cancer in the world. In India, oral cancer is associated with tobacco habit and is preceded in the majority of cases by premalignant lesions. Cancer detection programs for oral cancer are based on the premise that earlier the cancer is diagnosed, better is the outcome. Another approach to cancer control is to identify potentially malignant or pre-malignant lesions, and treat them to prevent transformation. Patients and Methods Between November 1999 and April 2000, 4 patients with oral leukoplakia were recruited. After obtaining informed consent, intralesional tetra-O-methyl nordihydroguaiarectic acid (M4N) was injected in doses of 20 $\mathrm{mg} /$ day for 5 days. After completion of this treatment, patients underwent surgical resection of the lesion in 3 cases and biopsy alone in 1 case. Toxicity was monitored by WHO toxicity criteria. Response was recorded as change in surface area, necrosis, pathological response, molecular response, complication, and recurrences. Results All the four lesions were located on the buccal mucosa three lesions were excised while the forth regressed completely with a 5 day M4N treatment. At the end of 27 and 33 months one patient each developed a new lesion. Down regulation of cdc2 was seen in 3 and of survivin in 2 patients. Both the patients with new lesions showed cdc 2 downregulation while one showed down regulation of survivin. Conclusions Intralesional M4N appears well tolerated using an intralesional dose of up to $100 \mathrm{mg}(20 \mathrm{mg} /$ day $)$ with no evidence of acute or delayed toxicity. The drug has demonstrated inhibition of cellular production of cdc2 and Survivin, a strongly pro-apoptotic response. The advantage of present technique is in its simplicity of administration, as these intralesional injections can be administered on day care bases.

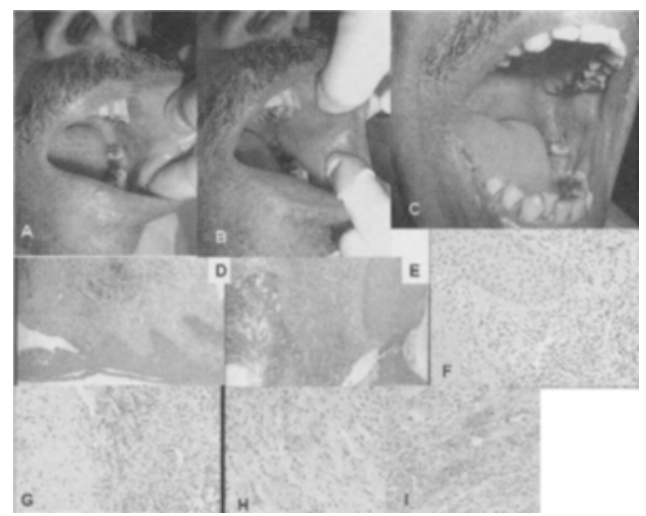

Figure showing the result of treatment with intralesional tetra-Omethyl nordihydroguaiarectic acid (M4N) in a case or oral leukoplakia A) pre treatment B) post treatment $\mathrm{C}$ ) at 1 year D) pre treatment histopathology E) post treatment Histopathology F) CDC 2 pretreatment G) CDC2 post treatment $H$ ) Survivin pre treatment I) survivin post treatment

\section{P258}

AZD2171 in combination with Paclitaxel is an effective therapy for Oral Cavity Squamous Cell Carcinoma in a murine orthotopic model F. Gomez-Rivera, * A. Santillan-Gomez, D. Fooshee, K. Gallagher, M. Zhao, A. Sung, M. Gu, S. Jasser, J.N. Myers. Head and Neck Surgery, MD Anderson Cancer Center, Houston, TX.

Introduction: Novel approaches are necessary for patients with squamous carcinoma of head\&neck. Vascular endothelial growth factor (VEGF) has been shown to play a critical role in this process and the receptor-2 (VEGFR-2) and 3 are its principal receptors in blood vessel and lymphatic endothelial cells respectively. Its inhibition is an appealing adjunct to current cytotoxic therapies. We evaluated the effect of AZD2171 on orthotopically established oral squamous cell carcinoma (OCSCC) in athymic nude mice, alone or in combination with paclitaxel. Methods: Four randomized groups of 12 animals each were used: control, AZD2171, paclitaxel and combination. Results: Treatment of mice bearing OCSCC tumors led to differences in mean tumor size between control(84.8 mm3), paclitaxel(75.4 mm3), AZD2171(52.4 mm3) and combination $(45.8 \mathrm{~mm} 3)$ groups. Groups that included AZD2171 were associated with tumor volume reduction $(\mathrm{p}=0.028)$. The median survival for the control, paclitaxel, AZD2171 and combination groups were 14, 36, 36, and 49 days. The difference between the groups was statistically significant by log-rank test $(\mathrm{p}<0.001)$. The lymph node metastases rates were $38 \%, 45 \%, 50 \%$ and $17 \%$ for the control, paclitaxel, AZD2171 and combination groups respectively $(\mathrm{p}=0.36$ ). Microvessel density by $\mathrm{CD} 31$ positive area was significantly lower for AZD2171 $(\mathrm{p}=0.007)$ and combination groups $(\mathrm{p}=.04)$ when compared to control group $(\mathrm{p}=0.59)$. Similar results were obtained for lymphatic 
microvessel density, measured by Lyve-1(+) staining. The apoptosis rate as measured by TUNEL staining counts, was significantly higher for the combination group $(\mathrm{p}=0.02)$. Conclusions: The main effect of AZD2171 correlated with inhibition of angiogenesis and lymphangiogenesis. There was a reduction in the lymph node metastases rate, although it did not reach statistical significance. AZD2171 effectively inhibits tumor growth and in combination with paclitaxel, also prolongs survival in an orthotopic model of OCSCC. Acknowledgment: AZD2171 was provided by AstraZeneca Pharmaceuticals and the National Cancer Institute Cancer Therapy Evaluation Program (NCI-CTEP), $\mathrm{NIH}$.

\section{P259}

T Cell Receptor Gene Therapy Targeting a Native Melanoma Antigen Can Impact Established Tumor Growth in a Murine Model J.D. Abad,* R.A. Morgan, S.A. Rosenberg. National Cancer Institute, Surgery Branch, Bethesda, MD.

Adoptive cell transfer therapy using tumor infiltrating lymphocytes for patients with metastatic melanoma has demonstrated significant objective response rates. One major limitation of these current therapies is the ability to isolate tumor reactive lymphocytes for patient treatment. Genetic engineering of peripheral blood lymphocytes with retroviral vectors encoding tumor antigen specific $\mathrm{T}$ cell receptors bypasses this restriction. To better evaluate the efficacy of T cell receptor gene therapy, a murine treatment model was developed. The genes encoding the pmel-1 T cell receptor targeting the B16 melanoma antigen, gp100, was used to generate a bicistronic retroviral vector. Transduction of CD8+ enriched C57BL/6 lymphocytes resulted in efficient pmel-1 T cell receptor expression ( $>90 \%)$ and gp 100 tetramer binding $(>35 \%)$ by flow cytometry. Lymphocytes transduced with this retrovirus specifically recognized and lysed gp100 pulsed target cells. Upon transfer into irradiated, B16 tumor-bearing mice, the genetically engineered lymphocytes expanded in vivo and significantly reduced tumor growth. These results demonstrate $\mathrm{T}$ cell receptor gene therapy targeting a native tumor antigen can successfully impact the growth of established tumors. This model may be a powerful tool for evaluating future $\mathrm{T}$ cell receptor gene transfer-based strategies.

\section{P260}

Clinical value of PET in melanoma patients with increasing levels of serum S-100B and negative conventional work-up L.H. Smit, ${ }^{1 *}$ J.D. De Vries, ${ }^{1}$ O.E. Nieweg, ${ }^{1}$ E.F. Comans, ${ }^{2}$ J.M. Bonfrer, ${ }^{1}$ R.A. Valdés Olmos, ${ }^{1}$ G.C. De Gast, ${ }^{1}$ A.A. Lammertsma, ${ }^{2}$ B.B. Kroon. ${ }^{1}$ 1. netherlands cancer institute, Amsterdam, Netherlands; 2. Free University Medical Centre, Amsterdam, Netherlands.

The $\mathrm{S}-100 \mathrm{~B}$ protein is progressively used as a tumor marker in melanoma patients. Its clinical utility has been described in determining prognosis, monitoring treatment and for early detection of recurrent disease. Positron emission tomography with fluor-18 deoxy-glucose (PET) is another relatively new diagnostic method used in melanoma patients to diagnose metastatic disease, to monitor treatment and to provide additional information when conventional imaging techniques are not conclusive. We determined serum S-100B values during routine follow-up of melanoma patients. The additional value of PET was studied prospectively in patients with an elevated S-100B in whom no recurrence could be detected with the conventional techniques. Patients with an elevated S-100B value on routine follow-up visit but with no signs of disease on physical examination were selected. Twenty-eight of such patients had no signs of disease on MRI of the brain, scintigraphy of the bone and CT of chest and abdomen. These patients underwent a whole-body PET scan. Hot spots on the PET scan were verified by means of fine-needle aspiration cytology. Additional imaging and follow-up were used as gold standard when no tissue confirmation could be obtained. The PET study was scored as false negative when tumor activity was identified within three months after a negative scan. PET was true-negative in fourteen patients and false-positive in three. PET was positive in the remaining eleven patients and all of them indeed were proven to have recurrent disease. In ten of these eleven patients, the PET findings led to treatment: chemotherapy in three patients and surgery in seven. Three of the surgically treated patients are alive without disease after 24 months, 56 months and 64 months. Their metastases were located in the small bowel, lung and retroperitoneum. Serum S-100B can identify patients with recurrent melanoma at an early stage and PET can localize these tumor sites when conventional imaging techniques fail. The sensitivity of PET in this situation is
$100 \%$ and the specificity is $82 \%$. The PET findings resulted in early treatment of $38 \%$ of this patient group.

\section{P261}

\section{Surgical Resection for Bulky or Recurrent Axillary Metastatic} Melanoma S.T. McElearney, ${ }^{*}$ C.L. Slingluff. University of Virginia, Charlottesville, $V A$.

Background: Bulky axillary melanoma can cause pain, functional impairment, and skin breakdown. Resection of advanced disease may provide palliation of symptoms and improved quality of life, and possibly may reduce tumor burden impacting survival. Methods: Patients who underwent axillary dissection for bulky or recurrent axillary metastases of melanoma between 1995 and 2006 at our institution were identified from a prospectively collected database. Inclusion criteria included axillary adenopathy $5 \mathrm{~cm}$ or greater, neurovascular invasion, or recurrence after a prior axillary complete lymph node dissection (CLND). Survival follow-up data were supplemented from the Social Security Death Index. Results: Forty patients with bulky axillary disease were reviewed. Fifteen $(38 \%)$ had vascular invasion requiring full or partial resection of axillary vein, $14(35 \%)$ had nerve invasion with $8(20 \%)$ requiring resection of thoracodorsal nerve, and $16(40 \%)$ had recurrent disease following prior CLND. Twelve (30\%) patients presented with brachial plexus symptoms. Axillary adenopathy size was a mean of $6.6 \mathrm{~cm}$, ranging up to $17 \mathrm{~cm}$. Surgery was most often performed as an outpatient, though hospital stays ranged from 0 to 5 days. $28(70 \%)$ had no other known disease and were resected with curative intent, while $12(30 \%)$ were resected for palliation despite known extra-axillary disease. Median follow-up for survival was 29 months for all patients and 43 months (SE 6) excluding patients who died. Of those resected for cure, axillary recurrence occurred in $8(29 \%)$, and mean survival was 48 months (SE 5) after the last CLND. With palliative resection, only two $(17 \%)$ recurred in that axillary basin during follow-up, and mean survival was 8 months after the last CLND. Conclusion: In patients with bulky or recurrent axillary adenopathy as the only site of known metastasis, long-term survival can be achieved with aggressive surgical resection. Even in patients with other known disease, resection of bulky axillary adenopathy can provide regional control and palliation, with minimal morbidity.

\section{P262}

Sentinel Node versus Therapeutic Lymph Node Dissection A.C. Van Akkooi,* M.G. Bouwhuis, M. Kliffen, A.N. Van Geel, C. Verhoef, P.I. Schmitz, J.H. De Wilt, A.M. Eggermont. Surgical Oncology, Erasmus Medical Center - Daniel den Hoed Cancer Center, Rotterdam, Zuid-Holland, Netherlands.

Background: Sentinel Node (SN) status is the most important prognostic factor for overall survival (OS) in stage I/II melanoma patients. However, the therapeutic value remains unclear. Some non-randomized retrospective trials suggest a survival benefit, but the methodology of these studies contained various errors. Interim results of the MSLT-I trial suggested a $13 \%$ survival benefit for SN positive patients compared to patients that underwent a therapeutic lymph node dissection (TLND). We recently reported that SN-positive patients with submicroscopic involvement of the SN (clusters of cells $<0.1$ $\mathrm{mm}$ ) had a distant recurrence rate of only $9 \%$ at 5 years, which is as good as $\mathrm{SN}$-negative patients. We thus hypothesized that these SN patients may be considered SN-negative. $\mathrm{SN}+$ patient populations differ from TLND patients if one includes submicroscopic metastases. Patients and Methods: Here we compare outcome of CLND in SN+ patients with outcome in TLND patients with palpable nodes, treated in Rotterdam. Survival rates were calculated from date of primary excision. Patients with primary melanomas on extremities or trunk were included; patients with unknown primaries were excluded. We identified 188 patients; 124 TLND patients (' 82 - '05) and 64 CLND patients (' 97 - '05). Median follow-up was 56 and 37 months, respectively. There were no significant differences between both groups regarding gender, Breslow thickness, ulceration and the site of the primary. Results: On univariate analysis site of the primary tumor (extremity versus trunk $)(\mathrm{P}<0.001)$, Breslow thickness $(\mathrm{P}=0.005)$, ulceration $(\mathrm{P}<0.001)$ were prognostic for $\mathrm{OS}$. On multivariate analysis, both site and ulceration remained independent prognostic factors for OS. There was a non-significant $13 \%$ difference in OS for the CLND compared to the TLND group (figure $1 \mathrm{~A})(\mathrm{P}=0.12)$. Excluding $\mathrm{SN}$ patients with submicrometastases $(n=15)$ reduced the difference in OS to $6 \%$ (figure $1 \mathrm{~B})(\mathrm{P}=0.42)$. Conclusion: The present study did not show a significant survival benefit for 
SN+CLND compared to TLND, especially not when patients with submicrometases were excluded.

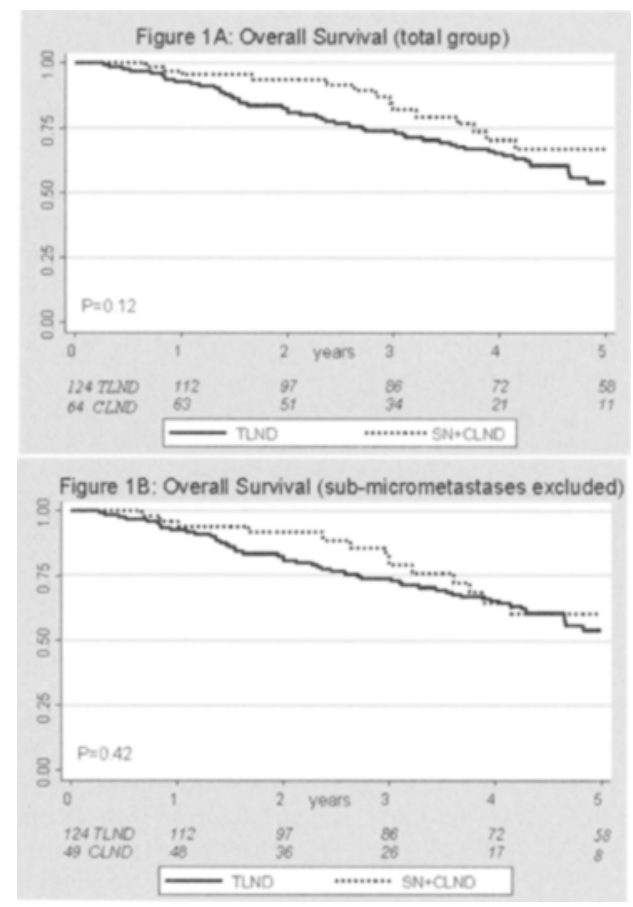

\section{P263}

Time Trends and Variables Associated with Compliance with Melanoma Treatment Guidelines in a Community Teaching Hospital J. Erickson, ${ }^{2 *}$ J. Velasco, ${ }^{3}$ T.J. Hieken. ${ }^{1}$ 1. Surgery, Rush North Shore Medical Center, Skokie, IL; 2. Rush University Medical Center,

Chicago, IL; 3. Rush Medical College, Chicago, IL.

Introduction: Wide variation in the surgical treatment of melanoma occurs, despite efforts to standardize care. Failure to apply these standards may lead to inaccurate staging, increased morbidity, a poor outcome, or be cost-ineffective. We undertook this study to evaluate the treatment of our melanoma patients and identify factors associated with variance from the National Comprehensive Cancer network (NCCN) recommendations for excision margins and sentinel lymph node biopsy with selective lymph node dissection. Methods: We retrospectively accessed Cancer Registry data on 252 clinically node negative melanoma cases. Treatment data was confirmed by individual review of all pathology reports and operative notes. Statistical analysis was performed using an SAS software package. Results: Margins of excision conformed to NCCN guidelines in $88 \%$ of T $0,90 \%$ of T1 and $65 \%$ of T2-4 tumors. Overtreatment was $>$ under-treatment for T0-T1 melanomas ( $7 \%$ vs. $4 \%$ ) whereas under-treatment was > over-treatment for thicker melanomas (T2-4, 32\% vs. $3 \%$ ). Lymph node staging was performed in $11 \%$ of T $1 \mathrm{a}, 64 \%$ of $\mathrm{T} 1 \mathrm{~b}, 72 \%$ of T2-3 and $50 \%$ of T 4 cases. Completion lymph node dissection was performed after a positive sentinel lymph node biopsy in $83 \%$ of cases. Treatment by a surgical oncologist was $95 \%$ and $90 \%$ compliant with margin and lymph node staging guidelines vs. other practitioners in $45 \%$ and $62 \%$ of cases $(\mathrm{p}<0.001)$. Documented compliance with margin guidelines improved from $45 \%$ to $72 \%$ for $1995-1999$ vs. $2000-2004$ and lymph node staging from $71 \%$ to $83 \%$ (all $\mathrm{p}<0.01$ ). Greater adherence to NCCN guidelines was seen with age $<80$ years, upper extremity and thinner melanomas (all $\mathrm{p}<0.01$ ). Conclusions: These data suggest that despite dissemination of treatment algorithms for the management of melanoma patients, treatment compliance with NCCN guidelines was suboptimal at our community teaching hospital. Treatment directed by a surgical oncologist showed the highest rate of adherence to national standards. Further investigation is needed to determine how to provide high-quality care to the greatest number of melanoma patients.

\section{P264}

Use of Nanochannel Membranes to Regulate the Release of Cytokines from Biodegradable Reservoirs N.B. Jones, ${ }^{*}$ G. Lesinski, L.J. Lee, H. He, M. Ferrari, W.E. Carson, III. Surgical Oncology, The Ohio State University, Columbus, $\mathrm{OH}$.

Locally recurrent melanoma tumors are often unresectable due to their size and/or location and are also resistant to radiation and chemotherapy. The local administration of cytokines can have beneficial effects in this setting, but improved delivery systems are required. By affixing a nanochannel $(<100 \mathrm{~nm})$ membrane to a custom-designed biodegradable reservoir $(4.2$ by $4.2 \mathrm{~mm})$ we have been able to create a functional nanodevice that exhibits linear (zero-order) release. We hypothesize that the local release of immunotherapeutic agents from a biodegradable nanochannel delivery system (nDS) will improve the ability of cytokines to exert an anti-tumor effect on unresectable melanoma lesions. Interferon-alpha (IFN) was chosen as the payload molecule as it is an FDA-approved treatment for melanoma that has only modest anti-tumor activity when administered systemically to mice with subcutaneous B16 tumors ( $1 \times 10^{\wedge} 6$ cells). $\mathrm{nDS}$ were loaded and tested for in vitro release of IFN at various time points using a commercial ELISA. The biological activity of the released IFN on immune cells and Mel39 melanoma cells was assessed via flow cytometric analysis for phosphorylated STAT1, a transcription factor that is critical for the anti-tumor activity of IFN. nDs exhibited linear release of glucose and lysozyme in vitro. Linear release of IFN in vitro was also observed from nDS with reservoirs made of poly(epsilon-caprolactone (PCL) and poly(lactic-co-glycolic acid (PLGA). Normal T cells and Mel39 cells exhibited increased levels of P-STAT1 when exposed to the output of IFN-loaded nDS as opposed to PBS-loaded devices. Systemic release of IFN was demonstrated in serum samples from normal mice 48 hours post-implantation of IFNloaded $\mathrm{nDS}$ but not in mice bearing control $\mathrm{nDS}$. The inflammatory reaction to implanted devices was minimal. B16 tumor growth was reduced in mice bearing locally implanted IFN-loaded nDS as compared to mice bearing control nDS or mice receiving daily i.p. injections of IFN $\left(2 \times 10^{\wedge} 4 \mathrm{U}\right)$ or PBS. In summary, locally implanted, biodegradable nDS are able to release cytokines at a constant rate and exert an anti-tumor effect in a murine model of melanoma.

\section{P265}

Quality of life after sentinel lymph node biopsy in patients with cutaneous melanoma M. De Vries, ${ }^{1}{ }^{*}$ J.E. Hoekstra-Weebers, ${ }^{2}$ R.J. Van Ginkel, ${ }^{1}$ J.S. Rietman, ${ }^{1}$ H.J. Hoekstra. ${ }^{1}$ 1. Surgery, UMCG, Groningen, Netherlands; 2. University of Groningen, Groningen, Groningen, Netherlands.

Introduction The aim of this study was to asses the quality of life after axillary and inguinal sentinel lymph node biopsy (SLNB) with or without completion lymph node dissection (CLND) in patients with cutaneous melanoma. Methods Between 1995 and 2003, 242 axillary and inguinal SLNBs were performed for cutaneous melanoma. There were 127 patients eligible for this retrospective study; 46 patients died and 69 patients were excluded. Informed consent was obtained from 116 patients and these patients entered the study. Quality of life was measured using the EORTC QLQ-C30, the McGill Pain Questionnaire and the Groningen activity restriction scale. Postoperative complications and lymphedema were also analysed. Results Median follow-up was 56 (4-94) months. Median age was 50 (18-77) years and the median Breslow thickness $2.0(1-13) \mathrm{mm}$. CLND was performed in 27 patients (23\%) with a tumourpositive SLN. The remaining 89 patients underwent SLNB alone. More postoperative complications were found in the CLND group than in the SLNB group alone $(13$ vs $5 ; \mathrm{p}<0.001)$. There were also more patients with lymphedema in the CLND group compared to the SLNB group alone (10 vs 8; $p$ $<0.001$ ). However, there were no differences in global quality of life, pain and activity restrictions between the SLNB group and the CLND group. ANOVA showed differences between the groups only for role functioning $(p=0.02)$ and a trend for physical functioning $(p=0.05)$ and fatigue $(0.05)$. Bonferroni test showed that these differences were most likely caused by the difference between the axillary SLNB and the axillary CLND. Conclusion The overall quality of life after axillary and inguinal SLNB, in patients with melanoma, is good. However, when CLND was performed in case of a tumourpositive SLNB, there was a poorer outcome on quality of life scale and symptom scales compared to SLNB alone. This difference was most likely caused by axillary CLND. 


\section{P266}

“Gloves and Socks" Melanoma: Does Histology Make a Difference? E. Soudri, ${ }^{1}$ M. Feinmesser, ${ }^{1}$ J. Schachter, ${ }^{2}$ R. Gutman, ${ }^{3}$ H. Gutman. ${ }^{1}{ }^{*}$ 1. Rabin Medical Center/Beilinson Campus and Tel Aviv University, Sackler School of Medicine, Petach Tikva, Israel; 2. Sheba Medical Center/Tel Aviv Unversity SAckler School of Medicine, Tel Hashomer, Israel; 3. Free-lance statistician, Petach Tikva, Israel.

Introduction: Acral lentiginous melanoma (ALM) is reported to be associated with low survival rates. This study compares the clinical course of ALM to that of non-ALM hand and foot melanoma, and to other extremity melanoma patients. Patients and Methods: A retrospective review of prospectively collected data of 168 patients with cutaneous melanoma of the extremities (ALM - $\mathrm{N}=29$, non-ALM - $\mathrm{N}=16$, other extremity $-\mathrm{N}=123$ ) operated on between $07 / 1993$ and $11 / 2005$ in a tertiary referral center. All known melanoma prognosticators were analyzed for their impact on disease-free and disease-specific survival at 53 months median follow-up. Results: Age distribution of the three groups was significantly different (Figure) ALM patients were older (median 70) than non-ALM (median 59) and other extremity (median 55) patients ( $\mathrm{p}=$ 0.015 ). Tumor characteristics, sentinel lymph node status, disease stage, and disease-free and disease-specific survival rates were similar for the ALM and non-ALM groups. However, other extremity melanoma patients, presented with significantly thinner (median $1.6 \mathrm{~mm}$ VS $2.87 \mathrm{~mm}$ and $2.85 \mathrm{~mm}$ ) lesions, fewer positive sentinel lymph nodes ( $10 \%$ VS $38 \%$ and $27 \%$ respectively), lower tumor stage and consequently, significantly better disease-specific and diseasefree survival $(p=0.0006, p=0.0001)$. The "Gloves and Socks" lesions almost lacked peri-tumoral lymphocytic infiltration. Multivariate analysis identified only tumor thickness $(p=0.017)$, stage $(p=0.00001)$, and patient age $(p=0.012)$, to be independent prognosticators for disease-specific survival. Conclusions: "Gloves and Socks" melanomas occur in older patients and are diagnosed at a more advanced loco-regional stage than melanomas in other extremity sites. Regardless of ALM histology, these patients fair significantly worse. Delayed diagnosis due to old age and difficult-to-see sites, and biological factors (low peri-lesional lymphocytic infiltration) are factors associated with reduced survival

Age at Diagnosis Distribution Density by Melanoma Histology and Location

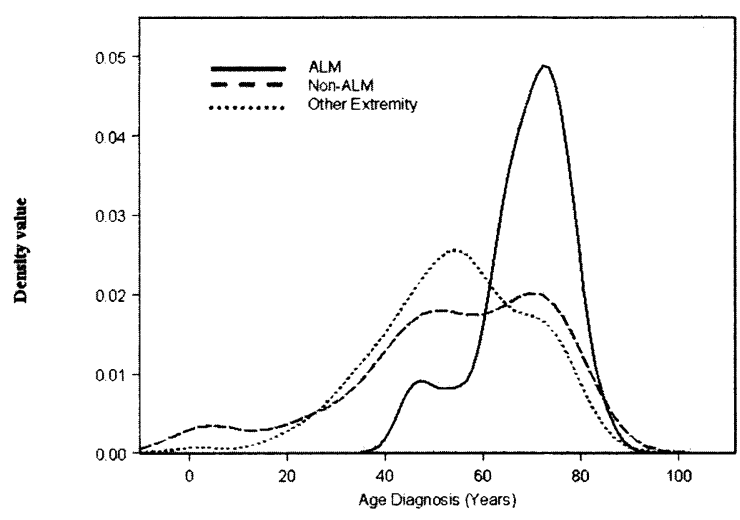

P267

Microscopic Satellitosis in Patients with Primary Cutaneous Melanoma T.F. Kimsey, ${ }^{*}$ T. Cohen, A. Patel, M.S. Brady. Surgery, Memorial Sloan-Kettering Cancer Center, New York, NY.

INTRODUCTION: Microscopic satellitosis in primary cutaneous melanoma is uncommon. Patterns of recurrence and the role of sentinel lymph node biopsy in this subset of patients has not been well-defined. METHODS: We reviewed a prospective database of patients with cutaneous melanoma at our center from 1996 to 2005 . Clinical and pathologic data were reviewed to determine the prognostic implications of microscopic satellitosis and define the role of SNL mapping in this setting. RESULTS: We identified 162 patients who presented to MSKCC with cutaneous melanoma containing satellitosis. Fourteen had no follow up, and 110 presented with advanced disease and were excluded from review. Thirty-eight patients presented for initial surgical management. These patients were reviewed. Most of the patients were male (28/38, $74 \%$ ), and the median age was 61 years (range 17 to 94 ). The most common site of the primary lesion was the extremity $(55 \%)$ followed by the trunk (29\%) and the head and neck (16\%). The median Breslow depth was $5.35 \mathrm{~mm}$ (range 1.9 to 17.3$)$. Most had lymphovascular invasion (68\%); $32 \%$ had perineural invasion. Three patients underwent wide excision (WE), while 35 underwent WE and sentinel lymph node (SLN) biopsy. In these patients with clinically localized disease, $63 \%$ developed recurrence at a median time to recurrence of 8 months. The most common site of initial recurrence was systemic $(46 \%)$ followed by in transit $(25 \%)$, nodal $(17 \%)$, and local $(12 \%)$. The 5 year disease free survival was $35 \%$. SLN metastases were found in 27 of the 35 patients $(77 \%)$. Of these patients, $21(78 \%)$ recurred with a median time to recurrence of 8 months. The most common site of initial recurrence in SLN positive patients was systemic $(48 \%)$ followed by nodal $(24 \%)$. A total of 13 patients had intermediate depth melanomas (1.9 to $3.8 \mathrm{~mm}$ ). Six $(46 \%)$ recurred. CONCLUSIONS: Microscopic satellitosis is a poor prognostic feature of primary melanoma. The risk of SLN metastasis is higher in this group of patients than any previously defined. Regional nodal staging and completion lymphadenectomy for SLN positive patients is unlikely to impart a therapeutic benefit in these patients

\section{P268}

Length of Stay and Recovery to Normal Function after Surgery for Metastatic Melanoma to Abdominal and Retroperitoneal Viscera L.T. Dengel, ${ }^{*}$ C.L. Slingluff, Jr.. Department of Surgery, University of Virginia Health System, Charlottesville, VA.

INTRODUCTION: Metastatic melanoma to abdominal and retroperitonea viscera carries a poor prognosis. There can be reluctance to offer surgical management for such patients; it may be valuable to understand the impact of surgery on return to normal function. METHODS: Review of a prospectively maintained database was performed to identify patients managed at our institution with metastatic melanoma to abdominal or retroperitoneal viscera who were operated on with an intent to resect all or most disease from September 1999 to August, 2006. Survival data were supplemented from the Social Security Death Index. RESULTS: Nineteen patients underwent surgery for metastasis to small bowel $(58 \%)$, liver $(11 \%)$, adrenal $(11 \%)$, pancreas $(5 \%)$, stomach $(5 \%)$, spleen $(5 \%)$, or rectum $(5 \%)$. The diagnosis was made as a result of clinical symptoms in $80 \%$ of cases, with the remainder were detected by imaging. Thirteen $(68 \%)$ had R0 resections. The hospital length of stay ranged from 3 to 14 days (median 7 days). There was $100 \%$ perioperative survival. Surgical complications occurred in only 4 patients: 2 were readmitted for postoperative bleeding, and 2 were treated as outpatients for wound infections. Initial follow-up was performed a median of 6 weeks post-operatively, when 13 patients $(68 \%)$ reported being back to baseline activity and function, 4 patients $(21 \%)$ experienced only minimal symptoms and post-operative deconditioning, and only 2 patients $(11 \%)$ were significantly limited by fatigue, anorexia or recurrent distant disease. Experimental melanoma vaccines were included in management of 16 patients $(84 \%)$. Median follow-up was 18 months (range 3 - 78) from the date of surgery at which time 13 of the 19 patients $(68 \%)$ were still alive, with actual 1 and 3 year survival rates of $63 \%, 26 \%$, respectively, and with three patients alive at 56,72 , and 78 months since surgery. CONCLUSION: In selected cases, surgery may have both palliative benefit and curative potential for patients with visceral metastases of melanoma. The morbidity can be very low. Surgical management of such patients should be encouraged in appropriate clinical settings.

\section{P269}

Routine Staging of Asymptomatic Patients with Ulcerated T3 and T4 Primary Melanomas Does not Detect Distant Metastases J. Schwartz, ${ }^{2}$ D.R. Murray, ${ }^{2}$ D.H. Lawson, ${ }^{1}$ D.A. Kooby, ${ }^{2}$ C.A. Staley, ${ }^{2}$ M. Rizzo, ${ }^{2}$ A. Hestley, ${ }^{2}$ G.W. Carlson, ${ }^{2}$ K.A. Delman. ${ }^{2 *}$ 1. Emory University School of Medicine, Division of Medical Oncology, Atlanta, GA; 2. Emory University School of Medicine, Division of Surgical Oncology, Atlanta, GA.

Jean-Claude Schwartz, Douglas R. Murray, David H. Lawson, David A. Kooby, Charles A. Staley, Monica Rizzo, Andrea Hestley, Grant W. Carlson, Keith A. Delman Introduction: Recent reports have indicated that routine imaging of asymptomatic, sentinel lymph node (SLN) positive patients with 
melanoma may be unwarranted. These studies did not specifically analyze the subset of patients with thick, ulcerated lesions, some of whom may have been node-negative but still have distant disease. The aim of this study was to determine the utility of radiologic staging in patients who presented with thicker, ulcerated lesions, regardless of SLN status. Methods: Utilizing a prospectively collected database from the division of surgical oncology, we identified patients who underwent SLN biopsy from 1994-2005 for thick and/or ulcerated lesions (AJCC T3b, T4a or T4b). The incidence of positive radiographic staging evaluations (MRI brain, CT chest, abdomen and pelvis or whole body PET-CT) was analyzed. Imaging was performed within 3 months of initial diagnosis to determine the utility of routine staging in this subset of patients. Results: 274 patients were identified with AJCC T3b, T4a or T4b lesions. 82 (34\%) had positive SLNs and $182(66 \%)$ were SLN negative. No patient with a negative SLN biopsy had evidence of distant metastases on imaging. Two (2\%) patients with positive SLN biopsies had significant imaging findings. Both of these patients had $\mathrm{T} 4 \mathrm{~b}$ lesions, representing $6 \%$ of all patients with $\mathrm{T} 4 \mathrm{~b}$ lesions. Overall, 0.8 percent $(2 / 264)$ of all patients who present with T3b, T4a, or T4b disease had radiologically detectable disease within 3 months of diagnosis. Conclusions: There is likely no role for routine imaging of asymptomatic melanoma patients at the time of initial diagnosis. The only subset of patients that may benefit from empiric staging are those with $\mathrm{T} 4 \mathrm{~b}$ lesions who are ultimately determined to be SLN positive. However, the majority of patients who present with ulcerated T3 or T4 melanomas may proceed to SLN biopsy and, if indicated, completion lymph node dissection without preoperative radiologic staging.

\section{P270}

Identification and Outcome of Regional Nodal Metastasis in Children with Melanoma: The MD Anderson Cancer Center Experience A.A. Hayes-Jordan, * J.E. Gershenwald, J.E. Lee, P.M. Mansfield, M.I. Ross, K.P. Lally, C.S. Cox, V.E. Prieto, C.E. Herzog. Surgical Oncology and Pediatrics, MD Anderson Cancer Center, Houston, TX.

Background: Despite an increase in adult melanoma, melanoma in the pediatric population remains rare. Sentinel node biopsy for the staging of patients with melanoma was routinely adopted beginning in 1992; in adults, this technique has significantly improved detection of occult melanoma metastases within regional lymph nodes. However, the impact of sentinel node biopsy (SLNB) in the staging of melanoma in children is largely unknown. We report our experience with identification of melanoma regional nodal metastasis in the pediatric population in the period since 1992. Methods: We performed a retrospective chart review of all patients seen at one institution between January 1, 1992 and December 31, 2002 in whom a diagnosis of melanoma was made prior to age 18 . Results: There were 88 primary melanomas in 76 patients. Patients' age ranged from 11 months to 17 years: 39 patients (51\%) were 14 or under, and 37 patients ( $49 \%$ ) were $15-17$ years. Thirty-two patients had T1 lesions, $17 \mathrm{~T} 2,10 \mathrm{~T} 3$, and 9 patients had T4 lesions. Of 41 patients who underwent intraoperative lymphatic mapping and SLNB, $12(29 \%)$ had metastatic melanoma identified in at least one sentinel node. When stratified by tumor thickness, $0 / 8(0 \%), 2 / 14(14 \%), 7 / 10(70 \%)$, and $3 / 5(60 \%)$ of patients with $\mathrm{T} 1, \mathrm{~T} 2, \mathrm{~T} 3$, and T4 lesions, respectively, had a positive SLNB. After a median follow-up of 53 months, 72 patients (95\%) are alive without disease (NED), 7 patients $(9 \%)$ had recurrence, and 4 patients $(5 \%)$ have died. All four patients who died developed distant metastatic disease and all were between the ages of 15 and 17 years. Of patients 15 to 17 years, $19 \%$ had stage 2,3 , or 4 disease compared to $39 \%$ of patients $<14$ years of age. Conclusion: Melanoma patients less than 14 years have an increased incidence of lymph node metastasis compared to those 15 to 17 years. No patient less than 15 years old has, to date, died of metastatic melanoma. These data suggest that SLNB provides important staging information for all children. Melanoma in older teenagers exhibits a different biologic behavior compared to melanoma in younger children.

Pediatric Melanoma

\begin{tabular}{|c|c|c|}
\hline & $<14$ years & $15-17$ years \\
\hline Stage 0 & $20 \%(8 / 39)$ & $22 \%(8 / 37)$ \\
\hline 1 & $41 \%(16 / 39)$ & $59 \%(22 / 37)$ \\
\hline 2 & $13 \%(5 / 39)$ & $8 \%(3 / 37)$ \\
\hline 3 & $26 \%(10 / 39)$ & $8 \%(3 / 37)$ \\
\hline 4 & 0 & $3 \%(1 / 37)$ \\
\hline
\end{tabular}

\section{P271}

PRE-EXENTERATIVE CHEMOTHERAPY, A NOVEL THERAPEUTIC APPROACH FOR PATIENTS WITH PERSISTENT OR RECURRENT CERVICAL CANCER C.M. Lopez, ${ }^{*}$ I.M. Chang Romàn, D.F. Cantú, R.F. Dolores Velázquez, A. Dueñas González. Surgical Oncology, National Cancer Institute at Mexico, Mexico, DF, Mexico, DF., Mexico.

Background: Most cervical cancer patients with pelvic recurrent or persistent disease are not candidates for exenteration, therefore they only receive palliative chemoterapy. Here we report the results of a novel treatment modality for these patients pre-exenterative chemotherapy-under the rational that the shrinking of the pelvic tumor would allow its resection. Methods: Patients with recurrent or persistent disease and no evidence of systemic disease, considered not be candidates for pelvic exenteration because of the extent of pelvic tumor received 3-courses of platinum-based chemoterapy. Response was evaluated by CT scan and bimanual pelvic examination; however the decision to perform exenteration relied on the physical findings. Toxicity to chemoterapy was evaluated with standard criteria. Survival was analyzed with the Kaplan-Meier method. Results: Seventeen patients were studied. The median number of chemoterapy courses was 4. there were 9 patients who responded to chemoterapy, evaluated by bimanual examination and underwent pelvic exenteration. Four of them had pathological complete response. Eigth patients did not respond and were not subjected to surgery. One patient died due to exenteration complications. At a median follow-up of 11 months, the median survival for the whole group was 11 months, 3 months in the non-operated and 32 months in those subjected to exenteration. Conclusion: Pre-exenterative chemoterapy is an alternative for cervical cancer patients that are no candidates for exenteration because of the extent of the pelvic disease. Its place in the management of recurrente disease needs to be investigated in randomized studies, however, its value for offering long-term survival in some of these patients with no other option than palliative care must be stressed.

\section{P272}

Survival of Patients with Pancreatic Cancer Predicted using Machine Learning Techniques J. Hayward, ${ }^{2 *}$ S. Alvarez, ${ }^{3}$ C. Ruiz, ${ }^{2}$ J. Tseng, ${ }^{1}$ M. Sullivan, ${ }^{1}$ G.F. Whalen.' ${ }^{1}$. UMASS Medical School, Worcester, MA; 2. Worcester Polytechnic Institute, Worcester, MA; 3. Boston College, Boston, MA.

Introduction: We wished to determine whether predictive techniques developed within the academic discipline of "machine learning," may be useful analyz ing small, but "dense" clinical data sets. Our hypothesis is that such techniques may be more accurate than standard multivariate regression. Method: Analysis of a retrospectively populated database designed with 190 fields considered important for clinical decision making in 91 patients with adenocarcinoma of the pancreas evaluated and treated by the Division of Surgical Oncology. Computations were performed using the Waikato Environment for Knowledge Analysis ("Weka"). Machine learning and standard multivariate regression techniques were used to construct predictive models based on randomly selected patient records. !0-fold cross-validation was repeated 10 times to provide a statistically robust performance assessment for each technique used in model building. The primary outcome measure was a comparison of survival time (number of months and in discrete ranges; $<6$ months, 6-12 months, $>12$ months) predicted by each model. Results: The best performing machine learning technique tested - " 2 nearest neighbor with bagging" outperformed linear regression for prediction of survival time with a higher correlation coefficient $(0.65 \mathrm{vs} 0.54)$ as well as lower mean absolute and root mean squared regression error $(p<0.005)$. Machine learning techniques using discretized survival ("1-nearest neighbor with bagging") achieved an accuracy of 0.57 vs 0.49 achieved by logistic regression. Conclusions: Machine learning techniques can provide better estimates of survival time in patients with adenocarcinoma of the pancreas than does standard multivariate regression. Ongoing work will evaluate potential contributions of machine learning to clinical decision making.

\section{P273}

Total Mesometrial Radical Resection (TMRR) in extended hysterectomy for early and locally advanced cervical cancers C.M. Lopez, ${ }^{*}$ I.M. Chang Román, D.F. Cantú, J.G. De la Garza, A. Dueñas González. Surgical Oncology, National Cancer Institute at Mexico, Mexico, DF., Mexico, DF., Mexico.

Surgical damage to the pelvic autonomic nerves during radical hysterectomy is thought to be responsible for considerable morbidity Objectives: The 
aim of our study was to give a surgical results (morbi-mortality) of this procedure.Both the incidence of locoregional recurrences and of late sequelae from intraoperative pelvic autonomic nerve damage in the treatment of rectal cancer have been reduced by total mesorectal excision (TME). We claim to achieve similar improvements with a corresponding concept to the surgical treatment of cervical cancer. Methods: We have developed the TMRR to remove the uterus and proximal vagina together with their integrated mesentery as an ontogenetic entidy. Lymph node dissection is extended and meticulously to additional pelvis sites. We studied histopathological features, intra- and early postoperative complications, late sequelae and oncological outcome in our patients treated with TMRR. Results: Since 10/2000 until 07/2006, 25 patients with cervical cancer, stages IA2 ( $\mathrm{n}=1), \operatorname{IB} 1(\mathrm{n}=13), \operatorname{IB} 2(\mathrm{n}=3), \operatorname{IIA}(\mathrm{n}=5)$, and $\operatorname{IIB}(n=3)$ were treated with TMRR and Extended Lymph node dissection.Median pathological tumor size was $2.5 \mathrm{~cm}(0.4-8 \mathrm{~cm}$.Mean duration of surgery was $4.3 \mathrm{~h}(\mathrm{r}=3.5-5.20)$. Median estimated blood loss was $350 \mathrm{ml}$ and median hospital stay was 4.2 days. Intraoperative complications not ocurred. In all but one the resection margins were negative. Mean number of removed pelvic lymph nodes was 35 (more or less 7),periaortic lymph node count was 10.Four patients $(16 \%)$ had positive nodes.By $8 / 2006$ one patient presented a distance recurrence. No severe long-term impairment of pelvis viscera functions related to autonomic nerve damage was observed by clinical examination.Four-year relapse-free and overall survival probabilities were $94 \%$ resp. $85 \%$.Any patient received adjuvant treatment. Conclusions:Based on these preliminary results TMRR achieves a superior therapeutic index in the surgical treatment of cervical cancer.We therefore this TMRR is feasible in locally advanced cervical cancer patients and promisory alternative for development countries without resources of radiotherapy equipment.

\section{P274}

Presacral Tumors: A Single Institution Experience J.L. Poggio, ${ }^{1 *}$ D.M. Schaffzin, ${ }^{1}$ R. Akbari, ${ }^{1}$ J. Casas-Ganem, ${ }^{2}$ R.D. Landmann, ${ }^{2}$ J.D. Guillem, ${ }^{1}$ P.B. Paty, ${ }^{1}$ J.H. Healey, ${ }^{2}$ P.J. Boland, ${ }^{2}$ W.D. Wong. ${ }^{1}$ 1. Department of Surgery, Section of Colon and Rectal Surgery, Memorial Sloan-Kettering Cancer Center, New York, NY; 2. Department of Surgery, Section of Orthopedic Service, Memorial Sloan-Kettering Cancer Center, New York, NY.

INTRODUCTION: Presacral tumors are a rare and diverse group of diseases that comprise the potential presacral space. Because of their relative rarity, confusion exists about their surgical treatment and outcome. We reviewed the surgical management and outcome of patients treated for these tumors at our institution. METHODS: Between January 1, 1992 and October 30, 2005, multiple databases were reviewed from the tumor registry and service databases. Retrospective chart data was collected pertaining to patient, operative, and outcome factors. RESULTS: Between January 1, 1992 and October 30, 2005 , a total of 112 patients were identified who met entry criteria for this study. A total of 58 were male. Mean age was 45.3 years. A total of 32 different diagnoses were identified. The most frequent diagnoses were Chordoma $(\mathrm{n}=30)$, Retrorectal Cysts (Tailgut cysts, Teratomas, Epidermoids Cysts, $\mathrm{n}=18$ ), Neurogenic Tumors (Schwannoma, Ependymoma, Neuroblastoma, $\mathrm{n}=10$ ), Giant Cell Tumors $(n=9)$, and Chondrosarcoma $(n=8)$. Forty five $(76.2 \%)$ of these patients required combined multidisciplinary procedures. Sixty one $(59.2 \%)$ underwent sacrectomy. For those with malignant tumors, actuarial 5yr survival was $94 \%$ following R0 resection $(\mathrm{n}=51)$ compared with $65 \%$ following incomplete $(\mathrm{R} 1 / \mathrm{R} 2)$ resection $(\mathrm{n}=21)$. Survival was significantly worse in the cohort with incomplete gross resection $(p<0.01)$. Functional disturbances including ambulatory $(\mathrm{p}<0.05)$ and genitourinary dysfunction $(\mathrm{p}<0.05)$ occurred in patients undergoing sacrectomy above the S3 level. Eleven $(18 \%)$ of the 61 patients who underwent sacrectomy had a concomitant proctectomy and colostomy. Fifteen $(30 \%)$ of the patients who had the rectum preserved required a bowel regimen for evacuation, and three $(6 \%)$ had frank incontinence. Rectal resection also predicted urinary dysfunction postoperatively $(p<0.05)$. CONCLUSION: Presacral tumors are rare but surgically challenging entities. Complete resection is the goal and is associated with significant survival advantage. Functional consequences ensue if sacrectomy above S3 is required. A multidisciplinary approach is warranted for select patients requiring complex surgical resection of these unusual tumors.

\section{P275}

Patterns of Recurrence After Conservative Treatment of Squamous Cell Carcinoma of the Anus P. Luna, ${ }^{*}$ M.L. Ramirez-Ramirez, S.E. Rodriguez, A. Cravioto, H. Martinez, S. Labastida. Surgical Oncology, Instituto Mexicano del Seguro Social, México D.F., Distrito Federal, Mexico.

Background. Squamous cell carcinoma of the anus (SCCA) is a rare tumor. Few information about the recurrence pattern does exist. Objective. To evaluate the treatment results and recurrence patterns with conservative treatment. Material and Methods. Between 1975 and 2005, 176 patients with diagnosis of SCCA were treated. Pretreatment evaluation included: physical examination, anoscopy, chest X-ray and CT scan of abdomen and pelvis. Patients were staged according the AJCC-UICC classification. Treatment consisted in radiotherapy (RT) to the pelvis at doses of $45 \mathrm{~Gy}$ in 20 fractions +20 Gy boost to the perineum. Other patients received chemotherapy (CRT) $(5-\mathrm{FU} 1,000 \mathrm{mg} / \mathrm{m} 2$ continuous infusion, days $1-5$ and $28-32+$ Mitomycin-C $10 \mathrm{mg} / \mathrm{m} 2$ days $1-28$ of RT). Response was evaluated by biopsies 8-12 weeks after RT or RT-CRT. Results. There were 112 females and 64 males. Mean age was 60.8 years. RT was administered to 96 patients and C-RT to 77 . Initial tumor stage in the RT group were: I $(n=6)$, II $(n=53)$ and III $(n=37)$ and in the C-RT group were: I $(\mathrm{n}=4)$, (II $(\mathrm{n}=42)$ and III $(\mathrm{n}=31)$. Overall local control in RT and C-RT groups were: $59.8 \%$ and $71.8 \%$, respectively $(p=0.06)$. Inguinal and distant failures occurred in $6.2 \%$ and $19.5 \%$ of patients treated with RT, whereas in $0 \%$ and $7.7 \%$ of those treated with CRT $(\mathrm{p}=0.02)$. Five-year disease-free survival of patients treated with RT was $47 \%$, whereas in those treated with CRT was $67 \%$ $(\mathrm{p}=0.02)$. Five-year disease-free survival of patients with stages I, II, IIIA and IIIB treated with RT was $90 \%, 59 \%, 32.5 \%$ and $22 \%$, whereas in those treated with CRT was $100 \%, 88 \%$ and $52 \%$ and $69 \%$.Significant differences between stages II and IIIB ( $p=0.02)$, but not in stages I and IIIA. Risk factors for local, inguinal and distant recurrence were: tumor stage $(\mathrm{p}=0.0004)$ and male gen$\operatorname{der}(p=0.002)$ and administration of RT $(p=0.002)$. Colostomy free survival in those treated with RT and C-RT was as follows: stage I-II and III, $54 \%$ and $35 \%$, whereas $84 \%$ and $69 \%(\mathrm{p}=0.03)$, respectively. Conclusion. Chemo-radiotherapy in the treatment of SCCA is associated with better local, inguinal, distant and colostomy free survival than those treated with RT.

\section{P276}

Salvage Radical Surgical Treatment Procedure for Persistent or Recurrent Squamous Cell Carcinoma of the Anal Canal P. Luna, M.L. Ramírez Ramírez, S. Rodríguez, H. Martínez, A. Cravioto, S. Labastida. Surgical Oncology, Instituto Mexicano del Seguro Social, México D.F., Distrito Federal, Mexico.

Background. Radiotherapy (RT) or chemoradiotherapy (CRT) are the standard treatment for squamous cell carcinoma of the anus (SCCA). Local or inguinal failures are frequently isolated and amenable to salvage radical surgery. However, the results are disappointing. Purpose. To evaluate the results of salvage radical surgery after conservative treatment for SCCAC. Materials and Methods. Between 1975 and 2005, 176 patients with SCCA were treated; 43 developed persistent or recurrent local or inguinal disease. Thirty one were treated with RT at mean doses of $5700 \mathrm{cGy}$ and 12 received CRT based on 5$\mathrm{FU}+$ Mitomycin-C. Local or groin failure were classified according to the disease-free interval in: 1$)$ persistent disease $(n=31)$ when was diagnosed within the 6 months of RT or CRT and, 2) recurrent disease $(n=12)$ when recurrence was documented $>6$ months. Multivariate analysis was performed to find risk factors associated with local recurrence and survival. Results. There were 30 females and 13 males with a mean age of 60 years. Primary tumor stages were: II $(n=21)$ and III $(n=22)$. Patients with persistent disease underwent abdominoperineal resection (APR) $(n=20)$, pelvic exenteration $(P E)(n=4)$ and groin dissection $(\mathrm{GD})(\mathrm{n}=4), \mathrm{APR}+\mathrm{GD}(\mathrm{n}=3)$. Patients with recurrent disease underwent APR $(n=8), P E(n=3)$ and APR + GD $(n=1)$. Postoperative complications was observed in 19 patients (44\%), no operative mortality occurred. After a median follow-up of 52 months local recurrence was observed $16 \%$ of patients with persistent disease, whereas in $42 \%$ with recurrent disease $(\mathrm{p}=0.02)$. Inguinal and distant recurrence was observed in three patients each $(9 \%)$ with persistent disease, whereas in three $(25 \%)$ and four patients $33 \%)$ with recurrent disease $(\mathrm{p}=0.02)$. Five-year survival in patients with persistent disease was $75 \%$, whereas $14 \%$ in those with recurrent disease $(\mathrm{p}=0.00001)$. Risk factors associated with local recurrence and five-year survival were: primary tumor stage and recurrent disease $(\mathrm{p}=0.04$ and 0.001$)$. Conclusion. Salvage radical 
surgery is an effective treatment for patients with persistent disease, for those with recurrent disease remains controversial.

\section{P277}

Treatment of Malignant Peritoneal Mesothelioma: A Single Institution Experience T.J. Kennedy, ${ }^{1}$ D. Labow, ${ }^{2}$ M.R. Weiser, ${ }^{1}$ P. Paty, ${ }^{1}$

D. Coit. ${ }^{1}$ 1. Surgical Oncology, Memorial Sloan Kettering Cancer Center, New York, NY; 2. Mount Sinai Medical Cenetr, New York, NY.

Introduction: Peritoneal mesothelioma is a rare malignancy characterized by peritoneal progression and poor clinical outcome. There is currently no uniformly accepted treatment modality for malignant peritoneal mesothelioma patients. The aim of this study was to review a contemporary single institution experience employing a more conservative approach for patients with this disease. Methods: We identified 30 patients treated for peritoneal mesothelioma from 1989 to 2000. Patient, tumor, and treatment variables were recorded. Overall survival was estimated by the Kaplan-Meier method. Factors associated with improved outcome were identified by logrank test. Results: There were 20 females and 10 males with mean age of 54.3 years. Four patients had history of asbestos exposure and 12 patients had history of tobacco use. The most common presenting symptoms were abdominal pain, ascites, weight loss and distension. Overall, $70 \%$ of patients underwent $>75 \%$ tumor debulking but less than $10 \%$ of patients underwent multiple organ resections or extensive peritonectomy. The overall morbidity rate was $13 \%$ with no patient requiring a reoperation; operative mortality rate was $3 \%$. Only $56 \%$ of patients received postoperative intraperitoneal chemotherapy with cisplatin and mitomycin C. Median follow up for all patients was 56.7 months. Median survival was 73.6 months with an overall projected 5 year survival greater than $50 \%$. Among patients alive at last followup, 8 were NED, and 7 were AWD. The most significant predictors of survival were female sex $(\mathrm{P}=0.04)$, age at diagnosis of $\angle 60$ years $(\mathrm{P}=0.005)$, treatment with postoperative intraperitoneal chemotherapy $(\mathrm{P}=0.03)$, tumor debulking $>75 \%(\mathrm{P}=0.02)$ and absence of metastatic disease $(\mathrm{P}=0.0006)$. Conclusion: Although peritoneal recurrence in mesothelioma is common, prolonged overall survival is possible. Less aggressive treatment regimens with tumor debulking and intraperitoneal chemotherapy are associated with acceptable survival with limited morbidity and mortality. Favorable outcome is associated with female sex, age $<60$ years, lack of metastatic disease at presentation, $>75 \%$ tumor debulking and treatment with intraperitoneal chemotherapy.

\section{Survival Curve}

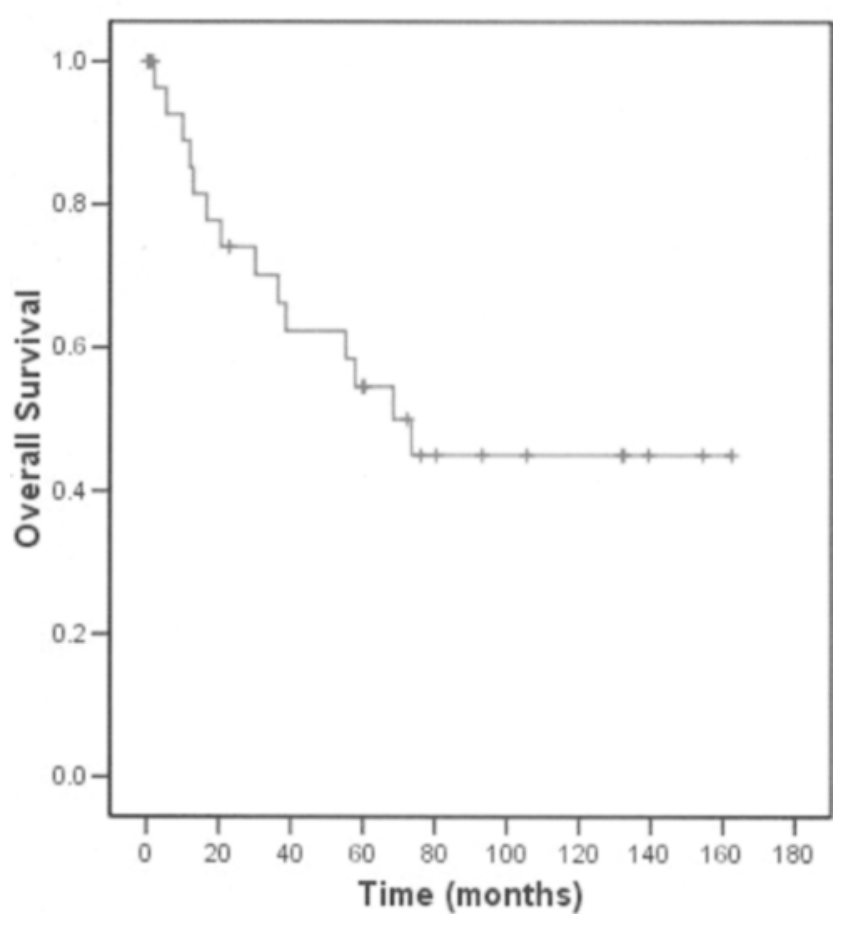

\section{P278}

Splenectomy Ameliorates Hematologic Toxicity of Intraperitoneal Hyperthermic Chemotherapy V. Soo, ${ }^{*}$ T. Bradley, P. Shen, J.H. Stewart, G.B. Russell, E.A. Levine. Surgical Oncology, Wake Forest University, Winston-Salem, NC.

Background: Peritoneal carcinomatosis (PC) is a terminal disease which has recently been treated with cytoreductive surgery and intraperitoneal hyperthermic chemotherapy (IPHC). This approach has been associated with prolonged survival and quality of life in select patients. To date, there has been no study investigating the effect of splenectomy on hematotoxicity in patients undergoing IPHC. Methods: Between December 2003 and March 2006, 100 patients with peritoneal carcinomatosis underwent first IPHC at our institution. All patients underwent cytoreduction to resect all gross tumor followed by IPHC using Mitomycin with a standard protocol. This included splenectomy when there was gross tumor involvement of the organ. The chemotherapy was delivered at $40^{\circ} \mathrm{C}$ for $120 \mathrm{~min}$. Postoperatively, patients were treated with Neupogen when their white blood cell (WBC) counts were $<4$. Hematologic adverse events were graded using the common toxicity criteria scale from $0-5$, with 5 being most severe. Results: There were 51 patients who underwent splenectomy (51\%). Cytopenia contributed to death in splenectomy 4 patients $(7.8 \%)$ and 1 patient who did not undergo splenectomy $(2.3 \%)$. In splenectomy patients the average WBC nadir was 5.6, the average absolute neutrophil count was 11.9 , the average platelet nadir was 179.5 , and the average hemoglobin (Hb) nadir was 8.4. In patients who didn't undergo splenectomy, the average WBC nadir was 4.2 , the average absolute neutrophil count was 9.2 , the average platelet nadir was 168.3 , and the average $\mathrm{Hb}$ nadir was 7.6. Neupogen was administered in $49 \%$ patients who did not receive a splenectomy, versus $27 \%$ of patients who did receive a splenectomy ( $p$-value $<0.025$ ). The difference in blood transfusions between the two populations was not statistically significant. Conclusions: Splenectomy appears to ameliorate the hematologic toxicity in patients with peritoneal carcinomatosis undergoing IPHC. Splenectomy significantly reduces the number of patients who require post-operative growth factor support. This suggests that while splenectomy may be associated with morbidity, it ameliorates neutropenia following IPHC.

\section{P279}

Reconstruction Following Total Scapulectomy: Humeral Suspension versus Scapular endoprosthesis T. Pritsch, ${ }^{1 *}$ J. Bickels, ${ }^{2}$ D. Brooks, ${ }^{1}$ T. Winberg, ${ }_{1}^{1}$ M.M. Malawer. ${ }^{1}$ 1. Orthopedic Onclology, Washington Hospital Center, Washington, DC; 2. National Unit of Orthopedic Oncology, Tel-Aviv Sourasky Medical Center, Tel-Aviv, Israel.

Humeral suspension was the most popular reconstructive modality following total scapulectomy until the beginning of the 1990's. The need for better functional and cosmetic results has subsequently led to the development of the scapular endoprosthesis; however, controversies regarding its functional superiority exist. The purpose of this study was to compare the clinical outcomes of scapular endoprosthetic reconstruction and humeral suspension. We analyzed the medical records of 31 consecutive patients who underwent total scapulectomy between 1979-2003. Reconstruction included humeral suspension and scapular endoprosthesis in 16 and 15 patients respectively. Endoprosthetic reconstruction was not available before 1992 and was performed only if the rhomboids, latissimus dorsi, deltoid, and trapezius muscles were preserved. Functional outcomes were assessed using the American Musculoskeletal Tumor Society System (MSTS). Functional outcomes and range of motion following scapular endoprosthetic reconstruction were significantly superior to humeral suspension. The MSTS scores of the scapular endoprosthesis group were Good-to-Excellent in 10 patients, fair in 4 and poor in 1 patient. Seven patients were able to abduct their shoulders more than 40 degrees and two had less than 20 degrees of active abduction. The MSTS scores of the humeral suspension group were Good-to-Excellent in two patients, fair in seven and poor in seven. Eleven patients could not actively abduct their shoulders more than 20 degrees, and none had more than 40 degrees of shoulder abduction. Patients with scapular endoprosthesis had superior cosmetic results, and unlike the majority of the humeral suspension patients, did not wear shoulder pads or customized orthoses for cosmetic purposes. Scapular endoprosthetic reconstruction has led to better functional and cosmetic results as compared to humeral suspension. We consequently recommend performing scapular endo- 
prosthetic reconstruction following total scapulaectomy whenever the rhomboids, latissimus dorsi, deltoid, and trapezius muscles are preserved

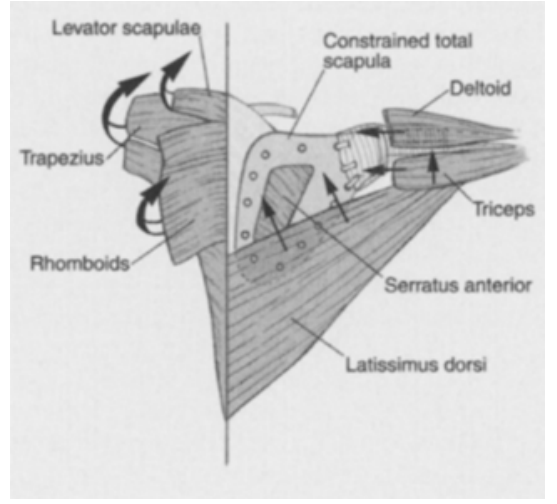

\section{P280}

Long term effects of imatinib therapy and impact of surgery in patients (pts) with $\mathrm{CD117}(+)$ gastrointestinal stromal tumors (GIST)without early progression on imatinib P. Rutkowski, ${ }^{1 *}$ Z. Nowecki, ${ }^{1}$ U. Grzesiakowska, ${ }^{1}$ W. Michej, ${ }^{1}$ M. Debiec-Rychter, ${ }^{2}$ A. Wozniak, ${ }^{3}$ P. Nyckowski, ${ }^{4}$ C. Osuch, ${ }^{5}$ M. Foszczynska, ${ }^{6}$ W. Ruka. ${ }^{1}$ 1. Soft Tissue/Bone Sarcoma and Melanoma, Cancer Center Warsaw, Warsaw, Poland; 2. Department of Human Genetics, University of Leuven, Leuven, Belgium; 3. Medical University of Gdansk, Gdansk, Poland; 4. Medical University of Warsaw, Warsaw, Poland; 5. Iagiellonian University, Cracow, Poland; 6. Regional Oncological Center Szczecin, Szczecin, Poland.

The aim of the study was to analyze the outcomes of treatment of inoperable/metastatic GIST CD117(+) pts, who demonstrated initially at least stabilization of disease during imatinib therapy (IM). Methods: We identified 196 pts in GIST Registry with advanced GIST treated with IM in the dose of 400$800 \mathrm{mg}$ [09/2001-07/2006] without early $(<4$ months) progression on IM. Median follow-up time was 25 months (range:5-59). Results: The estimated 3 -year overall and progression-free survival (OS,PFS; calculated from the date of start of IM) were $78 \%$ and $61 \%$, respectively. Median OS was not reached and estimated median PFS was 46 months. Overall 33 pts (16.8\%) died (due to progressive disease-PD). The disease progression was seen in $66 \mathrm{pts}(33.7 \%)$ with median time of 17.8 months (late resistance). Radiological complete remissions (CR) during IM were rare ( 15 cases; $7.7 \%$ ). Exon 11 KIT mutations were detected in 46 of 64 cases $(71.8 \%)$ with known mutational status of tumors. Overall 39 pts $(19.9 \%)$ underwent surgery of residual disease (35 R0/R1 resections) followed by further IM and in this group we observed significantly longer OS (3-yr: $100 \%)$ and DFS (3-yr: $95 \%)$ and only 4 PD (10\%). The negative predictive factors for PFS in the entire group of pts according to univariate analysis were $(p<0.05)$ : baseline high neutrocyte count, baseline poor WHO performance status $>1$, baseline low albumin level, mutations other than exon 11 , primary overtly malignant disease, primary tumor size $>10 \mathrm{~cm}$, mitotic index $>10 / 50 \mathrm{HPF}$, baseline low hemoglobin level and lack of surgical treatment. We identified 4 factors negatively affecting PFS significant in multivariate analysis $(p<0.05)$ : baseline poor WHO performance status $>1$, baseline low hemoglobin level, lack of surgical treatment and mitotic index $>10 / 50 \mathrm{HPF}$. Conclusions: We confirmed that many advanced GIST pts, who did not demonstrate early resistance, benefit from imatinib therapy for a prolonged time, while secondary progressions are observed. The selected group of pts, who had undergone resection of residual disease during imatinib treatment, demonstrated excellent outcomes.

\section{P281}

Sacral Tumous: A Challenge in Management K. Narayanan,* H.S. Bhatoe, S. Gupta, P. Singh, S. Kapoor, G. Rajagopal. Surgical Oncology, Army Hospital (R\&R), New Delhi, India.

Tumours of the sacrum are rare and varied. The anatomical location of the bone, at the pivot of the pelvic skeleton playing a significant role in stability and weight transmission, vascularity, proximity to important structures and neural issues makes the resection of the sacrum a challenge. We present a single institution experience of 12 sacral lesions in adults treated over the past 5 years. The presentation of the lesions were either as masses, back pain or neural compressive features with lower limb and cauda eqina syndrome. Preoperative pathology was established by fine needle aspiration or core biopsy. The diagnosis encountered include plasmacytoma, ependymoma, chordoma, giant cell tumour and schwannoma. All patients underwent CECT and contrast enhanced MRI of the pelvis and 3-D reconstruction of the images to plan the extent of resection and reconstruction. Highly vaslar lesions were embolized preoperatively. Lesions not involving the proximal 2 pieces of the sacrum were treated by excision alone. Five patients needed spinopelvic stabilization with TSRS sextant pedicular screw system for restoring spinopelvic continuity. Surgery needed 2 sittings in 2 and recurrences occurred in two patients which was treated by re-excision. There was no mortality in hospital. Average blood loss was $1800 \mathrm{ml}$ in the embolozed group and $2500 \mathrm{ml}$ in the non embolized group. Implant extrusion and skin breakdown needing a second reconstructive procedure in two patients. Of the 4 who presented with neurological deficits all but one showed significant improvement. Tenpatients needed post operative adjuvant radiotherapy. Multidisciplinary planning of resection technique, embolization before surgery, planning the reconstruction of the joint and skin cover and preservation of neural structures are essential to successful outcome. Three dimensional reconstruction of the pelvis and tumour was extremely helpful in planning adequacy of exposure and resection. The authors share their experience in managing the rare group of tumours and review experience of others.

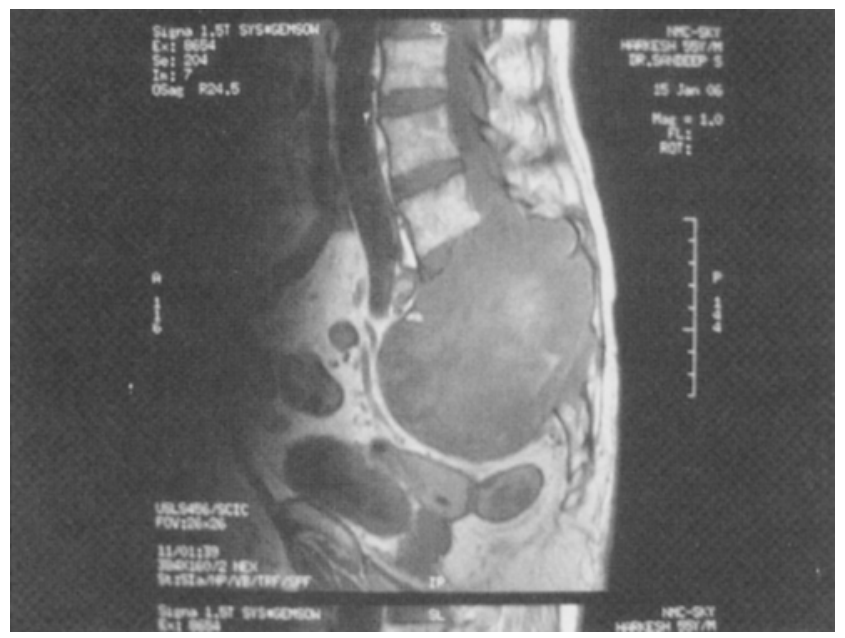

\section{P282}

Dermatofibrosarcoma Protuberans: Surgical Modality, Margin Status, and Outcome A. Meguerditchian, ${ }^{1 *}$ B. Lema, ${ }^{2}$ S. Lopian, ${ }^{3}$ J. Wang, ${ }^{2}$ W.G. Kraybill, ${ }^{1}$ N. Zeitouni, ${ }^{1}$ J.M. Kane III. ${ }^{1}$ 1. Surgical Oncology, Roswell Park Cancer Institution, Buffalo, NY; 2. State University of New York - Buffalo, Buffalo, NY; 3. Rochester Institute of Technology, Rochester, NY.

INTRODUCTION: Dermatofibrosarcoma protuberans (DFSP) is a slow growing spindle cell tumor with a high local recurrence rate but low metastatic potential. Although wide local excision (WLE) is the potentially curative therapy, ideal margin width is not standardized and Mohs' microsurgery (MMS) has emerged as an alternative. The purpose of this study is to assess the relative merits of WLE vs. MMS for the treatment of DFSP. METHODS: Retrospective review of all patients treated for DFSP between 1971-2006 at a single institution. Patient demographics, tumor features, surgical modality (WLE 
vs. MMS), final pathology, and clinical outcome were evaluated. RESULTS: Forty-eight patients treated for DFSP were identified. Median age was 40 years (range 12-74), 87.5\% were Caucasian, and $56.5 \%$ were female. Forty-five cases were primary and 3 were recurrent. Twenty-eight patients were treated by WLE: 13 extremity, 10 trunk, and 5 head and neck lesions. Twenty patients were treated by MMS: 5 extremity, 9 trunk and 6 head and neck lesions. In the WLE group, median tumor size was $8.4 \mathrm{~cm}$ (range $0.5-17.0$ ) and median margin width was $2 \mathrm{~cm}$ (range $1-3$ ). In the MMS group, median tumor size was $7.5 \mathrm{~cm}$ (range $4-14$ ) and the median number of margin excisions required for a negative margin (starting with $1 \mathrm{~cm}$ ) was 2 (range $1-4)$. Median OR time was 77 minutes for WLE (range 40 - 270) and 232.5 minutes for MMS (range 82 - 655). Complex closure techniques were required $21.4 \%$ for WLE and $65 \%$ for MMS. Positive resection margins were present in 25\% (7/28) of WLE and $0 \%$ of MMS. At a median follow-up of 63 months for WLE (range $1.5-494.3$ ) and 50 months for MMS (range $0.6-147$ ), the local recurrence rate was $3.6 \%$ $(1 / 28)$ for WLE (a previously recurrent tumor) and $0 \%$ for MMS. No deaths were attributable to DFSP. CONCLUSION: Despite a higher rate of positive margins with WLE, MMS and WLE appear to be equivalent in regard to local control for DFSP. MMS is resource intensive and may produce a more complex defect. However, it may be beneficial for recurrent DFSP or in cosmetically/anatomically challenging areas.

\section{P283}

Evaluation of different absorbable matrix compounds to harbour autologous fibroblasts for closure of soft tissue defects after sarcoma resection P. Hohenberger, ${ }^{1 *} \mathrm{C}$. Syring, ${ }^{2} \mathrm{M}$. Seifert. ${ }^{3} 1$. Division of Surgical Oncology and Thoracic Surgery, Faculty of Medicine, Mannheim, Germany; 2. Department of Biotechnology, German Institute of Cell and Tissue Replacement (DIZG), Berlin, Germany; 3. Institute of Medical Immunology Charite, Berlin, Germany.

Problem: Following sarcoma resection combined with irradiation wound healing problems often occur and reauire pedicled of free flaps. If flaps are not available or fail, tissue engineering could provide a valuable option for volume reconstitution. Since degradation of many materials is a constraint for the use of engineered constructs, we aimed at developing a carrier with a predictable outcome. Methods: The suitability of different carriers was assessed with human dermal fibroblasts, since they represent a major cell type of soft tissue. Proliferation was analysed on acellular dermis, collagen gels, polyglactic pads, multilayer polypropylene nets and cell culture plasticware using fibroblasts obtained from different donors. Fibroblasts were incubated in collagenase, filtered, centrifugated, seeded, and later populated (density 15,700 cells $/ \mathrm{cm} 2$ ). The intracellular activity of LDH was used to measure the number of viable cells attached to the carrier and analysis of its stability was performed by resistance to collagenase digestion. Statistics : $t$-test, $p<0.05$ significant. Results: The highest $\mathrm{LDH}$ at $2.9 \pm 0.3 / \mathrm{cm} 2$ was measured in fibroblasts cultivated on acellular dermis as cultures exhibited a significantly higher cell number than other carriers. Coloured pads released toxic side products. The resistance of the carrier to digestion was analysed by volume measurements after being populated over different periods of time. Acellular dermis was not significantly contracted displaying a volume retention of $97.1 \pm 0.6 \%$. Histological assessment revealed no structural changes of the dermal matrix. Collagen gels showed another picture beeing contracted to $76.9 \pm 3.7 \%$ of its original volume already after $24 \mathrm{~h}$ incubation and to $65.4 \pm 6.3 \%$ at day 4 , whereas all other carriers showed no stability. Conclusion: Human allogeneic acellular dermis turned out to be a safe biological carrier for soft tissue engineering. Its high stability, resistance to contraction and enzymatic digestion opens new opportunities to fill-up soft tissue defects after sarcoma treatment in combination with patient autologous fibroblasts.

\section{P284}

Surgical Resection for Retroperitoneal Sarcomas: Analysis of Factors Determining Outcome J.R. Ouellette, * F.F. Amersi, R. Alban, J. Altura, N.P. McAndrew, A.W. Silberman. Surgical Oncology, Cedar Sinai Medical Center - Samuel Oschin Comprehensive Cancer Institute, Los Angeles, $C A$.

Background. Patients with intermediate or high-grade retroperitoneal sarcomas (RS) have poor outcomes with high local recurrence rates. Aggressive surgical resection provides the most effective therapy. We performed a retrospective analysis of aggressive surgical resection of patients with RS to eval- uate long-term outcomes. Methods: Between 1986 and 2005, retroperitoneal surgical resections were performed on 73 pts, of which 48 pts with RS were identified. Of these pts, $58.3 \%$ had surgical resection for the primary tumor and $41.7 \%$ were for recurrences. Cox regression univariate analysis considered the following variables: gender, tumor size, tumor location, histology, grade, vascular involvement, margins, and adjuvant therapy. Records of all patients were reviewed with particular attention to local recurrence, overal and disease-free survival. All resections were performed by the same surgeon. Results: Of the 48 pts, $51 \%$ were male, $49 \%$ were female, with a mean age of 62 (range 20-85). The most common pathology was either liposarcoma $(44.2 \%)$ or leiomyosarcoma $(23.3 \%)$. Mean tumor size was $16.9 \mathrm{~cm}$, with $83.8 \%$ being either intermediate or high-grade. In-continuity organ resections were performed in $88.4 \%$, with $72.1 \%$ requiring multi-organ resections. Colon was the most common solid organ resected $(67.4 \%)$, followed by kidney $(44.2 \%)$, and small bowel (18.6\%). Complete gross resection (R0 or R1) was accomplished in $79.1 \%$ of pts. Seven patients required multiple resections for recurrences. Overall 1,3 , and 5 yr survival was $96 \%, 85 \%$ and $38 \%$, respectively, for patients with resections of the primary tumor; however, overall survival was lower for patients who underwent resections for recurrent disease, with 1,3 , and 5 yr survival rates of $93 \%, 38 \%$, and $19 \%$, respectively (Table). Tumor size and grade were found to be significant predictors of overall survival. Conclusion: Aggressive multi-organ surgical resection is safe and effective at improving long-term survival, in patients with primary RS. Some pts with recurrent RS can also have improved long-term survival with surgical resection.

\begin{tabular}{|c|c|c|c|c|}
\hline & Contiguous Organ Resection(\%) & \# of Pts & \# Complete Resection (\%) & Survival (\%) \\
\hline Primary RS & 81.5 & 28 & 74.1 & 38 \\
\hline RecurrentRS & 100 & 20 & 35.3 & 19 \\
\hline
\end{tabular}

\section{P285}

Percutaneous Cryoablation of Lung Tumors in Nonsurgical Candidates-Initial Results A.A. Mavanur, ${ }^{*}$ P.D. Allmendinger, ${ }^{2}$ J.E. Foster, ${ }^{2}$ R. Lowe. ${ }^{2}$ 1. University of Connecticut, Farmington, CT; 2. Hartford Hospital, Hartford, CT.

INTRODUCTION: There is a paucity of data in the literature regarding cryoablation of lung tumors in patients who are poor surgical candidates. The objective of our study was to determine the feasibility and safety of percutaneous cryoablation of lung tumors in this group of patients and also determine the tumor response to this modality of treatment. METHODS: A retrospective chart review of patients who had undergone a CT guided percutaneous cryoablation of lung tumor was performed. Patients were identified from an interventional radiology database. RESULTS: 36 patients underwent 42 procedures from May 2004 to June 2006. 20 were female with a mean age of 58.6 years. 16 patients had primary tumors $(44 \%), 8$ had recurrent tumors after previous surgery (23\%) and 12 had metastatic disease (33\%). The most common reason for nonoperability was poor lung function or nonresectable tumor. Size of the tumors varied from $0.9 \mathrm{cms}$ to $5 \mathrm{cms}$ with an average of $2.8 \mathrm{cms}$ The patient with the $0.9 \mathrm{cms}$ mass refused surgery. The procedure was successfully completed in all patients $(\mathrm{N}=42) .35(83 \%)$ had pleuritic chest pain and $21(50 \%)$ had a dry cough post procedure. Pneumothorax was identified in 24 instances $(57 \%$ ). In 14 of these a pigtail catheter was placed in the interventional radiology suite immediate post procedure. The remaining 10 were observed and half of these needed a delayed chest tube. One patient with a superficial tumor developed a broncho-pleural fistula after cryoablation. Hemoptysis was observed in $4(9.5 \%)$ instances. There was no procedure related mortality. A follow up PET CT is available in 26 patients with a mean follow up of 10 months (Range 4-24 months). 5 had recurrence of tumor activity, 6 had minimal activity at tumor site possibly due to post-treatment inflammation. The remainder (15) had no activity in the lung fields on PET scan. CONCLUSIONS: We have demonstrated that percutaneous cryoablation of lung tumors is a viable option for control of lung tumors in patients who are not surgical candidates. This procedure can be performed safely with no mortality and minimal morbidity. 


\section{P286}

Comparison of Transhiatal and Transthoracic esophagectomy for carcinomas of the gastroesophageal junction J.D. Amortegui, * J.L. Bell, T.E. Gaines. University of Tennessee, Knoxville, TN.

Introduction: Whether esophagectomy for malignancy should be performed via transthoracic (TT) or transhiatal (TH) approach remains unknown, to add more confusion to this topic, some groups have recommended an abdominal approach for lesions in the gastric cardia. The objective of this study is to compare the TT and TH approaches for tumors limited to the gastroesophageal junction (GEJ). Methods: 10-year retrospective review of TT and TH esophagectomies for carcinomas of the distal esophagus, GEJ and gastric cardia. Analysis was by intention to treat. Results: There were $24 \mathrm{TT}$ and $22 \mathrm{TH}$ esophagectomies. There were no significant differences in age, gender, use of alcohol or tobacco, location of the tumor, pathologic type, grade, stage, use of chemotherapy and radiation therapy, ASA class, preoperative albumin or associated comorbidities between groups. The TT group had a significant longer operative time (430 $\mathrm{min}$ vs $328 \mathrm{~min}, \mathrm{p}=0.002$ ), fewer days free of ventilatory support at 10 days $(8.2$ vs $9.8 p=0.04)$ and at 20 days $(17.1$ vs $19.8 p=0.05)$, less days out of the ICU at 20 days $(13.9$ vs $16.7 \mathrm{p}=0.05)$. The TT group also required more transfusions $(2.9 \pm 3.2$ vs $0.6 \pm 1$ units of $P R B C, p=0.003)$. There were no significant differences in the total number of postoperative complications per group (TT $1.8 \pm 2.1$ vs TH $1.1 \pm 1.3 \mathrm{p}=0.1$ ), time to tolerate clear diet by mouth (TT $8.9 \pm 4$ vs TH $9.1 \pm 4.1 \mathrm{p}=0.9$ ), mortality at 30 days (TT 0 and TH 0 ), hospital mortality (TT $1 / 24$ vs TH $0 / 22 \mathrm{p}=0.5$ ) or total length of stay (TT $18.1 \pm$ 11.6 vs TH $14.7 \pm 6.9 \mathrm{p}=0.2$ ). The 5 -year survival was similar $56.6 \% \mathrm{TH}$ and $33.2 \%$ TT (Log Rank 0.45 ). Conclusion: Although the morbidity, mortality and 5 -year survival is similar for TT and TH esophagectomies, the latter is associated with less need for ventilatory support, ICU stay and transfusions. We recommend the TH approach for tumor of the GEJ when feasible.

\section{P287}

Should Pneumonectomy be Avoided in Patients with Locally Advanced Non-Small Cell Lung Cancer Following Induction Chemotherapy? F.M. Shamji, ${ }^{1}$ T.A. D'Amato, ${ }^{2 *}$ A.S. Ashrafi ${ }^{3}$ D.E. Maziak, ${ }^{1}$ S.R. Sundaresan, ${ }^{1}$ D. AL-Shehlb, ${ }^{1}$ A. El-Sherif, ${ }^{4}$ P.F. Ferson, ${ }^{4}$ J.D. Luketich, ${ }^{4}$ R.J. Landreneau. ${ }^{4}$ 1. Ottawa Civic Hospital, Ottawa, ON, Canada; 2. Department of Surgery, Jefferson Medical College, Philadelphia, PA; 3. Niagara Health System, St. Catharines, ON, Canada; 4. University of Pittsburgh Medical Center, Pittsburgh, PA.

Background: Pneumonectomy is a high-risk procedure. Recent data from the S9900 trial implied an un-acceptable early mortality with pneumonectomy following neo-adjuvant chemotherapy thereby raising skepticism for this surgical option for patients with regionally advanced non-small cell lung cancer (NSCLC). Complications related to pneumonectomy and pre-operative chemotherapy remain important issues in the management of these patients. Methods: A retrospective review of 207 resected NSCLC patients undergoing pneumonectomy over a 15 -year period was undertaken. Patients were matched for gender, laterality, pulmonary function, and co-morbidities other than neo-adjuvant chemotherapy. Complications defined as respiratory failure; pneumonia, empyema and bronchopleural fistula (BPF), adverse cardiac events and $30 \mathrm{~d}$ mortality, were analyzed for associations with laterality neo-adjuvant chemotherapy. Results: Of 207 patients, 57 received induction chemotherapy and 151 surgery alone. Age was moderately predictive of early death $[\mathrm{HR}=1.24$ for age $>70 \mathrm{y}, \mathrm{p}=0.059]$, and respiratory failure was highly predictive of $<30$ day mortality $[H R=148, p=0.0001]$. Overall $30 \mathrm{~d}$ mortality $(7.2 \%)$ did not differ between induction chemotherapy and surgery alone. [see Table]. Conclusions: Morbidity and early mortality following pneu- monectomy after induction chemotherapy appears acceptable. Surgery for regionally advanced NSCLC should still be considered for patients following preoperative chemotherapy, and in contrast to the S9900 data; pneumonectomy should remain an option in future neo-adjuvant trials for stage IIIA disease. Caution with $\mathrm{R}$ pneumonectomy irrespective of induction therapy is recommended.

Outcome Following Pneumonectomy

\begin{tabular}{|c|c|c|c|c|c|c|}
\hline Oucome & Right & Left & Fischer's Exact & Chemotherapy & Surgery Only & Fischer's Exact \\
\hline N & 91 & 116 & $\mathrm{p}$ & 57 & 151 & $\mathrm{p}$ \\
\hline & \% & \% & & * & \% & \\
\hline Bronchpleural Fistula & 18 & 1.4 & 0.0018 & 10 & 5.8 & 0.42 \\
\hline Respiratory Failure & 23 & 6.9 & 0.019 & 18 & 12 & 0.52 \\
\hline Pneumonia & 20 & 9.7 & 0.25 & 10 & 14 & 0.76 \\
\hline Arryhmia & 26 & 32 & 0.41 & 29 & 30 & 0.53 \\
\hline 30 day Morality & 14.7 & 3.7 & 0.038 & 8.8 & 6.7 & 0.7 \\
\hline Median Survival a & $1.5 y$ & $2.0 \mathrm{y}$ & 0.91 & $1.9 \mathrm{y}$ & $1.2 y$ & 0.32 \\
\hline
\end{tabular}

\section{P288}

Partial penectomy for patients with squamous cell carcinoma of the penis: the Memorial Sloan-Kettering experience T. Koppie, ${ }^{*}$ R. Korets, M.E. Snyder, P. Russo. Memorial Sloan Kettering Cancer Center, New York, NY.

PURPOSE: To present our institution's experience with squamous cell carcinoma (SCC) of the penis, with analysis of oncologic efficacy and survival MATERIALS AND METHODS: Between 1989 and 2005, we identified 32 consecutive patients (median age 61) with SCC of the penis managed with partial penectomy. Clinicopathologic variables were examined, and actuarial and disease-specific survival were determined. RESULTS: Pathologic stage of the primary tumor was pTis in 1 patient $(3 \%)$, pT1 in $11(34 \%)$, pT2 in $16(50 \%)$, and pT3 in $4(13 \%)$. Pathologic grade was well differentiated in 9 patients $(28 \%)$, moderately differentiated in $20(63 \%)$, and poorly differentiated in 2 $(6 \%)$. Twenty-five patients ( $78 \%)$ underwent inguinal lymph node dissection, with $15(60 \%)$ demonstrating nodal metastases. Twenty-two patients $(69 \%)$ underwent pelvic lymph node dissection; 21 were negative for pelvic nodal metastases and one had grossly positive nodes. One patient developed local recurrence. After a mean follow-up of 34 months, actuarial survival was $56 \%$. Numbers of patients alive and disease-free were $9(75 \%)$ and $11(55 \%)$ in the low-stage and advanced-stage groups, and $8(89 \%)$ and $12(60 \%)$ in the well and moderately differentiated groups. Both patients with poorly differentiated disease died of disease within 12 months from presentation. CONCLUSIONS : Partial penectomy for SCC of the penis provides excellent local control, with low recurrence rate and acceptable maintenance of urinary and sexual function. Outcomes are generally poor, however, for patients with metastases, even in moderately differentiated disease. Future studies are needed to identify a reliable method of predicting regional metastases 